\title{
Handbook of Positive Psychology, Religion, and Spirituality
}

\section{Editors}

Edward B. Davis

School of Psychology, Counseling, and Family Therapy

Wheaton College

Wheaton, IL, USA

Everett L. Worthington, Jr.

Psychology Department

Virginia Commonwealth University

Richmond, VA, USA

Sarah A. Schnitker

Psychology and Neuroscience Department

Baylor University

Waco, TX, USA 
To my beloved family -Meghan, Graham, Madeleine, Hayes, and Rachel. Thanks for always supporting my "nerdy work" (aka scholarly research). I love y'all with all my heart and am deeply grateful for each of you. The greatest joy of my life is getting to love and be loved by you. Thank you for inspiring me daily to nurture a meaning ful spirituality and the virtues that emanate from it.

-E. B. D.

We all need people to open doors to admit us into new professional arenas. Rodney $K$. Goodyear (at that time Editor of the Journal of Counseling and Development) and Stanley $R$. Strong (one of my research and counseling mentors, who is now deceased) both invited me into mainstream psychology religious and spiritual research that went beyond Christian psychology. I'm so grateful to them and to my students who journeyed with me.

-E. L.W.

To my husband, Seth Robins, and the healthcare administrators and workers around the world who have drawn upon religious and spiritual resources to exhibit courage, compassion, perseverance, and patience during the global COVID-19 pandemic.

-S. A. S. 


\section{Foreword}

To date, two areas of behavioral science have informed one another too seldomlypositive psychology and the psychology of religion and spirituality. Each subfield has now accumulated decades of empirically grounded wisdom on the practices and processes by which people can best forge their better selves. In uniting these two subfields, this innovative Handbook of Positive Psychology, Religion, and Spirituality leverages the accumulated evidence from each subfield and builds the case for further synergies between them. Whether people seek to be more joyful or peaceful, or more centered or moral, these seekers will be better served by deeper, more frequent, and more substantive cross-fertilization between these two scientific domains.

This scholarly fusion is well-timed. In an era of rising threat and mushrooming uncertainty, increasing numbers of pandemic-weary people are struggling to nurture and maintain their mental well-being and hope. Growing social divisiveness has eroded civility worldwide, undermining many people's faith in humanity. Making matters worse, all manner of scandals and greed have surfaced within traditional religious institutions. Eroded public trust in these institutions thus has often made them unattractive sources of solace. Against this backdrop, increasing numbers of people who describe themselves as "spiritual but not religious" appear more likely to mix-and-match their own self-styled spiritual practices than to accept-or even explore - the longstanding prebundled practices that are offered by established religions.

Both personally and professionally, I find the fusion of positive psychology with the science of religion and spirituality to be generative. Having dedicated my career to establishing the science of positive emotions, I frequently use the insight that positive emotions both broaden and build to guide me toward personal spiritual practices that most effectively open my heart and mind and build my resilience and resolve. Seen through these lenses, nature hikes, meditation, 
and slow stretch yoga have become soul-satisfying "go-to" practices when I feel unmoored. By contrast, religious or spiritual practices that leave me feeling cold fail to sustain my attention.

In my professional life, seeds to integrate the science of religion and spirituality into my research program on positive psychology were first planted in 2010, when I accepted the invitation to become a Templeton Research Fellow at the Danielsen Institute at Boston University. My charge was to explore the intersection of religious and psychological well-being. The ensuing six lectures I delivered at Boston University became the framework for my 2013 book, Love 2.0 (Penguin, www.PositivityResonance.com), which in turn became a roadmap for my team's work in our Positive Emotions and Psychophysiology (PEP) Lab at the University of North Carolina at Chapel Hill (www.PositiveEmotions.org). More seeds were sown in 2012 when Patty Van Cappellen joined the PEP Lab, at first as a visiting doctoral student and eventually as a post-doctoral fellow and assistant research professor. She brought a deep scholarly understanding of religion and spirituality to the table, and since then, we together have investigated how religion and spirituality shape and are shaped by positive emotions. Dr. Van Cappellen's contributions to the subfields of positive psychology and the psychology of religion and spirituality have since been honored with multiple early career awards, and I am fortunate to continue to learn from and with her.

The accumulating evidence, from my and others' research teams, has convinced me that both domains of inquiry — positive psychology and the science of religion and spiritualityunpack a multitude of complementary practices and processes that help people, relationships, and communities flourish. Flourishing, by definition, fuses individual psychological well-being with contributions to the greater societal good, thereby providing foundations for morality and spirituality. As I put it in my book Love 2.0: 
I've been particularly drawn to religious writings that shine a spotlight on experiences of oneness and connection, because those are part of the signature of love [a.k.a., positivity resonance]. In these moments, borders seem to evaporate and you feel part of something far larger than yourself, be it nature, eternity, humanity, or the divine. ... Following in [William] James's footsteps, I take spirituality to revolve around expansive emotional moments like these. ... [As James wrote in 1902], "Feeling is the deeper source of religion, and ... philosophical and theological formulas are secondary products, like translations of a text into another tongue."

I especially resonate with how ...George Vaillant...equates spirituality with positive emotions, noting that these states are what connect you to others, to the divine, and over time help you attain wisdom and maturity. [As Vaillant (2009)] succinctly concludes "Love is the shortest definition of spirituality I know."

These potent, boundary-blurring and heart-expanding experiences of positivity resonance that you share with others are not merely an academic concept or a poetic flourish. Positivity resonance changes your biochemistry in ways scientists are only just beginning to grasp... In this way, love and health cocreate each other in your life. At the same time, this reciprocal, upward spiral dynamic between micro-moments of love and lasting changes in your health forges a path toward your higher spiritual sense of oneness.

My team has since documented, through randomized field experiments, that when people begin a spiritual practice, like loving-kindness meditation, they build up their flourishing mental health. Likewise, when they begin a positive psychology practice, like forging more everyday moments of social connection, they build up their spirituality. Yet current understandings of 
these mutual influences barely scratch the surface. Further integration of these subfields will take us deeper, as is one of the central theses of this Handbook. To the science of religion and spirituality, for instance, positive psychology can bring fresh hypotheses about biopsychosocial mechanisms. To positive psychology, the science of religion and spirituality can bring fresh concepts and measures to better capture ineffable experiences that seekers and believers might characterize as divine, sacred, or spirit-filled. It will also be vital to investigate the processes by which secular practices become sacred and whether and to what degree such sanctification increases the benefits of those practices.

In short, this new Handbook offers fresh seeds to germinate in the minds of researchers, practitioners, and laypeople worldwide. In the coming decades, when these ideas have fully taken root as tested hypotheses across wide-ranging research projects and real-world settings, we will have accumulated greater scientific understanding, better science, and more impactful applications of that science. Humanity sorely needs deeper scientific understandings_-based on evidence gathered across continents, cultures, and social classes - of the practices and processes that support human flourishing. For some, that science might inspire a course correction in their life's journey. In aggregate, those course corrections stand to uplift all of humanity.

\section{Barbara L. Fredrickson}

Chapel Hill, North Carolina

December 2021 


\section{Acknowledgements}

There are many people and groups we wish to thank for how they contributed to this Handbook. First, we would like to thank the culturally and religiously diverse cast of authors who wrote its chapters. You all are incredibly talented and busy people, and we are grateful for how you devoted your time, expertise, and wisdom to this project. You did so in the midst of the global COVID-19 pandemic — which affected us all but affected some of you particularly acutely — and you graciously responded to the editorial feedback and requests of three scrupulous editors. We are delighted with the chapters you wrote and are thankful for the ways that you drew on your virtues of wisdom, patience, and grit to bring them to fruition.

Next, we would like to thank the John Templeton Foundation (JTF), Templeton World Charity Foundation, and Templeton Religion Trust, which have generously supported not only this volume (JTF Grant 61865) but also the lion's share of the theory and research that comprises it. We are especially grateful to the various Templeton program officers and executives who have helped shape and shepherd our and our colleagues' work at the positive psychology of religion and spirituality (PPRS) intersections over the years, including Nick Gibson, Richard Bollinger, Kimon Sargeant, Andrew Serazin, Ellen Morgan, Bonnie Poon Zahl, Chris Stewart, and Erik Gjesfjeld. You have been instrumental in building the theory, research, and practice foundations for the integrative PPRS field that is the focus of this book.

We also would like to thank our colleagues and friends in the American Psychological Association's Division 36 (Society for the Psychology of Religion and Spirituality) and at our respective work institutions-Wheaton College (EBD), Virginia Commonwealth University (ELW), and Baylor University (SAS). You all have generously supported us in this project and in our broader careers, and this Handbook would not be possible without your faithful support. 
We thank our Editors and Project Coordinators at Springer, especially Sharon Panulla, Sofia Geck, and Srividya Subramanian. We are grateful for your enthusiastic support of this project and the editorial wisdom that you and your team brought to helping refine, publish, and promote it.

In addition, we are grateful to all our wonderful colleagues and students who have journeyed with us during our careers. It has been a joy and privilege to collaborate with you in building the foundations of this PPRS field. We are particularly thankful for our friends and colleagues in the Institute for Research on Psychology and Spirituality (IRPS), including Pete Hill, Todd Hall, Clark Campbell, Doug Daugherty, Liz Hall, Julie Exline, Don Davis, Joshua Hook, Daryl Van Tongeren, Alexis Abernethy, Bob Emmons, Jo-Ann Tsang, Steve Sandage, Liz Mancuso, Keith Edwards, Steve Porter, John Williams, Dave Wang, Mark McMinn, Jason McMartin, Kathy Johnson, Pam King, Cynthia Eriksson, Carissa Dwiwardani, Wade Rowatt, Charlotte Witvliet, Joe Bulbulia, Justin Barrett, Kevin Masters, and many others. You all have helped immensely in stimulating, challenging, and enriching our work over the years, and we are deeply grateful for you. In many ways, our IRPS group's esprit de corps has fueled our passion to devote our careers to this PPRS field.

In addition to these dear friends in IRPS, we are grateful to the other key colleagues and students who have contributed so meaningfully to our lives and work. Ward would like to thank Andy Yarborough, Jamie Aten, Gary R. Collins, Beth Brokaw, Corné Bekker, Mark Yarhouse, Glen Moriarty, Jen Ripley, Steve Parker, Bruce Bartholow, Jim Laffoon, Crystal Park, Pehr Granqvist, Carissa Sharp, Theresa Tisdale, Nancy Crawford, Tyler Lefevor, Richard Cowden, Job Chen, Laura Captari, Laura Shannonhouse, Stacey McElroy-Heltzel, Joe Currier, Kevin Ladd, Michiel Van Elk, Jordan LaBouff, Terri Watson, Ben Pyykkonen, Jake Johnson, Cynthia 
Neal Kimball, Virginia Shaffer, Austin Lemke, Andrew Cuthbert, Kevin Glowiak, and Ethan Lacey.

Ev would like to thank so many people that we're afraid it would extend the book's length beyond the possibility of even lifting it. He is extremely grateful to all his former student colleagues, from whom he has learned so much and to whom he owes a deep debt of gratitude. He also has over 25 international colleagues with whom he is currently collaborating, and they have been very inspirational to him. He also truly appreciates the many, many colleagues widespread throughout the United States. He would like to acknowledge two who were particularly formative in his career and whom the three of us - and countless others-are grieving their loss in 2021—Larry Crabb, Jr. and Gary R. Collins. We know they are happy with their Lord, and yet we miss them.

Sarah is also overwhelmed with gratitude for all the colleagues who have shaped and sharpened her thinking throughout the years. She is especially grateful to her primary earlycareer mentors—Peter Hill, Bob Emmons, and Justin Barrett—who taught her to think expansively and creatively about how to build a field. She additionally is so very grateful to the members of her Science of Virtues lab at Baylor; they manage the day-to-day activities of research, which allows Sarah to take on these types of editorial activities. In particular, she is thankful for her grant manager Lizzy Davis' leadership and support on so many levels.

We also would like to thank and congratulate each other. It has been a true privilege and honor to work on this project with such dear friends and respected colleagues. We have worked together harmoniously throughout this labor of love, and we are grateful for how we have sharpened, inspired, and supported each other. We also are grateful for the reliable internet connection at the public library and McDonald's about a mile from Ev's home, without which 
Sarah and Ward would not have been able to communicate with Ev. (He had let his annual subscription to the Pony Express expire just prior to starting this book.)

Sarah and Ev are particularly grateful to Ward, who did more than his share of the work. Thank you, Ward. When this book is published, you'll deserve to take a long, well-deserved victory lap. And then a long, well-deserved nap. How you managed to care for three wonderful kids, engage in loving husbandly interactions, and still keep us and all the authors on point is a modern-day miracle.

Most importantly, we are deeply grateful for our families - our spouses, children, grandchildren (for Ev at any rate), parents, and siblings - who have enriched our lives and been essential people who contribute to our own flourishing. We are grateful for their patience with us as we nerd out on work, travel to nerd conventions, lose track of time while writing or editing, and copyedit errors on emails or grocery lists. We are especially grateful for your support of our meaningful work even amidst a global pandemic. You are the ones who inspire us most to help build a positive psychology of religion and spirituality.

As we close this account of our acknowledgements in pulling together a book on religion, spirituality, and positive psychology, we must acknowledge that we each attribute all that is good to God. We pray this book will be contribute meaningfully to the faith, hope, and love of people around the world. 
Table of Contents

Foreword

Barbara L. Fredrickson

Acknowledgements

Part 1: Historical and Theoretical Considerations

Chapter 1: Integrating Positive Psychology and the Psychology of Religion and Spirituality: Transcending Coexistence to Potentiate Coevolution

Edward B. Davis, Everett L. Worthington, Jr., Sarah A. Schnitker, Kevin J. Glowiak, Austin W. Lemke, and Chase Hamilton

Chapter 2: Positive Psychology and the Psychology of Religion and Spirituality in Historical Perspective

James M. Nelson and Noelle Canty

Chapter 3: On the Integration of Positive Psychology and the Psychology of Religion and Spirituality: Logical, Normative, and Methodological Questions

Steven L. Porter, Jason Baehr, Tenelle Porter, and Robert C. Roberts

Chapter 4: Virtues in Positive Psychology and the Psychology of Religion and Spirituality

Juliette L. Ratchford, Mason S. Ming, and Sarah A. Schnitker

Chapter 5: Theories of Health and Well-Being Germane to a Positive Psychology of Religion and Spirituality

Douglas A. MacDonald

Chapter 6: Meaning as a Framework for Integrating Positive Psychology and the Psychology of Religiousness and Spirituality

Crystal L. Park and Daryl R. Van Tongeren

\section{Part 2: Methodological Considerations}

Chapter 7: Measurement at the Intersection of Positive Psychology and the Psychology of Religion and Spirituality

Peter C. Hill, Nicholas DiFonzo, C. Eric Jones, and Justin S. Bell

Chapter 8: Methodological Diversity in Positive Psychology and the Psychology of Religion and Spirituality

Jo-Ann Tsang, Rosemary L. Al-Kire, Edward B. Davis, Hilary N. Alwood, and

Wade C. Rowatt 


\section{Part 3: Cultural Considerations}

Chapter 9: Cultural Considerations in Positive Psychology and the Psychology of Religion and Spirituality

Jacqueline S. Mattis

Chapter 10: Positive Psychology and Christianity

Adam S. Hodge, Joshua N. Hook, Jichan J. Kim, David K. Mosher, Aaron T. McLaughlin, Don E. Davis, and Daryl R. Van Tongeren

Chapter 11: Positive Psychology and Judaism

Mark Schiffman, Aaron Cherniak, Eliezer Schnall, Suzanne Brooks, Steven Pirutinsky, and Devora Shabtai

Chapter 12: Living the Good Life: An Islamic Perspective on Positive Psychology

Seyma N. Saritoprak and Hisham Abu-Raiya

Chapter 13: Positive Psychology and Hinduism

Kamlesh Singh, Mahima Raina, and Doug Oman

Chapter 14: Positive Psychology and Buddhism

Seth Zuihō Segall and Jean L. Kristeller

Chapter 15: Positive Psychology and Religion/Spirituality Across Cultures in Europe, Non-U.S. North America, and South America

Clàudia Rossy, María Gámiz, Silvia Recoder, Iris Crespo, Maria Fernández-Capo, Edward B.

Davis, and Ethan K. Lacey

Chapter 16: Positive Psychology and Religion/Spirituality Across Cultures in Africa, Asia, and Oceania

Richard G. Cowden, Victor Counted, and Man Yee Ho

Part 4: Developmental Considerations

Chapter 17: Religion, Spirituality, and Youth Thriving: Investigating the Roles of the Developing Mind and Meaning-Making

Pamela Ebstyne King, Susan Mangan, and Rodrigo Riveros

Chapter 18: Religious/Spiritual Development and Positive Psychology: Toward an Integrative Theory

Edward B. Davis, James M. Day, Philip A. Lindia, and Austin W. Lemke

Part 5: Happiness and Well-Being

Chapter 19: The Scientific Study of Life Satisfaction and Religion/Spirituality

Elizabeth Krumrei Mancuso and Rosemond Travis Lorona 
Chapter 20: The Scientific Study of Positive Emotions and Religion/Spirituality

Patty Van Cappellen, Ruixi Zhang, and Barbara L. Fredrickson

Chapter 21: The Scientific Study of Positive Psychology, Religion/Spirituality, and Physical Health

Kevin S. Masters, Julia K. Boehm, Jennifer M. Boylan, Kaitlyn M. Vagnini, and

Christina L. Rush

Chapter 22: The Scientific Study of Positive Psychology, Religion/Spirituality, and Mental Health

Edward P. Shafranske

Part 6: Character Strengths and Virtues

Chapter 23: The Scientific Study of Religion/Spirituality, Forgiveness, and Hope Melissa Washington-Nortey, Everett L. Worthington, Jr., and Rihana Ahmed

Chapter 24: Religion/Spirituality and the Twin Virtues of Humility and Gratitude Madalyn R. Cauble, Iman Abdulkadir Said, Aaron T. McLaughlin, Sarah Gazaway, Daryl R. Van Tongeren, Joshua N. Hook, Ethan K. Lacey, Edward B. Davis, and Don E. Davis

Chapter 25: Theological Virtues, Health, and Well-Being: Theory, Research, and Public Health

Katelyn N. G. Long and Tyler J. VanderWeele

Part 7: Clinical and Applied Considerations

Chapter 26: Integrating Positive Psychology, Religion/Spirituality, and a Virtue Focus Within Culturally Responsive Mental Healthcare

Laura E. Captari, Steven J. Sandage, Richard A. Vandiver, Peter J. Jankowski, and

Joshua N. Hook

Chapter 27: Meaningfulness and Religious/Spiritual Meaning Systems at Work: A Multilevel Framework

Bryan J. Dik and Alexandra J. Alayan

Chapter 28: Positive Psychology and Religiousness/Spirituality in the Context of Couples and Families

Annette Mahoney, Jay R. Chinn, and James S. McGraw

Chapter 29: Positive Psychology in the Context of Religious Communities

David C. Wang, Mark R. McMinn, Zachary Wood, and Collin Lee

Chapter 30: Building Spiritual Fortitude and Resilience Following Disaster: Synthesizing the Contributions of Positive Psychology and Religion/Spirituality

Laura E. Captari, Laura Shannonhouse, Jamie D. Aten, and Jordan D. Snyder 


\section{Part 8: Field Unification and Advancement}

Chapter 31: Future Directions for the Positive Psychology of Religion and Spirituality Edward B. Davis, Sarah A. Schnitker, Everett L. Worthington, Jr., and Ethan K. Lacey 


\section{List of Contributors}

Hisham Abu-Raiya School of Social Work, Tel Aviv University, Tel Aviv, Israel,

hisham@tauex.tau.ac.il

Rihana Ahmed Psychology Department, Virginia Commonwealth University, Richmond, VA, USA, ahmedrj@vcu.edu

Alexandra J. Alayan Department of Psychology, Colorado State University, Fort Collins, CO, USA, allie.alayan@ colostate.edu

Rosemary L. Al-Kire Department of Psychology and Neuroscience, Baylor University, Waco, TX, USA, marah_al-kire1@baylor.edu

Hilary N. Alwood Department of Psychology and Neuroscience, Baylor University, Waco, TX, USA, hilary_alwood1@baylor.edu

Jamie D. Aten Humanitarian Disaster Institute, Wheaton College, Wheaton, IL, USA, jamie.aten@wheaton.edu

Jason Baehr Department of Philosophy, Loyola Marymount University, Los Angeles, CA, USA, jason.baehr@1mu.edu

Justin S. Bell Department of Psychology, Rochester Institute of Technology, Rochester, NY, USA, jsb2350@rit.edu

Julia K. Boehm Department of Psychology, Chapman University, Orange, CA, USA, jboehm@chapman.edu

Jennifer M. Boylan Department of Health and Behavioral Sciences, University of Colorado Denver, Denver, CO, USA, jennifer.boylan@ucdenver.edu

Suzanne Brooks Azrieli Graduate School of Jewish Education and Administration, Yeshiva University, New York, NY, USA, sebrooks@yu.edu 
Noelle Canty Department of Psychology, Valparaiso University, Valparaiso, IN, USA, noelle.canty@valpo.edu

Laura E. Captari The Albert and Jessie Danielsen Institute, Boston University, Boston, MA, USA, lcaptari@bu.edu

Madalyn R. Cauble College of Education and Human Development, Georgia State University, Atlanta, GA, USA, mcauble1@ @student.gsu.edu

Aaron Cherniak Department of Psychology, Stockholm University, Stockholm, Sweden, aaron.cherniak@ post.idc.ac.il

Jay R. Chinn Department of Psychology, Bowling Green State University, Bowling Green, OH, USA, chinnj@bgsu.edu

Victor Counted School of Psychology, Western Sydney University, Penrith, Australia, connect@ victorcounted.org

Richard G. Cowden Human Flourishing Program, Harvard University, Boston, MA, USA, rcowden@fas.harvard.edu

Iris Crespo Basic Sciences Department, Universitat Internacional de Catalunya (UIC),

Barcelona, Spain, icrespo@uic.es

Don E. Davis Department of Counseling and Psychological Services, Georgia State University, Atlanta, GA, USA, ddavis88@gsu.edu

Edward B. Davis School of Psychology, Counseling, and Family Therapy, Wheaton College, Wheaton, IL, USA, ward.davis@ wheaton.edu

James M. Day Psychological Sciences Research Institute, Universite Catholique de Louvain, Ottignies-Louvain-la-Neuve, Belgium, james.day@uclouvain.be 
Nicholas DiFonzo Department of Psychology, Roberts Wesleyan College, Rochester, NY, USA, nicholas.difonzo@gmail.com

Bryan J. Dik Department of Psychology, Colorado State University, Fort Collins, CO, USA, Bryan.Dik@ColoState.edu

Maria Fernández-Capo Basic Sciences Department, Universitat Internacional de Catalunya (UIC), Barcelona, Spain, mariafc@uic.es

Barbara L. Fredrickson Department of Psychology and Neuroscience, University of North Carolina, Chapel Hill, NC, USA, blf@unc.edu

María Gámiz Basic Sciences Department, Universitat Internacional de Catalunya (UIC), Barcelona, Spain, mariagamiz@uic.es

Sarah Gazaway College of Education and Human Development, Georgia State University, Atlanta, GA, USA, sgazaway1@ @student.gsu.edu

Kevin J. Glowiak School of Psychology, Counseling, and Family Therapy, Wheaton College, Wheaton, IL, USA, kjglowiak86@gmail.com

Chase Hamilton School of Psychology, Counseling, and Family Therapy, Wheaton College, Wheaton, IL, USA, chase.hamilton3030@gmail.com

Peter C. Hill Rosemead School of Psychology, Biola University, La Mirada, CA, USA, peter.hill@biola.edu

Man Yee Ho Department of Social and Behavioural Sciences, City University of Hong Kong, Hong Kong, China, my.ho@cityu.edu.hk

Adam S. Hodge Department of Psychology, University of North Texas, Denton, TX, USA, adamhodge@my.unt.edu 
Joshua N. Hook Department of Psychology, University of North Texas, Denton, TX, USA, joshua.hook@unt.edu

Peter J. Jankowski Counseling Program, Bethel University, Saint Paul, MN, USA, pjankows@ bethel.edu

C. Eric Jones Psychology Department, Regent University, Virginia Beach, VA, USA, ejones@ regent.edu

Jichan J. Kim Department of Psychology, Liberty University, Lynchburg, VA, USA, jjkim5@liberty.edu

Pamela Ebstyne King Thrive Center for Human Development, School of Psychology \& Marriage and Family Therapy, Fuller Theological Seminary, Pasadena, CA, USA, pamking@fuller.edu

Jean L. Kristeller Psychology Department, Indiana State University, Terre Haute, IN, USA, Jean.Kristeller@indstate.edu

Ethan K. Lacey School of Psychology, Counseling, and Family Therapy, Wheaton College, Wheaton, IL, USA, ethan.lacey@ my.wheaton.edu

Collin Lee Rosemead School of Psychology, Biola University, La Mirada, CA, USA, collin.lee@biola.edu

Austin W. Lemke School of Psychology, Counseling, and Family Therapy, Wheaton College, Wheaton, IL, USA, austin.lemke@my.wheaton.edu

Philip A. Lindia School of Psychology, Counseling, and Family Therapy, Wheaton College, Wheaton, IL, USA, philip.lindia@my.wheaton.edu

Katelyn N. G. Long Department of Epidemiology, Harvard T.H. Chan School of Public Health, Boston, MA, USA, knlong@ @sph.harvard.edu 
Rosemond Travis Lorona Department of Psychology, Point Loma Nazarene University, San Diego, CA, USA, rlorona@ pointloma.edu

Douglas A. MacDonald College of Liberal Arts and Education, University of Detroit Mercy, Detroit, MI, USA, macdonda@udmercy.edu

Annette Mahoney Department of Psychology, Bowling Green State University, Bowling

Green, OH, USA, amahone@bgsu.edu

Elizabeth Krumrei Mancuso Social Science Division, Seaver College, Pepperdine University, Malibu, CA, USA, elizabeth.mancuso@ pepperdine.edu

Susan Mangan Thrive Center for Human Development, School of Psychology \& Marriage and Family Therapy, Fuller Theological Seminary, Pasadena, CA, USA, susan.a.mangan@gmail.com

Kevin S. Masters Department of Psychology, University of Colorado Denver, Denver, CO, USA, kevin.masters@ucdenver.edu

Jacqueline S. Mattis Department of Psychology, Rutgers University-Newark, Newark, NJ, USA, jacqueline.mattis@ rutgers.edu

James S. McGraw Department of Psychology, Bowling Green State University, Bowling Green, OH, USA, jsmcgra@bgsu.edu

Aaron T. McLaughlin Department of Counseling and Psychological Services, Georgia State University, Atlanta, GA, USA, amclaughlin9@student.gsu.edu

Mark R. McMinn Department of Psychology, George Fox University, Newberg, OR, USA, mmcminn@georgefox.edu

Mason S. Ming Department of Psychology and Neuroscience, Baylor University, Waco, TX, USA, Mason_Ming1@baylor.edu 
David K. Mosher Department of Psychology, University of North Texas, Denton, TX, USA, davidmosher@my.unt.edu

James M. Nelson Department of Psychology, Valparaiso University, Valparaiso, IN, USA, ¡im.nelson@valpo.edu

Doug Oman School of Public Health, University of California-Berkeley, Berkeley, CA, USA, dougoman@berkeley.edu

Crystal L. Park Department of Psychological Sciences, University of Connecticut, Storrs, CT, USA, crystal.park@uconn.edu

Steven Pirutinsky Graduate School of Social Work, Touro College, New York, NY, USA, stevenpirutinsky@gmail.com

Steven L. Porter Rosemead School of Psychology and Talbot School of Theology, Biola University, La Mirada, CA, USA, steve.porter@biola.edu

Tenelle Porter Department of Psychology, University of Pennsylvania, Philadelphia, PA, USA, tenelle.porter@gmail.com

Mahima Raina Jindal School of Psychology and Counselling, O.P. Jindal Global University, Sonipat, India, mahima.raina@gmail.com

Juliette L. Ratchford Science of Virtues Laboratory, Department of Psychology and Neuroscience, Baylor University, Waco, TX, USA, juliette_ratchford1@ baylor.edu

Silvia Recoder Basic Sciences Department, Universitat Internacional de Catalunya (UIC), Barcelona, Spain, $\underline{\text { srecoder@uic.es }}$

Rodrigo Riveros Thrive Center for Human Development, School of Psychology \& Marriage and Family Therapy, Fuller Theological Seminary, Pasadena, CA, USA, riverosm@usc.edu 
Robert C. Roberts Department of Philosophy, Baylor University, Waco, TX, USA,

Robert_Roberts@baylor.edu

Clàudia Rossy Basic Sciences Department, Universitat Internacional de Catalunya (UIC),

Barcelona, Spain, crossy@uic.es

Wade C. Rowatt Department of Psychology and Neuroscience, Baylor University, Waco, TX,

USA, Wade_Rowatt@baylor.edu

Christina L. Rush Department of Psychology, University of Colorado Denver, Denver, CO,

USA, christina.rush@ucdenver.edu

Iman Abdulkadir Said College of Education and Human Development, Georgia State

University, Atlanta, GA, USA, isaid2@ student.gsu.edu

Steven J. Sandage, Ph.D. The Albert and Jessie Danielsen Institute, Boston University, Boston,

MA, USA, ssandage@bu.edu

Seyma N. Saritoprak Department of Psychological Sciences, Case Western Reserve

University, Cleveland, OH, USA, seyma.saritoprak@case.edu

Mark Schiffman Azrieli Graduate School of Jewish Education and Administration, Yeshiva

University, New York, NY, USA, mordechai.schiffman@yu.edu

Eliezer Schnall Department of Psychology, Yeshiva University, New York, NY, USA, schnall@yu.edu

Sarah A. Schnitker Science of Virtues Laboratory, Department of Psychology and

Neuroscience, Baylor University, Waco, TX, USA, $\underline{\text { Sarah_Schnitker@baylor.edu }}$

Seth Zuihō Segall Independent Scholar, sethzuihosegall@gmail.com

Devora Shabtai Center for Education Studies, University of Warwick, Coventry, England, Devora-Shabtai.Greer@warwick.ac.uk 
Edward P. Shafranske Graduate School of Education \& Psychology, Pepperdine University, Los Angeles, CA, USA, edward.shafranske@pepperdine.edu

Laura Shannonhouse Department of Counseling and Psychological Services, Georgia State University, Atlanta, GA, 1shannonhouse@gsu.edu

Kamlesh Singh Department of Humanities and Social Sciences, Indian Institute of Technology Delhi, New Delhi, India, singhk.iitd@gmail.com

Jordan D. Snyder Department of Psychology, University of Wisconsin-Parkside, Kenosha, WI, snyderj@uwp.edu

Jo-Ann Tsang Department of Psychology and Neuroscience, Baylor University, Waco, TX, USA, JoAnn_Tsang@baylor.edu

Kaitlyn M. Vagnini Department of Psychology, University of Colorado Denver, Denver, CO, USA, kaitlyn.vagnini@ucdenver.edu

Patty Van Cappellen Social Science Research Institute, Duke University, Durham, NC, USA, patty.vancappellen@duke.edu

Daryl R. Van Tongeren Department of Psychology, Hope College, Holland, MI, USA, vantongeren@hope.edu

Tyler J. VanderWeele Departments of Epidemiology and Biostatistics, Harvard T.H. Chan School of Public Health, Boston, MA, USA, tvanderw@hsph.harvard.edu

Richard A. Vandiver The Albert and Jessie Danielsen Institute, Boston University, Boston, MA, USA, richardv@bu.edu

David C. Wang Rosemead School of Psychology, Biola University, La Mirada, CA, USA, david.wang@biola.edu 
Melissa Washington-Nortey Psychology Department, Virginia Commonwealth University, Richmond, VA, USA, melissa.washington-nortey@kcl.ac.uk

Zachary Wood Rosemead School of Psychology, Biola University, La Mirada, CA, USA, zachary.b.wood@biola.edu

Everett L. Worthington, Jr. Psychology Department, Virginia Commonwealth University, Richmond, VA, USA, eworth@vcu.edu

Ruixi Zhang Social Science Research Institute, Duke University, Durham, NC, USA, ruixi.zhang@duke.edu 


\section{Chapter 1:}

Integrating Positive Psychology and the Psychology of Religion and Spirituality: Transcending Coexistence to Potentiate Coevolution

Edward B. Davis ${ }^{1}$, Everett L. Worthington, Jr. ${ }^{2}$, Sarah A. Schnitker ${ }^{3}$, Kevin J. Glowiak ${ }^{1}$, Austin W. Lemke ${ }^{1}$, and Chase Hamilton ${ }^{1}$

${ }^{1}$ School of Psychology, Counseling, and Family Therapy, Wheaton College

${ }^{2}$ Psychology Department, Virginia Commonwealth University

${ }^{3}$ Department of Psychology and Neuroscience, Baylor University

\section{Author Note}

We have no known conflicts of interest to disclose.

This publication was made possible through the support of Grant 61865 from the John Templeton Foundation. The opinions expressed in this publication are those of the authors and do not necessarily reflect the views of the John Templeton Foundation.

Correspondence concerning this chapter should be addressed to Edward B. Davis, Wheaton College, 501 College Ave, Wheaton, IL 60187. Email: ward.davis@ wheaton.edu. 


\begin{abstract}
This chapter sets the stage for the Handbook of Positive Psychology, Religion, and Spirituality. First, we examine the cumulative growth of the positive psychology and psychology of religion/spirituality (R/S) fields, including their degree of existing overlap and their typical publication outlets. Next, we discuss why enhanced integration of these fields would be mutually beneficial and would potentiate their coevolution toward greater scientific and societal impact. Then we acknowledge potential barriers to the integration of these fields and offer recommendations for transcending those barriers. Finally, we preview the organization of this handbook and make suggestions to guide reading it.
\end{abstract}

Keywords: positive psychology, religion, spirituality, integration, collaboration 


\section{Chapter 1: Integrating Positive Psychology and the Psychology of Religion and Spirituality: Transcending Coexistence to Potentiate Coevolution}

It was a fall day in 1930, and famous American philosopher and historian William Durant was raking leaves in his yard. A despondent stranger walked up and shocked Durant by confessing he was planning to commit suicide unless Durant could give him "one good reason" to live. Flustered, Durant gave a feeble reply: "I bade him get a job—-but he had one; to eat a good meal—but he was not hungry; he left visibly unmoved by my arguments" (Smith, 2017, p. 19). Durant was so haunted by the man's question that he wrote over 100 of the brightest minds of his time, asking each luminary to answer the question "What is the meaning or worth of human life?" (Durant, 1933, p. 3). He compiled their answers in On the Meaning of Life (Durant, 1933), published in the wake of World War I and heart of the Great Depression (Smith, 2017).

Questions about life's meaning have vexed humans across history. For millennia, philosophers and religious scholars led discourse on the topic, but since its inception in the late $19^{\text {th }}$ century, psychology has contributed to this discourse as well. Psychology's contribution budded with the work of William James, a founding figure both in mainstream psychology and the psychology of religion and spirituality (R/S; i.e., "the empirical or academic study of spiritual experience or organized religion from a psychological perspective," VandenBos, 2015, p. 860). James (1890/2011) presciently warned psychology from becoming "a psychology without a soul" (p. 7), experiencing a kind of etymological amnesia by forgetting its Greek root words psyche and logos literally mean "the study of the soul" (Pillsbury \& Pennington, 1942, p. 2). Unfortunately, James's advice was unheeded. Psychology largely ignored the scientific study of R/S until the 1960s, when interest was reinvigorated (Hood, 2012). 
Yet another of psychology's roots remained largely neglected until the end of the $20^{\text {th }}$ century. In his 1998 Presidential Address to the American Psychological Association (APA), Martin E. P. Seligman (1999) averred: "It's my belief that since the end of World War II, psychology has moved too far away from its original roots, which were to make the lives of all people more fulfilling and productive, and too much toward the important, but not all-important, area of curing mental illness" (p. 559). In response, he issued a clarion call for a "positive psychology" (Seligman, 1999, p. 561) that would redress this imbalance, helping psychology reclaim its mission by reorienting psychological science and practice toward understanding and promoting human strength and flourishing (Seligman, 1999). Several predecessors (such as William James, Abraham Maslow, and Carl Rogers) had already called for psychology to focus more on positive mental health, optimal functioning, growth, and human potential, but it usually is Seligman who is credited with catalyzing the positive psychology field in 1998 (Hart, 2021).

\section{Positive Psychology and the Psychology of Religion and Spirituality}

Positive psychology is the "field of psychological theory and research that focuses on the psychological states (e.g., contentment, joy), individual traits or character strengths (e.g., intimacy, integrity, altruism, wisdom), and social institutions that enhance subjective well-being and make life most worth living" (VandenBos, 2015, p. 810). In developing the Values in Action taxonomy that became the theoretical foundation of positive psychology, Peterson and Seligman (2004) consulted scholars and exhaustively searched the scholarly and historical literatures. They drew from the writings of religious scholars and moral philosophers across time, cultures, and faith traditions. R/S was one of the 24 character strengths that emerged in their taxonomy.

However, mainstream psychology has historically adopted a "noninteractive stance" (Jones, 1994, p. 184) toward R/S, perhaps because psychologists generally are much less 
religious or spiritual than the overall population (Shafranske \& Cummings, 2013), often do not have much formal training or competence in R/S (Vieten \& Lukoff, 2021), and frequently hold skeptical (or even biased) attitudes toward R/S (Gergen, 2009; Jones, 1994). But how much has positive psychology adopted this noninteractive stance toward R/S, given that R/S is one of the core character strengths (Peterson \& Seligman, 2004) and is robustly linked to health and wellbeing (Koenig et al., 2012)? In many ways, this question is what sparked the current handbook.

With this Handbook of Positive Psychology, Religion, and Spirituality, we particularly sought to accomplish three goals: (a) examine the degree of overlap between the positive psychology and psychology of R/S fields, (b) summarize and synthesize the relevant theoretical and empirical literature at these intersections, and (c) catalyze the integration of these fields and thereby potentiate their coevolution toward greater scientific and societal impact. The purpose of this introductory chapter is to set the stage for your evaluation of whether this book achieves these lofty goals. First, we describe the cumulative growth of the two fields, including how much overlap exists and where their research is published. Second, we outline reasons why the increased integration of positive psychology and the psychology R/S would be reciprocally beneficial. Third, we discuss potential barriers to this integration and suggest ways to transcend them. Lastly, we preview the handbook and recommend ways to glean the most as you read it.

\section{Existing Trends and Overlap}

To examine existing trends and overlap, we conducted two systematic literature searches in the APA's PsycINFO database. Both searches were conducted on December 31, 2020 and only used the standardized index terms available in the APA Thesaurus of Psychological Index Terms (APA, 2020). The following four index terms were the only ones available to use for positive psychology: "positive psychology", "virtue", "happiness", and "well-being"; the only 
three available for the psychology of R/S were "religion", "spirituality", and "faith". We constrained our search to index terms, because using a controlled (standardized) vocabulary is generally recommended, due to countless ways researchers can describe related concepts (Soto, 2017). Additionally, for these searches, we used the search field "DE" (Descriptors), because doing so ensured the retrieval of entries that were focused on a specific concept (rather than entries that contained a keyword anywhere in an entry, regardless of that entry's focus).

In Table 1.1, we present the results of the first search, which identified the cumulative number of academic articles and book entries that focused on positive psychology topics, psychology of R/S topics, and both types of topics. Entries are presented by year, starting with 1998, when Seligman gave his APA Presidential Address on positive psychology. From 1998 to $2020,48,623$ articles and book entries focused on at least one of the indexed positive psychology topics; 26,192 on R/S topics; and 1,783 (2.4\% of the collective 73,032 entries) on both.

Table 1.2 displays results of the second search, which examined a selection of premier psychology journals to see how much they each published articles on positive psychology topics, R/S topics, or both, between 1998 and 2020. Among the selected 23 journals (most of which were among the top-ranked psychology journals in the 2019 Journal Citation Reports [Clarivate Analytics, 2020]), the proportion of articles focusing on positive psychology varied widelyfrom $0.5 \%$ to $7.2 \%$. By comparison, the proportion of articles on R/S was consistently low $(0.0 \%$ to $2.0 \%)$, and the proportion on both $\mathrm{R} / \mathrm{S}$ and positive psychology was extremely low $(0.0 \%$ to $0.4 \%)$. Of the 56,400 articles published collectively across these journals, only $387(0.7 \%)$ were on R/S and $31(0.1 \%)$ were on both R/S and positive psychology. Even in the top niche journals in these fields, the proportion of articles on both topics was low, ranging from 3.9\% (35/889) in Journal of Positive Psychology to 13.2\% (69/522) in Psychology of Religion and Spirituality. 


\section{Integrating the Fields of Positive Psychology and Psychology of R/S}

There is growing scientific evidence that, across a wide variety of complex systems, integration ("the linkage of differentiated elements," Siegel, 2020, p. 461) is a central marker and mechanism of flourishing (Siegel, 2020). Indeed, we approach the current handbook with the belief that increased integration of positive psychology and the psychology of R/S will not only lead each field toward greater flourishing but also will lead to greater flourishing in mainstream psychology and society. Yet first we explore reasons why such integration is even possible.

\section{Why Can We Integrate These Fields?}

They often have similar aims. The overall aims of mainstream psychology are to (a) enhance scientific understanding of the human mind and behavior and (b) use this understanding to benefit society and improve people's lives (APA, 2011; Bermant et al., 2011). Similarly, the central aims of positive psychology are to advance scientific understanding of human strengths and flourishing and then use that understanding to benefit people, institutions, and societies (Hart, 2021; Seligman \& Csikszentmihalyi, 2000). Likewise, the main aims of the psychology of $\mathrm{R} / \mathrm{S}$ are (a) to enhance scientific understanding of spirituality ("search for or relationship with the sacred," Harris et al., 2018, p. 1) and religion ("search for significance that occurs within the context of established institutions designed to facilitate spirituality," Pargament et al., 2013, p. 15) and (b) use that understanding to benefit society and improve people's lives (Pargament, 2013). In sum, because positive psychology and the psychology of R/S have resonant aims (with each other and with the aims of mainstream psychology), they can be integrated readily. Both fields are working toward the same goals_ - advancing understanding and improving lives.

They have similar foundations. Next, both positive psychology and the psychology of $\mathrm{R} / \mathrm{S}$ are dedicated to the empirical study of the human mind and behavior; thus, they share a 
fundamental methodology (empirical science) and focus (human mind and behavior). They also have similar historical and philosophical origins, dating back to the classical and medieval periods (e.g., the writings of Aristotle, Plato, and early and medieval Christian authors; see Chapter 2, this volume). Moreover, each has historically explored the foundations of morality, ethics, and virtues (see Chapters 3 and 4, this volume). Further, there historically has been much overlap in these fields' epistemological assumptions (Nelson \& Slife, 2012; Snyder et al., 2021).

They have similar emphases. In the modern era, both fields have resonant emphases as well. For instance, positive psychology focuses on the study and promotion of subjective experiences and individual traits that enhance well-being, as well as on the social institutions that facilitate these experiences and traits (Seligman, 2011; Seligman \& Csikszentmihalyi, 2000). Similarly, the psychology of R/S studies people's search for the sacred and the social contexts and institutions that facilitate this search (Pargament et al., 2013). Both fields also emphasize practical applications (in clinical, workplace, and other contexts; Donaldson et al., 2020; Pargament, 2013) and issues relevant to people across cultures and time (health, well-being, meaning, virtues, positive emotions, and relationships; Seligman, 2011; Vaillant, 2008).

\section{Why Should We Integrate These Fields?}

Clearly, we can integrate these fields, but should we? In the inaugural article of The Journal of Positive Psychology, Linley et al. (2006) asserted that positive psychology "can prosper through integration [with other fields and with mainstream psychology], rather than wither through isolation" (p. 5), and they outlined strategies for accomplishing that goal. Likewise, many scholars (Emmons \& Paloutzian, 2003; Jones, 1994; Pargament et al., 2013) have argued that the psychology of R/S will prosper to the degree it becomes more integrated with other disciplines, with other psychology subfields, and with mainstream psychology. 
Greater integration will benefit both fields. By "integration" we do not mean the two subfields will become indistinguishable. Rather, we are suggesting these differentiable fields can achieve more interconnection, becoming increasingly intersecting circles on a Venn diagram in which their overlap represents a truly shared space of dialogue, synergy, and collaboration. As shown in Tables 1.1 and 1.2, this shared intersection is currently quite minimal.

If positive psychology and the psychology R/S achieve greater integration, it will be mutually beneficial. Because positive psychology already has a substantial platform in the scientific literature (Rusk \& Waters, 2013) and public sphere (Donaldson et al., 2020), its integration with the psychology of R/S could permit the latter to have greater visibility and impact. Similarly, because positive psychology is grounded firmly in the highest standards of scientific measurement and methodology (Peterson \& Seligman, 2004; Seligman \& Csikszentmihalyi, 2000), its integration with the psychology of R/S could enhance the scientific rigor of the latter's studies, measures, and methodologies (see Chapters 7 and 8, this volume).

Positive psychology will benefit from increased integration as well. For billions of people across the globe, R/S is a major source of meaning (Park, 2010), identity (Hays, 2016), growth (Tedeschi et al., 2018), and resilience (Pargament \& Cummings, 2010). Nonetheless, R/S has received relatively little attention within positive psychology (Rusk \& Waters, 2013; Snyder et al., 2021). Increased integration of these fields would enable positive psychology to enhance its scientific understanding of how people from diverse cultures and traditions draw on R/S to nurture positive emotional and relational experiences, create and sustain a sense of meaning, cultivate and enhance their well-being, and cope with and grow from adversity (Pargament, 2013; Vaillant, 2008). It also would enable positive psychology to draw on well-validated measures of R/S and the expertise of R/S scholars and practitioners (Pargament, 2013). 
Greater integration will benefit mainstream psychology. Additionally, the broader field of psychology would benefit. Indeed, the APA's vision statement (the change APA aspires to bring in the world) is "a strong, diverse, and unified psychology that enhances knowledge and improves the human condition" (APA, 2011, p. 4, emphasis added). Within mainstream psychology, R/S is recognized as an important facet of human diversity (Hays, 2016; Vieten \& Lukoff, 2021), yet as shown in Table 1.2, premier psychology journals still do not publish many articles on R/S. This dearth represents an enormous opportunity for positive psychology and the psychology of R/S. Because the link between R/S and well-being is so well-established (Koenig et al., 2012; Lefevor et al., 2021), research and practice at the intersections of R/S and positive psychology can help psychology fulfill its mission of improving people's lives. For example, greater integration of these fields can help psychology grow in scientific understanding of how $\mathrm{R} / \mathrm{S}$ can enhance the flourishing of people, institutions, and societies. It also can help develop and refine spiritually integrated interventions that are evidence-based and designed both to alleviate problems and actualize potentials (Pargament, 2013; see Chapter 26, this volume).

Greater integration will benefit society. Ultimately, the increased integration of positive psychology and the psychology of R/S will benefit society. Research suggests the largest influence on someone's well-being is the country in which they live (Geerling \& Diener, 2020). Nations can draw on scientific R/S research to enhance the well-being of their citizens individually and the flourishing of their society collectively (Diener \& Seligman, 2004, 2018). People who are higher in well-being tend to live healthier and longer lives, have more positive and rewarding relationships, be more economically prosperous and productive, feel greater meaning and purpose in life, and exhibit better citizenship and civic engagement. Additionally, countries with higher collective well-being tend to experience greater collective economic, 
environmental, social, and societal flourishing (Diener \& Seligman, 2018; Diener \& Tay, 2015). When it comes to R/S, empirical evidence suggests R/S may exhibit its strongest effects on people's well-being via its influence on people's social support (Geerling \& Diener, 2020), meaning/purpose in life (Jebb et al., 2020), and positive emotions (Van Cappellen et al., 2016). These effects are especially pronounced for people in societies characterized by difficult life circumstances (e.g., low safety, income, life expectancy, and basic need fulfillment; Diener et al., 2011). Increased research at the intersections of positive psychology and the psychology of R/S could have a particularly strong impact on those societies and their communities and citizens.

Early in the positive psychology movement, Seligman and Csikszentmihalyi (2000) envisioned that "a psychology of positive human functioning will arise that achieves a scientific understanding and effective interventions to build thriving in individuals, families, and communities" (p. 13). Advancing scientific understanding and practical interventions at the intersections of positive psychology and R/S will help make this dream a reality. In so doing, the coevolution of these fields can promote their respective and collective actualization.

\section{Barriers to Integrating Positive Psychology and the Psychology of Religion and Spirituality Personal and Professional Unfamiliarity with Religion and Spirituality}

In general, psychologists are not very religious or spiritual (Shafranske \& Cummings, 2013). In the U.S., roughly $90 \%$ of people believe in God and $75 \%$ say R/S is a very or fairly important part of their lives (Gallup, n.d.; Pew Research Center, 2017). However, only 30\% of psychologists believe in God, and just 50\% indicate R/S is very or fairly important (Delaney et al., 2013; Shafranske \& Cummings, 2013). Although over $80 \%$ of psychologists believe R/S is beneficial to mental health (Delaney et al., 2013), only 20\% to 30\% receive explicit professional training in R/S competencies (Hathaway, 2013; Vieten \& Lukoff, 2021). This lack of personal 
and professional familiarity with $\mathrm{R} / \mathrm{S}$ is presumably one barrier that has limited the integration of the psychology of R/S field with both positive and mainstream psychology (Jones, 1994).

\section{Skepticism Toward and Potential Bias Against Religion and Spirituality}

Furthermore, psychology has historically exhibited considerable skepticism toward R/S, perhaps due to the dominant influences of positivism, naturalism, and materialism (Jones, 1994; Shafranske \& Cummings, 2013; Slife \& Reber, 2009). This skepticism creates a barrier between R/S and both mainstream psychology and positive psychology. In fact, some scholars have even averred that mainstream psychology is fundamentally biased against R/S (Gergen, 2009; Slife \& Reber, 2009). This skepticism and possible bias may be one explanation for why there currently is so little incorporation of R/S into positive psychology research (see Table 1.1; Rusk \& Waters, 2013) and so little R/S research published in premier psychology journals (see Table 1.2).

\section{Skepticism Toward and Potential Bias Against Positivity}

Correspondingly, one possible barrier to the integration of positive psychology with mainstream psychology and psychology of R/S research might be humans' evolutionarily adapted negativity bias ("propensity to attend to, learn from, and use negative information far more than positive information," Vaish et al., 2008, p. 383). This bias helps explain why people across the world are often more psychophysiologically reactive to negative than positive news content (Soroka et al., 2019). This negativity bias likely contributes to mainstream psychology’s tendency to focus on negatively valenced phenomena such as distress, disease, and dysfunction (Seligman, 1999; Seligman \& Csikszentmihalyi, 2000). Another consequence of this tendency may be a bias against positivity, especially when it comes to publishing manuscripts or funding projects that focus on positive topics (e.g., strengths, virtues, resilience, and well-being) or processes (e.g., growth, optimal functioning, flourishing, and actualization). Psychology’s 
potential bias against positivity may be so strong that it fuels skepticism toward various forms of positivity that are encountered in scientific research, clinical practice, and everyday life (e.g., posttraumatic growth; Tedeschi et al., 2018). This skepticism and bias may impede the integration of positive psychology and the psychology of R/S.

\section{Recommendations for Transcending These and Related Barriers}

Despite these barriers, integration between positive psychology and psychology of R/S is possible. Several scholars have proposed theoretical accounts for how mainstream psychologists can better engage the study of R/S (Cresswell, 2014; Gergen, 2009; Jones, 1994; Paloutzian \& Park, 2021; Slife \& Reber, 2009) and positive psychology (Hill \& Hall, 2018; Snyder et al., 2021). These accounts often begin with identifying value conflicts (Yarhouse \& Johnson, 2013), self-assessing biases embedded in one's own worldview assumptions (e.g., about ontology, anthropology, universalism, and morality; Hill \& Hall, 2018) and philosophical assumptions (e.g., about epistemology and about whether theism and scientific naturalism are compatible; Nelson \& Slife, 2012; Slife \& Reber, 2009, 2021), and then working to transcend these biases.

Although people may adopt a variety of approaches for interaction across disciplines and subdisciplines (e.g., Jones [1994] describes critical-evaluative, constructive, and dialogical approaches to interactions between R/S and psychology), there is recent convergence on approaches that emphasize social constructionism, cultural diversity, and lived experiences. For example, Cresswell (2014) argues psychologists should adopt a pragmatic cultural-psychology approach that focuses on "inductive understandings of realities shaped in everyday practices within communities as opposed to naturalist laws" (p. 137), partly to avoid the "nothing but_-" reductionism William James sought to redress. Hence, in this handbook, we devote considerable attention to theory, methodological assumptions, cultural diversity, and practical applications. 


\section{Outline of the Handbook}

The handbook is divided into eight parts: history/theory (6 chapters), method (2 chapters), culture (8 chapters), development (2 chapters), well-being (4 chapters), character strengths/virtues ( 3 chapters), applications ( 5 chapters), and field advancement (1 chapter).

\section{Part 1: Historical and Theoretical Considerations}

The six chapters comprising the first part of the book will lay the foundation for understanding the ways the psychology of R/S and positive psychology overlap. The present chapter offers an orientation to the topic and the book, and then Nelson and Canty (Chapter 2) offer an overview of each field's history, including how those interact. In Chapter 3, Porter and colleagues explore philosophical questions regarding whether these fields can and should be integrated, as well as philosophical reasons why methodological pluralism is a promising paradigm for integration. Next, Ratchford et al. (Chapter 4) examine the intersections of virtue theory and research in these fields, and MacDonald (Chapter 5) reviews each field's dominant theories of health and well-being. Park and Van Tongeren (Chapter 6) propose that meaning is a framework for integrating science and practice in these two fields, and they offer suggestions for guiding this process. Throughout Part 1, authors explore motives, models, and methods for bringing together these currently disconnected fields. They approach integration historically, philosophically, and theoretically, and they argue that virtues, health/well-being, and meaning are focal areas by which these subfields can become unified more fully and synergistically.

\section{Part 2: Methodological Considerations}

Two chapters comprise this section, and each reveals the shared commitment that positive psychology and the psychology of R/S have to empirical methods. In Chapter 7, Hill et al. review existing measurements in these fields and offer recommendations for using those tools 
and techniques in research and practice. Tsang and colleagues (Chapter 8) summarize existing methodologies utilized in each field and (like Porter et al. in Chapter 3) suggest methodological diversity is a promising strategy for achieving greater integration and impact. Overall, in Part 2, the authors help lay the methodological groundwork for the rest of the handbook.

\section{Part 3: Cultural Considerations}

Similarly, the next section helps lay the cultural groundwork for the book. Mattis (Chapter 9) discusses how various cultural groups have grappled with matters of virtue, justice, and well-being, including how religious/spiritual institutions and individuals have successfully (and unsuccessfully) promoted virtue, justice, and well-being worldwide. This leads to chapters exploring the intersections of positive psychology with each of the world's major religions: Christianity (Hodge et al., Chapter 10), Judaism (Schiffman et al., Chapter 11), Islam (Saritoprak \& Abu-Raiya, Chapter 12), Hinduism (Singh et al., Chapter 13), and Buddhism (Segall \& Kristeller, Chapter 14). The section's last two chapters examine the geographically different cultural contexts of science and practice at the intersections of positive psychology and the psychology of R/S. Rossy and colleagues (Chapter 15) focus on the regions of Europe, non-U.S. North America, and South and Central America. Cowden and colleagues (Chapter 16) look at the regions of Africa, Asia, and Australia-Oceania. Taken together, in Part 3, authors unpack the cultural nuances and complexities embedded in science and practice at these intersections.

\section{Part 4: Developmental Considerations}

But these nuances and complexities are not limited to matters of culture, faith tradition, or geography, as Part 4 illustrates through its focus on human development. Like in Chapter 6, King and colleagues (Chapter 17) adopt a meaning-making framework to discuss how R/S can help promote the thriving of children and adolescents. In Chapter 18, Davis and colleagues propose 
Positive R/S Development theory, an integrative theory that explains how R/S develops and interacts with well-being across the lifespan. In these chapters, we see how science and practice at the intersections of R/S and positive psychology must adopt a developmentally sensitive framework, even as they adopt the culturally responsive frameworks highlighted in Part 3.

\section{Part 5: Happiness and Well-Being}

Parts 5 and 6 shift the discussion of positive psychology and R/S toward particular topics of study. Part 5 looks at the topics of happiness and well-being. Mancuso and Lorona (Chapter 19) review existing theory and research on the relationship between life satisfaction and R/S, including the nuances that affect the directionality and dynamics of this relationship. Likewise, Van Cappellen and colleagues (Chapter 20) synthesize existing theory and research on the link between positive emotions and R/S, with a focus on the self-transcendent emotions of awe, gratitude, compassion, and love. The other chapters in this section consider the intersections between R/S and both physical health (Masters et al., Chapter 21) and mental health (Shafranske, Chapter 22), including the directionality and influencers of these relationships.

\section{Part 6: Character Strengths and Virtues}

In Part 6, the topical discussion pivots to specific character strengths and virtues. This section begins with chapters exploring theory and research at the intersections of R/S with two sets of related virtues: (a) forgiveness and hope (Washington-Nortey et al., Chapter 23) and (b) humility and gratitude (Cauble et al., Chapter 24). Then, in Chapter 25, Long and VanderWeele examine another set of related virtues — the theological virtues of faith, hope, and love — but they do so from a public health perspective. In so doing, they help transition to the next section, which is practical and applied in focus. 


\section{Part 7: Clinical and Applied Considerations}

Part 7 looks at applications in particular domains. Captari and colleagues (Chapter 26) focus on clinical and applied interventions at the intersections of R/S and positive psychology. The next four chapters look at these intersections in other applied contexts: work (Dik \& Alayan, Chapter 27), couple and family relationships (Mahoney et al., Chapter 28), faith communities (Wang et al., Chapter 29), and disasters and humanitarian aid (Captari et al., Chapter 30).

\section{Part 8: Field Unification and Advancement}

In Chapter 31, we summarize key themes that emerged across the book. We propose unifying positive psychology and psychology of R/S into an integrated field-the positive psychology of R/S - and make recommendations for science, practice, and funding in this field.

\section{Conclusion and Suggestions}

We hope this review of the topic and handbook whets your appetite for the chapters that follow. We encourage you to approach this book as an intellectual meal to savor slowly and mindfully. Yet we recognize you might not be satisfied fully with the buffet, because there are many unanswered questions. As this chapter's analysis of publishing trends reveals, there currently is not much overlap between the positive psychology and psychology of R/S fields, but there is exciting potential for them to become more unified in science and practice. We encourage you to approach this handbook with that vista of possibility in mind. Search for reasons it might be beneficial for positive psychology to integrate R/S more into its theorizing, empirical research, and practical applications. Look for ways religious/spiritual individuals and institutions might benefit from positive psychology's theories, research, and applied tools. Ultimately, we hope these suggestions will inform what you "eat" and digest from this book, so that it can help guide you to new horizons of discovery in your research, practice, and life. 


\section{References}

American Psychological Association. (2011). Impact: APA and APA services strategic plan. https://www.apa.org/about/apa/strategic-plan

American Psychological Association. (2020). APA thesaurus of psychological index terms, July 2020 update. Author.

Bermant, G., Talwar, C., \& Rozin, P. (2011). To celebrate positive psychology and extend its horizons. In K. M. Sheldon, T. B. Kashdan, \& M. F. Steger (Eds.), Designing positive psychology: Taking stock and moving forward (pp. 430-438). Oxford University Press.

Clarivate Analytics. (2020). 2019 journal citation reports. Author.

Cresswell, J. (2014). Can religion and psychology get along? Toward a pragmatic cultural psychology of religion that includes meaning and experience. Journal of Theoretical and Philosophical Psychology, 34(2), 133-145. https://doi.org/10.1037/a0033042

Delaney, H. D., Miller, W. R., \& Bisonó, A. M. (2013). Religiosity and spirituality among psychologists: A survey of clinician members of the American Psychological Association. Spirituality in Clinical Practice, 1(S), 95-

106. https://doi.org/10.1037/2326.4500.1.S.95

Diener, E., \& Seligman, M. E. P. (2004). Beyond money: Toward an economy of well-being. Psychological Science in the Public Interest, 5(1), 1-31. https://doi.org/10.1111/j.0963$\underline{7214.2004 .00501001 . x}$

Diener, E., \& Seligman, M. E. P. (2018). Beyond money: Progress on an economy of well-being. Perspectives on Psychological Science, 13(2), 171-175. https://doi.org/10.1177/1745691616689467 
Diener, E., \& Tay, L. (2015). Subjective well-being and human welfare around the world as reflected in the Gallup World Poll. International Journal of Psychology, 50(2), 135-149. https://doi.org/10.1002/ijop.12136

Diener, E., Tay, L., \& Myers, D. G. (2011). The religion paradox: If religion makes people happy, why are so many dropping out? Journal of Personality and Social Psychology, 101(6), 1278-1290. https://doi.org/10.1037/a0024402

Donaldson, S. I., Csikszentmihalyi, M., \& Nakamura, J. (2020). Positive psychological science: Improving everyday life, well-being, work, education, and societies across the globe $\left(2^{\text {nd }}\right.$ ed.). Routledge.

Durant, W. (1933). On the meaning of life. Williams \& Norgate.

Emmons, R. A., \& Paloutzian, R. F. (2003). The psychology of religion. Annual Review of Psychology, 54, 377-402.

Gallup. (n.d.). Religion. Retrieved February 25, 2021, from https://news.gallup.com/poll/1690/Religion.aspx

Geerling, D. M., \& Diener, D. (2020). Effect size strengths in subjective well-being research. Applied Research in Quality of Life, 15, 167-185. https://doi.org/10.1007/s11482-018$\underline{9670-8}$

Gergen, K. J. (2009). The problem of prejudice in plural worlds. Journal of Theoretical and Philosophical Psychology, 29(2), 97-101. https://doi.org/10.1037/a0017817

Harris, K.A., Howell, D.S. \& Spurgeon, D.W. (2018). Faith concepts in psychology: Three 30year definitional content analyses. Psychology of Religion and Spirituality, 10(1), 1-29. http://dx.doi.org/10.1037/rel0000134

Hart, R. (2021). Positive psychology: The basics. Routledge. 
Hathaway, W. (2013). Pathways toward graduate training in the clinical psychology of religion and spirituality. In K. I. Pargament, A. Mahoney, \& E. P. Shafranske (Eds.), APA handbook of psychology, religion, and spirituality (Vol. 2, pp. 635-649). American Psychological Association. https://doi.org/10.1037/14046-033

Hays, P. A. (2016). Addressing cultural complexities in practice ( ${ }^{\text {rd }}$ ed.). APA.

Hill, P. C., \& Hall, M. E. L. (2018). Uncovering the good in positive psychology. In N. Brown, T. Lomas, \& F. Eiroa-Orosa (Eds.), Routledge international handbook of critical positive psychology (pp. 245-262). Routledge.

Hood, R. W., Jr. (2012). The history and current state of research on psychology of religion. In L. J. Miller (Ed.), The Oxford handbook of psychology and spirituality (pp. 7-20). Oxford University Press. https://doi.org/10.1093/oxfordhb/9780199729920.013.0001

James, W. (1890/2011). The principles of psychology (Vols. 1 \& 2). Digireads.com.

Jebb, A. T., Morrison, M., Tay, L., \& Diener, E. (2020). Subjective well-being around the world: Trends and predictors across the life span. Psychological Science, 31(3), 293-305. https://doi.org/10.1177/0956797619898826

Jones, S. L. (1994). A constructive relationship for religion with the science and profession of psychology: Perhaps the boldest model yet. American Psychologist, 49(3), 184-199. https://doi.org/10.1037/0003-066X.49.3.184

Koenig, H. G., King, D. E., \& Carson, V. B. (2012). Handbook of religion and health (2 $2^{\text {nd }}$ ed.). Oxford University Press.

Lefevor, G. T., Davis, E. B., Paiz, J. Y., \& Smack, A. C. P. (2021). The relationship between religiousness and health among sexual minorities: A meta-analysis. Psychological Bulletin. Advance online publication: https://doi.org/10.1037/bul0000321 
Linley, P. A., Joseph, S., Harrington, S., \& Wood, A. M. (2006). Positive psychology: Past, present, and (possible) future. Journal of Positive Psychology, 1(1), 3-16. https://doi.org/10.1080/17439760500372796

Nelson, J., \& Slife, B. (2012). Theoretical and epistemological foundations. In L. Miller (Ed.), Oxford handbook of psychology and spirituality (pp. 21-35). Oxford University Press.

Paloutzian, R. F., \& Park, C. L. (2021). The psychology of religion and spirituality: How big the tent. Psychology of Religion and Spirituality, 13(1), 3-13. http://dx.doi.org/10.1037/rel0000218

Pargament, K. I. (Ed.). (2013). APA handbook of psychology, religion, and spirituality (Vol. 1 \& 2). American Psychological Association. https://10.1037/14045-001 and https://10.1037/14045-002

Pargament, K. I., \& Cummings, J. (2010). Anchored by faith: Religion as a resilience factor. In J. Reich, A. Zautra, \& J. Hall (Eds.), Handbook of adult resilience (pp. 193-210). Guilford.

Pargament, K. I., Mahoney, A., Exline, J. J., Jones, J. W. \& Shafranske, E. P. (2013).

Envisioning an integrative paradigm for the psychology of religion and spirituality. In K. I. Pargament (Ed.), APA handbook of psychology, religion, and spirituality (Vol. 1, pp. 3-19). American Psychological Association. https://10.1037/14045-001

Park, C. L. (2010). Making sense of the meaning literature: An integrative review of meaning making and its effects on adjustment to stressful life events. Psychological Bulletin, 136(2), 257-301. https://doi.org/10.1037/a0018301

Peterson, C., \& Seligman, M. E. P. (2004). Character strengths and virtues: A handbook and classification. American Psychological Association. 
Pew Research Center (2017). The global religious landscape. Author. https://www.pewforum.org/2012/12/18/global-religious-landscape-exec/

Pillsbury, W. B. \& Pennington, L. A. (1942). Introduction to general psychology. Handbook of general psychology (pp. 2-18). Dryden Press.

Rusk, R. D., \& Waters, L. E. (2013). Tracing the size, reach, impact, and breadth of positive psychology. The Journal of Positive Psychology, 8(3), 207-221. https://doi.org/10.1080/17439760.2013.777766

Seligman, M. E. P. (1999). The president's address. American Psychologist, 54(8), 559-562.

Seligman, M. E. P. (2011). Flourish. Simon and Schuster.

Seligman, M. E. P., \& Csikszentmihalyi, M. (2000). Positive psychology: An introduction. American Psychologist, 55(1), 5-14. https://doi.org/10.1037/0003-066X.55.1.5

Shafranske, E. P., \& Cummings, J. P. (2013). Religious and spiritual beliefs, affiliations, and practices of psychologists. In K. I. Pargament (Ed.), APA handbook of psychology, religion, and spirituality (Vol. 2, pp. 23-41). APA. https://10.1037/14045-002

Siegel, D. J. (2020). The developing mind ( $3^{\text {rd }}$ ed.). Guilford Press.

Slife, B. D., \& Reber, J. S. (2009). Is there a pervasive implicit bias against theism in psychology? Journal of Theoretical and Philosophical Psychology, 29(2), 63-79.

Slife, B. D., \& Reber, J. S. (2021). Against methodological confinement: Toward a pluralism of methods and interpretations. Psychology of Religion and Spirituality, 13(1), 14-18. http://dx.doi.org/10.1037/rel0000274

Smith, E. E. (2017). The power of meaning: The true route to happiness. Ebury Digital.

Snyder, C. R., Lopez, S. J., Edwards. L. M., \& Marques, S. C. (Eds.). (2021). The Oxford handbook of positive psychology ( $3^{\text {rd }}$ ed.). Oxford University Press. 
Soroka, S., Fournier, P., \& Nir, L. (2019). Cross-national evidence of a negativity bias in psychophysiological reactions to news. Proceedings of the National Academy of Sciences, 116(38), 18888-18892. https://doi.org/10.1073/pnas.1908369116

Soto, M. (2017, January 19). APA PsycInfo expert tip: Searching by keyword, index term, and more. https://blog.apapubs.org/2017/01/19/psycinfo-expert-tip-searching-by-keyword$\underline{\text { index-term-and-more/ }}$

Tedeschi, R. G., Shakespeare-Finch, J., Taku, K., \& Calhoun, L. G. (Eds.). (2018). Postraumatic growth: Theory, research, and applications. Routledge.

Vaillant, G. E. (2008). Spiritual evolution: A scientific defense of faith. Broadway Books. Vaish, A., Grossmann, T., \& Woodward, A. (2008). Not all emotions are created equal: The negativity bias in social-emotional development. Psychological Bulletin, 134(3), 383403. https://doi.org/10.1037/0033-2909.134.3.383

Van Cappellen, P., Toth-Gauthier, M., Saroglou, V., \& Fredrickson, B. L. (2016). Religion and well-being: The mediating role of positive emotions. Journal of Happiness Studies, 17, 485-505. https://doi.org/10.1007/s10902-014-9605-5

VandenBos, G. R. (Ed.). (2015). APA dictionary of psychology (2 ${ }^{\text {nd }}$ ed.). American Psychological Association. http://dx.doi.org/10.1037/14646-000

Vieten, C., \& Lukoff, D. (2021). Spiritual and religious competencies in psychology. American Psychologist. Advance online publication. https://doi.org/10.1037/amp0000821

Yarhouse, M. A., \& Johnson, V. (2013). Value and ethical issues: The interface between psychology and religion. In K. I. Pargament (Ed.), APA handbook of psychology, religion, and spirituality (Vol. 2, pp. 43-70). APA. https://doi.org/10.1037/14046-003 


\section{Table 1.1}

Cumulative Number of Academic Articles and Book Entries on Positive Psychology and the Psychology of Religion/Spirituality

\begin{tabular}{|c|c|c|c|c|c|c|c|c|c|}
\hline \multirow[t]{2}{*}{ Year } & \multicolumn{4}{|c|}{ Positive psychology $(n=48,623)$} & \multicolumn{3}{|c|}{ Psychology of R/S $(n=26,192)$} & \multirow[t]{2}{*}{ Total } & \multirow[t]{2}{*}{ Both $(\%)$} \\
\hline & Positive psychology ${ }^{a}$ & Virtues ${ }^{b}$ & Happiness ${ }^{\mathrm{c}}$ & Well-being $^{\mathrm{d}}$ & Religion $^{\mathrm{e}}$ & Spirituality $^{\mathrm{f}}$ & Faith $^{\mathrm{g}}$ & & \\
\hline 1998 & 0 & 2 & 64 & 399 & 180 & 176 & 11 & 792 & $10(1.3)$ \\
\hline 1999 & 0 & 4 & 137 & 831 & 346 & 380 & 26 & 1,616 & $27(1.7)$ \\
\hline 2000 & 10 & 10 & 259 & 1,413 & 572 & 664 & 46 & 2,756 & $56(2.0)$ \\
\hline 2001 & 29 & 14 & 379 & 2,038 & 823 & 968 & 63 & 3,979 & $79(2.0)$ \\
\hline 2002 & 101 & 24 & 523 & 2,893 & 1,141 & 1,339 & 93 & 5,529 & $134(2.4)$ \\
\hline 2003 & 183 & 36 & 668 & 3,780 & 1,551 & 1,777 & 125 & 7,318 & $178(2.4)$ \\
\hline 2004 & 341 & 42 & 821 & 4,780 & 2,010 & 2,277 & 175 & 9,436 & $226(2.4)$ \\
\hline 2005 & 413 & 58 & 1,018 & 5,817 & 2,584 & 2,927 & 225 & 11,734 & $281(2.4)$ \\
\hline 2006 & 534 & 66 & 1,241 & 7,235 & 3,379 & 3,728 & 293 & 14,783 & $359(2.4)$ \\
\hline 2007 & 675 & 91 & 1,527 & 8,685 & 4,140 & 4,510 & 369 & 17,953 & $439(2.4)$ \\
\hline 2008 & 835 & 114 & 1,856 & 10,268 & 4,889 & 5,280 & 432 & 21,214 & $518(2.4)$ \\
\hline 2009 & 1,066 & 136 & 2,215 & 11,908 & 5,710 & 6,074 & 501 & 24,692 & $609(2.5)$ \\
\hline 2010 & 1,271 & 198 & 2,600 & 13,707 & 6,493 & 6,822 & 586 & 28,337 & $689(2.4)$ \\
\hline 2011 & 1,539 & 274 & 3,031 & 15,668 & 7,328 & 7,668 & 729 & 32,321 & $815(2.5)$ \\
\hline 2012 & 1,787 & 385 & 3,458 & 17,876 & 8,238 & 8,641 & 935 & 36,760 & $945(2.6)$ \\
\hline 2013 & 2,167 & 529 & 4,071 & 20,681 & 9,165 & 9,617 & 1,139 & 42,018 & $1,066(2.5)$ \\
\hline 2014 & 2,601 & 700 & 4,623 & 23,494 & 10,200 & 10,529 & 1,352 & 47,359 & $1,205(2.5)$ \\
\hline 2015 & 2,956 & 877 & 5,121 & 26,236 & 11,069 & 11,347 & 1,597 & 52,367 & $1,325(2.5)$ \\
\hline 2016 & 3,318 & 952 & 5,637 & 29,148 & 11,783 & 12,053 & 1,687 & 57,133 & $1,403(2.5)$ \\
\hline 2017 & 3,634 & 1,050 & 6,048 & 31,921 & 12,510 & 12,786 & 1,734 & 61,702 & $1,529(2.5)$ \\
\hline 2018 & 3,963 & 1,160 & 6,461 & 34,658 & 13,232 & 13,469 & 1,816 & 66,190 & $1,630(2.5)$ \\
\hline 2019 & 4,197 & 1,247 & 6,929 & 37,474 & 13,789 & 14,115 & 1,910 & 70,542 & $1,722(2.4)$ \\
\hline $2020^{\#}$ & 4,297 & 1,295 & 7,144 & 39,157 & 14,111 & 14,477 & 1,962 & 73,032 & $1,783(2.4)$ \\
\hline
\end{tabular}

Note. R/S = religion/spirituality. Entries were identified in PsycINFO, using the following search terms: "DE "Positive Psychology"; ${ }^{b} D E$ "Virtue"; cDE "Happiness"; ${ }^{\mathrm{d}} \mathrm{DE}$ "Well Being"; ${ }^{\mathrm{e}} \mathrm{DE}$ "Religion"; fDE "Spirituality"; 'DE "Faith". For all searches, the only limiters were "excludes dissertations" and that the Source Types were either "academic journals" or "books."

\#Up through December 31, 2020. 


\section{Table 1.2}

Positive Psychology (PP) and Psychology of Religion and Spirituality (PoRS) Articles in Select Psychology Journals, 1998 to 2020

\begin{tabular}{|c|c|c|c|c|c|}
\hline Journal & 2-year IF & Total articles & PP articles & PoRS articles & PP+PoRS articles \\
\hline Personality and Social Psychology Review & 12.321 & 453 & $19(4.2)$ & $9(2.0)$ & $2(0.4)$ \\
\hline Perspectives on Psychological Science & 8.275 & 1,008 & $46(4.6)$ & $6(0.6)$ & $0(0.0)$ \\
\hline American Psychologist & 6.536 & 4,094 & $141(3.4)$ & $30(0.7)$ & $1(0.0)$ \\
\hline Journal of Personality and Social Psychology & 6.315 & 3,423 & $218(6.4)$ & $37(1.1)$ & $2(0.1)$ \\
\hline Current Directions in Psychological Science & 5.110 & 1,548 & $46(3.0)$ & $9(0.6)$ & $1(0.1)$ \\
\hline Child Development & 4.891 & 3,290 & $66(2.0)$ & $14(0.4)$ & $0(0.0)$ \\
\hline Journal of Consulting and Clinical Psychology & 4.632 & 2,481 & $28(1.1)$ & $8(0.3)$ & $1(0.0)$ \\
\hline Journal of Counseling Psychology & 3.697 & 1,290 & $80(6.2)$ & $17(1.3)$ & $4(0.3)$ \\
\hline Journal of Personality & 3.667 & 1,311 & $95(7.2)$ & $25(1.9)$ & $2(0.2)$ \\
\hline Journal of Clinical Child and Adolescent Psychology & 3.656 & 1,693 & $9(0.5)$ & $0(0.0)$ & $0(0.0)$ \\
\hline Attachment \& Human Development & 2.656 & 741 & $12(1.6)$ & $3(0.4)$ & $0(0.0)$ \\
\hline Psychological Trauma & 2.595 & 1,233 & $31(2.5)$ & $25(2.0)$ & $2(0.2)$ \\
\hline Counseling Psychologist & 2.263 & 1,078 & $52(4.8)$ & $19(1.8)$ & $2(0.2)$ \\
\hline Journal of Clinical Psychology & 2.138 & 2,805 & $59(2.1)$ & $56(2.0)$ & $2(0.1)$ \\
\hline Total $(\%$ of total $)$ & & 56,400 & $1,780(3.2)$ & $387(0.7)$ & $31(0.1)$ \\
\hline Journal of Positive Psychology (2006-2020) & 3.819 & 889 & -- & $35(3.9)$ & $35(3.9)$ \\
\hline Psychology of Religion and Spirituality (2009-2020) & 2.367 & 522 & $69(13.2)$ & -- & $69(13.2)$ \\
\hline
\end{tabular}

Note. IF = impact factor from the 2019 Journal Citation Reports (Clarivate Analytics, 2020). 


\title{
Chapter 2: Positive Psychology and the Psychology of Religion and Spirituality in Historical Perspective
}

\author{
James M. Nelson and Noelle Canty \\ Department of Psychology, Valparaiso University
}

\begin{abstract}
Author Note
We have no known conflict of interest to disclose.

Correspondence concerning this article should be addressed to James M. Nelson, Department of Psychology, 1001 Campus Drive South, Valparaiso, Indiana 46483. Email

jim.nelson@valpo.edu
\end{abstract}




\begin{abstract}
The fields of positive psychology (PP) and the psychology of religion/spirituality (PRS) have much in common, both in terms of their areas of interest and the presuppositions they bring to their work. For example, PP and PRS are both rooted in the philosophies of positivism (which assumes all knowledge must be empirically verified using the scientific method) and naturalism (which assumes there are no realities beyond the natural, material world). Both PP and PRS have much to offer society and the scientific community, but their historic roots in positivistic naturalism currently limit this potential, both in terms of concepts and methods. In this chapter, we argue that for PP and the PRS to coevolve and flourish, they must transcend their perhaps often unaware — but staunch—commitment to positivistic naturalism. In particular, the fields of PP and PRS need to draw inclusively and meaningfully from the methodological, conceptual, and experiential insights of philosophical and religious traditions. Doing so will help PP and PRS broaden the scope of what they each consider meaningful, possible, desirable, and transformative. Ironically, a greater appreciation of the past will enable both fields to have greater scientific, societal, and practical impact in the future.
\end{abstract}

Keywords: positive psychology, psychology of religion and spirituality, history, virtue, philosophy 


\section{Chapter 2: Positive Psychology and the Psychology of Religion and Spirituality in Historical Perspective}

A pastoral care team from a local church located in a Christian-minority area of the U.S. recently visited an elderly member, Jane. Suffering pain from terminal cancer, she was bedridden and preparing to enter hospice care. The team expected despondency, but she was deeply content, grateful that she was in good hands. Her main concern was for the daughter of a fellow church member who was also fighting cancer. The visit concluded happily when the team promised to visit her friend and joined her in prayer.

Contemporary psychologists have much to offer in understanding and helping this extraordinary woman. Scientists in the field of positive psychology (PP) focus on advancing scientific understanding of virtues, strengths, and well-being, so they could help the pastoral care team by sharing relevant research findings about how and why people's character virtues often enhance their well-being (see Ratchford et al., Chapter 4, this volume). PP practitioners could help by offering research-supported interventions to help Jane cope with her illness, prepare for death, and enact her virtues during her remaining time. In the same way, scientists in the psychology of religion/spirituality (PRS) field — which focuses on the scientific study of people's search for sacred meaning and connection—could share helpful scientific findings about how people's religiousness/spirituality reliably leads to growth in virtues and enhanced well-being (see Davis et al., Chapter 18, Appendix 18.S2, this volume; Long \& VanderWeele, Chapter 25, Appendix 25.S2, this volume). Practitioners from the PRS field could share spiritually integrated psychological interventions that could help Jane to enhance her well-being (see Captari et al., Chapter 26, this volume). There are also rich possibilities for collaborations between the fields (Barton \& Miller, 2015; see also Davis et al., Chapter 1, this volume). 
An understanding of history provides two critical pieces of information that help us understand the potentials and challenges facing these fields. First, PP and PRS have shared methodological commitments that have emerged historically, and although these commitments offer some advantages, they are based on dated philosophical positions that can hinder research and its practical application. Second, although history reveals that interest in positive human states and their connections to religious or spiritual life goes back many centuries and incorporates many insightful ideas, researchers in PP and PRS have sometimes misread and redefined foundational concepts like "happiness" or "spirituality," leading to problems in research and practice (Martin, 2007). It is always desirable for scientists and practitioners to look carefully at their presuppositions and methods so that they do not hinder progress toward valued, important goals. This chapter takes a somewhat critical tone, not because we do not appreciate the terrific successes of PP and PRS, but because we hope historical material in this chapter will help individuals in these fields better realize their distinct and shared goals.

\section{Historical Development of PP and PRS}

PP and PRS involve exciting attempts to understand important but often neglected aspects of human experience and behavior, potentially generating knowledge of great benefit individuals and society. The current incarnation of PP has its official beginnings in the late $20^{\text {th }}$ century with the work of Seligman and Csikszentmihalyi (2000), whereas PRS has slightly older

roots going back to the work of William James and his contemporaries in the late $19^{\text {th }}$ century.

In early PP, the goal was helping individuals or groups achieve what Martin Seligman (2002) called "gratification" through strengthening latent, natural characteristics. For Seligman, the definition of the good life is "using your signature strengths to obtain abundant gratification in the main realms of one's life" (p. 262). In later writing, Seligman (2011) seems to prefer to 
define the good life through using the term and concept of "well-being" (pp. 5-29). The goal of PP then became the understanding and enhancement of well-being. By comparison, PRS is "the empirical or academic study of spiritual experience or organized religion from a psychological perspective," including "ways in which religious faith affects the behaviors and cognitive processes of believers" (VandenBos, 2015, p. 860). Both fields make frequent use of universal categories and are deeply committed to utilizing the scientific method, as practiced in mainstream psychology. This method is a historical development with strengths and limitations.

\section{Positivism and the Development of Method}

With origins in the early and later 20th century, science in PP and PRS typically assumes the philosophical viewpoint of positivistic naturalism (PN). Unrelated etymologically to the term "positive" in PP, PN is a cluster of beliefs about science and the world that developed in the $16^{\text {th }}$ through $20^{\text {th }}$ centuries, at the time psychology was attempting to define its place as a science.

Positivism assumes that all knowledge of reality must be empirically verified using the scientific method, and naturalism assumes there are no realities beyond the material world, which operates according to fixed, natural laws. Naturalistic assumptions are particularly tricky in the field of PRS, because these assumptions often conflict with beliefs of the religious traditions they study.

In the Early Modern period, science was redefined through the work of scientists and philosophers such as René Descartes. Their viewpoints encouraged the development of universally applicable models based on perceived general natural laws (Dijksterhuis, 1961; Nelson \& Slife, 2013; Wootton, 2015). The view of humans as machines, promoted by writers like Descartes and de lá Mettrie, also emerged at this time, leading to scientific atomism, the idea that people can be understood by disassembling them and studying component parts. 
The increasing emphasis on objectivity and empiricism in Early Modern and Enlightenment thought eventually reached its peak in the philosophy of science known as positivism. Positivism was a creation of the early $19^{\text {th }}$ century philosopher Auguste Comte, who argued that an idea can be true only if it is backed by empirical data obtained by an investigator who is a detached, impartial observer. He believed that human enquiry was passing through "primitive" stages of religious and philosophical thinking, eventually to be replaced by scientific enquiry that would propel human advancement. This view of history led to scientism, the belief that only the scientific method can produce true knowledge (Comte, 1839). Because history was seen as positively progressive, there was little to be learned from studying the past, other than to produce triumphal accounts about how we are moving to a glorious future (Seligman, 2019).

Adherents of PN trust a basic method of organizing knowledge. This method is thought to give them correct feedback about their perceptions, but it automatically invalidates other types of knowledge. It limits inquiry only to statements directly verifiable by sense perception; other types of statements are judged "metaphysical" or "armchair speculation" and thus meaningless (Feigl, 1949). Mechanistic models eliminate ideas of final purpose or transcendence, rejecting philosophy and theology as valid ways of knowing about the world (Dijksterhuis, 1961). PN can thus become not just a philosophy of science but a kind of alternative worldview.

The sole focus of naturalism is on universal laws, which marks a large departure from previous thinkers such as Aristotle. Aristotle argued the principles of eudaimonia or the good life — the foundations of ethics—are natural and thus necessary. Because these principles are a certain knowledge accessible to reason, they were viewed as epistēme, a kind of scientific knowledge (NE, VI.iii.2-4). However, Aristotle felt such principles were largely inadequate by themselves, because applying them in specific situations required practical reasoning and virtue 
(phronessis), the principles of which are not "scientific" truths because what is best changes based on particular situations (NE, VI.v.1-4). A scientific statement thus can never completely capture what the virtuous person needs to know in daily pursuit of the good life in a particular context. Taking this view of Jane's situation, one would argue that any set of universal statements will not provide much of what is needed for Jane to live a good life in her current circumstances.

In PN, moral statements are not viewed as facts but instead are seen as expressions of emotion or attempts to rouse them (Ayer, 1952, pp. 102-108). This emotivist position is a type of moral nihilism, where moral positions have no mind-independent enduring reality and are outside of the possibility of direct scientific study (Hunter \& Nedeliski, 2018; cf. Willard, 2018). One can scientifically study the effects of a particular behavior or belief, but science by itself cannot support claims about if these effects are good or bad. In the emotivist view, behaviors are bad only if they become the occasion for either producing offense or undesirable feelings. This would be in contrast with Jane's point of view, which focuses on her obligations to others rather than on her feelings. Interestingly, this view of emotions is philosophical (and not scientific) viewpoint that automatically rejects absolute moral knowledge. It also leads PP to a paradoxical position whereby virtue is subjective to individuals but also a universal, acultural phenomenon.

A key point is that these methods are not necessary to scientific practice. Rather, they are one of a set of possible choices regarding what constitutes good science, including scientific investigations of interest to psychologists in PP and PRS.

\section{Effects of PN for PP}

Although PN has been debunked in moral philosophy and rejected in the general philosophy of science (Willard, 2018), it remains the unspoken position in mainstream psychology and much of Western culture. Many researchers in PP and PRS would not identify as 
positivists, but they continue to practice (or unconsciously hold to) many tenets of PN. Their research often focuses on stating universal laws that apply across individuals and situations, limiting how the results can be applied to particular cases. Feelings of scientism and disdain for philosophical and traditional religious perspectives limit the ability of researchers to appreciate, understand, and engage concepts and successful methods from the past. A mechanistic/atomistic view of the human person often leads to unrealistically decontextualized and simplistic results, in contrast to more complex models found in some excellent recent PP and PRs studies (Kashdan \& Steger, 2011; McNulty \& Fincham, 2012). There is frequent discomfort with the idea that there might be universal moral truths or spiritual phenomena that influence our well-being.

Atomism and reductionism can be beneficial, as they motivate and simplify scientific study, but they also have limitations and even incoherencies. For instance, Hunter and Nedelisky (2018) note that happiness levels were high in Hitler youth groups. Shall we thus put everyone in Hitler youth groups as a "positive" intervention? Most would say no, because such a decision misunderstands the full implications of such a move. From a religious and philosophical point of view, we can say that the way of life in which the Hitler youth groups participated involves a violation of other goods that we value, such as respect for human beings regardless of ethnicity, handicaps, or religion (Ivtzan et al., 2016, p. 11). Atomistic research in PP has these limitations.

The case of Jane raises similar issues. It is impossible to understand her virtue of gratitude separately from her altruistic character, her religious faith that was nurtured in the context of her faith community, and the way of life that she has lived for many years. For her, religion is not just an activity taken up for its health benefits, but something that gives her a connectedness and prudence that reaches beyond the sum total of her other virtues. This leads to further questions. Why does her character development seem unlimited, despite the limitations of 
death and suffering? What is the role of her religious community and friends in this process? PN limits PP's ability to research these issues, because it often negates the objective desirability of a religious way of life and the ability of this life to carry one beyond "normal" in character development. Furthermore, even if we knew the general answers to these questions, we would still lack the knowledge of how to apply those principles to others in different situations. The attempts in PP to develop specific interventions (e.g., the work of Enright on forgiveness) are a step in the right direction (see Captari et al., Chapter 26, this volume), but these systems often provide inadequate guidance for how they can be deployed in the way of life of specific individuals. As Aristotle argued, what works for Jane may be unlikely to work for others.

\section{Effects of PN for PRS}

For much the first half of the $20^{\text {th }}$ century, despite a promising beginning with works of researchers like William James and Edwin Starbuck, negative views toward religion (which are endemic to PN) abounded, as seen in works by Sigmund Freud and James Leuba. These views suppressed research in PRS. Freud (1927/1961) argued that religious experience had no veridicality, was a liability, and was an emotional defense mechanism against powerlessness, and Leuba (1912) argued that psychology could assist in the formation of a way of life to take the place of religion. Leuba believed all religious experiences or needs were simply psychological phenomena that should be studied by psychologists using positivist, scientific methods (and not by religious practitioners or theologians, whom he viewed as ignorant and biased). This stance provided little incentive for psychologists to study religion, except as a kind of psychopathology.

The undermining of religious and philosophical ways of thought created a vacuum of ways to address issues of human struggle. Faith in medicine replaced them. The $18^{\text {th }}$ and $19^{\text {th }}$ centuries saw the widespread establishment of mental hospitals and the explosion of diagnostic 
categories, such as in the works of Pinel and other writers (e.g., Pinel, 1807). Moral questions were explicitly discussed from a medical perspective, as in the work of James Prichard (1835) on "moral insanity" (pp. 12-26). In the $20^{\text {th }}$ century, the medicalization of what were formerly human problems continued, despite protests (Horowitz, 2002; Jahoda, 1958).

Many researchers in PP and PRS are aware of at least some of these deficiencies.

However, professional training in psychology deeply embeds PN attitudes and methods into the thinking of budding psychologists, and even those who recognize the problems have difficulty moving beyond them. For instance, most scholars would reject the PN idea that the measures they use in studies somehow fully capture the underlying phenomena; however, researchers often speak or act as if the measured phenomena are now understood and universal naturalistic principles have been uncovered, assuming for instance the meaning of a particular phenomenon is the same or similar for all individuals and across all cultures, situations, and time periods.

\section{Historical Reactions and Resistance to PN}

Many $20^{\text {th }}$ century psychologists were unhappy with psychodynamic psychology and PN. They tried to develop alternatives, notably in the "third force" of humanistic psychology. In the first edition of Motivation and Personality, Maslow (1954) articulated a vision to move the field "toward a positive psychology" (pp. 353-363) that would study a widened set of problems. Challenging the positivist orthodoxy and psychology's sole focus on pathology, he later advocated a holistic and moral perspective based on the positive potential of the human person. This new psychology would abandon the atomistic approach and be attentive to the interactions of individual problems within the context of the well-being of the human character.

Existential, phenomenological, transpersonal, and some personality psychologists worked with goals similar to those of Maslow (Froh, 2004). For instance, Gordon Allport argued that 
even though universal "nomothetic" laws might exist and be describable, they were so general as to be worthless in understanding the individual in their "idiographic" reality (Allport, 1961, pp. 8-9). However, humanistic psychology's rejection of the PN worldview and its methods led to marginalization within professional psychology, so that its ideas are rarely referenced today even within PP (Rich, 2018). Founders of PP like Martin Seligman have sometimes claimed that the PP's movement beyond focus on pathology is a new undertaking, although this claim has been disputed (Held, 2005), and Seligman appears to have stepped back from this claim at times.

In one of the founding documents of PRS, The Varieties of Religious Experience, William James (1902/1961) displayed discomfort with the PN emphasis on natural law, quantification, and mechanistic models; he preferred the qualitative method of case study. James affirmed the usefulness of religion, as perceived by those who participated in his case studies, and he took the view that suffering, indecision, and conflict could lead to personal growth. However, he also subscribed to the PN view of moral nihilism - the idea that the truth values of religious feelings could only be evaluated based on a pragmatic standard of their effects.

After psychodynamic psychology was dethroned in the 1960s, PRS became an academic field. On the organizational side, psychologists with personal religious commitments who did not share the PN allergy to religion formed professional groupings, beginning with the American Catholic Psychological Association in the late 1940s and eventually becoming Division 36 in the American Psychological Association (Reuder, 1999). Intellectual developments included the work of Gordon Allport, who developed the concepts of intrinsic and extrinsic religious motivation, which he used to challenge the accepted connection between religion and prejudice (Allport \& Ross, 1967). Researchers and statisticians like Richard Gorsuch $(1984,1988)$ soon developed sophisticated measurement techniques for phenomena related to religion, leading to an 
explosion in empirical enquiry and publications. This research also challenged PN stereotypes of religion as psychopathology, as it empirically demonstrated that religious participation had many advantages (Bonelli \& Koenig, 2013). Gorsuch also pointed out the limitations of nomothetic naturalistic research, arguing that ideographic inquiry focused on the individual and their context was essential. However, even in its study of religion as advantageous, PRS accepted the constraints of PN methodology and its bracketing of moral issues (Hood, 2012). This strategic move bypassed psychology's opposition to non-PN viewpoints and helped it succeed.

\section{The Current Situation}

In theory, PP are PRS undermine the PN view of religion and philosophy because PRS treats religion as a worthwhile object of study, and PP allows moral virtues inspired by philosophy to form the basis for experimentation and treatment. Both areas set out to objectify their areas of study, but this objectification still assumes the naturalistic and atomistic approach of PN (Fernández-Rios \& Novo, 2012; Hill \& Hall, 2018). PP and PRS make their objects of study more objective by developing broad abstract categories like "spirituality" in order to generalize about aspects of happiness and spirituality that are purportedly universal in every culture. The approach is commonly atomistic, where for instance ethical and religious categories are separated from each other in research, even though historically their integration in the human personality was viewed as necessary. In their theorizing and research, positive psychologists frequently follow an atomistic line, which for instance focuses on one virtue without regard to other virtues or their practical context, contrary to Aristotelian virtue ethics (Vaccarezza, 2017).

In order to maintain acceptance and relevance, PP has historically been forced to deal with medical models of the good life, which can view the good life as "the phenomena of a superstate of good mental health, well beyond and above the mere absence of disability illness 
[but which] has yet to be scientifically demonstrated ... In contrast, the benefits of disease prevention and control have been tangibly demonstrated” (Barton, 1958, pp. 112-113). In consequence, many past and recent articles in PP tout benefits to physical or mental health as a reason for pursuing PP practices, which could be taken to imply a kind of implicit endorsement of medical model thinking. In the medical model, the case of Jane would revolve around possible diagnoses, rather than problems in living or exceptional aspects of her life, and in so doing, it would miss vital aspects of Jane's exceptionality.

\section{History and the Development of Concepts in PP and PRS}

Modern psychology overall has relatively little interest in human potential, especially as it is related to religion, leaving the field quite thin in terms of native concepts and theory. In order to correct this, PP and PRS have mined the resources of philosophy and theology. However, PN assumes these traditions are inferior ways of knowing the world, so PP and PRS have frequently rechristened and altered ancient terms and concepts with more acceptable, secular meanings. Unfortunately, this can undermine our ability to form the deep and clear definitions necessary for research (Reich \& Hill, 2008), and it precludes a common understanding even of basic concepts like PP (Lazarus, 2003). The rechristening of the terms "happiness" (eudaimonia) and "passion" (pathē) are especially important.

\section{The Good Life, Virtue, and Happiness}

Positive psychologists often argue their field has two approaches to understanding the good life. These are a hedonic approach (where the good life is associated with happiness or well-being) and a eudaimonic approach that connects it with the development of positive virtues.

Early treatments of emotions like happiness appeared in the work of Carol Ryff, and the hedonic approach has been more recently championed by Ed Diener (2000) under the construct 
"well-being," which he has advanced as a scientific approach to studying happiness. Diener defines well-being as a universal variable involving "peoples' positive evaluations of their lives, includes positive emotion, engagement, satisfaction, and meaning" (Diener \& Seligman, 2004, p. 1). In theory, this is a very broad concept, but in practice the term well-being is often associated with feeling good or positive subjective experiences, and discussions of issues related to life meaning were relatively few until Seligman's (2011) PERMA model. In more recent work, Diener and other positive psychologists have argued for the broadening of the well-being concept so it can better be applied to the benefit of individuals and societies.

The eudaimonic approach of Seligman and others is different in some ways, but fundamentally it is not very different from the hedonic approach. It spends considerable time talking about character development and virtue, but like the hedonic approach, the good life ultimately comes down to "subjective well-being," which is often conceptualized as an individual feeling state, even though the concept can also be applied at a group or societal level. This perspective leads research to largely coalesce around a subjective, emotivist point of view that tends to emphasize an individualistic view of the person (Ivtzan et al., 2016).

The emphasis on feelings or subjective experience can also be found in PRS discussions. In the $19^{\text {th }}$ century, modernist writers like Schleiermacher (1897) saw feelings as the center of religion. This idea was key in the work of William James (1902/1961), who argued that a psychological understanding of religion must focus on "religious feelings and religious impulses" (p. 22). James (1902/1961) identified two types of religion, one of "healthy mindedness" (connected to a straightforward experience of "religious happiness") and the other of the "sick soul" with a deeper emotional experience of "the depressed and melancholy" (connected to an acute awareness of evil; pp. 78-142). Psychologists could talk about the 
religious experience but not talk about the power of religious practices. In this situation, "spirituality" can become a more key topic than "religion," and both can be objectively studied and measured based mainly on categorizing the types of feelings people feel during their experiences. This kind of division must be approached cautiously, because for people like Jane, spirituality and religion are intertwined.

The PP understanding of eudaimonia may be based on a misunderstanding of the Classical concept. For Aristotle, eudaimonia was the positive goal of life. The term literally means "gifted by a good daimon," but translations from the past couple of centuries misleadingly render the term as "happiness." Eudaimonia actually equates with the good life (eu zên), a life lived with our natural final cause or purpose in mind (NE I.iv.2). Eudaimonia is thus not an emotion or feeling. It is a form of life (de tithemen ergon zōe tina) that involves the "exercise of the soul's powers" according to a rational principle (logos) and virtue (aretē) across a lifetime (NE, I.vii.14-16), where virtue is a disposition to act in praiseworthy ways (NE, I.xiii.20). The end of virtue is virtue itself, just as the best life is one in which a person's natural, latent faculties are exercised to the fullest ability in accordance with reason. This could be a pleasant life (hêdus), but any pleasure is a byproduct of living a rational life, not life's goal (NE, I.viii.10-11). Importantly, it is a state of being and way of life lived in the context of a community (koinonia politike ; Slife \& Richardson, 2008) and cannot be thought of in strictly individualistic terms. A key aim of Classical philosophers was the attempt to create communities that would not just discuss eudaimonia as an idea but develop it as a way of life for its followers (Hadot, 1995).

Early Christian thinkers likewise believed that human flourishing, eudaimonia or beatitudo, does not consist of bodily pleasure; instead, it consists of finding personal or communal meaning as given by God in the present moment, which may or may not have an 
accompanying sense of enjoyment (Aquinas, ST, Ia-IIae Q2 A6, A8; Q3 A8). A good life can be had even when one does not have pleasurable feelings, if one is finding meaning in their life. Beatitudo and eudaimonia are related to the kind of life we live and the meaning we find in it, so happiness can be found even in the midst of suffering - as is amply demonstrated in Jane's case.

As part of its relational view of persons and the good life, Christianity attempted to create communities that would promote the development of a good life — not just to a select few but to all who desire it. Judeo-Christian thought holds that we are created in the image of God (eikona theou), and as a result, human potential both transcends imagination and the human condition itself. Our potential as created in the image of God is both a gift and a goal (Nellas, 1987). The term deification (theōsis) describes the process of the human person becoming more like the objectively good Divinity, through God and the person mutually approaching (perichōrēsis) each other (Maximos Confessor, 1857-1858). It leads to a reconstruction of our nature beyond individual human effort, dependent on grace and aided by the Christian community. The human person was intrinsically relational, both with God and other humans (Nelson \& Slife, 2017).

\section{Virtues, Vices, Passions, and Emotions}

From its modern inception, PP has attempted an analysis of virtues and positive feelings. A key work was that of Peterson and Seligman (2004), who made an inventory of strengths, called Values in Action, based on a selective review of materials from the Western, South, and East Asian traditions, using the atomistic criteria that "each element can be defined and measured independently of the other elements" (p. 2). The definitions of the values usually differed from their original meaning, and Peterson and Seligman (2004) admitted "it proved beyond our ability to specify a reasonable theory" that would underlie a taxonomy (p. 6). The virtues are viewed as isolated scientific entities in that they can be measured, and they are thus 
claimed to express intrinsic and universal truth. Individual instances of religion/spirituality in history are summarized in one word, such as transcendence (Slife \& Richardson, 2008). Such operational definitions can be highly reliable, but they may have questionable validity.

The PP analysis of virtue differs substantially from the Classical view. For Aristotle, virtues (aretē) are habits that operate in unison, forming the stable character traits (hexis) necessary for eudaimonia. For example, acquiring prudence (phronessis) is impossible without possessing the other virtues (NE, VI.xii.10). A unity-of-virtues view allowed Aristotle to claim that health is associated with a harmonious balance of the virtues, not the pursuit of individual virtues. He also argued that it was impossible to understand virtues without considering vices, habits that undermine our pursuit of the good life. Many early Christian authors were trained in Classical thought and creatively synthesized the ideas of virtues, practical reasoning (phronesis) in individual situations, and eudaimonia with Christian theology. As in Aristotle, negative characteristics needed to be discussed in order to identify and encourage positive growth better.

Another difference between Classical and PP concepts revolves around the terms "passions" (pathē) and "emotions." Classical authors were primarily interested in the passions, which were viewed not just as emotions but as complex cognitive and feeling states that interfered with the good life. Christian thinkers developed sophisticated mental practices to eliminate the barriers to flourishing produced by inordinate passions, sin, and ordinary human limitations. This changed in the Early Modern period, when the term passions (with its philosophical and theological linkages) began to be replaced by the term emotion (Descartes, 1909; Dixon, 2003). Emotions like pleasure became the key to eudaimonia, and the PN reduction of ethics to emotion tied the worth of virtue to feelings (Willard, 2018). Note that this is not a scientific "truth" but a historically conditioned position that we may or may not adopt. 


\section{Effects of Conceptual Developments on PP and PRS}

In PN, a universal human nature selfishly prioritizes individual survival and happiness, which transferred to the Western cultural movement of modernism as a value for individualism (Sugarman, 2007; Taylor, 1989). The growing restlessness in PP seems related to discomfort with these roots in inherited modernist individualism and the problematic methodology of atomistically separating virtues from each other. Post-Enlightenment sources are the only ones that tend to make the cut, and pre-Enlightenment sources tend to be interpreted within $20^{\text {th }}$ or $21^{\text {st }}$ century ideological priorities, apart from the relationality of persons and virtues.

Some researchers in PP want to move the field in new directions. The use of concepts like forgiveness could derive consistent meanings based on Classical concepts. Paul Wong (2011, p. 69) has suggested a "PP 2.0" that would focus on meaning and other issues. Some authors also feel uncomfortable with the current emphasis on positive emotions, neglecting to address negative emotions and vices and the positive value of suffering in developing virtue (Kidd, 2015; Kristjánsson, 2013, pp. 86-150; Lazarus, 2003). In addition, a rising chorus notes how most PP research has been conducted within the cultural framework of the Western values and methods of the 20th and $21^{\text {st }}$ centuries, especially in the individualistic focus on subjective happiness rather than communal flourishing (Koop \& Fave, 2013). An allergy to religious and philosophical ways of thinking is problematic, because religious and philosophical traditions can provide conceptual categories and structures that can strengthen psychological investigations into questions involving life's meaning (Ivtzan et al., 2016; Kaczor, 2017). Concern over decontextualized models has led some researchers to begin exploring the use of qualitative methods, although in practice many of these studies diverge from basic tenets of this type of research, as used in other social science disciplines like anthropology. 


\section{Conclusion}

Many helpful premodern ideas about the human person and various ways of life are difficult to access today. A PN approach, based in antiquated philosophy of science, rejects a historical understanding of how the good life can be studied and achieved in various religious and cultural contexts. A movement away from positivist views of virtues - and towards an interdisciplinary pool of Classical and Christian or other religious strategies (e.g., material drawn from Buddhism) for maximizing positive potential—could benefit PP and PRS research and practice. This chapter has offered a foundation for correcting these problems in PP and PRS.

PP could improve its potential contributions to therapy as it moves away from universal naturalistic understandings of virtue to definitions more appropriate to diverse contexts. With less vague generalization and more basis in specific historical and religious examples of virtues and religious practices and beliefs, PP and PRS will be more empirical. An understanding of the complexity of virtues can reconcile the points of view of the psychologist and the patient more quickly and enable a patient to become more proficient in virtuous habits appropriate to their practical individual situation. As informed by PRS, one key PP goal should be helping people cultivate objectively good virtues or habits that work together harmoniously to support character stability and enhance health and well-being (see Davis et al., Chapter 18, this volume).

Jane is a rare example of a person who lives the good life under extraordinary circumstances. Wouldn't it be wonderful if PP and PRS could make Jane's resources accessible to others? This $21^{\text {st }}$ century potential cannot be attained using approaches mired in $19^{\text {th }}$ century prejudices. Instead, PP and PRS can work together to bring the good life to an ever-expanding number of people in our global society. Patients like Jane, positive and concerned about the needs of others even in circumstances that often dampen character, are realistic possibilities. 


\section{References}

Allport, G. (1961). Pattern and growth in personality. Holt, Rinehart, and Winston.

Allport, G. W., \& Ross, J. M. (1967). Personal religious orientation and prejudice. Journal of Personality and Social Psychology, 5(4), 432-443. https://doi.org/10.1037/h0021212

Aquinas, T. (2012). Summa theologiae, Prima Secundae, 1-70 (L. Shapcote, Tr., J. Mortensen \& E. Alarcón, Eds.). Aquinas Institute for the Study of Sacred Doctrine.

Aristotle. (1926). Nicomachean ethics (H. Rackham, Tr.). Harvard University Press.

Ayer, A. (1952). Language, truth and logic. Dover Publications.

Barton, W. (1958). Viewpoint of a clinician. In M. Jahoda, Current concepts of positive mental health (pp. 111-119). Basic Books. https://doi.org/10.1037/11258-007

Barton, Y., \& Miller, L. (2015). Spirituality and positive psychology go hand in hand: An investigation of multiple empirically derived profiles and related protective benefits. Journal of Religion and Health, 54, 829-843. https://doi.org/10.1007/s10943-015-0045-2

Bonelli, R. \& Koenig, H. (2013). Mental disorders, religion and spirituality 1990 to 2010: A systematic evidence-based review. Journal of Religion and Health, 52, 657-673. https://doi.org/10.1007/s10943-013-9691-4

Comte, A. (1839). Cours de philosophie positive, Tome quatrième. Bachelier, Libraire pour les Mathématiques.

Descartes, R. (1909). Les passions de l'ame. In C. Adam \& P. Tannery (Eds.), Euvres de Descartes, XI.djvu (pp. 301-488). Léopold Cerf.

Diener, E. (2000). Subjective well-being: The science of happiness and a proposal for a national index. American Psychologist, 55(1), 34-43. https://doi.org/10.1037//0003-066X.55.1.34 
Diener, E. \& Seligman, M. (2004). Beyond money: Toward an economy of well-being. Psychological Science in the Public Interest, 5(1), 1-31. https://doi.org/10.1111/j.0963-7214.2004.00501001.x

Dijksterhuis, E. (1961). The mechanization of the world picture: Pythagoras to Newton (C. Dikshoorn, Tr.). Princeton University Press.

Dixon, T. (2003). From passions to emotions: The creation of a secular psychological category. Cambridge University Press.

Feigl, H. (1949). Operationism and scientific method. In H. Feigl, \& W. Sellars (Eds.), Readings in philosophical analysis (pp. 498-509). Appleton-Century-Crofts.

Fernández-Rios, L. \& Novo, M. (2012). Positive psychology: Zeigeist (or spirit of the times) or ignorance (or disinformation) of history? International Journal of Clinical and Health Psychology, 12(2), 333-344.

Freud, S. (1927/1961). The future of an illusion. W.W. Norton.

Froh, J. (2004). The history of positive psychology: Truth be told. NYS Psychologist, 16, 18-20. https://www.nyspa.org/page/NYSPsychologist

Gorsuch, R. (1984). Measurement: The boon and bane of investigating religion. American Psychologist, 39(3), 228-236. https://doi.org/10.1037/0003-066X.39.3.228

Gorsuch, R. (1988). Psychology of religion. Annual Review of Psychology, 39, 201-221. https://doi.org/10.1146/annurev.ps.39.020188.001221

Hadot, P. (1995). Philosophy as a way of life. Blackwell.

Held, B. (2005). The "virtues" of positive psychology. Journal of Theoretical and Philosophical Psychology, 25(1), 1-34. https://doi.org/10.1037/h0091249 
Hill, P. \& Hall, M. (2018). Uncovering the good in positive psychology: Toward a worldview conception that can help positive psychology flourish. In N. Brown, T. Lomas \& F. Eiroa-Orosa (Eds.), The Routledge international handbook of positive psychology (pp. 245-262). Routledge. https://doi.org/10.4324/9781315659794-19

Hood, R. (2012). The history and current state of research on psychology of religion. In L. Miller (Ed.), The Oxford Handbook of Psychology and Spirituality (pp. 7-20). Oxford University Press. https://doi.org/10.1093/oxfordhb/9780199729920.013.0001

Horowitz, A. (2002). Creating mental illness. University of Chicago Press.

Hunter, J. \& Nedelisky, P. (2018). Science and the good: The tragic quest for the foundations of morality. Yale University Press.

Ivtzan, I., Lomas, T., Hefferon, K. \& Worth, P. (2016). Second wave positive psychology. Routledge.

Jahoda, M. (1958). Current concepts of positive mental health. Joint Commission on Mental Illness and Health Monograph Series No. 1. Basic Books.

James, W. (1902/1961). The varieties of religious experience. Collier.

Kaczor, C. (2017). On definitions and traditions. Psychology of Religion and Spirituality, 9(3), 296-298. http://dx.doi.org/10.1037/rel0000128

Kashdan, T. \& Steger, M. (2011). Positive psychology: Where did it come from, where is it going? In K. Sheldon, T. Kashdan \& M. Steger (Eds.), Designing positive psychology: Taking stock and moving forward (pp. 9-21). Oxford University Press. https://doi.org/10.1093/acprof:oso/9780195373585.003.0002

Kidd, I. J. (2015). Transformative suffering and the cultivation of virtue. Philosophy, Psychiatry, \& Psychology, 22(4), 291-294. https://doi.org/10.1353/ppp.2015.0048 
Koop, H. \& Fave, A. (Eds.). (2013). Well-being and cultures: Perspectives from positive psychology. Springer.

Kristjánsson, K. (2013). Virtues and vices in positive psychology: A philosophical critique. Cambridge University Press.

Lazarus, R. (2003). The Lazarus manifesto for positive psychology and psychology in general. Psychological Inquiry, 14(2), 173-189. https://doi.org/10.1207/S15327965PLI1402_04

Leuba, J. H. (1912). A psychological study of religion: Its origin, function and future. Macmillan.

Martin, M. (2007). Happiness and virtue in positive psychology. Journal for the Theory of Social Behavior, 37(1), 89-103. https://doi.org/10.1111/j.1468-5914.2007.00322.x

Maslow, A. (1954). Motivation and personality. Harper \& Row.

Maximos Confessor. (1857-1858). Questions ad Thalassium de scriptura sacra. In J.P.-Migne (Ed.) Patrologiae cursus completus, Series Graeca, Tomus XC (cols. 244-785). Imprimerie Catholique.

McNulty, J. \& Fincham, F. (2012). Beyond positive psychology? Toward a contextual view of psychological processes and well-being. American Psychologist, 67(2), 101-110. https://doi.org/10.1037/a0024572

Nellas, P. (1987). Deification in Christ: Orthodox perspectives on the nature of the human person. St. Vladimir's Seminary Press.

Nelson, J. \& Slife, B. (2013). Theoretical and epistemological foundations of the psychology of religion. In L. Miller (Ed.), Oxford Handbook of Psychology and Spirituality (pp. 21-35). Oxford University Press. https://doi.org/10.1093/oxfordhb/9780199729920.013.0002 
Nelson, J. \& Slife, B. (2017). A new positive psychology: A critique of the movement based on early Christian thought. The Journal of Positive Psychology, 12, 459-467. http://dx.doi.org/10.1080/17439760.2016.1228006

Peterson, C., \& Seligman, M. E. P. (2004). Character strengths and virtues: A handbook and classification. American Psychological Association.

Pinel, P. (1807). Nosographie Philosophique, ou la Methode de l'Analyse Applicquee a la Médecine, 3 Tome (Troisèmé édition revue, corrigée et augmentée). Chez J. A. Brosson.

Prichard, J. (1835). A treatise on moral insanity and other disorders affecting the mind. Sherwood, Gilbert, and Piper.

Reich, K. \& Hill, P. (2008). Quo vadis psychology of religion? Introduction to the special section. Archive for the Psychology of Religion, 30, 5-18. https://doi.org/10.1163/157361208X316935

Reuder, M. (1999). A history of Division 36 (Psychology of Religion). In D. Dewsbury (Ed.), Unification through division: Histories of the divisions of the American Psychological Association (Vol. 4, pp. 91-108). American Psychological Association. https://doi.org/10.1037/10340-004

Rich, G. (2018). Positive psychology and humanistic psychology: Evil twins, sibling rivals, distant cousins, or something else? Journal of Humanistic Psychology, 58(3), 262-283. https://doi.org/10.1177/0022167817698820

Schleiermacher, F. (1897). Der christliche Glaube nach den Grundsätzen der evangelischen Kirche. O. Hendel.

Seligman, M. (2002). Authentic happiness: Using the new positive psychology to realize your potential for lasting fulfillment. Free Press. 
Seligman, M. (2011). Flourish. Free Press.

Seligman, M. (2019). Positive psychology: A personal history. Annual Review of Clinical Psychology, 15, 1-23. https://doi.org/10.1146/annurev-clinpsy-050718- 095653

Seligman, M. \& Csikszentmihalyi, M. (2000). Positive psychology: An introduction. American Psychologist, 55(1), 5-14. https://doi.org/10.1037//0003-066X.55.1.5

Slife, B. \& Richardson, F. (2008). Problematic ontological underpinnings of positive psychology: A strong relational alternative. Theory \& Psychology, 18(5), 699-723. https://doi.org/10.1177/0959354308093403

Sugarman, J. (2007). Practical rationality and the questionable promise of positive psychology. Journal of Humanistic Psychology, 47(2), 175-197. https://doi.org/10.1177/0022167806297061

Taylor, C. (1989). Sources of the self. Cambridge University Press.

Vaccarezza, M. (2017). The unity of the virtues reconsidered: Competing accounts in philosophy and positive psychology. Review of Philosophy and Psychology, 8, 637-651. http://doi.org/10.1007/s13164-017-0334-7

VandenBos, G. (Ed.). (2015). APA Dictionary of Psychology ( $2^{\text {nd }}$ ed.). American Psychological Association.

Willard, D. (2018). The disappearance of moral knowledge (S. Porter, A. Preston, \& G. Ten Elshof Eds.). Routledge.

Wong, P. (2011). Positive psychology 2.0: Toward a balanced interactive model of the good life. Canadian Psychology, 52(2), 69-81. https://doi.org/10.1037/a0022511

Wootton, D. (2015). The invention of science: A new history of the scientific revolution. HarperCollins. 


\title{
Chapter 3:
}

On the Integration of Positive Psychology and the Psychology of Religion/Spirituality: Logical, Normative, and Methodological Questions

\author{
Steven L. Porter ${ }^{1}$, Jason Baehr ${ }^{2}$, Tenelle Porter ${ }^{3}$, and Robert C. Roberts ${ }^{4}$ \\ ${ }^{1}$ Rosemead School of Psychology and Talbot School of Theology, Biola University \\ ${ }^{2}$ Department of Philosophy, Loyola Marymount University \\ ${ }^{3}$ Department of Psychology, University of Pennsylvania \\ ${ }^{4}$ Department of Philosophy, Baylor University
}

\begin{abstract}
Correspondence concerning this chapter should be addressed to Steven L. Porter, Rosemead School of Psychology, Biola University, 13800 Biola Ave, La Mirada, CA 90639. Email: steve.porter@biola.edu
\end{abstract}




\begin{abstract}
When it comes to the integration of positive psychology and the psychology of religion/spirituality $(\mathrm{R} / \mathrm{S})$, there are three second-order, philosophical questions that need answering: Can these two fields be integrated? Should these two fields be integrated? And, if so, how is it best to integrate these two fields? Although this chapter touches on the logical and normative questions, it is the third, methodological question that receives the greatest attention. We argue that although, from a philosophical perspective, there are no methodological barriers to integrating these two subfields, there is a methodological bonanza in their integration. The golden opportunity is for integrative researchers to abandon a methodological exclusivism that privileges the research methods of the natural sciences in favor of a methodological pluralism that critically engages the philosophical schools and religious/spiritual traditions within which features of human well-being and religious/spiritual life are located. This more eclectic epistemology will provide a broader evidential basis for integrative conclusions and will help connect those conclusions to the realities and complexities of human lives.

Keywords: positive psychology, psychology of religion and spirituality, philosophical psychology, integration, methodology
\end{abstract}




\section{Chapter 3: On the Integration of Positive Psychology and Psychology of Religion/Spirituality: Logical, Normative, and Methodological Questions}

In one way or another, every human person lives out answers to four fundamental questions of human existence: What is real? What is the good life? What does it mean to be a good person? And, how does one become a good person? Even if one's answers are that there are no right answers, that will surely manifest in one's life. Others may be absolutely certain they have arrived at the truth regarding these matters, and perhaps in part due to that certainty, their answers can lead to a lack of empathy for those who disagree. Still, there will be persons who find their way into a vision of reality, the good life, the good person, and the process of becoming a good person that strike many as beautiful and compelling. Clearly, much is at stake when answering these fundamental human questions, and we need all the help we can get to arrive at answers that are good, better, and best. Although we can make do without knowledge, it helps when we are guided aright. To that end, research and theory coming out of the interaction of positive psychology and the psychology of religion/spirituality (R/S) holds great promise.

With the existential import of this discussion in mind, we investigate three second-order, philosophical questions that overlay any attempt to integrate theoretically these two (and any other) fields of study (Porter, 2004). First, we have the logical question: can these fields be integrated? Second, we address the normative question: should these fields be integrated? And, lastly, we approach the methodological question: how should these fields be integrated?

\section{The Logical Question: Can These Fields Be Integrated?}

Is there a logical relationship between positive psychology and psychology of R/S, such that meaningful theoretical integration is possible? By meaningful theoretical integration we have in mind interaction among the two fields' concepts, theories, methodologies, data, and 
practical implications, with the ultimate aim of increased understanding of human functioning. It is important to highlight that our focus is the theoretical integration of ideas from these respective fields and not, for instance, the clinical integration of interventions from these two fields. Whereas there are important things to be said about clinical and other forms of applied integration, we are concerned with the prior issue of the interrelationship of ideas. The logic, then, of such integration is quite clear: we need (a) two demarcated fields of study, (b) that are both attempting to make truth-claims about the nature of reality, and (c) the bringing together of which may yield increased understanding. Let us approach these items in turn.

When it comes to (a) — having two clearly demarcated fields of study—psychologists of $\mathrm{R} / \mathrm{S}$ are attempting to "come to an understanding of the psychological bases of religious belief, experience, and behavior" (Emmons \& Paloutzian, 2003, p. 377), whereas positive psychology is seeking to come to an understanding of hedonic and eudaimonic well-being (Ryan \& Deci, 2001). Given these descriptions, it would be sensible to think that the study of one field would naturally involve the other, because at some level, most religions/spiritualities are concerned with questions of human well-being, and contrariwise, investigation into human well-being will eventually have to consider the place of R/S, if any. Despite reasonable overlapping in this manner, we can sensibly demarcate these two subject matters: one field is primarily attentive to the psychology of human well-being (whether or not that includes R/S), and the other is mainly focused on the psychology of R/S (whether or not that includes notions of human well-being).

Besides having two clearly demarcated fields, we also need to ensure that (b) both subdisciplines are attempting to make truth-claims about a unified reality. The only manner in which the claims of one field can bear logical relations to the claims of another field is if both claims refer to an interconnected reality. Some might balk at the realism assumed in this way of 
countenancing integration. However, at this juncture, we simply mean to distinguish fields that are attempting to describe a real world from those areas of study that are by definition fictional or antirealist. Although it might be metaphorically rich, for instance, to integrate the Harry Potter series with the study of plate tectonics, the Harry Potter series is a work of fiction that is not attempting to describe the nature of the actual world. Rather, Rowling's books construct a fantasy world, whereas plate tectonics attempts to describe the actual structure of the earth's crust. It is not that the wonderful world of wizardry and the presumably wonderful world of plate tectonics are so far apart. Rather, it is that they are referencing two logically distinct worlds, one of which does not actually exist. Although the fictitious world of Harry Potter might end up providing a metaphor that aids in real-world plate tectonics, and Rowling's understanding of plate tectonics might make for a great scene in the Harry Potter stories, there would be something illogical about trying to expand our knowledge of, for instance, an earthquake earlywarning device by pouring over Book Five of the Harry Potter series. Of course, it would not be illogical to do so if we discovered that Rowling had carefully studied plate tectonics and was attempting to advance a theory about earthquake early-warning devices in her otherwise fictional Book Five. But that just shows the point: the only manner in which the claims of one field can be logically related to the claims of another field is if both fields are making claims that refer to a unified reality. Because researchers in both positive psychology and the psychology of R/S put forward claims about actual human persons and their functioning in real life, things look good for there being a logical relation between these two areas of investigation.

And yet, there is another, more vexing assumption of realism in what has been said thus far. Psychologists study agreed-upon constructs of human psychological phenomena and, it might be thought, not the psychological phenomena themselves. Indeed, on some metaphysical 
accounts, all that exists is a socially constructed reality with no "real" world beyond agreed-upon conceptualizations (Burr, 2015). If positive psychologists are constructing worlds of hedonic/eudaimonic well-being and psychologists of R/S are constructing worlds of religious and spiritual ideas, experiences, and behavior, how would one determine whether there can be logical relationships between these two socially constructed "realities"?

In response, we distinguish between moderate and radical social constructionism. The latter, which philosophers call "antirealism," is the view that we have no access to the "realities" that our conceptual schemes "refer" to- - the "realities" are in fact fictions of our own making. The moderate view maintains that human conceptualizations of the world are socially embedded and, therefore, socially influenced interpretations or models of reality. This way of understanding social constructionism is not antirealist about the nature of reality itself. Rather, the idea is that there exists a mind-independent reality that can be accurately understood and described through human thought and language, to greater and lesser degrees. Moderate social constructionism appreciates the subjective and social nature of the mind-world relationship, while maintaining that claims about reality still have a truth-value. Consequently, logical relations such as deductive entailment, inductive inference, and probabilistic/statistical reasoning are tenable.

A radical social constructionism adds an antirealist ontological claim to this moderate view. The radical social constructionist maintains there is no mind-independent reality beyond humanly agreed-upon conceptualizations. Social constructions make things what they are in such a way there is no nonconstructed court of appeals for the truth or falsity of human perspectives about the world (Gergen, 1999). If radical social constructionism is true (although it is difficult to know how we could determine that), then logical relations would be constituted by social agreement as well. If enough participants consented to use language to discuss these two areas of 
discourse, then it would be "logical" to do so based on social agreement. In principle, there could be no objection to this move. For the current chapter to proceed on this basis would be for us to try to persuade you, the reader, to join us in thinking about these issues in the manner we do. Because epistemic goods— - like truth, understanding, and knowledge — are no longer the goal, we would have to proffer some sort of pragmatic motivation for our call to solidarity (Rorty, 1985). Of course, a problem arises when this pragmatic motivation for social agreement (and/or what is socially agreed upon) requires criticism. If all reality is socially constructed, there is no basis to critique those social constructions of reality that do harm or otherwise fail to achieve agreement.

Fortunately, there is another way to proceed - via embracing moderate social construction alongside a commitment to ontological realism about human psychological phenomena (Boghossian, 2006; Richardson \& Guignon, 2008). Although it is essential to appreciate that human knowers are subjectively interpreting reality through culturally embedded and socially influenced interpretive frameworks, that admission does not bar humans from attempting to collect evidence and develop theories that to varying degrees approximate the way people are. On this view, the second condition for integration to be logically possible is secured: (b) both positive psychology and psychology of R/S are making truth claims about reality.

With the first two conditions met, we turn to consider whether the last condition holds: (c) whether the bringing together of the two fields may yield increased understanding of human psychology. First, a word about "bringing together." The simplest way to bring these fields together is for those researching positive psychology to include religious/spiritual concepts and variables in their research and vice versa for psychologists of R/S. Presumably, this strategy will largely consist of testing for interrelationships between features of human well-being (e.g., virtues and positive emotions) and features of human religious/spiritual life (e.g., meditation and 
perceived experiences of God). Will such testing yield increased understanding of human nature and functioning? Presumably the answer is, yes. Even if there is no statistically significant relationship between positive psychological qualities and religious/spiritual life, that finding itself would be breaking news. Of course, if there are significant correlations, that too would be important. But with these considerations we now will turn from the logical question—can we integrate these two fields? - to the normative question, would it be valuable to do so?

\section{The Normative Question: Should These Fields Be Integrated?}

Although we contend there are no logical barriers to the meaningful theoretical integration of positive psychology and psychology of R/S, we now turn to exploring the value of such integration. That we can integrate does not mean that we necessarily ought to. Because intradisciplinary integration is a theoretical affair, the normative question is whether such integration will further human understanding in ways that are perceived as value-added.

On this score, there appears much in favor of the integration of positive psychology and R/S and little to say against it. Again, because humans cannot avoid bumping up against issues of human well-being and questions of religious and spiritual significance, understanding the interrelationships between these two domains of life has an immediate appeal. And there is good reason to think there are important interconnections. For one, many historically situated philosophical and religious/spiritual schools of thought have maintained that there are significant connections between ethics and religious belief, virtue and spiritual practice, human flourishing and religious behavior, and so on (e.g., Hare, 2009). A variety of psychological studies already suggest relevant connections (e.g., Davis et al., 2012; Schnitker et al., 2017; VanderWeele, 2017; Wilkins et al., 2012; for reviews, see Davis et al., Appendix 18.S2, this volume). It would be of immense benefit if psychological study could help locate reliable and unreliable 
religious/spiritual paths to virtuous, meaningful lives. And because increasing numbers of people are deidentifying with religion (e.g., Van Tongeren et al., 2021), it would also be helpful to determine how well-being is affected in the absence of religion and/or spirituality.

Beyond the benefits of increased understanding of personal development, there are an array of social ills that have to do with a disconnect between religion/spirituality and human flourishing. For instance, the term "religiously motivated" far too often qualifies things like racism, gender discrimination, homophobia, ethnic cleansing, hate crimes, terrorism, and so on. It is difficult to think of a domain of contemporary life that is more fraught with animosity than moral failures by religious persons and institutions. If the integration of these two areas can help realign religion and justice, that would bring tremendous good (e.g., Palmer \& Burgess, 2020).

Although the likelihood of valuable integration looms large, we also need to consider normative reasons against integration. Perhaps one reason is the track record of religion alluded to above. It seems there are far too many cases of religious persons and institutions being on the wrong side of justice. Hunter and Nedelisky (2020) have noted the "epic failure of religion to provide a unifying and peaceable solution to the problems of difference and complexity in the modern world" (p. 212). One might think that given this failure, positive psychology needs to steer clear of religion in order to make progress in conceptualizing and promoting human flourishing. And yet, there is a more complex story to tell about the historical relation of religion to human well-being. Religion has not only perpetuated injustice. It has also inspired reform movements and helped produce social reformers (Palmer \& Burgess, 2020). Rather than neglecting its study, the continued threat of religiously fueled violence and the promise of religiously motivated compassion should lead to prioritizing the study of R/S in relation to human flourishing. 


\section{The Methodological Question: How Should These Fields Be Integrated?}

Having seen that we can and should bring together the study of positive human qualities and religion/spirituality, our final query is how best to do so. Initially, things look rather straightforward in this regard. Seeing that both fields utilize a range of similar quantitative and qualitative methods (see Tsang et al., Chapter 8, this volume), combining research programs should be fairly seamless. In principle, researchers in each field can include the theories, constructs, measures, and data from the other field, with the goal of testing relationships between features of well-being and religious/spiritual life. Voila, successful theoretical integration!

The very existence of this volume attests to a more complicated situation. For instance, some have suggested that positive psychology research tends to be more rigorous than R/S research, so for there to be effective integration, there needs to be an increase in the rigor of psychology of R/S (Worthington et al., in press). If that claim is true, the way forward is clear: psychologists of R/S need to up their scholarly game. Yet that is a practical matter, not a philosophical one. Indeed, whatever rigor-related difference exists between the two fields, both disciplines operate within the same social scientific milieu that tends to value randomized controlled experiments as the gold standard (McCall \& Green, 2004). How closely each field's research designs measure up to that standard is itself an empirical question.

However, lingering in the background of this discussion, there is a philosophical question about method that is relevant to these fields' integrative potential. The question can be put like this: Why think features of hedonic/eudaimonic well-being and religious/spiritual life are adequately captured by the empirical methods of psychology, particularly quantitative-statistical methods? Obviously, this question is a skeptical one (as philosophers are wont to do), and it may seem like it is headed in the wrong direction for a volume defending the integration of positive 
psychology and R/S. Although the question is skeptical, our suggested way forward is constructive. One answer to how we might best integrate these two subfields is to encourage an integrative methodology that goes beyond narrowly empirical sources/methods and includes nonquantitative sources/methods, in order to capture more of the irreducible nature of the phenomena in question. In what follows, we first propose an integrative methodology and then turn to several considerations in its favor.

\section{The Nature of Methodological Pluralism}

The integrative approach we have in mind—what has been called methodological pluralism (Roth, 1987; cf. Gantt \& Melling, 2009; Slife \& Reber, 2021)—might be thought of as a broaden-and-build epistemology that allows premises from philosophy, religious/spiritual traditions, phenomenological experience, and other nonquantitative sources into the evidence base, alongside quantitative-statistical findings. Obviously, this is a more radical sort of mixedmethods approach that goes beyond simply the call for the more frequent use of qualitative methods (e.g., Davis et al., 2016; Gergen et al., 2015).

To be clear, our call for methodological pluralism is consistent with the desire for more rigor in research. Psychology should do empirical science as rigorously as possible. It is just that psychology should also do scientia as rigorously as possible. The Latin scientia signals a premodern view of knowledge in which evidence from philosophy, religion, historical traditions, phenomenological experience, and the like entered rational investigation, alongside quantitative evidence. Ours is a call for positive psychologists and psychologists of R/S to take this broader expanse of evidence into consideration when coming to their conclusions, in the same manner that at least some philosophers and religious scholars take on board the empirical evidence of the sciences when coming to their philosophical and religious conclusions. Our proposal then for 
how best to integrate positive psychology and psychology of R/S is to be even more integratively inclusive and move towards inter or even transdisciplinary integration (McGrath, 2019). This would involve looking to virtue ethics, moral philosophy, existentialism, Aristotelianism, Confucianism, Thomism, personalism, Darwinism, phenomenology, analytic theology, Buddhist thought, Jewish theology, Islamic theology, Hindu theology, religious studies, philosophical naturalism, and so on. Again, these perspectives would be consulted not only in conceptualizing the phenomena to be studied (and generating meaningful research questions and testable hypotheses) but also in interrogating the empirical results.

In this effort, we heed the call of Emmons and Paloutzian (2003) who recommended a "multilevel interdisciplinary paradigm" (p. 395) for research in the psychology of R/S. They argued: "A single disciplinary approach is incapable of yielding comprehensive knowledge of phenomena as complex and multifaceted as spirituality" (p. 395). Building off this claim, Belzen and Hood (2006) proposed that a multilevel interdisciplinary paradigm "cannot be achieved without acceptance of...methodological pluralism" (p. 6). They identified three major types of methods. The first type includes the familiar quantitative-statistical methods of psychology. The second type "includes research methods from a broader social-scientific range" (Belzen \& Hood, 2006, p. 10), such as qualitative interviews, ethnographies, and biographical analysis. The third type "relies heavily on data and insights from disciplines like history, theology, literature, and cultural studies” (Belzen \& Hood, 2006, p. 10). Although Belzen and Hood (2006) acknowledged that many topics in psychology can get along well with using the first type of research methodology alone, they contended that the personal nature of R/S (and we would add hedonic/eudaimonic well-being) require the second and third types of methods as well. 
An openness to methodological pluralism can be found within positive psychology as well. Schnitker and Emmons (2017) have argued that researchers from positive psychology "have neglected to fully engage theological and philosophical perspectives throughout the research process" (p. 239). They envision fostering "cross-disciplinary conversation that engages current scholarship in psychology of religion and spirituality, positive psychology, theology, and philosophy" (pp. 239-240). Similarly, Rich (2017) has called for positive psychology to embrace a broad range of qualitative and interdisciplinary methods that are "by nature more open and pluralistic than the quantitative approach associated with logical positivism" (p. 229). Lomas and colleagues (2020) have forecasted a coming "third wave" of positive psychology that involves an "epistemological broadening" (p. 1) that "goes beyond the boundaries of psychology to incorporate knowledge and research methodologies from a broad range of fields" (p. 11).

In championing methodological pluralism, our negative concern is with methodological exclusivism. By methodological exclusivism, we mean the tendency to exclude sources of knowledge that do not meet the standards of the quantitative-statistical methods. Rich (2017) has gotten at the spirit of this sort of exclusivism in his encouragement to psychological researchers to be "vigilant against any sort of 'physics envy' in which they mistakenly feel that using numeric data and quantitative methods in itself makes them more 'scientific"' (p. 222; cf. Friedman \& Brown, 2018). Below we contend that this exclusivism is driven, in part, by a lingering hangover from $20^{\text {th }}$ century logical positivism as well as the cultural authority that is found in some settings by hewing to the experimental protocols of the natural sciences (that is, scientism; see Nelson \& Canty, Chapter 2, this volume). Richardson and Guignon (2008) have argued that positive psychology problematically assumes scientism, and the instead have recommended thinking of positive psychology as an "interpretive social science rather than an 
aggressively 'scientific' and heavily instrumental one” (p. 623). They have suggested: “An interpretive psychology would draw on a wider array of methods or approaches to understanding," including "[c]ultural history, theoretical and philosophical analysis, some degree of cultural and political engagement, spiritual experiences and disciplines, to mention just a few, along with varied forms of quantitative and qualitative research" (p. 623).

Our point is not to generate skepticism regarding quantitative-statistical methodologies but rather to point out the epistemic inadequacy of such methodologies alone. People filter scientific findings through their personal experience, family history, cultural traditions, philosophical points of view, religious/spiritual outlooks, and political commitments. "There must be something wrong with the study" or "This research must be biased" are tempting and all-too-frequent responses when the proclaimed results of a study do not match up with what people deeply feel or have reasons to believe true. "We believe in science," says the popular poster, but many people do not believe in scientism, where scientism refers to the view that science-backed findings are the only source of knowledge and/or automatically override any other claims to knowledge. This latent skepticism is particularly at play when it comes to positive psychology and psychology of R/S, given how central human well-being and R/S are to personal experience and to culturally embedded traditions. Doubts over quantitative-statistical findings can be soothed when they are considered alongside evidence from philosophy, religion, history, and phenomenological investigation. Drawing off this broader evidence-base can provide confirmation, explanation, elaboration, and/or qualification of the empirical results.

There are at least two ways such interdisciplinary integration might unfold. First, the researcher might allow philosophical, religious, and spiritual premises into their evidence base and argumentation. This is to do research from an explicit point-of-view, which many have 
argued is inescapable (see Gantt \& Melling, 2009). The philosophical naturalist, the Ignatian, the Zen Buddhist, the humanist, the Aristotelian, the Confucian, and so on announce that their research is being conducted based on certain principles from those traditions, both in the conceptualization of the study and in the evaluation of the empirical results. The second integrative route is for the researcher to bracket any explicit point-of-view and hypothetically consider additional sources of evidence in the discussion of the empirical results. For instance, a study that shows that mindfulness practices decrease anxiety can be interrogated from the perspectives of Zen Buddhism, contemplative Christianity, and philosophical naturalism. The value here is to consider the interpretations, objections, and affirmations of one or more salient perspective. The researcher can discuss these matters hypothetically: if one is a Zen Buddhist, one will likely respond in such-a-such way, but if one is a philosophical naturalist, one will likely respond in this other manner. On either route, the empirical findings are not presented either in an epistemological vacuum or within a presumed secular frame (Taylor, 2007).

\section{Four Considerations in Favor of Methodological Pluralism}

Having described methodological pluralism, we propose four considerations in its favor.

The first is a reminder that, within contemporary psychology, any tendency to privilege quantitative-statistical methods at the exclusion of nonquantitative approaches is unwarranted (Nelson \& Slife, 2012). Many have argued that the social sciences tended towards a methodological unity with the natural sciences in part due to the historical rise (in the 1920s and 1930s) of what came to be known as "logical positivism" championed by a group of philosophers referred to as the Vienna Circle (Gergen et al., 2015; Nelson, 2006; Robinson, 1995; Toulmin \& Leary, 1985). For instance, a series of eight monographs entitled "Unified Science" were written by those associated with the Vienna Circle. Each volume sought to extend 
the quantitative experimental designs of the natural sciences to the social sciences. The first volume, written in 1932 by Otto Neurath, was titled "Unified Science and Psychology" (Sorell, 1994, pp. 12-13). This attempt to unify experimental methods was rooted in logical positivism's "verificationism." The principle of verificationism stated roughly that "all statements are either analytic (and thus tautological), empirical (and thus verified by observation), or meaningless" (Toulmin \& Leary, 1985, p. 603). Because the natural sciences possessed the most stringent methods of controlled observation, the developing social sciences adopted those methods to avoid meaninglessness. Even though logical positivism and its verificationist criterion were eventually subjected to serious critique, Roth (1987) has noted the social sciences "have retained a fundamentally positivistic conception of knowledge, a conception that identifies knowledge with the results of natural science" (p. 116), and Nelson (2006) has claimed that logical positivism became the "de-facto epistemology for psychology" (p. 210; cf. Sorrell, 1994).

This lingering adherence to a defunct positivism (and the scientism it funds) is unwarranted. It bears reminder there were at least three crucial problems that led to the demise of verificationism. First, moral statements — such as, "racism is wrong" or "honesty in research is good"-are unverifiable by sense experience and therefore rendered meaningless (Willard et al., 2018). It was absurd to maintain that calls for racial justice and honest reporting of data are meaningless emotional pleas, and to maintain these pleas seriously undermines the values needed for a functional science. Second, various notions required for science-such as causation, universal generalizations, and atomic/subatomic particles-were themselves unverifiable. The verifiability principle undermined core elements of the empirical science that positivists meant to uphold (Misak, 1995). The third problem was that logical positivism's verificationist criterion could not itself be empirically verified. The proposition that "only propositions that are logically 
true or can be verified by empirical facts should count as meaningful" is not itself logically true nor can it be verified by empirical facts. Indeed, the claim itself is not an empirical claim but a universal claim about what makes statements meaningful (Plantinga, 2018). According to the verificationist criterion, the criterion itself is meaningless. No doubt empirical verification is an important source of evidence (and quantitative-statistical methods are one way to provide such evidence), but to maintain that it is the only valid source of evidence is unjustifiable.

A second consideration in favor of looking beyond quantitative-statistical methods is that showing the reliability of these methods depends on nonquantitative arguments. For instance, the existence of mind-independent reality, the notion that language refers to reality, the identification of standards of a good theory, the reliability of sense-perception, memory, testimony, and rational intuition are each foundational to trusting quantitative-statistical methods, yet these matters cannot be determined by those methods (De Haro, 2020). Other forms of investigation, such as conceptual analysis, phenomenological experience, rational intuition, and principles of evidential reasoning are required. As De Haro (2020) has concluded: “The scientific quest presupposes having a number of philosophical issues settled first: or, at least, it presupposes engaging with the various conceptual options, and taking a stance on them" (p. 310).

A third consideration in favor of a methodological pluralism emerges from the distinction between etic and emic research (Pike, 1967). Etic research utilizes more distal constructs that are generalizable across multiple contexts, whereas emic research is a more proximal approach that takes an insider, tradition-specific perspective (Hall et al., 2018). Watson et al. (2011) have argued that both etic and emic approaches are necessary in the psychology of R/S, with emic research lending greater cultural validity. Cultural validity would certainly be an instrumental good of emic methods, but presumably emic research is likely to have greater cultural acceptance 
because it is paying attention to the broader evidential base that is already accepted within its context of study. This broader and contextualized evidential base is data in the sense of the Latin datum, that which is "given." Emic researchers can take seriously what counts as legitimate sources of evidence within their research context, thereby providing additional indicators of unobservable constructs and additional explanatory evidence for connections between variables. One reason to take the evidential base of one's research context seriously is that it opens up the possibility of deeper contextualized understanding of the phenomena in question. Another reason is that, within that context, there may be evidence that is relevant to the quantitative-statistical results. And yet, why provisionally trust the evidence that is culturally accepted within a social setting? For one thing, to do otherwise would be to privilege the agreed-upon evidential sources within the researcher's context, and unless there is good reason to do so, this privileging is arbitrary. Second, although contextualized systems of thought can be horribly wrong, there is some reason to think that living, historically situated traditions have stood the test of time for good reason. This leads us to the last consideration in favor of methodological pluralism.

Living religious, spiritual, and philosophical traditions are embodied ways of life that ideally refine practical wisdom over time. McDowell (1996) has characterized a tradition as "a store of historically accumulated wisdom about what is a reason for what" (p. 126). In the context of discussing traditions, McDowell (1996) noted that the rise of modern individualism brings with it a loss or devaluation of the idea that immersion in a tradition might be a respectable mode of access to the real. Instead it comes to seem incumbent on each individual thinker to check everything for herself. When particular traditions seem ossified or hidebound, that encourages a fantasy that one should discard reliance on tradition altogether, whereas the right response would be to 
insist that a respectable tradition must include an honest responsiveness to reflective criticism. (pp. 98-99) MacIntyre (1984)—a well-known proponent of this idea—has contended that a "living" tradition is "an historically extended, socially embodied argument" (p. 222) in which traditions are refined over time through interaction with people within and outside the tradition. Although MacIntyrean notions of tradition-dependent rationality and incommensurability seem to go too far, it remains true that human moral and religious lives are embedded in lived, culturally situated traditions (Hill \& Hall, 2018). Methodological pluralism is built into communities of practice, which are subject to internal refinement such that "immersion in a tradition might be a respectable mode of access to the real" (McDowell, 1996, p. 89).

Acknowledging the reality of the social embeddedness of human moral and spiritual life (and the potential for internal correction) does not on its own offer a reason to think these historically situated traditions accurately conceptualize the good life and the good person. However, it does make it plausible to think that they offer a coherent narrative of the world that is, to varying degrees, in touch with reality. The refined narratives within these traditions offer accounts of the interrelationships among beliefs, practices, virtues, and ultimate purpose. This provides space for positive psychology and the psychology of R/S to engage jointly and meaningfully with religious and philosophical traditions as important interlocutors, attending to what is "given" within those traditions — on those traditions" own terms. A helpful analogy is that of a crime scene investigator who takes in eyewitness testimony, footage from nearby security cameras, footprints at the scene, psychological profiles, DNA evidence, and even the local psychic. The investigator does not lay aside their own evaluative and methodological filters, but they are willing to countenance purported evidence from sources that they may later find good 
reason to overturn. So, this is not the metaphysical claim that one can only make sense of human well-being and religious/spiritual life from within a particular tradition. Rather, it is the epistemological claim that there are sources of evidence available from within particular traditions that are neglected unless methodological pluralism is embraced intentionally and practiced meaningfully.

\section{Conclusion}

What if humanity is not on the verge of apocalypse but instead moral and spiritual breakthrough? Indeed, perhaps we are on the verge of such a breakthrough in part because we are on the verge of apocalypse. As Camus' The Plague illustrates, there is nothing like the inevitable demise of humanity to get humanity's attention. When death and disaster loom, possibilities of other-worldly transcendence and this-worldly well-being are piqued. Specifically, what if we are on the verge of a moral and spiritual breakthrough that requires the integrated resources of positive psychology, the psychology of R/S, and additional evidential resources found within philosophical, religious, and spiritual traditions? One early proponent of a pluralist method, William James (1909), wrote: "Let empiricism once become associated with religion, as hitherto, through some strange misunderstanding, it has been associated with irreligion, and I believe that a new era of religion as well as of philosophy will be ready to begin” (p. 314).

MacIntyre (1984) famously ended After Virtue with these lines: "We are waiting not for a Godot, but for another-doubtless very different-St. Benedict” (p. 255). If McIntyre is right that we need communities like St. Benedict's to help structure our moral and religious/spiritual lives, perhaps we also need those communities to understand properly the relationship between $\mathrm{R} / \mathrm{S}$ and human well-being. If so, alongside another St. Benedict, perhaps we also wait for another-doubtless very different—William James. 


\section{References}

Belzen, J. A., \& Hood, R. W. (2006). Methodological issues in the psychology of religion: Toward another paradigm? The Journal of Psychology, 140(1), 5-28. https://doi.org/10.3200/JRLP.140.1.5-28

Boghossian, P. (2006). Fear of knowledge: Against relativism and constructivism. Oxford University Press.

Burr, V. (2015). Social constructionism. In J. Wright (Ed.), International encyclopedia of the social \& behavioral sciences (pp. 222-227). Elsevier.

Davis, D. E., Hook, J. N., Van Tongeren, D. R., Gartner, A. L. \& Worthington Jr., E. L. (2012). Can religion promote virtue?: A more stringent test of the model of relational spirituality and forgiveness. The International Journal for the Psychology of Religion, 22(4), 252-266. https://doi.org/10.1080/10508619.2011.646229

Davis, E. B., Cuthbert, A. D., Hays, L. W., Aten, J. D., Van Tongeren, D. R., Hook, J. N., Davis, D. E., \& Boan, D. (2016). Using qualitative and mixed methods to study relational spirituality. Psychology of Religion and Spirituality, 8(2), 92-98. https://doi.org/10.1037/rel0000046

De Haro, S. (2020). Science and philosophy: A love-hate relationship. Foundations of Science, 25, 297-314. https://doi.org/10.1007/s10699-019-09619-2

Emmons, R. A., \& Paloutzian, R. F. (2003). The psychology of religion. Annual Review of Psychology, 54, 377-402. https://doi.org/10.1146/annurev.psych.54.101601.145024

Friedman, H. L., \& Brown, N. J. L. (2018). Implications of debunking the "critical positivity ratio" for humanistic psychology. Journal of Humanistic Psychology, 58(3), 239-261. http://dx.doi.org/10.1177/0022167818762227 
Gantt, E. E., \& Melling, B. S. (2009). Science, psychology, and religion: An invitation to Jamesian pluralism. The Journal of Mind and Behavior, 30(3), 149-164. https://www.jstor.org/stable/43854246

Gergen, K. J. (1999). An invitation to social construction. Sage.

Gergen, K. J., Josselson, R., \& Freeman, M. (2015). The promises of qualitative inquiry. American Psychologist, 70(1), 1-9. http://dx.doi.org/10.1037/a0038597

Hall, M. E. L., Shannonhouse, L., Aten, J., McMartin, J., \& Silverman, E. J. (2018). Religionspecific resources for meaning-making from suffering: defining the territory. Mental Health, Religion \& Culture, 21(1), 77-92. https://doi.org/10.1080/13674676.2018.1448770

Hare, J. (2009). God and morality: A philosophical history. Wiley-Blackwell.

Hill, P. C. \& Hall, M. E. L. (2018). Uncovering the good in positive psychology: Toward a worldview conception that can help positive psychology flourish. In N. J. L. Brown, T. Lomas, \& F. J. Eiroa-Orosa (Eds.), The Routledge international handbook of critical positive psychology (pp. 245-262). Routledge.

Hunter, J. D. \& Nedelisky, P. (2020). Science and the good: The tragic quest for the foundations of morality. Yale University Press.

James, W. (1909). A pluralistic universe. Longmans, Green, and Company.

Lomas, T., Waters, L., Williams, P., Oades, L. G., \& Kern, M. L. (2020). Third wave positive psychology: Broadening towards complexity. The Journal of Positive Psychology. Advance online publication. $\underline{\text { https://doi.org/10.1080/17439760.2020.1805501 }}$

McCall, R. B., \& Green, B. L. (2004). Beyond the methodological gold standards of behavioral research: Considerations for practice and policy. SRCD Social Policy Report, 18(2), 3-19. 
McDowell, J. (1996). Mind and world. Harvard University Press.

McGrath, A. E. (2019). The territories of human reason: Science and theology in an age of multiple rationalities. Oxford University Press.

MacIntyre, A. (1984). After virtue: A study in moral theory ( $3^{\text {rd }}$ ed.). University of Notre Dame.

Misak, C. J. (1995). Verificationism: Its history and prospects. Routledge.

Nelson, J. M. (2006). Missed opportunities in dialogue between psychology and religion. Journal of Psychology and Theology, 34(3), 205-216.

Nelson, J. M. \& Slife, B. D. (2012). Theoretical and epistemological foundations. In L. J. Miller (Ed.), The Oxford handbook of psychology and spirituality (pp. 21-35). Oxford University Press.

Palmer, M. D., \& Burgess, S. M. (2020). The wiley blackwell companion to religion and social justice. John Wiley \& Sons.

Pike, K. L. (1967). Language in relation to a unified theory of the structure of human behavior ( $2^{\text {nd }}$ ed.). Mouton.

Plantinga, A. (2018). “Scientism: Who needs it?” In J. de Ridder, R. Peels, R. van Woudenberg (Eds.), Scientism: Prospects and perils (pp. 220-232). Oxford University Press.

Porter, S. L. (2004). Wesleyan quadrilateral as a theory of integration. Journal of Psychology and Theology, 32, 190-199.

Richardson, F. C. \& Guignon, C. (2008). Positive psychology and philosophy of social sicence. Theory \& Psychology 18(5), 605-627. https://doi.org/10.1177/0959354308093398

Rich, G. J. (2017). The promise of qualitative inquiry for positive psychology: Diversifying methods. The Journal of Positive Psychology, 12(3), 220-231. https://doi.org/10.1080/17439760.2016.1225119 
Robinson, D. N. (1995). An intellectual history of psychology ( $3^{\text {rd }}$ ed.). University of Wisconsin Press.

Rorty, R. (1985). Solidarity or objectivity? In J. Rajchman and C. West (Eds.), Postanalytic philosophy (pp. 3-19). Columbia University Press.

Roth, P. A. (1987). Meaning and method in the social sciences. A case for methodological pluralism. Cornell University Press.

Ryan, R. M., \& Deci, E. L. (2001). On happiness and human potentials: A review of research on hedonic and eudaimonic well-being. Annual Review of Psychology, 52, 141-166. https://doi.org/10.1146/annurev.psych.52.1.141

Schnitker, S. A., \& Emmons, R. A. (2017). The psychology of virtue: Integrating positive psychology and the psychology of religion. Psychology of Religion and Spirituality, 9(3), 239-241. http://dx.doi.org/10.1037/rel0000133

Schnitker, S. A., Houltberg, B., Dyrness, W., \& Redmond, N. (2017). The virtue of patience, spirituality, and suffering: Integrating lessons from positive psychology, psychology of religion, and Christian theology. Psychology of Religion and Spirituality, 9(3), 264275. https://doi.org/10.1037/rel0000099

Slife, B. D., \& Reber J. S. (2021). Against methodological confinement: Toward a pluralism of methods and interpretations. Psychology of Religion and Spirituality, 13(1), 14-18. http://dx.doi.org/10.1037/rel0000274

Sorell, T. (1994). Scientism: Philosophy and the infatuation with science. Taylor \& Francis.

Taylor, C. (2007). A secular age. Harvard University Press.

Toulmin, S. \& Leary, D. (1985). The cult of empiricism in psychology and beyond. In S. Koch \& D. Leary (Eds.), A century of psychology as science (pp. 594-617). McGraw-Hill. 
Van Tongeren, D. R., DeWall, C. N., Chen, Z., Sibley, C. G., \& Bulbulia, J. (2021). Religious residue: Cross-cultural evidence that religious psychology and behavior persist following deidentification. Journal of Personality and Social Psychology, 120(2), 484503. https://doi.org/10.1037/pspp0000288

VanderWeele, T. J. (2017). Religious communities and human flourishing. Current Directions in Psychological Science. 26(5), 476-481. https://doi.org/10.1177/0963721417721526

Watson, P. J., Chen, Z., \& Hood Jr., R.W., (2011). Biblical foundationalism and religious reflection: Polarization of faith and intellect oriented epistemologies within a Christian ideological surround. Journal of Psychology and Theology, 39(1), 111-121.

Wilkins, T. A., Piedmont, R. L., \& Magyar-Russell, G. M. (2012). Spirituality or religiousness: Which serves as the better predictor of elements of mental health? Research in the Social Scientific Study of Religion. 23, 53-73. https://doi.org/10.1163/9789004229549_004

Willard, D., Porter, S. L., Preston, A., \& Ten Elshof, G. (2018). The disappearance of moral knowledge. Routledge.

Worthington, E. L., Jr., Cowden, R. G., Davis, E. B., \& Exline, J. J. (in press). Virtues in positive psychology and the psychology of religion and spirituality: Existing overlap and promising possibilities. In L. J. Miller (Ed.), Handbook of the psychology of religion and spirituality ( $2^{\text {nd }}$ ed.). Oxford University Press. 


\title{
Chapter 4:
}

Virtues in Positive Psychology and the Psychology of Religion and Spirituality

\author{
Juliette L. Ratchford, M.A. ${ }^{1}$ \\ Mason S. Ming, M.A. ${ }^{1}$ \\ Sarah A. Schnitker, Ph.D. ${ }^{1}$
}
${ }^{1}$ Science of Virtues Laboratory, Department of Psychology and Neuroscience, Baylor University, Waco, TX, USA

Correspondence concerning this chapter should be addressed to Juliette L. Ratchford.

Email: julietteratchford@ gmail.com 


\begin{abstract}
In this chapter, we explore the overlap and intersections of research on virtue within the fields of positive psychology and the psychology of religion and spirituality. Research involving the study of virtue in tandem with religion and spirituality is presented, in order to guide future scholarship at the crossroads of positive psychology, religion, and spirituality. Intersections including trait taxonomies, cultural ideals, cultural practices, goal pursuit, context-specific emotions, and narrative identity make virtue research an optimal hub for integration. This chapter is applicable to work in faith communities, psychoeducation, and clinical work. For faith communities, it summarizes empirical research into the ways religion and spirituality can help or undermine virtue development. For people seeking psychoeducation, we outline the history of virtue research in tandem with the psychology of religion and spirituality, providing historical context for understanding virtue development. Finally, for clinical practitioners, we describe the strengths-based model of clinical practice — introduced by humanistic psychology and popularized by positive psychology — which shifted away from the medical model of psychology that dominated the twentieth century.
\end{abstract}

Keywords: Virtue, Religion, Spirituality, Character Strengths, Meaning Making 


\section{Chapter 4:}

\section{Virtues in Positive Psychology and the Psychology of Religion and Spirituality}

Take a moment to think of the most virtuous person you know. Ask yourself: What virtues do they possess? What makes them virtuous? How did they become virtuous? How religious or spiritual is this virtuous person? What role does religion/spirituality seem to play in the embodiment, expression, and enhancement of their virtuous character?

The purpose of this chapter is to explore such questions by reviewing and synthesizing virtue theory and research from two fields of study—positive psychology and the psychology of religion and spirituality $(\mathrm{R} / \mathrm{S})$. Historically, both fields have aimed to promote scientific understanding and practical cultivation of virtues (Schnitker \& Emmons, 2017). However, as shown in Chapter 1 (see Table 1.1 and 1.2), since positive psychology's inception in 1998, virtues have received considerably less scholarly attention than other key concepts (such as wellbeing or happiness), and journals have been reluctant to publish research on the intersections between positive psychology and R/S. In the current chapter, we first discuss reasons why virtues represent an optimal hub for integrating positive psychology and the psychology of R/S. Next, we explore places of intersection between the two fields in relation to the study of virtue. Finally, we discuss practical applications and implications of integrating virtue research and practice. Throughout the chapter, we summarize theories and research on virtues.

\section{Psychology of Religion and Spirituality, Positive Psychology, and Virtue}

Following the attacks on the World Trade Center and Pentagon on September 11, 2001, the psychology of R/S garnered major research attention, as seen in the six-fold increase in the frequency of "religion" or "spirituality" being used in article titles and abstracts from 2005 to 2014, compared to prior decades (Paloutzian, 2017a). The study of R/S now spans multiple 
subdisciplines and countries, as researchers worldwide investigate the continued importance of R/S across cultures (Paloutzian, 2017b). But interest in the psychological study of virtues and religion did not originate in recent decades. For example, during his 1906 presidential address to the American Psychological Association, William James (the father of American psychology who investigated religious beliefs and conversion experiences throughout his career) proposed two empirical questions: "(a) What were the limits of human energy? and (b) How could this energy be stimulated and released so it could be put to optimal use?" (Rathunde, 2001, p. 136).

Psychological interest in virtue and R/S waned in the mid-1920s and 1930s. For the study of virtue, this lack of interest was catalyzed by Hartshorne and May's (1928-1930) empirical studies, which found a dearth in consistent truth-telling across different honesty-relevant situations and thereby launched the person-situation debate. This debate involved a situationist critique that challenged the assumption enduring personality characteristics actually exist and thus led to a relative stalemate in virtue research until the latter part of the $20^{\text {th }}$ century. That stalemate coincided with the waning study of R/S until the 1960s, influenced by such factors as the separation of psychology and philosophy departments, the scientific model of psychology being based on positivist scientific principles, and the fears of "taboo" topics that veered into philosophical or theological directions (Emmons \& Paloutzian, 2003).

The co-occurring sporadic research histories of virtue and R/S suggest that researchers might associate the two constructs to some degree. Indeed, much of the literature that examines the benefits and detriments of R/S considers virtue as an outcome of interest (Ratchford et al., 2021a). Likewise, the scientific study of virtues has regularly consulted philosophical and religious writings to inform construct creation, definition articulation, and theory development (Peterson \& Seligman, 2004). Hence, this chapter explores virtues from both positive 
psychological and religious/spiritual lenses. In fact, we argue that virtues are an optimal hub for integrating the positive psychology and psychology of R/S fields.

\section{Virtues: An Optimal Hub for Integration}

Virtues can be generally defined as "dispositional deep-seated habits that contribute to flourishing and that occur in activities with the following three features: they are done well, not done poorly, and in accordance with the right motivation and reason" (Ratchford et al., 2021b, p. 8). ${ }^{1}$ Virtues provide a unique context for integrating psychology of R/S and positive psychology. Religious texts from Buddhist, Christian, and Islamic traditions are replete with references to virtues such as courage, justice, humanity, temperance, wisdom, and transcendence (Wade, 2010). Also, positive psychology’s conceptualizations of virtue often derived from religious traditions. For example, the virtue of the Protestant work ethic - emphasizing hard work, discipline, and thrift — has become increasingly secularized and universal (Kalemci \& Tuzun, 2019), but it was historically rooted in the specific faith tradition of Protestant Christianity.

Many virtues studied in positive psychology are pulled directly from religious/spiritual teachings. For instance, gratitude to God (Rosmarin et al., 2011) and grace (Graves, 2017) are explicitly religious constructs and have received growing empirical attention. Other examples, which probably include both religious/spiritual and secular influences, include chastity (Hardy \& Willoughby, 2017), hope (King et al., 2020), humility (Davis et al., 2017), and forgiveness

(Worthington \& Wade, 1999). Considering the historical enmeshment between R/S and virtues, a serious inclusion of religious/spiritual understandings of virtue would profit the broader scholarly study of virtues. Pluralistic multidisciplinary work among theologians, psychologists, anthropologists, and philosophers may provide a more accurate "thick" contextual understanding

\footnotetext{
${ }^{1}$ This conceptualization of virtue ties well with the Positive Religious and Spiritual Development (PRSD) theory presented in Davis et al. (Chapter 18) of this volume.
} 
of R/S and virtue, as each discipline brings a unique perspective to the narrative (Graves, 2017).

Even after considering the many converging points, the psychology of R/S and positive psychology have hesitated to cooperate fully in virtue research. For various reasons, each has resisted drawing upon the rich knowledge and perspectives the other has to offer. Perhaps researchers in these fields have hesitated to cooperate due to concerns about pathologizing the human condition, worries about forcing R/S on secular individuals, or assumptions that one field might begin to subsume the other. At times this hesitation may even be based in mistrust and prejudice. Regardless of the reasons, these fields have much to offer one another, and researchers have thus begun integrating R/S into their work with virtues (e.g., Schnitker \& Emmons, 2017). Our aim is to highlight key intersections between positive psychology and the psychology of $\mathrm{R} / \mathrm{S}$, within the context of virtues research. Given that virtues are a component of the personality system, we organize these intersections around commonly held, virtue-relevant components of personality and context: trait taxonomies, cultural ideals, cultural practices, goal pursuits, context-specific emotions, and narrative identities (McAdams \& Pals, 2006).

\section{Intersection on Trait Taxonomies}

Examination of virtues as personality traits is a common point of intersection between positive psychology and psychology of R/S. Perhaps the most salient example of integration is Peterson and Seligman's (2004) Values in Action Inventory of Strengths (VIA-IS). Seligman, widely considered the father of positive psychology, oversaw a massive project to classify human strengths and virtues into a cohesive taxonomy of flourishing that could be used as a companion to extant taxonomies of languishing (e.g., the Diagnostic and Statistical Manual $[D S M])$. To do so, Peterson and Seligman delved into Jewish, Christian, Muslim, Buddhist, and

Hindu religious texts and thought. McGrath (2015) followed up on their work by taking millions 
of globally representative responses to the VIA-IS and using factor analytic methods to organize the virtues into three categories: caring, inquisitiveness, and self-control. However, some scholars have raised concerns about the VIA-IS's viability as a virtue measure (Snow, 2019), arguing it has not yet demonstrated adequate construct equivalence (measurement invariance) to verify its assumption that its assessed virtues hold universal meanings within and across cultures.

Additionally, in the HEXACO honesty-humility factor (Lee \& Ashton, 2004), R/S and virtue-related concepts have been proposed as potential sixth factors of personality, unique from other well-established high order Big 5 personality traits (i.e., Neuroticism, Extraversion, Openness, Agreeableness, Conscientiousness; McCrae \& Costa, 2008). The HEXACO measure only considers the virtues of honesty and humility; it is by no means an exhaustive measure of all virtues, nor was it intended to be. However, because the HEXACO assesses specific virtues, it is useful to consider how R/S is associated with the HEXACO. Indeed, researchers consistently find that trait honesty-humility is moderately associated with R/S (Aghababaei et al., 2014).

Psychology of R/S research in the late 1990s created trait conceptualizations of spirituality orthogonal to Big Five traits (Piedmont, 1999). Trait spirituality has also been reliably linked with virtues like forgiveness (Leach \& Lark, 2004), humility (Davis et al., 2017), patience (Schnitker et al., 2017), and thrift (Ratchford et al., 2021a). As research continues to develop in the realm of spirituality, the definition of spirituality has broadened to encompass secular, nonreligious people who engage in self-transcendent practices (Koenig, 2008). This may pose an issue in conducting research on $\mathrm{R} / \mathrm{S}$ and virtue, because under this broader definition, spirituality may overlap entirely with virtue. Further empirical inquiry is necessary to determine the extent of conflation between trait spirituality and specific trait virtues.

\section{Intersection on Cultural Ideals}


Although it is tempting to consider virtues as universal, Snow (2019) has argued that virtues are culturally dependent, and researchers ought to be more sensitive to cross-cultural variability in the development, composition, and expression of virtues. This possibility is evidenced when assessments validated with Western populations do not generalize to nonWestern cultures. For example, after failing to authenticate the six-factor VIA-IS structure in a Chinese sample, Duan et al. (2012) created a three-factor Chinese Virtues Questionnaire that more validly assessed virtues in Chinese culture. Likewise, virtues can be understood differently within a single nation. For example, in the U.S., humility is related to more liberal transcendent values (e.g., benevolence; universalism) or more conservative conformity values (e.g., conformity; tradition), depending on whom you ask (Schwartz et al., 2012). Indeed, scholars are finding that virtue conceptualization depends heavily on person-context relations (Fowers et al., 2021), and the influence of culture on virtue development and expression cannot be ignored.

Community, family, and culture — especially religious culture—are major players in virtue development. Religious institutions are influential cultural settings, and differences among religious cultures lead people to prize some values over others. For example, predominantly Hindu cultures such as India value thrift—-the wise use and distribution of resources—more so than other cultures (e.g., in North America and Europe; Choenni, 2011). Additionally, social support from one's religious/spiritual context (such as being involved in a religious community) can be influential in virtue development as well. For instance, King and Furrow (2004) found that involvement in religious activities led to increased empathy and altruism among adolescents who had relationships with coreligionists (who shared beliefs and values in common with them).

\section{Intersection on Practices and Interventions}

Although not the only contexts to do so, religious traditions and communities offer some 
of the most readily accessible, shared sacred practices and meaning-making frameworks (Park, 2005) that are regularly coupled with opportunities to develop virtue (Schnitker et al., 2019a). Many activities that promote virtue development are religious and transcendent in nature, including religious/spiritual practices such as prayer, meditation, and service attendance. However, not all practices or strivings are created equal (King, 2008). Sanctified strivingspractices or goals imbued with sacred significance (such as sanctifying one's marriage) — lead to greater goal pursuit and social support for goals (Mahoney et al., 2005). Indeed, when otherwise ordinary habits or earthly relationships are imbued with sacred meaning (e.g., deciding to spend more time with one's partner because your relationship is perceived as sacred), they tend to bolster virtue development (e.g., commitment, love, and fidelity; see Chapter 28, this volume).

Religious communities are particularly good at encouraging individuals to engage in behavioral practices (King, 2008) that regularly foster virtues. For example, prayer has been shown to increase gratitude (Lambert et al., 2009), meditation can increase compassion (Boellinghaus et al., 2013), and diet regulation — a behavior similar to religious fasting — can improve self-regulation (Muraven, 2010), a capacity some researchers consider the underlying mechanism of all virtues (McCullough \& Willoughby, 2009; Root Luna et al., 2017). In fact, some argue that R/S leads to virtues, which in turn lead to well-being (see Krause et al., 2019).

Unfortunately, in a review of positive psychological interventions, Rye et al. (2013) found that very few positive psychology interventions explicitly incorporated R/S, even when doing so would have been relatively easy. Although some positive psychology interventions overlap with R/S practices in their activation of meaning-making, it is still unclear whether religious, spiritual, moral, or instrumental forms of meaning change the impact of the interventions (Schnitker et al., 2021). However, research has found that meaning that is self- 
transcendent (i.e., beyond-the-self) increases the efficacy of interventions (Yeager et al., 2014).

\section{Intersection on Self-Transcendent Positive Emotions}

Self-transcendent positive emotions (e.g., awe, gratitude, and love) are associated with both positive psychology and the psychology of R/S and may serve as a bridge between R/S and virtue. They tend to activate and increase spirituality (Van Cappellen et al., 2013). For example, in one study, church attendance activated the emotion of love, which in turn activated spontaneous generosity, as participants were more willing to share a hypothetical lottery prize with others (Van Cappellen et al., 2016). Gratitude, perhaps the most often studied virtue in positive psychology, is also a historically religious construct. In longitudinal and experimental studies, Lambert et al. (2009) found the religious practice of prayer activated gratitude, and more frequent prayer led to subsequently increased gratitude. But self-transcendent positive emotions are not limited to activating warmth-based virtues like compassion and generosity. For example, higher levels of daily gratitude as a self-transcendent positive emotion (but not happiness) have been associated with higher self-control and patience (i.e., being able to wait well when necessary), likely by helping people recognize the benefits and benefactors in their lives (Dickens \& DeSteno, 2016). Likewise, experimental manipulations to induce gratitude in comparison to neutral or happy emotions increase financial patience (DeSteno et al., 2014).

Self-transcendent positive emotions may also serve to motivate a person to engage both in spirituality and virtuous behavior, which may be why many positive psychology interventions seek to activate self-transcendent positive emotions. For example, loving-kindness meditation increases daily positive emotions, which in turn increase meditation practices in an upward spiral (Fredrickson et al., 2017; see Van Cappellen et al., this volume). This upward spiral of both the intervention and the experience of positive emotions is also found in gratitude interventions 
(Lambert et al., 2009). Interventions framed in a R/S context (e.g., prayer) have demonstrated greater effect in eliciting experiences of gratitude than interventions not framed in that context (Lambert et al., 2009), suggesting that contextualizing interventions as religious/spiritual may be particularly advantageous to virtue development (see Captari et al., Chapter 26, this volume).

\section{Intersection on Goal Pursuits}

Goals—what people habitually are trying to do or want to accomplish—have been assessed in research in both subfields. In the psychology of R/S, much of the research regarding goals has focused on ultimate concerns, which are goals that in some fashion are concerned with R/S (goals such as deepening one's relationship with God or spending more time reading religious texts; Emmons \& Paloutzian, 2003). The presence of explicitly spiritual goals is associated with greater well-being and adaptive goal pursuit (Emmons \& Schnitker, 2013). However, goals need not be explicitly spiritual to be imbued with spiritual significance, which is referred to as sanctification. For example, people regularly imbue parenting, marriage, and work with sacred significance. Research suggests both theistic and nontheistic sanctification of goals

produces better goal outcomes and higher well-being when the goal pursuit is successful (Kusner et al., 2014; Mahoney \& Pargament, 2005), but in goal failure, sanctification can lead to greater decrements in well-being because the loss is perceived as a desecration (Pargament et al., 2005).

Examining the relative conflict and harmony among a person's various goals has proven fruitful in predicting the incidence of spiritual transformation and change; in a longitudinal study on adolescents who were involved in a week-long religious service trip, goals were assessed prior to attending the trip. Researchers found that adolescents who ascribed less meaning to their goals and experienced conflict amongst their goals before the trip were more likely to have a religious transformation experience (e.g., conversion or recommitment) during the trip than 
adolescents who found their existing goals more meaningful (Schnitker et al., 2019b). This finding suggests that R/S functions to organize the meaning-making system and offer a sense of meaning to people who do not have it already (Park \& Van Tongeren, Chapter 6, this volume).

In much the same way, positive psychologists have recently investigated virtues through the lens of goal pursuit. A longitudinal study on university students found that people who are more virtuous (specifically patient) in the pursuit of their goals tend to exert more effort in that pursuit and find the goals more meaningful (Thomas \& Schnitker, 2017). Additionally, goals that were more meaningful to people predicted subsequent virtuous goal pursuit. This finding suggests that, as people engage strivings, meaning and virtue have an upward spiral effect on each other, similar to self-transcendent positive emotions. These findings regarding virtue, R/S, and meaning suggest the importance of meaning and meaning-making for both the study of virtue and of R/S. Additional work is needed to integrate goal-based approaches to assessing R/S and virtues. For example, experimental studies could test how manipulations to imbue the goal of developing virtues with sacred meaning affects virtue outcomes (Williams et al., 2021).

\section{Intersection on Narrative Identities}

Narrative identities often undergird virtue development and expression as well. Narrative identity is an individual's ever-evolving life story about who they are and how they came to be who they are becoming (McAdams, 2006). Although not all narrative identities are religious in nature, narrative identities linked to religious traditions provide unique virtue-promoting, meaning-making frameworks (Park, 2005), wherein individuals are motivated to behave morally, transcend themselves, and find meaning (King et al., 2020). Although all narrative identities provide some sort of meaning structure for characteristic adaptations, religious/spiritual narrative identities (i.e., stories people have in their minds about the sacred meaning and transcendent 
experiences of their lives) provide people with sacred meaning and lead to the sanctification of their actions (Bronk, 2014). An identity shaped by transcendence, ultimacy, or sacredness is an especially powerful motivator for activities that are perceived to be virtuous in the moral framework of the person's religious tradition. Moreover, life narratives with redemptive arcs, which are promoted by many faith traditions, are related to higher levels of generativity in older adults (McAdams, 2006). Thus, when individuals embed their narrative identity into a religious tradition, it can take on a powerful transcendent component that is supported by that community. Applications for Clinicians and Practitioners in Secular and Religious/Spiritual Settings

Identifying points of convergence and divergence between the scientific study of virtues in the psychology of R/S and positive psychology is a useful scholarly exercise. This sort of targeted comparison also reveals potential applications in both secular and R/S settings.

Applications in secular settings. As clinicians and practitioners (e.g., educators, therapists, and youth workers) integrate virtues into their methods, they must be strategic about where they attempt to intervene. Should practitioners try to influence the development of the traits that support virtues, focus on cultivating self-transcendent positive emotions, or help people construct virtue-supportive narrative identities? It is likely that interventions will be most successful when they attend to all three (although the efficacy of this is yet to be empirically tested). Interventions at the trait level of personality are perhaps the most difficult because of high trait stability (even though trait-level changes are potentially long-lasting and deeply rewarding; see Roberts et al., 2017), whereas interventions at other levels of personality (such as narrative identity) are more readily accessible. For example, forgiveness and hope interventions tend to focus on changing emotion-regulation strategies and relational schemas at the level of what people are doing (e.g., specific behaviors, feelings, and thoughts; Wade et al., 2014). 
Many current virtue interventions avoid discussion or use of R/S. This approach may stem from positive psychology's humanistic grounding (Waterman, 2013), but it may result in users' inability to integrate the intervention into their meaning systems, which could limit the longevity of the intervention's effects. It is hard for an intervention to be effective if it does not fit into a user's meaning-making and identity systems. Virtue interventions do not need to prescribe a meaning system, but they should attempt to map readily onto people's evolving narrative identities and already existing meaning systems. By doing so, these interventions could have more potent and persistence effects. Connecting virtue-relevant habits to a person's narrative identity can foster a purpose for motivating that habit, which may thereby promote virtue development (Schnitker et al., 2019a; see Davis et al., Chapter 18, this volume). Of course, certain cultures will value some virtues over others, so what constitutes virtue in one culture may not be considered moral or virtuous in another culture (see Mattis, Chapter 9, this volume).

Hence, when working with individuals, practitioners should keep in mind that virtues are culturally derived and culturally embedded. Accordingly, clinicians and practitioners utilizing positive psychology interventions in a secular setting need to consider their patients' cultural and religious/spiritual background. Clients may value certain virtues over others, depending on their cultural background and religious perspectives. Biases toward certain virtues may also differ from the clinician's views. Furthermore, it is important to reiterate that most of the research on positive psychology interventions has been conducted in Western populations, so the crosscultural effectiveness of them often has not yet been demonstrated. In short, virtue-focused clinical practice must be culturally sensitive and responsive. Let us take the case of the virtue of forgiveness and R/S. If a fictitious client Mark does not highly value forgiveness as a virtue, but his clinician Judy is of a Judeo-Christian faith that places great emphasis on forgiveness, then 
there may be conflict between Judy and Mark in cases where Mark has been wronged by someone in his life. To be culturally sensitive and responsive, Judy should ask Mark what his cultural values are and let those values take precedence in the clinical relationship and goals.

Applications in religious/spiritual settings. In their work with people, religious leaders (clergy, religious educators, etc.) may also find that positive psychology interventions are helpful tools. Religious leaders might find it helpful to tailor positive psychological interventions to their congregation's needs (see Chapter 26 and Chapter 29, this volume). For example, a gratitude intervention that asks individuals to list three positive things that went well during the day could be tailored to ask congregants to write down three positive things for which they are grateful to God. Indeed, experimental research suggests framing gratitude journaling as a prayer leads to more potent effects among religious people (Schnitker \& Richardson, 2019). Similarly, praying for a romantic partner or friend (in comparison to describing the partner to a parent or thinking positive thoughts about a friend) leads to greater forgiveness across time (Lambert et al, 2010). Ideally, additional research will test these sorts of virtue-development activity modifications for other virtues and religious activities beyond prayer for efficacy and provide support for their use.

Many positive psychology interventions overlap with spiritual practices, and leaders can look to the science to see what has been efficacious for most people. However, long-standing, effective spiritual practices (e.g., prayer and meditation) should not be misappropriated for their virtue intervention functions. For religious leaders and adherents, spiritual practices have spiritual functions that extend well beyond their psychological effects. Science can help inform religious practitioner choices but should not replace them. How to intervene is dependent on the values and goals of the intervention participant, which must be discussed within the context of the person's holistic needs and the relationship between the practitioner and participant. 
Moreover, caution should be exercised to avoid common pitfalls, such as assuming all interventions can be used into a new setting without contextualization or culturally needed adaptation (Hendriks et al., 2019). Likewise, practitioners should be aware that positive psychology interventions touted to build virtue may have unintended and undesirable side effects. For example, mindfulness interventions sometimes reduce caring moral responses to harmful behaviors (Schindler et al., 2019) and can increase self-focus and self-enhancement bias (Gebauer et al., 2018). Despite these pitfalls, positive psychology interventions that are properly understood and tailored to fit meaning systems can become powerful aids for cultivating growth.

\section{Conclusion}

As demonstrated throughout this chapter, the study of virtue in positive psychology and the psychology of R/S run parallel to each other and often converge. Points of convergence and divergence provide numerous applications to clinical and applied work regarding the ways to intervene, the integration of virtue and R/S in interventions, the application of interventions to an individual's meaning system, and the overlap between R/S and virtue interventions. The research presented throughout this chapter shows a rich history of virtue research and highlights a fruitful line of integrative research to come. We found multiple areas of intersection for R/S and positive psychology on virtue, including trait taxonomies, cultural ideals, cultural practices, goal pursuits, context-specific emotions, and narrative identities. Our hope is that the work presented within this chapter may illuminate conversations for individuals, families, and communities around the questions we posed at the beginning of the chapter, such as: What makes a person virtuous, and what role can $R / S$ play in the embodiment, expression, and enhancement of virtuous character? 


\section{References}

Aghababaei, N., Wasserman, J. A., \& Nannini, D. (2014). The religious person revisited: Crosscultural evidence from the HEXACO model of personality structure. Mental Health, Religion \& Culture, 17(1), 24-29. https://doi.org/10.1080/13674676.2012.737771

Boellinghaus, I., Jones, F. W., \& Hutton, J. (2013). Cultivating self-care and compassion in psychological therapists in training: The experience of practicing loving-kindness meditation. Training and Education in Professional Psychology, 7, 267-277. https://doi.org/10.1037/a0033092

Bronk, K. (2014). Purpose in life: A critical component of optimal youth development. Springer.

Choenni, C. E. S. (2011). Integration Hindustani style? On the migration, history and diaspora of Hindustanis. Free University of Amsterdam.

Davis, D. E., Hook, J. N., McAnnally-Linz, R., Choe, E., \& Placeres, V. (2017). Humility, religion, and spirituality: A review of the literature. Psychology of Religion and Spirituality, 9(3), 242-253. https://doi.org/10.1037/rel0000111

DeSteno, D., Li, Y., Dickens, L., \& Lerner, J. S. (2014). Gratitude: A tool for reducing economic impatience. Psychological Science, 25(6), 1262-1267. https://doi.org/10.1177\%2F0956797614529979

Dickens, L., \& DeSteno, D. (2016). The grateful are patient: Heightened daily gratitude is associated with attenuated temporal discounting. Emotion, 16(4), 421-425. https://doi.org/10.1037/emo0000176

Duan, W., Ho, S. M., Yu, B., Tang, X., Zhang, Y., Li, T., \& Yuen, T. (2012). Factor structure of the Chinese Virtues Questionnaire. Research on Social Work Practice, 22(6), 680-688. https://doi.org/10.1177\%2F1049731512450074 
Emmons, R. A., \& Paloutzian, R. F. (2003). The psychology of religion. Annual Review of Psychology, 54(1), 377-402. https://doi.org/10.1146/annurev.psych.54.101601.145024

Emmons, R. A., \& Schnitker, S. A. (2013). Gods and goals: Religion and purposeful action. In R. F. Paloutzian and C. L. Park (Eds.), Handbook of the Psychology of Religion and Spirituality ( $2^{\text {nd }}$ ed., pp. 256-273). Guilford Press.

Fowers, B. J., Carroll, J. S., Leonhardt, N. D., \& Cokelet, B. (2021). The emerging science of virtue. Perspectives on Psychological Science, 16(1), 118-147. https://doi.org/10.1177\%2F1745691620924473

Fredrickson, B. L., Boulton, A. J., Firestine, A. M., Van Cappellen, P., Algoe, S. B., Brantley, M. M., Kim, S. L., Brantley, J., \& Salzberg, S. (2017). Positive emotion correlates of meditation practice: A comparison of mindfulness meditation and loving-kindness meditation. Mindfulness, 8(6), 1623-1633. https://doi.org/10.1007/s12671-017-0735-9

Gebauer, J. E., Nehrlich, A. D., Stahlberg, D., Sedikides, C., Hackenschmidt, A., Schick, D., Stegmaier, C. A., Winfelder, C. C., Bruk, A., \& Mander, J. (2018). Mind-body practices and the self: Yoga and meditation do not quiet the ego but instead boost selfenhancement. Psychological Science, 29(8), 1299-1308. https://doi.org/10.1177\%2F0956797618764621

Graves, M. (2017). Grace and virtue: Theological and psychological dispositions and practices. Psychology of Religion and Spirituality, 9, 303-308. https://doi.org/10.1037/rel0000129

Hardy, S. A., \& Willoughby, B. J. (2017). Religiosity and chastity among single young adults and married adults. Psychology of Religion and Spirituality, 9, 285-295. https://doi.org/10.1037/rel0000112

Hartshorne, H., \& May, M.A. (1928-1930). Studies in the nature of character. Vol. 1: Studies in 
deceit. Vol. 2 (with J.B. Maller, 1929), Studies in service and self-control. Vol. 3 (with F. K. Shuttleworth, 1930), Studies in the organization of character. Macmillan.

Hendriks, T., Warren, M. A., Schotanus-Dijkstra, M., Hassankhan, A., Graafsma, T., Bohlmeijer, E., \& de Jong, J. (2019). How WEIRD are positive psychology interventions? A bibliometric analysis of randomized controlled trials on the science of well-being. The Journal of Positive Psychology, 14(4), 489-501. https://doi.org/10.1080/17439760.2018.1484941

Kalemci, R. A., \& Tuzun, I. K. (2019). Understanding protestant and Islamic work ethic studies: A content analysis of articles. Journal of Business Ethics, 158(4), 999-1008. https://doi.org/10.1007/s10551-017-3716-y

King, P. E. (2008). Spirituality as fertile ground for positive youth development. In R. M. Lerner, R. W. Roeser, \& E. Phelps (Eds.), Positive youth development and spirituality: From theory to research (pp. 55-73). Templeton Foundation Press.

King, P. E., \& Furrow, J. L. (2004). Religion as a resource for positive youth development: Religion, social capital, and moral outcomes. Developmental Psychology, 40(5), 703713. https://doi.org/10.1037/0012-1649.40.5.703

King, P. E., Schnitker, S. A., \& Houltberg, B. (2020). Religious groups and institutions as a context for moral development. In L. A. Jensen (Ed.), Oxford Handbook of Moral Development (pp. 592-612). Guilford Press.

Koenig, H. G. (2008). Concerns about measuring "spirituality" in research. The Journal of Nervous and Mental Disease, 196(5), 349-355. https://doi.org/10.1097/NMD.0b013e31816ff796

Krause, N., Hill, P. C., \& Ironson, G. (2019). Evaluating the relationships among religion, social 
virtues, and meaning in life. Archive for the Psychology of Religion, 41(1), 53-70. https://doi.org/10.1177\%2F0084672419839797

Kusner, K. G., Mahoney, A., Pargament, K. I., \& DeMaris, A. (2014). Sanctification of marriage and spiritual intimacy predicting observed marital interactions across the transition to parenthood. Journal of Family Psychology, 28(5), 604-614.

https://doi.org/10.1037/a0036989

Lambert, N. M., Fincham, F. D., Braithwaite, S. R., Graham, S. M., \& Beach, S. R. (2009). Can prayer increase gratitude? Psychology of Religion and Spirituality, 1(3), 139-149. https://doi.org/10.1037/a0016731

Lambert, N. M., Fincham, F. D., Stillman, T. F., Graham, S. M., \& Beach, S. R. (2010). Motivating change in relationships: Can prayer increase forgiveness? Psychological Science, 21(1), 126-132. https://doi.org/10.1177\%2F0956797609355634

Leach, M. M., \& Lark, R. (2004). Does spirituality add to personality in the study of trait forgiveness? Personality and Individual Differences, 37(1), 147-156. https://doi.org/10.1016/j.paid.2003.08.007

Lee, K., \& Ashton, M. C. (2004). Psychometric properties of the HEXACO personality inventory. Multivariate Behavioral Research, 39(2), 329-358. https://doi.org/10.1207/s15327906mbr3902_8

Mahoney, A. \& Pargament, K. I. (2005). A higher purpose: The sanctification of strivings in a community sample. International Journal for the Psychology of Religion, 15(3), 239262. https://doi.org/10.1207/s15327582ijpr1503_4

Mahoney, A., Pargament, K. I., Cole, B., Jewell, T., Magyar, G. M., Tarakeshwar, N., MurraySwank, N. A., \& Phillips, R. (2005). A higher purpose: The sanctification of strivings in a 
community sample. The International Journal for the Psychology of Religion, 15(3), 239-262. https://doi.org/10.1207/s15327582ijpr1503_4

McAdams, D. P. (2006). The redemptive self: Generativity and the stories Americans live by. Research in Human Development, 3, 81-100. https://doi.org/10.1080/15427609.2006.9683363

McAdams, D. P., \& Pals, J. L. (2006). A new Big Five: Fundamental principles for an integrative science of personality. American Psychologist, 61(3), 204-217. https://doi.org/10.1037/0003-066X.61.3.204

McCrae, R. R., \& Costa, P. T., Jr. (2008). The five-factor theory of personality. In O. P. John, R. W. Robins, \& L. A. Pervin (Eds.), Handbook of personality: Theory and research., 3rd ed. (pp. 159-181). Guilford Press.

McCullough, M. E., \& Willoughby, B. L. (2009). Religion, self-regulation, and self-control: Associations, explanations, and implications. Psychological Bulletin, 135(1), 69-93. https://doi.org/10.1037/a0014213

McGrath, R. E. (2015). Integrating psychological and cultural perspectives on virtue: The hierarchical structure of character strengths. The Journal of Positive Psychology, 10(5), 407-424. https://doi.org/10.1080/17439760.2014.994222

Muraven, M. (2010). Building self-control strength: Practicing self-control leads to improved self-control performance. Journal of Experimental Social Psychology, 46(2), 465-468. https://doi.org/10.1016/j.jesp.2009.12.011

Paloutzian, R. (2017b). Psychology of religion in the world. Revista Pistis Praxis, 9(1), 15-30. http://dx.doi.org/10.7213/2175-1838.09.001.DS01

Paloutzian, R. F. (2017a). Psychology of religion in global perspective: Logic, approach, 
concepts. The International Journal for the Psychology of Religion, 27(1), 1-13. https://doi.org/10.1080/10508619.2017.1241529

Pargament, K. I., Magyar, G. M., Benore, E., \& Mahoney, A. (2005). Sacrilege: A study of sacred loss and desecration and their implications for health and well-being in a community sample. Journal for the Scientific Study of Religion, 44(1), 59-78. https://doi.org/10.1111/j.1468-5906.2005.00265.x

Park, C. L. (2005). Religion as a meaning-making framework in coping with life stress. Journal of Social Issues, 61(4), 707-729. https://doi.org/10.1111/j.1540-4560.2005.00428.x

Peterson, C., \& Seligman, M. E. P. (2004). Character strengths and virtues: A handbook and classification. Oxford University Press.

Piedmont, R. L. (1999). Does spirituality represent the sixth factor of personality? Spiritual transcendence and the five-factor model. Journal of Personality, 67(6), 985-1013. https://doi.org/10.1111/1467-6494.00080

Ratchford, J. L., Pawl, T., Jeffrey, A., \& Schnitker, S. A. (2021b). What is virtue? Using philosophy to refine psychological definition and operationalization. Manuscript under review.

Ratchford, J. L., Schnitker, S. A., \& Reppas, P. (2021a). The virtue of thrift: A person-centered conceptualization and measure development. Journal of Happiness Studies: An Interdisciplinary Forum on Subjective Well-Being, 22, 385-411. http://doi.org/10.1007/s10902-020-00235-7

Rathunde, K. (2001). Toward a psychology of optimal human functioning: What positive psychology can learn from the "experiential turns" of James, Dewey, and Maslow. Journal of Humanistic Psychology, 41, 135-153. 
https://doi.org/10.1177\%2F0022167801411008

Roberts, B. W., Luo, J., Briley, D. A., Chow, P. I., Su, R., \& Hill, P. L. (2017). A systematic review of personality trait change through intervention. Psychological Bulletin, 143(2), 117-141. https://doi.org/10.1037/bul0000088

Root Luna, L. M., Van Tongeren, D. R., \& Witvliet, C. V. (2017). Virtue, positive psychology, and religion: Consideration of an overarching virtue and an underpinning mechanism. Psychology of Religion and Spirituality, 9, 299-302. https://doi.org/10.1037/rel0000127

Rosmarin, D. H., Pirutinsky, S., Cohen, A. B., Galler, Y., \& Krumrei, E. J. (2011). Grateful to God or just plain grateful? A comparison of religious and general gratitude. The Journal of Positive Psychology, 6(5), 389-396. https://doi.org/10.1080/17439760.2011.596557

Rye, M. S., Wade, N. G., Fleri, A. M., \& Kidwell, J. E. M. (2013). The role of religion and spirituality in positive psychology interventions. In K. I. Pargament, A. Mahoney, \& E. P. Shafranske (Eds.), APA handbook of psychology, religion, and spirituality (Vol. 2): An applied psychology of religion and spirituality (pp. 481-508). American Psychological Association. https://doi.org/10.1037/14046-025

Schindler, S. A., Pfattheicher, S., \& Reinhard, M. A. (2019). Potential negative consequences of mindfulness in the moral domain. European Journal of Social Psychology, 49(5), 10551069. https://doi.org/10.1002/ejsp. 2570

Schnitker, S. A., \& Emmons, R. A. (2017). The psychology of virtue: Integrating positive psychology and the psychology of religion. Psychology of Religion and Spirituality, 9(3), 239-241. http://dx.doi.org/10.1037/re10000133

Schnitker, S. A., King, P. E., \& Houltberg, B. (2019a). Religion, spirituality, and thriving: Transcendent narrative, virtue, and telos. Journal of Research on Adolescence, 29(2), 
276-290. https://doi.org/10.1111/jora.12443

Schnitker, S. A., Ratchford, J. L., Emmons, R. A., \& Barrett, J. L. (2019b). High goal conflict and low goal meaning are associated with an increased likelihood of subsequent religious transformation in adolescents. Journal of Research in Personality, 80, 38-42. https://doi.org/10.1016/j.jrp.2019.04.004

Schnitker, S. A., \& Richardson, K. L. (2019). Framing gratitude journaling as prayer amplifies its hedonic and eudaimonic well-being, but not health, benefits. The Journal of Positive Psychology, 14, 427-439. http://doi.org/10.1080/17439760.2018.1460690

Schnitker, S. A., Shubert, J., Ratchford, J. L., Lumpkin, M., Houltberg, B. J. (2021). Mixed results on the efficacy of the CharacterMe smartphone app to improve self-control, patience, and emotional regulation competencies in adolescents. Frontiers in Psychology, 12, 586713. https://doi.org/10.3389/fpsyg.2021.586713

Schwartz, S. H., Cieciuch, J., Vecchione, M., Davidov, E., Fischer, R., Beierlein, C., \& DirilenGumus, O. (2012). Refining the theory of basic individual values. Journal of Personality and Social Psychology, 103(4), 663-688. https://doi.org/10.1037/a0029393

Snow, N. E. (2019). Positive psychology, the classification of character strengths and virtues, and issues of measurement. The Journal of Positive Psychology, 14(1), 20-31. https://doi.org/10.1080/17439760.2018.1528376

Thomas, R. M., \& Schnitker, S. A. (2017). Modeling the effects of within-person characteristic and goal-level attributes on personal project pursuit over time. Journal of Research in Personality, 69, 206-217. https://doi.org/10.1016/j.jrp.2016.06.012

Van Cappellen, P., Saroglou, V., Iweins, C., Piovesana, M., \& Fredrickson, B. L. (2013). Selftranscendent positive emotions increase spirituality through basic world 
assumptions. Cognition \& Emotion, 27(8), 1378-1394.

https://doi.org/10.1080/02699931.2013.787395

Van Cappellen, P., Saroglou, V., \& Toth-Gauthier, M. (2016). Religiosity and prosocial behavior among churchgoers: Exploring underlying mechanisms. The International Journal for the Psychology of Religion, 26(1), 19-30. https://doi.org/10.1080/10508619.2014.958004

Wade, N. G., Hoyt, W. T., Kidwell, J. E., \& Worthington Jr, E. L. (2014). Efficacy of psychotherapeutic interventions to promote forgiveness: A meta-analysis. Journal of Consulting and Clinical Psychology, 82(1), 154-170. https://doi.org/10.1037/a0035268

Wade, R. (2010). Bridging Christianity, Islam, and Buddhism with virtue ethics. In K.

Engebretson, M. de Souza, G. Durka, \& L. Gearon (Eds.) International handbook of inter-religious education (pp. 313-324). Springer.

Waterman, A. S. (2013). The humanistic psychology-positive psychology divide: Contrasts in philosophical foundations. American Psychologist, 68(3), 124-133. https://doi.org/10.1037/a0032168

Williams, E. G., Ratchford, J. L., Pawl, T., Balkaya-Ince, M., \& Schnitker, S. A. (2021). Selftranscendent moral motivation and virtue development. Manuscript in preparation.

Worthington, E. L., Jr., \& Wade, N. G. (1999). The psychology of unforgiveness and forgiveness and implications for clinical practice. Journal of Social and Clinical Psychology, 18, 385-418. https://doi.org/10.1521/jscp.1999.18.4.385

Yeager, D. S., Henderson, M. D., Paunesku, D., Walton, G. M., D'Mello, S., Spitzer, B. J., \& Duckworth, A. L. (2014). Boring but important: A self-transcendent purpose for learning fosters academic self-regulation. Journal of Personality and Social Psychology, 107(4), 559-580. https://doi.org/10.1037\%2Fa0037637 


\title{
Chapter 5: Theories of Health and Well-Being Germane to a \\ Positive Psychology of Religion and Spirituality
}

\author{
Douglas A. MacDonald \\ Department of Psychology, University of Detroit Mercy
}

\begin{abstract}
Author Note
Douglas A. MacDonald (D) https://orcid.org/0000-0002-2100-1526
\end{abstract}

The author has no conflicts of interest to disclose.

The author would like to acknowledge and thank Edward (Ward) B. Davis, Everett Worthington, and Ethan Lacey for their support in the completion of this chapter and supporting materials.

Correspondence concerning this chapter should be addressed to Douglas A. MacDonald, University of Detroit Mercy, 4001 W. McNichols Road, Detroit MI 48221-3038. Email:

macdonda@udmercy.edu or pneumaticscope@gmail.com 


\begin{abstract}
The chapter provides an overview of existing theories of health and well-being that are relevant for a positive psychology of religion and spirituality. Emphasis is given to theories that have an empirical basis or whose main constructs are operationalized through validated measurement instruments. This chapter covers several theories focused on spiritual well-being, holistic health, perceived wellness, eudaimonia (psychological well-being, self-determination theory, and eudaimonic identity theory), virtues and character strengths, and the PERMA model of wellbeing. To help facilitate programmatic research and greater synergy between the psychology of religion and spirituality, positive psychology, and mainstream psychological science, the chapter briefly describes Koenig's (2012), MacDonald's (2009), and VanderWeele's (2017) theories that attempt to integrate available research into directional causal models.
\end{abstract}

Keywords: spirituality, religion, well-being, health, positive psychology 


\section{Chapter 5: Theories of Health and Well-Being Germane to a Positive Psychology of Religion and Spirituality}

The time for a positive psychology of religion and spirituality has arrived, for at least two reasons. First, scholarly interest in the influence of religiousness and spirituality on health and well-being has shown a marked increase in recent decades (Koenig et al., 2012; Miller, in press; Paloutzian \& Park, 2013; Pargament, 2013). Second, empirical and theoretical developments in positive psychology have characterized religiousness and spirituality both as drivers and expressions of well-being (Lee et al., 2021; Vitters $\varnothing$, 2016). Because these two subdisciplines have similar aims (Davis et al., Chapter 1, this volume) and have been said to go "hand in hand" (Barton \& Miller, 2015, p. 829), movement toward the formalization of a positive psychology of religion and spirituality seems warranted (see Davis et al., Chapter 31, this volume).

For a positive psychology of religion and spirituality to make meaningful scientific progress, it is important to take inventory of the work that has been done. There is a particular need to consolidate knowledge and clarify possible directions for theory and inquiry. With this in mind, the purpose of this chapter is to provide an overview of some available theories and models related to individual-level ${ }^{2}$ religiousness, spirituality, health, and well-being. I emphasize theories whose core constructs are operationalized with measurement tools or have a strong empirical foundation tied to a naturalistic scientific stance. Due to space limitations, only select theories are discussed, but readers are encouraged to explore the many other promising theories out there (e.g., Dodge et al., 2012; Lee et al., 2021; see also Davis et al., Chapter 18, this volume).

\footnotetext{
${ }^{2}$ For multilevel theories and research on religiousness, spirituality, health, and well-being, see King et al. (Chapter 17, this volume), Davis et al. (Chapter 18, this volume), Long and VanderWeele (Chapter 25, this volume), Dik and Alayan (Chapter 27, this volume), Mahoney et al. (Chapter 28, this volume), and Wang et al. (Chapter 30, this volume).
} 


\section{Definitions}

To facilitate clarity of expression, it is worthwhile to provide some working definitions of this chapter's main concepts. In this chapter, the term religiousness will be used rather than religion. As argued by Paloutzian and Park (2021), the term religiousness is more in line with a scientific psychology because it can be "understood as human behavior, including its affective, attitudinal, and cognitive aspects" (p. 3). Following their lead, religiousness is defined as "an individual's or group's understanding, enacting, and being vis-à-vis religion" (Paloutzian \& Park, 2021, pp. 3-4). Spirituality is considered to be a broad, multidimensional, and unique domain of human functioning that is superordinate to, but inclusive of, religiousness (MacDonald, 2017; MacDonald et al., 2015). More precisely, spirituality is defined as a natural aspect of human functioning [that] relates to a special class of non-ordinary experiences and the beliefs, attitudes, and behaviors that cause, co-occur, and/or result from such experiences. The experiences themselves are characterized as involving states and modes of consciousness [that] alter the functions and expressions of self and personality and impact the way in which we perceive and understand ourselves, others, and reality as a whole. (MacDonald et al., 2015, p. 5)

There are numerous definitions and conceptualizations of health, well-being, and related terms (see Appendix 5.S1, Table 5.S1). In this chapter, health and well-being are framed in positive psychological terms and are defined as positive appraisal of one's quality of life and capacity for self-determination and self-fulfillment, as applied to all domains of functioning (e.g., physical, psychological, social, and spiritual). Although arguments might be made that health and well-being should be treated as distinct constructs, available factor analytic research suggests that measures of such concepts and related terms (e.g., wellness) tend to contribute to the same 
factors or components (see MacDonald, 2018). Thus, it appears defendable on empirical grounds to use these concepts as synonyms in this chapter, except where needed to describe a theory.

\section{Theories of Health and Well-Being}

A perusal of the literature suggests that extant theories of health/well-being may be organized into two loose categories. In the first, the theories involve defining and elucidating health/well-being specifically within the domain of spirituality (i.e., spiritual well-being). In essence, these are theories that start with spirituality and then broaden the concept to include health/well-being as it relates to the unfolding and manifestation of spirituality developmentally. In the second category, spirituality is conceptualized as an aspect or facet of broader conceptualizations of health/well-being (i.e., it is viewed as one of several expressions of health and well-being). The following discussion is organized accordingly.

\section{Spirituality as Well-Being}

As noted by Moberg (1984), interest in spiritual well-being (SWB) effectively started 50 years ago thanks to the 1971 White House Conference on Aging. Participants in this conference and its technical committee aimed to provide a theoretical framework for SWB that could be used to facilitate policy development and support inquiry in a variety of areas including but not limited to religious research, quality of life, and holistic health (Moberg, 1971). At the core of this framework is the committee's definition of the term spiritual, which they defined as a person's “inner resources especially [their] ultimate concern, the basic value around which all other values are focused, the central philosophy of life — whether religious, anti-religious, or nonreligious — which guides a person's conduct, the supernatural and nonmaterial dimensions of human nature" (Moberg, 1971, p. 3). Extending from this definition, SWB was seen as a dynamic and lifelong process of spiritual growth that involves addressing basic human needs to 
cope with suffering, adversity, existential uncertainty, and psychosocial and personological change across the lifespan. Moberg (1984) elaborated that, within this framework, SWB is considered to be (a) multidimensional, (b) related to but distinct from religiousness, and (c) manifested in all contexts of life and not just within institutional religious settings. It is noteworthy that Moberg also affirmed the definition of SWB developed by the National Interfaith Coalition on Aging (1975). In this definition, SWB was defined as "the affirmation of life in a relationship with God, self, community, and the environment that nurtures and celebrates wholeness" (quoted from Moberg, 1984, p. 352). In both cases, Moberg (1984) opined that although these definitions may have some practical value, they are too broad to permit their use in empirical research on SWB. He therefore called for the development of assessment tools that would measure the various facets of SWB.

With respect to its facets, Moberg (1984) acknowledged that SWB has "possibly hundreds of components" (p. 352). Despite the construct's complexity, Moberg (1984) followed through with his call by using survey data (obtained in the U.S. and Sweden) to construct several indices he proffered could be used to study SWB empirically. In particular, he took items from the survey and completed an exploratory factor analysis. Based upon observed factor loadings and analysis of item content, he labeled the factors Christian Faith, Self-Satisfaction, Personal Piety, Subjective Spiritual-Well-Being, Optimism, Religious Cynicism, and Elitism. In his interpretation, Moberg viewed the first four factors as having both theoretical value and empirical support with his data, whereas the latter three factors appeared conceptually and statistically weak and possibly mislabeled. Subsequent research using Moberg's indices has provided evidence supporting their convergent and discriminant validity (MacDonald, 2000a). 
Although the development of empirical indices is noteworthy in its own right, Moberg made other contributions as well (e.g., Moberg, 1979; Moberg \& Brusek, 1978). The most significant of these is a theory he developed that serves as the basis for one of the most widely used measures of SWB, the Spiritual Well-Being Scale (Paloutzian \& Ellison, 1982; Ellison, 1983). In this theory, SWB is construed in terms of vertical and horizontal dimensions. The vertical dimension is referred to as religious well-being, which is defined as well-being manifested in one's perceived relationship to a higher power (i.e., God). The horizontal dimension is called existential well-being and refers to well-being expressed in the form of purpose, meaning in life, and life satisfaction—but without any overtly religious elements. In this model, SWB is operationalized as the combination of these two dimensions. The psychometric properties of the Spiritual Well-Being Scale have been studied extensively, and the measure has demonstrated good evidence of reliability and convergent and criterion validity (Paloutzian et al., 2021). However, evidence supporting the factorial validity of the scale — and by extension the two-dimensional model it purportedly measures — has been less favorable (Sterner et al., 2021).

Another more recently developed approach to SWB and its measurement has its roots in the 1970s, especially the National Interfaith Coalition on Aging's (1975) definition mentioned above. This theory is the four-domain model of spiritual health and well-being that serves as the conceptual basis for the Spiritual Well-Being Questionnaire (Gomez \& Fisher, 2003), Spiritual Health and Life Orientation Measure (Fisher, 2010), and 4-item Spiritual Well-Being Index (Fisher \& Ng, 2017). As its name implies, the four-domain model defines SWB as consisting of four facets: Personal (i.e., relation to one's self, with an emphasis on life meaning, purpose, and values), Communal (i.e., relation with others, involving morality, religion, culture, and a positive perception of humanity), Environmental (i.e., sense of connection, appreciation, and nurturing 
orientation toward nature), and Transcendental (i.e., relation of self to a transcendent power or dimension). These domains are seen as dynamic and interconnected, such that they change over time and mutually influence one another. However, the motivational core of the model, and the primary driver by which the fulfillment of SWB can be attained, is an intentional desire for selfdevelopment that is congruent with personal meaning and purpose in life. The successful realization of SWB is manifested through the experience of bliss and internal harmony (Fisher, 2010). The measures based on this conceptual model have been used in several studies and demonstrate acceptable evidence of reliability and validity (Fisher, 2010; Fisher \& Ng, 2017).

\section{Spirituality as a Component of Well-Being}

Theories in this next group tend to incorporate religiousness/spirituality as one expression of multicomponential conceptualizations of health/well-being. We begin with Stoudenmire et al.'s (1985) model of optimal functioning and the measure they developed to assess it — the Holistic Living Inventory. Motivated by the desire to address ambiguities in the definition and measurement of holistic living, Stoudenmire and colleagues (1985) drew from theories of holism and optimal functioning adopted by different organizations, such as the American Holistic Medical Association and the Institute of Religion and Health. They constructed a four-facet model of holistic living that includes physical, emotional, mental, and spiritual dimensions. Optimal functioning was defined specific to each component. The physical dimension was defined as enhancement of physical fitness through responsible lifestyle (e.g., diet, exercise) practices and self-monitoring and regulation of one's physical health and functioning. The emotional dimension was seen as enhancement of emotional self-satisfaction through positive and responsible behavioral choices and the avoidance or mitigation of negative emotions such as depression, anger, and anxiety. The mental dimension involved the enhancement of mental 
developmental potentials through intellectual pursuits, the increased appreciation of aesthetics, and the minimization of irrational attitudes. Lastly, the spiritual dimension involved fostering a sense of oneness with a higher power and actively developing and adhering to an ethical system.

Although the Holistic Living Inventory has demonstrated reasonably good evidence of validity and reliability (Stoudenmire et al., 1985), it has been criticized for its length and item complexity. This led to the development of the Mental, Physical, and Spiritual Well-Being Scale (Vella-Brodrick \& Allen, 1995), which has demonstrated stronger psychometric properties and an improved theoretical foundation. For instance, the model underlying the Holistic Living Inventory does not specify how its four dimensions relate to each other to enable integrated, optimal functioning. Conversely, Vella-Brodrick and Allen (1995) not only provide clear definitions of their three identified domains of well-being, but they also state explicitly that holistic well-being involves "the balanced nourishment of mind, body, and spirit" (p. 661). Their introduction of balance into the conceptualization of holistic well-being makes it clear that holistic health/well-being depends on equally meaningful attention to all facets of well-being.

Another theory of interest is Adams et al.'s (1997) wellness model, which serves as the basis of their Perceived Wellness Survey (PWS). This model draws from prior theories of positive health, with an emphasis on Dunn's (1961) systems approach and Antonovsky's (1988) notion of salutogenesis (i.e., an approach to health that focuses on factors that contribute to wellbeing, particularly in the face of adversity and stress). Adams and colleagues (1997) conceptualize perceived wellness as "a multidimensional, salutogenic construct" (p. 209) wherein overall wellness is facilitated and maintained through the reciprocal integration and homeostasis of well-being's dimensions. There are six dimensions that comprise the model: physical wellness (i.e., perception of, and expectations for, positive physical health), 
psychological wellness (i.e., expectation of positive outcomes in life), social wellness (i.e., perception of social support), emotional wellness (i.e., positive self-esteem), intellectual wellness (i.e., perception of optimal engagement in activities that are intellectually stimulating), and spiritual wellness (i.e., a sense of life purpose and belief in the existence of a unifying force). As an operationalization of the wellness model, the Perceived Wellness Survey has demonstrated evidence of acceptable reliability and validity and shown promise in research (Adams et al., 1997; Harari et al., 2005; MacDonald, 2018; Rothmann \& Ekkerd, 2007).

A final set of theories are well-established in positive psychology proper and may be characterized as representing related but distinct research traditions within that subdiscipline. First are theories of eudaimonic well-being. With its roots traceable to ancient Greece and particularly the work of Aristotle (e.g., Aristotle, 2002), eudaimonia is an approach to well-being that is seen as analogous to notions of self-actualization. At its core, eudaimonic well-being is seen as living life in an authentic, self-directed, engaged, and meaningful manner. It is worth noting that in his formulation of the concept of eudaimonia, Aristotle placed primary emphasis on ethics, virtue, and wisdom and did not make overt ties to anything spiritual. However, some scholars have noted that the concept later underwent a "Christianization," resulting in it incorporating transcendence (a widely recognized spiritual concept; Boyce-Tillman, 2020).

In positive psychology, major theories aligned with eudaimonia include psychological well-being theory (Ryff, 1989, 2018; Ryff \& Keyes, 1995; Ryff \& Singer, 2008), selfdetermination theory (Ryan et al., 2008), and eudaimonic identity theory (Waterman, 2011; Waterman et al., 2010; see Appendix 5.S1, Table 5.S2 for summaries of each of these major theories). None of these theories includes much by way of overt religious or spiritual content, but each of them incorporates meaning and purpose in life, albeit to differing degrees. 
Meaning and purpose is a relatively common element in definitions and measures of spirituality and/or SWB as well (e.g., Paloutzian \& Ellison, 1982; see Park \& Van Tongeren, Chapter 6, this volume). Given this content overlap, care must be exercised when interpreting empirical relations between spirituality and eudaimonic well-being, because correlations are likely inflated when relying on instruments that assess meaning/purpose as part of each construct (for discussions of this issue, see Garssen et al., 2016; Koenig, 2008; MacDonald, 2017, 2018). Notwithstanding such problems, scholarly attention has been given to the relation and place of spirituality within eudaimonic theory (e.g., Pargament et al., 2016), and scholars have proposed extending eudaimonic theories to incorporate spirituality in a manner that extends beyond meaning and purpose alone (e.g., van Dierendonck, 2004).

Next, there are positive psychological theories that frame well-being in terms of values, virtues, and character. Perhaps the most influential of these is Peterson and Seligman's (2004) Values in Action (VIA) nosology of character strengths and virtues. In developing this system, Peterson and Seligman completed an extensive survey of the literature with the intention of identifying strengths and virtues that appeared invariant across history and cultures and were universally seen as contributing to the development of good moral character. Their efforts resulted in the creation of a classification system and several associated measures, chiefly including the 240-item VIA Inventory of Strengths. The system itself provides six classes of core virtues, under which are 24 character strengths. The virtues are wisdom/knowledge, courage, humanity, justice, temperance, and transcendence. Within the last virtue (transcendence), religiousness and spirituality is placed as a character strength. It is described as involving faith and purpose and as manifesting through the adoption of a belief system regarding one's life and the universe as having meaning. It more specifically entails having an understanding of one's 
place within the universe in a manner that informs one's conduct and serves as a source of comfort (Park et al., 2004). The VIA Inventory of Strengths and the broader scientific study of virtues and strengths have garnered a lot of attention in positive psychology (e.g., Stichter \& Saunders, 2019; see also Ratchford et al., Chapter 4, this volume). There also is good evidence supporting the reliability and validity of the VIA Inventory of Strengths (Peterson \& Seligman, 2004; Ruch et al., 2010) and its more recent revision (McGrath \& Wallace, 2021).

One last theory deserves mention - the PERMA model of flourishing proposed by Seligman (2011). PERMA is an acronym that stands for Positive emotions, Engagement,

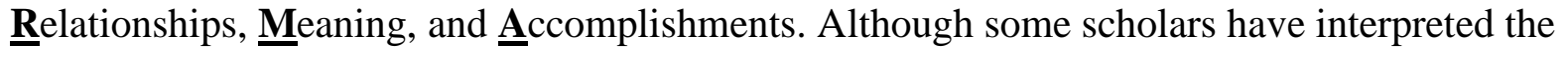
model as yet another definition of well-being (e.g., Goodman et al., 2018), Seligman (2018) has argued the model's five elements are best understood as the building blocks of well-being and not as well-being per se. A measure of the elements, called the PERMA-Profiler, has been developed and exhibited satisfactory evidence of reliability and validity (Butler \& Kern, 2016).

\section{The Need for an Integrative Scientific Theory}

All the theories discussed so far in this chapter were selected because of their potential to contribute to empirical research. Based upon this sampling, one might have the impression there is a more than sufficient theoretical footing to guide a positive psychology of religion and spirituality. However, this impression would not be wholly accurate for at least two key reasons. First, extant theories are disparate in terms of their underlying emphases as to the place of spirituality within well-being and vice-versa, a problem that is exacerbated by the fact the definitions of spirituality and well-being often blend in ways that create confusion as to whether spirituality is a contributor to_-versus a manifestation of —well-being. This is particularly the case when it comes to meaning and purpose (MacDonald, 2017, 2018). Second and more 
importantly, there are indications the psychology of religion and spirituality itself is not wholly clear on what it should study and how best to study it (Linfield, 2021). There is discussion and debate about how the subdiscipline can be brought into greater accord with psychological science. Paloutzian and Park (2021), two prominent figures in the field, have suggested a multilevel interdisciplinary meaning systems approach holds promise for guiding such efforts.

The existing research on spirituality, religion, health, and well-being provides reasonably fertile ground for the development of testable theories that can guide a rigorous positive psychology of religion and spirituality. One current challenge is finding a way to rectify and integrate the myriad theories that are presently available. The research well-establishes the empirical links between religiousness, spirituality, and well-being. As such, we are now at a point where we need to move to the level of identifying causal influences and mechanisms that contribute to well-being outcomes. More specifically, there is a clear need to recognize and incorporate biological, social, psychological (including cognitive, affective, experiential, and personality factors), behavioral, and developmental factors into theoretical frameworks, in order to enable researchers and practitioners to understand the complexity, structure, and dynamics of religiousness and spirituality and its influence on health/well-being. Fortunately, there are some candidate theories that hold promise to serve as starting points for programmatic research. Due to space constraints, I mention three here, but the reader is encouraged to explore the literature for others (e.g., Davis et al., Chapter 18, this volume).

First, Koenig (2012) and Koenig et al. (2012) present impressively detailed theoretical models specifying causal pathways between religiousness and mental and physical health. Figure 5.1 shows a simplified, adapted version of the model applicable to Western religions (i.e., Christianity, Judaism, and Islam) and presenting positive mental health as the outcome variable. 
Second is MacDonald's (2009) bio-social-psychological model (see Figure 5.2), which was devised using the comprehensive, five-dimensional measurement model of spirituality developed by MacDonald (2000a, 2000b). In particular, after a survey of available theory and research to identify what correlates with and contributes to each of the five dimensions, MacDonald suggested the dimensions could be configured to create a directional causal model that starts with social (religiousness) and biological (spiritual experience) determinants, which in turn influence psychological development and ultimately well-being. Finally, VanderWeele's (2017) human flourishing theory (see Figure 5.3) is a model of overall well-being in which well-being (which he uses synonymously with the term flourishing) is defined as "a state in which all aspects of a person's life are good... [because the person is] doing or being well in the following five broad domains of human life: (i) happiness and life satisfaction; (ii) health, both mental and physical; (iii) meaning and purpose; (iv) character and virtue; and (v) close social relationships" (p. 8149). (Since then, a sixth domain has been added—financial and material stability [Gallup, 2021].) VanderWeele's (2017) theory highlights four major causal pathways to these six facets of wellbeing: family, work, education, and religion/spirituality. VanderWeele (2017) derived this theory through a rigorous review of the longitudinal, experimental, and quasi-experimental literature, and he and Gallup (2021) have developed and refined a well-validated, cross-culturally applicable measure of it — the Global Flourishing Study Questionnaire (Gallup, 2021).

In each of these three models, there are some appealing features worth mentioning. For instance, each of these theories: (a) is copasetic with naturalistic science (e.g., their variables, mechanisms, and outcomes are construed in ways that do not require the use of nebulous or metaphysically loaded concepts that are difficult to define and measure with precision; Park \& Paloutzian, 2021), (b) takes culture and cultural differences into consideration with respect to 
how the models operate and/or apply to different populations (Gallup, 2021; Koenig, 2012;

Koenig et al., 2012; MacDonald et al., 2015; VanderWeele, 2017), and (c) is testable via the use of well-validated assessment tools (Gallup, 2021; MacDonald, 2000a, 2000b; VanderWeele, 2017). At the same time, each model offers a unique approach to the study of religiousness, spirituality, and health/well-being. For instance, Koenig's model focuses specifically on religiousness and incorporates biological, psychological, social, and environmental influences and dynamics. By comparison, MacDonald's model is domain-specific. Its variables have been characterized as defining the content domain of spirituality as unique from other functional domains, and the model's biological, social, and psychological components are construed as being embedded in the variables themselves. Last, VanderWeele's model is inclusive of many broad domains of human functioning and well-being (e.g., social, religious, spiritual, physical, psychological, and financial/material), has been developed and refined through rigorous empirical and cross-cultural research, and incorporates religiousness and spirituality as key contributors to overall flourishing and well-being.

\section{Conclusion}

For the past few decades, shared interest in religiousness, spirituality, health, and wellbeing has been growing in both the positive psychology and psychology of religion and spirituality fields. Given the state of the science, it appears that both subdisciplines have a lot to gain through the formalization of a positive psychology of religion and spirituality (see Davis et al., Chapter 31, this volume). It is hoped that the theories and models discussed in this chapter aid in facilitating new developments in research, practice, policymaking, and public health. 


\section{References}

Adams, T., Bezner, J., \& Steinhardt, M. (1997). The conceptualization and measurement of perceived wellness: Integrating balance across and within dimensions. American Journal of Health Promotion, 11(3), 208-218. https://doi.org/10.4278\%2F0890-1171-11.3.208

Antonovsky, A. (1988). Unraveling the mystery of health. Jossey-Bass.

Aristotle. (2002). Nicomachean ethics (C. Rowe \& S. Broadie, Trans.). Oxford University Press.

Barton, Y. A., \& Miller, L. (2015). Spirituality and positive psychology go hand in hand: An investigation of multiple empirically derived profiles and related protective benefits. Journal of Religion and Health, 54, 829-843. https://doi.org/10.1007/s10943-015-0045-2

Boyce-Tillman, J. (2020). An ecology of eudaimonia and its implications for music education. In G. D. Smith \& M. Silverman (Eds.), Eudaimonia (pp. 71-88). Routledge.

Butler, J., \& Kern, M. L. (2016). The PERMA-Profiler: A brief multidimensional measure of flourishing. International Journal of Wellbeing, 6(3), 1-48. https://doi.org/10.5502/ijw.v6i3.526

Dodge, R., Daly, A., Huyton, J., \& Sanders, L. (2012). The challenge of defining wellbeing. International Journal of Wellbeing, 2(3), 222-235. https://doi.org/10.5502/ijw.v2i3.4

Dunn, H. (1961). High level wellness. Vernon.

Ellison, C. W. (1983). Spiritual well-being: Conceptualization and measurement. Journal of Psychology and Theology, 11(4), 330-340. https://doi.org/10.1177/009164718301100406

Fisher, J. (2010). Development and application of a spiritual well-being questionnaire called SHALOM. Religions, 1(1), 105-121. https://doi.org/10.3390/rel1010105

Fisher, J., \& Ng, D. (2017). Presenting a 4-Item Spiritual Well-Being Index (4-ISWBI). Religions, 8(9), Article 179. https://doi.org/10.3390/rel8090179 
Gallup. (2021). Global Flourishing Study Questionnaire development report. Author. https://hfh.fas.harvard.edu/files/pik/files/globalflourishingstudy_report.pdf

Garssen, B., Visser, A., \& de Jager Meezenbroek, E. (2016). Examining whether spirituality predicts subjective well-being: How to avoid tautology. Psychology of Religion and Spirituality, 8(2), 141-148. https://doi.org/10.1037/rel0000025

Gomez, R., \& Fisher, J. W. (2003). Domains of spiritual well-being and development and validation of the Spiritual Well-Being Questionnaire. Personality and Individual Differences, 35, 1975-1991. https://doi.org/10.1016/S0191-8869(03)00045-X

Goodman, F. R., Disabato, D. J., Kashdan, T. B., \& Kauffman, S. B. (2018). Measuring wellbeing: A comparison of subjective well-being and PERMA. Journal of Positive Psychology, 13(4), 321-332. https://doi.org/10.1080/17439760.2017.1388434

Harari, M. J., Waehler, C. A., \& Rogers, J. R. (2005). An empirical investigation of a theoretically based measure of perceived wellness. Journal of Counseling Psychology, 52(1), 93-103. https://doi.org/10.1037/0022-0167.52.1.93

Koenig, H. G. (2008). Concerns about measuring "spirituality" in research. Journal of Nervous and Mental Disease, 196, 349-355. https://doi.org/10.1097/NMD.0b013e31816ff796

Koenig, H. G. (2012). Religion, spirituality, and health. International Scholarly Research Notices. Article 278730. https://doi.org/10.5402/2012/278730

Koenig, H. G., King, D. E., \& Carson, V. B. (2012). Handbook of religion and health (2 ${ }^{\text {nd }}$ ed.). Oxford University Press.

Lee, M. T., Kubzansky, L. D., \& VanderWeele, T. J. (Eds.). (2021). Measuring well-being: Interdisciplinary perspectives from the social sciences and humanities. Oxford University Press. 
Linfield, K. J. (Ed.). (2021). Psychology of religion and spirituality: What should we study and how? Introducing this special section. Psychology of Religion and Spirituality, 13(1), 12. http://dx.doi.org/10.1037/rel0000401

MacDonald, D. A. (2000a). Spirituality: Description, measurement, and relation to the Five Factor Model of personality. Journal of Personality, 68(1), 153-197. https://doi.org/10.1111/1467-6494.t01-1-00094

MacDonald, D. A. (2000b). The Expressions of Spirituality Inventory: Test development, validation and scoring information. Unpublished test manual.

MacDonald, D. A. (2009). Identity and spirituality: Conventional and transpersonal perspectives. International Journal of Transpersonal Studies, 28, 86-106. https://doi.org/10.24972/ijts.2009.28.1.86

MacDonald, D. A. (2017). Commentary on "Existential well-being: Spirituality or well-being?" Journal of Nervous and Mental Disease, 205(3), 242-248. https://doi.org/10.1097/NMD.0000000000000649

MacDonald, D. A. (2018). Taking a closer look at well-being as a scientific construct: Delineating its conceptual nature and boundaries in relation to spirituality and existential functioning. In N. J. L. Brown, T. Lomas, \& F J. Eiroa-Orosa (Eds.), The Routledge international handbook of critical positive psychology (pp. 26-52). Routledge.

MacDonald, D. A., Friedman, H. L., Brewczynski, J., Holland, D., Salagame, K. K. K., Mohan, K. K., Gubrij, Z. O., \& Cheong, H. W. (2015). Spirituality as a scientific construct: Testing its universality across cultures and languages. PLOS One, 10(3), Article e0117701. https://doi.org/10.1371/journal.pone.0117701 
McGrath, R. E., \& Wallace, N. (2021). Cross-validation of the VIA Inventory of StrengthsRevised and its short forms. Journal of Personality Assessment, 103(1), 120-131. https://doi.org/10.1080/00223891.2019.1705465

Miller, L. J. (Ed.). (in press). Oxford handbook of psychology and spirituality (2 ${ }^{\text {nd }}$ ed.). Oxford University Press.

Moberg, D. O. (1971). Spiritual well-being: Background and issues. White House Conference on Aging. https://files.eric.ed.gov/fulltext/ED057348.pdf

Moberg, D. O. (Ed.). (1979). Spiritual well-being: Sociological perspectives. University Press of America.

Moberg, D. O. (1984). Subjective measures of spiritual well-being. Review of Religious Research, 25(4), 351-364. https://doi.org/10.2307/3511368

Moberg, D. O., \& Brusek, P. (1978). Spiritual well-being: A neglected subject in quality of life research. Social Indicators Research, 5, 303-323. https://doi.org/10.1007/BF00352936

National Interfaith Coalition on Aging. (1975). Spiritual well-being: A definition. Author.

Paloutzian, R. F., Agilkaya-Sahin, Z., Bruce, K. C., Kvande, M. N., Malinakova, K., ... You, S.K. (2021). The Spiritual Well-Being Scale (SWBS): Cross-cultural assessment across 5 continents, 10 languages, and 300 studies. In A. Ai, P. Wink, R. F. Paloutzian, \& K. A. Harris (Eds.), Assessing spirituality in a diverse world (pp. 413-444). https://link.springer.com/chapter/10.1007/978-3-030-52140-0_17

Paloutzian, R. F., \& Ellison, C. W. (1982). Loneliness, spiritual well-being and quality of life. In L. A. Peplau \& D. Perlman (Eds.), Loneliness (pp. 224-237). John Wiley \& Sons.

Paloutzian, R., \& Park, C. (2013). Handbook of the psychology of religion and spirituality (2 ${ }^{\text {nd }}$ ed.). Guilford Press. 
Pargament, K. I. (Ed.). (2013). APA handbook of psychology, religion, and spirituality [2 volumes]. American Psychological Association. https://doi.org/10.1037/14045-000

Pargament, K. I., Wong, S., \& Exline, J. J. (2016). Wholeness and holiness: The spiritual dimension of eudaimonics. In J. Vitters $\emptyset$ (Ed.), Handbook of eudaimonic well-being (pp. 379-394). Springer.

Park, C., \& Paloutzian, R. (2021). Is the psychology of religion and spirituality a science? Yes. Psychology of Religion and Spirituality, 13(1), 24-25. https://doi.org/10.1037/rel0000403

Park, N., Peterson, C., \& Seligman, M. E. P. (2004). Strengths of character and well-being. Journal of Social and Clinical Psychology, 23(5), 603-619. https://doi.org/10.1521/jscp.23.5.603.50748

Peterson, C., \& Seligman, M. E. P. (2004). Character strengths and virtues: A handbook and classification. American Psychological Association; Oxford University Press.

Rothmann, S., \& Ekkerd, J. (2007). The validation of the perceived wellness survey in the South African police service. SA Journal of Industrial Psychology, 33(3), 35-42.

Ruch, W., Proyer, R. T., Harzer, C., Park, N., Peterson, C., \& Seligman, M. E. P. (2010). Values in Action Inventory of Strengths (VIA-IS): Adaptation and validation of the German version and the development of a peer-rating form. Journal of Individual Differences, 31(3), 138-149. https://doi.org/10.1027/1614-0001/a000022

Ryan, R. M., Huta, V., \& Deci, E. L. (2008). Living well: A self-determination theory perspective on eudaimonia. Journal of Happiness Studies, 9, 139-170. https://doi.org/10.1007/s10902-006-9023-4 
Ryff, C. D. (1989). Happiness is everything, or is it? Explorations on the meaning of psychological well-being. Journal of Personality and Social Psychology, 57(6), 10691081. https://doi.org/10.1037/0022-3514.57.6.1069

Ryff, C. D. (2018). Eudaimonic well-being: Highlights from 25 years of inquiry. In K. Shigemasu, S. Kuwano, T. Sato, \& T. Matsuzawa (Eds.), Insights from psychology: Proceedings of the $31^{\text {st }}$ Internatinal Congress of Psychology (pp. 375-395). Wiley.

Ryff, C. D., \& Keyes, C. L. M. (1995). The structure of psychological well-being revisited. Journal of Personality and Social Psychology, 69(4), 719-727. https://doi.org/10.1037/0022-3514.69.4.719

Ryff, C. D., \& Singer, B. H. (2008). Know thyself and become what you are: A eudaimonic approach to psychological well-being. Journal of Happiness Studies, 9(1), 13-39. https://doi.org/10.1007/s10902-006-9019-0

Seligman, M. E. P. (2011). Flourish. Simon \& Schuster.

Seligman, M. E. P. (2018). PERMA and the building blocks of well-being. Journal of Positive Psychology, 13(4), 333-335. https://doi.org/10.1080/17439760.2018.1437466

Sterner, W. R., Hall, S., \& Burkholder, D. (2021). An examination of confirmatory factor analytic models of the Spiritual Well-Being Scale. The Journal of Counselor Preparation and Supervision, 14(2). https://repository.wcsu.edu/jcps/vol14/iss2/3

Stichter, M., \& Saunders, L. (2019). Positive psychology and virtue: Values in action. Journal of Positive Psychology, 14(1), 1-5. https://doi.org/10.1080/17439760.2018.1528381

Stoudenmire, J., Batman, D., Pavlov, M., \& Temple, A. (1985). Validation of a Holistic Living Inventory. Psychological Reports, 57, 303-311. https://doi.org/10.2466/pr0.1985.57.1.303 
van Dierendonck, D. (2004). The construct validity of Ryff's Scales of Psychological WellBeing and its extension with spiritual-well-being. Personality and Individual Differences, 36(3), 629-643. https://doi.org/10.1016/S0191-8869(03)00122-3

VanderWeele, T. J. (2017). On the promotion of human flourishing. PNAS, 114(31), 8148-8156. https://doi.org/10.1073/pnas.1702996114

Vella-Brodrick, D. A., \& Allen, F. C. L. (1995). Development and psychometric evaluation of the Mental, Physical, and Spiritual Well-Being Scale. Psychological Reports, 77, 659674. https://doi.org/10.2466/pr0.1995.77.2.659

Vitters $\varnothing$ J. (Ed.). (2016). Handbook of eudaimonic well-being. Springer.

Waterman, A. S. (2011). Eudaimonic identity theory: Identity as self-discovery. In S. J. Schwartz, K. Luyckx, \& V. L. Vignoles (Eds.), Handbook of identity theory and research (pp. 357-379). Springer.

Waterman, A. S., Schwartz, S. J., Zamboanga, B. L., Ravert, R. D., Williams, M. K., Agocha, V. B., Kim,. S. Y., \& Donnellan, M. B. (2010). The Questionnaire for Eudaimonic WellBeing: Psychometric properties, demographic comparisons, and evidence of validity. Journal of Positive Psychology, 5, 41-61. https://doi.org/10.1080/17439760903435208 


\section{Figure 5.1}

\section{A Simplified Version of Koenig's (2012) Model of Religiousness and Positive Mental Health}

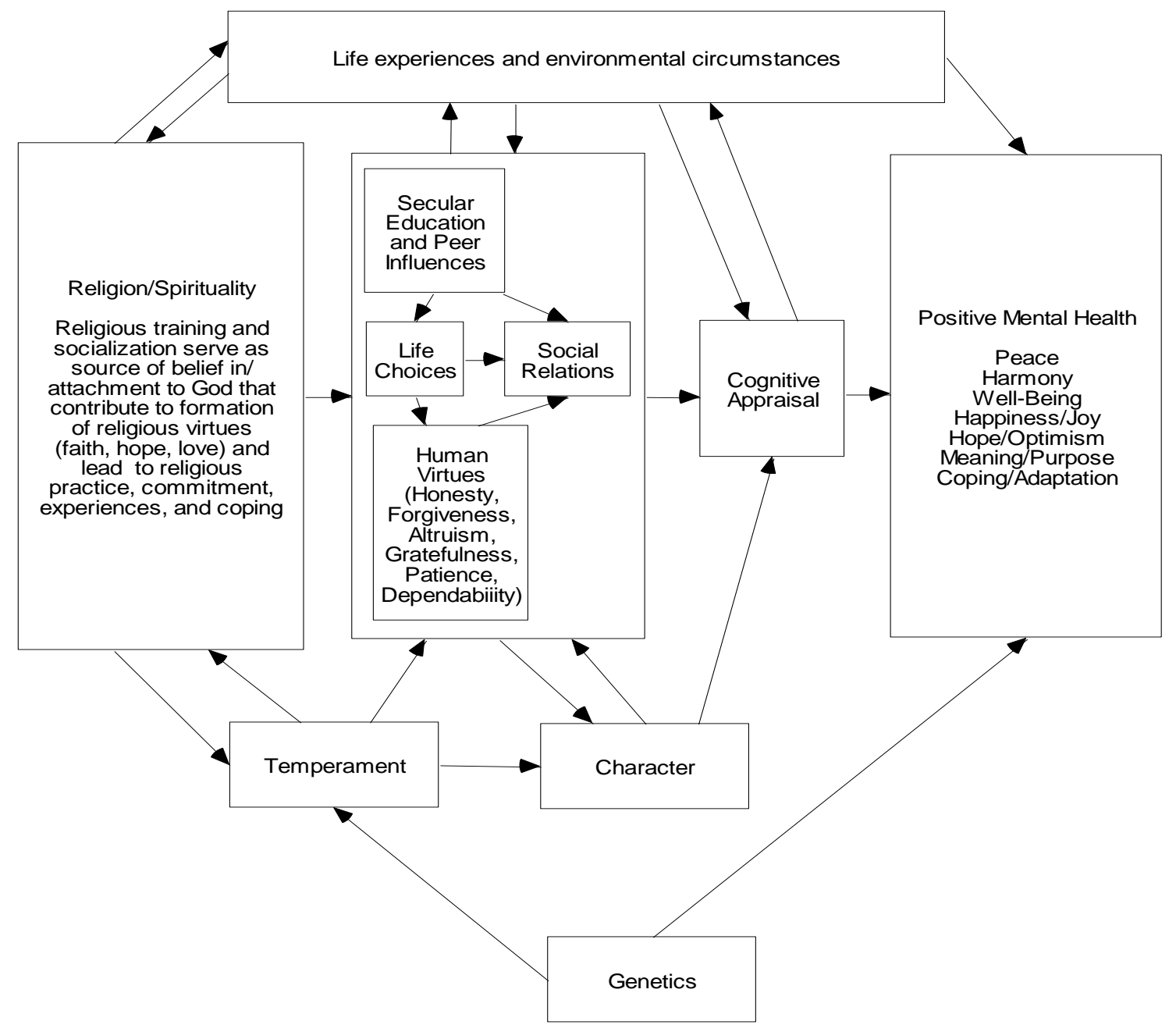

Note. Figure adapted from Koenig (2012). This figure shows Koenig's model based on Western religious traditions.

There are separate models based on Eastern religious and Secular Humanist traditions. All three models in their full forms can be found in Koenig et al. (2012). 


\section{Figure 5.2}

\section{A Visual Depiction of MacDonald's (2009) Bio-Social-Psychological Model of Spirituality and}

\section{Well-Being}
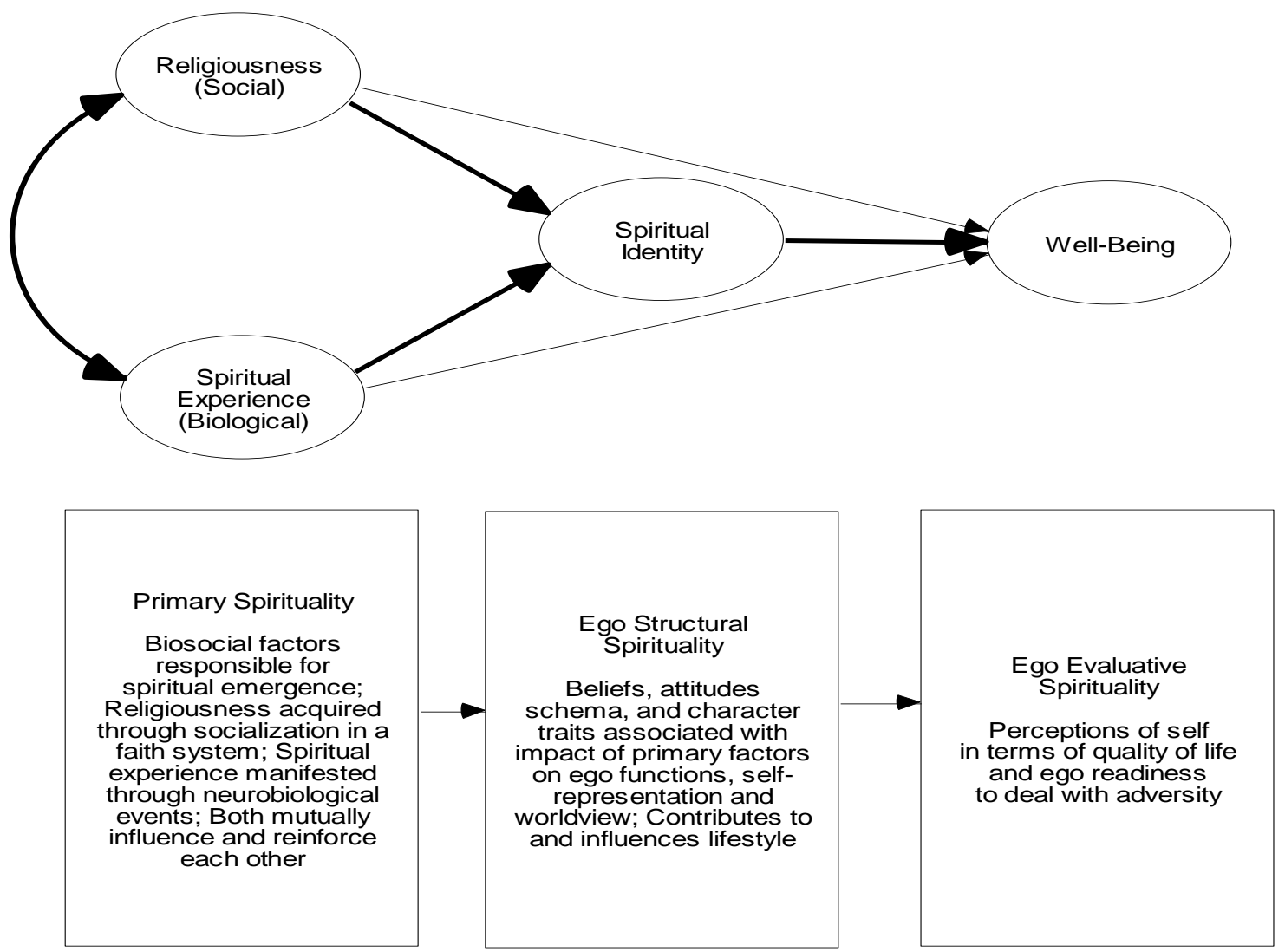

Note. Figure adapted from MacDonald (2009). The top part of the figure shows a directional structural model, with the thick-lined arrows denoting the main directional pathways. The bottom part of the figure provides an alternative and more theory-informed version of the model. 


\section{Figure 5.3}

VanderWeele's (2017) Theory of Human Flourishing (Well-Being)

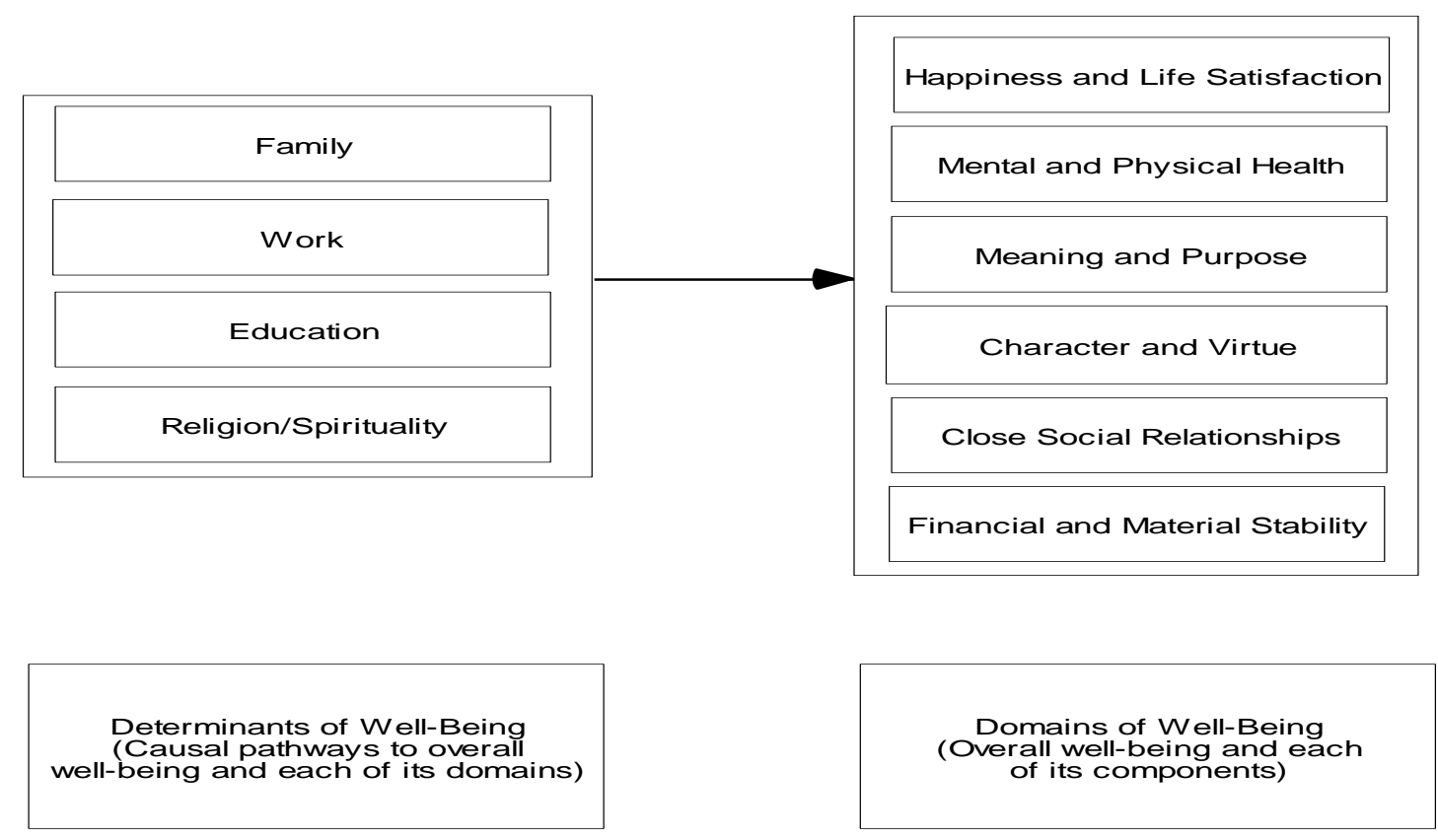

Note. VanderWeele's (2017) human flourishing theory is a model in which well-being (seen as synonymous with flourishing) is defined as "a state in which all aspects of a person's life are good" (VanderWeele, 2017, p. 8149). Specifically, the person is "doing or being well in the following [six] broad domains of human life: (i) happiness and life satisfaction; (ii) health, both mental and physical; (iii) meaning and purpose; (iv) character and virtue; close social relationships [and (vi) financial and material stability]" (VanderWeele, 2017, p. 8149; see also Gallup, 2021). Each of these domains is theorized as being desired universally (i.e., they are applicable to all cultures), and as typically serving as an end in itself. There are four main determinants of overall well-being and its facets (domains): family, work, education, and religion/spirituality (Gallup, 2021; VanderWeele, 2017). 


\title{
Chapter 6: Meaning as a Framework for Integrating Positive Psychology and the Psychology of Religiousness and Spirituality
}

\author{
Crystal L. Park \\ University of Connecticut \\ Daryl R. Van Tongeren \\ Hope College
}

Correspondence concerning this chapter should be addressed to Crystal L. Park, Department of Psychological Sciences, University of Connecticut, 406 Babbidge Road, Unit 1020, Storrs, CT 06269. Email: crystal.park@uconn.edu 


\begin{abstract}
Both the subfields of positive psychology (PP) and the psychology of religiousness and spirituality (PRS) are producing interesting, relevant, and impactful research, but very little integration of this research occurs between these subfields. We propose that a meaning system framework may be useful in unifying science and practice at the intersection of PP and PRS. In this chapter, we describe this meaning systems framework and summarize each subfield's key meaning-related areas of focus, methods, and applications. We then highlight the convergences and divergences in these key areas of both subfields. We conclude that meaning is a unifying construct around which future theory and empirical research on PP and PRS can coalesce, and we propose some specific directions for a fruitful integration of these two subfields.
\end{abstract}

Keywords: meaning, religion, spirituality, positive psychology 


\section{Chapter 6: Meaning as a Framework for Integrating Positive Psychology and the Psychology of Religiousness and Spirituality}

Psychological science often reflects the contemporary trends and currents of culture. Two prominent subfields within psychology—positive psychology (PP) and the psychology of religiousness and spirituality (PRS) — appear especially relevant at this historical and cultural inflection point. The search for a deeper sense of mooring and a more authentic life is a persistent theme in human nature (Bland, 2020). Because PP and PRS are substantially invested in understanding meaningful human experience (Kim-Prieto, 2014), each may hold answers to this prevailing search for meaning. PP aims to highlight and capitalize on the strengths of individuals and communities to promote well-being, and PRS provides a critical perspective on individuals as they seek meaning and transcendent connection. The need for increased collaboration between researchers in these subfields (to integrate their respective bodies of knowledge) is clear (see Davis et al., Chapter 1, this volume). Synergistic collaboration can propel both subfields forward, generate actionable advances in knowledge, and address pressing existential questions that could improve the human condition.

Unfortunately, to date, little collaborative research has brought the knowledge and perspectives of these two subfields together. In this chapter, we propose to apply a meaning systems framework to help integrate these subfields. Because both emphasize many meaningrelevant aspects in their theories and empirical work, meaning provides a unifying scaffolding. This chapter uses a global meaning framework to review the contemporary conceptual and empirical work on meaning in both areas, allowing us to identify convergences and divergences between the two subdisciplines, as well as promising opportunities for future cross-pollination.

\section{Bringing PP and PRS Together: A Meaning-Focused Approach}


By definition, PP focuses primarily on positive aspects of psychology (Schnitker et al., 2017), and from its founding, PP has been especially focused on applications, with the special emphasis on promoting well-being. PP's focus on the "positive" features of human functioning inherently narrows its areas of potential intersection with other fields. By comparison, PRS has generally been open to both positive and negative aspects of psychological phenomena. For example, Allport and his colleagues (Allport \& Ross, 1967) sought to identify which aspects of religiousness promoted versus protected against prejudice. Taken together, these two subfields of psychology—PP and PRS—are quite distinct, yet they clearly overlap in many ways.

To bring them together, we consider these two subfields using the model of global meaning as a unifying framework. Meaning plays a central role in both PP and PRS (see Davis et al., Chapter 1, this volume), so it provides a natural bridge to integrate these complementary but largely separate subdisciplines. Meaning has been conceptualized as the constellation of an individuals' general orienting systems - the frameworks of knowledge and motivation through which people understand and navigate their lives (Park, 2010). Although there are undoubtedly cultural variations in the expression of orienting systems, meaning systems consist of three distinct aspects—beliefs, goals and values, and the subjective sense of meaning in life. Global beliefs are individuals' fundamental views of the world and other people and their identity in that world. Global goals are individuals' unique hierarchies of motives and values. The extent to which people feel their experiences are congruent with their global beliefs and goals gives rise to their subjective sense of meaning in life (see Park, 2010). In the sections below, we use this global meaning framework to compare and contrast the approaches of PP and PRS, including the topics, methods, and applications of their work.

We organize our chapter around three primary areas of overlap: content, methods, and 
applications. First, we discuss the meaning-related topics of study in both PP and PRS, explaining how each subfield approaches that area of inquiry and how each perspective is both similar and unique. Next, we discuss the various methods that PP and PRS researchers use to study meaning. Finally, we review applications of meaning-based research in PP and PRS, highlighting areas of convergence.

\section{Major Meaning-Related Topics of Study in PP and PRS}

\section{Beliefs}

PP tends to focus on people's beliefs about themselves and their (earthly) futures. In PP, two core beliefs that are often studied are optimism and growth mindset. Optimism refers to generalized positive expectancies (Scheier \& Carver, 2018), and it has been associated with a myriad of mental and physical health benefits, including lower depression, anxiety, and stress, as well as lower rates of cardiovascular disease and even mortality (for review, see Scheier \& Carver, 2018). Growth mindset refers to the belief that individuals' abilities can be developed (versus the belief that these abilities are fixed; Yeager \& Dweck 2020). Although initially focused on intellectual abilities, the study of growth- versus fixed-mindset beliefs has encompassed other domains, including health habits and interpersonal skills. Research has demonstrated generally favorable effects that stronger growth-mindset beliefs have on a wide range of academic outcomes and well-being indices (Yeager \& Dweck, 2020).

Within PRS, conceptualizations of religiousness often highlight the importance of beliefs in differentiating religious from nonreligious individuals (and religious individuals who ascribe to different religions). PRS also frequently examines how people's religious beliefs affect various cognitive, emotional, and behavioral processes. In PRS, studies of belief tend to focus on people's metaphysical beliefs (e.g., existence and nature of God or the afterlife; Jong et al., 2019) 
and beliefs about God's role in their daily lives (Wilt et al., 2017). Religious beliefs have been studied explicitly (e.g., whether people endorse belief in God) and implicitly (e.g., God-related IAT, intuitive mindset; Park \& Carney, 2021). Some research has been conducted on religious beliefs about suffering and God's role in it (Hale-Smith et al., 2012). However, although beliefs are considered central to religiousness, they have been studied relatively seldomly (Park, 2020).

\section{Cognitive Processing}

Relative to cognitions (beliefs), cognitive processing has not been a large focus of PP research. PP has paid relatively little attention to how people process information. One relevant line of research within PP is the broaden-and-build theory, which avers that positive emotions can influence thinking processes (Frederickson, 2001). One aspect of this theory maintains that individuals' thought-action repertoires can be broadened through positive emotions (resulting, for example, from play or exploration). Through this broadening, individuals can discover novel and creative ideas, actions, and social ties, thereby building their tangible, social, psychological, and spiritual resources. These personal resources can serve as reserves on which individuals can later draw to enhance their coping and well-being (see Van Cappellen et al., this volume).

Within PRS, the cognitive science of religion subfield has highlighted many aspects of cognitive processes and how religious meaning operates within these processes (White, 2021). For example, Pyysiäinen (2013) and Davis et al. (2018) have described how the two parallel processing systems - sometimes referred to as intuitive and reflective (or systems 1 and 2; Stanovich et al., 2014) — may support different types of religious and nonreligious thinking. Some research has suggested religious beliefs are positively associated with intuitive/irrational thinking and inversely associated with rational thinking (Pennycook et al., 2016).

\section{Goals}


Goals were an early focus of PP, and indeed they are a natural fit for that field, given PP's general future orientation. Positive psychologists have focused not only on the motivations and values involved in goal pursuit but also on the contents of those goals (e.g., materialistic vs. nonmaterialistic; Kasser, 2016). An early PP volume by Schmuck and Sheldon (2001) presented fundamental perspectives on the importance of goals within PP. Although goals remain a continued area of study in PP, especially as they relate to happiness and life meaning (Schnell, 2009), theories and empirical work on goals are not a central feature of contemporary PP.

PRS has had relatively little to say about life goals. Some research has explored the notion of spiritual strivings (spiritual goals that can provide meaning, significance, and agency), which are ultimately related to higher levels of emotional well-being (Schnitker \& Emmons, 2013). For many, religious goals may be their primary or ultimate aim (e.g., to serve God, to live as a good and faithful person), and many goals can be made holy through sanctification (Mahoney et al., 2021). However, this topic has not been a major focus in PRS, and the limited work that has been done has generally not considered the roles of religious goals within a larger perspective of individuals' goals (Schnitker \& Emmons, 2013). Some recent work has demonstrated that religious believers report deriving more meaning from their relationships and religiousness, whereas atheists have fewer and less conventional sources of meaning, even though relationships remain their primary source of meaning (Nelson et al., 2021).

\section{Values}

Values are the aspect of global meaning comprising abstract ideals or principles (e.g., freedom, helpfulness) that guide behavior (Maio et al., 2003). PP lacks a strong focus on a broad set of values; however, from the birth of PP, the related study of character strengths or virtues has been a central focus (Peterson \& Seligman, 2004; see Ratchford et al., Chapter 4, this 
volume). Peterson and Seligman (2004) created a taxonomy of character strengths and virtues, along with an assessment tool (the Values in Action Inventory of Strengths) that is still widely used to measure them. However, although this approach is considered to be "values in action," the construct of values is not directly addressed in most research on character strengths.

Despite their pervasive presence in major world religions, values have received little attention in PRS research either. Although a few PRS studies have focused on values, these studies generally focus on specific values rather than a full complement such as that outlined by Schwartz (1994; Sandy et al., 2017). For example, prior PRS work has examined the role of religion in worldview conflict (Brandt et al., 2019), including how people ranging in religious fundamentalism are prejudiced toward dissimilar others (Brandt \& Van Tongeren, 2017) and how religious individuals defend their values when under threat (e.g., Van Tongeren et al., 2021a). Only recently has PRS examined the interplay between religion and values (Van Tongeren et al., 2021b), such as moral foundations (Van Tongeren et al., in press) and Schwartz's values (Schwadel et al., 2021).

PRS researchers focusing on values and virtues could make a greater impact by integrating this work within the broader framework of values elaborated elsewhere in psychology. In addition, both PP and PRS researchers might usefully profit from elaborating how central constructs such as happiness or transcendence relate to different value profiles. This line of research would be one way to tap into the cultural influences and differences of different groups, given that values are a central cultural influence on behavior (Miles, 2015).

\section{Motivation}

Within PP, approaches to motivation tend to stress individuals' inherent inclination towards growth and development; these models of growth are collectively termed organismic 
theories. Chief among current organismic motivational theories is self-determination theory, which avers that humans have three basic needs: autonomy (volition), relatedness (connecting to and feeling important to others), and competence (effectiveness and mastery; Ryan et al., 2008). Self-determination theory is concerned with the development and influence of intrinsic (inherently rewarding to the person) and extrinsic (reward derived by someone or something outside the person) sources of motivation, especially as those sources of motivation relate to meeting people's basic needs and guiding their decision-making (Maurer \& Daukantaitè, 2020).

Another motivational construct prominent in PP is hope (see Chapter 23, this volume). Within PP, hope is a trait-like variable that refers to individuals' characteristic ways of thinking about their goals, specifically the motivation to move toward goals and to have actionable ways to achieve them, sometimes called "the will" and "the way." Thus, within PP, hope describes individuals' sense of agency towards their goals (Snyder, 2002). Many studies have demonstrated favorable associations of hope with physical and mental health (Scioli et al., 2016).

In contrast to PP's broad examinations of motivation, the study of motivation within PRS tends to focus narrowly on people's orientations towards their religious life. That is, the primary motivations studied within PRS are intrinsic and extrinsic religiousness, as originated with Allport (Allport \& Ross, 1967) and described above. Within PRS, intrinsic motivation regarding religiousness refers to the extent to which religion is the central motive for one's life, whereas extrinsic religious motivation refers to the extent to which one is religious for some ulterior motive, such as comfort or social integration. This distinction between intrinsic and extrinsic motivation is echoed in self-determination theory and is compatible with it. Although originally developed in the context of studying prejudice, intrinsic and extrinsic religious motivations have been shown to be related to many other important domains, including interpersonal relationships 
and mental and physical health (see Park, 2021, for an overview).

\section{Subjective Sense of Meaning in Life}

Eudaimonia, the Aristotelian perspective on happiness or flourishing, is a core topic in PP. In Seligman's (2011) PERMA theory of well-being, one pillar of flourishing is the feeling that life has meaning and purpose. Many studies within PP have suggested that effectively using one's primary character strengths makes life feel more meaningful (Allan, 2015). PP studies have examined the extent to which people experience life as meaningful and the conditions that promote this sense of meaningfulness. Across diverse samples and measures, researchers have found that people generally feel their lives are "pretty meaningful" (Heintzelman \& King, 2014, p. 561). Many predictors of this subjective sense of meaningfulness in life have been identified, including social relationships, religious faith, positive affect, and an environment that is predictable and structured (Heintzelman \& King, 2014; Heintzelman et al., 2013).

In PRS, the subjective sense of meaning in life has received surprisingly little research attention (Park, 2013). PRS theories often hypothesize that religion provides a strong sense of

meaning in the form of comprehensibility, purpose, and mattering (George \& Park, 2017). Some studies have compared atheists and religious believers on (e.g., Nelson et al., 2021)—or examined associations of strength of different aspects of religiousness with (e.g., FioRito et al., 2021) — meaning in life. These studies have generally found positive relationships between religiousness and subjective sense of meaning.

\section{PP and PRS: Convergence and Divergence of Major Meaning-Related Topics}

This brief overview suggests that meaning is a central topic in both PP and PRS. Research on beliefs, cognitive processes, goals, values, motivations, and subjective sense of meaning in life have been studied by both fields for many years. Yet this work has seldom 
overlapped, even though these meaning-related topics provide a rich terrain for cross-pollination. PRS perspectives on optimism, for example, might provide insights into how beliefs in God's nature and involvement in the world lead to developing and maintaining optimism.

In addition, neither PP nor PRS has a strong contemporary focus on studying life goals. Thus, theory and research on life goals might be a useful place for developing research on meaning that integrates perspectives from PP and PRS (Schnitker \& Emmons, 2013; see also Davis et al., Chapter 18, this volume). For example, theories and constructs from PRS, such as specific religious beliefs, intrinsic and extrinsic religious motives, and sanctification processes, could be woven into studies of the construction and modification of goal hierarchies and the occurrence and resolution of goal violations in important life domains (Park, 2013). Another potential point of intersection for PP and PRS is the notion that the depth of a person's sources of meaning can range from "demoralizingly shallow to awesomely transcendent" (Reker \& Wong, 1988; Steger, 2021, p. 1724). Hence, both PP and PRS researchers might focus on the depth or quality of sources of meaning as a way to integrate their respective approaches to meaning. Finally, we acknowledge that these intersections are embedded in particular cultural contexts, and future work could examine not only the specific cultural variations of these processes but also the degree to which some of these dimensions are universal or pancultural.

\section{Methodological Approaches to Studying Meaning: Design and Method}

Another potential area of convergence between PP and PRS is in their methods. Finding compatibility in their approaches to meaning-related constructs could build bridges between these fields. In this section, we examine methodological approaches to studying meaning in PP and PRS, along on two dimensions: design and data source (see also Tsang et al., this volume).

Design. The most common design implemented by both PP and PRS researchers is cross- 
sectional correlational (see Tsang et al., this volume). Cross-sectional analyses of associations between constructs of interest are likely employed so often because of the ease by which such designs can be executed with convenience samples. Although such designs are often necessary first steps in a research program, they suffer from the inherent limitations of precluding temporal sequencing, and they are unable even to hint at causal explanations for observed relationships. Nonetheless, cross-sectional correlational approaches are frequently used in both subfields.

Fortunately, longitudinal designs are becoming more common in both PP and PRS. Such investigations can draw from existing datasets designed to answer other questions (e.g., Sibley \& Bulbulia, 2012) or involve studies specifically conducted to examine meaning-focused questions over time (e.g., Abe, 2016). The benefit of such time-series designs is the ability to identify whether the degree of a particular construct at one time predicts the presence (and degree) of other constructs at future timepoints, suggesting a temporal ordering of the variables. Yet as with cross-sectional correlational designs, causal relationships cannot be established definitively, given the role of potential third variables or alternative explanations (cf. VanderWeele et al., 2020). Hence, the gold standard for many research questions is the experimental design.

In both PP and PRS, experimental designs are important for advancing understanding of the causal mechanisms involved in psychological processes. For example, within PRS, a great deal of studies have involved using social cognitive priming to activate constructs of interest experimentally (e.g., Van Tongeren et al., 2021a). Within PP, experimental work on meaning has been more limited and consists mostly of experiments examining small "mini-interventions" (e.g., writing a letter of gratitude to induce feelings of meaningfulness; Van Tongeren et al., 2016). Future experimental work may be particularly valuable in examining meaning-related constructs bridging the interests of PP and PRS. 
Another research approach worth mentioning here is a meta-analytic review. Intended to provide an overview of extant research and a "state of the science" regarding a particular effect or relationship, meta-analyses depend in part on the amount of existing data available to inform the research question. Given the recency of PP relative to PRS, meta-analyses related to meaning appear more frequently on PRS-related topics, such as religious coping (e.g., Ano \& Vasconcelles, 2005) and the association between religion and well-being (e.g., Jim et al., 2015; Lefevor et al., 2021). PP, founded much later than PRS, has a smaller corpus of work, resulting in fewer meta-analytic papers on meaning-related constructs (e.g., Fischer \& Chalmers, 2008).

Data sources. A common (and relatively easy) data source is the self-report survey, in which participants provide responses to questions or items administered by the researchers. Such data can be qualitative (i.e., descriptive written or oral accounts), quantitative (i.e., numerical responses), or mixed methods (i.e., a combination of both qualitative and quantitative data). Qualitative data can be particularly powerful for revealing the depth of people's ability to make meaning (e.g., Davis et al., 2019). Self-report surveys are quite common in both PP and PRS. Whereas the benefits of expediency, convenience, and scalability often impel researchers to rely on self-report sources of data collection, the obtained data may be biased or inaccurate. For those reasons, researchers might consider relying on behavioral studies, in which they directly examine participants' behavior, including specific actions, physiological responses (e.g., cortisol, galvanic skin conductance, blood pressure, or heart rate variability), or more intensive objective observations (e.g., fMRI studies). Studies assessing easily observable behavioral indicators are common in both PP and PRS, whereas physiological observations are relatively rare in both.

For both PP and PRS, surveys and behavioral studies often take place in controlled environments via laboratory studies. However, PRS research also examines meaning-related 
constructs in field studies, such as examining how people experiencing illness or trauma make meaning (Park et al., 2008). Other field studies examine individuals in their daily lives, using such methods as daily diary (which typically assess participants once daily over the course of several days) and experience sampling methodologies (which signal participants to respond to prompts in the midst of their daily lives, usually multiple times per day for several days). Field studies have been more common in PP than PRS. Finally, considerations regarding the particular cultural context within which data are sampled - and encouraging cross-cultural research — are important for catalyzing research at this nexus.

\section{Applications of Meaning-Related Concepts}

Since its founding, PP has embraced as its explicit mandate "to make life better for all people" (Seligman, 1999, p. 559), and a robust literature is available on interventions to promote strengths and flourishing. Unlike many psychological interventions focused on those in distress or at high risk of psychological difficulties, PP interventions are often conducted with the general population. Some of this work centers on promoting meaning in life, based on the assumption that enhanced meaning will promote happiness. PP interventions to enhance meaning have been shown to be modestly successful (e.g., Gander et al., 2016). In addition to attempting to bolster meaning, intervention trials have been conducted to increase hope (Rye et al., 2013). In addition, PP has generated a large literature on applying PP in the workplace, some of which involves the concept of "meaningful work" (Steger et al., 2012), which is associated with meaning in life and has been shown to buffer effects of work stress on meaning in life (Allan et al., 2016). Although PP interventions have been used extensively in schools, almost none of this work directly focuses on meaning (Chodkiewicz \& Boyle, 2017; see King et al., Chapter 17, this volume).

In contrast to PP, PRS as a discipline has generally been oriented towards advancing 
scientific understanding rather than practical applications. Thus, relatively few PRS studies have directly attempted to apply theory or empirical work to intervene with general populations. Within specific clinical populations, however, PRS has generated substantial interest. For example, many religious/spiritual interventions have been studied for their efficacy in helping substance-abusing populations (Captari et al., Chapter 26, this volume). Similarly, some clinical religious/spiritual interventions have been studied among people with physical health problems (Gonçalves et al., 2017), but they rarely have focused directly on meaning. In the workplace, PRS researchers have studied vocational calling, the process of connecting one's work with one's deepest life meaning and religious roots (Dik \& Alayan, Chapter 27, this volume).

By comparison, PP explicitly aims to improve people's lives, and it is therefore quite application-oriented. Meaning-focused applications for healthy populations and those with identified challenges may be an obvious place for integrating these subfields (Rye et al., 2013). In particular, PP and PRS research could collaboratively develop and test the effectiveness of spiritually integrative, meaning-focused interventions. Such interventions could focus on not only meaning in life but also beliefs, cognitive processes, goals, values, and motivations. In other topic areas, some intervention studies have compared secular and spiritual versions (e.g., Wachholtz et al., 2017); this approach may work well in testing PP- and PRS-relevant interventions. Another application with clinical relevance is studying strengths and virtues. Cultivating and capitalizing on individuals' strengths has been a focus of prevention and intervention efforts (Jankowski et al., 2020). To date, these interventions have rarely integrated spirituality, but bringing spirituality into virtue-based models of change is a promising direction.

\section{Overall Summary and Conclusions}

Meaning has been a central feature of both PP and PRS, but little research has integrated 
these two subfields. We see several promising areas for bridging these two areas and promoting collaboration. First, the intersection of religious virtues or character strengths and their association with global meaning is a new frontier. To what degree do some people experience gratitude toward God or hold supernatural hope, and how might those emotions and beliefs affect the goals people set, their motivation to reach such goals, and the values that help sustain them along the way? Second, PP and PRS might naturally intersect in the domain of coping with stress and trauma: how do religious beliefs about the divine and the nature of suffering interact with individual differences in humility or optimism, especially in the process of metabolizing negative life experiences and potentially experiencing growth? What roles do PP constructs, such as courage and awe, play in how people make meaning during adversity? Third, we suspect that both subfields might leverage their common focus on transcending the self; PP often focuses on connections with others, whereas PRS focuses on connections with the divine or nature. How might these experiences of transcendence similarly affect global beliefs, goals, or values?

Finally, PP may be moving toward a "second wave" (Wong, 2011), in which it adopts a more balanced approach to human functioning by acknowledging both the "dark" and "light" side of human life. As it does, PRS may offer many insights. PRS is particularly attuned to existential questions and considerations, and work at the intersection of existential-PP and PRS seems particularly promising. We encourage researchers to integrate meaning across PP and PRS perspectives, using some of the sophisticated study designs and data sources described above, as well as using cutting-edge methods such as artificial intelligence approaches. PP and PRS are thriving scholarly domains. Given their conceptual composition and trajectories, we contend that they can profitably advance by leveraging their common focus on meaning. Collaborative endeavors can catalyze future work and help answer some of life's more pressing questions. 


\section{References}

Abe, J. A. A. (2016). A longitudinal follow-up study of happiness and meaning-making. The Journal of Positive Psychology, 11(5), 489-498. https://doi.org/10.1080/17439760.2015.1117129

Allan, B. A. (2015). Balance among character strengths and meaning in life. Journal of Happiness Studies, 16(5), 1247-1261. https://doi.org/10.1007/s10902-014-9557-9

Allan, B. A., Douglass, R. P., Duffy, R. D., \& McCarty, R. J. (2016). Meaningful work as a moderator of the relation between work stress and meaning in life. Journal of Career Assessment, 24(3), 429-440. https://doi.org/10.1177/1069072715599357

Allport, G. W., \& Ross, J. M. (1967). Personal religious orientation and prejudice. Journal of Personality and Social Psychology, 5(4), 432-443. https://doi.org/10.1037/h0021212

Ano, G. G., \& Vasconcelles, E. B. (2005). Religious coping and psychological adjustment to stress: A meta-analysis. Journal of Clinical Psychology, 61(4), 461-480. https://doi.org/10.1002/jclp.20049

Bland, A. M. (2020). Existential givens in the COVID-19 Crisis. Journal of Humanistic Psychology, 60(5), 710-724. https://doi.org/10.1177/0022167820940186

Brandt, M. J., Crawford, J. T., \& Van Tongeren, D. R. (2019). Worldview conflict in daily life. Social Psychological and Personality Science, 10, 35-43. https://doi.org/10.1177\%2F1948550617733517

Brandt, M. J., \& Van Tongeren, D. R. (2017). People both high and low in religious fundamentalism are prejudiced toward dissimilar groups. Journal of Personality and Social Psychology, 112, 76-97. https://doi.org/10.1037/pspp0000076

Chodkiewicz, A. R., \& Boyle, C. (2017). Positive psychology school-based interventions: A 
reflection on current success and future directions. Review of Education, 5(1), 60-86. https://doi.org/10.1002/rev3.3080

Davis, E. B., Granqvist, P., \& Sharp, C. (2018). Theistic relational spirituality: Development, dynamics, health, and transformation. Psychology of Religion and Spirituality. Advance online publication. https://doi.org/10.1037/rel0000219

Davis, E. B., Kimball, C. N., Aten, J. D., Andrews, B., Van Tongeren, D. R., Hook, J. N., Davis, D. E., Granqvist, P., \& Park, C. L. (2019). Religious meaning making and attachment in a disaster context: A longitudinal qualitative study of flood survivors. The Journal of Positive Psychology, 14(5), 659-671. https://doi.org/10.1080/17439760.2018.1519592

FioRito, T. A., Abeyta, A. A., \& Routledge, C. (2021). Religion, paranormal beliefs, and meaning in life. Religion, Brain \& Behavior, 11(2), 139-146. https://doi.org/10.1080/2153599X.2020.1824938

Fischer, R., \& Chalmers, A. (2008). Is optimism universal? A meta-analytical investigation of optimism levels across 22 nations. Personality and Individual Differences, 45(5), 378382. https://doi.org/10.1016/j.paid.2008.05.008

Fredrickson, B. L. (2001). The role of positive emotions in positive psychology: The broadenand-build theory of positive emotions. American Psychologist, 56(3), 218-226. https://doi.org/10.1037/0003-066X.56.3.218

Gander, F., Proyer, R. T., \& Ruch, W. (2016). Positive psychology interventions addressing pleasure, engagement, meaning, positive relationships, and accomplishment increase well-being and ameliorate depressive symptoms. Frontiers in Psychology, 7, 686. https://doi.org/10.3389/fpsyg.2016.00686

George, L. S., \& Park, C. L. (2017). The multidimensional existential meaning scale: A tripartite 
approach to measuring meaning in life. Journal of Positive Psychology, 12, 613-627. https://doi.org/10.1080/17439760.2016.1209546

Gonçalves, J. P. D. B., Lucchetti, G., Menezes, P. R., \& Vallada, H. (2017). Complementary religious and spiritual interventions in physical health and quality of life: A systematic review of randomized controlled clinical trials. PloS One, 12(10), e0186539. https://doi.org/10.1371/journal.pone.0186539

Hale-Smith, A., Park, C. L., \& Edmondson, D. (2012). Measuring beliefs about suffering: Development of the Views of Suffering Scale. Psychological Assessment, 24, 855-866. https://doi.org/10.1037/a0027399

Heintzelman, S. J., \& King, L. A. (2014). Life is pretty meaningful. American Psychologist, 69(6), 561-574. https://doi.org/10.1037/a0035049

Heintzelman, S. J., Trent, J., \& King, L. A. (2013). Encounters with objective coherence and the experience of meaning in life. Psychological Science, 24(6), 991-998. https://doi.org/10.1177\%2F0956797612465878

Jankowski, P. J., Sandage, S. J., Bell, C. A., Davis, D. E., Porter, E., Jessen, M., Motzny, C. L., Ross, K. V., \& Owen, J. (2020). Virtue, flourishing, and positive psychology in psychotherapy: An overview and research prospectus. Psychotherapy, 57(3), 291-309. https://doi.org/10.1037/pst0000285

Jim, H. S., Pustejovsky, J. E., Park, C. L., Danhauer, S. C., Sherman, A. C., Fitchett, G., Merluzzi, T. V., Munoz, A. R., George, L., Snyder, M. A., \& Salsman, J. M. (2015). Religion, spirituality, and physical health in cancer patients: A meta-analysis. Cancer, 121(21), 3760-3768. https://doi.org/10.1002/cncr.29353

Jong, J., Halberstadt, J., Bluemke, M., Kavanagh, C., \& Jackson, C. (2019). Death anxiety, 
exposure to death, mortuary preferences, and religiosity in five countries. Scientific Data, 6(1), 1-5. https://doi.org/10.1038/s41597-019-0163-x

Kasser, T. (2016). Materialistic values and goals. Annual Review of Psychology, 67, 489-514. https://doi.org/10.1146/annurev-psych-122414-033344

Kim-Prieto, C. (2014). Introduction: Positive psychology of religion across traditions and beliefs. In C. Kim-Prieto (Ed.), Religion and spirituality across cultures (pp. 1-18). Springer. https://doi.org/10.1007/978-94-017-8950-9

Lefevor, G. T., Davis, E. B., Paiz, J. Y., \& Smack, A. C. P. (2021). The relationship between religiousness and health among sexual minorities: A meta-analysis. Psychological Bulletin. Advance online publication. https://doi.org/10.1037/bul0000321

Mahoney, A., Wong, S., Pomerleau, J. M., \& Pargament, K. I. (2021). Sanctification of diverse aspects of life and psychosocial functioning: A meta-analysis of studies from 1999 to 2019. Psychology of Religion and Spirituality. Advance online publication. https://doi.org/10.1037/rel0000354

Maio, G. R., Olson, J. M., Bernard, M. M., \& Luke, M A. (2003). Ideologies, values, attitudes, and behavior. In J. Delamater (Ed.), Handbook of social psychology (pp. 283-308). Kluwer Academic and Plenum Publishers.

Maurer, M. M., \& Daukantaitè, D. (2020). Revisiting the organismic valuing process theory of personal growth: A theoretical review of Rogers and its connection to positive psychology. Frontiers in Psychology, 11, 1706. https://doi.org/10.3389/fpsyg.2020.01706

Miles, A. (2015). The (re) genesis of values: Examining the importance of values for action. American Sociological Review, 80(4), 680-704. https://doi.org/10.1177/0003122415591800 
Nelson, T. A., Abeyta, A. A., \& Routledge, C. (2021). What makes life meaningful for theists and atheists? Psychology of Religion and Spirituality, 13(1), 111-118. http://dx.doi.org/10.1037/rel0000282

Park, C. L. (2010). Making sense of the meaning literature: An integrative review of meaning making and its effects on adjustment to stressful life events. Psychological Bulletin, 136, 257-301. https://doi.org/10.1037/a0018301

Park, C. L. (2013). Religion and meaning. In R. F. Paloutzian \& C. L. Park (Eds.), Handbook of the psychology of religion and spirituality ( $2^{\text {nd }}$ ed., pp. 357-379). Guilford Press.

Park, C. L. (2020). Finally, some well-deserved attention to the long-neglected dimension of religious beliefs: Suggestions for greater understanding and future research. Religion, Brain, and Behavior, 10, 191-197. https://doi.org/10.1080/2153599X.2018.1532452

Park, C. L. (2021). Intrinsic and extrinsic religious motivation: Retrospect and prospect. International Journal for the Psychology of Religion, 31, 213-222. https://doi.org/10.1080/10508619.2021.1916241

Park, C. L., \& Carney, L. M. (2021). Religious head versus heart beliefs: Measurement development and validation. Psychology of Religion and Spirituality. Advance online publication. https://doi.org/10.1037/re10000352

Park, C. L., Edmondson, D., Fenster, J. R., \& Blank, T. O. (2008). Meaning making and psychological adjustment following cancer: The mediating roles of growth, life meaning, and restored just-world beliefs. Journal of Consulting and Clinical Psychology, 76(5), 863-875. https://doi.org/10.1037/a0013348

Pennycook, G., Ross, R. M., Koehler, D. J., \& Fugelsang, J. A. (2016). Atheists and agnostics are more reflective than religious believers: Four empirical studies and a meta-analysis. 
PloS one, 11(4), e0153039. https://doi.org/10.1371/journal.pone.0153039

Peterson, C., \& Seligman, M. E. P. (2004). Character strengths and virtues: A handbook and classification. American Psychological Association.

Pyysiäinen, I. (2013). Cognitive science of religion: State-of-the-art. Journal for the Cognitive Science of Religion, 1(1), 5-28. https://doi.org/10.1558/jcsr.v1i1.5

Reker, G. T., \& Wong, P. T. P. (1988). Aging as an individual process: Toward a theory of personal meaning. In J. E. Birren \& V. L. Bengston (Eds.), Emergent theories of aging (pp. 214-246). Springer.

Ryan, R. M., Huta, V., \& Deci, E. L. (2008). Living well: A self-determination theory perspective on eudaimonia. Journal of Happiness Studies, 9(1), 139-170. https://doi.org/10.1007/s10902-006-9023-4

Rye, M. S., Wade, N. G., Fleri, A. M., \& Kidwell, J. E. M. (2013). The role of religion and spirituality in positive psychology interventions. In K. I. Pargament, A. Mahoney, \& E. P. Shafranske (Eds.), APA handbooks of psychology, religion, and spirituality (Vol. 2) (pp. 481-508). American Psychological Association. https://doi.org/10.1037/14046-025

Sandy, C. J., Gosling, S. D., Schwartz, S. H., \& Koelkebeck, T. (2017). The development and validation of brief and ultrabrief measures of values. Journal of Personality Assessment, 99(5), 545-555. https://doi.org/10.1080/00223891.2016.1231115

Scheier, M. F., \& Carver, C. S. (2018). Dispositional optimism and physical health. American Psychologist, 73(9), 1082-1094. https://doi.org/10.1037/amp0000384

Schmuck, P. E., \& Sheldon, K. M. (2001). Life goals and well-being: Towards a positive psychology of human striving. Hogrefe \& Huber Publishers.

Schnell, T. (2009). The Sources of Meaning and Meaning in Life Questionnaire (SoMe): 
Relations to demographics and well-being. The Journal of Positive Psychology, 4(6), 483-499. https://doi.org/10.1080/17439760903271074

Schnitker, S. A., \& Emmons, R. A. (2013). Spiritual striving and seeking the sacred: Religion as meaningful goal-directed behavior. International Journal for the Psychology of Religion, 23(4), 315-324. https://doi.org/10.1080/10508619.2013.795822

Schnitker, S. A., Houltberg, B., Dyrness, W., \& Redmond, N. (2017). The virtue of patience, spirituality, and suffering: Integrating lessons from positive psychology, psychology of religion, and Christian theology. Psychology of Religion and Spirituality, 9(3), 264-275. https://doi.org/10.1037/rel0000099

Scioli, A., Scioli-Salter, E. R., Sykes, K., Anderson, C., \& Fedele, M. (2016). The positive contributions of hope to maintaining and restoring health: An integrative, mixed-method approach. The Journal of Positive Psychology, 11(2), 135-148.

https://doi.org/10.1080/17439760.2015.1037858

Schwadel, P., Hardy, S. A., Van Tongeren, D. R., \& DeWall, C. N. (2021). The values of religious nones, dones, and sacralized Americans: The links between changes in religious affiliation and Schwartz values. Journal of Personality, 89(5), 867-882. https://doi.org/10.1111/jopy.12620

Schwartz, S. H. (1994). Are there universal aspects in the structure and contents of human values? Journal of Social Issues, 50, 19-45. https://doi.org/10.1111/j.1540$\underline{4560.1994 . t b 01196 . x}$

Seligman, M. E. P. (1999). The president's address (Annual Report). American Psychologist, 54, $559-562$.

Seligman, M. E. P. (2011). Flourish. Simon \& Schuster. 
Sibley, C. G., \& Bulbulia, J. (2012). Faith after an earthquake: A longitudinal study of religion and perceived health before and after the 2011 Christchurch New Zealand earthquake. PloS One, 7(12), e49648. https://doi.org/10.1371/journal.pone.0049648

Snyder, C. R. (2002). Hope theory: Rainbows in the mind. Psychological Inquiry, 13(4), 249275. https://doi.org/10.1207/S15327965PLI1304_01

Stanovich, K. E., West, R. F., \& Toplak, M. E. (2014). Rationality, intelligence, and the defining features of Type 1 and Type 2 processing. In J. W. Sherman, B. Gawronski, \& Y. Trope (Eds.), Dual-process theories of the social mind (pp. 80-91). Guilford Press.

Steger, M. F. (2021). Meaning in life: A unified model. In C. R. Snyder, S. J. Lopez, L. M. Edwards, \& S. C. Marques (Eds.), The Oxford handbook of positive psychology $\left(3^{\text {rd }}\right.$ ed., pp. 1720-1730). Oxford University Press. https://doi.org/10.1093/oxfordhb/9780199396511.013.56

Steger, M. F., Dik, B. J., \& Duffy, R. D. (2012). Measuring meaningful work: The Work and Meaning Inventory (WAMI). Journal of Career Assessment, 20, 322-337. https://doi.org/10.1177/1069072711436160

VanderWeele, T. J., Mathur, M. B., \& Chen, Y. (2020). Outcome-wide longitudinal designs for causal inference: A new template for empirical studies. Statistical Science, 35(3), 437466. https://doi.org/10.1214/19-STS728

Van Tongeren, D. R., Davis, E. B., Hook, J. N., Davis, D. E., \& Aten, J. D. (2021a). Existentially threatening stimuli increase religious cognitive dissonance among the less intrinsically religious. Psychology of Religion and Spirituality, 13(3), 298-303. https://doi.org/10.1037/rel0000296

Van Tongeren, D. R., DeWall, C. N., Chen, Z., Sibley, C. G., \& Bulbulia, J. (2021b). Religious 
residue: Cross-cultural evidence that religious psychology and behavior persist following deidentification. Journal of Personality and Social Psychology, 120(2), 484-503. https://doi.org/10.1037/pspp0000288

Van Tongeren, D. R., DeWall, C. N., Hardy, S. A., \& Schwadel, P. (in press). Religious identity and morality: Evidence for religious residue and decay in moral foundations. Personality and Social Psychology Bulletin. https://doi.org/10.1177\%2F0146167220970814

Van Tongeren, D. R., Green, J. D., Davis, D. E., Hook, J. N., \& Hulsey, T. L. (2016). Prosociality enhances meaning in life. The Journal of Positive Psychology, 11, 225-236. https://doi.org/10.1080/17439760.2015.1048814

Wachholtz, A. B., Malone, C. D., \& Pargament, K. I. (2017). Effect of different meditation types on migraine headache medication use. Behavioral Medicine, 43(1), 1-8. https://doi.org/10.1080/08964289.2015.1024601

White, C. (2021). An introduction to the cognitive science of religion. Routledge.

Wilt, J. A., Exline, J. J., Lindberg, M. J., Park, C. L., \& Pargament, K. I. (2017). Theological beliefs about suffering and interactions with the divine. Psychology of Religion and Spirituality, 9(2), 137-147. https://doi.org/10.1037/rel0000067

Wong, P. T. P. (2011). Positive psychology 2.0: Towards a balanced interactive model of the good life. Canadian Psychology/Psychologie Canadienne, 52(2), 69-81. https://doi.org/10.1037/a0022511

Yeager, D. S., \& Dweck, C. S. (2020). What can be learned from growth mindset controversies? American Psychologist, 75(9), 1269-1284. https://doi.org/10.1037/amp0000794 


\title{
Chapter 7:
}

Measurement at the Intersection of Positive Psychology and the Psychology of

\section{Religion/Spirituality}

\author{
Peter C. Hill \\ Rosemead School of Psychology, Biola University \\ Nicholas DiFonzo \\ Roberts Wesleyan College \\ C. Eric Jones \\ Regent University \\ Justin S. Bell \\ Rochester Institute of Technology
}

Correspondence concerning this chapter should be addressed to Peter C. Hill, Rosemead School of Psychology, Biola University, 13800 Biola Ave, La Mirada, CA 90639. Email: peter.hill@biola.edu 


\begin{abstract}
A catalogue and exploration of measures at the intersection of virtues and character strengths (VCS) and the psychology of religion/spirituality (R/S) can inform theoretically interesting and practically valuable questions involving the VCS-R/S relationship. We review a select group of measures in the psychology of R/S through the lens of positive psychology's six core virtues (wisdom/knowledge, courage, humanity, justice, temperance, and transcendence) and 24 character strengths, as articulated by Peterson and Seligman (2004). To accomplish this goal, we examined 200 measures in the psychology of R/S and determined whether or not each of the 24 character strengths is also assessed by the scale. For example, a scale measuring daily spiritual experiences also measures love (a character strength associated with the virtue of humanity), appreciation of beauty and excellence, and gratitude (character strengths of the virtue of transcendence). Additionally, we explore how religious/spiritual context may shape conceptualization and measurement of VCS constructs, thus affording a clearer understanding of both the virtue and the religious/spiritual context. We caution that drawing conclusions without a consideration of the religious/spiritual worldview context of the populations being studied may risk a distorted understanding of VCS. Online access to characterological ratings of 200 religious/spiritual scales is provided.
\end{abstract}

Keywords: measurement, religion, spirituality, virtue, character strengths 


\section{Chapter 7: Measurement at the Intersection of Positive Psychology and the Psychology of Religion/Spirituality}

In this chapter, we review measures at the intersection of positive psychology and the psychology of religion/spirituality $(\mathrm{R} / \mathrm{S})$. We do this by viewing measures from the psychology of R/S through the lens of virtues and character strengths (VCS), as formulated in the seminal work of Peterson and Seligman (2004). That taxonomy had 24 character strengths that were organized into 6 virtues: wisdom/knowledge, courage, humanity, justice, temperance, and transcendence. In this chapter, we identify VCS that are assessed by extant religious/spiritual measures and make the case that the religious/spiritual context should be taken into account when applying the VCS taxonomy to assessing religiously/spiritually committed individuals. For example, the Attachment to God Scale, which ostensibly assesses God attachment (i.e., God as safe haven and secure base, seeking proximity to God, and responding to separation from God), also assesses courage (specifically, the character strength of bravery, e.g., "My relationship with God gives me the courage to face new challenges"), and temperance (specifically, the character strength of self-regulation, e.g., "When I face difficulties, I turn to God"; Sim \& Loh, 2003).

To identify VCS assessed in religious/spiritual scales, we examined a conceptually diverse sample of 200 psychology of R/S measures by rating each scale on its assessment of the 24 VCS. Ratings were made independently, and disagreements were resolved through discussion. Consensus ratings are presented in an online table that is organized by the six virtues and related character strengths. See Appendix 7.S1 for the complete Table of VCS Ratings of R/S Scales. A detailed description of its methods and the list of scales associated with each character strength is in Appendix 7.S2. In the current chapter, we review a selection of these scales and explore how each virtue's character strengths are conceptualized in religious/spiritual contexts. 
One thesis of this chapter is that for people who are religiously or spiritually committed, a thorough understanding of virtue must take their religious/spiritual worldview into account. This does not mean a religious/spiritual lens is always necessary in conceptualizing virtue. Secular philosophy, particularly grounded in the Aristotelian tradition, has provided much clarity to the study of virtue. However, there is an undeniable linkage between R/S and virtue (see Ratchford et al., Chapter 4, this volume). For example, in a study of eight religious and moral philosophies (Confucianism, Taoism, Buddhism, Hinduism, Athenian philosophy, Judaism, Islam, and Christianity), Dahlsgaard et al. (2005) found that all the core virtues were expressed in the major writings of multiple religious and philosophical traditions. In fact, the authors pointed out that even though the core virtues are not meant to reflect any single tradition, all six core virtues were identified eight centuries earlier (dubbed the Seven Heavenly Virtues) by the Catholic theologian Aquinas in his Summa Theologiae: four by name (Wisdom, Courage, Justice, and Temperance) and two implicitly as the theological virtues of Faith/Hope (Transcendence) and Love (Humanity; see Long \& VanderWeele, Chapter 25, this volume). Thus, it is reasonable to assume that contemporary R/S measures assess each core virtue and their related character strengths.

A catalogue and exploration of measures at the intersection of VCS and R/S can inform theoretically interesting questions, many with significant practical import. For example, what is "the extent to which religion and spirituality help foster [or impede] those human strengths and virtues that lie at the heart of what makes a life well lived" (Hood et al, 2018, p. 452)? More broadly, by identifying, highlighting, and clarifying points of VCS-R/S intersection, this chapter can inform interdisciplinary cross-pollination and dialogue (see Cowden et al., Chapter 16 this volume). 
For many reasons, a "VCS-colored-lenses" approach to identifying and understanding measures at this intersection is justified. First, the VCS-R/S intersection is already on the positive psychology map. Because most existing theories of VCS emerged from the writings of religious thinkers and moral philosophers (Dahlsgaard et al., 2005), the scientific study of VCS originated, at least in part, in intersection with R/S. Second, VCS have been given a place of honor in the positive psychology map. That is, because VCS are intrinsically linked to questions about the good life (a life worth living), VCS have become foundational and ubiquitous concepts in positive psychology, the science of human flourishing. Third, using VCS-colored lenses is productive; it enables the perception of key VCS ingredients in a culturally rich and complex religious/spiritual stew. This is so because constructs of R/S (e.g., religious beliefs, experiences, and practices) naturally involve, reflect, or produce a number of virtues, such as when a comprehensive set of religious beliefs affords a sense of perspective (e.g., "perspective" is a character strength in the Peterson and Seligman [2004] virtue category of "wisdom/knowledge").

The downside of such productivity is the danger of reductionism—reducing religious/spiritual phenomena to the additive combinations of the VCS that comprise them. Religious/spiritual phenomena are not merely collations of VCS ingredients. For example, with VCS-colored lenses, the abstracted character strength of love can be perceived as part of what is being measured by religious/spiritual scales of spiritual experience (e.g., "I feel loved by God"). However, the phenomenological spiritual experience of being loved by God (the whole) is more than simply love plus spirituality (the sum of its parts). Indeed, we contend that, for religiously/spiritually committed people, their religious/spiritual worldview helps define and nurture VCS; in our culinary metaphor, VCS ingredients are often cultivated in the soil of R/S. 


\section{VCS in Religious/Spiritual Concepts and Measures}

We organize the rest of this chapter according to the six core virtues, as conceived by Peterson and Seligman (2004): wisdom/knowledge, courage, humanity, justice, temperance, and transcendence. For each virtue, we point to connections between character strengths and constructs in the psychology of R/S. Next, we focus on a subset of these VCS-R/S connections by exploring how particular character strengths are conceptualized and measured in religious/spiritual contexts and then review a selection of religious/spiritual scales that assess those strengths. We draw on insights gained from our VCS-focused ratings of 200 conceptually and religiously diverse scales from the psychology of R/S. Some character strengths are frequently represented in the reviewed measures, whereas others are hardly considered. Scholars and practitioners working at the intersection of R/S and VCS can access the complete Table of VCS Ratings of R/S Scales and Lists of R/S Scales by VCS in Appendix 7.S1 and Appendix 7.S2.

\section{Wisdom and Knowledge}

Peterson and Seligman (2004) conceptualize the virtue of wisdom/knowledge as "cognitive strengths that entail the acquisition and use of knowledge" (p. 29), especially the use of such strengths for the common good. Wisdom/knowledge is comprised of the character strengths of creativity, curiosity, open-mindedness, love of learning, and seeing things in a perspective that makes sense to oneself and others, and it can be both fostered and inhibited by $\mathrm{R} / \mathrm{S}$. The character strength of creativity is evident in religious problem solving or as a collective strength (such as in religious communities that emphasize creative expressions of worship). Curiosity is manifested in research on indigenous, new age, and transpersonal religious/spiritual orientations. Open-mindedness is reflected in research examining quest religious orientation and religious/spiritual struggles and doubts. Love of learning includes people's efforts to gain 
knowledge and understanding of their own and others' R/S. Perspective is germane to religious/spiritual ideologies, worldviews, and beliefs. We discuss VCS connections with quest orientation, religious/spiritual struggle and doubt, and religious/spiritual beliefs more closely.

Quest religious orientation, the willingness to face complex existential issues (Batson et al., 1993), requires open-mindedness. In this orientation, tentativeness reflects $R / S$ maturity. If flexibility is viewed as a hallmark of mental health, then quest reflects an open-minded toleration of ambiguity that marks wisdom and R/S maturity. An example item from the Quest Scale (Batson \& Schoenrade, 1991) is "As I grow and change, I expect my religion to also grow and change," and one from the Religious Maturity Scale (Dudley \& Cruise, 1990) is "Important questions about the meaning of life do not have simple or easy answers; therefore, faith is a developmental process."

Quest orientation is also emblematic of religious/spiritual struggles and doubts. Indeed, religious/spiritual questing, struggles, and doubts often increase following exposure to tragedy, as profound existential questions might press for a spiritual transformation that involves a new system of meaning (Krauss \& Flaherty, 2001). Example measures include the Religious Doubts Scale (Altemeyer, 1988) that assesses the extent one has religious doubts (e.g., "Doubts about the existence of a benevolent, good God, caused by the suffering or death of someone I knew.") and the Quest scale (Batson \& Schoenrade, 1991; e.g., "I have been driven to ask religious questions out of growing awareness of the tensions in my world and in my relationship to my world."). Religious/spiritual beliefs are the propositions that help one make sacred sense of people, events, and the cosmos; they afford perspective. Example items from the Buddhist Beliefs and Practices Scale (Emavardhana \& Tori, 1997) are "I believe in the theory of karma and rebirth" and "I think the cessation of suffering occurs when the Noble Eightfold Path is followed." 


\section{Courage}

Peterson and Seligman (2004) define the virtue of courage as "emotional strengths that involve the exercise of the will to accomplish goals in the face of opposition, external or internal" (p. 29). It consists of character strengths of bravery (valor), persistence, integrity, and vitality (enthusiasm). Strengths of persistence and bravery are involved in moral behavior and religious coping; integrity, in intrinsic religious orientation; and vitality, in religious/spiritual well-being. We will discuss VCS connections with intrinsic orientation and religious coping.

As an internalized, paramount desire to serve the object of one's sacred devotion, a key element of intrinsic religious orientation is integrity-moral behavior and expressed R/S that are consistent with internalized sacred devotion (Allport, 1950). The intrinsically oriented religious person maintains their integrity and authenticity, even in the face of derision or disappointment. Example items from the Religious Orientation Scale (Allport \& Ross, 1967) are "I try hard to carry my religion over into all my other dealings in life" and "The prayers I say when I am alone carry as much meaning and personal emotion as those said by me during services."

Several measures of religious coping, such as Pargament et al.'s (1998) positive religious coping subscale, involve the character strengths of bravery and persistence. Example items include: "Tried to put my plans into action together with God" and "Tried to see how God might be trying to strengthen me in this situation." An example from the Pakistani Religious Coping Practices Scale (Khan \& Watson, 2006) is "Gave Sadaqah—charity—in the name of Allah."

\section{Humanity}

Peterson and Seligman (2004) define humanity as a core virtue of "interpersonal strengths that involve tending and befriending others" (p. 29). Its character strengths are love, kindness, and social intelligence. This virtue "relies on doing more than what is only fair-showing 
generosity even when an equitable exchange would suffice, kindness even if it cannot (or will not) be returned, and understanding even when punishment is due" (p. 37). Love is germane to God representations and attachment to God, whereas kindness is implicated in religious attitudes, moral behavior, and R/S social support. Social intelligence is involved in religious caregiving. We examine VCS connections with God representations, attachment to God, and R/S support. God representations, one's mental representations of God, involve one's perceptions of the character of God, especially with respect to the character strength of love. God-representation scales include Johnson et al.'s (2015) 18-item list of adjectives of a benevolent God (e.g., caring, generous, gracious) and an authoritarian God (e.g., commanding, stern, controlling). Another example is Benson and Spilka's (1973) Loving God items (e.g., accepting, loving, approving).

Attachment to God, the extent to which a person perceives God as a safe haven from distress and a source of relational and emotional security, is grounded in the character strength of love. Attachment to God theory is rooted in the assumption people have an attachment system that impels them to develop internal working models of relational-emotional patterns that are formed primarily during early childhood interactions with caregivers. Researchers have found that God often serves as an attachment figure (Granqvist, 2020). Most measures of attachment (including attachment to God) emphasize assessing insecure attachment patterns of avoidance and anxiety. Two attachment to God scales are Rowatt and Kirkpatrick's (2002; e.g., "God seems to have little or no interest in my personal affairs" [avoidance]; “God's reactions to me seem to be inconsistent" [anxiety]) and Beck and McDonald's (2004; "I prefer not to depend too much on God" [avoidance]; "I often worry about whether God is pleased with me" [anxiety]).

Of course, some religious traditions do not emphasize a theistic notion of a personal God, but for such traditions, the humanity virtue is still relevant. For example, Buddhism suggests that 
attachment (to anything or anyone) is unhealthy. Indeed, one of the four Noble Truths of Buddhism is that suffering arises from attachment to desires. In this faith tradition, attachment is "a mental affliction that distorts the cognition of its object by exaggerating its admirable qualities and screening out its disagreeable qualities" (Sahdra et al., 2010, p. 116). In other words, perceptions of an attachment object—which are by nature distorted and illusory—interfere with authentic relationship with that object. In this religious/spiritual worldview, the character strength of love requires nonattachment ("release from mental fixations," Sahdra et al., 2010, p. 116): release from fixating on a relationship (i.e., desiring it) brings about a sense of security that actually facilitates the relationship. Therefore, nonattachment should "enhance relatedness, compassion, and well-being because... the need to influence relationship partners or life events to fit some static mold is no longer present" (Sahdra et al., 2010, p. 117). Sahdra et al. developed the Nonattachment Scale and found that nonattachment was indeed negatively associated with both anxious and avoidant attachment (considerably stronger with anxious), and it was positively related to empathy, generosity, self-compassion, and well-being. Example items include "I have a hard time appreciating others' successes when they outperform me" (reverse-scored) and "I do not have to hang on to the people I love at all costs; I can let them go if they wish to go." Religious/spiritual support is an aspect of kindness and love in religious/spiritual relationships and communities. Bjorck and Maslim's (2011) Multi-Faith Religious Support Scale assesses perceived support from one's religious community, from religious leaders, and from God (with an adolescent version subsequently published). Originally developed among Muslim women, the scale's validity has been supported with other groups (e.g., Korean-speaking Protestants). Example items are "Other participants in my religious group care about my life and situation" and "I can turn to my religious leaders for advice when I have problems." 


\section{Justice}

Peterson and Seligman (2004) define the virtue of justice as "civic strengths that underlie healthy community life" (p. 30). It includes the character strengths of citizenship, fairness, and leadership. Peterson and Seligman (2004) argue this virtue is unique in that its character strengths can be considered as strengths among people (they pertain to community living), whereas the strengths of humanity are strengths between people (they pertain to interpersonal relationships). The character strength of fairness is relevant to extrinsic religious orientation, religious/spiritual attitudes about prejudice, and religious/spiritual attitudes about social justice. Citizenship and leadership are implicated in religious/spiritual community identity and religious/spiritual organizational attributes. We discuss VCS connections with religious/spiritual prejudice, extrinsic religious orientation, social justice attitudes, and organizational attributes. Prejudice, of course, is fundamentally related to fairness. Prejudice among religious people is what prompted Allport (1950) to distinguish between those who use their religion instrumentally as a means to another end — extrinsic religious orientation — and those who live their religion as an end in itself-intrinsic religious orientation. Extrinsically oriented religious persons were more racially prejudiced than intrinsically oriented persons. Example items from the Religious Orientation Scale's extrinsic subscale are (Allport \& Ross, 1967) are “Occasionally I find it necessary to compromise my religious beliefs in order to protect my social and economic well-being" and "The primary purpose of prayer is to gain relief and protection."

Many religious traditions emphasize themes of social justice. Attitudes about social justice pertain to issues involving fairness regarding the welfare of less powerful groups and persons; social justice includes concerns about poverty, immigration, discrimination, and hunger. A brief measure developed for use with Christians is the Sanctification of Social Justice Scale 
(Todd et al., 2014) with items such as "God wants Christians to work for social justice" and "God wants Christians to confront discrimination so that everyone can be successful." Religious/spiritual organizational attributes, such as the climate, cohesion, leadership, and communication in a faith community or institution, are germane to citizenship and leadership. In this area, faith communities and institutions are assessed in terms of group and organizational dynamics. For example, the Congregation Climate Scales (Pargament et al., 1983) include subscales assessing sense of community (e.g., "Members treat each other as family, for example, visiting the sick, celebrating anniversaries, etc.”), organizational clarity (good leadership; "Our church has clearly stated goals for the future"), stability ("It is usually not a problem finding teachers for religious education classes"), and openness to change ("Members are willing to share and listen to different points of view"). The role of good R/S leadership is also assessed in the Congregation Satisfaction Questionnaire (Silverman et al., 1983), with items asking whether the leader is well-informed, creative, dedicated, and receptive to new ideas.

\section{Temperance}

Peterson and Seligman (2004) define the virtue of temperance as "strengths that protect against excess" (p. 30). It includes the character strengths of forgiveness/mercy, humility/ modesty, prudence, and self-regulation, most of which are central concepts across many faith traditions. Humility and forgiveness are respectively involved in R/S-grounded humility and forgiveness, religious practices and moral behavior are involved in prudence, and self-control and religious/spiritual coping are germane to self-regulation. Here we will examine VCS connections with R/S-grounded humility, forgiveness, and moral behavior.

Naturally, $R / S$-grounded humility refers to when the character strength of humility is rooted in a religious/spiritual worldview. Broadly, humility is defined as a nondefensive 
willingness to view oneself accurately (including owning one's limitations), an other-oriented (rather than self-focused) interpersonal stance, a low concern for personal status, and a nondefensive teachability (see McElroy et al., 2019, for a review of humility measures). R/Sgrounded humility bases these components in one's religious/spiritual beliefs, practices, and relationships, especially people's perceived relationship to God. For example, the Theistic Intellectual Humility Scale (Hill et al., 2021) assesses intellectual humility on a vertical plane (e.g., "My understanding of the world depends on God revealing things to me"; "I don't need to know everything because God is in control") and a horizontal plane ("I'm not always sure my interpretations of the Bible are right"). The similarly grounded Spiritual Humility Scale (Davis et al., 2010a) uses informant-ratings (e.g., "He/she accepts his/her place in relation to the Sacred."). $R / S$-grounded forgiveness likewise refers to when the character strength of forgiveness is rooted in a religious/spiritual worldview. Forgiveness is often based in R/S, whether as a central component in seeking the path of righteousness for Hindus (Klostermaier, 1994), as part of forbearance and compassion for Buddhists (Higgins, 2001), as a response to divine forgiveness for Jews and Christians (Rye et al., 2000), or as a command from Allah for Muslims. For instance, the State Sanctification of Forgiveness Scale (Davis et al., 2012) takes religious/spiritual motives into account; example items are "God wants me to forgive the person who hurt me" and "If I don't forgive the person who hurt me, my spiritual life will suffer." The Relational Engagement of the Sacred for Transgressions scale (Davis et al., 2010b) assesses "the extent to which victims actively engage a relationship with the Sacred to deal with a specific transgression" (p. 288; "I tried to view him/her as a child of God"; "I tried to pray for him/her."). Moral behavior involves the character strength of self-regulation. Religious texts and traditions speak a lot about self-regulation, broadly defined as exerting control over one's 
responses in order to pursue goals and maintain standards such as moral injunctions, norms, and ideals. Baumeister and Exline (1999) claim that self-control is personality's "moral muscle" (p. 1170) and state that "virtues seem based on the positive exercise of self-control, whereas sin and vice often revolve around failures of self-control" (p. 1175). In fact, the frequently documented connection of R/S with physical and mental health may be due largely to R/S's emphasis on selfregulation (McCullough \& Willoughby, 2009). Many religious/spiritual measures involve the character strength of self-regulation, such as "When I face a problem in life, I believe that I am being punished by Allah for bad actions I did" (Psychological Measure of Islamic Religiousness scale; Abu-Raiya et al., 2008) and "God helps me to keep from drinking when I have a lot of problems” (Alcohol-related God Locus of Control Scale for Adolescents; Goggin et al., 2007).

\section{Transcendence}

Peterson and Seligman (2004) defined the virtue of transcendence as "strengths that forge connections to the large universe and provide meaning" (p. 30), and it includes the character strengths of appreciation of beauty and excellence, gratitude, hope, humor, and spirituality. The appreciation of beauty and excellence is relevant to mysticism and transcendent experiences; gratitude is germane to grace; hope is implicated in religious/spiritual coping and death transcendence; and spirituality is relevant to religious/spiritual insight, transcendent experiences, and God representations. We will examine VCS connections with religious/spiritual insight, transcendent experiences, and God representations.

Of note to positive psychologists, it is first worthwhile to dwell momentarily on the potentially transformative power of strong transcendence. Strong transcendence is "an aspect of human life or experience that involves encounters with things that defy human comprehension, understanding, and control" (Nelson, 2009, p. 548). Life is sometimes filled with enigmas; loved 
ones die and babies are born, the innocent suffer while the guilty prosper, freak accidents happen whereas serendipitous discoveries are made. Such woeful—and wonderful—slices of life often invite larger existential questions. Religious/spiritual thinkers contend that close encounters of the strong-transcendence kind (e.g., with transcendent reality, sacred beings or forces, or God) have the power to respond to life's enigmas and transform people in powerful and positive ways. Thus, transcendence - as abstractly conceived in the VCS frame, relates to aspects of personal transformative encounters with strong transcendence - as holistically conceived by psychologists of R/S. These aspects include religious/spiritual insight (e.g., meaning, religious coping, belief, faith maturity, God concepts, and God attributions), transcendent experiences (e.g., awe, wonder, prayer, meditation, and mysticism), changed future expectations (e.g., hope, optimism, and death transcendence), and transformed relational emotions and postures (e.g., submission, acceptance, gratitude, trust, joy, contentment, comfort, and peace).

Religious/spiritual insight, which involves the character strength of perspective, deserves special attention here because of positive psychology's increasingly meaning-oriented focus, including its emphasis on the dialectic between the positives and negatives of life (a shift that has been referred to as "Positive Psychology 2.0," Wong, 2011, p. 69). R/S is, of course, a wellknown source of meaning, and meaning making is a central facet of R/S (Park \& Van Tongeren, this volume; Davis et al., Chapters 1 and 18, this volume). Furthermore, a sense of meaning in life significantly mediates the positive relationship between R/S and subjective well-being (Steger \& Frazier, 2005). In short, R/S and positive psychology already intersect at meaning.

One multidimensional measure that taps into insight inherent in transcendent encounters is the Spiritual Orientation Inventory (Elkins et al., 1988; see also Lazar, 2021). This measure originates within a framework of spirituality, which is defined as "a way of being and 
experiencing that comes about through awareness of a transcendent dimension and that is characterized by certain identifiable values in regard to self, others, nature, life, and whatever one considers to be the Ultimate" (Elkins et al., 1988, p. 10). Its items on the Meaning and Purpose in Life subscale assess spiritually based meaningful insights (e.g., "Whether or not it is always clear to us, the universe is unfolding in a meaningful, purposeful manner" and "My belief that there is a transcendent, spiritual dimension gives meaning to my life"). Items on its Sacredness of Life subscale assess insights into spiritually derived meaning from even mundane activities (e.g., "Even such activities as eating, work, and sex have a sacred dimension to them" and "I do not divide life into sacred and secular; I believe all of life is infused with sacredness"). Its items on the Awareness of the Tragic subscale assess meaning accruing from adverse life experiences (e.g., "I have grown spiritually as a result of pain and suffering”).

Within a religious/spiritual framework, transcendent experiences-which are germane to the appreciation of beauty and excellence - also deserve notice, particularly because of positive psychology's long-standing engagement with mindfulness, a practice originally intended to promote awareness of transcendent reality. Mindfulness is typically defined as nonjudgmental awareness of present experience (Langer \& Moldoveanu, 2000), but this conception often is stripped of its religious/spiritual moorings, which originated in Buddhism and Christianity (Sharf, 2014). However, mindfulness researchers and therapies are increasingly reintegrating mindfulness within a holistic religious/spiritual framework (Purser \& Milillo, 2014). Thus, R/S and positive psychology also intersect at transcendent experiences. The Spiritual Orientation Inventory (Elkins et al., 1988) assess experiences with strong transcendence by asking about awareness of a Transcendent Dimension (e.g., "I have had transcendent, spiritual experiences in 
which...”: “...I felt deeply and intimately loved by something greater than I” and “...deeper aspects of truth seem to have been revealed").

God representations (i.e., one's mental representations of a deity) are related to the character strengths of spirituality and perspective, and they are directly relevant to encounters with strong transcendence. Dual-process conceptualizations of God representations are particularly interesting, because they highlight the difference between cognitive-doctrinal representations of God ("head knowledge")— one facet of transcendent understanding/insight--and affective-experiential representations of God ("heart knowledge") — which is one facet of transcendent relational emotion (see the theoretical framework and review of 73 measures by Sharp et al., 2021). For example, Zahl and Gibson (2012) asked Christian adults to consider positive (e.g., kind, responsive, approachable) and critical (e.g., critical, judgmental, controlling) adjectives of God in two different ways. Respondents were asked to indicate "whether it was descriptive of what they 'should believe that God is like' (intended to capture doctrinal representations), or what they 'personally feel that God is like' (intended to capture experiential representations)" (p. 220). This resulted in two subscales assessing doctrinal representations (of God as positive and critical) and two assessing experiential representations (God as positive and critical). Only experiential representations of God as positive were predictive of life satisfaction.

\section{Conclusion: The Religious/Spiritual Varieties of Positive Psychology}

In this chapter, we examined scales at the intersection of positive psychology and the psychology of R/S. Our intent was to aid scholars and practitioners by identifying VCS assessed with religious/spiritual measures and by making VSC-R/S connections explicit. We accomplished this goal by viewing measures from the psychology of R/S through the lens of VCS - a foundational and ubiquitous construct in positive psychology. By exploring the scales 
featured in this chapter, we also demonstrated how — at the level of measurement — constructs in the psychology of R/S can enhance our understanding of VCS. More broadly, we have shown how VCS measurement may be shaped within religious/spiritual contexts. We drew on insights gained from our VCS-focused ratings of 200 diverse scales from the psychology of R/S, the complete set of which is available in the Table of VCS Ratings of R/S Scales and Lists of R/S Scales by VCS in Appendix 7.S1 and Appendix 7.S2.

We conclude with a note of caution. There is a sense that the metaphor of “intersection" - around which this chapter and volume are centered—is a misnomer. Different conceptualizations of VCS are grounded in different religious/spiritual worldviews, and because measurement follows conceptualization, measurement ought to reflect these religious/spiritual worldview differences (Hill \& Hall, 2017). Had William James been a positive psychologist of R/S who was writing for this volume, perhaps he would have entitled his chapter The Religious/Spiritual Varieties of Positive Psychology.

Consider, for example, the concept of well-being, which is perhaps the overriding VCS concept to which all virtues and character strengths are indicators. Well-being is conceived-and therefore should be measured-differently among the different religions. Well-being in Buddhism, Christianity, Confucianism, Hinduism, Sufism, and Taoism, for example, is flavored with the virtues of social harmony over personal expressiveness (Joshanloo, 2014; Uchida et al., 2004). Likewise, Muslim concepts of happiness are grounded in Islam, which emphasizes virtues of piety, fear of God, and submission to God's will, as expressed in the Shari'ah (i.e., "the divine law"; Joshanloo, 2013). Within Islam, ultimate happiness comes in part from liberation from the flesh, suggesting that hedonic well-being scales measuring the frequency and intensity of positive emotion might be invalid when used with Muslims. In this vein, Abu-Raiya and 
Pargament (2011) noted that empirical research with Muslims needs to attend to Islam; otherwise it will risk distortion. Their note applies to research assessing any religious/spiritual population.

Similarly, because of the abstracted nature of positive psychology's formulation of VCS, the risk of reductive distortion is inherent in negotiating the "intersection" of VCS and the psychology of R/S. Thus, when dealing with specific populations, such as people who adhere to religious/spiritual traditions, it is important that researchers and practitioners use positive psychology measures that validly assess such populations - not on positive psychology's assumed cross-culturally universal terms but in the idiographically defined terms of the people they are assessing. A consideration of psychology of R/S measures will aid this endeavor. 


\section{References}

Abu-Raiya, H., Pargament, K. I., Mahoney, A., \& Stein, C. (2008). A psychological measure of Islamic religiousness: Development and evidence of reliability and validity. The International Journal for the Psychology of Religion, 18, 291-315. https://doi.org/10.1080/10508610802229270

Abu-Raiya, H., \& Pargament, K. I. (2011). Empirically based psychology of Islam: Summary and critique of the literature. Mental Health, Religion \& Culture, 14(2), 93-115. https://doi.org/10.1080/13674670903426482

Allport, G. W. (1950). The individual and his religion. MacMillan.

Allport, G. W., \& Ross, J. M. (1967). Personal religious orientation and prejudice. Journal of Personality and Social Psychology, 5, 447-457. https://doi.org/10.1037/h0021212

Altemeyer, B. (1988). Enemies of freedom. Jossey-Bass.

Batson, C., \& Schoenrade, P. (1991). Measuring religion as quest: Reliability concerns. Journal for the Scientific Study of Religion, 30, 430-447. https://doi.org/10.2307/1387278

Batson, C. D., Schoenrade, P., \& Ventis, W. L. (1993). Religion and the individual: A socialpsychological perspective. Oxford University Press.

Baumeister, R. F., \& Exline, J. J. (1999). Virtue, personality, and social relations: Self-control as the moral muscle. Journal of Personality, 67, 1165-1194. https://doi.org/10.1111/1467$\underline{6494.00086}$

Beck, R., \& McDonald, A. (2004). Attachment to God: The Attachment to God Inventory, tests of working model correspondence, and an exploration of faith group differences. Journal of Psychology and Theology, 32, 92-103. https://doi.org/10.1177/009164710403200202 
Benson, P., \& Spilka, B. (1973). God image as a function of self-esteem and locus of control. Journal for the Scientific Study of Religion, 12, 297-310.

Bjorck, J. P., \& Maslim, A. A. (2011). The Multi-Faith Religious Support Scale: Validation with a sample of Muslim women. Journal of Muslim Mental Health, 1, 62-80. https://doi.org/10.3998/jmmh.10381607.0006.105

Dahlsgaard, K., Peterson, C., \& Seligman, M. E. P. (2005). Shared virtue: The convergence of valued human strengths across culture and history. Review of General Psychology, 9(3), 203-213. https://doi.org/10.1037/1089-2680.9.3.203

Davis, D. E., Hook, J. N., Van Tongeren, D. R., \& Worthington, E. L., Jr. (2012). Sanctification of forgiveness. Psychology of Religion and Spirituality, 4(1), 31-39. https://doi.org/10.1037/a0025803

Davis, D. E., Hook, J. N., Worthington, E. L., Van Tongeren, D. R., Gartner, A. L., \& Jennings, D. J. (2010a). Relational spirituality and forgiveness: Development of the Spiritual Humility Scale (SHS). Journal of Psychology and Theology, 38, 91-100.

Davis, D. E., Hook, J. N., Worthington, E. L., Jr., Van Tongeren, D. R., . . Norton. L. (2010b). Relational spirituality and dealing with transgressions: Development of the Relational Engagement of the Sacred for a Transgression (REST) scale. International Journal for the Psychology of Religion, 20, 288-302. https://doi.org/10.1080/10508619.2010.507699

Dudley, M. G., \& Cruise, R. J. (1990). Measuring religious maturity: A proposed scale. Review of Religious Research, 32, 97-109. https://doi.org/10.2307/3511758

Elkins, D. N., Hedstrom, L. J., Hughes, L. L., Leaf, J. A., \& Saunders, C. (1988). Toward a humanistic-phenomenological spirituality: Definition, description, and measurement. Journal of Humanistic Psychology, 28, 5-18. https://doi.org/10.1177/0022167888284002 
Emavardhana, T., \& Tori, C. D. (1997). Changes in self-concept, ego defense mechanisms, and religiosity following seven-day Vipassana meditation retreats. Journal for the Scientific Study of Religion, 36, 194-206. https://doi.org/10.2307/1387552

Goggin, K., Murray, T. S., Malcarne, V. L., Brown, S. A., \& Wallston, K. A. (2007). Do religious and control cognitions predict risky behavior? I. Development and validation of the Alcohol-related God Locus of Control Scale for Adolescents (AGLOC-A). Cognitive Therapy and Research, 31, 111-122. https://doi.org/10.1007/s10608-006-9091-0

Granqvist, P. (2020). Attachment in religion and spirituality: A wider view. Guilford Press.

Higgins, R. (2001). Buddhists practice forgiveness: Mindful suffering. Christian Century, 118, 9-10. https://www.christiancentury.org/article/mindful-suffering

Hill, P. C., \& Hall, M. E. L. (2017). Uncovering the good in positive psychology: Toward a worldview conception that can help positive psychology flourish. In N. J. L. Brown, T. Lomas, \& F. J. Eiroá-Orosa (Eds.), The Routledge international handbook of critical positive psychology (pp. 245-262). Routledge.

Hill, P. C., Hall, M. E. L., Wang, D. C., \& Decker, L. A. (2021). Theistic intellectual humility and well-being: Does ideological context matter? Journal of Positive Psychology, 16(2), 155-167. https://doi.org/10.1080/17439760.2019.1689424

Hood, R. W., Jr., Hill, P. C., \& Spilka, B. (2018). The psychology of religion: An empirical $\operatorname{approach}\left(5^{\text {th }}\right.$ ed.). Guilford.

Johnson, K. A., Okun, M. A., \& Cohen, A. B. (2015). The mind of the Lord: Measuring authoritarian and benevolent God representations. Psychology of Religion and Spirituality, 7(3), 227-238. https://doi.org/10.1037/rel0000011 
Joshanloo, M. (2013). A comparison of Western and Islamic conceptions of happiness. Journal of Happiness Studies, 14(6), 1857-1874. https://doi.org/10.1007/s10902-012-9406-7

Joshanloo, M. (2014). Eastern conceptualizations of happiness: Fundamental differences with Western views. Journal of Happiness Studies, 15(2), 475-493. https://doi.org/10.1007/s10902-013-9431-1

Khan, Z. H., \& Watson, P. J. (2006). Construction of the Pakistani Religious Coping Practices Scale. The International Journal for the Psychology of Religion, 16, 101-112. https://doi.org/10.1207/s15327582ijpr1602__2

Klostermaier, K. K. (1994). A survey of Hinduism. State University of New York Press.

Krauss, S. W., \& Flaherty, R. W. (2001). The effects of tragedies and contradictions on religion as a quest. Journal for the Scientific Study of Religion, 40(1), 113-122.

Langer, E., \& Moldoveanu, M. (2000). The construct of mindfulness. Journal of Social Issues, 56, 1-9. https://doi.org/10.1111/0022-4537.00148

Lazar, A. (2021). The Spiritual Orientation Inventory (SOI): A multidimensional measure of humanistic spirituality. In A. L. Ai, P. Wink, R. F. Paloutzian, \& K. A. Harris (Eds.), Assessing spirituality in a diverse world (pp. 249-269). Springer Cham.

McCullough, M. E., \& Willoughby, B. L. B. (2009). Religion, self-regulation, and self-control: Associations, explanations, and implications. Psychological Bulletin, 135, 69-93. https://doi.org/10.1037/a0014213

McElroy-Heltzel, S. E., Davis, D. E., DeBlaere C., Worthington, E. L., Jr. \& Hook, J. N. (2019). Embarrassment of riches in the measurement of humility: A critical review of 22 measures. Journal of Positive Psychology, 14, 393-404. https://doi.org/10.1080/17439760.2018.1460686 
Nelson, J. M. (2009). Psychology, religion, and spirituality: Springer Science \& Business Media.

Pargament, K. I., Silverman, W. H., Johnson, S. M., Echemendia, R. J., \& Snyder, S. (1983). The psychosocial climate of religious congregations. American Journal of Psychology, 11, 351-381. https://doi.org/10.1007/BF00894054

Pargament, K. I., Smith, B. W., Koenig, H. G., \& Perez, L. (1998). Patterns of positive and negative religious coping with major life stressors. Journal for the Scientific Study of Religion, 37, 710-724. https://doi.org/10.2307/1388152

Peterson, C., \& Seligman, M. E. P. (2004). Character strengths and virtues: A handbook and classification. Oxford University Press.

Purser, R. E., \& Milillo, J. (2014). Mindfulness revisited: A Buddhist-based conceptualization. Journal of Management Inquiry, 24(1), 3-24. https://doi.org/10.1177/1056492614532315

Rowatt, W. C., \& Kirkpatrick, L. A. (2002). Two dimensions of attachment to God and their relation to affect, religiosity, and personality constructs. Journal for the Scientific Study of Religion, 41, 637-651. https://doi.org/10.1111/1468-5906.00143

Rye, M., Pargament, K., Ali, M., Beck, G., Dorff, E., Hallisey, C., . . Williams, J. (2000). Religious perspectives on forgiveness. In M. McCullough, K. Pargament, C. Thoresen (Eds.), Forgiveness: Theory, research, and practice (pp. 17-40). Guilford Press.

Sahdra, B. K., Shaver, P., \& Brown, K. (2010). A scale to measure nonattachment: A Buddhist complement to Western research on attachment and adaptive functioning. Journal of Personality Assessment, 92(2), 116-127. https://doi.org/10.1080/00223890903425960

Sharf, R. H. (2014). Is mindfulness Buddhist? (and why it matters). Transcultural Psychiatry, 52(4), 470-484. https://doi.org/10.1177/1363461514557561 
Sharp, C., Davis, E., George, K., Cuthbert, A., Zahl, B., Davis, D., Hook, J., \& Aten, J. (2021). Measures of God representations: Theoretical framework and critical review. Psychology of Religion and Spirituality, 13(3), 340-357. https://doi.org/10.1037/rel0000257

Silverman, W. H., Pargament, K. I., Johnson, S. M., Echemendia, R. J., \& Snyder, S. (1983). Measuring member satisfaction with the church. Journal of Applied Psychology, 68, 664677. https://doi.org/10.1037/0021-9010.68.4.664

Sim, T. N., \& Loh, B. (2003). Attachment to God: Measurement and dynamics. Journal of Social and Personal Relationships, 20, 373-389. https://doi.org/10.1177/0265407503020003006

Steger, M. F., \& Frazier, P. (2005). Meaning in life: One link in the chain from religiousness to well-being. Journal of Counseling Psychology, 52, 574-582. https://doi.org/10.1037/0022-0167.52.4.574

Todd, N. R., Houston, J., \& Odahl-Ruan, C. A. (2014). Preliminary validation of the Sanctification of Social Justice Scale. Psychology of Religion and Spirituality, 6, $245-$ 256. https://doi.org/10.1037/a0036348

Uchida, Y., Norasakkunkit, V., \& Kitayama, S. (2004). Cultural constructions of happiness: Theory and empirical evidence. Journal of Happiness Studies, 5, 223-239. https://doi.org/10.1007/s10902-004-8785-9

Wong, P. T. (2011). Positive psychology 2.0: Towards a balanced interactive model of the good life. Canadian Psychology/Psychologie Canadienne, 52(2), 69-81.

Zahl, B. P., \& Gibson, N. J. (2012). God representations, attachment to God, and satisfaction with life: A comparison of doctrinal and experiential representations of God in Christian young adults. The International Journal for the Psychology of Religion, 22(3), 216-230. https://doi.org/10.1080/10508619.2012.670027 


\title{
Chapter 8:
}

Methodological Diversity in Positive Psychology and the Psychology of Religion and Spirituality

\author{
Jo-Ann Tsang ${ }^{1}$ \\ Rosemary L. Al-Kire ${ }^{1}$ \\ Edward B. Davis ${ }^{2}$ \\ Hilary N. Alwood ${ }^{1}$ \\ Wade C. Rowatt ${ }^{1}$ \\ ${ }^{1}$ Department of Psychology and Neuroscience, Baylor University \\ ${ }^{2}$ School of Psychology, Counseling, \& Family Therapy, Wheaton College
}

Corresponding author: Jo-Ann Tsang, JoAnn_Tsang@ Baylor.edu. 


\begin{abstract}
Although psychologists were certainly not the first to study flourishing, virtues, spirituality, and religiousness, one of their key contributions has been to examine these constructs using the scientific method. Complex concepts such as gratitude, humility, spirituality, and religiousness present unique challenges to researchers, requiring them to utilize equal doses of scientific rigor and methodological ingenuity. In this chapter, we describe some of these efforts in both positive psychology and the psychology of religion/spirituality (R/S). Specifically, we provide examples of research using correlational cross-sectional data, longitudinal data, experiments, field research, and qualitative and mixed methods. We then discuss the strengths and weaknesses of using each approach to study positive psychological and religious/spiritual constructs. We note some advances in technology that may open new directions for research, and we discuss future directions for the fields, including issues of research transparency and the need for cross-cultural research.
\end{abstract}

Keywords: religiousness, spirituality, positive psychology, research methodology, open science 


\section{Chapter 8: Methodological Diversity in Positive Psychology and the Psychology of Religion and Spirituality}

Whereas the psychology of religion/spirituality (R/S) has historically focused both on the positive and negative outcomes of religious beliefs and institutions relevant to the divine or sacred (e.g., Hill et al., 2000), positive psychology has focused more specifically on topics related to flourishing, including well-being and resilience (Snyder et al., 2021). Despite differences in topics of study, these fields share commonalities in their research methods and methodological diversity (or lack thereof; see Table 8.1). Both fields largely emphasize quantitative, cross-sectional data, providing an efficient but incomplete picture of very complex phenomena. In this chapter, we describe various measures and research designs used in the psychological study of R/S and positive psychology, and we outline the strengths and weaknesses of each. We also discuss emerging research and data collection issues (e.g., open science practices and cross-cultural concerns), and we suggest ways the fields of positive psychology and the psychology of R/S can increase methodological rigor and diversity. Throughout this chapter, we use specific research examples in positive psychology and psychology of R/S, but in no way is our brief review meant to be exhaustive.

\section{What Kinds of Measures?}

The measures that researchers use have important and often unexamined effects on the conclusions researchers can draw. Asking individuals if they are spiritual or forgiving, for example, has the potential to elicit very different answers compared to observing individuals' spiritual or forgiving behavior. Below we summarize some strengths and limitations of measures that are commonly used in the fields of positive psychology and the psychology of R/S. 


\section{Self-Reports}

Both fields rely heavily on self-report measures (Yaden et al., 2018; see Hill et al., Chapter 7, this volume, for a review), which enable the efficient measurement of multiple dimensions of complex constructs. However, self-reports can be subject to self-presentation bias, especially when measuring socially desirable constructs (Tourangeau \& Yan, 2007) such as religiousness (Presser \& Stinson, 1998) and well-being (Heintzelman et al., 2015). Several reviews have noted positive relationships between social desirability and individual differences in religiousness (e.g., Gebauer et al., 2017), leading some to question whether religious people only present themselves as more altruistic (Galen, 2012) and less prejudiced (Batson et al., 1993). Self-reports might be particularly problematic for certain concepts such as humility, given that "self-reports of high levels of humility may ironically indicate a lack of humility" (D. E. Davis et al., 2010, p. 246). Because of a heavy reliance on self-reports, these two fields may actually be studying how religious and happy people think they should be, rather than how religious and happy people truly are.

One solution is to control statistically for social desirability (e.g., Saroglou et al., 2005, Study 4). However, it is difficult to distinguish between self-presentation and actual high levels of desirable behaviors (e.g., Heintzelman et al., 2015), so partialling out social desirability may remove legitimate variance from other measures. Fortunately, another solution lies in the use of informant reports.

\section{Informant Reports}

Friends, family members, and acquaintances of religious individuals report that those individuals tend to display more empathy (Łowicki \& Zajenkowski, 2019), agreeableness (McCullough et al., 2003), and prosociality (Saroglou et al., 2005) than their less-religious peers. 
Informant reports have also been used for constructs such as patience (Shubert et al., 2020) and humility (D. E. Davis et al., 2010). Not only might informant reports circumvent participants' social desirability biases, but discrepancies between self- and informant-reports themselves can predict variance in well-being (Shubert et al., 2020).

Although useful, informant-reports are also limited. Informant- and self-reports are based on different information (Vazire, 2010). For instance, Meagher et al. (2020) noted that informant ratings of humility were based on observable conciliatory behaviors, whereas self-ratings of humility were likely based more on knowledge about one's own thoughts; these types of discrepancies could lead to different behavioral correlates of peer- and self-rated humility. This limitation may also apply to other constructs with cognitive and relational components, such as religiousness, self-regulation (Hardy et al., 2020), or forgiveness. Additionally, Galen et al. (2014) argued that informant reports of relationships between religiousness and prosociality were based in part on stereotypes that religious individuals were more helpful. Vazire's (2010) selfother knowledge asymmetry model incorporates these and other explanations for degrees of selfother rating (dis)agreement. In contrast, Helzer et al. (2014) ruled out the heuristic confound in informant reports of moral character, and they found results suggesting that these informants were less subject to presentation biases.

\section{Behavioral Measures}

Behavioral measures can address social desirability by providing covert and psychologically costly measures of relevant constructs (Batson et al., 1993). Yet behavioral measures are quite rare in positive psychology and psychology of R/S. Many assessments of religious behavior are actually self-reports of behaviors, such as religious attendance (Presser \& Stinson, 1998) or prayer frequency (Masters \& Spielmans, 2007). In one exception, Grossoehme 
et al. (2011) coded written prayers for styles of religious coping. Some online archival data can also be considered "behavioral," such as the content of online sermons (e.g., Graham et al., 2009) and online search information related to R/S and positive psychology constructs (MacInnis \& Hodson, 2015). Behavioral measures can also be assessed in the laboratory, such as the use of reciprocity to measure gratitude (Tsang \& Martin, 2017) and physical distance from a conflictdiscussion partner to study humility (J. C. Wright et al., 2018).

Despite their strengths, behavioral measures should complement and not replace selfreports. Self-reports can provide more nuanced pictures of psychological constructs. For example, self-report measures of gratitude (McCullough et al., 2002) can assess gratitude with more complexity, compared to reciprocity behavior (Tsang \& Martin, 2017). Alone, behavioral measures might have ambiguous validity; reciprocity could be the result of gratitude but also could reflect compliance to norms. Moreover, because behaviors can be particularly sensitive to context, they should not be used as sole indicators of individual differences (Dang et al., 2020).

\section{Implicit Measures}

Self-report measures are most relevant when assessing conscious, easily accessible attitudes. In contrast, implicit measures purport to assess unconscious attitudes in ways that are less affected by self-presentation. For example, an implicit measure of humility (relative to arrogance) assesses individuals' reaction times when associating humble and arrogant words with self and other words (Rowatt et al., 2006). Religious beliefs and self-concepts can also be measured implicitly (LaBouff et al., 2010). Measures such as the Implicit Association Test (Shariff et al., 2008) and the Affect Misattribution Procedure (Ross et al., 2019) have been utilized to assess the strength of people's associations between supernatural words and concepts of truelfalse and real/imaginary. 


\section{Physiological Measures}

In both R/S and positive psychology, physiological measures can uncover mechanisms and functions of constructs. Brain imaging technology has shed light on brain structures involved in religiousness (Maselko, 2013) and in character virtues such as patience (van den Bos et al., 2014). Other physiological measures (e.g., blood pressure, heart rate, and galvanic skin response) have been used to study constructs such as spirituality (Newberg, 2014) and gratitude (Ginty et al., 2020). Chemical markers like high oxytocin may be relevant to religiousness (Sasaki et al., 2011) and forgiveness (Worthington et al., 2015), and low cortisol may be relevant to optimism (Nicolson et al. 2020).

Physiological indicators are indirect measures that can provide insight into the embodied nature of psychological phenomena. Because the processes assessed are automatic, they are less contaminated by self-presentation biases. As with behavioral measures, physiological measures are often paired with other assessment methods in order to enhance their validity. Despite their strengths, physiological measures are costly to administer, both in terms of time and resources, especially when compared to other methods such as self-report surveys. Moreover, particular care should be taken to choose appropriate control or comparison groups (Newberg, 2014).

\section{What Kinds of Studies?}

Even the best measures cannot compensate for lack of rigor in research design. Below we summarize the strengths and weaknesses of several quantitative research designs that are utilized in R/S and positive psychology studies, as well as a few qualitative and mixed-methods designs.

\section{Cross-Sectional, Correlational Research}

The vast majority of research in these two fields are cross-sectional, correlational studies (see Table 8.1). Many key variables, such as religious affiliation or dispositional hope, are 
challenging to manipulate experimentally. As an example, most studies on religiousness and depression utilize cross-sectional, correlational data (Koenig et al, 2012). These studies usually show an inverse relationship between religiousness and depression, consistent with a protective effect. However, because of the causal ambiguity embedded in cross-sectional research, it may also be that depressed people have less motivation to attend religious services (Maselko et al., 2012). Likewise, there may be confounding variables (such as personality; Mihaljevic et al., 2016) that are causing associations between religiousness and depression.

\section{Longitudinal Research}

Longitudinal studies measure variables at different points in time, removing some causal ambiguity. They can enable researchers to see (a) if earlier levels of one variable affect later levels of another, (b) if there are bidirectional temporal associations between variables, and (c) if there are between-person differences in within-person trajectories across time. As an example, Hardy and colleagues (2020) utilized growth curve modeling to uncover positive bidirectional relationships in within-person variation in religiousness and both cognitive and emotional selfregulation over time. Studies that include several measures of predictor and outcome variables over time (and control for covariates) are better able to test for directionality and reciprocal relationships (VanderWeele et al., 2016; see also Davis et al., Chapter 18, Appendix 18.S2, this volume; Long \& VanderWeele, Chapter 25, this volume, Appendix 25.S2).

Experience sampling and daily diary methodologies are two longitudinal methods that help examine within-person processes and have been gaining traction in the fields of positive psychology and the psychology of R/S. For example, Balkaya-Ince et al. (2020) used an experience sampling approach when investigating the daily and momentary fluctuations in Muslim American adolescents' self-reported religious identity in relation to civic engagement 
and maternal religious socialization. They collected data at multiple (random) time points within a day by sending a signal to the participants' cell phones. Benefits to the experience sampling approach include increased ecological validity (Hektner et al., 2006) and limiting recall bias that may occur in cross-sectional self-report studies (Shiffman et al., 2008), but self-report bias may still persist with this methodological approach (Hofmann \& Patel, 2015).

\section{Experimental Research}

Although true experiments were initially rare in $\mathrm{R} / \mathrm{S}$, the use of religious primes in experiments has become more common. For example, experiments have identified causal relationships between religious salience and prosociality (Shariff et al., 2016). Positive psychology has utilized experiments since its inception, yet the proportion of experimental studies is still incredibly small relative to other nonexperimental designs (see Table 8.1). Much of experimental positive psychology has focused on interventions to promote outcomes like happiness and well-being (Carr et al., 2020).

Despite its advantages for causal inference, experimental research has its own challenges. Behavioral priming results have been notoriously difficult to replicate (Cesario, 2014), and questions remain about the strength of religious priming effects (van Elk et al., 2015), especially among those who do not identify as religious. The careful consideration of control groups is also of paramount importance (Galen, 2012).

\section{Qualitative and Mixed-Methods Research}

Although quantitative research is useful for theory testing, it may lose much of the richness and complexity inherent in religious/spiritual and positive psychological constructs. Qualitative and mixed-methods research can provide the depth that quantitative research might miss. Qualitative research involves studying phenomena in their natural setting and trying to 
understand their subjective meanings. These studies collect observational, interview, textual, or audiovisual data and then analyze it using approaches such as grounded theory, phenomenology, narrative analysis, ethnography, case study (Creswell \& Creswell, 2018), thematic analysis (Braun \& Clark, 2006), and consensual qualitative research (Hill et al., 1997). Mixed-methods research combines elements of both qualitative and quantitative research to capitalize on the strengths of each (Johnson et al., 2007).

Quantitative research has generally dominated in the psychology of R/S (Hood, 2012) and positive psychology fields (Snyder et al., 2021). Yet since the 1990s, the number and rigor of qualitative psychological studies of R/S and positive psychology has increased substantially (E. B. Davis et al., 2016; Snyder et al., 2021). Qualitative methods hold promise for the empirical study of R/S and positive psychology because many of these phenomena are multidimensional, multilayered, context-sensitive, and culturally embedded (Loewenthal, 2013; Pedrotti \& Edwards, 2017), and qualitative methods often can capture these aspects better than quantitative methods alone (E. B. Davis et al., 2016).

Similarly, mixed-methods research is becoming more common in the psychology of R/S and positive psychology (E. B. Davis et al., 2016; Snyder et al., 2021). Mixed-methods research tends to be costly in terms of resources, but it draws on strengths of qualitative and quantitative methods to develop a more complete and contextualized understanding (Creswell \& Creswell, 2018). For example, in a large mixed-methods study, Delle Fave et al.'s (2011) qualitative data revealed evidence that across six countries in Europe, Africa, and Australia, laypeople defined happiness as "a condition of psychological balance and harmony" (p. 185), and they mainly associated their happiness and meaning with the quality of their family and social relationships (but not with their religiousness or spirituality). Likewise, the study's quantitative data revealed 
that participants' R/S did not contribute significantly to their subjective sense of happiness, meaning, or satisfaction with life.

\section{What Kind of Setting? Field Research}

Research setting is crucial when considering methodological diversity. In both fields, most research is conducted in the laboratory or via online surveys; very little field research exists either in psychology of R/S (B. R. E. Wright, 2018) or positive psychology (see Table 8.1). Field experiments provide causal tests with higher ecological validity compared to lab experiments. Although fieldwork provides rich data and information about a specific context, it may be limited in its generalizability (Anczyk et al., 2019; cf. B. R. E. Wright, 2018). Field research can be useful in reaching diverse populations and in understanding phenomena not well-captured with student samples or in the lab (e.g., religiously motivated violence; Ginges et al., 2011).

One example of fieldwork in R/S is the study of religious rituals (see Hobson et al., 2018, for a review). Rituals often lead to positive outcomes, including enhanced regulation of emotions, goals, and social connections (Hobson et al., 2018). In one cross-cultural field experiment, participants who engaged in a "high-ordeal" (i.e., physically taxing/painful) religious ritual were more generous and held more inclusive social identities than those who engaged in a "low-ordeal" ritual (Xygalatas et al., 2013).

\section{What's New? Computational Methods, Cross-Cultural Data, and Open Science Computational Methods}

Through the use of new computational methods, researchers can utilize large amounts of data readily available online (Yaden et al., 2018). For instance, language data derived from social media can be analyzed with natural language processing tools such as Linguistic Inquiry and Word Count (LIWC; Pennebaker et al., 2015). Ritter and colleagues (2014) analyzed data from 
16,000 Twitter users and found that Christians used more positive and fewer negative words compared to atheists. Computational methods can help researchers make efficient use of large amounts of pre-existing data in natural settings.

\section{Cross-Cultural Data}

Researchers in R/S and positive psychology are also making use of online data repositories, such as the Association of Religion Data Archives (ARDA: www.theARDA.com), International Social Survey Programme (http://issp.org/), and Gallup World Poll (https://www.gallup.com/analytics/318875/global-research.aspx). These provide national and international data, enabling cross-religious and cross-country comparisons. For instance, Diener et al. (2011) utilized the Gallup World Poll to investigate the moderating effect of societal circumstances on the relationship between religiousness and subjective well-being. These data repositories provide researchers invaluable opportunities to examine questions cross-culturally.

\section{Open Science}

Openness and transparency are emerging concerns in scientific research (Aalbersberg et al., 2018). Open science practices encourage researchers to share materials and data, preregister hypotheses and methods, attempt replication in independent labs, and verify that peer-reviewed publications have been appropriately vetted. Open science practices can be used in quantitative (Charles et al., 2019) and qualitative research (Haven et al., 2020). Preregistering hypotheses is becoming the norm (Nosek \& Lindsay, 2018). Many journals are adopting open-science policies that include making materials and data available (Lindsay, 2017; see https://topfactor.org/), and several journals (including the Journal of Positive Psychology, International Journal for the Psychology of Religion, and Archive for Psychology of Religion) support open science badges and/or registered reports. We hope more positive psychology and R/S journals follow suit. 
Researchers can benefit from teaming together to conduct and replicate studies. In an innovative collaboration, Landy et al. (2020) led 15 teams testing five research questions (about moral judgment, negotiation, and implicit cognition) using two large samples of participants. Effect sizes varied dramatically (and in opposite directions) when different labs tested the same research questions. Thankfully, the days of publishing single studies based on unrepresentative convenience samples without replication seem to be nearly over.

\section{What's Next?}

In what methodological directions should psychology of R/S and positive psychology move? We present four suggestions. First, both fields should increase their use of qualitative data and mixed-method studies in order to capture the complexity and culturally embedded nature of important constructs. Qualitative and mixed methods are particularly important when researchers are exploring understudied topics (e.g., spiritual resilience), complex phenomena (e.g., suffering), cultural nuances, and cross-cultural comparisons (E. B. Davis et al., 2016).

In the quantitative realm, researchers should increase their use of theory-driven experiments and longitudinal studies, thereby permitting better investigation of causal mechanisms and directionality. Much experimental work in both fields focuses on the effects of religious priming or positive interventions. Additional basic research is needed to investigate variables that cause or change R/S and positive psychological constructs. Furthermore, researchers need to utilize more implicit, behavioral, and physiological measures, especially when studying constructs that may be highly susceptible to social desirability bias.

Researchers also need to increase their use of open science practices, and journals in psychology of R/S and positive psychology need to continue to promote (or require) these practices. Especially in these fields, where topics such as R/S may still be highly controversial 
(Charles et al., 2019) and interventions related to positive psychology may be broadly applied, it becomes important to adopt practices such as preregistration and open science badges. Doing so will greatly improve the quality, credibility, and replicability of research.

Last, researchers must attend to sample diversity in addition to methodological diversity. The majority of psychology studies, including those in positive psychology and the psychology of R/S, utilize unrepresentative samples (i.e., WEIRD—Western, Educated, Industrialized, Rich, Democratic samples), which limits generalizations we can make about human nature or possible cross-cultural universals (Henrich et al., 2010). The increased use of online data and computational methods can facilitate increased sample diversity. More attention to this issue is urgently needed in order to move beyond merely the psychology of Western R/S and virtues.

\section{Conclusions}

As the study of religiousness, spirituality, and positive psychology increase in prominence, the time is ripe to re-evaluate these two fields' methodological diversity. New technology is increasing researchers' ability to look inside the brain, collect large amounts of online data from diverse samples, conduct research with the utmost integrity and generosity, and network with other researchers across the world. Researchers have more opportunities than ever to expand these fields to explore a richer, more complex psychology of religiousness, spirituality, virtue, and well-being — across a wider variety of religions, spiritualities, and cultures. 


\section{References}

Aalbersberg, I. J., Appleyard, T., Brookhart, S., Carpenter, T., Clarke, M., Curry, S., ... \& Freedman, L. (2018). Making science transparent by default; introducing the TOP statement. $\underline{\text { https://osf.io/sm78t }}$

Anczyk, A., Grzymała-Moszczyńska, H., Krzysztof-Świderska, A., \& Prusak, J. (2019). The replication crisis and qualitative research in the psychology of religion. International Journal for the Psychology of Religion, 29(4), 278-291.

https://doi.org/10.1080/10508619.2019.1687197

Balkaya-Ince, M., Cheah, C. S. L., Kiang, L., \& Tahseen, M. (2020). Exploring daily mediating pathways of religious identity in the associations between maternal religious socialization and Muslim American adolescents' civic engagement. Developmental Psychology, 56(8), 1446-1457. https://doi.org/10.1037/dev0000856

Batson, C. D., Schoenrade, P. A., \& Ventis, W. L. (1993). Religion and the individual: A social-psychological perspective. Oxford University Press.

Braun, V., \& Clarke, V. (2006). Using thematic analysis in psychology. Qualitative Research in Psychology, 3(2), 77-101. https://doi.org/10.1191/1478088706qp063oa

Carr, A., Cullen, K., Keeney, C., Canning, C., Mooney, O., Chinseallaigh, E., \& O’Dowd, A. (2020). Effectiveness of positive psychology interventions: A systematic review and meta-analysis. The Journal of Positive Psychology. Advance online publication. https://doi.org/10.1080/17439760.2020.1818807

Cesario, J. (2014). Priming, replication, and the hardest science. Perspectives on Psychological Science, 9(1), 40-48. https://doi.org/10.1177/1745691613513470 
Charles, S. J., Bartlett, J. E., Messick, K. J., Coleman, T. J., III, \& Uzdavines, A. (2019). Researcher degrees of freedom in the psychology of religion. The International Journal for the Psychology of Religion, 29(4), 230-245. https://doi.org/10.1080/10508619.2019.1660573

Creswell, J. W., \& Creswell, J. D. (2018). Research design: Qualitative, quantitative, and mixed methods approaches ( $5^{\text {th }}$ ed.). Sage.

Dang, J., King, K. M., \& Inzlicht, M. (2020). Why are self-report and behavioral measures weakly correlated? Trends in Cognitive Sciences, 24(4), 267-269. https://doi.org/10.1016/j.tics.2020.01.007

Davis, D. E., Worthington, E. L., Jr., \& Hook, J. N. (2010). Humility: Review of measurement strategies and conceptualization as personality judgment. The Journal of Positive Psychology, 5(4), 243-252. https://doi.org/10.1080/17439761003791672

Davis, E. B., Cuthbert, A. D., Hays, L. W., Aten, J. D., Van Tongeren, D. R., Hook, J. N., Davis, D. E., \& Boan, D. (2016). Using qualitative and mixed methods to study relational spirituality. Psychology of Religion and Spirituality, 8(2), 92-98. https://doi.org/10.1037/rel0000046

Delle Fave, A., Brdar, I., Freire, T., Vella-Brodrick, V., \& Wissing, M. (2011). The eudaimonic and hedonic components of happiness. Social Indicators Research, 100(2), 185-207. https://doi.org/10.1007/s11205-010-9632-5

Diener, E., Tay, L., \& Myers, D. G. (2011). The religion paradox: If religion makes people happy, why are so many dropping out? Journal of Personality and Social Psychology, 101(6), 1278-1290. https://doi.org/10.1037/a0024402

Galen, L.W. (2012). Does religious belief promote prosociality?: A critical examination. 
Psychological Bulletin, 138, 876-906. https://doi.org/10.1037/a0028251

Galen, L. W., Williams, T. J., \& Ver Wey, A. L. (2014). Personality ratings are influenced by religious stereotype and ingroup bias. The International Journal for the Psychology of Religion, 24(4), 282-297. https://doi.org/10.1080/10508619.2013.837658

Gebauer, J. E., Sedikides, C., \& Schrade, A. (2017). Christian self-enhancement. Journal of Personality and Social Psychology, 113, 786-809. https://doi.org/10.1037/pspp0000140

Ginges, J., Atran, S., Sachdeva, S., \& Medin, D. (2011). Psychology out of the laboratory: The challenge of violent extremism. American Psychologist, 66(6), 507-519. https://doi.org/10/1037/a0024715

Ginty, A. T., Tyra, A. T., Young, D. A., John-Henderson, N. A., Gallagher, S., \& Tsang, J. C. (2020). State gratitude is associated with lower cardiovascular responses to acute psychological stress: A replication and extension. International Journal of Psychophysiology, 158, 238-247. https://doi.org/10.1016/j.ijpsycho.2020.10.005

Graham, J., Haidt, J., \& Nosek, B. A. (2009). Liberals and conservatives rely on different sets of moral foundations. Journal of Personality and Social Psychology, 96(5), 1029-1046. https://doi.org/10.1037/a0015141

Grossoehme, D. H., Jacobson, C. J., Cotton, S., Ragsdale, J. R., VanDyke, R., \& Seid, M. (2011). Written prayers and religious coping in a paediatric hospital setting. Mental Health, Religion \& Culture, 14(5), 423-432. https://doi.org/10.1080/13674671003762693

Hardy, S. A., Baldwin, C. R., Herd, T., \& Kim-Spoon, J. (2020). Dynamic associations between religiousness and self-regulation across adolescence into young adulthood. Developmental Psychology, 56(1), 180-197. https://doi.org/10.1037/dev0000841 
Haven, T. L., Errington, T. M., Gleditsch, K. S., van Grootel, L., Jacobs, A. M., Kern, F. G., ... \& Mokkink, L. B. (2020). Preregistering qualitative research: a Delphi study. International Journal of Qualitative Methods, 19. https://doi.org/10.1177/1609406920976417

Heintzelman, S. J., , Trent, J., \& King, L. A. (2015). Revisiting desirable response bias in well-being reports. The Journal of Positive Psychology, 10(2), 167-178.

\section{https://doi.org/10.1080/17439760.2014.927903}

Helzer, E. G., Furr, R. M., Hawkins, A., Barranti, M., Blackie, L. E. R., \& Fleeson, W. (2014). Agreement on the perception of moral character. Personality and Social Psychology Bulletin, 40 (2), 1698-1710. DOI: 10.1177/0146167214554957

Hektner, J. M., Schmidt, J. A., \& Csikszentmihalyi, M. (2006). Experience sampling method: Measuring the quality of everyday life. Sage.

Henrich, J., Heine, S. J., \& Norenzayan, A. (2010). The weirdest people in the world?. Behavioral and Brain Sciences, 33(2-3), 61-83. https://doi.org/10.1017/S0140525X0999152X

Hill, C. E., Thompson, B. J., \& Williams, E. N. (1997). A guide to conducting consensual qualitative research. The Counseling Psychologist, 25, 517-572. https://doi.org/10.1177/0011000097254001

Hill, P. C., Pargament, K. I., Hood, Jr., R. W., McCullough, M. E., Swyers, J. P., et al. (2000). Conceptualizing religion and spirituality: Points of communality, points of departure. Journal for the Theory of Social Behavior, 30(1), 51-77. https://doi.org/10.1111/1468$\underline{5914.00119}$

Hobson, N. M., Schroeder, J., Risen, J. L., Xygalatas, D., \& Inzlicht, M. (2018). The psychology of rituals: An integrative review and process-based framework. Personality and Social 
Psychology Review, 22(3), 260-284. https://doi.org/10.1177/1088868317734944

Hofmann, W., \& Patel, P. V. (2015). SurveySignal. Social Science Computer Review, 33(2), 235-253. https://doi.org/10.1177/0894439314525117

Hood, R. W., Jr. (2012). The history and current state of research on psychology of religion. In L. J. Miller (Ed.), The Oxford Handbook of Psychology and Spirituality (pp. 7-20). Oxford University Press. https://doi.org/10.1093/oxfordhb/9780199729920.013.0001

Johnson, R. B., Onwuegbuzie, A. J., \& Turner, L. A. (2007). Toward a definition of mixed methods research. Journal of Mixed Methods, 1(2), 112-133. https://doi.org/10.1177\%2F1558689806298224

Koenig, H. G., King, D., \& Carson, V. (Eds.). (2012). Handbook of religion and health. University Press.

LaBouff, J. P., Rowatt, W. C., Johnson, M. K., Thedford, M., \& Tsang, J. A. (2010). Development and initial validation of an implicit measure of religiousness-spirituality. Journal for the Scientific Study of Religion, 49(3), 439-455. https://doi.org/10.1111/j.1468-5906.2010.01521.x

Landy, J., Jia, M., Ding, I., Viganola, D., Tierney, W., Dreber, A., Johannesson, M., Pfeiffer, T., ... Uhlmann, E. L. (2020). Crowdsourcing hypothesis tests: Making transparent how design choices shape research results. Psychological Bulletin, 146(5), 451-479. https://doi.org/10.1037/bul0000220

Lindsay, D. S. (2017). Sharing data and materials in psychological science. Psychological Science, 28(6), 699-702. https://doi.org/10.1177/0956797617704015

Loewenthal, K. M. (2013). Religion, spirituality, and culture: Clarifying the direction of effects. In K. I. Pargament (Ed.), APA Handbook of Psychology, Religion, and Spirituality (Vol. 
1, pp. 239-255). American Psychological Association. https://doi.org/10.1037/14045-013

Łowicki, P., \& Zajenkowski, M. (2019). Religiousness is associated with higher empathic concern: Evidence from self- and other-ratings. Psychology of Religion and Spirituality, 13(2), 127-135. http://doi.org/10.1037/rel0000299

MacInnis, C. C., \& Hodson, G. (2015). Do American states with more religious or conservative populations search more for sexual content on Google? Archives of Sexual Behavior, 44, 137-147. https://doi.org/10.1007/s10508-014-0361-8

Maselko, J. (2013). The neurophysiology of religious experience. In K. I. Pargament, J. J. Exline, \& J. W. Jones (Eds.), APA Handbook of Psychology, Religion, and Spirituality (Vol. 1, pp. 205-220). American Psychological Association.

Maselko, J., Hayward, R. D., Hanlon, A., Buka, S., \& Meador, K. (2012). Religious service attendance and major depression: A case of reverse causality? American Journal of Epidemiology, 175(6), 576-583. https://doi.org/10.1093/aje/kwr349

Masters, K. S., \& Spielmans, G. I. (2007). Prayer and health: Review, meta-analysis, and research agenda. Journal of Behavioral Medicine, 30(4), 329-338. https://doi.org/10.1007/s10865-007-9106-7

McCullough, M. E., Emmons, R. A., \& Tsang, J. (2002). The grateful disposition: A conceptual and empirical topography. Journal of Personality and Social Psychology, 82, 112-127. https://doi.org/10.1037/0022-3514.82.1.112

McCullough, M. E., Tsang, J., \& Brion, S. (2003). Personality traits in adolescence as predictors of religiousness in early adulthood: Findings from the Terman Longitudinal Study. Personality and Social Psychology Bulletin, 29(8), 980-991. https://doi.org/10.1177\%2F0146167203253210 
Meagher, B. R., Leman, J. C., Heidenga, C. A., Ringquist, M. R., \& Rowatt, W. C. (2020). Intellectual humility in conversation: Distinct behavioral indicators of self and peer ratings. The Journal of Positive Psychology, 16(3), 417-429. https://doi.org/10.1080/17439760.2020.1738536

Mihaljevic, S., Aukst-Margetic, B., Karnicnik, S., Vuksan-Cusa, B., \& Milosevic, M. (2016). Do spirituality and religiousness differ with regard to personality and recovery from depression? A follow-up study. Comprehensive Psychiatry, 70, 17-24. https://doi.org/10.1016/j.comppsych.2016.06.003

Newberg, A. B. (2014). The neuroscientific study of spiritual practices. Frontiers in Psychology, 5, 215. https://doi.org/10.3389/fpsyg.2014.00215

Nicolson, N. A., Peters, M. L., Meevissen, Y. M. (2020). Imagining a positive future reduces cortisol response to awakening and reactivity to acute stress. Psychoneuroendocrinology, 116, Article 104677. https://doi.org/10.1016/j.psyneuen.2020.104677

Nosek, B. A., \& Lindsay, D. S. (2018). Preregistration becoming the norm in psychological science. APS Observer, 31(3), 19-21.

Pedrotti, J. T., \& Edwards, L. M. (2017). Cultural context in positive psychology: History, research, and opportunities for growth. In M. A. Warren, \& S. I. Donaldson (Eds.), Scientific Advances in Positive Psychology (pp. 257-287). Praeger/ABC-CLIO.

Pennebaker, J. W., Boyd, R. L., Jordan, K., \& Blackburn, K. (2015). The development and psychometric properties of LIWC 2015. The University of Texas at Austin.

Presser, S., \& Stinson, L. (1998). Data collection mode and social desirability bias in selfreported religious attendance. American Sociological Review, 63(1), 137-145. https://doi.org/10.2307/2657486 
Ritter, R. S., Preston, J. L., \& Hernandez, I. (2014). Happy tweets: Christians are happier, more socially connected, and less analytical than atheists on Twitter. Social Psychological and Personality Science, 5(2), 243-249. https://doi.org/10.1177\%2F1948550613492345

Ross, R. M., Brown-Iannuzzi, J. L., Gervais, W. M., Jong, J., Lanman, J. A., McKay, R., \& Pennycook, G. (2019). Measuring supernatural belief implicitly using the Affect Misattribution Procedure. Religion, Brain \& Behavior, 10(4), 393-406. https://doi.org/10.1080/2153599X.2019.1619620

Rowatt, W. C., Powers, C., Targhetta, V., Comer, J., Kennedy, S., \& Labouff, J. (2006). Development and initial validation of an implicit measure of humility relative to arrogance. The Journal of Positive Psychology, 1(4), 198-211. https://doi.org/10.1080/17439760600885671

Saroglou, V., Pichon, I., Trompette, L., Verschueren, M., \& Dernelle, R. (2005). Prosocial behavior and religion: New evidence based on projective measures and peer ratings. Journal for the Scientific Study of Religion, 44(3), 323-348. https://doi.org/10.1111/j.1468-5906.2005.00289.x

Sasaki, J. Y., Kim, H. S., \& Xu, J. (2011). Religion and well-being: The moderating role of culture and the oxytocin receptor (OXTR) gene. Journal of Cross-Cultural Psychology, 42(8), 1394-1405. https://doi.org/10.1177\%2F0022022111412526

Shariff, A. F., Cohen, A. B., \& Norenzayan, A. (2008). The devil's advocate: Secular arguments diminish both implicit and explicit religious belief. Journal of Cognition and Culture, 8(3-4), 417-423. https://doi.org/10.1163.156853708X358245

Shariff, A. F., Willard, A. K., Andersen, T., \& Norenzayan, A. (2016). Religious priming: A meta-analysis with a focus on prosociality. Personality and Social Psychology Review, 
20(1), 27-48. https://doi.org/10.1177/1088868314568811

Shiffman, S., Stone, A. A., \& Hufford, M. R. (2008). Ecological momentary assessment. Annual Review of Clinical Psychology, 4, 1-32. https://doi.org/10.1146/annurev.clinpsy.3.022806.091415

Shubert, J., Ratchford, J., Houltberg, B., \& Schnitker, S. (2020). Differentiation as discrepancies in adolescent's perceptions of patience: An illustration of response surface analysis. Journal of Positive Psychology. Advance online publication. https://doi.org/10.1080/17439760.2020.1858334

Snyder, C. R., Lopez, S. J., Edwards, L. M, \& Marques, S. C. (Eds.). (2021). The Oxford Handbook of Positive Psychology ( ${ }^{\text {rd }}$ ed.). Oxford University Press.

Tourangeau, R., \& Yan, T. (2007). Sensitive questions in surveys. Psychological Bulletin, 133(5), 859-883. https://doi.org/10.1037/0033-2909.133.5.859

Tsang, J., \& Martin, S. R. (2017). Four experiments on the relational function of gratitude. The Journal of Positive Psychology, 14(2), 188-205. https://doi.org/10.1080/17439760.2017.1388435

van den Bos, W., Rodriguez, C. A., Schweitzer, J. B., \& McClure, S. M. (2014). Connectivity strength of dissociable striatal tracts predict individual differences in temporal discounting. The Journal of Neuroscience, 34(31), 10298-10310. https://doi.org/10.1523/JNEUROSCI.4105-13.2014

VanderWeele, T. J., Jackson, J. W., \& Li, S. (2016). Causal inference and longitudinal data: A case study of religion and mental health. Social Psychiatry and Psychiatric Epidemiology, 51, 1457-1466. DOI 10.1007/s00127-016-1281-9

van Elk, M., Matzke, D., Gronau, Q., Guang, M., Vandekerckhove, J., \& Wagenmakers, E. 
J. (2015). Meta-analyses are no substitute for registered replications: A skeptical perspective on religious priming. Frontiers in Psychology, 6, 1365. https://doi.org/10.3389\%2Ffpsyg.2015.01365

Vazire, S. (2010). Who knows what about a person? The self-other knowledge asymmetry (SOKA) model. Journal of Personality and Social Psychology, 98(2), 281-300. https://doi.org/10.1037/a0017908

Worthington, E. L., Jr., Lavelock, C., Witvliet, C. v.-O., Rye, M., Tsang, J., \& Toussaint, L. (2015). Measures of forgiveness: Self-report, biological, chemical, and behavioral indicators. In G. J. Boyle, D. H. Saklofske, \& G. Matthews (Eds.), Measures of Personality and Social Psychological Constructs (pp. 474-502). Elsevier. https://doi.org/10.1016/N978-0-12-386915-9.00017-6

Wright, B. R. E. (2018). Field experiments in religion: A dream whose time has come. Journal for the Scientific Study of Religion, 57(2), 193-205. https://doi.org/10.1111/jssr.12509

Wright, J. C., Nadelhoffer, T., Thomson Ross, L., \& Sinnott-Armstrong, W. (2018). Be it ever so humble: Proposing a dual-dimension account and measurement of humility. Self and Identity, 17(1), 92-125. https://doi.org/10.1080/15298868.2017.1327454

Xygalatas, D., Mitkidis, P., Fischer, R., Reddish, P., Skewes, J., Geertz, A. W., Roepstorff, A., \& Bulbulia, J. (2013). Extreme rituals promote prosociality. Psychological Science, 24(8), 1602-1605. https://doi.org/10.1177\%2F0956797612472910

Yaden, D. B., Eichstaedt, J. C., \& Medaglia, J. D. (2018). The future of technology in positive Psychology: Methodological advances in the science of well-being. Frontiers in Psychology, 9, 962. https://doi.org/10.3389/fpsyg.2018.00962 


\section{Table 8.1}

Methodology of Empirical Articles on Positive Psychology (PP) and the Psychology of Religion and Spirituality (PoRS), 2000-2020

\begin{tabular}{|c|c|c|c|c|c|c|c|c|}
\hline \multirow[t]{2}{*}{$\begin{array}{l}\text { Methodological } \\
\text { feature }\end{array}$} & \multicolumn{2}{|c|}{$\begin{array}{l}\text { Total empirical articles } \\
\quad(N=1,664,896)\end{array}$} & \multicolumn{2}{|c|}{$\begin{array}{l}\text { Articles on PP } \\
(N=28,915)\end{array}$} & \multicolumn{2}{|c|}{$\begin{array}{l}\text { Articles on PoRS } \\
\quad(N=10,555)\end{array}$} & \multicolumn{2}{|c|}{$\begin{array}{l}\text { Articles on PP and PoRS } \\
\qquad(N=986)\end{array}$} \\
\hline & $n$ & $\%$ & $n$ & $\%$ & $n$ & $\%$ & $n$ & $\%$ \\
\hline Quantitative study & $1,381,133$ & 83.0 & 24,422 & 84.5 & 7,494 & 71.0 & 826 & 83.8 \\
\hline Qualitative study & 184,222 & 11.1 & 3,747 & 13.0 & 2,963 & 28.1 & 139 & 14.1 \\
\hline Mixed methods study & 43,371 & 2.6 & 1,005 & 3.5 & 513 & 4.9 & 36 & 3.7 \\
\hline Longitudinal design & 141,469 & 8.5 & 3,205 & 11.1 & 711 & 6.7 & 68 & 6.9 \\
\hline Cross-sectional design & $1,523,427$ & 91.5 & 25,710 & 88.9 & 9,844 & 93.3 & 918 & 93.1 \\
\hline Experimental study & 97,599 & 5.9 & 1,182 & 4.1 & 231 & 2.2 & 24 & 2.4 \\
\hline Field study & 18,164 & 1.1 & 263 & 0.9 & 164 & 1.6 & 4 & 0.4 \\
\hline
\end{tabular}

Note. Search results are based on a series of PsycINFO searches conducted on January 4, 2021, using the limiters of "Peer Reviewed" and "2000-2020" for Publication Year, "Empirical Study" for Methodology, and "Peer Reviewed Journal" for Publication Type.

Searches for PP used the search term "DE (Positive Psychology) OR (Virtue) OR (Happiness) OR (Well Being)", and searches for PoRS used the search term "DE (Religion) OR (Spirituality) OR (Faith)". For each methodological feature, the number of studies was determined based on how the PsycINFO database classified the study's methodology; therefore, each indicated percentage should be viewed as an approximation. 


\title{
Chapter 9:
}

Cultural Considerations in Positive Psychology and the Psychology of Religion and Spirituality

\author{
Jacqueline S. Mattis \\ Department of Psychology, Rutgers University-Newark
}

\begin{abstract}
Author Note
Jacqueline S. Mattis iD https://orcid.org/0000-0002-6714-9163
\end{abstract}

Correspondence concerning this article should be addressed to Jacqueline S. Mattis, 360

Martin Luther King, Jr. Drive, Newark, NJ 07103. Email: jacqueline.mattis@,rutgers.edu 


\begin{abstract}
Research in positive psychology and the psychology of religion and spirituality has been largely acultural, acontextual, and apolitical. However, social scientists interested in positive human development- have long recognized that culture plays a central and indelible role in shaping human behavior. In this chapter, I highlight clarifying definitions of culture. I explore the link between culture, religiosity, spirituality, and positive psychological development. I argue that extant approaches to the study of culture, religiosity/spirituality, and positive development are limited in that they do not sufficiently account for structural and sociopolitical contributors to these relationships. I also posit that existing models do not typically account for the power dynamics that shape positive psychological and prosocial outcomes. Drawing on the Socioecological, Transactional model of Religiosity and Spirituality (SET-RS; Urban et al., 2020), I propose a new conceptual frame that addresses these gaps. This new framework, the Integrative model of Culture, Religiosity, and Spirituality in Positive Development (CRSPD), can guide future research on the relations among culture, religiosity, spirituality, and prosocial development.
\end{abstract}

Keywords: culture, religiosity, spirituality, positive psychology, context 


\section{Chapter 9: Cultural Considerations in Positive Psychology and the Psychology of Religion and Spirituality}

On June 17, 2015, Dylann Roof entered Emanuel African Methodist Episcopal (AME) Church in Charleston, South Carolina during a Bible study class. Senior Pastor and State Senator Clementa C. Pinckney and his members welcomed Roof to the church. Soon after the Bible study began, Roof opened fire, injuring three members of the church and killing Rev. Pinckney and eight others. Roof, a White supremacist, targeted the church because its members are Black, and because of the church's history of civil rights activism. Aligning his actions with a contentious national Presidential election that deliberately fomented racial tensions, Roof hoped the murders would incite a race war. In the throes of their own suffering and grief, members of Emanuel AME church were called upon to issue public statements of forgiveness. Many community members rejected calls to forgive Roof, noting that African Americans have too often been asked to forgive people who have harmed them. Forgiveness aside, members of Emanuel AME joined with other African American leaders to initiate a national conversation on faith, honesty, hope, healing, and justice. This virtuous response resulted from at least three factors: (a) peoples' strong connection with their identities as Christians and as members of a cultural institution with deep roots; (b) deeply held cultural norms of centering on God and faith; and (c) a conviction that authentic faith requires radical hope, compassion, and mercy, as well as a genuine commitment to justice.

This chapter grapples with a key question: How might we draw upon these kinds of realworld events to develop ecologically sound models of the relations among culture, religion, and prosocial development? I argue that if psychology is serious about understanding the relations among culture, religion, spirituality, and human goodness, we must develop frameworks that attend to the role of power, structural factors, and sociopolitical realities in prosocial outcomes. I begin by reflecting on the meanings of culture, religiosity, and spirituality, and I conclude by 
offering such a framework. Throughout this chapter I use the term prosocial behavior to refer to voluntary actions intended to benefit others (Pfattheicher et al., 2022).

\section{What is Culture?}

Anthropologist Clifford Geertz (1973) defined culture as a "historically transmitted pattern of meanings embodied in symbols, a system of inherited conceptions expressed in symbolic forms by means of which men communicate, perpetuate, and develop their knowledge about and attitudes toward life" (p. 89). Cultural values, beliefs, practices, products, and identifications share certain features. They are transmitted across generations. They are fairly stable. They are both implicit (nonconscious, nonarticulated) and explicit (conscious, articulated). They are learned, shared, and normalized by those within the cultural system (Kroeber \& Kluckhohn, 1952; Swartz, 2001). The dimensions of culture (beliefs, practices, and products) also have specific functions. They make the world understandable and predictable for individuals and collectives. They guide relations among the members of the group and provide members with the knowledge and resources needed to solve both mundane and extraordinary challenges (Hofstede, 2001; Javidan et al., 2006). They also serve as "the collective programming of the mind that distinguishes the members of one group or category of people from another" (Hofstede, 2001, p. 9). In sum, culture is a multidimensional, transgenerational phenomenon that shapes every aspect of life, including what individuals and groups think, how they behave, what they produce, how they structure their communities and physical environments, who they think they are, how they relate to people inside and outside their group, and how they navigate the vicissitudes of life.

It is worth pausing here to clarify what culture is not. First, culture is neither static nor discrete (García Coll, 2020). Symbols, values, practices, and other dimensions of culture may change as groups interact within and across geographic and virtual spaces (Fischer, 2009; García Coll, 2020). As such, cultural accommodation, cultural hybridity, and acculturation are important 
parts of the conversation, especially in a global context in which migration and encounters through virtual spaces are increasingly common (Fischer, 2009). Culture is also developmental. How people understand and engage particular cultural values, beliefs, or practices changes with age, developmental period, and cohort/generational status. As such, forgiveness may not have the same meaning or manifestations for cohorts of South Africans who lived with the daily brutality of Apartheid that it has for younger cohorts who were born and raised in the post-Apartheid era.

Second, culture is not synonymous with minoritized and marginalized ethnoracial or socioeconomic statuses. Minoritization, marginalization, poverty, and wealth are social positions that derive from enactments of power. In her critique of scholarship on Haiti and Haitian culture, Anthropologist Gina Ulysse (2015) cautioned against uncritically equating culture with these social statuses. Ulysse (2015) asserted that poverty and crisis are so ubiquitous in media representations of Haiti that people have come to equate these aspects of life with Haitian culture. However, these outcomes are largely long-term consequences of colonization. Haitian culture is not the sum of Haitian despair. Haitian culture is evident in the content of the rich body of stories, folk art, music, heroes, relational values, and accomplishments of its citizens (Ulysse, 2015).

The need to distinguish between culture and social position is vital as we consider recent calls to study socioeconomic status (SES) as a form of culture (e.g., see Kraus et al., 2020). The call to equate culture with SES reifies the problematic logic of Oscar Lewis's (1966) work on the so-called "culture of poverty." Equating SES with culture ignores the reality that, at least in the United States, SES correlates strongly with race and ethnicity (Williams, 1996). As such, classed assertions tend to become entangled with ethnoracial assertions. More importantly, when culture is conflated with statuses such as SES, we shift attention away from history and power, and we tend to misattribute to culture outcomes and processes that are more accurately attributed to legacies of structural and or contextual inequity, including structural racism and colonialism 
(Malherbe, 2020). Without attention to these latter forces, researchers risk attributing to culture sets of values, attitudes, and behaviors that may actually reflect intra- or transgenerational responses to chronic disadvantage, inequity, and trauma. In sum, researchers interested in the study of culture must be careful both to define culture and to avoid construct contamination (Hall et al., 2016). We must be sure that we are measuring culture (e.g., historically transmitted values, norms, and practices) rather than marginalization, SES, or similar constructs.

\section{Religion/Spirituality and Culture}

Defining culture is the first step in understanding the relations among culture, religiosity/spirituality, and positive outcomes. However, this work also requires us to define religiosity and spirituality and to clarify how these constructs are linked to culture. Religiosity refers to belief in a higher power (e.g., God), a relationship with that higher power, and engagement in practices (e.g., prayer, religious service attendance) that point to a commitment to one's beliefs (Mattis, 2000; Zinnbauer et al., 1997). Spirituality refers to a quest after the sacred (Pargament, 2007), a belief in the transcendent nature of life, and the translation of those beliefs into a connection with others, including God, humans, spirits, and nature (Mattis, 2000).

Religion and spirituality are cultural systems (Cohen \& Neuberg, 2019; Geertz, 2006). As cultural systems, religious and spiritual systems are replete with historically transmitted products (e.g., stories, songs) and practices (e.g., rituals) that evoke emotions, set expectations, guide behavior, and define people's identities (Geertz, 2006). Importantly, religious and spiritual values,

symbols, and practices achieve power through their connection to a higher authority (e.g., God) and to beliefs about ultimate ends (e.g., salvation, divine punishment).

Here it is worth noting some of the limitations that plague work on the psychology of religion/spirituality. First, much of the psychological research on religion and spirituality tends to be Christocentric (Saroglou et al., 2020). Thus far, psychology has paid relatively little attention to 
the beliefs, symbols, and practices of people from non-Christian traditions, including practitioners of indigenous religions. A second limitation is that, unlike our colleagues in anthropology, psychologists have paid little attention to the reality that, in many cultures, religiosity/spirituality includes belief in range of spiritual beings, including ancestors, saints, and angels, as well as belief in the power of objects such as charms (Pew Research Center, 2010). For many people, these spirits, religious symbols, and sacred objects provide meaningful guidance, protection, healing, hope, wisdom, and courage (Mageo \& Howard, 1996; Pew Research Center, 2010).

Third, psychologists often treat religious/spiritual identities and practices as distinct and bounded. Scholars tend to assume, for example, that people who identify as religious only embrace the beliefs and practices of a single religious/spiritual group. However, this assumption ignores the reality that religious syncretism-i.e., mixing religious beliefs, practices, and symbols from two or more religious systems - is a common feature of religious/spiritual life (Sigalow, 2016). Indeed, roughly $24 \%$ of Americans attend services outside their religion (Pew Research Center, 2009).

Importantly, syncretism has been a common response to oppressive groups' attempted imposition of religion or spirituality. Cultural groups that are subject to coercion have often infused dominant systems of faith with icons, stories, values, and practices that preserved their own indigenous beliefs, values, and identities (Mageo \& Howard, 1996). Given this history of syncretism, researchers interested in the interplay between culture and religion/spirituality must remember that people who share the same religious identification (e.g., Christian) but have different cultural histories may embrace substantively different beliefs, symbols, and practices.

Finally, psychologists interested in religion/spirituality often have not accounted for the fact that, in response to the challenges they face, different cultural groups have reimagined the meanings of religious texts and developed unique modes of religious expression (e.g., religious music, patterns of worship, and modes of prayer). For example, in response to slavery, 
colonization, and persistent racism, African-American, Latinx, and Latin-American Christians have developed liberationist readings of the Bible. Liberation theologies emphasize themes of love, hope, resistance, and justice, and they imagine God and Christ as committed to dismantling injustice in the world (Barreto \& Sirvent, 2019; Cone, 2010; Lincoln \& Mamiya, 1990). These readings of the Bible have allowed marginalized Black, Latinx, and Latin-American communities to expose the contradictions and evil that are endemic in using religion to justify the subjugation of others. Within the U.S., Black liberationists have animated the outreach and activism at the heart of America's Civil Rights and justice movements (Cone, 2010; Lincoln \& Mamiya, 1990). Importantly, cultural differences in peoples' readings of religious texts have implications both for the ways that religious/spiritual institutions function and for peoples' expectations of religious institutions. Indeed, African Americans are more likely than Americans from other ethnoracial groups to report their religious leaders preach about issues of equity and social justice. African Americans are also more likely to report that religious institutions have a moral imperative to address social justice issues directly (Pew Research Center, 2021a). Cultural and ethnoracial differences in institutional functioning and in peoples' expectations of religious institutions may be amplified by the fact that religious institutions often are highly segregated, at least in the U.S. The overwhelming majority (80\%) of Americans attend religious services in racially segregated settings (Dougherty et al., 2020). This level of segregation supports the reinforcement of religious/spiritual beliefs, values, and practices over time.

\section{Values, Culture, Religiosity, and Positive Behavior}

Cultural psychologists have been particularly focused on identifying core values that either are universal or help differentiate cultural groups. They then seek to understand how those values align with peoples' identities, roles, choices, and actions (Betancourt \& López, 1993; Pepper et al., 2010; Rokeach, 1969; Schwartz, 2010). Although the work on culture and values is 
large and robust, little of that work has centered on religiosity/spirituality. Recently, researchers interested in positive psychology have begun to explore the links among culture, religion, and values (Schimmel, 2000). Dahlsgaard et al. (2005), for example, mined the written texts of some of the world's major religions to identify core values, virtues, and character strengths in these religious systems. They suggested that six character strengths—courage, justice, humanity, temperance, wisdom, and transcendence - are commonly represented in these major religions, albeit to different degrees. Khodayarifard et al. (2016) similarly analyzed Islamic texts and identified an Islamic bent toward optimism, gratitude, and a view of God as a benevolent force. Other work has examined ethnoracial differences in values and sought to determine whether these differences are accounted for by group differences in religiosity. Taking this approach, Krause and Hayward (2015) found that, relative to their White counterparts, African Americans feel more compassion for strangers, and they provide more emotional support and more instrumental help to strangers. These differences in prosocial orientation appeared to be explained in part by African Americans' greater religious commitment, gratitude to God, and humility (Krause \& Hayward, 2015). This emerging line of work supports the contention that the study of values provides important insights on culture and behavior (see Park \& Van Tongeren, Chapter 6, this volume).

Although values are important, cultural theorists remind us that values are only one dimension of culture (Swartz, 2001) and provide us with only a slice of understanding culture (Inglehart \& Baker, 2000). Moreover, Javidan et al. (2006) have cautioned that values-centered studies of culture are guided by two common but empirically untested assumptions: (a) the ecological values assumption and (b) the onion assumption. The ecological values assumption highlights researchers' tendency to believe values are especially important aspects of culture and studying the values of individuals is the most meaningful and robust way to understand culture. The onion assumption calls attention to the tendency to believe that what individuals, 
organizations, and nations say they value is a good predictor of how they actually behave. Importantly, findings on the link between values and behaviors are mixed. For example, in multinational studies, values and behavior often are either unrelated or related in unexpected ways (Hofstede et al., 1990; Javidan et al., 2006). These mixed findings warrant scholarly attention.

For a host of reasons, studies that use values to predict prosocial behavior may fail to detect relations or may yield unexpected findings. It might be difficult to discern which specific cultural values (or combination of values) are most relevant for predicting particular outcomes. The values and behaviors under investigation also may not be measured accurately. This issue of measurement is especially relevant in cross-cultural research, because cultural values (such as happiness) do not always carry the same meaning across cultural contexts (e.g., see Joshanloo, 2014). Values-based models may also fail because the roster of values to which researchers attend is usually limited by the interests and perspectives of White, Western scholars (Taras et al., 2009). Values that exist in one cultural context may have no analogue in another, yet may be essential for explaining prosocial behavior. For example, the South African concept of ubuntu ("being self through others") has no clear parallel in American culture, but it explains generosity and other prosocial outcomes among South Africans (Mugumbate \& Nyanguru, 2013). Excluding culturally important values from studies of behavior will naturally lead to poorer scientific outcomes.

Evidence suggests another important reason why values-based models of behavior may ultimately fall short is because values alone may be insufficient to explain behavior fully. Cultural values are often strongly related to macrolevel variables. For example, individualism and collectivism tend to correlate highly with macrolevel structural variables, such as level of industrialization, gross national product, birth rate, and rates of literacy (van de Vijver \& Leung, 2000). These correlational patterns suggest models that seek to link culture and behavior may be incomplete if they fail to include structural and sociopolitical factors. An emerging body of cross- 
cultural research highlights the links among structural/sociopolitical conditions, religiosity/spirituality, and prosocial outcomes. For instance, using longitudinal data from the European Values Study, Storm (2016) found that people who live in countries that have a history of being governed fairly and effectively are more likely to behave prosocially (e.g., they are more likely to be honest), because they anticipate they can rely on the government to catch and punish violators. In these national contexts, religiosity tends to be less strongly correlated with prosocial behavior. However, in European countries where people believe that structures of control (such as the government) are weak, illegitimate, or nonexistent, religious communities and God become central sources of moral authority (Storm, 2016). In these national settings, religiosity tends to be more strongly associated with prosocial behavior. Further, there is evidence the cultural orientations that inform people's experience of structural conditions and their experience of religion may help to explain why people behave prosocially. For example, altruism and honesty are highest in European nations where people have a cultural orientation toward showing respect for authority (including governmental and divine authority) and a strong cultural awareness of being surveilled by figures of authority, including God and the government (Doebler, 2015; Galen, 2012; Shariff \& Norenzayan, 2007; Storm, 2016). Taken together, these findings highlight the need for conceptual models of prosocial development that account for structural, sociopolitical, and religious factors, when it comes to explaining positive outcomes.

\section{Culture, Religiosity/Spirituality, Power, and Goodness}

On one level, efforts to understand the interplay among culture, religiosity/spirituality, and structure mean paying attention to "1) religions as cultures in and of themselves, 2) religions as subcultures of national cultures, 3) national cultures as subcultures of transnational religious

regions of the world, 4) religions and national cultures as conflicting with each other, and 5) new 
blends of religious and national culture resulting from globalization" (Cohen \& Neuberg, 2019, p. 857). On another level, efforts to understand these relations entail attending to history and power.

Centering history, power, politics, and justice in conversations about culture, religiosity, spirituality, and human goodness should not feel alien. Indeed, religious and spiritual texts (such as the Bible) are replete with stories of the tenuous relationships among ruling classes, powerful leaders, and vulnerable populations. Moreover, history demonstrates that across time, religious individuals and institutions have often weaponized faith in the service of maintaining individual and in-group privilege and power. As such, religion and religious extremism have often been at the root of war, colonization, national invasions, forcible conversions, enslavement, genocide, oppression, and acts of inhumanity (Iannaccone \& Berman, 2006). These aspects of religious life are evident in contemporary life. In fact, a 2021 study found that 43 countries reported high levels of social hostilities (e.g., terrorist activity, kidnappings, murders, and assaults) related to religion (Pew Research Center, 2021b). Religious institutions and religious communities also have been targets of discrimination. Indeed, 180 countries reported one or more instances of government harassment of religious groups. For example, governments have prohibited the display of religious symbols, mandated or prohibited head coverings, detained people for engaging in worship, and used technology to surveil members of particular religious groups (Pew Research Center, 2021b). Importantly, however, the same religious/spiritual beliefs that have been used by some individuals and institutions to justify domination and harm have been used by others to justify extraordinary acts of compassion, generosity, forgiveness, and justice-oriented activism.

The benefit of accounting for culture, religiosity/spirituality, and power in studies of prosocial development is evident in research on prosocial development among African American/Black adults in the U.S. For African-American/Black communities, organizational religious involvement is a particularly robust predictor of a host of prosocial behaviors, including 
altruism, volunteerism, and community and civic engagement (Grayman-Simpson, \& Mattis, 2013; Gutierrez \& Mattis, 2014; Lincoln \& Mamiya, 1990). The prosocial leanings of Black youth and adults is credited to a strong cultural orientation toward communalism within AfricanAmerican/Black communities, as well as to Black cultural theologies that emphasize love, hope, justice, and liberation (Cone, 2010; Gilkes, 2001). These cultural orientations have been especially important for African Americans because racism created harmful structural conditions that blocked people from opportunities to access key resources. African-American religious institutions have historically filled gaps in care by providing educational, financial, and other tangible supports to members of the African-American community (Lincoln \& Mamiya, 1990). Although religiosity/spirituality is a robust predictor of prosocial behavior among African Americans, the relations between culture, religiosity and prosocial behavior is complicated by structural factors. In settings marked by inequality and unjust use of coercive power (e.g., threats of arrest), prosocial behaviors are common, but these behaviors are not always related to religiosity/spirituality in simple or direct ways. For example, Mattis et al. (2008) found that in overpoliced neighborhoods, helping others sometimes invited suspicion or placed helpers at risk for being harassed or otherwise punished by structures of power, including the police. Research on forgiveness reveals similar complexities in the relations among culture, religiosity/spirituality, structural factors, and prosocial outcomes. For instance, Gallagher (2002) found that even when culturally and religiously grounded vehicles for interpersonal and intergroup forgiveness (e.g., the Truth and Reconciliation Commission in South Africa) were available, many individuals who were survivors of structural violence found it impossible to forgive (Gallagher, 2002). The brutal history of racism in South Africa — and devastating impact of this system within and across generations of people - made it difficult for many people to imagine forgiving without any conversation about punishment, reparations, and the ceding of power. Taken together, these findings highlight the need for 
models that center structural/sociopolitical conditions, power, and history in dialogues about relations among culture, religiosity/spirituality, and prosocial outcomes. Next, I propose such a theory—-the Culture, Religiosity/Spirituality and Positive Development (CRSPD) model.

\section{A New Model of Culture, Religiosity/Spirituality, and Prosocial Development}

The proposed framework offered here builds on Mattis et al.'s (2019) Socioecological, Transactional framework for explaining the study of Religiosity and Spirituality and prosocial development in urban settings (SET-RS Urban). The SET-RS Urban is a place-based model of prosocial outcomes that begins with an appreciation that religiosity/spirituality (e.g., religious institutions, religious/spiritual ideologies, and religious/spiritual family and friends) help shape prosocial outcomes. However, the SET-RS model posits the link between religiosity/spirituality and positive outcomes is complicated by macro-, meso- and micro-level factors. Specifically, sociopolitical conditions that are characteristic of urban areas (e.g., pervasive inequality and segregation) promote structural arrangements (e.g., policies) that create risk, vulnerability, stress, and resources for urban communities and residents. The risks, stresses, and resources that people experience depend in part on their identities (e.g., race) and social positions (e.g., SES, caste).

Prosocial outcomes depend in part on how individuals, families, and communities are impacted by—and make meaning of—structural risks and stressors, as well as by people's perceptions of contextual and situational cues. Prosocial outcomes also depend on individuallevel (e.g., empathic personality orientation), family-level (e.g., family models of compassion), and community- or organizational-level factors (e.g., involvement in volunteerism). Finally, the likelihood that individuals, organizations, and social groups will express certain prosocial behaviors may depend on whether they are acknowledged, punished, or rewarded for their prosocial behaviors when they engage in such behaviors. For example, several factors might motivate an undocumented immigrant to advocate publicly for the rights of vulnerable groups, 
such as that immigrant's faith, empathic personality orientation, and cultural and family orientations toward collectivism. However, when and how the immigrant does advocacy work may be informed by fears about arrest and the consequences of such an outcome for their family.

The Culture, Religiosity/Spirituality, and Positive Development (CRSPD) model advances the SET-RS Urban model by highlighting the role of culture in prosocial development. CRSPD (Figure 9.1) retains the SET-RS model's focus on sociopolitical and structural conditions, risks, stresses, assets, and opportunities, as well as individual, community, institutional, and contextual contributions to prosocial development. Yet CRSPD is not limited to urban settings. Furthermore, CRSPD introduces five features of culture into these inter-relationships: time, dimension of culture, modes of cultural expression, level of ecology, and sites of cultural expression.

Time. In its attention to time, CRSPD highlights the importance of change. The model elevates the reality that culture's influence on behavior is historical and current, and culture's processes of influence are developmental. This approach draws attention to factors such as personal changes in belief and national and generational shifts toward secularization or religious conservatism. Time is represented as a factor that operates across all aspects of the model.

Dimensions. The model requires us to remain mindful of the various dimensions of culture that may be examined in any study of development. CRSPD theory draws our attention to the fact we have the flexibility to study numerous manifestations of culture, including values, symbols (e.g., images), texts, gestures, practices, and rituals.

Modes. The CRSPD framework encourages researchers to ask how (i.e., in what format) culture is expressed. Culture can be expressed in a variety of ways, including visually, orally, emotionally, cognitively, behaviorally, and relationally. 
Levels. CRSPD theory requires that we attend to the reality that culture operates at multiple levels (i.e., on multiple planes of the ecology), including the national, community, institutional, relational, and individual levels.

Sites. Finally, CRSPD theory takes a more granular approach to the question of where culture is expressed. Within any level of ecology, culture is expressed at different sites. For example, at the individual level, religious and prosocial sensibilities might be expressed on the body through tattoos. Similarly, gurdwaras may express religious and prosocial values in the built environment, by posting messages such as "All Are Welcome Here" on their public billboards.

By calling attention to these five features of culture, CRSPD theory allows researchers and clinicians to stay true to current conceptualizations of culture as a historically transmitted, multidimensional, intra- and transgenerational phenomenon that is expressed in our values, practices, and products. Furthermore, by deliberately focusing our attention on dimensions, sites, modes, and levels of expression in each area (e.g., religiosity/spirituality, community, individual), the model intends to encourage researchers to see aspects of culture and cultural functioning (e.g., the manifestations of religiosity and prosociality in music) that might otherwise remain invisible.

\section{Conclusion and Conceptual and Methodological Considerations}

The definitions and conceptualizations of culture, religion, and spirituality that dominate psychology, and by extension this work, are not universal. They reflect the thinking of Western (and generally White) scholars from a particular subset of industrialized societies (Dueck et al., 2017). These definitions prevail because of the cultural politics of knowledge production and dissemination. Indeed, publication in social science texts requires that scholars (including scholars of color, indigenous, and non-Western scholars) cite Western (and generally White) scholars. Westerners and White scholars are, however, under no similar obligation to cite scholars of color, indigenous scholars, or non-Western scholars. Disrupting these hegemonic practices will be 
critical to building a truly inclusive approach to the study of culture, religiosity/spirituality, and prosocial development. Scholars in positive psychology and the psychology of religion and spirituality must be reflective about the ways in which disciplinary practices, assumptions, measures, and methods reify cultural dynamics that exclude, erase, and silence others.

As we move toward a more culturally inclusive approach to prosocial development, we must ensure that studies offer clear definitions of culture. We must identify the specific aspects of culture that contribute to prosocial outcomes (Diemer \& Gore, 2009; Taras et al., 2009). Efforts to identify and measure the aspects of culture that are at play should, of course, be primarily emically driven. That is, members of the cultural groups that are the focus of inquiry must centrally inform our thinking about the issues being studied. Finally, the conceptual frameworks that guide our work must be ecologically valid. In that regard, our frameworks must account for the ways that religiosity/spirituality and prosociality are informed by structural/sociopolitical factors, power, and more proximal factors (including personality and family and peer religious socialization).

The CRSPD framework is one important step towards accounting for these factors. Importantly, CRSPD lends itself to the use of various research designs, methods, and analytic strategies. For instance, the model lends itself to the use of longitudinal and cross-sectional designs, multilevel modeling strategies, and analyses of change. CRSPD also lends itself to the use of qualitative methods and analyses, including the use of interviews and archival analysis of cultural artifacts (e.g., music, clothing, social media postings). It is worth noting that studies of material culture have advantages in that, unlike self-report approaches, they do not presume that people are conscious of the cultural values and beliefs by which they operate (Taras et al., 2009).

CRSPD theory also has clinical implications. It of course is consistent with meta-analytic evidence that clinical interventions are more effective when they are adapted to the client's culture and when psychotherapists demonstrate cultural competence (Soto et al., 2018). In 
addition, it recognizes that interventions certainly need to be clear on the religious and prosocial values, norms, and behaviors they intend to support and promote. However, CRSPD theory highlights the need to clarify the values, norms, and behaviors that already exist across various levels of people's social ecologies. Interventions also must attend to the multiple sites in which people express and enact prosocial values. Importantly, CRSPD theory draws attention to the fact that because interventions exist in a sociopolitical context, the efficacy of interventions may depend on understanding historical and structural factors that support or work against desired outcomes of those interventions. For example, the model highlights the reality that individuals and groups who are the focus of interventions may need (or demand) acknowledgement of and accountability for harms done to them by others, in order for them to engage in and benefit from interventions. At best, interventions that ignore sociopolitical context may ring hollow and be of limited value; at worst, they may further harm, oppress, and marginalize the very people we are seeking to support.

If scholars and clinicians in positive psychology and the psychology of religion and spirituality are truly committed to culturally grounded models of prosocial outcomes, it is imperative that we be critically reflective about the ways we do our work. It is also important that we expand our methodological and analytic toolkits so that we can more fully and thoughtfully study culture and work with people in culturally responsive ways. Our work will be richer if we collaborate with scholars and practitioners whose perspectives and skills can deepen our work. 


\section{References}

Barreto, R., \& Sirvent, R. (2019). Decolonial Christianities: Latinx and Latin American perspectives. Palgrave MacMillan.

Betancourt H., \& López S. R. (1993). The study of culture, ethnicity, and race in American psychology. American Psychologist, 48, 629-637. https://doi.org/10.1037/0003$\underline{066 X .48 .6 .629}$

Cohen, A. B., \& Neuberg, S. (2020). Religious cultures and religious conflict. In D. Cohen \& S. Kitayama (Eds.), Handbook of cultural psychology (2 ${ }^{\text {nd }}$ ed., pp. 857-875). Guilford Press.

Cone. J. (2010). A Black theology of liberation. Orbis Books.

Dahlsgaard, K., Peterson, C., \& Seligman, M. E. P. (2005). Shared virtue: The convergence of valued human strengths across culture and history. Review of General Psychology, 9, 203213. https://doi.org/10.1037/1089-2680.9.3.203

Diemer, M., \& Gore, P. (2009). Culture and assessment: Nomothetic and idiographic considerations. The Career Development Quarterly, 57, 342-347. https://doi.org/10.1002/j.2161-0045.2009.tb00120.x

Doebler, S. (2015). Love thy neighbor? Relationships between religion and racial intolerance in Europe. Politics and Religion, 8, 745-771. https://doi.org/10.1017/S1755048315000607

Dougherty, K. D., Chaves, M., \& Emerson, M. O. (2020). Racial diversity in U.S. congregations, 1998-2019. Journal for the Scientific Study of Religion, 59, 651-662. https://doi.org/10.1111/jssr.12681

Dueck, A., Ansloos, J., Johnson, A., \& Fort, C. (2017). Western cultural psychology of religion: Alternatives to ideology. Pastoral Psychology, 66, 397-425. https://doi.org/10.1007/s11089$\underline{016-0731-3}$ 
Fischer, R. (2009). Where is culture in cross-cultural research? An outline of a multilevel research process for measuring culture as a shared meaning system. International Journal of CrossCultural Management: CCM, 9, 25-49. https://doi.org/10.1177/1470595808101154

Galen, L. W. (2012). Does religious belief promote prosociality? A critical examination. Psychological Bulletin, 138(5), 876-906. https://doi.org/10.1037/a0028251

Gallagher, S. (2002). "I want to say: / Forgive me": South African discourse and forgiveness. PMLA, 117(2), 303-306. http://www.jstor.org/stable/823277

García Coll, C. (2020). Globalization, culture, and development: Are we ready for a paradigm shift? Human Development, 64, 245-249. https://doi.org/10.1159/000512903

Geertz, C. (1973). The interpretation of cultures. Basic Books.

Geertz, C. (2006). Religion as a cultural system. In M. Banton (Ed.), Anthropological approaches to the study of religion (pp. 1-46). Routledge.

Gilkes, C. T. (2001). If it wasn't for the women: Black women's experience and womanist culture in church and community. Orbis Books

Grayman-Simpson, N., \& Mattis, J.S. (2013). "If it wasn't for the church....” Organizational religiosity and informal community helping among African American adults. Journal of African American Studies, 17, 243-252. https://doi.org/10.1007/s12111-012-9213-6

Gutierrez, I. A., \& Mattis, J. S. (2014). Factors predicting volunteer engagement among urbanresiding African American women. Journal of Black Studies, 45, 599-619. https://doi.org/10.1177/0021934714543189

Hall G. C. N., Yip T., \& Zárate, M. A. (2016). On becoming multicultural in a monocultural research world: A conceptual approach to studying ethnocultural diversity. American Psychologist, 71(1), 40-51. https://doi.org/10.1037/a0039734

Hofstede, G. (2001). Culture's consequences ( $2^{\text {nd }}$ ed.). Sage. 
Hofstede, G., Neuijen, B., Ohayv, D. D., \& Sanders, G. (1990). Measuring organizational cultures: A qualitative and quantitative study across twenty cases. Administrative Science Quarterly, 35, 286-316.

Iannaccone, L. R., \& Berman, E. (2006). Religious extremism: The good, the bad, and the deadly. Public Choice, 128(1/2), 109-129. https://doi.org/10.1007/s11127-006-9047-7

Inglehart, R., \& Baker, W. E. (2000). Modernization, cultural change, and the persistence of traditional values. American Sociological Review, 65, 19-51.

Javidan, M., House, R., Dorfman, P., Hanges, P. J., \& de Luque, M. S. (2006). Conceptualizing and measuring cultures and their consequences: A comparative review of GLOBE's and Hofstede's approaches. Journal of International Business Studies, 37, 897-914.

https://doi.org/10.1057/palgrave.jibs.8400234

Joshanloo, M. (2014). Eastern conceptions of happiness: Fundamental differences with Western views. Journal of Happiness Studies, 15, 475-493.

Khodayarifard, M., Ghobari-Bonab, B., Akbari-Zardkhaneh, S., Zamanpour, E., Zandi, S., \& Derakhshan, M. (2016). Positive psychology from Islamic perspective. International Journal of Behavioural Science, 2, 1-7.

Kraus, M. W., Callaghan, B., \& Ondish, P. (2020). Social class as culture. In D. Cohen \& S. Kitayama (Eds.), Handbook of cultural psychology ( $2^{\text {nd }}$ ed., pp. 721-747). Guilford Press.

Krause, N., \& Hayward, R. D. (2015). Race, religion, and virtues. The International Journal for the Psychology of Religion, 25, 152-169. https://doi.org/10.1080/10508619.2014.916591

Kroeber, A. L., \& Kluckhohn, C. (1952). Culture: A critical review of concepts and definitions. Papers. Peabody Museum of Archaeology \& Ethnology, Harvard University, 47(1), viii, 223.

Lewis, O. (1966). The culture of poverty. Scientific American, 215(4), 19-25. 
Lincoln, C. E., \& Mamiya, L. H. (1990). The Black church in the African American experience. Duke University Press.

Mageo, J., \& Howard, A. (1996). Spirits in culture, history, and mind. Routledge.

Malherbe, N. (2020). Articulating liberation psychologies of culture. Journal of Theoretical and Philosophical Psychology, 40(4), 203-218. https://doi.org/10.1037/teo0000126

Mattis, J. (2000). African American women's definitions of spirituality and religiosity. Journal of Black Psychology, 26, 101-122. https://doi.org/10.1177/0095798400026001006

Mattis, J., Grayman, N., Cowie, S., Winston, C., Watson, C., \& Jackson, D. (2008). Intersectional identities and the politics of altruistic care in a low-income, urban community. Sex Roles, 59, 418-428. https://doi.org/10.1007/s11199-008-9426-2

Mattis, J., Palmer, G., \& Hope, M. (2019). Where our bright star is cast: Religiosity, spirituality, and positive Black development in urban landscapes. Religions, 10, Article 654. https://doi.org/10.3390/rel10120654

Mugumbate, J., \& Nyanguru, A. (2013). Exploring African philosophy: The value of ubuntu in Social Work. African Journal of Social Work, 3, 82-100.

Pargament, K. I. (2007). Spiritually integrated psychotherapy. Guilford Press.

Pepper, M., Jackson, T., \& Uzzell, D. (2010). A study of multidimensional religion constructs and values in the United Kingdom. Journal for the Scientific Study of Religion, 49, 127-146.

Pew Research Center (2010). Tolerance and tension: Islam and Christianity in Subsaharan Africa. https://www.pewforum.org/2010/04/15/executive-summary-islam-and-christianity-in-sub$\underline{\text { saharan-africa/ }}$

Pew Research Center (2021a). Faith among Black Americans. https://www.pewforum.org/2021/02/16/faith-among-black-americans/ 
Pew Research Center (2021b). Globally, social hostilities related to religion decline in 2019, while government restrictions remain at highest levels. https://www.pewforum.org/2021/09/30/globally-social-hostilities-related-to-religiondecline-in-2019-while-government-restrictions-remain-at-highest-levels/

Pew Research Centers Religion Public Life Project RSS (2009). Many Americans mix multiple faiths. https://www.pewforum.org/2009/12/09/many-americans-mix-multiple-faiths/

Pfattheicher, S., Asbjørn Nielsen, Y., \& Thielmann, I. (2022). Prosocial behavior and altruism: A review of concepts and definitions. Current Opinion in Psychology, 44, 124-129 https://doi.org/10.1016/j.copsyc.2021.08.021

Rokeach, M. (1969). Part I. Value systems in religion. Review of Religious Research, 11, 3-23.

Saroglou, V., Clobert, M., Cohen, A., Johnson, K., Ladd, K., et al. (2020). Believing, bonding, behaving, and belonging: The cognitive, emotional, moral, and social dimensions of religiousness across cultures. Journal of Cross-Cultural Psychology, 51, 551-575. https://doi.org/10.1177/0022022120946488

Schimmel. S. (2000). Vices, virtues, and sources of human strength in historical perspective. Journal of Social and Clinical Psychology, 19, 137-150.

Schwartz, S. H. (2010). Basic values: How they motivate and inhibit prosocial behavior. In P. Mikulincer \& P. Shaver (Eds.), Prosocial motives, emotions, and behavior: The better angels of our nature (pp. 221-241). American Psychological Association.

Shariff, A. F., \& Norenzayan, A. (2007). God is watching you: Priming God concepts increases prosocial behavior in an anonymous economic game. Psychological Science, 18(9), 803809. https://doi.org/10.1111/j.1467-9280.2007.01983.x

Sigalow, E. (2016). Towards a sociological framework of religious syncretism in the United States. Journal of the American Academy of Religion, 84, 1029-1055. 
Soto, A., Smith, T. B., Griner, D., Rodríguez, M. D., \& Bernal, G. (2018). Cultural adaptations and therapist multicultural competence: Two meta-analytic reviews. Journal of Clinical Psychology, 74, 1907-1923. https://doi.org/10.1002/jclp.22679

Storm, I. (2016). Morality in context: A multilevel analysis of the relationship between religion and values in Europe. Politics and Religion, 9, 111-138.

https://doi.org/10.1017/S1755048315000899

Swartz, M. J. (2001). On the substance and uses of culture's elements. Cross-Cultural Research, 35, 179-200. https://doi.org/10.1177/106939710103500205

Taras, V., Rowney, J., \& Steel, P. (2009). Half a century of measuring culture: Review of approaches, challenges, and limitations based on the analysis of 121 instruments for quantifying culture. Journal of International Management, 15, 357-373. https://doi.org/10.1016/j.intman.2008.08.005

Ulysse, G. A. (2015). Why Haiti needs new narratives: A post-quake chronicle. Wesleyan University Press.

van de Vijver, F. J. R., \& Leung, K. (2000). Methodological Issues in Psychological Research on Culture. Journal of Cross-Cultural Psychology, 31(1), 33-51. https://doi.org/10.1177/0022022100031001004

Williams, D. R. (1996). Race/ethnicity and socioeconomic status: Measurement and methodological issues. International Journal of Health Services, 26, 483-505. https://doi.org/10.2190/U9QT-7B7Y-HQ15-JT14

Zinnbauer, B. J., Pargament, K. I., Cole, B., Rye, M. S., Butter, E. S., Belavich, T. G., Hipp, K. M., Scott, A. B., \& Kadar, J. L. (1997). Religion and spirituality: Unfuzzying the fuzzy. Journal for the Scientific Study of Religion, 36, 549-564. https://doi.org/10.2307/1387689 


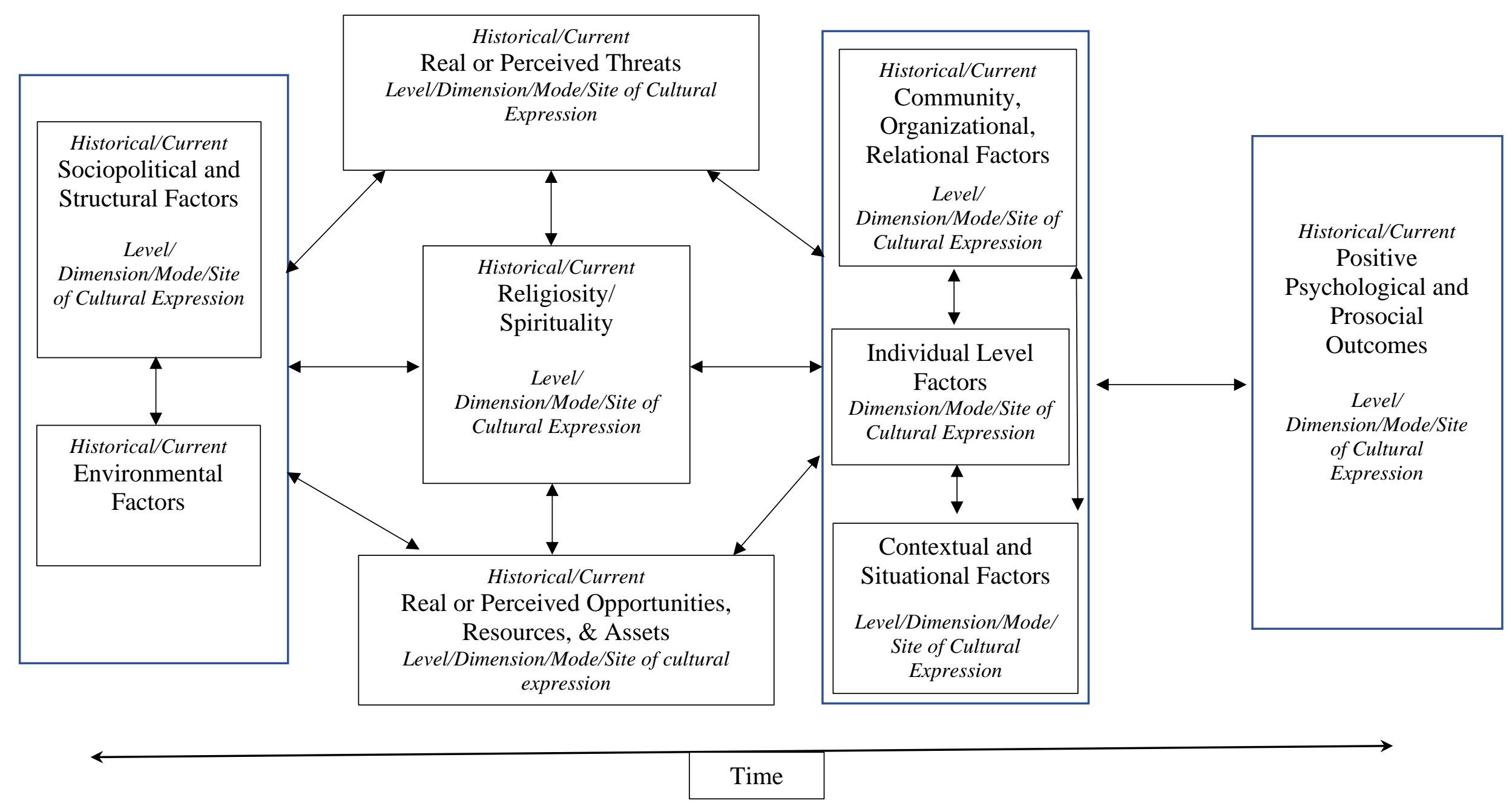

Figure 9.1. Integrative model of the role of Culture, Religiosity, Spirituality in Positive Development (CRSPD). 


\section{Chapter 10:}

\section{Positive Psychology and Christianity}

Adam S. Hodge ${ }^{1}$, Joshua N. Hook ${ }^{1}$, Jichan J. Kim² ${ }^{2}$ David K. Mosher ${ }^{3}$, Aaron T. McLaughlin ${ }^{4}$, Don E. Davis ${ }^{4}$, and Daryl R. Van Tongeren ${ }^{5}$

${ }^{1}$ Department of Psychology, University of North Texas

${ }^{2}$ Department of Psychology, Liberty University

${ }^{3}$ Department of Psychology, Abilene Christian University

${ }^{4}$ Department of Counseling and Psychological Services, Georgia State University

${ }^{5}$ Department of Psychology, Hope College

Correspondence concerning this chapter should be addressed to Adam S. Hodge. Email: adamhodge@my.unt.edu 


\begin{abstract}
Both theoretical reflection and empirical research have purported a mutual exchange of benefits for the collaboration between positive psychology (PP) and Christianity. However, the varied belief systems and worldviews held among adherents of distinct Christian traditions may interfere with practical collaborative efforts between PP and Christianity. In this chapter, we explore important considerations for effective collaboration between PP and Christianity. Namely, we argue that Christianity can provide a framework for what individuals should value and what virtues a person should pursue, whereas PP can provide the tools and methodology for how to pursue the goals that are identified by Christianity. We first review the geographic distribution and demographic composition of Christians around the world, emphasizing the value of context when working with Christian populations. Second, we highlight fundamental beliefs and values that may influence how Christians interpret and utilize empirical findings from the field of PP. Third, we discuss how Christian beliefs, traditions, and doctrines influence our understandings of well-being, health, and virtue development. Throughout the chapter, we discuss strategies and implications for positive collaboration between the fields of PP and Christianity.
\end{abstract}

Keywords: Christianity, positive psychology, religion, spirituality, virtue 


\section{Positive Psychology and Christianity}

The relationship between psychology and Christianity has often been fraught with tension, antagonism, and mutual suspicion (Hodge, Hook, Davis, \& McMinn, 2020), but that does not have to be the case. Indeed, Martin Luther King, Jr. (1963/2010) famously asserted:

Science investigates; religion interprets... The two are not rivals. They are complementary. Science keeps religion from sinking into the valley of crippling irrationalism and paralyzing obscurantism. Religion prevents science from falling into the marsh of obsolete materialism and moral nihilism. (p. 4)

As this quote illustrates, people have specific beliefs and worldviews about how psychology and Christianity may, or may not, work together. Yet there is substantial overlap in the goals and values of positive psychology (PP) and the goals and values of Christianity, leading to a rise in scholarly attention on their potential intersections. Two notable examples of this trend include a recent special issue of the Journal of Positive Psychology entitled "Christian Positive Psychology" (Johnson, 2017) and a recent book The Science of Virtue: Why Positive Psychology Matters to the Church (McMinn, 2017). The current chapter explores these intersections as well.

Given the prevalence of Christianity globally and the current trends in the geographic distribution of Christianity, we hope to contribute to the growing literature that critiques the foundational assumption that PP is value-neutral (Prinzing, 2021) and that advances the argument that religious/spiritual (R/S) thought can provide a foundation, or worldview, from which to base future empirical study in PP (Hill \& Hall, 2018). (See Table 10.1 for a summary of reasons why PP and Christianity could and should work together.) To accomplish this goal, we first (a) provide a brief overview of the geographic distribution and demographic composition of Christians around the world and (b) highlight how a changing religious landscape will likely 
affect collaborative efforts between PP and Christianity over time. We then review fundamental beliefs and values among Christians that may influence how Christians utilize empirical findings from the field of PP. We conclude the chapter by discussing how Christian beliefs, traditions, and doctrines may influence one's understanding of well-being, health, and virtue.

\section{Geographic Distribution and Demographic Composition of Christians around the World}

The global landscape of Christianity is rapidly changing. As of 2010, there were an estimated 2.18 billion people globally who identified as Christian (approximately one-third of the population at the time), making it the largest world religion, compared to Islam ( $23 \%$ of the world's population), Hinduism (15\%), Buddhism (7\%), Judaism (0.2\%), folk religions (6\%), and other religions (1\%). Recent projections suggest the world's Christian population will continue to increase, albeit modestly, through 2060, with much of the growth occurring in sub-Saharan Africa (Pew Research Center, 2017).

Regarding current trends of the geographic distribution of Christians, recent projections estimate a shift in balance will take place within the next 30 to 40 years. Christians in the SubSaharan Africa regions will likely increase and bypass the proportion of Christians in Western society. This shift in balance is largely due to both an aging Christian population and a growing rate of individuals deidentifying from religion in the West (Pew Research Center, 2017).

Because of the changing geographic landscape of Christianity, it is important to consider how these shifts are likely to affect collaborative efforts between PP and Christianity. Religion, culture, and society are highly intertwined, resulting in differences in how religion is practiced and understood in different geographic locations (Cohen et al., 2016). Not only can religion influence society (e.g., Christianity's influence on U.S. culture; Cohen \& Hill, 2007), but society can also influence religious practice (e.g., the influence of U.S. culture on American Christianity; 
Cohen et al., 2005). Additionally, research has suggested that conceptualizations of happiness vary across time and culture (Oishi et al., 2013), so it is quite possible that religious beliefs, values, and traditions contribute to this evolving view of what constitutes a "good life." The interconnections between Christian worldviews and localized cultures may set the stage for researchers to consider how different PP constructs may be perceived by different groups. Thus, it is important to keep in mind how geographical, cultural, and national contexts may influence religious practices and specific beliefs, all of which can impact the dynamics between PP and Christianity, as these trends will likely continue to shift over time.

\section{Organizational/Denominational Structure, Beliefs, and Religious Practices}

It is important to emphasize that Christianity is not monolithic. Therefore, it is difficult to describe how Christians (as a whole) might interact with PP. A common strategy used to distinguish groups of Christians is to look at denominational differences based on organizational structures, beliefs, and practices. In 2010, over half of the 2.18 billion Christians around the world belonged to the Roman Catholic church, 36.5\% to Protestant churches, $11.9 \%$ to Orthodox churches, and $1.3 \%$ to denominations that did not fit within these categories (e.g., Mormon, Jehovah's Witness; Pew Research Center, 2011). Currently, there are over 300 Christian denominations in the United States alone, and this number is constantly changing (Rhodes, 2015). Despite the nearly impossible task of concisely distinguishing Christian denominations from one another, we highlight important theological differences (both between and within Christian denominations and leaders) that might influence how Christians approach PP. 


\section{Denominational and Individual Differences}

A recent project surveyed Christian ministry leaders to explore factors that may influence Christian leaders' views on integrating psychological research and practice into Christian church ministry. First, Hodge, Hook, Davis, and McMinn (2020) explored broad denominational differences by collapsing all denominations into three groups: (a) Roman Catholic, (b) Mainline Protestant (i.e., denominations that are associated with the National Council of Churches and have historic roots and influence in American society; Hadaway \& Marler, 2006), and (c) Evangelical Protestant (i.e., an umbrella group of Protestant churches that affirm the doctrine of regeneration, emphasize evangelism, and affirm the authority and historicity of the Bible;

FitzGerald, 2017). Hodge et al. (2020) found differences between denominational groups when it came to the prevalence of social/community concerns, perceived barriers between psychology and church ministry, and perceived compatibility between psychology and church ministry. Mainline Protestant and Catholic leaders identified fewer barriers and more compatibility than Evangelical Protestants. Mainline Protestant leaders identified more social/community concerns than Evangelical Protestant leaders. However, many denominational differences disappeared when controlling for other factors (e.g., political conservatism, intratextual fundamentalism).

Second, to explore these findings further within the same sample of Christian leaders, McLaughlin et al. (in press) utilized a person-centered analytical approach and found three subgroups of Christian church leaders, based on differences in theological worldviews: (a) theologically conservative leaders, (b) theologically moderate leaders, and (c) theologically progressive leaders. In several areas of ministry, theologically moderate and theologically progressive leaders demonstrated a greater motivation to draw on psychological science than did theologically conservative leaders. Of note, theologically moderate and theologically progressive 
pastors were interspersed across the broad denominational groups used in Hodge, Hook, Davis, and McMinn (2020; i.e., Evangelical Protestant, Mainline Protestant, Catholic). Taken together, these findings highlight the importance of individual differences when exploring theological beliefs among Christians, rather than assuming homogeneity across Christian traditions.

\section{Important Dimensions that Distinguish Christians}

Worthington (1988) identified three important value dimensions by which highly committed adherents in the Abrahamic traditions evaluate and develop their values, beliefs, and doctrines: (a) authority of human leaders, (b) authority of Scripture or doctrine, and (c) religious group norms. Where people locate themselves on these value dimensions vary by denomination and individual. However, their stance on these dimensions likely informs the crucial beliefs, values, and doctrines that are central to them.

\section{Authority of Human Leaders}

The authority of human leaders relates to one's viewpoint regarding which people or institutions should be esteemed as having authority over one's life. This assumes that religious people, namely Christians, might follow the lead of authority. When considering collaborative efforts between PP and Christianity, it is important to understand how Christians view human spiritual authority. The social standing of a psychologist — even a Christian psychologist — might not hold much authority in some Christian groups if group members are skeptical of the natural and social sciences or give greater authority to religious leaders (e.g., a pastor or the Pope). The psychologist's viewpoints may be disregarded if they run contrary to those of religious leaders.

For collaboration, psychologists could align with church leaders and help church leaders understand and share psychological findings (and their potential impact) in their church. To be effective, this type of collaboration will likely need to involve considerable humility and 
openness on the part of both psychologists and Christian leaders, as they honor the expertise and authority each holds within their spheres of study and influence.

\section{Authority of Scripture or Doctrine}

Authority of Scripture or doctrine is especially relevant to Christian worldviews. Many Christian groups purport that the Bible, and occasionally other Christian literature, depending on the tradition, has special value and authority over and above other sources of information (see Hood et al., 2005, for a review on intratextuality and its relationship to fundamentalism). Thus, many groups (including Christian-identifying counselors and clinicians; see McMinn et al., 2010) view psychology and the natural sciences as separate belief systems from Christianity, and they may debate how much epistemological authority to attribute to theology or science.

Christian psychologists have written extensively on efforts to integrate psychology and Christianity (e.g., Johnson, 2010; Neff \& McMinn, 2020). For example, in Johnson’s (2010) book on five views of psychology and Christian integration, some Christian integrationists draw

a distinct line between biblical authority and psychology, prioritizing the notion that Scripture is sufficient to address psychological concerns (i.e., the Biblical Counseling view), whereas others see psychology and theology explaining different levels of truth (i.e., truth can be discovered from scientific disciplines such as psychology that may not be readily observable in Christian tradition and doctrine; the Levels-of-Explanation view). Of course, there are individual differences in how Christians (both Christian psychologists and lay people alike) approach psychological science, but it is fair to assume that beliefs on the authority and sufficiency of the Bible are often important in informing how Christians view psychology.

Regarding collaborative efforts between PP and Christianity, if epistemic authority is ascribed to theology, positive psychologists may be reticent to engage in collaborative efforts 
where their work must be subservient to the other party's theology. On the other hand, if epistemic authority is ascribed to psychology, then theologians may perceive Scripture as being distorted by the current science of the time (Porter, 2010). Regardless of where one potential collaboration partner stands on their view of the authority of Scripture, collaboration is possible when the other party's view of the authority of the Bible is understood, valued, and respected.

\section{Religious Group Norms}

Religious group norms involve one's loyalty to members of their group (e.g., the church, denomination, congregation, etc.) and exclusion of people outside of one's self-referential group. Worthington (1988) has provided an example of this value dimension when describing how neoorthodox churches emphasized ecumenism (i.e., promoting unity among Christian churches) in the twentieth century, which ran contrary to the practice of drawing specific denominational lines based on doctrine, ecclesiastical authority, and scriptural authority.

Regarding collaborative efforts, one might consider how Christian group norms may help or hinder a Christian from engaging in PP interventions and research (Rye et al., 2013). Religious individuals are more likely to consult with their religious leader than a psychologist or medical doctor (Chalfant et al., 1990), and clergy have been called the "gatekeepers" to the professional practice of psychology (Gorsuch \& Meylink, 1988). Some Christians may have very little desire to engage in PP practice unless they perceive the PP contribution is consistent with the norms of their religious-identity group.

Furthermore, a Christian may be intrigued by aspects of PP or want to explore PP interventions, yet they might not feel comfortable engaging in these practices, due to the perception that the "secular" intervention would conflict with group norms. For example, a Christian with higher levels of religious commitment or fundamentalism may feel uncomfortable 
with certain mindfulness-meditation practices (Williams et al., in press). Therefore, it is important to be conscious of a Christian client's assumptions underlying therapeutic interventions and to assess Christian clients' level of trust in their psychotherapist's ability to adapt these interventions to match the client's R/S beliefs respectfully and adequately.

Notably, PP researchers have recognized the hesitancy of some Christians in approaching psychological science, and these researchers have begun to explore how PP interventions could be implemented in Christian settings. There is a growing body of research suggesting that targeted PP interventions can be beneficial for Christian communities, including interventions promoting gratitude (Uhder et al., 2017), wisdom (McLaughlin et al., 2018), humility (Cuthbert et al., 2018), grace (Bufford et al., 2018), and forgiveness (Toussaint et al., 2020). Thus, in their work, PP researchers and practitioners could recognize the strong influence of Christian beliefs and group norms and identify ways to work with potential Christian groups and clients in a culturally humble and clinically sensitive way. This does not mean the scientific approach has to change but rather that the researcher or clinician may take into consideration how the participants or clients might respond to the intervention, based on their unique Christian beliefs and practices.

To be sure, there are other important values and beliefs held by Christians. However, the aforementioned beliefs provide a strong foundation for improving discussion regarding the intersection of PP and Christianity. It is important for psychologists to be aware of the values and beliefs held by their Christian clients, in order to understand better how their Christian clients might perceive their presenting problems or the purpose of a PP intervention. On the other hand, it is important for Christian church leaders to tolerate some level of metaphysical or worldview difference with positive psychologists, in order to see how PP research or interventions might be used or adapted to help their communities experience greater well-being and flourishing. 


\section{Christian Understanding of Well-Being, Health, and Virtue}

To this point, we have not directly highlighted the overlap between PP and Christianity, although we have alluded to this overlap (given the relatively high volume of scholarly literature on the subject). In the following section, we outline how PP and Christianity share overlapping interests regarding well-being, health, and virtue, but we also identify differences in how the two fields approach these subjects (see Table 10.2 for an overview). Given that PP is value-laden (Prinzing, 2021), we also use the following section to explore how Christian values might be utilized to (a) provide a framework for future empirical inquiry into PP constructs and (b) serve as a motivator to encourage more individuals to engage with PP research and practice. In other words, Christianity provides a framework that informs adherents about what values and virtues are worth pursuing, but it does not necessarily describe how to achieve these goals. On the other hand, PP provides an empirical base for how to develop virtues and character strengths, but it may not directly address why pursuing virtues and well-being is important or meaningful.

To begin, when it comes to pursuing ultimate human flourishing or well-being, what Christians consider important may look different from how PP measures and assesses wellbeing. For example, PP typically examines well-being on two fronts: (a) a hedonic pathway towards well-being (i.e., pursuing pleasure and escaping pain) and (b) a eudaimonic pathway towards well-being (i.e., development of virtues that lead to the good life; Ryan \& Deci, 2001). Christianity is most strongly aligned with a eudaimonic pathway, such that pursuing a virtuous life is thought to help a Christian become more Christ-like (Nelson \& Slife, 2017), by having the in-dwelling Christ formed more into their character (traditional Christian character formation).

A major difference in how positive psychologists view eudaimonic well-being compared to Christians is the motivation behind why an individual would pursue virtuous living. PP 
research and practice tend to examine virtues independently (e.g., examining gratitude distinctly from humility). The end goal for the positive psychologist may be understanding and promoting internal and relational benefits of virtues. This runs contrary to dominant Christian thinking that conceptualizes virtues as interrelated and simultaneously developing (e.g., increases in gratitude accompany increases in humility and vice versa), with the ultimate goals of virtue development being that one is better able to worship God and to love God and others (Nelson \& Slife, 2017).

Additionally, some Christians may also believe that virtues are gifts that develop within one's connection to God—perhaps through God's agency—rather than being developed through sheer willpower or in response to one's individual agency. For example, the "fruit of the spirit" listed in Galatians 5:22-23 (e.g., love, patience, self-control) map directly onto virtues that are widely studied in PP. One common interpretation of this passage suggests that Christians naturally produce these virtues as their Christian faith grows and they become more like Christ. (The flip-side of this perspective would be that non-Christians are unlikely to produce these fruits [virtues] due to a lack of connection with God.) An alternate perspective is that God initiates the pursuit of virtue, but human agency is needed to build the virtue (James 1:4, 12, 2127; Eph 4:22-24). Therefore, depending on their understanding of God's role in strengthening virtue development, Christians may differ in what they believe about how non-Christians may develop virtues or benefit from behaving virtuously.

However, more nuanced views about how non-Christians (people outside of a relationship with God) may grow in virtues and receive benefits from acting virtuously are possible. Even so, it is important to consider how a Christian's view on virtue development might differ, depending on how they view the locus of control for virtue development. This may lead to differing views on whether positive change in a person's life occurs through divine 
intervention (e.g., God providing a believer the ability to exercise self-control when it comes to substance use) or whether these changes can be boiled down to agentic self-regulatory processes and outcomes. Christians may attribute positive physical and mental health changes to God, whereas positive psychologists, using empirical methods, are bound by the rules of psychological science and cannot introduce non-naturalistic explanatory causes of behavior change (e.g., positive psychologists are unable to measure the amount God's assistance in helping an individual develop virtues). It may promote more fruitful collaboration if psychologists and Christian leaders might increasingly view the cultivation of virtue as a mystery of collaboration between both divine influence and human engagement (e.g., grace; Bufford et al., 2018).

Collaborative efforts that hold space for this dialectic may be helpful in increasing wellbeing and health outcomes in a wider ministerial context as well. For example, Pennington and Hackney (2017) have made a convincing case that Judeo-Christian texts, namely the Bible, are deeply embedded with themes that pertain to people who are seeking happiness and well-being through their approach to God. Pennington and Hackney (2017) suggest that, in the Bible, God provides moral commands that temporarily function to reduce levels of hedonic well-being in order to help humans experience life to the full (i.e., eudaimonic well-being). Thus, viewing Christianity as primarily a restriction on one's behavior to achieve a place of right-standing with God is limited. A Christian PP framework is helpful for providing a theological rationale for why striving to live a virtuous life (e.g., practicing self-regulation) is worthwhile for Christians.

Furthermore, McMinn (2017) has suggested the church needs PP to (a) build a stronger bridge between science and Christianity and (b) provide practical application to Christian philosophy and teaching. Constructs of PP (e.g., well-being, forgiveness) map well onto Christian language and ideals (e.g., God's forgiveness, biblical insistence on unilateral human 
forgiveness). This fact is no coincidence, as early theoretical development of PP considered world religions' prescriptions for the good and virtuous life (Peterson \& Seligman, 2004). For Christians, finding ways to connect science with their Christian beliefs is likely important in making sense of the world and discerning sources of truth. PP perspectives that utilize scientific approaches to developing virtues and behaviors in pursuit of well-being could be useful for Christians as they consider more deeply how they strive toward spiritual development and ideals of Christian living.

PP and Christianity can also have different viewpoints on the origin and practice of virtue (Beck \& Haugen, 2013). For example, Titus (2017) has illustrated how contemporary PP seeks to describe character strengths and virtue under the assumption that positive human nature promotes one to act morally. On the other hand, a Christian approach to PP, through the writings of Thomas Aquinas, provides a multidimensional conceptualization of virtue, where a person's disposition, behavior, and reasoning for behaving virtuously are all taken into consideration. The actual list of character strengths and virtues may not be that different in PP compared to Christianity. Christian-based reasoning for why a Christian should act virtuously is deeply rooted in the belief that Christians should care for themselves, others, and God. PP-based reasoning is rooted in helping people live and flourish, rather than merely exist (Keyes \& Haidt, 2003).

Furthermore, the beliefs held by Christianity may encourage people to engage virtuously in a way that PP may unintentionally, or intentionally, ignore. Roberts (2017) has outlined how a person's perception of virtue may be altered by their religious beliefs, which could lead to higher motivation for behaving virtuously, compared to contemporary PP theories. It is here that Christianity has much to offer the field of PP, by providing motivation for pursuing a virtuous lifestyle beyond the goal of being a moral, ethical, and happy person. Research has generally 
supported the notion that there is a positive relationship between general religion/spirituality and virtues such as forgiveness (Davis et al., 2013), humility (Davis et al., 2017), and gratitude (Tsang et al., 2012). However, the relationships are often complex, and someone's motivation to be virtuous may not actually result in the person actually behaving virtuously. Even so, it appears that identifying Christian motivations to live virtuous lives would help positive psychologists find ways to motivate Christian clients to pursue virtue development and well-being.

Lastly, there is a tradition within PP in which Christian thought has had a significant impact on the study of virtue. In the introduction of his book, McMinn (2017) outlined that many of the leading researchers on forgiveness identify as Christian, as do some of the major leaders studying gratitude and humility. These researchers identify values within the Christian faith and find strategies that may encourage many people — both inside and outside of the Christian faith— to pursue well-being and positive relationships through the development of virtue and character strengths. Another clear example of this strong collaborative relationship between PP and Christianity is the recent empirical research that has strengthened psychology's theoretical and empirical understanding of grace. Using theological underpinnings of grace from numerous religious backgrounds, but largely drawing from Judeo-Christian traditions, Emmons et al. (2017) provided a definition of grace that considers the role of social obligation (i.e., being unobligated to offer acceptance to an undeserving person) as a distinguishing factor that separates grace from other virtues (e.g., forgiveness, compassion). By this definition, God is the ultimate giver of grace, as there is no obligation for God to provide acceptance to people.

In a recent narrative review surveying the extant empirical literature on grace $(k=61)$, Hodge, Hook, Davis, Van Tongeren, et al. (2020) found that grace is often used interchangeably with general spirituality, divine intervention, and other related virtues (e.g., forgiveness 
likelihood) in psychological research on grace (which has largely been conducted by Christian psychologists). Grace is foundational to the Christian religion. Yet many Christians and Christian psychologists may struggle to determine what makes grace unique from other prosocial attitudes and behaviors, perhaps diluting their understanding of the cognitive and emotional impact grace might have in a person's life. This is where PP can help Christianity. PP can help (a) demonstrate empirically how Christian virtues may impact an individual's sense of well-being and (b) explore how Christian virtues may differentially promote the development of other virtues.

\section{An Example of Fruitful Collaboration}

Perhaps the best way to illustrate how PP and Christianity could work together to benefit one another is through an example. Forgiveness is a popular virtue within $\mathrm{PP}$, and it is also a central aspect of the Christian faith. PP has found a strong relationship between forgiveness and health/well-being (Worthington et al., 2007). However, some people may perceive that unforgiveness is an effective coping mechanism in certain situations, even though unforgiveness may lead to subsequent stress and anxiety. In certain situations, positive psychologists may struggle to help motivate people to engage in PP's forgiveness interventions, whereas Christianity provides direct commands for a person to forgive routinely (Matthew 18:21-22).

On the other hand, some Christians may struggle with forgiveness and seek additional resources on how to forgive, or they may feel like they have truly made a decision to forgive (i.e., decisional forgiveness), but they are confused about why they still experience negative emotions regarding the offense (i.e., they have not experienced emotional forgiveness). In the PP field, there are many models of forgiveness that have a strong empirical foundation (e.g., REACH Forgiveness, Forgive for Good; Toussaint et al., 2020), and these resources could help Christians pursue, and perhaps better understand, the virtues they feel God wants them to pursue. 
Here, PP and Christianity could work well together in that Christianity could provide a framework to understand why individuals may be motivated to engage in virtue development and pursue well-being (at least for nearly 1/3 of the world's population), and PP could utilize Christian tradition and a Christian understanding of virtue to identify and explore other mechanisms of virtue development that might be impactful for the PP field as a whole.

\section{Conclusion}

PP and Christianity have many shared goals, and recent scholarly efforts have demonstrated how the two fields might become better friends and collaborators. The global landscape and organizational/denominational structure of Christianity is steadily changing, and these changes bring new challenges and opportunities to enhance efforts of collaboration. In light of this, we have sought to convey a message to both psychologists and Christian leaders. The world continues to become more interconnected and diverse, and it is important for positive psychologists to be sensitive to and to learn about the beliefs and perspectives of both Christian clientele and potential partners for collaboration. Perhaps with better communication and humility, PP research and practice will be enhanced by interacting with Christian traditions, practices, institutions, and beliefs. Similarly, we encourage Christian ministry leaders and lay individuals to be open to new insights from PP regarding virtue development and human flourishing. Worldview differences will continue to arise and be a challenge. Not everyone will come to value integrating PP into theology or Christian living. But, if one decides such integration is warranted, there are numerous ways for psychological concepts to be integrated into Christian living and ministry. Thus, there is ample reason for PP and Christianity to continue striving toward positive collaboration, acknowledging that both fields can benefit substantially from one another (McMinn, 2017). 


\section{References}

Beck, R., \& Haugen, A. D. (2013). The Christian religion: A theological and psychological review. In K. I. Pargament, J. J. Exline, \& J. W. Jones (Eds.), APA Handbook of Psychology, Religion, and Spirituality (Vol. 1): Context, Theory and Research (p. 697711). American Psychological Association. https://doi.org/10.1037/14045-039

Bufford, R. K., McMinn, M. R., Moody, J. A., \& Geczy-Haskins, L. (2018). The effects of grace interventions in church communities. The Journal of Positive Psychology, 13(5), 512521. https://doi.org/10.1080/17439760.2017.1350740

Chalfant, H. P., Heller, P. L., Roberts, A., Briones, D., Aguirre-Hochbaum, S., \& Farr, W. (1990). The clergy as a resource for those encountering psychological distress. Review of Religious Research, 31, 305-313. http://dx.doi.org/10.2307/3511620

Cohen, A. B., Hall, D. E., Koenig, H. G., \& Meador, K. G. (2005). Social versus individual motivation: Implications for normative definitions of religious orientation. Personality and Social Psychology Review, 9, 48-61. https://doi.org/10.1207/s15327957pspr0901_4

Cohen, A. B., \& Hill, P. C. (2007). Religion as culture: Religious individualism and collectivism among American Catholics, Jews, and Protestants. Journal of Personality, 75, 709-742. https://doi.org/10.1111/j.1467-6494.2007.00454.x

Cohen, A. B., Wu, M. S., \& Miller, J. (2016). Religion and culture: Individualism and collectivism in the East and West. Journal of Cross-Cultural Psychology, 47(9), 12361249. https://doi.org/10.1177/0022022116667895

Cuthbert, A. D., Davis, E. B., Aten, J. D., Short, A., Yarborough, C. A., Lavelock, C. R., Worthington, E. L., Jr., Davis, D. E., Hook, J. N., \& Van Tongeren, D. R. (2018). Cultivating humility in religious leaders: The effectiveness of a spiritually integrated 
positive psychology intervention. Spirituality in Clinical Practice, 5(4), $227-$ 239. https://doi.org/10.1037/scp0000185

Davis, D. E., Hook, J. N., McAnnally-Linz, R., Choe, E., \& Placeres, V. (2017). Humility, religion, and spirituality: A review of the literature. Psychology of Religion and Spirituality, 9(3), 242-253. https://doi.org/10.1037/rel0000111

Davis, D. E., Worthington, E. L., Jr., Hook, J. N., \& Hill, P. C. (2013). Research on religion/spirituality and forgiveness: A meta-analytic review. Psychology of Religion and Spirituality, 5(4), 233-241. https://doi.org/10.1037/a0033637

Emmons, R. A., Hill, P. C., Barrett, J. L., \& Kapic, K. M. (2017). Psychological and theological reflections on grace and its relevance for science and practice. Psychology of Religion and Spirituality, 9(3), 276-284. https://doi.org/10.1037/rel0000136

FitzGerald, F. (2017). The evangelicals: The struggle to shape America. Simon \& Schuster.

Gorsuch, R. L., \& Meylink, W. D. (1988). Toward a co-professional model of clergypsychologist referral. Journal of Psychology and Christianity, 7, 22-31.

Hadaway, C. K., \& Marler, P. L. (2006). Growth and decline in the mainline. In C. H. Lippy (Ed.), Faith in America: Changes, challenges, and new directions (Vol. 1, p. 1-24). Praeger. https://ixtheo.de/Record/133045524X

Hill, P. G., \& Hall, M. E. L. (2018). Uncovering the good in positive psychology: Toward a worldview conception that can help positive psychology flourish. In N. J. L. Brown, T. Lomas, \& F. J. Eiroa-Orosa (Eds.), The Routledge international handbook of critical positive psychology (p. 245-262). Routledge/Taylor \& Francis Group. https://psycnet.apa.org/record/2017-42568-016 
Hodge, A. S., Hook, J. N., Davis, D. E., \& McMinn, M. R. (2020). Attitudes of religious leaders toward integrating psychology and church ministry. Spirituality in Clinical Practice, 7(1), 18-33. http://dx.doi.org/10.1037/scp0000200

Hodge, A. S., Hook, J. N., Davis, D. E., Van Tongeren, D. R., Bufford, R. K., Bassett, R. L., \& McMinn, M. R. (2020). Experiencing grace: A review of the empirical literature. The Journal of Positive Psychology. Advanced online publication. https://doi.org/10.1080/17439760.2020.1858943.

Hood, R. W., Hill, P. C., \& Williamson, W. P. (2005). The psychology of religious fundamentalism. Guilford. https://psycnet.apa.org/record/2005-07173-000

Johnson, E. L. (Ed.). (2010). Psychology and Christianity: Five views (2 $2^{\text {nd }}$ ed.). InterVarsity Press.

Johnson, E. L. (2017). Towards a Christian positive psychology: Introduction to the special issue. The Journal of Positive Psychology, 12(5), 425-426. https://doi.org/10.1080/17439760.2016.1228011

Keyes, C. L. M., \& Haidt, J. (Eds.). (2003). Flourishing: Positive psychology and the life welllived. American Psychological Association. https://doi.org/10.1037/10594-000

King, M. L., Jr. (1963/2010). Strength to love. Fortress Press.

McLaughlin, A. T., Davis, D. E., Hook, J. N., Hodge, A. S., Choe, E., McMinn, M. C., \& Worthington, E. L. Jr. (in press). Approaches to understanding and aligning with religious leaders: A latent profile analysis of religious leaders' worldviews. Spirituality in Clinical Practice. Advanced online publication. https://doi.org/10.1037/scp0000258

McLaughlin, P. T., McMinn, M. R., Morse, M., Neff, M. A., Johnson, B., Summerer, A., \& Koskela, N. (2018). The effects of a wisdom intervention in a Christian congregation. 
The Journal of Positive Psychology, 15(2), 502-511.

https://doi.org/10.1080/17439760.2017.1350739

McMinn, M. R. (2017). The science of virtue: Why positive psychology matters to the church. Brazos Press.

McMinn, M.R., Staley, R.C., Webb, K.C., \& Seegobin, W. (2010). Just what is Christian counseling anyway? Professional Psychology: Research and Practice, 41, 391-397. https://doi.org/10.1037/a0018584

Neff, M. A., \& McMinn, M. R. (2020). Embodying integration: A fresh look at Christianity in the therapy room. InterVarsity Press.

Nelson, J. M., \& Slife, B. D. (2017). A new positive psychology: A critique of the movement based on early Christian thought. The Journal of Positive Psychology, 12(5), 459-467. https://doi.org/10.1080/17439760.2016.1228006

Oishi, S., Graham, J., Kesebir, S., \& Galinha, I. (2013). Concepts of happiness across time and cultures. Personality and Social Psychology Bulletin, 39(5), 559-577. https://doi.org/10.1177/0146167213480042.

Pennington, J. T., \& Hackney, C. H. (2017). Resourcing a Christian positive psychology from the Sermon on the Mount. The Journal of Positive Psychology, 12(5), 427-435. https://doi.org/10.1080/17439760.2016.1228008

Peterson, C., \& Seligman, M.E.P. (2004). Character strengths and virtues: A handbook and classification. American Psychological Association and Oxford University Press.

Pew Research Center (2011, December). Global Christianity: A report on the size and distribution of the world's Christian population. https://assets.pewresearch.org/wpcontent/uploads/sites/11/2011/12/Christianity-fullreport-web.pdf. 
Pew Research Center (2017, April 5). The changing global religious landscape: Babies born to Muslims will begin to outnumber Christian births by 2035; people with no religion face a birth dearth. https://www.pewforum.org/2017/04/05/the-changing-global-religiouslandscape/.

Porter, S. L. (2010). Theology as queen and psychology as handmaid: The authority of theology in integrative endeavors. Journal of Psychology and Christianity, 29(1), 3-14.

Prinzing, M. M. (2021). Positive psychology is value laden-It's time to embrace it. The Journal of Positive Psychology, 16(3), 289-297. https://doi.org/10.1080/17439760.2020.1716049

Rhodes, R. (2015). The complete guide to Christian denominations: Understanding the history, beliefs, and differences. Harvest House Publishers.

Roberts, R. C. (2017). Virtues and belief in God. The Journal of Positive Psychology, 12(5), 480-488. https://doi.org/10.1080/17439760.2016.1228003

Ryan, R. M., \& Deci, E. L. (2001). On happiness and human potentials: A review of research on hedonic and eudaimonic well-being. Annual Review of Psychology, 52, 141-166. http://dx.doi.org/10.1146/annurev.psych.52.1.141

Rye, M. S., Wade, N. G., Fleri, A. M., \& Kidwell, J. E. M. (2013). The role of religion and spirituality in positive psychology interventions. In K. I. Pargament, A. Mahoney, \& E. P. Shafranske (Eds.), APA handbook of psychology, religion, and spirituality (Vol. 2): An applied psychology of religion and spirituality (pp. 481-508). American Psychological Association. https://doi.org/10.1037/14046-025

Titus, C. S. (2017). Aquinas, Seligman, and positive psychology: A Christian approach to the use of the virtues in psychology. The Journal of Positive Psychology, 12(5), 447-458. https://doi.org/10.1080/17439760.2016.1228005 
Toussaint, L. L., Griffin, B. J., Worthington, E. L., Jr., Zoelzer, M., \& Luskin, F. (2020).

Promoting forgiveness at a Christian college: A comparison of the REACH forgiveness and forgive for good methods. Journal of Psychology and Theology, 48(2), 154-165. https://doi.org/10.1177/0091647120911109

Tsang, J.-A., Schulwitz, A., \& Carlisle, R. D. (2012). An experimental test of the relationship between religion and gratitude. Psychology of Religion and Spirituality, 4(1), 4055. https://doi.org/10.1037/a0025632

Uhder, J., McMinn, M. R., Buford, R. K., \& Gathercoal, K. (2017). A gratitude intervention in a Christian church community. Journal of Psychology and Theology, 45(1), 46-57. https://doi.org/10.1177/009164711704500104

Williams, N., Shoidiya-Zeumalt, S., Gazaway, S., Chang, C. Y., McLaughlin, A., \& Davis, D. (in press). Religious and spiritual concerns of mindfulness: Implications for utilizing mindfulness in counseling. Journal of Psychology and Christianity.

Worthington, E. L., Jr. (1988). Understanding the values of religious clients: A model and its application to counseling. Journal of Counseling Psychology, 35(2), 166-174. https://doi.org/10.1037/0022-0167.35.2.166

Worthington, E.L., Witvliet, C. V. O., Pietrini, P., \& Miller, A. (2007). Forgiveness, health, and well-being: A review of evidence for emotional versus decisional forgiveness, dispositional forgivingness, and reduced unforgiveness. Journal of Behavioral Medicine, 30(4), 291-302. http://dx.doi.org/10.1007/s10865-007-9105-8 


\section{Table 10.1}

Reasons Why Positive Psychology (PP) and Christianity Could and Should Work Together

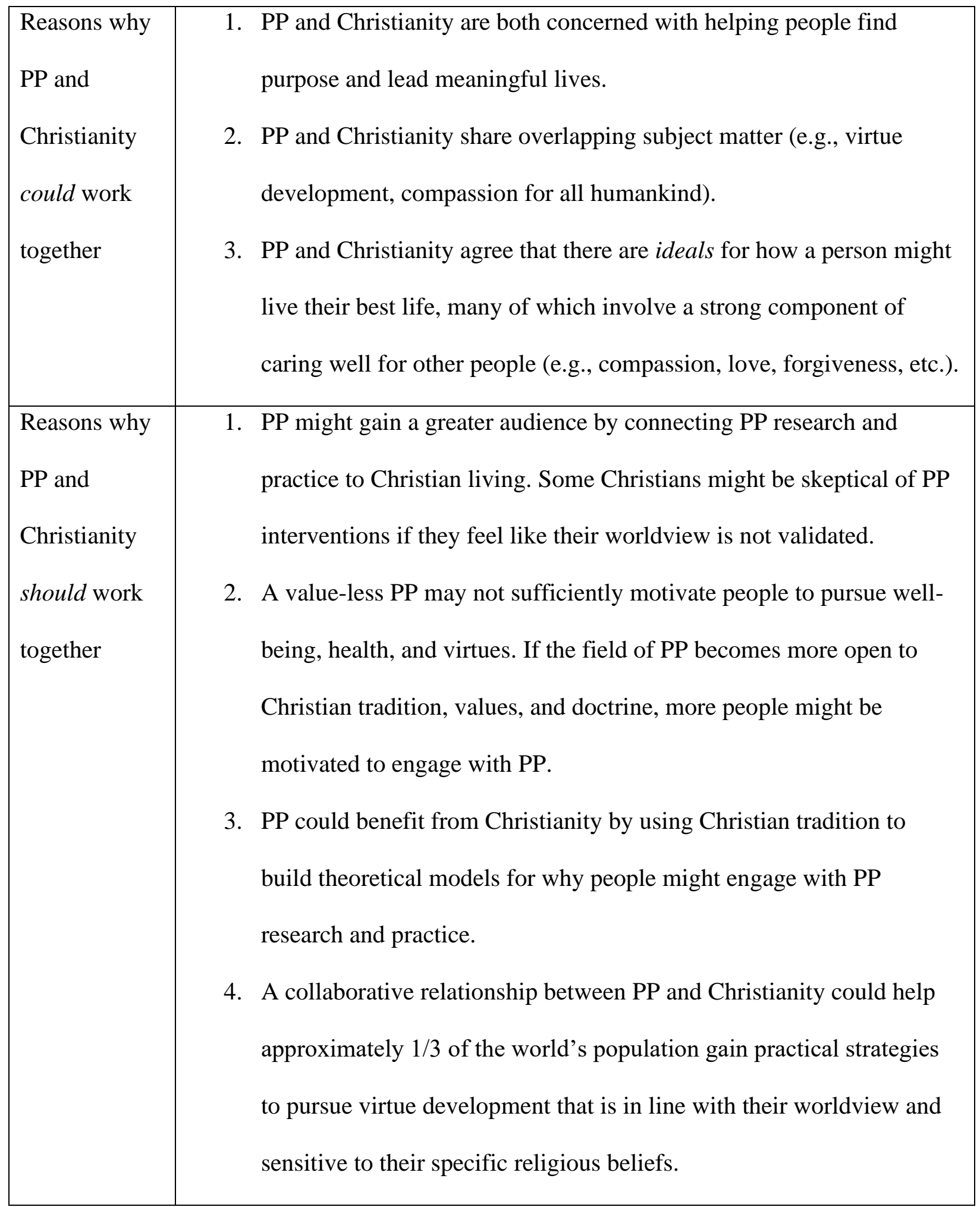


Table 10.2

Comparison of Well-Being, Health, and Virtue in Positive Psychology (PP) and Christianity

\begin{tabular}{|c|c|c|}
\hline & Positive Psychology & Christianity \\
\hline \multirow[t]{2}{*}{$\begin{array}{l}\text { Well-being } \\
\text { and health }\end{array}$} & $\begin{array}{l}\text { Emphasizes both hedonic and } \\
\text { eudaimonic well-being }\end{array}$ & $\begin{array}{l}\text { Values eudaimonic well-being, which } \\
\text { develops from a strong relationship } \\
\text { with God }\end{array}$ \\
\hline & $\begin{array}{l}\text { Motivation to pursue well-being and } \\
\text { health is based on the notion that it is } \\
\text { best for humans to flourish, rather than } \\
\text { merely exist (Keyes \& Haidt, 2003) }\end{array}$ & $\begin{array}{l}\text { Virtues develop simultaneously within } \\
\text { a relationship with God; God is viewed } \\
\text { as the catalyst of virtue development } \\
\text { Motivation to pursue well-being and } \\
\text { health is based on pursuing spiritual } \\
\text { development and the ideals of Christian } \\
\text { living }\end{array}$ \\
\hline Virtues & $\begin{array}{l}\text { Virtue lists are derived from religious } \\
\text { traditions and ancient philosophies } \\
\text { (Peterson \& Seligman, 2004) }\end{array}$ & $\begin{array}{l}\text { Virtues are prescribed by God (e.g., } \\
\text { fruit of the spirit; three theological } \\
\text { virtues of faith, hope, and love; Beck \& } \\
\text { Haugen, 2013) as values to pursue }\end{array}$ \\
\hline & $\begin{array}{l}\text { Virtues are treated as (mostly) } \\
\text { unrelated character traits that may or } \\
\text { may not work together to promote } \\
\text { positive outcomes }\end{array}$ & $\begin{array}{l}\text { Virtues are treated as interrelated } \\
\text { constructs that have broad applications } \\
\text { and meaning (e.g., love incorporates } \\
\text { other virtues such as altruism, } \\
\text { compassion, forgiveness, etc.) }\end{array}$ \\
\hline
\end{tabular}




\title{
Chapter 11: Positive Psychology and Judaism
}

\author{
Mark Schiffman ${ }^{1}$ \\ Aaron Cherniak $^{2}$ \\ Eliezer Schnall ${ }^{1}$ \\ Suzanne Brooks ${ }^{1}$ \\ Steven Pirutinsky ${ }^{3}$ \\ Devora Shabtai ${ }^{4}$
}

\author{
${ }^{1}$ Yeshiva University \\ ${ }^{2}$ Stockholm University \\ ${ }^{3}$ Touro College \\ ${ }^{4}$ University of Warwick
}

Correspondence concerning this chapter should be addressed to Mark Schiffman. Email: mordechai.schiffman@yu.edu 


\begin{abstract}
Many core tenets of positive psychology and the psychology of religion/spirituality (R/S) are essential concepts within the rich literature and culture of Jewish tradition. Judaism, with its long history of texts, traditions, and practices, can substantively contribute to the developing field of positive psychology. In this chapter, we explore happiness, character, and spirituality within a Judaic framework, opening opportunities for integrative theory, research, and clinical practice. From a theoretical standpoint, these Judaic models provide useful ways of conceptualizing positive psychology constructs. On a pragmatic level, researchers and practitioners can utilize these ideas in their work with the Jewish population, enhancing their efficacy and cultural competence. The chapter includes a general description of Jewish tradition and relevant demographics for context and then summarizes the theory, research, and practice of happiness, character, and spirituality, noting practical suggestions and future directions.
\end{abstract}

Keywords: Judaism, spirituality, flourishing, happiness, character 


\section{Chapter 11: Positive Psychology and Judaism}

In the second half of the twentieth century, the late Rabbi Menachem Mendel Schneerson led a sect of ultra-Orthodox Jews who are known as Lubavitch Hasidim. Over the course of his life, Rabbi Schneerson underwent profoundly difficult personal hardship. Born in the Russian empire in 1902, he lived through pogroms, two world wars, a typhus epidemic, the persecution and exile of his father, violent political revolutions, the murder of his brother, grandmother, and other relatives by the Nazis, and a life of childlessness (Kalmenson, 2019). Yet due to being steeped in thousands of years of traditional Jewish teachings, Rabbi Schneerson developed what Kalmenson (2019) describes as a "positivity bias," which he cultivated by "actively seeking the positive aspect or opportunity in any given situation, believing deeply in God's ultimate goodness and immanent presence, and living with purpose, responsibility, and meaning” (p. 19).

Although Jewish teachings throughout the ages are vast and the lived experiences of Jews varied, it is helpful to frame them through the prism of the "positivity bias." Through embodying Judaic values, many Jews have been enabled to live spiritually rich and positive lives, despite extraordinary hardships and persecution. Jewish thought, beliefs, and practices have millennialong histories that can inform the comparatively new field of positive psychology. Collaborations between positive psychologists and Jewish philosophers, leaders, and adherents is recommended to understand Jewish concepts of well-being and spirituality. Such collaborations may further enrich the field of positive psychology and provide practitioners with additional resources to inform research and practice. Towards that goal, this chapter is a brief introduction to Judaism and the Jewish people, followed by a discussion of aspects of Jewish tradition and culture that are pertinent for positive psychology and the psychology of religion/spirituality (R/S).

\section{Introduction to Judaism}


With historical roots stretching back nearly 4,000 years, Judaism is a monotheistic religion that affirms belief in one transcendent and immanent God who, as recounted in the Pentateuch, created the world and entered into a covenantal relationship with the Jewish people. After miraculously redeeming the Children of Israel from bondage in Egypt, God revealed Himself to them at Mount Sinai and presented them with the Torah. Besides the Pentateuch, the Jewish biblical canon also includes 19 later works of the Prophets and the Hagiographa. The Prophets weave ethical exhortations into the story of the tribes of Israel as they entered the Land of Israel, established the monarchy of King David, built the Temple, were exiled from Israel in 586 BCE, and returned to build the Second Temple. The Hagiographa includes books such as Psalms, Job, and Proverbs, which contain ethics, wisdom, and spiritual guidance (Baskin, 2011). Within traditional Judaism, the written biblical texts are supplemented by the Oral Law, which elucidates and elaborates on the written text, explaining and interpreting the laws and narrative of the Bible. Shortly after the destruction of the Second Temple in $70 \mathrm{CE}$, the Oral Law was compiled and organized in the form of the Mishna and other literary works. Scholarly rabbinic debates concerning these texts were collected and redacted in the Talmud until approximately the seventh centuries CE (Hezser, 2004). During the Middle Ages, a plethora of rabbinic works emerged, encompassing Hebrew Bible and Talmud commentary, law, poetry, Jewish philosophy (comparing Judaism with Neoplatonic and Aristotelian concepts), and Kabbalah (Tirosh-Samuelson, 2003). During this period, the different cultural traditions of Sephardim (Jews from the Iberian Peninsula, North Africa, and the Middle East) and Ashkenazim (Jews from Northern and Eastern Europe) developed (Ben-Shalom, 2004).

In the modern period, in response to the Enlightenment, the streams of Judaism that comprise the $21^{\text {st }}$ century landscape emerged. Although currently there are several 
denominations, each with its own subcategories (see Baskin, 2011), we focus primarily on three: Orthodox, Conservative, and Reform. Orthodox Jews generally follow Jewish law (known as Halakha) and tradition as it has been interpreted in the Talmud and practiced throughout the ages. The Conservative movement also refers to traditional Jewish law as a source for contemporary practice but is more open to revision and adaptation of codified Jewish law. Major distinctions between Conservative and Orthodox practice relate to issues of egalitarianism in worship, Sabbath observance, and adherence to dietary laws (Baskin, 2011). Reform Judaism (or Liberal or Progressive Judaism) does not believe that Jewish law is binding, and it is thus more likely to alter traditional concepts to conform with modern values (Gehl, 2014).

\section{Demographics}

Over four-fifths of the close to 15 million Jews worldwide live in the U.S. and Israel (DellaPergola, 2020). Depending on how the term Jewish is defined, there are close to 7 million Jewish people in the U.S. (Sheskin \& Dashefsky, 2020). Current estimates indicate that approximately $37 \%$ identify as Reform, $17 \%$ as Conservative, $9 \%$ as Orthodox, $32 \%$ as nondenominational, and 4\% with smaller denominations (Pew Research Center, 2021). In Israel, there are around 6.6 million Jews (DellaPergola, 2020), but their denominations differ — with 22\% identifying as Orthodox, 29\% as Masorti (most similar in practice to Conservative Jews in North America), and 49\% as Hiloni (secular; Pew Research Center, 2016). Smaller Jewish communities are also found throughout the world, including in Canada, United Kingdom, Argentina, Russia, Germany, Australia, and Brazil (DellaPergola, 2020).

\section{Judaism and Positive Psychology}

In contrast to the disease model (which emphasizes pathology), positive psychology focuses on human strengths, wellness, and fulfillment (Seligman et al., 2005). Jewish thought 
and tradition are similarly replete with perspectives on psychological health and well-being (see Pirutinsky, 2020). In this section, we survey the Jewish tradition on the topics of (a) happiness flourishing, (b) character strengths, and (c) spirituality. This brief yet broad overview shows the relevance of a Judaic framework to positive psychology. On the whole, Jewish values, beliefs, and actions encourage a spiritually integrated, strength-based, and PERMA (Positive emotion,

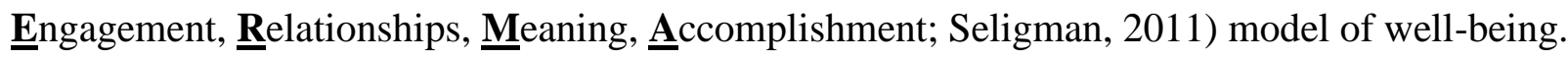

\section{Happiness and Flourishing}

The positive psychology literature generally incorporates two types of happiness, each rooted in Greek philosophy: hedonism (concerned with pleasure, comfort, and enjoyment) and eudaimonia (focused on pursuing complex goals that are meaningful to self and society; Delle Fave et al., 2011). Although the original focus of positive psychology gravitated closer to a hedonic view, the PERMA model aims to integrate eudaimonia through a focus on meaning and engagement (Seligman, 2011). We highlight four elements of the Jewish conceptualization of happiness that can contribute to positive psychology's broader conceptualizations of happiness.

Multiple Perspectives. It is impossible to describe a single Jewish view of happiness, as there are multiple approaches, with varying emphases. This point is captured in the fact that one of the biblical Hebrew words that is most closely associated with happiness is ashrei (TiroshSamuelson, 2003). As Jonathan Sacks (2014) contends, the word is written in plural form, indicating that the most appropriate translation would be "happinesses," signifying that happiness is not unidimensional. Different paths to happiness are reflected in the Hebrew Bible, rabbinic works, and later Jewish philosophical writings. Pelcovitz and Pelcovitz (2014) highlight the diverse Judaic understandings of happiness by pointing to several synonyms used for it in Jewish literature (simchah, sasson, gilah, rinah, ditzah, chedva) and by identifying the nuances 
of each word. Research and practice in this area is likely to be most fruitful when acknowledging the individual and contextual nature of happiness, along with its varied sources and expressions.

Blending of Pleasure and Meaning. The diversity within the Jewish conceptualization of happiness incorporates elements of pleasure and enjoyment and integrates them into a larger framework of meaning and self-transcendence. One example of this duality relates to the celebration and customs surrounding the Jewish holidays. On these special days that occur annually, Jews are enjoined to celebrate with festive meals. But this obligation is far from purely hedonistic. These festivals incorporate a spiritual component with the experience of celebrating "before God" (Deuteronomy 16:15), and they are meant to involve providing for the material welfare of the society's poor and vulnerable. Otherwise, as Maimonides states, the "meal is not a rejoicing in a divine commandment, but a rejoicing in his own stomach" (Twersky, 1972, p. 108). Thus, Judaism recognizes that happiness has both hedonic and eudaimonic elements, emphasizing transcending the self by helping others and by acknowledging the presence of God.

Importance of Action. Another important element of the Judaic notion of happiness is the role of law and ritual and its relationship to the inner life. Cohen et al. (2013) posit that Judaism emphasizes action, in contrast to beliefs and the cultivation of internal states. However, this emphasis on action does not diminish from the importance of belief and internal development. Rather, Jewish tradition suggests it is through committed action that an inner state is cultivated. Jewish spiritual-ethics, known as mussar, emphasize the importance of not being content with mindless acts by accentuating a focus on the inner life. Yet the internal state itself is not enough. It is only through the ritualized act that the proper inner state can be developed and actualized. Ritualized acts both should be done with joy and produce joy (Fishbane, 1995). 
Happiness is Not the Goal. According to several prominent modern Jewish thinkers, happiness is an important but not central value in Judaism (Soloveitchik, 1983; Sacks 2014). It is not the telos as it may be in an Aristotelian eudaimonic conceptualization. Although serving God should be done with joy (Deuteronomy 28:47; Psalms 100:2) and that service should lead to a joyous life (Psalms 19:9), serving God through the commandments is the ultimate goal. Judaism, in Sacks' (2014) words, involves "the pursuit of holiness, not the pursuit of happiness" (p. 32). Happiness may come as a result of living according to the will of God, but it is not the aim.

These Judaic notions of happiness provide an important counterpoint to the dangers of a self-absorbed, overly hedonic happiness. Judaic happiness does not negate the place of pleasure but insists pleasure must be balanced with self-transcendence; balancing internal and external aspects of happiness offers a well-rounded perspective. It also helps us consider that happiness should not be pursued as its own goal but should be experienced in pursuit of a higher value.

\section{Character Strengths}

Commandment. Following the eudaimonic approach, positive psychology encourages the cultivation of character strengths as part of living the good life (Peterson \& Seligman, 2004). The development of character strengths is also essential in the Judaic tradition, with one major difference from the Aristotelian framework. For Aristotle, happiness comes from arriving at a proper disposition by subordinating passions to human reason. According to the Hebrew Bible and the Talmudic sages, human reason plays a role in character formation. Still, within Judaism, it is ultimately to God's laws that one subordinates passions and appetites, not to human reason (Tirosh-Samuelson, 2003). Thus, the character strengths accentuated in the Judaic tradition involve developing personal discipline and ethical behavior for the purpose of fulfilling God's commandments. Not only are traits important for fulfilling the commandments, but character 
development itself is seen as an independent biblical commandment of emulating God (Blau, 2000). This perspective can transform character development into a deeply spiritual endeavor.

Virtues and Strengths. Peterson and Seligman (2004) surveyed the literature of various philosophical and religious traditions and identified six core virtues they hypothesized must be present for someone to be considered of good character (transcendence, wisdom, courage, humanity, justice, and temperance). They further conceptualized 24 character strengths, which they described as the processes or mechanisms that define the virtues. In their limited treatment of Judaic texts, they identified some, but not all, of the 24 strengths. Yet Schnall et al. (2014) contend that all 24 character strengths are evident in the classic texts of Judaism. They surveyed the Torah literature related to the five character strengths in the virtue of transcendence: hope, humor, gratitude, spirituality, and appreciation of beauty. Here, we survey the remaining virtues (wisdom, courage, humanity, justice, and temperance), followed by a discussion of spirituality.

Wisdom. The books of Psalms and Proverbs are replete with references to the importance of wisdom and knowledge of the world and of God. The sages viewed the learning of Torah as an essential, daily commandment to which they devoted considerable time and energy (TiroshSamuelson, 2003). Judaism recognizes various forms of wisdom and methods for cultivating it. Two primary paradigms include the rationalist perspective and the Kabbalistic tradition. Medieval rationalists like Maimonides believed wisdom is achieved through knowledge of Torah and nature (Hartman, 2010). The Kabbalistic tradition espouses that wisdom is reached through contemplation of God's nature and direct experience of His presence (Scholem et al., 2007).

Courage. The importance of bravery in battle is explicit in the Hebrew Bible (e.g., Deuteronomy 31:6; Joshua 1:6). Talmudic sages also conceptualized courage as a moral and psychological strength, both in the ethical and the spiritual realms. As Ben Zoma states in 
Mishna Avot (4:1): "Who is strong? He who conquers his desires." In addition, the traits of vitality and enthusiasm were encouraged in the context of performing the commandments. For example, Abraham is described as performing God's will with zeal; he serves as a role model for Jews to perform the commandments with alacrity (Genesis 22:3; Talmud Pesachim 4a).

Humanity. Many biblical and Talmudic works extol the virtue of humanity, which include strengths and traits that manifest in caring relationships with others (Peterson \& Seligman, 2004). Traits like love and kindness are emblematic of how God relates to the world and thus must be emulated through the process of imitatio Dei. This requirement to emulate God creates what Wurzburger (1994) calls an ethic of responsibility, wherein Jews are commanded to help others through gemilut chasadim (acts of kindness), including giving charity to the poor, providing hospitality to strangers, visiting the sick, comforting mourners, facilitating marriage, and redeeming captives. In fact, according to Rabbi Akiva (Jerusalem Talmud Nedarim 9:3) "love thy neighbor like yourself" (Leviticus 19:17) is the greatest principle in the Torah.

Justice. The virtue of justice, including the character strengths of fairness and leadership, also plays a vital role in Judaism. A just judicial system is mandated in the Hebrew Bible, as is stated "justice, justice you shall pursue" (Deuteronomy 16:20). Businesspeople are commanded to be honest and perform their jobs with integrity (Leviticus 19:35). Abraham argues before God, asking God to be fair in His treatment of Sodom and Gomorrah (Genesis 18:13-23), thereby serving as a paradigm for fighting for what is right and just (Sacks, 2007). Moses and others serve as examples of leadership, and several contemporary works outline lessons of leadership from the Bible (Brown, 2013; Sacks, 2015) and Talmud (Schnall \& Greenberg, 2012).

Temperance. Character strengths incorporated in the virtue of temperance (forgiveness, prudence, humility, and self-control) play a prominent role in Jewish literature. Granting 
forgiveness is encouraged, and someone who refrains from doing so to one who sincerely repents is considered cruel (Talmud Bava Kamma 92a). In a strong endorsement of prudence, the Talmud states, "Who is wise? He [or she] who foresees the consequences [of their actions]" (Talmud Tamid 32a). In addition, humility is identified as the crown jewel of a trait encouraged by the sages (Tirosh-Samuelson, 2003). Finally, many commandments encourage exercising self-control when it comes to eating and sexual relations. Based on this fact, Cohen et al. (2013) conceptualize self-control as a possible protective factor that may account for some of the positive correlations between Jewish R/S and both mental and physical health, an idea which has found empirical support (e.g., McCullough \& Willoughby, 2009; Pirutinsky, 2014).

\section{Spirituality}

As defined by Peterson and Seligman (2004), spirituality is demonstrated by people who possess clear beliefs about the meaning and purpose of the cosmos and believe in a transcendent, nonphysical element that infuses their life with a higher purpose and impacts their actions accordingly. Thus defined, spirituality relates to several Judaic concepts, such as the notion of divine providence, which incorporates the belief God is omniscient, guides both historical events and also everyday occurrences, rewards those who observe the commandments, and punishes those who do not (Schnall et al., 2014). A central example is the shema affirmation, which is recited daily and incorporates biblical passages about the unity and love of God, commitment to the commandments, and belief in reward and punishment (Deuteronomy 6:4-5 and 11:13-17). Moreover, in the mystical literature, everyday human behavior impacts the spiritual world, imbuing mundane and religious activities with cosmic, spiritual import (Scholem et al., 2007).

Prayer, identified by Peterson and Seligman (2004) as an essential component of spirituality, plays a central role within Judaism. In addition to ancient biblical accounts of 
meaningful prayers, the Talmud outlines the obligation for Jews to engage in formal prayer three times a day (Berakhoth 26b). According to some, prayer is the most significant form of divine service (Berakhoth 32b). Prayer is not limited to making requests for the fulfillment of physical needs; it also involves yearning for connection to the Divine (Schnall et al., 2014).

Moreover, although Peterson and Seligman (2004) counted spirituality as one of the 24 character strengths, the Jewish tradition conceptualizes spirituality as a meta-trait. The Talmudic sages encourage "doing everything for the sake of Heaven" (Mishna Avot 2:12), interpreted by later commentaries as an imperative to infuse even mundane activities with sanctity (Lamm et al., 1999). This notion would align with Piedmont's (1999) claim that spirituality is a distinct personality dimension that directs, drives, and selects behaviors in both secular and religious contexts. It is also consistent with Pargament and Mahoney’s (2005) sanctification theory, which contends that any object or action can be psychologically imbued with an element of sacredness.

\section{Research on Judaism, Positive Psychology, and Psychology of R/S}

Well-Being. There is limited but growing research related to Judaism and well-being, with varied outcomes (Cohen et al., 2013). Rosmarin, Krumrei, and Pargament (2010) surveyed both Jews and Christians and found that greater gratitude and spirituality were associated with reduced depression and anxiety in both groups. Similarly, Vilchinsky and Kravetz (2005) found that, among both religious and secular Israeli Jewish students, religious belief was negatively associated with psychological distress and positively associated with psychological well-being. Additionally, Pirutinsky et al. (2011) report that intrinsic religiosity moderated the association between poor physical health and depression among Jews. There also is evidence that Jewish practice prospectively predicts remission of depressive episodes (Pirutinsky \& Rosmarin, 2018). 
Character Strengths. The research related to character strengths within the Jewish population is limited. In a sample that included participants from different religious traditions, including Jews, Rosmarin et al. (2011) identified gratitude towards God as a construct distinct from general gratitude. They hypothesized that gratitude to God can cultivate transcendence and interconnectedness beyond general gratitude and may be experienced more frequently and in more diverse situations. They found that such spiritual gratitude uniquely predicted mental wellbeing over and above general gratitude. A subsequent study also found that gratitude to God is more enduring in the face of emotional distress than general gratitude (Rosmarin et al., 2016).

Spirituality. The aforementioned studies on gratitude fit a growing trend within the psychology of R/S that examines whether virtues contextualized in a religious belief system function differently from general character strengths. Prominent models of positive psychology present spirituality as one characteristic among others that compose a specific virtue, such as Peterson and Seligman's (2004) classification, which places spirituality as one of several character strengths that make up the virtue of transcendence. However, spirituality may be a component whose influence affects all other character strengths rather than a standalone trait. Similar to how framing gratitude in a religious framework of meaning changes the expression of gratitude, religious attributions can transform other strengths, such as granting forgiveness and expressing love. In that vein, Kor et al. (2019) administered several measures of both character strengths generally and spirituality specifically to 1,352 middle school Israeli adolescents ( $85 \%$ of the participants were Jewish). Their ensuing longitudinal factor analysis indicated that, at least among Jews, spirituality was a discrete factor independent of the classic tripartite model of intrapersonal, interpersonal, and intellectual strengths. In other words, spirituality is not simply a distinct character strength among many others that fits neatly into one virtue, but an overarching 
property that imbues all other character strengths with an additional layer of meaning. Spirituality as an independent factor remained stable over time and contributed to higher subjective well-being and prosociality. The study supports the contention that, within Judaism, spirituality can be conceptualized as a meta-trait that influences other character traits.

Internal/External. Another theme within the literature on spirituality is the distinction between internal and external manifestations of religious life, including how they relate to wellbeing. The body of research exploring these differences is mixed, and a review is beyond the scope of this chapter (e.g., Schnall et al., 2010, 2012). However, it appears that for some Jewish individuals, internal components of religiosity such as personal (as opposed to ritualized and codified) prayer, religious motivations, and attachment to God may be more reliable predictors of flourishing. For example, in a cross-sectional study, Levin (2013) found that, among 1,849 Israeli Jews, personal prayer was associated with elevated life satisfaction and well-being, but synagogue attendance and formal prayer were not. Relatedly, in another cross-sectional study, Lazar (2015) found that the quality of prayer - prayer behavior, prayer type, and belief in prayer-predicted elevated life satisfaction better than did attending formal prayer. On the other hand, Pirutinsky et al. (2011) reported that for non-Orthodox Jews, social support mediated (explained) the relationship between religiosity and coping with health difficulties.

Finally, furthering the distinction between internal and external components of R/S, Pirutinsky et al. (2020) investigated the congruence of Jewish adherents' implicit attitudes and explicit beliefs about God. They reported that congruent, positive, implicit attitudes and explicit beliefs predicted the highest levels of life satisfaction. The lowest levels of life satisfaction were found among those with positive implicit attitudes and low levels of explicit positive beliefs. 
This finding suggests that integrated conscious and nonconscious beliefs are an important factor in determining religions' impact on well-being (see Davis et al., Chapter 18, this volume).

Religious Coping. Additional research focuses on the use of religious coping mechanisms to enhance well-being. British Jews who utilized religious coping methods after a major stressor were more likely to report positive affect (Loewenthal et al., 2000). Rosmarin, Pargament, Krumrei, and Flannelly (2009) created and validated a Jewish Religious Coping Scale (JCOPE) and found that positive religious coping predicted lower levels of anxiety, and negative religious coping predicted higher levels of depression and anxiety. The JCOPE added concepts particular to Judaism, such as "I look forward to Shabbat," "I talk to my rabbi," and "I try to do Mitzvot (good deeds)." Rosmarin, Pargament, and Mahoney (2009) created a Trust in God Scale, based on an $11^{\text {th }}$-century Jewish text, which draws on the strengths of spirituality, particularly trust in an omnipresent and benevolent God. These researchers found that divine trust was related to higher personal happiness and lower depression and anxiety. Based on these concepts, they developed a spiritually integrated treatment for anxiety. A subsequent study found that those who participated in this spiritually integrated treatment reported reduced worry, stress, depression, and intolerance of uncertainty (Rosmarin et al., 2010).

Based on the literature in the broader population, there are several conceptual theories that can explain the healing power of religious coping in Judaism. First, the strengthened interpersonal connections utilized by religious individuals in times of distress, such as speaking with one's rabbi, may increase a sense of ethnic identity and communal support (Pirutinsky \& Mancuso, 2011). Relatedly, the cycle of religious life (e.g., Sabbath and holiday meals, congregational service) may provide opportunities to develop a sense of belonging and a social support system, although limited research suggests that this mechanism may be more relevant to 
non-Orthodox Jews (Pirutinsky et al., 2011). Second, adherence to Jewish practice may strengthen one's sense of higher, spiritual purpose in life (see Park \& Van Tongeren, Chapter 6, this volume). These religious practices may also provide a potent form of behavioral activation that can protect against disengagement, sadness, and depression (Krumrei et al., 2013; Pirutinsky \& Rosmarin, 2018). Finally, prayer and other efforts to connect with God can foster positive emotions which can protect against worry and despair that emerge from anticipating further pain and helplessness (Rosmarin, Pargament, \& Mahoney, 2009; see Chapter 20, this volume).

\section{Implications and Applications for Clinical Practice}

Clinicians working with a Jewish client must be aware of Jewish interdenominational diversity and refrain from assumptions about Jewish R/S merely because the client identifies as Jewish (Schiffman, 2016). Even within a particular denomination, Schnall (2006) notes that "Orthodox Jewry is a diverse group, with many subgroups, and that members of the subgroups differ to a greater or lesser degree in their language, diet, worldview, dress, and even religious practice" (p. 277). The same is true of Reform Judaism (Gehl, 2014) and other subgroups.

Based on the framework and research presented, assuming a Jewish client is receptive, it may be beneficial to integrate religious themes within a therapeutic or coaching context. Clinicians can enhance commitment and motivation to therapeutic tasks, as well as bolster their efficacy, by framing happiness within a meaningful religious framework, explicitly framing therapeutic goals as a functional path to the fulfillment of commandments and connection to God, and sanctifying and spiritualizing the entire enterprise of character development.

Clinicians can also use biblical and rabbinic statements or other culturally familiar concepts to discuss tenets of positive psychology. Spiritually integrated clinical treatments often draw on biblical verses and religious proverbs to facilitate the acceptance of new positive 
cognitions (Pargament, 2007). In addition, religious stories may play a prominent role in treatment (Schnall et al., 2016). Positive psychology interventions drawing on Jewish tradition can utilize these same sources.

Finally, the distinction in the research between internal and external components of R/S has important implications. Interventions that aim to integrate Jewish tradition and positive psychology may benefit from addressing both the explicit (more doctrinal) and implicit (more experiential) aspects of spirituality. For example, an intervention utilizing traditional texts to advance ideas regarding gratitude or transcendence may be more effective when coupled with activities that promote personalized experiences such as prayer, meditation, and journaling.

\section{Implications and Applications for Research}

Research related to positive psychology and the psychology of R/S within the Jewish population is scarce. Judaism differs from other religions in significant ways (Cohen et al., 2013), and research specifically targeting Jewish variables is warranted. There is a clear need for more methodical and targeted research programs to help advance this field.

For example, to propel a more robust research agenda in the Jewish community, Levin (2013) called on researchers to stratify analyses denominationally. Cohen et al. (2013) noted that lack of denominational differentiation limits what conclusions can be drawn about potential influences. In a move in this direction, Cherniak et al. (2021) investigated how differences between the denominations, including centrality of ritual practice and gender-based obligations in ritual, may modify the link between R/S and well-being. Relatedly, researchers can take Beck and Haugen's (2013) suggestion to use empirical methods to explore the theological assumptions of Christians and do the same with Jews, which would assist in identifying to what extent the textual heritage and beliefs are internalized and serve as factors to increase well-being. 
Additionally, researchers could follow the lead of Rosmarin, Pargament, Krumrei, and Flannelly (2009) in generating measures that specifically reflect the character of Jewish R/S and other positive psychology constructs. Future research can also build on Rosmarin et al. (2011) and explore how other character strengths in addition to gratitude, such as forgiveness, humility, and compassion, may present differently when infused with religious/spiritual meaning.

Although this chapter continued the theoretical groundwork linking positive psychology's virtues to concepts in Judaism, there is an additional need to analyze the nuances of each trait through the prism of classic Judaic literature and culture. Similarly, more systematic applied research that will further develop and evaluate clinical and educational interventions is necessary. A collaboration between positive psychologists and Jewish studies scholars, Jewish community leaders, and those who integrate Jewish thought and practices into their lives, could lead to greater understanding and application of millennia-old Judaic wisdom.

\section{Conclusion}

The field of positive psychology is enhanced by taking into account the nuanced differences in relationships between psychology and well-being for each religion. Understanding Jewish conceptualizations of happiness, character, and spirituality deepens and further enlightens our understanding of the broader mechanisms by which virtues and character traits enhance emotional well-being and functioning. This benefit is bidirectional, as positive psychology, with its cutting-edge scientific research and empirically supported strategies, offers innovative angles and practical strategies for the practice of Judaism. Judaism, with its close to 4,000 years of history, offers tried and true wisdom and perspective that can enhance the field of positive psychology. Integration of the two has begun, yet there is still much more work to be done. 


\section{References}

Baskin, J. R. (2011). The Cambridge dictionary of Judaism and Jewish culture. Cambridge University Press.

Beck, R., \& Haugen, A. D. (2013). The Christian religion: A theological and psychological review. In K. I. Pargament, J. J. Exline, \& J. W. Jones (Eds.), APA handbook of psychology, religion, and spirituality (Vol. 1): Context, theory, and research (pp. 697-

711). American Psychological Association. https://doi.org/10.1037/14045-039

Ben-Shalom, M. (2004). Medieval Jewry in Christendom. In M. Goodman (Ed.) The Oxford handbook of Jewish studies. (pp. 153-192). Oxford University Press.

Blau, Y. (2000). The implications of a Jewish virtue ethic. Torah U-Madda Journal, 9, 19-41.

Brown, E. (2013). Leadership in the wilderness: Authority and anarchy in the book of Numbers. Maggid Books.

Cherniak, A. D., Pirutinsky, S., \& Rosmarin, D. H. (2021). Does gender moderate effects of religion on anxiety? [Unpublished manuscript]. Department of Psychology, Stockholm University, Stockholm, Sweden.

Cohen, A. B., Gorvine, B. J., \& Gorvine, H. (2013). The religion, spirituality, and psychology of Jews. In K. I. Pargament, J. J. Exline, \& J. W. Jones (Eds.), APA handbook of psychology, religion, and spirituality (Vol. 1): Context, theory, and research (pp. 665679). American Psychological Association. https://doi.org/10.1037/14045-037

DellaPergola, S. (2020). World Jewish Population, 2019. In American Jewish yearbook 2019 (pp. 263-353). Springer.

Delle Fave A., Massimini F., \& Bassi M. (2011). Psychological selection and optimal experience across cultures: Social empowerment through personal growth. Springer. 
Fishbane, M. (1995). The inwardness of joy in Jewish spirituality. Boston University Studies in Philosophy and Religion, 16, 71-88.

Gehl, N. (2014). Reform Judaism. In Leeming D.A. (Ed.) Encyclopedia of psychology and religion (pp. 1480-1482). Springer. https://doi.org/10.1007/978-1-4614-6086-2_9253

Hartman, D. (2010). Maimonides: Torah and philosophic quest (Expanded Edition). JPS.

Hezser, C. (2004). Classical Rabbinic literature. In M. Goodman (Ed.) The Oxford handbook of Jewish studies. (pp. 115-140). Oxford University Press.

Kalmenson, M. (2019). Positivity bias: Practical wisdom for positive living: Inspired by the life and teachings of the Lubavitcher Rebbe. Ezra Press.

Kor, A., Pirutinsky, S., Mikulincer, M., Shoshani, A., \& Miller, L. (2019). A longitudinal study of spirituality, character strengths, subjective well-being, and prosociality in middle school adolescents. Frontiers in Psychology, 10, 377. https://doi.org/10.3389/fpsyg.2019.00377

Krumrei, E. J., Pirutinsky, S., \& Rosmarin, D. H. (2013). Jewish spirituality, depression, and health: An empirical test of a conceptual framework. International Journal of Behavioral Medicine, 20(3), 327-336. https://doi.org/10.1007/s12529-012-9248-z

Lamm, N., Brill, A., \& Carmy, S. (1999). The religious thought of Hasidism: Text and commentary. Yeshiva University Press.

Lazar, A. (2015). The relation between prayer type and life satisfaction in religious Jewish men and women: The moderating effects of prayer duration and belief in prayer. The International Journal for the Psychology of Religion, 25(3), 211-229. https://doi.org/10.1080/10508619.2014.920603 
Levin, J. (2013). Religious behavior, health, and well-being among Israeli Jews: Findings from the European Social Survey. Psychology of Religion and Spirituality, 5(4), 272-282. https://doi.org/10.1037/a0032601

Loewenthal, K. M., MacLeod, A. K., Goldblatt, V., Lubitsh, G., \& Valentine, J. D. (2000). Comfort and joy? Religion, cognition, and mood in Protestants and Jews under stress. Cognition and Emotion, 14(3), 355-374. https://doi.org/10.1080/026999300378879

McCullough, M. E., \& Willoughby, B. L. B. (2009). Religion, self-regulation, and self-control: Associations, explanations, and implications. Psychological Bulletin, 135(1), 6993. https://doi.org/10.1037/a0014213

Pargament, K. I. (2007). Spiritually integrated psychotherapy: Understanding and addressing the sacred. Guilford Press.

Pargament, K. I., \& Mahoney, A. (2005). Sacred matters: Sanctification as a vital topic for the psychology of religion. The International Journal for the Psychology of Religion, 15(3), 179-198. https://doi.org/10.1207/s15327582ijpr1503_1

Pelcovitz, R., \& Pelcovitz, D. (2014). Life in the balance: Torah perspectives on positive psychology. Shaar Press.

Peterson, C., \& Seligman, M. E. P. (2004). Character strengths and virtues: A handbook and classification. American Psychological Association.

Pew Research Center (2016). Israel's Religiously Divided Society. Author.

Pew Research Center. (2021). Jewish Americans in 2020. Author.

Piedmont, R. L. (1999). Does spirituality represent the sixth factor of personality? Spiritual transcendence and the five-factor model. Journal of Personality, 67(6), 985-1013. https://doi.org/10.1111/1467-6494.00080 
Pirutinsky, S. (2014). Does religiousness increase self-control and reduce criminal behavior? A longitudinal analysis of adolescent offenders. Criminal Justice and Behavior, 41(11), $1290-1307$.

Pirutinsky, S. (2020). I created the evil inclination and I created Torah its antidote: An indigenous Jewish psychology. In T. A. Sisemore \& J. J. Knabbs (Eds.), The psychology of world religions and spiritualties: An indigenous perspective (pp. 59-84). Templeton University Press.

Pirutinsky, S., Cherniak, A. D., \& Rosmarin, D. H. (2020). Implicit and explicit attitudes towards God and life satisfaction. Psychology of Religion and Spirituality, 12(4), 387392. https://doi.org/10.1037/rel0000250

Pirutinsky, S., \& Mancuso, A. F. (2011). Who are we? Social identity and psychological wellbeing. Graduate Student Journal of Psychology, 13, 39-44.

Pirutinsky, S., \& Rosmarin, D. H. (2018). Protective and harmful effects of religious practice on depression among Jewish individuals with mood disorders. Clinical Psychological Science, 6(4), 601-609. https://doi.org/10.1177/2167702617748402

Pirutinsky, S., Rosmarin, D. H., Holt, C. L., Feldman, R. H., Caplan, L. S., Midlarsky, E., \& Pargament, K. I. (2011). Does social support mediate the moderating effect of intrinsic religiosity on the relationship between physical health and depressive symptoms among Jews? Journal of Behavioral Medicine, 34(6), 489-496. https://doi.org/10.1007/s10865$\underline{011-9325-9}$

Rosmarin, D. H., Krumrei, E. J., \& Pargament, K. I. (2010). Are gratitude and spirituality protective factors against psychopathology? International Journal of Existential Positive Psychology, 3(1), 799-808. 
Rosmarin, D. H., Pargament, K. I., Krumrei, E. J., \& Flannelly, K. J. (2009). Religious coping among Jews: Development and initial validation of the JCOPE. Journal of Clinical Psychology, 65(7), 670-683. https://doi.org/10.1002/jclp.20574

Rosmarin, D. H., Pargament, K. I., \& Mahoney, A. (2009). The role of religiousness in anxiety, depression, and happiness in a Jewish community sample: A preliminary investigation. Mental Health, Religion and Culture, 12(2), 97-113. https://doi.org/10.1080/13674670802321933

Rosmarin, D. H., Pargament, K. I., Pirutinsky, S., \& Mahoney, A. (2010). A randomized controlled evaluation of a spiritually integrated treatment for subclinical anxiety in the Jewish community, delivered via the Internet. Journal of Anxiety Disorders, 24(7), 799808. https://doi.org/10.1037/rel0000021

Rosmarin, D. H., Pirutinsky, S., Cohen, A. B., Galler, Y., \& Krumrei, E. J. (2011). Grateful to God or just plain grateful? A comparison of religious and general gratitude. The Journal of Positive Psychology, 6(5), 389-396. https://doi.org/10.1080/17439760.2011.596557

Rosmarin, D. H., Pirutinsky, S., Greer, D., \& Korbman, M. (2016). Maintaining a grateful disposition in the face of distress: The role of religious coping. Psychology of Religion and Spirituality, 8(2), 134-140. https://doi.org/10.1037/rel0000021

Sacks, J. (2007). To heal a fractured world: The ethics of responsibility. Schocken.

Sacks, J. (2014). Happiness: A Jewish perspective. Journal of Law and Religion, 29 (1), 30-47. Sacks, J. (2015). Lessons in leadership: A weekly reading of the Jewish Hebrew Bible. Maggid. Schnall, E. (2006). Multicultural counseling and the Orthodox Jew. Journal of Counseling and Development, 84, 276-282. https://doi.org/10.1002/j.1556-6678.2006.tb00406.x 
Schnall, E., Eichenbaum, B., \& Abramowitz, Y. (2016). Jewish stories in mental health counseling. Journal of Creativity in Mental Health, 11, 12-26. https://doi.org/10.1080/15401383.2015.1130667

Schnall, E., \& Greenberg, M. J. (2012). Groupthink and the Sanhedrin: An analysis of the ancient court of Israel through the lens of modern social psychology. Journal of Management History, 18, 285-294. https://doi.org/10.1108/17511341211236228

Schnall, E., Kalkstein, S., Fitchett, G., Salmoirago-Blotcher, E., Ockene, J., Tindle, H. A., Thomas, A., Hunt, J. R., \& Wassertheil-Smoller S. (2012). Psychological and social characteristics associated with religiosity in women's health initiative participants. Journal of Religion and Health, 51, 20-31. https://doi.org/10.1007/s10943-011-9549-6

Schnall, E., Schiffman, M., \& Cherniak, A. (2014). Values that transcend: Positive psychology in Jewish texts and tradition. In C. Kim-Prieto (Ed.), Religion and spirituality across cultures (pp. 21-45). Springer.

Schnall, E., Wassertheil-Smoller S., Swencionis, C., Zemon, V., Tinker, L., O’Sullivan, M. J., Van Horn, L., \& Goodwin, M. (2010). The relationship between religion and cardiovascular outcomes and all-cause mortality in the women's health initiative observational study. Psychology and Health, 25, 249-263. https://doi.org/10.1080/08870440802311322

Schiffman, M. (2016). Incorporating Jewish texts with REBT in the treatment of clinical anger. Journal of Rational-Emotive \& Cognitive-Behavior Therapy, 34(3), 225-239. https://doi.org/10.1007/s10942-016-0239-x

Scholem, G., Garb, J., \& Idel, M. (2007). Kabbalah. In M. Berenbaum \& F. Skolnik (Eds.), Encyclopaedia Judaica (2 ${ }^{\text {nd }}$ ed., Vol. 11, pp. 586-692). Macmillan Reference. 
Seligman, M. (2011). Flourish. Free Press.

Seligman, M. E., Steen, T. A., Park, N., \& Peterson, C. (2005). Positive psychology progress: Empirical validation of interventions. American Psychologist, 60(5), 410-421. https://doi.org/10.1037/0003-066x.60.5.410

Sheskin, I. M., \& Dashefsky, A. (2020). United States Jewish Population, 2019. In American Jewish Year Book 2019 (pp. 135-231). Springer.

Soloveitchik, J. D. (1983). Halakhic man (1 ${ }^{\text {st }}$ English ed.). Jewish Publication Society.

Tirosh-Samuelson, H. (2003). Philosophy and kabbalah: 1200-1600. In D. H. Frank and O.

Leaman (Eds.). The Cambridge companion to medieval Jewish philosophy (pp. 218-257). Cambridge University Press. https://doi.org/10.1017/ccol0521652073.011

Twersky, I. (Ed.). (1972). A Maimonides reader. Behrman House.

Vilchinsky, N., \& Kravetz, S. (2005). How are religious belief and behavior good for you? An investigation of mediators relating religion to mental health in a sample of Israeli Jewish students. Journal for the Scientific Study of Religion, 44(4), 459-471. https://doi.org/10.1111/j.1468-5906.2005.00297.x

Wurzburger, W. S. (1994). Ethics of responsibility: Pluralistic approaches to covenantal ethics. Jewish Publication Society. 


\section{Chapter 12:}

\section{Living the Good Life: An Islamic Perspective on Positive Psychology}

Seyma N. Saritoprak, PhD

Case Western Reserve University

Hisham Abu-Raiya, $\mathrm{PhD}$

Tel Aviv University

Correspondence concerning this chapter should be addressed to Seyma N. Saritoprak.

Email: seyma.saritoprak@case.edu 


\begin{abstract}
This chapter aims to explore Muslims' religion/spirituality and discuss the factors that are important to consider in scientific research and clinical practice at the intersections of positive psychology, religion, and spirituality. The discussion is based on the theological teachings of the Islamic religion, theoretical speculations regarding the links between Islam and positive psychology, and the relevant findings of empirical studies conducted with Muslim samples. The chapter is composed of five sections. The first is an overview of the geographic distribution, demographic composition, and intrafaith diversity of Muslims around the world. The second section reviews key Muslim religious/spiritual beliefs, teachings, practices, and texts. The third section discusses common Muslim understandings of virtues, health, and well-being. The fourth section reviews potential implications for science and practice at the intersections of positive psychology, religion, and spirituality. The final section offers recommendations for guiding research and practice when working with Muslims at these intersections.
\end{abstract}

Keywords: Islam, Muslims, religion, spirituality, positive psychology 


\section{Chapter 12: Living the Good Life: An Islamic Perspective on Positive Psychology}

Islam means submission to the will of God. In the present chapter, we describe Islam as a religion and explore its connections to positive psychology by reviewing research that examines positive psychological constructs as experienced by Muslims. We do so in five major sections. First, we overview the reach of Islam throughout the world. Then we review key Muslim religious/spiritual beliefs, teachings, practices, and texts. In the third section, we discuss common Muslim understandings of virtues, health, and well-being. In the penultimate section, we review potential implications for science and practice at the intersections of positive psychology (PP) and religion/spirituality (R/S). Finally, we make recommendations for guiding research and practice when working with Muslims at these intersections.

\section{The Worldwide Reach of Islam}

The world's Muslim population is large and growing fast. Muslims compose about 1.8 billion of the world's current population (24\%) and are projected to represent $31 \%$ of individuals worldwide by 2060 (Pew, 2017). Muslims can vary in demographics, depending on geography, ethnicity, and language; thus, they are an inherently diverse population. For example, Muslims in the United States are the most ethnically and racially diverse faith community (Mogahed \& Chouhoud, 2017). There are two major sects in Islam: Sunni and Shi'a. About $85 \%$ of Muslims identify as Sunni Muslims, whereas 15\% identify as Shi'a Muslims. Historically, the division between Sunni (meaning "people of tradition") and Shi'a (meaning "followers of Ali") Muslims stems from the disagreement regarding the successor of Prophet Muhammad. Following the death of Prophet Muhammad, a majority of Muslims (later referred to as Sunni Muslims) believed the successor should be appointed through consensus, whereas a minority of Muslims (later referred to as Shi'a Muslims) believed the successor should be 
Prophet Muhammad's cousin, due to kinship. Although there are differences between these two major branches of Islam, Sunni and Shi'a Muslims predominantly abide by the same religious doctrine.

Similarly, although the Muslim population's tremendous diversity reflects inevitable variations, there are central Islamic beliefs and practices that unite Muslims around the globe. For instance, a recent worldwide survey found that Islam was reported to be very important to Muslims (69\% to 99\%) living in Africa, the Middle East, and South Asia; likewise, in the United States, $66 \%$ of Muslims described Islam as very important to them (Pew Research Center, 2018). Although Islam may play a substantial role in the lives of many Muslims, it is important to keep in mind that there both are Muslims who do not practice Islam and Muslims who practice Islam to varying degrees. Thus, an important caveat for the current chapter is that the information presented is framed from an Islamic perspective but will not apply equally to all Muslims. The majority of this chapter's content will apply most squarely to practicing Muslims.

\section{Key Islamic Beliefs and Practices}

Key Islamic beliefs and practices are derived from two primary sources: the Qur'an and

the hadith. Muslims believe the Qur'an is the direct Word of God, which was revealed in Arabic to Prophet Muhammad over the span of 23 years (610 to $632 \mathrm{CE}$ ). The Qur'an is an integral part of many Muslims' lives, and it establishes the overarching meaning framework for guiding Muslims as they seek to live a good life. Correspondingly, the hadith are sayings and actions of Prophet Muhammad that were recorded and transmitted over centuries. The hadith are a major source for understanding legal and theological Islamic tradition. In sum, Muslims rely on both the Qur'an and Prophet Muhammad's life, actions, and sayings to guide their lives.

Islam emphasizes both faith and practice. First, six articles of faith establish the main doctrines of Islam. The first article of faith emphasizes belief in the existence and oneness of 
God (tawheed). Tawheed is the backbone of Islam and is based on the tenet that God has no equal or offspring and is the sole entity of worship. The second article of faith is the belief in angels. Angels are viewed as immortal entities who carry out God's commands and can serve as messengers between God and humans. The third article refers to believing in the holy scriptures of God (Qur'an, Torah, Gospel, and Psalms). Belief in all four books is an essential component of Islamic faith. The Qur'an acknowledges prior revelations but is proclaimed to be the final revelation that completes prior holy scriptures.

The fourth article of faith is the belief in the prophets and messengers of God (e.g., Moses, Jesus, and Abraham), including the belief that Prophet Muhammad is the final messenger of God. The fifth article is the belief in the Day of Judgment. According to Islamic thought, the Day of Judgment is the day when humans will ultimately be resurrected, held accountable for all deeds and actions, and accordingly receive reward or punishment. The sixth article of faith is believing in Divine decree, which proposes that all events (whether good or bad) occur within God's Will, Power, and Knowledge. At the same time, belief in Divine decree does not negate human will; Muslims are responsible for their actions, which can govern their destiny.

In addition, the five pillars of Islam were formulated as the fundamentals of worship and the primary obligations of a practicing Muslim. The first pillar, shahadah, is the verbal profession of faith, which refers to the statement "There is no god but God [Allah] and Muhammad is the messenger of God"; this pillar underscores the monotheistic nature of Islam (Esposito, 2002). The second pillar, salah, refers to the daily obligatory prayers. Muslims are prescribed to offer five prayers that take place at certain times of the day (dawn, noon, midafternoon, sunset, and evening). The obligatory prayers involve a series of physical movements such as standing, kneeling, bowing, and touching the ground with one's forehead. 
When completing salah, Muslims orient themselves towards the Ka'bah-the holy shrine. The five daily prayers help Muslims cultivate a God-conscious mindset amid their worldly concerns and help nurture a sense of submission, connection, and gratitude towards God (Esposito, 2002).

The third pillar, zakat (translated as purification), is the obligatory act of almsgiving. Zakat refers to the fortunate giving to the less fortunate. Muslims with financial ability are required to donate $2.5 \%$ of their yearly wealth and assets to individuals of less fortunate socioeconomic backgrounds. The fourth pillar, syiam, is fasting during the month of Ramadan. Around the globe, Muslims who have the health and ability to do so are required to fast daily (from dawn to dusk) throughout the ninth month of the lunar calendar (Ramadan). Importantly, this fasting not only involves abstaining from eating and drinking but also involves abstaining from behaviors considered sinful, such as gossiping, lying, and cheating. Ramadan is intended to be a month of spiritual reflection (Esposito, 2002), and it also promotes a sense of social solidarity — both locally and globally. The fifth pillar, hajj, is the pilgrimage to Mecca. Muslims who have the physical and financial means are required to visit the holy shrine Ka'bah, located in Mecca, Saudi Arabia. Taking part in hajj is believed to cleanse a Muslim of past sins and to renew and deepen their faith. It is often considered one of the highest forms of worship.

\section{Common Muslim Understandings of Virtues, Health, and Well-Being}

\section{The Good Life}

An Islamic understanding of health and well-being maintains that a good life is primarily achieved through living in accordance with Islamic teachings. Thus, studying PP from a Muslim perspective must entail the study of virtues drawn both from PP and the Islamic religion. Nevertheless, it is noteworthy that Muslims consider Islam as much more than a religion. Through the Qur'an and hadith, Islam is viewed as a comprehensive social, psychological, and 
spiritual guide that governs all domains of a Muslim's life. Both beliefs and behaviors (practices) are seen as central, interconnected components in living a good life along the path of God, as reflected in the Qur'anic verse "Whosoever acts righteously—whether a man or a woman —and embraces belief, We will surely grant him a good life; and will surely grant such persons their reward according to the best of their deeds" (16:97). Even so, living a good life is not necessarily an end goal for Muslims; instead, Muslims' end goal is to obtain God's pleasure. Thus, Muslims view the quest to live a good life as a lifelong journey.

\section{Islamic Faith and Well-Being}

Numerous studies have found an association between religiousness and psychological well-being among Muslims (e.g., Abu-Raiya \& Agbaria, 2016). For example, data gathered from large, Kuwaiti-Muslim youth samples has revealed evidence that overall religiousness is related to higher happiness (Abdel-Khalek, 2007; $N=5,042$ ) and life satisfaction (Baroun, 2006; $N=$ 941), as well as lower depression and anxiety (Abdel-Khalek, 2007). Similarly, research among Pakistani Muslims $(N=200)$ has found that Islamic spirituality is associated with more positive mood and higher meaning in life (Khan et al., 2015). Moreover, among Muslim undergraduates in England $(N=60)$, taking part in religious activities was ranked as their most important source of personal meaning, on average (Aflakseir, 2012). However, there is a dearth of literature examining more specific Islamic beliefs and practices that may contribute to well-being. Hence, the following section proposes some common Islamic beliefs and practices that may foster health and well-being from an Islamic perspective. Wherever possible, we incorporate discussion of prior empirical studies of R/S and psychological outcomes among Muslims. 


\section{Trust in God}

Prominent Islamic scholars have highlighted tawakkul (reliance on or putting trust in God) as a construct of high spiritual virtue among Muslims (Al-Ghazzali, 1972). The Qur'an frequently encourages Muslims to put their trust in God, as reflected in the verse "Put your trust in Allah: Allah is sufficient as Guardian" (3:33). Trusting God is viewed as particularly central in the face of distressing life events, and it is the ultimate framework for receiving blessings in the worldly life and in eternal life (Huda et al., 2019). For example, Prophet Muhammad stated "If you trusted Allah with due tawakkul [i.e., reliance and trust in God], He would provide you sustenance as He provides for the birds; they go out in the morning with empty stomachs and come back in the evening with full stomachs" (Al-Tirmidhi, 1975, p. 573).

Data on tawakkul among Muslims is limited to a few qualitative studies, yet the available research suggests tawakkul may play a frequent role in Muslims' coping with adversity. For example, qualitive data from Palestinian Muslim women diagnosed with breast cancer revealed that tawakkul played an important role in their positive coping and coming to terms with their illness (Hammoudeh et al., 2017). Similarly, Iranian Muslims diagnosed with end-stage renal disease reported their tawakkul helped foster a greater sense of peace and tolerance of their difficulties (Shahgholian \& Yousefi, 2015). Iranian Muslim patients with hemophilia also indicated that tawakkul helped them in their coping and problem solving (Rambod et al., 2019). Islamic scholars have suggested tawakkul can foster positive attitudes toward God, a feeling of security during adversity, and a sense of peace (Khodayarifard et al., 2016).

\section{Worship}

From an Islamic perspective, worshiping God is a fundamental component of well-being (Joshanloo, 2013). The Qur'an indicates: “And I did not create the jinn and mankind except to worship Me" (51:56), which underscores the Islamic belief that the act of worshiping God is the 
primary reason for the creation of humankind. Similarly, Prophet Muhammad stated: "Goodness and comfort are for him who worships his Lord in a perfect manner and serves his master sincerely" (Al-Bukhari, 2001, p. 149). Within the Islamic tradition, worshiping God may entail any deed that is conducted in accordance with Islamic teachings, such as (but certainly not limited to) praying, fasting, giving charity, beginning a meal with bismillah (in the name of God), and visiting a sick person. Everyday activities that are mundane in nature can take on a deeper sense of meaning when completed with the intentional purpose of worship. Indeed, a recent meta-analysis in the psychology of R/S field revealed the sanctification of various aspects of life is linked with better positive psychosocial adjustment (Mahoney et al., 2021).

Additionally, worship can foster both a sense of connectedness with God and a sense of transcendence. According to Ibn Sina — a historically prominent Islamic philosopher and physician - true happiness is attained through the knowledge and contemplation of God (Awaludin, 2017). From an Islamic perspective, human nature is inherently inclined toward knowing and worshiping God; thus, a harmonious psychological state is viewed as achievable through connecting meaningfully with God (Rothman \& Coyle, 2018). For example, a qualitative study of Jordanian Muslims with heart disease found that participants viewed worshiping God as central to their life, and their faith in God and engagement in prayer fostered a sense of hope, inner strength, meaning and purpose in life, and acceptance of self-responsibility (Nabolsi \& Carson, 2011). Furthermore, a quantitative study of Canadian Muslims found that spiritual experiences of perceived connectedness with God were related to higher prayer frequency and life satisfaction (Albatnuni \& Koszycki, 2020).

\section{Gratitude}

Throughout the Qur' an and hadith, practicing the virtue of gratitude is encouraged. For example, one of many verses reminding Muslims to express gratitude is the Qur'anic verse "So 
remember Me; I will remember you. And be grateful to Me and do not deny Me" (2:152). In fact, practicing gratitude is thought to lead to increased favor and blessings, as reflected in the verse “And [remember] when your Lord proclaimed, 'If you are grateful, I will surely increase you [in favor]"” (14:7). Along similar lines, Prophet Muhammad stated: "Allah is pleased with His servant if, when he eats something, he thanks Allah for it, and when he drinks something, he thanks Allah for it" (Muslim, 1967, p. 2095), suggesting gratitude enhances God's pleasure.

There are a few studies of gratitude among Muslims. In a study of Muslim (and Hindu) young adults $(N=152)$ in India, trait gratitude was associated with higher psychological wellbeing (Anas et al., 2015). Similarly, a study of Muslim university students in Pakistan $(N=230)$ found that trait gratitude predicted higher life satisfaction (Perveen \& Yasin, 2017). In a longitudinal study of earthquake survivors $(N=310)$ in Indonesia (a predominantly Muslim country), trait gratitude was associated with lower concurrent posttraumatic stress at 8 months postdisaster, even after controlling for personality and coping-related factors (Lies et al., 2014).

\section{Patience}

Patience $(s a b r)$ is prescribed throughout the Qur' an and hadith, and as such, it is a core Islamic virtue. Patience is seen as both the ability to restrain oneself from what is discouraged (or prohibited) and as an essential ingredient for pursuing goals while practicing self-control and endurance (El-Aswad, 2014). The Qur'an indicates that believers who practice patience will be “attainers of success" (23:111). The Qur'an underscores the notion that goodness comes from engaging in patience, as reflected in the verse "But only those who are steadfast in patience, only those who are blessed with great righteousness, will attain to such goodness" (41:35).

Correspondingly, the hadith encourages patience in the face of hardships and promises Muslims that God will forgive their sins in response to their patience: "Never a believer is stricken with a 
discomfort, an illness, an anxiety, a grief or mental worry or even the pricking of a thorn but Allah will expiate his sins on account of his patience" (Al-Bukhari, 2001, p. 114).

Psychological studies of patience among Muslims are scarce. However, a qualitative study of Pakistani Muslims with chronic illness $(N=31)$ found that patience helped alleviate participants' anger and support their meaning-making coping with their illness (Mir \& Sheikh, 2010). Furthermore, in a quantitative study of Muslim survivors of a volcanic disaster in Indonesia $(N=68)$, participants in a patience- and prayer-focused spiritual intervention evidenced decreased psychopathological symptoms at post-treatment, relative to the control group (Uyun \& Witruk, 2017). Moreover, in a qualitative study of older Muslim women who immigrated to Belgium $(N=30)$, patience was identified as a centrally important virtue to enact in the face of suffering and as a means by which Muslims develop a closer relationship with God, obtain forgiveness of their sins, and enhance their well-being (Baeke et al., 2012).

\section{Forgiveness}

Concepts of forgiveness such as afw (pardon), safh (overlooking a wrong), and ghafir (erasing sin) are collectively mentioned 277 times in the Qur'an (McCullough et al., 2001). Because humans are inevitably prone to making mistakes, hurting each other, and engaging in transgressions, forgiveness is an essential virtue within Islam. God encourages Muslims to ask Him for forgiveness for wrongdoings, as evidenced by the verse "And whoever does a wrong or wrongs himself but then seeks forgiveness of Allah will find Allah Forgiving and Merciful" (4:110). Not only is seeking divine forgiveness from God encouraged, but practicing interpersonal forgiveness is encouraged as well. In fact, the Qur'an suggests divine forgiveness can be fostered through forgiving other humans, as reflected in the verse "Let them forgive and overlook, do you not wish that Allah should forgive you? For Allah is Oft- 
Forgiving, Most Merciful" (24:22). Likewise, Prophet Muhammad stated: "When you come to one who is in straitened circumstances forgive him, for perhaps God may forgive us" (AlBukhari, 2001, p. 176).

A few studies have found evidence that interpersonal forgiveness is associated with better psychological and spiritual outcomes. A qualitative study of Pakistani Muslims $(N=20)$ found that participants reported their practice of forgiveness led to greater happiness, life satisfaction, confidence, relaxation, positive social interactions, and spiritual development (Gull \& Rana, 2013). Similarly, in a quantitative study of Turkish Muslim university students $(N=$ 560), forgiveness predicted both higher life satisfaction and lower generalized anxiety (Ayten \& Karagoz, 2021). These findings were replicated in a large multinational Muslim sample $(N=$ 706), in which forgiveness was associated with higher life satisfaction, lower generalized anxiety, and more involvement in religious/spiritual practices (Abu-Raiya \& Ayten, 2019).

\section{Awareness of an Afterlife}

Belief in the afterlife is a fundamental component of the Islamic faith as well. The worldly life is viewed as a temporary place of residence prior to entering the eternal life. Life is understood as a place of trials and tests, and a Muslim's actions during these tests and trials are thought to determine whether they enter heaven or hell on the Day of Judgment, as mentioned in the Qur'an: "[He] who created death and life to test you [as to] which of you is best in deed" (67:2). The Qur'an promises “a great reward” (64:15) for Muslims who act in accordance with Islamic teachings in the face of such trials, promising that "the Hereafter is better than the first [life]" (93:4). Hence, Muslims often live with strong awareness of an afterlife; although one's worldly life can be good, Muslims' ultimate goal is to achieve well-being in the afterlife through obtaining God's pleasure in the worldly life. 
A few studies have examined the role of afterlife beliefs on Muslims' well-being. Living in awareness of the afterlife can possibly foster Muslims' sense of consciousness regarding their behaviors, helping promote delayed gratification, circumspect perspective-taking, and thoughtful choices in accordance with Islamic teachings. For example, Muslims can reframe their worries as being temporary worldly concerns (Hamdan, 2008). In a similar way, a qualitative study of Muslim Moroccan women $(N=15)$ found it was common for these women to view death as a liberation from an unfair worldly life and as a transition to a more peaceful and sincere life (Ahaddour et al., 2019). In a quantitative study with an elderly Pakistani Muslim sample, having positive afterlife beliefs was associated with higher religiousness and lower depression symptoms (Ghayas et al., 2021). Moreover, a qualitative study of Somali refugees ( $N$ $=42$ ) found that participants described their works (actions) in this life as helping them earn a good afterlife (McMichael, 2002).

\section{Implications for Science and Practice}

Studies examining the role of specific Islamic beliefs and practices on Muslims' wellbeing are scarce within the field of psychology. The available literature predominantly involves qualitative studies or cross-sectional studies examining the association between general Islamic religiousness and well-being. Although such studies are beneficial in recognizing the links between the Islam religion and well-being, there is little to no longitudinal or experimental evidence examining the causal direction of this relationship (i.e., whether Islamic religiousness enhances well-being, well-being leads to increased religiousness, or both). There also is very limited examination of the effects of specific Islamic beliefs and practices on well-being. Additionally, studies of Islamic spiritual or psychological interventions are sparse, so there 
currently is little practical guidance for whether, when, and how Muslims might benefit from certain interventions (e.g., Muslim-accommodated forms of evidence-based interventions).

Furthermore, there is a need for examining the Qur'an and hadith more systematically to identify religiously indigenous PP constructs (e.g., ways of understanding and cultivating virtue and well-being from a distinctly Islamic perspective). For example, a content analysis of the Qur'an, Riaz (2015) identified 41 categories of PP-relevant constructs (e.g., contentment, humility). Similar studies may help develop and refine a positive Islamic psychology framework.

Currently, most of the PP literature is grounded in Western ideas and ideals, which are often overly secular and culturally bound, thereby limiting their validity, utility, and generalizability among (a) Muslims generally, (b) specific subgroups of Muslims (e.g., Sunni and Shi'a Muslims), and (c) Muslims in particular cultures around the world. Regarding the latter point, given the geographic and cultural diversity among Muslims, there is a pressing need for culture-focused theory and research at the intersections of PP, Islamic religiousness, and wellbeing (see Mattis, Chapter 9, this volume). For instance, cultural psychologists have called for the development of indigenous PP models that better capture the strengths, values, ideas, and positive characteristics of often neglected non-Western cultural groups (Lambert et al., 2015).

Preliminary findings from intervention studies highlight the potential promise of developing distinctly Islamic PP interventions for use with Muslims. For example, in a quasiexperimental study of Muslim women with multiple sclerosis $(N=40)$, participants in a sevensession Islamic PP program (designed to improve participants' relationships with themselves, others, and the world) evidenced higher gains in self-reported quality of life than people in the no-treatment control group (Saeedi et al., 2015). Furthermore, researchers examining Iranian Muslim participants $(N=65)$ with breast cancer found that participants who were assigned to a 
6-week spiritual well-being intervention group endorsed significantly higher postintervention spiritual well-being in comparison to the participants assigned to the control group (which was psychoeducational in nature; Jafari et al., 2013). However, the study did not include a secular form of psychotherapy as an active comparison group. Similarly, in qualitative data from a pilot study with Somali Muslims $(N=26)$, participants in an Islamic-accommodated, trauma-focused intervention reported they benefited from the incorporation of Islamic beliefs and practices (e.g., Prophetic traditions, verses from the Qur'an, prayer to God) into the treatment (Zoellner et al., 2021); however, it is worth noting that this pilot study lacked a control condition.

Moreover, establishing an evidence-based wellness intervention for practice will require bridging together Islamic beliefs and practices within a psychological model that is effective in promoting well-being. For example, researchers have suggested that well-being can be facilitated through Acceptance and Commitment Therapy (ACT) within an Islamic context, given theoretical commonalities between ACT and Islam (Tanhan, 2019). Nevertheless, empirical studies are necessary to establish such posited links. Notably, Captari et al. (2018) recently conducted a meta-analysis investigating the efficacy of R/S-modified psychotherapy and found that R/S-adapted psychotherapy was as effective as standard secular forms of psychotherapy in promoting psychological outcomes and was more effective in fostering spiritual outcomes. Similarly, treatments adapting and incorporating cultural values have exhibited larger effect sizes in comparison to treatments that do not modify methods (Soto et al., 2018). Integrating elements of one's R/S and culture into standards forms of psychotherapy may be particularly relevant for Muslims, given the modally salient role of Islam and culture in their lives, identities, and worldviews. 
Despite several interventionists who have accommodated treatments to Muslim beliefs and practices, limited randomized controlled trials have demonstrated that Muslimaccommodated treatments are equally or more effective than nonaccommodated treatments. For example, a study examining a culturally and religiously adapted form of CBT revealed that Pakistani participants $(N=137)$ assigned to the treatment group endorsed significantly greater improvements in depression, anxiety, somatic, and disability scores compared to the participants in the control group (Naeem et al., 2015). However, the study did not examine a standard (nonadapted) form of CBT. Thus, the available studies stress the need for more rigorous research in order to develop, test, and refine evidence-based, Muslim-accommodated interventions. In particular, randomized controlled trials are needed to test whether gains made by accommodating to Islam are as or more effective than standard (i.e., "secular") treatments.

\section{Recommendations for Guiding Research and Practice}

By incorporating specific Islamic beliefs and practices into treatment, clinicians working with Muslims in diverse settings might help foster Muslim clients' well-being and sense of living a good life; however, more scientific data is needed to establish support for such claims. To begin with, mental health providers, whether Muslim or non-Muslim, may inquire the degree to which Islamic beliefs and practices are important in a client's everyday life. For non-Muslim clinicians, additional steps may be necessary to become familiar with their client's belief system. Given the central relevance of Islam for practicing Muslims, clinicians are encouraged to develop their religious/spiritual clinical competencies (Vieten \& Lukoff, 2021). For example, mental health providers can learn how to explore clients' religious/spiritual sources of support and strength while also addressing clients' religious/spiritual struggles. Given the geographic diversity of the Muslim population, clinicians should also be mindful of the role of culture. Such 
information will foster culturally sensitive explanations of a client's behaviors, thoughts, and emotions, as well as an understanding of what may (or may not) be adaptive (Abu-Raiya, 2015).

As part of culturally responsive practice, clinicians can draw on Islamic concepts to promote clients' well-being. Promoting well-being through an Islamic perspective could include the incorporation of Qur'anic verses and the sayings and actions of Prophet Muhammad. Doing so might help clients connect better with the content of psychotherapy. For example, clients may be encouraged to discuss how specific Qur'anic verses or hadith teachings relate to their perception of life in general, their approach to their current experience, and their psychological and spiritual well-being (Abu-Raiya, 2015). Specific interventions may include developing R/Sfocused client goals (such as enhancing one's relationship with God), dialoguing about how the presence of an afterlife relates to clients' making meaning of their current circumstances, or exploring how Islamic concepts of patience and trust in God can promote clients' well-being. However, it is important to note that there is a dearth of clinical research with Muslims, and further research is necessary in order to ascertain the effectiveness of such possible interventions. Importantly, research suggests that imams (Muslim religious leaders) can play a prominent role in promoting healthy behaviors among Muslim communities and that imams may even serve as an alternative to behavioral health providers in providing support for mental health-related concerns (Padela et al., 2011). Imams can provide faith-based interventions on an array of topics, given their knowledge in Islamic theology and their expertise in Muslim practices. Thus, they can play a role in fostering well-being in R/S-grounded ways. Although there is little available empirical data on mosque-based interventions, one qualitative study found that mosque members expressed interest in imam-led sermons on health education (Vu et al., 2018); the participants interviewed in this study viewed the mosque as a space to target a wider 
community audience, and they reported having a preference for imams who are more knowledgeable in health-related topics. Thus, prior to disseminating information, it may be important for imams to work closely with mental health consultants to receive training and education on well-being related constructs from a psychological and scientific perspective.

Finally, studies examining Muslim and/or non-Muslim chaplains have revealed evidence that Muslim patients in a hospital setting often incorporate religious beliefs and practices in the face of their illness (Abu-Ras \& Laird, 2011). Thus, chaplains working with Muslim patients in hospital settings can help promote psychological well-being within the context of patients' physical illness. In a qualitative study focusing on Muslims with chronic illness in Iran, a chaplain visiting patients in the home and the hospital noted the importance of having R/Sfocused discussions that help chronically ill Muslim patients make meaning of their health status (Irajpur \& Moghimiyan, 2018). Muslims in this study who were patients with chronic illness reported a need to engage in prayer to build a relationship with God and a need for Divine forgiveness (from God) and interpersonal forgiveness (from others) to help them feel a sense of peace and acceptance in the face of their death (Irajpur \& Moghimiyan, 2018). In facilitating conversations about specific Islamic beliefs and practices, chaplains can help address possible emotional and/or spiritual distress while also helping foster a sense of peace and comfort.

\section{Conclusion}

The study of positive psychology from an Islamic perspective continues to be an avenue for growth in both research and clinical practice. The constructs proposed in the current chapter can contribute to the development of R/S-accommodated psychological interventions tailored for Muslims. Through more rigorous research and systematic studies of Islam and well-being, psychology can play a vital role in helping Muslims live a good life. 


\section{References}

Abdel-Khalek, A. M. (2007). Religiosity, happiness, health, and psychopathology in a probability sample of Muslim adolescents. Mental Health, Religion and Culture, 10(6), 571-583. https://doi.org/10.1080/13674670601034547

Abu-Raiya, H. (2015). Working with religious Muslim clients: A dynamic, Qura'nic-based model of psychotherapy. Spirituality in Clinical Practice, 2(2), 120-

133. https://doi.org/10.1037/scp0000068

Abu-Raiya, H., \& Agbaria, Q. (2016). Religiousness and subjective well-being among IsraeliPalestinian college students: Direct or mediated links? Social Indicators Research, 126(2), 829-844. https://doi.org/10.1007/s11205-015-0913-x

Abu-Raiya, H., \& Ayten, A. (2019). Religious involvement, interpersonal forgiveness and mental health and well-being among a multinational sample of Muslims. Journal of Happiness Studies, 21, 3051-3067. https://doi.org/10.1007/s10902-019-00213-8

Abu-Ras, W., \& Laird, L. (2011). How Muslim and non-Muslim chaplains serve Muslim patients? Does the interfaith chaplaincy model have room for Muslims’ experiences? Journal of Religion and Health, 50(1), 46-61. https://doi.org/10.1007/s10943-010-9357-4

Aflakseir, A. (2012). Religiosity, personal meaning, and psychological well-being: A study among Muslim students in England. Pakistan Journal of Social and Clinical Psychology, 10(1), 27-31.

Ahaddour, C., Broeckaert, B., \& Van den Branden, S. (2019). "Every soul shall taste death." Attitudes and beliefs of Moroccan Muslim women living in Antwerp (Belgium) toward dying, death, and the afterlife. Death Studies, 43(1), 41-55. 
https://doi.org/10.1080/07481187.2018.1437096

Albatnuni, M. \& Koszycki, D. (2020). Prayer and well-being in Muslim Canadians: Exploring the mediating role of spirituality, mindfulness, optimism, and social support. Mental Health, Religion \& Culture, 23(10), 912-927. https://doi.org/10.1080/13674676.2020.1844175

Al-Bukhari, M. B. I. (2001). Sahih al-Bukhari. Dar Tawq al-Najat.

Al-Ghazzali, A. M. (1972). Revival of religious science (Translation of Ihya ulum Al-din by Bankey Behari). Sufi Publishing Company.

Al-Tirmidhi, M. B. I. (1975). Sunan al-Tirmidhi. Mustafa Babi al-Halabi.

Anas, M., Aijaz, A., \& Nazam, F. (2015). Relationship between gratitude and psychological well-being among youths. Indian Journal of Health \& Wellbeing, 6(5), 517-519.

Awaludin, A. (2017). Martin Seligman and Avicenna on happiness. Tasfiyah, 1(1), 1-30. http://dx.doi.org/10.21111/tasfiyah.v1i1.1840

Ayten A., \& Karagoz, S. (2021). Religiosity, spirituality, forgiveness, religious coping as predictors of life satisfaction and generalized anxiety: A quantitative study on Turkish Muslim university students. Spiritual Psychology and Counseling, 6(1), 7-28. http://dx.doi.org/10.37898/spc.2021.6.1.130

Baeke, G., Wils, J. P., \& Broeckaert, B. (2012). "Be patient and grateful”: Elderly Muslim women's responses to illness and suffering. Journal of Pastoral Care \& Counseling, 66(3), 1-9. https://doi.org/10.1177/154230501206600305

Baroun, K. A. (2006). Relations among religiosity, health, happiness, and anxiety for Kuwaiti adolescents. Psychological Reports, 99(3), 717-722. https://doi.org/10.2466/PR0.99.3.717-722 
Captari, L. E., Hook, J. N., Hoyt, W., Davis, D. E., McElroy-Heltzel, S. E., \& Worthington Jr, E. L. (2018). Integrating clients' religion and spirituality within psychotherapy: A comprehensive meta-analysis. Journal of Clinical Psychology, 74(11), 19381951. https://doi.org/10.1002/jclp.22681

El-Aswad, E. S. (2014). Patience in Sunni Muslim worldviews. In D. A. Leeming (Ed.), Encyclopedia of psychology and religion (pp. 1318-1321). Springer Science and Business.

Esposito, J. L. (2002). What everyone needs to know about Islam. Oxford University Press.

Ghayas, S., Batool, S. S., \& Adil, A. (2021). Relationship between religiosity and depression level of Pakistani elderly population: Mediational role of afterlife belief. Trends in Psychology, 29(1), 1-11. https://doi.org/10.1007/s43076-020-00021-7

Gull, M., \& Rana, S. A. (2013). Manifestation of forgiveness, subjective well being and quality of life. Journal of Behavioural Sciences, 23(2), 17-36.

Hamdan, A. (2008). Cognitive restructuring: An Islamic perspective. Journal of Muslim Mental Health, 3(1), 99-116. https://doi.org/10.1080/15564900802035268

Hammoudeh, W., Hogan, D., \& Giacaman, R. (2017). From a death sentence to a disrupted life: Palestinian women's experiences and coping with breast cancer. Qualitative Health Research, 27(4), 487-496. https://doi.org/10.1177/1049732316628833

Huda, M., Sudrajat, A., Muhamat, R., Teh, K. S. M., \& Jalal, B. (2019). Strengthening divine values for self-regulation in religiosity: Insights from Tawakkul (trust in God). International Journal of Ethics and Systems, 35(3), 1-23. https://doi.org/10.1108/IJOES-02-2018-0025

Irajpur, A., \& Moghimiyan, M. (2018). Dimensions of the spiritual needs of Muslim chronic 
patients: A qualitative study. Journal of Muslim Mental Health, 12(2), 29-43. https://doi.org/10.3998/jmmh.10381607.0012.203

Jafari, N., Farajzadegan, Z., Zamani, A., Bahrami, F., Emami, H., Loghmani, A., \& Jafari, N. (2013). Spiritual therapy to improve the spiritual well-being of Iranian women with breast cancer: A randomized controlled trial. Evidence-Based Complementary and Alternative Medicine, 2013, 1-9. https://doi.org/10.1155/2013/353262

Joshanloo, M. (2013). A comparison of Western and Islamic conceptions of happiness. Journal of Happiness Studies, 14(6), 1857-1874. https://doi.org/10.1007/s10902-012-9406-7

Khan, Z. H., Watson, P. J., Naqvi, A. Z., Jahan, K., \& Chen, Z. J. (2015). Muslim experiential religiousness in Pakistan: Meaning in life, general well-being and gender differences. Mental Health, Religion \& Culture, 18(6), 482-491. https://doi.org/10.1080/13674676.2015.1079602

Khodayarifard, M., Ghobari-Bonab, B., Akbari-Zardkhaneh, S., \& Zandi, S. (2016). Positive psychology from Islamic perspective. International Journal of Behavioral Sciences, 10(1), 29-34.

Lambert, L., Pasha-Zaidi, N., Passmore, H.-A., \& York Al-Karam, C. (2015). Developing an indigenous positive psychology in the United Arab Emirates. Middle East Journal of Positive Psychology, 1(1), 1-23.

Lies, J., Mellor, D., \& Hong, R. Y. (2014). Gratitude and personal functioning among earthquake survivors in Indonesia. The Journal of Positive Psychology, 9(4), 295-305. https://doi.org/10.1080/17439760.2014.902492

Mahoney, A., Wong, S., Pomerleau, J. M., \& Pargament, K. I. (2021). Sanctification of diverse 
aspects of life and psychosocial functioning: A meta-analysis of studies from 1999 to 2019. Psychology of Religion and Spirituality. Advance online publication. https://doi.org/10.1037/rel0000354

McCullough, M. E., Pargament, K. I., \& Thoresen, C. E. (2001). Forgiveness: Theory, research, and practice. Guilford Press.

McMichael, C. (2002). "Everywhere is Allah's place": Islam and the everyday life of Somali women in Melbourne, Australia. Journal of Refugee Studies, 15(2), 171-188.

Mir, G., \& Sheikh, A. (2010). “Fasting and prayer don't concern the doctors... they don't even know what it is": Communication, decision-making and perceived social relations of Pakistani Muslim patients with long-term illnesses. Ethnicity \& Health, 15(4), 327-342. https://doi.org/10.1080/13557851003624273

Mogahed, D., \& Chouhoud, Y. (2017). American Muslim poll 2017: Muslims at the crossroads. Institute for Social Policy and Understanding.

Muslim, H. N. (1967). Sahih Muslim. Dar Ihya al-Turath al-Arabi.

Nabolsi, M. M., \& Carson, A. M. (2011). Spirituality, illness and personal responsibility: The experience of Jordanian Muslim men with coronary artery disease. Scandinavian Journal of Caring Sciences, 25(4), 716-724. https://doi.org/10.1111/j.1471-6712.2011.00882.x

Naeem, F., Gul, M., Irfan, M., Munshi, T., Asif, A., Rashid, S., Khan, M. N. S., Ghani, S., Malik, A., Aslam, M., Farooq, S., Husain, N., \& Ayub, M. (2015). Brief culturally adapted CBT (CaCBT) for depression: A randomized controlled trial from Pakistan. Journal of Affective Disorders, 177, 101-107. https://doi.org/10.1016/j.jad.2015.02.012

Padela, A. I., Killawi, A., Heisler, M., Demonner, S., \& Fetters, M. D. (2011). The role of imams 
in American Muslim health: Perspectives of Muslim community leaders in Southeast Michigan. Journal of Religion and Health, 50(2), 359-373.

https://doi.org/10.1007/s10943-010-9428-6

Perveen, A., \& Yasin, M. G. (2017). Materialism and life satisfaction in Muslim youth: Role of gratitude and religiosity. Pakistan Journal of Psychological Research, 32(1), 231-245.

Pew Research Center. (2017). The changing global religious landscape. Retrieved from https://www.pewforum.org/2017/04/05/the-changing-global-religious-landscape/.

Pew Research Center. (2018). How religious commitment varies by country among people of all ages. Retrieved from https://www.pewforum.org/2018/06/13/how-religious-commitmentvaries-by-country-among-people-of-all-ages/.

Rambod, M., Sharif, F., Molazem, Z., \& Khair, K. (2019). Spirituality experiences in hemophilia patients: A phenomenological study. Journal of Religion and Health, 58(3), 992-1002. https://doi.org/10.1007/s10943-018-0621-3

Riaz, M. N. (2015). Portrayal of positive psychological capital in Qur'an. Al-Idah, 31(2), 37-73.

Rothman, A., \& Coyle, A. (2018). Toward a framework for Islamic psychology and psychotherapy: An Islamic model of the soul. Journal of Religion and Health, 57(5), 1731-1744. https://doi.org/10.1007/s10943-018-0651-x

Saeedi, H., Nasab, S. H. M., Zadeh, A. M., \& Ebrahimi, H. A. (2015). The effectiveness of positive psychology interventions with Islamic approach on quality of life in females with multiple sclerosis. Biomedical and Pharmacology Journal, 8(2), 965-970. https://dx.doi.org/10.13005/bpj/848

Shahgholian, N., \& Yousefi, H. (2015). Supporting hemodialysis patients: A phenomenological 
study. Iranian Journal of Nursing and Midwifery Research, 20(5), 626-633. http://dx.doi.org/10.4103/1735-9066.164514

Soto, A., Smith, T. B., Griner, D., Domenech Rodríguez, M., \& Bernal, G. (2018). Cultural adaptations and therapist multicultural competence: Two meta-analytic reviews. Journal of Clinical Psychology, 74(11), 1907-1923. https://doi.org/10.1002/jclp.22679

Tanhan, A. (2019). Acceptance and commitment therapy with ecological systems theory: Addressing Muslim mental health issues and wellbeing. Journal of Positive School Psychology, 3(2), 197-219. https://doi.org/10.47602/jpsp.v3i2.172

Uyun, Q., \& Witruk, E. (2017). The effectiveness of sabr (patience) and salat (prayer) in reducing psychopathological symptoms after the 2010 Merapi eruption in the region of Yogyakarta, Indonesia. Trends and Issues in Interdisciplinary Behavior and Social Science, 165-174. http://dx.doi.org/10.1201/9781315269184-28

Vieten, C. \& Lukoff, D. (2021). Spiritual and religious competencies in psychology. American Psychologist. Advance online publication. https://doi.org/10.1037/amp0000821

Vu, M., Muhammad, H., Peek, M. E., \& Padela, A. I. (2018). Muslim women's perspectives on designing mosque-based women's health interventions: An exploratory qualitative study. Women \& Health, 58(3), 334-346. https://doi.org/10.1080/03630242.2017.1292344

Zoellner, L. A., Bentley, J. A., Feeny, N. C., Klein, A. B., Dolezal, M. L., Angula, D. A., \& Egeh, M. H. (2021). Reaching the Unreached: Bridging Islam and Science to Treat the Mental Wounds of War. Frontiers in Psychiatry, 12, Article 599293. https://doi.org/10.3389/fpsyt.2021.599293 


\title{
Chapter 13:
}

\section{Positive Psychology and Hinduism}

\author{
Kamlesh Singh ${ }^{1}$, Mahima Raina ${ }^{2}$, and Doug Oman ${ }^{3}$ \\ ${ }^{1}$ Dept. of Humanities \& Social Sciences, Indian Institute of Technology Delhi (India) \\ 2 Jindal School of Psychology and Counselling, O.P. Jindal Global University, Sonipat, \\ India \\ ${ }^{3}$ School of Public Health, University of California, Berkeley
}

Correspondence concerning this chapter should be addressed to Kamlesh Singh. Email: singhk.iitd@gmail.com 


\begin{abstract}
Hinduism encompasses much heterogeneity within its many schools of thoughts and practice. However, the common thread that binds these multiple perspectives is this handbook's main topic of inquiry - human well-being and happiness. Hindu thought has always deliberated on the process, nature, conditions, and practices that lead to a fulfilled life. In this chapter, we begin by explaining Hinduism's core beliefs and tenets that guide daily living. Many extant psychological publications on Hinduism explicate psychological ideas embedded in tradition. We draw comparisons between the Hindu worldview and broadly Western-based positive psychology concepts like well-being and the self. The chapter also expands on select Hindu models of human flourishing. The chapter further explains the recent empirical evidence on Hindu constructs, including well-being enhancing strategies, in relation to several positive psychology constructs such as well-being and flourishing. Finally, in the light of the reviewed empirical evidence, we discuss some directions and recommendations for future research at the intersections of Hindu religious literature and positive psychology.
\end{abstract}

Keywords: Hinduism, Positive Psychology, Yoga, Meditation, Bhagavad Gītā 


\section{Chapter 13: Positive Psychology and Hinduism}

Hindus represent 15 percent of the global population, with 99 percent of Hindus concentrated in the Asia Pacific region, especially in India, Nepal, and Mauritius. The remaining 1 percent of Hindus are scattered across Europe, Latin America, North America, the Middle East, and Africa. Hinduism is the third largest religion in the world, after Christianity and Islam (Oman \& Paranjpe, 2020). It encompasses a very broad and diverse array of traditions, rituals, practices, beliefs, and philosophies that originated in South Asia. Hinduism regards the four Vedas as the fountainhead of its wisdom, but it also reveres several other important texts such as the Purānasas, Laws of Manu, Bhagavad Gītā, Rāmāyaṇa, and Mahābhārata. Collectively, these texts inform, direct, and provide insights on morals, ethics, human functioning, well-being practices, and proper conduct. Specific practices, rituals, and beliefs vary across different states in India, but the overarching aim of these practices and rituals is more or less uniform.

Indian psychology, as derived mainly from the classical Hindu thoughts and practices, has global relevance and significant implications for human functioning, suffering, health, and well-being (Oman \& Paranjpe, 2020). Mainstream psychology's overall shift towards studying well-being has intensified interest in Hindu practices as well, as is evident in the growing body of research on Hindu practices such as meditation and yoga. This empirical scrutiny has corroborated the potential of Hindu thought and practice to both explain and enhance well-being.

Yet intersections between Hinduism and positive psychology still lack adequate visibility in academic discourse. Rao and Paranjpe (2016) have noted two contributing factors: (a) Indian psychology or Hindu sources emphasize theory and concepts, rather than the prototypical forms of evidence recognized by modern science; and (b) psychology, as practiced in academic and professional spaces in modern India, replicates and imitates the Western models that proliferated 
as Western-trained scholars began working in universities across India. The latter may be called mainstream psychology, which is contrasted with an Indian psychology that is based on native ideas and practices that developed over millennia in the Indian subcontinent. In recent decades, psychology researchers in India have undertaken systematic scholarship to restore balance and recover access to insights transmitted by Hinduism and other Indian traditions, an effort often called the "Indian Psychology Movement" (Oman \& Paranjpe, 2020, p. 178; see also

Cornelissen, 2002). Contributors to this movement report that underlying different indigenous Indian or "Dharmic" traditions such as Hinduism, Buddhism, and Jainism, there is a "unifying thread that runs across these different systems binding them together in significant ways [and giving] Indian thought its identity," thereby warranting "Indian Psychology" as a broad term that encompasses psychologies embedded in multiple faith traditions (Rao \& Paranjpe, 2016, p. 9).

The present chapter focuses on Hinduism, offering an overview of intersections between Hinduism and positive psychology. We first compare and contrast modes of inquiry used within Indian versus mainstream psychology. Second, we explain two influential conceptualizations of flourishing that are derived from traditional Hindu thought. Third, we review empirical studies on selected Hindu concepts and practices, describing their relationship with various well-being indicators. After sketching dissemination efforts by a variety of nonacademic institutions in India and abroad, we conclude by discussing implications and suggestions for broadening scholarship on the intersections of Hinduism and positive psychology.

\section{Hindu View of the World and Human Nature}

\section{Meaning of Well-Being: Hindu (Indian) Psychology vs. Mainstream Positive Psychology}

Hindu spiritual thought has the aim of elevating human functioning, which resonates with positive psychology's aim of promoting human flourishing. Positive psychology has been 
responsible for expanding the meaning of human well-being beyond absence of illness to include physical, mental, emotional and spiritual well-being. However, the meaning and experience of every psychological attribute of human existence is moderated by sociocultural factors. For example, in Western culture and extant positive psychology scholarship, well-being is usually defined in terms of hedonia (pleasure) and eudaimonia (personal fulfilment; Ryan \& Deci, 2001). Under this broad umbrella of hedonia and eudaimonia, several models of well-being have been postulated, including psychological and social well-being (Ryff, 1989; Keyes, 1998, 2005), as well as the PERMA model of well-being that reflects an amalgamation of positive emotion,

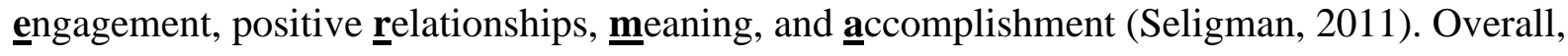
two noteworthy features of Western concepts of well-being are (a) an emphasis on the biopsycho-social viewpoint of well-being (Ryan \& Deci, 2001) and (b) a focus on the external conditions of well-being, such as interpersonal relationships and social engagement. In addition, the Hindu (Indian) conception of well-being directly emphasizes the influence of consciousness, viewed as transcendent in nature and often called sat-chit-ananda (defined in Hindu thought as the inner state of truthfulness, bliss, and consciousness; Srivastava \& Misra, 2011). Hindu philosophers have avidly discussed the nature and conditions of happiness, satisfaction, and fulfilment, including and how to attain and sustain this state (Srivastava \& Misra, 2011).

Moreover, the recommended process to attain and sustain the state of bliss and happiness is by knowing the self (see the next paragraph for detailed discussion on the meaning of self). In contrast to the mainstream psychological emphasis on identifying contingent well-being predictors such as relationships, traditional Hindu thought affirms that well-being is ultimately not contingent on any objective, tangible conditions (Salagame, 2013). Still, some concepts of humanistic psychology, such as self-actualization and the fully functioning individual, recognize 
the quest of achieving full human potential as an inherent individual motivation (D'Souza \& Gurin, 2016). Consistent with the humanistic view, positive psychology also recognizes the importance of human existence as multilayered, and it recognizes the importance of emotions, relationships, meaning, and accomplishments in healthy and optimal human functioning (Seligman, 2011; Singh \& Raina, 2020). In Hindu thought, attaining the state of sat-chit-ananda results in harmony in life (a sense of balance, flexibility, and meaningful mutual relations) and peace of mind (affective well-being and an experience of serenity and calmness; Singh at al., 2016). In sum, whereas Hindu thought posits self-inquiry and self-managing as a precursor for well-being, Western thought, spurred by a materialist worldview, has considered meaningful relationships and positive external experiences as precursors of well-being (Salagame, 2013).

\section{The Meaning of Self in Hinduism vs. Mainstream Positive Psychology}

Consistent with the Hindu emphasis on spiritual as opposed to material dimensions of human existence (Salagame, 2013), Hinduism also emphasizes a spiritual understanding of the self. The Western worldview uses notions such as self-concept and self-identity to explain the meaning of the self, which generally is viewed as a social-cognitive construction (Oyserman et al., 2012). In contrast, the Hindu notion of self clearly distinguishes the materially experienced human body (deha) from the spiritual existence of human life (jiva). One clarifying example is Hindu rituals of death: Rituals for the dead body before cremation are distinguished from rituals for the spiritual self (jiva), which are performed to facilitate the soul's journey (Salagame, 2013).

Similarly, although Hindu concepts of well-being center on holistic individual emancipation (which involves expansion or elevation of mind-body-consciousness as a whole), mainstream psychology has emphasized therapeutic adjustment as a practical goal and the creation of universally generalizable knowledge as its methodological ideal (Rao \& Paranjpe, 
2016). Although positive psychology and Hindu thought converge regarding well-being and optimal human functioning, their uses of concepts diverge, with the former relying heavily on scientific, reductionistic methodologies. More broadly, Hindu concepts of human nature and the sources of well-being are rooted in Sanskrit and related Indic languages, and many terms are “'Sanskrit [or other Indic language] non-translatables' [that] cannot be translated by a corresponding English word without reducing their original purport" (Choudry, 2017, p. 443; see also Choudry \& Vinayachandra, 2015; Malhotra \& Babaji, 2020). Bearing in mind such limitations, the next sections discuss some common Hindu models of well-being and how their constituent concepts are problematized and studied in Hinduism versus positive psychology.

\section{Hindu Models of Human Well-Being}

Bhawuk (2011) presented models of human flourishing and happiness drawn from Hindu texts. Here we discuss some key concepts and their intersections with positive psychology.

\section{Anasakti: The Root of Developing the Virtue of Temperance}

Perhaps the most influential Hindu model of flourishing is presented in the Bhagavad Gìtāa, a sacred text dating in its present form from approximately the $2^{\text {nd }}$ century BCE. The Bhagavad Gìtā stresses the attainment of sthitaprajña, which refers to being established in a state of wise equanimity or temperance. This state is attained when thoughts, emotions, and desires are managed effectively through niṣkāma karma or dispassionate action (Singh \& Raina, 2015). Using this basic tenet of Bhagavad Gītā, Bhawuk (2011) explains how thoughts, cognition, and behavior are intricately woven and how their interactions can affect human wellbeing and flourishing. He explains that when an embodied individual becomes attached to a goal, a strong desire to achieve the goal erupts. At this stage, the individual engages in action to achieve the goal, which can result in a plethora of emotional outcomes. For example, if the 
individual is successful in achieving the goal, it can result in a person experiencing greed to achieve more, and failure can result in anger. These emotional reactions serve as reinforcers that influence future decisions. An individual continues to be mired and burdened with emotions and thoughts, as desires are understood to be "fire that is never satiated" (Bhagavad Gītā, Verse 3.39). Desires have the capability to overpower an individual's intellect and mind, and fulfilment of one desire leads to the birth of another. Thus, according to the Bhagavad Gìtā, the only way to lead a happy and purposeful life is by managing our desires. To achieve this, individuals must pursue all their worldly duties and desires dispassionately (nișkäma karma). This essentially means pursuing every task and desire wholeheartedly but without personal expectation or embroilment. It is the shedding of attachment to the results. This can be achieved through meditation and contemplation, as we critically examine and question our desires and how they affect us. As we wean ourselves from attachment to results, the vices of human nature (namely, greed, anger, lust, pride, and attachment/ego) vanish, and we become established in a steady state of temperance. When we pursue our life dispassionately, we lead ourselves towards the habitual experience of temperance. In terms of positive psychology, the concept of Anasakti has some resonance with Peterson and Seligman's (2004) model of character strengths and virtuesspecifically the virtue of temperance, which encompasses the strengths of humility, forgiveness, and self-regulation (e.g., of emotional reactions to disappointments and insecurities).

\section{Yoga: The Path of Achieving Inner Balance}

Western popular culture recognizes a set of practices called "yoga," which are often presented as a psycho-physical therapy aiming to integrate the mind, body, and spirit. However, this Western yoga, that scholars have called "transnational anglophone yoga," is most often restricted only to āsanas or physical postures to gain body flexibility and strength (see Oman \& 
Paranjpe, 2020, pp. 191-192; Raina \& Singh, 2018). Although terms like jīvanmukti and kundalinī yoga are very popular in the West, the meanings of these terms have been lost in translation and are mostly restricted to body postures. Even with the often very limited modern understanding of yoga as involving primarily postures (äsanas), a plethora of research studies support the efficacy of yoga practice for human health and well-being (Ross et al., 2013; Ross \& Thomas, 2010; Sengupta, 2012; Simard \& Henry, 2009). However, the word yoga traditionally means a state of union achieved through various paths of unification. The Sanskrit word yoga is derived from the root "yuj" which means to bind together. The union here refers to the union of an individual soul with the absolute, often translated as God (De Michelis, 2004).

Due to our socialization, we are reinforced positively or negatively to respond to our environment in a conditioned manner. An individual responds to these desires in different ways, often leading to an experience of an ever-oscillating mind. The aștāinga yoga (literally, eightlimbed yoga), taught in the Patañjali Yogasütras, emphasizes cultivating mental focus through concentrative meditation and other practices. This practice leads to freedom from many common manifestations of mental instability, which itself is understood to be a root cause of unhappiness.

Many people within and outside contemporary India have come to regard Indian wisdom traditions as a fountainhead of immense knowledge on the meaning of self and on understanding the antecedents of human well-being and flourishing. However, given its complex nature, Hinduism's deeper concepts have sometimes been misunderstood as relevant only to mystics or sages who are committed to a spiritual path. Furthermore, mainstream Western psychology has long focused on pathology and has only recently begun to emphasize well-being (mostly since the emergence of positive psychology). For this reason, scientific understanding of many positive psychology constructs is still relatively nascent. Furthermore, perhaps due in part to the 
methodological differences noted earlier, there has been minimal exploration and assimilation of Indian psychological constructs into mainstream positive psychology until just recently.

\section{Self-Observation/Introspection: Different Methods Based on Different Philosophies}

Because the idea of the holistic self is embedded in the ethos of Hindu (Indian) thought, Hinduism uses first-person approaches of self-observation/introspection as its main tools for studying human functioning. Whereas mainstream psychology understands and uses observation to systematically record manifested behavior, within Indian thought, observation includes both internal/experiential self-observations and external/manifested observations. According to tradition, the complex theories of human cognition, flourishing, and well-being presented in classic Hindu texts were generated after careful self-observation of human processes on bodymind-consciousness levels (Cornelissen, 2011; Rao \& Paranjpe, 2016). Within Hinduism, consistent with the aims of knowing the self, the internal observations of the human mind and consciousness play a centrally important role in the process of human unfolding towards flourishing. Fortunately, psychologists have begun considering cultural nuances in understanding and explaining human functioning and have started to integrate the scientific methodologies and basic tenets of Hindu thought to advance understanding of human flourishing (Salagame, 2013).

\section{Using Scales to Assess Key Indian Constructs: Strengths and Weaknesses}

To bridge the methodological disconnect between Hindu wisdom (Indian psychology) and mainstream positive psychology, recent studies have focused on quantifying a variety of key Indian constructs. The scales have enabled researchers to identify meaningful correlations between some of these Indian constructs and other well-being indicators, and more importantly, they have allowed researchers to explore the culturally situated meaning of happiness and wellbeing among Hindus. In the next paragraphs, we discuss select Hindu measures that have been 
developed and describe their scores' correlations with existing positive psychology concepts.

In some studies, overall well-being has been operationalized as sat-cit-ānanda, which is traditionally understood as a natural state of every human being that involves inner happiness, bliss, and well-being — an emancipated state that every human strives to achieve (see Rao \& Paranjpe, 2016; Singh, Khari, et al., 2014). In developing a 17-item Sat-Cit-Ānanda Scale, Singh, Khari, et al. (2014) found evidence that sat-cit-ānanda is comprised of cit (or chit, consciousness), antahśakti (or antahshakti, inner strength), sat (truthfulness), and ānanda (bliss). These revealed factors indicated that cit or consciousness relates to taking responsibility for one's thoughts, emotions, speech, feelings, and actions. Sat or truthfulness is seeing positivity and good qualities of others, having the sense of belongingness with one another, seeing goodness in nature, and loving (all) people. Ananda or bliss refers to the state of mind when an individual ceases to be afflicted by reactivity to the thoughts, behaviors, and actions of others. Ananda is the ability to see events happening around us without getting swayed by them. Apart from theoretically defined sat-cit-ānanda (the three expected factors), another supporting factor emerged in statistical analysis—antahśakti, or inner strength, which was highly correlated with the other three factors. That factor encompassed items assessing energy, initiative, standing up for oneself, challenge in dealing with difficult people, and ways to achieve what you want to achieve. Sat-Chit-Ananda Scale scores have shown positive correlations with satisfaction with life, subjective happiness and flourishing, peace of mind, harmony in life, and experiences of positive emotion, and they have shown inverse correlations with experiences of negative emotion (Singh, Khari, et al., 2014; Singh et al., 2018).

\section{Assessing a Pathway to Well-Being}

A similar concept of well-being is offered in the Patañjali Yogasütras' presentation of 
așțānga yoga (eight-limbed yoga) as a pathway to a state of ultimate well-being and bliss. Scores on the newly developed Ashtanga Yoga Hindi Scale have shown correlations between aștāinga yoga practices and higher flourishing, greater positive emotions, and lower negative emotions (Raina \& Singh, 2018).

Furthermore, concepts such as anāsakti (detachment) have been conceptualized as a stress-coping mechanism wherein dispassionate action is thought to be associated with wellbeing (Singh \& Raina, 2015). Anāsakti refers to an ability to take motivated action without the expectation of rewards or punishment. It is characterized by faith in God, outcome vulnerability, empathy, persistence and fortitude, frustration tolerance, and emotional equipoise. Using the newly developed Anāsakti Scale, several researchers have found evidence that anāsakti is linked to the experience of lower stress-strain (Banth \& Talwar, 2012; Jha, 2002; Pande \& Naidu, 1992) and higher well-being (e.g., flourishing and positive emotions; Singh \& Raina, 2015).

Similarly, in Hindu thought, an absence of anāsakti and a presence of vikāras (affliction of mind) constrains one's ability to experience a state of bliss. Paralleling positive psychology's efforts to undo ill-effects of negative emotions, Hindu thought has identified five traditional vikāras or vices/internal enemies (Sharma \& Singh, 2016): lust (kama: intense desire or obsessive need for sensual gratification), anger (krodha: strong feelings of annoyance, displeasure, or hostility), attachment (moha: obsessive attachment to objects or people), greed (lobha: intense desire for material possessions), and pride/ego (ahamkara: vanity or grandiosity). Many empirical studies have found correlations between vikāras and negative psychosocial outcomes. For example, anger is positively related to depression, pride is associated with narcissism, and gratitude is inversely related to greed (Peterson \& Seligman, 2004; Sharma \& Singh, 2016). Sharma and Singh (2016) reported that the vikāras were related to the concurrent 
experience of higher negative emotions and lower positive emotions.

\section{Hindu Practices for Enhancing Well-Being}

In this section, while not losing sight of relevant concepts of health and well-being, we give primary attention to the practices for enhancing health that are recommended in Hindu thought. Along the way, we draw parallels between several of these traditional practices and their modern-day manifestations, including “yoga," mindfulness meditation, and others. We also briefly review empirical studies evaluating the efficacy of modern variants of these practices.

\section{Mind-Body Unity}

Hinduism encompasses multiple schools of thought that offer diverse perspectives and explanations of human functioning and flourishing. These perspectives are discussed as six systems of thought, often called philosophies or darśanas (views) in the Hindu tradition (Oman \& Paranjpe, 2020). Both within and between schools of thought, Hinduism has consistently applied itself to intellectual debates involving philosophical introspection (Salagame, 2013).

In the traditional Hindu view, bodily health and well-being are closely tied to advancement in spiritual pursuit. For example, a healthy, light body with glowing skin, sonorous voice, and fragrant smell are viewed as signs of spiritual progress (Oman \& Paranjpe, 2020). This holistic approach to health and well-being is the central tenet of Āyurveda, a 5000- yearold, comprehensive, traditional stream of medicine native to India. Āyurveda simultaneously considers both physical and psychological roots of an illness, and it emphasizes the unique physio-psychological constitution of an individual. Because the locus is the unique constitution of an individual, Āyurveda's approach differs from that of modern biomedical sciences, which focus on categories (Andreason, 2007). Āyurvedic treatments hinge on diet changes, herbs, aroma therapy, color therapy, yoga, meditation, and various detoxification techniques (e.g., nasal 
cleansing, enema, and mind-body rejuvenation techniques like herbal massages and steam baths). Modern science is increasingly considering the mind-body connection (McEwen, 2017), especially when it comes to understanding and treating chronic pain (Leader \& Corfield, 2008).

\section{Hindu Practices to Enhance Emotional, Mental, and Spiritual Well-Being}

Hinduism enlists several paths of self-growth and mastery by recommending practices that enhance positive human virtues. For example, practicing radical love and devotion towards the divine is encouraged. The spiritual practices associated with these values are bhajans, kìrtans, satsang (the practice of singing in the praise of the divine), and mantra japa ("short spiritual formula for calling up what is best and deepest in ourselves," Easwaran, 2008, p. 12).

Satsang, kīrtans, and bhajans are community practices (traditionally emerging from South Asia) that aim at personal and spiritual development but are practiced in a group setting. They are usually practiced as a group or community activity that also involves connecting with the community (through religious discourse) in the presence of an individual who has more advanced knowledge of these religious practices (e.g., a spiritual teacher or guru; Rybak et al., 2015). The group practice is understood not just as a means for spiritual emancipation but also as a way to foster healthier interpersonal relations, through strengthening social and family support (Singh, Jain, \& Singh, 2014). Mantra japa (the practice of mantra repetition or chanting) has been understood as a psychological tool that enables an individual to overcome a stressful situation by calming and destressing the mind. When practiced regularly, it has the potential of fostering resilience, thereby contributing to overall improvement in dealing with stress (Bormann et al., 2020; Oman \& Bormann, 2021). Mantra japa can be practiced at various times throughout the day, in between activities of daily living (Bormann et al., 2020), or it can be combined with meditation. Evidence indicates the practice fosters long-term psychological benefits such as the 
development of resilience, healthy coping, and lower depressive symptoms (Wolf \& Abell, 2003), effective management of mental health conditions including post-traumatic stress disorder, and improvement in chronic illnesses such as AIDS and cancer (Oman et al., 2020). The practice also induces a deep relaxation state in the brain and body (Harne et al., 2019). Hinduism also encourages self-inquiry, which involves learning about our true self and using the mind to study the very nature of mind. Self-inquiry is also referred to as the intellectual path towards enlightenment. The practice is used to enhance the value of wisdom and knowledge by emphasizing the use of reasoning skills. These skills are used for critical self-introspection, involving studying oneself and diligently developing and choosing healthy coping mechanisms.

The practices associated with this school of self-inquiry are svādhyāya and meditation. Svādhyāya means study of scriptures and self-reflection (Raina \& Singh, 2018). This selfreflection involves contemplation of one's motives, behaviors, and circumstances. Indeed, scores on the newly developed Swadhaya Scale have been linked to greater flourishing, higher positive emotions, and lower negative emotions (Singh \& Sahni, 2016). In addition, preliminary evidence suggests that Spiritually Augmented Cognitive Restructuring - a psychotherapeutic intervention that incorporates the practice of $s v \bar{a} d h y \bar{a} y a$-is effective in restructuring cancer patients' negative core beliefs through the use of spiritual teaching and practices. More broadly, the integration of spirituality with traditional therapy can enhance religious individuals' well-being (see Captari et al., Chapter 26, this volume), partly by aiding the process of overcoming selflimiting beliefs and assumptions and allowing for expansion of self-awareness (Cloninger, 2006). In Hinduism, this self-reflection or expansion of self-awareness is the basic tenet and nature of self-inquiry and svādhyāya.

Self-inquiry is also aided by meditation, which is an exercise that relies on two main 
classes of methods: (1) focused-attention meditation upon a single object, sound, concept, breath or experience and (2) open-monitoring meditation, involving nonintrusive, nonjudgemental monitoring of the content of experience from moment to moment. Both techniques have documented effects on regulation of emotions and attention (Lutz et al., 2008; Oman \& Bormann, 2021), partly via creation of a cognitive-control state that impacts how an individual allocates attention over time. Perhaps due to the efficacy of meditation for regulating of attention and emotion, its practice is commonly employed across all major branches of traditional yoga.

\section{Community-Level Spiritual Programs}

From a health-promotion perspective, it is important to recognize that support for engaging in Hindu practices is widely available internationally and in India. Many social organizations headed by faith leaders strive to help people understand and experience the benefits of traditional Hindu practices. Organizations such as the Art of Living, Heartfulness, Isha Foundation, and many others (see Appendix 13.S1) have a global presence and many followers, and they all strive toward a vision of enhancing people's health and well-being. They offer various in-person and sometimes online programs on yoga and meditation, and they facilitate access to health and well-being practices, especially among optimally functioning populations. Recent research has supported the effectiveness of such practices for enhancing a variety of well-being indicators, including mental health (e.g., reduced burnout) and physical health (e.g., increased heart rate variability; Arya et al., 2017, 2018; Rangasamy et al., 2019; Waghorne, 2014). Other groups, such as Ramakrishna Mission, Vivekananda mission, ISKCON, and Sivananda yoga, are worldwide spiritual movements that propagate Hindu philosophy and ideals and facilitate health and well-being practices in the community (e.g., Chaurey, 2000;

Seppälä et al., 2020). 


\section{Conclusion and Future Directions}

In this chapter, we have examined various practices, rituals, belief systems, and paths of self-development prescribed in Hinduism. We have also summarized the theory and research on how all these are related to an individual's physical-mental-social well-being, work, and overall community. In many of these, we have tied them to current positive psychology. Many of the practices have drawn interest across diverse cultures. It is noteworthy and not always understood that the meaning and spiritual relevance of many Hindu concepts and practices have often been lost in translation. Even so, growing empirical evidence supports the benefits of these practices for human well-being and for other outcomes of interest to positive psychology (e.g., virtues).

Hinduism and positive psychology intersect at several points and share the common goal of enhancing human well-being. Although positive psychologists or researchers in India are trained to seek this goal (especially through empirical research), faith leaders have been guided by accumulated traditional experience and wisdom based on what has been called "realizationderived knowledge" (Oman \& Singh, 2018, p. 172; see also discussion of parā vidyā in Oman \& Paranjpe, 2020). Appropriately merging these approaches could yield beneficial synergies that foster improved understanding and practice. Large disconnects persist regarding administration and implementation of these approaches, but progress is occurring. Further integration of these approaches on the practical or even epistemic levels might enhance motivation to engage more

confidently in practices deemed effective (Ford, 1992; Oman \& Singh, 2018). Similarly, research can support ongoing social uptake of these practices, ensuring that communities within and outside India continue to receive benefit from these time-tested, health-enhancing practices.

On the other hand, positive psychology practitioners in India need to be aware of the deeper effects of religion and culture that seep organically into the psyche. Instead of over- 
emphasizing Western-based principles and implementing them as is with Indian locals, it would be more appropriate to make sustained efforts towards clinical integration-tailoring psychotherapy to a client's religious/spiritual background and conducting research to explore the conditions under which spiritually integrated therapies can be more beneficial (e.g., Oman \& Bormann, 2021). Referral networks between traditional and modern practitioners may also be useful (e.g., Shields et al., 2016). Practitioners should overcome any mental barriers, imbibed through the conditioning of an education disproportionately steeped in Western thinking, that may undermine their ability to approach the issues informed by an emic understanding.

We also encourage positive psychologists to conduct further research on traditional yet ongoing practices to better grasp their mental health effects and implications for conceptualizing and conducting group therapies. Methods inspired by such indigenous practices, if effective, may potentially be low cost, therapeutic, foster social relationships, encourage community participation, and be serviceable as a form of mental health support for many clients in need.

As we have discussed, Hinduism places much focus on the self and on finding one's own unique identity and purpose. In addition, Hinduism offers many pathways to optimum human functioning and well-being; we have only discussed a few in this chapter. In the encounter between the very young field of positive psychology and the very old and deep tradition of Hinduism, we have identified many commonalities and overlaps as well as important differences. Research to date has only explored a small fraction of the important questions worth exploring. Further knowledge could foster helpful collaboration between Hinduism (Indian psychology) and positive psychology, for the public good. Together, positive psychology and Hindu traditional wisdom might foster a much-needed integration of empirical and experiential forms of learning, building on the best insights and strengths of each collaborative partner. 


\section{References}

Andreason, N. C. (2007). DSM and the death of phenomenology in America: An example of unintended consequences. Schizophrenia Bulletin, 33,1, 108-112. https://doi.org/10.1093/schbul/sb1054

Arya, N. K., Singh, K., \& Malik, A. (2017). Effect of Heartfulness Spiritual Practice based program on mental and physical health indicators. International Journal of Research in Management \& Social Science, 5, 91-103. https://doi.org/10.1016/j.ihj.2018.05.004

Arya, N. K., Singh, K., Malik, A., \& Mehrotra, R. (2018). Effect of Heartfulness cleaning and meditation on heart rate variability. Indian Heart Journal, 70, 50-55. https://doi.org/10.1016/j.ihj.2018.05.004

Banth, S., \& Talwar, C. (2012). Anasakti, the Hindu ideal, and its relationship to well-being and orientations to happiness. Journal of Religion and Health, 51,3, 934-946. https://doi.org/10.1007/s10943-010-9402-3

Bormann, J. E., Kane, J. J., \& Oman, D. (2020). Mantram repetition: a portable practice for being mindful. Mindfulness, 11(8), 2031-2033. https://doi.org/10.1007/s12671-020$\underline{01440-4}$

Bhawuk, D. (2011). Spirituality and Indian Psychology. Springer Science \& Business Media. https://doi.org/10.1007/978-1-4419-8110-3

Chaurey, A. (2000). Ramakrishna Mission initiative impact study: final report. United States. https://doi.org/10.2172/758770

Choudry, A. (2017). K. Ramakrishna Rao and Anand C. Paranjpe, psychology in the Indian tradition. Psychological Studies, 62(4), 439-442. https://doi.org/10.1007/s12646-017$\underline{0424-8}$ 
Choudry, A., \& Vinayachandra, B. K. (2015). Understanding happiness: The concept of sukha as ‘excellent space'. Psychological Studies, 60(3), 356-367. https://doi.org/10.1007/s12646$\underline{015-0319-5}$

Cloninger, C. R. (2006). The science of well-being: An integrated approach to mental health and its disorders. World Psychiatry, 5(2), 71. https://doi.org/10.1016/j.wpsyc.2012.05.019

Cornelissen, M. (2002). Pondicherry manifesto of Indian psychology. Psychological Studies, 47,1, 168-169. https://doi.org/10.1007/s12646-014-0248-8

Cornelissen, M. (2011). Foundations of Indian psychology Volume 2: Practical applications. Pearson Education India.

De Michelis, E.(2004). A history of modern yoga: Patañjali and Western esotericism. Continuum. https://doi.org/10.1525/nr.2006.9.3.141

D’Souza, J., \& Gurin, M. (2016). The universal significance of Maslow's concept of selfactualization. Humanistic Psychologist, 44(2), 210-214. https://doi.org/10.1037/hum0000027

Easwaran, E. (2008). The mantram handbook (5 ${ }^{\text {th }}$ ed.). Nilgiri Press.

Ford, M. E. (1992). Motivating humans. Sage. https://doi.org/10.4135/9781483325361

Harne, B. P., Bobade, Y., Dhekekar, R. S., \& Hiwale, A. (2019). SVM classification of EEG signal to analyze the effect of OM mantra meditation on the brain. In 2019 IEEE 16th India Council International Conference (INDICON) (pp. 1-4). IEEE. https://doi.org/10.1109/indicon47234.2019.9030339

Jha, M. K. (2002). Certain psychological correlates of asakti-anasakti. [Unpublished doctoral dissertation]. TM Bhagalpur University.

Keyes, C. L. M. (1998). Social well-being. Social Psychology Quarterly, 61(2), 121-140. 


\section{https://doi.org/10.2307/2787065}

Keyes, C. L.M. (2005). Mental illness and/or mental health? Investigating axioms of the complete state model of health. Journal of Consulting and Clinical Psychology, 73(3), 539-548. https://doi.org/10.1037/0022-006x.73.3.539

Leader, D., \& Corfield, D. (2008). Why do people get ill? Exploring the mind-body connection. Penguin. https://doi.org/10.1111/j.1752-0118.2008.00079_1.x

Lutz, A., Slagter, H. A., Dunne, J. D., \& Davidson, R. J. (2008). Attention regulation and monitoring in meditation. Trends in Cognitive Sciences, 12(4), 163-169. https://doi.org/10.1016/j.tics.2008.01.005

Malhotra, R., \& Babaji, S. D. (2020). Sanskrit non-translatables: The importance of Sanskritizing English. Manjul Publishing House.

McEwen, B. S. (2017). Integrative medicine: Breaking down silos of knowledge and practice an epigenetic approach. Metabolism: Clinical and Experimental, 69, 21-29. https://doi.org/10.1016/j.metabol.2017.01.018

Oman, D., Bormann, J. E., \& Kane, J. J. (2020). Mantram repetition as a portable mindfulness practice: Applications during the COVID-19 pandemic. Mindfulness. https://doi.org/10.1007/s12671-020-01545-w

Oman, D., \& Bormann, J. E. (2021). Mindfulness through mantram and inspired passage meditation: Toolkit for diversity. In S. K. Gupta (Ed.), Handbook of research on clinical applications of meditation and mindfulness-based interventions in mental health (pp. 214-236). IGI Global. https://doi.org/10.4018/978-1-7998-8682-2.ch014

Oman, D., \& Paranjpe, A. C. (2020). Psychology of Hinduism from the inside out. In T. A. Sisemore \& J. J. Knabb (Eds.), The psychology of world religions and spiritualities: An 
indigenous perspective (pp. 165-196). Templeton Press.

Oman, D., \& Singh, N. N. (2018). Combining Indian and Western spiritual psychology:

Applications to health and social renewal. Psychological Studies, 63(2), 172-180. https://doi.org/10.1007/s12646-016-0362-x

Oyserman, D., Elmer, K., \& Smith, G. (2012). Self, self-concept, and identity. In M. R. Leary \& J. P. Tangney (Eds.). Handbook of self and identity (pp. 69-104). Guilford. https://doi.org/10.1002/9780470998519.ch23

Pande, N., \& Naidu, R. K. (1992). Anasakti and health: A study of non-attachment. Psychology and Developing Societies, 4,1, 89-104. https://doi.org/10.1177/097133369200400106

Peterson, C., \& Seligman, M. E. (2004). Character strengths and virtues: A handbook and classification. Oxford University Press.

Raina, M., \& Singh, K. (2018). The Ashtanga Yoga Hindi Scale: An assessment tool based on Eastern philosophy of yoga. Journal of Religion and Health, 57, 12-25. https://doi.org/10.1007/s10943-015-0096-4

Rangasamy, V., Susheela, A. T., Mueller, A., Chang, T. F. H., Sadhasivam, S., \& Subramaniam, B. (2019). The effect of a one-time 15-minute guided meditation (Isha Kriya) on stress and mood disturbances among operating room professionals: A prospective interventional pilot study. F1000Research, 8(335), 1-12. https://doi.org/10.12688/f1000research.18446.1

Rao, K. R., \& Paranjpe, A. C. (2016). Psychology in the Indian tradition. Springer. https://doi.org/10.1007/978-81-322-2440-2

Ross, A., Friedmann, E., Bevans, M., \& Thomas, S. (2013). National survey of yoga practitioners: mental and physical health benefits. Complementary Therapies in 
Medicine, 21(4), 313-323. https://doi.org/10.1016/j.ctim.2013.04.001

Ross, A., \& Thomas, S. (2010). The health benefits of yoga and exercise: A review of comparison studies. Journal of Alternative and Complementary Medicine, 16(1), 3-12. https://doi.org/10.1089/acm.2009.0044

Ryan, R. M., \& Deci, E. L. (2001). On happiness and human potentials: A review of research on hedonic and eudaimonic well-being. Annual Review of Psychology, 52(1), 141-166. https://doi.org/10.1146/annurev.psych.52.1.141

Rybak, C., Sathaye, D., \& Deuskar, M. (2015). Group counseling and Satsang: Learning from Indian group practices. Journal for Specialists in Group Work, 40(2), 147-162. https://doi.org/10.1080/01933922.2015.1017064

Ryff, C. D. (1989). Happiness is everything, or is it? Explorations on the meaning of psychological well-being. Journal of Personality and Social Psychology, 57(6), 10691081. https://doi.org/10.1037/0022-3514.57.6.1069

Salagame, K. K. K. (2013). Perspectives on reality in Indian traditions and their implications for health and well-being. In A. Morandi, \& A.N.N. Nambi (Eds.), An integrated view of health and well-being: Bridging Indian and Western knowledge (pp. 39-57). Springer. https://doi.org/10.1007/978-94-007-6689-1_3

Seligman M. E. P. (2011). Flourish. Simon \& Schuster.

Sengupta, P. (2012). Health impacts of yoga and pranayama: A state-of-the-art review. International Journal of Preventive Medicine, 3(7), 444-458.

Seppälä, E. M., Bradley, C., Moeller, J., Harouni, L., Nandamudi, D., \& Brackett, M. A. (2020). Promoting mental health and psychological thriving in university students: A randomized controlled trial of three well-being interventions. Frontiers in Psychiatry, 11, 1-14. 
https://doi.org/10.3389/fpsyt.2020.00590

Sharma, S., \& Singh, K. (2016). Development and validation of Vikaras Hindi scale. Mental Health, Religion \& Culture, 19,5, 420-432. https://doi.org/10.1080/13674676.2016.1189891

Shields, L., Chauhan, A., Bakre, R., Hamlai, M., Lynch, D., \& Bunders, J. (2016). How can mental health and faith-based practitioners work together? A case study of collaborative mental health in Gujarat, India. Transcultural Psychiatry, 53(3), 368-391. https://doi.org/10.1177/1363461516649835

Simard, A. A., \& Henry, M. (2009). Impact of a short yoga intervention on medical students' health: A pilot study. Medical Teacher, 31(10), 950-952. https://doi.org/10.3109/01421590902874063

Singh, K., Jain, A., \& Singh, D. (2014). Satsang: A culture specific effective practice for wellbeing. In Marujo \& Neto (Eds.), Positive nations and communities (pp. 79-100). Springer. https://doi.org/10.1007/978-94-007-6869-7_5

Singh, K., Mitra, S., \& Khanna, P. (2016). Psychometric properties of Hindi version of peace of mind, harmony in life and sat-chit-ananda scales. Indian Journal of Clinical Psychology, 43(1), 58-64. https://doi.org/10.1007/978-94-007-6869-7_5

Singh, K., Khanna, P., Khosla, M., Rapelly M. \& Soni, A. (2018) Revalidation of the Sat-ChitAnanda Scale. Journal of Religion and Health, 57, 1392-1401. https://doi.org/10.1007/s10943-016-0328-2

Singh, K., Khari, C., Amonkar, R. S., Arya, N. K., \& Kumar, S. K. (2014). Development and validation of a new scale: Sat-Chit-Ananda Scale. International Journal on Vedic Foundations of Management, 1(2), 102-122. https://doi.org/10.1007/s10943-016-0328-2 
Singh, K. \& Raina, M. (2015). Development and validation of a test on Anasakti (nonattachment): An Indian model of well-being. Mental Health, Religion and Culture, 18, 715-725. https://doi.org/10.1080/13674676.2015.1084612

Singh, K., \& Raina, M. (2020). Demographic correlates and validation of PERMA and WEMWBS scales in Indian adolescents. Child Indicators Research, 13(4), 1175-1186. https://doi.org/10.1007/s12187-019-09655-1

Singh, K., \& Sahni, P. (2016). Swadhaya Scale: An Indian perspective. International Journal of Indian Psychology, 4(1), 5-24.

Srivastava, A. K., \& Misra, G. (2011). Cultural perspectives on nature and experience of happiness. In: A. K. Dalal \& G. Misra (Eds.), New directions in health psychology (pp. 109-131). Sage. https://doi.org/10.1177/097133360201400208

Waghorne, J. P. (2014). Engineering an artful practice: on Jaggi Vasudev's Isha Yoga and Sri Sri Ravi Shankar's art of living. In E. Goldberg \& M. Singleton (Eds.), Gurus of Modern Yoga (pp. 283-307). Oxford University Press. https://doi.org/10.1093/acprof:oso/9780199938704.003.0014

Wolf, D. B., \& Abell, N. (2003). Examining the effects of meditation techniques on psychosocial functioning. Research on Social Work Practice, 13,1, 27-42. https://doi.org/10.1177/104973102237471 


\title{
Chapter 14: Positive Psychology and Buddhism
}

\author{
Seth Zuihō Segall, Ph.D. ${ }^{1}$ and Jean L. Kristeller, Ph.D. ${ }^{2}$ \\ ${ }^{1}$ Independent Scholar \\ ${ }^{2}$ Department of Psychology, Indiana State University
}

Correspondence concerning this chapter should be addressed to Seth Zuihō Segall. Email: sethzuihosegall@gmail.com 


\begin{abstract}
Buddhism is a historically and culturally diverse religious and philosophical tradition that offers a positive vision of the human potential for ending suffering and for leading lives that are happy and good. In recent years, ideas drawn from the Buddhist tradition have found their way into Western psychology, informing new ways of understanding and promoting human well-being. These insights and tools include Buddhist ideas (and Buddhist-informed interventions) focused on mindfulness, radical acceptance, lovingkindness and self-compassion, self-transcendence, and the ability to disengage from unwholesome thought processes. In this chapter, we describe the diversity of Buddhist belief and practice, both in Asia and in the West. The core concepts common to distinct Buddhist traditions are reviewed, followed by an analysis of how these concepts relate to key principles of positive psychology. Research on Buddhist insights and intervention is reviewed, and contemporary clinical applications are discussed.

Keywords: Buddhism, positive psychology, spirituality, religion, mindfulness
\end{abstract}




\section{Chapter 14: Positive Psychology and Buddhism}

"Buddhism" refers to a broad range of traditions, beliefs, and practices that originated approximately 2,500 years ago in the teachings of Gautama Buddha and that have been instantiated in diverse cultures across history since then. Although these instantiations share several core ideas in common (e.g., the Four Noble Truths, Eightfold Noble Path, and Dependent Origination), they also differ in significant and meaningful ways.

At the same time, within all Buddhism's variations, we find beliefs and practices that do not fit easily within the culturally constructed Western category of "religion." Even though traditional forms of Buddhism have religious, spiritual, and supernatural elements, they also have elements of a cosmology, a philosophy, a psychology, and an ethical-spiritual path (i.e., way of life; Kristeller \& Rapgay, 2013). In this way, Buddhism shares similarities with the ancient Confucian, Daoist, and Greco-Roman traditions, which also were ways of life aimed at cultivating well-being derived from living in accord with virtue and wisdom (Hadot, 1995).

Although Positive Psychology (PP) is an empirically grounded secular undertaking, it shares an interest in promoting well-being that is common across these Confucian, Daoist, Greco-Roman, and Buddhist ways of life. Yet the question of how to incorporate the insights of a religious, philosophical, and ethical tradition like Buddhism into a secular, empirical undertaking like PP is fraught with difficulties. Helderman (2019) has explored the history of modern psychology's attempts to incorporate Buddhist insights, including the difficulties these attempts have entailed. Such difficulties include the negotiation of culturally constructed boundaries among religion, secularity, empiricism, medicine, spirituality, psychotherapy, and well-being. The ultimate goal of Buddhism is enlightenment (bodhi), which both resonates with and diverges from PP's emphasis on eudaimonia, well-being, and psychological health. 
With such richness in mind, the current chapter begins by reviewing the main trajectories of Buddhist thought throughout Asian history and during its transmission to the West, focusing on themes that are common to most forms of Buddhism and are most relevant to PP. Then we summarize how mainstream psychology and PP have incorporated these themes and modalities, how fruitful this cross-pollination has been, and issues that call for further inquiry.

\section{Early Buddhism}

Buddhism developed on the Indian subcontinent between 400 and 100 BCE, when its core tenets were first elaborated, orally transmitted, and transcribed. Although Buddhist beliefs and practices continued to develop over subsequent millennia, all classical schools of Buddhism agree on the core doctrines of the Four Noble Truths, Eightfold Noble Path, and Dependent Origination. Each of these doctrines is concerned with human suffering and how to remediate it.

\section{The Four Noble Truths}

The Buddha noted that human lives are suffused with suffering (dukkha). Some of this suffering is due to the existential contingencies of old age, sickness, and death. We also all must face situations in which our desires are frustrated or we must confront unwanted circumstances. Even when our desires are met, pleasure is fleeting, given that everything is impermanent.

The First Noble Truth states that suffering is an inextricable part of life. The Second Noble Truth avers that the cause of most suffering is our craving for pleasant sensations and our aversion toward unpleasant sensations. We try to cling to pleasures that cannot last and avoid unavoidable unpleasantness. The Third Noble Truth indicates that it is possible to eliminate craving and aversion by learning to observe them, rather than react to them. The Fourth Noble Truth identifies the Eightfold Noble Path as the path leading to the elimination of craving and aversion and thereby to the cessation of suffering (Gethin, 1998, pp. 59-80). 


\section{The Eightfold Noble Path}

The Eightfold Noble Path is the path to nibbana (nirvana in Sanskrit), an enlightened state in which craving, aversion, ignorance, and the cycle of rebirth are ended. The Path's eight elements are right view, intention, speech, conduct, livelihood, effort, mindfulness, and concentration. Right view is understanding the laws of karma - that our thoughts and actions have consequences for our well-being, both in this and future lifetimes. Right intention refers to intentions of nongreed, nonhatred, and nonharming. Right speech means desisting from lying, gossiping, and causing discord. Right conduct means avoiding killing, stealing, lying, sexual misconduct, and intoxication. Right livelihood means not engaging in harmful occupations. Right effort means initiating and maintaining wholesome mental states and preventing and stopping unwholesome ones. Right mindfulness means maintaining present-moment awareness of wholesome and unwholesome mental factors and their consequences. Right concentration refers to maintaining one-pointed concentration in meditation practice (Gethin, 1998, pp. 80-84).

\section{Dependent Origination}

Dependent Origination is the causal process though which ignorance, craving, and attachment lead to suffering. In this formula, sense contact with the external world leads to pleasant, unpleasant, or neutral feelings (vedenas). Pleasant feelings lead to craving (tanha) and attachment (upadana). In turn, craving and attachment embroil us in an endless cycle of suffering. Mindfulness of cravings and aversions can allow us to break free of this chain of causation (Williams, 2000, pp. 62-72). Dependent Origination shows we can release ourselves from the control of previously conditioned reactions and shift towards greater flexibility and wiser choices. We learn to pause and mindfully respond to situations rather than just react to them. 


\section{Buddhist Virtues}

Early Buddhism outlines sets of virtues to be cultivated. Chief among these virtues are the Divine Abodes (Brahmaviharas) of compassion, lovingkindness, equanimity, and joy in the good fortune of others (Rahula, 1959). There is also an additional set of virtues (Paramis) that, in addition to those already named, includes generosity, morality, renunciation, wisdom, energy, patience, truthfulness, and resolve (Bodhi, 2011), each of which is clearly aligned with PP.

\section{Non-Self (Anatta)}

The Buddha taught that human beings were bundles of psychophysical processes (kandhas) that were subject to continuous change and impermanence (and hence that there can be no unchanging soul or self [atman] that defines one's inner essence; Williams, 2000). We arise out of complex sets of causes and conditions that undergo change as a result of our actions and experiences. Our adult selves, for example, are quite different from our childhood selves.

\section{Later Indian Buddhism (100 BCE-1100 CE)}

Over the next millennium, Buddhism underwent significant elaborations on the Indian subcontinent, as it dialogued with other Indian schools of thought that were developing alongside it. These elaborations included the emergence of Mahayana Buddhism; the Madhyamika, Tathagatagarbha, and Yogacara schools of thought; and Tantric practice. This chapter cannot explore all these complex developments, but we will highlight a few aspects of some of them.

Mahayana Buddhism emerged between 100 BCE and 100 CE. It is marked by changes in the motivation for and the envisioned endpoint of the Buddhist path. Mahayana Buddhists aspire to free all beings from suffering. Rather than seeking personal nibbana, they aim at becoming bodhisattvas who delay their personal liberation until all beings first are saved. Mahayana 
Buddhism became the dominant school of Buddhism in China, Japan, Korea, and Vietnam and the foundation for the development of Vajrayana Buddhism in Tibet (Harvey, 1990).

Nagarjuna (c. 150-250 CE) developed the Madhyamika school that taught that all phenomena (dharmas) are empty (sunyata) of self-existence (Westerhoff, 2018). No phenomenon has an inner essence that makes it what it is; instead, phenomena arise out of their complex relationships with everything that exists. For example, people exist because of their historical relations to the sets of past causes and conditions from which they arose and their ongoing relations with their social, cultural, and natural worlds. Humans have no fixed inner essence apart from these. The same is true for all phenomena. Additionally, concepts only mean something as part of an interrelated web of concepts (e.g., something can only be big if something else is small).

Yogacara Buddhism taught our thoughts and actions leave karmic seeds (bija) that can develop, lie fallow, or wither in an unconscious storehouse (alaya vijñana), as a consequence of cultivating or abandoning them. It emphasized our experience of reality is not simply out thereit is interpreted, constructed, and projected by the mind (Westerhoff, 2018). Tantric Buddhism introduced sets of mantras (ritual phrases), mudras (body postures/gestures), energy meditations, visualizations, and devotional and antinomian practices designed to speed the path to enlightenment. Tantra had its largest influence on Vajrayana Buddhism in Tibet and Shingon Buddhism in Japan, but tantric practices can be found throughout all of Asia (Skilton, 1994).

\section{Buddhism Beyond India}

Early Indian Buddhism traveled southward to Sri Lanka (c. 300 BCE) and became the basis for the Theravada Buddhism of Sri Lanka, Thailand, Burma, Laos, and Cambodia. Mahayana Buddhism spread northward along the Silk Road from India to China (c. 50-200 CE) 
and Tibet around (c.700-900 CE; Skilton, 1994). Buddhism took on the local coloration of indigenous cultures in each country it was transmitted to. Chinese Buddhism, for example, was influenced by Daoism and Confucianism. New sects quickly arose, as Buddhism adapted to the needs of East Asian and Tibetan culture. Japan, for example, saw the emergence of the Pure Land, True Pure Land, Tendai, Kegon, Shingon, Soto Zen, Rinzai Zen, and Nichiren Buddhist sects. It is beyond the scope of this chapter to elaborate the differences among these new sects, except to note the important influence of Theravada vipassana, East Asian Zen, and Tibetan Vajrayana on Western culture, mainstream psychology, and PP.

The $19^{\text {th }}$ century saw attempts to modernize Asian Buddhism, largely as part of a complex response to Western colonialism (McMahan, 2008). The Sinhalese Buddhist revival (Prothero, 1996), Southeast Asian Vipassana (Braun, 2013), and Japanese Meiji Era Zen (Rutschman-Byler, 2014) are examples of modernized forms of Buddhism that came to influence Western mainstream psychology.

Buddhism came to America primarily through successive waves of Asian immigration during the $19^{\text {th }}$ and $20^{\text {th }}$ centuries. Most American Buddhists are Asian-descent Buddhists whose temples and churches provided a venue where their immigrant forbears could practice and transmit their culture (Han, 2021). Buddhism also came to America through European-descent travelers to Asia who brought Buddhist practices and beliefs back with them, as well as Asianborn teachers who taught Westerners in newly established practice centers. Early interest was also stimulated by the publications of Anglo-American popularizers, Beat Generation poets and novelists, and psychotherapists like Carl Jung, Erich Fromm, and Karen Horney (Fields, 1986). The predominantly non-Asian American Buddhist communities that arose from these later influences tend to emphasize lay meditative practice that includes extended meditation 
retreats. They tend to be skeptical of Buddhism's supernatural elements and to focus more on its psychological aspects, viewing Buddhism as a path to enhance people's well-being in meaningful ways (Segall, 2020).

\section{Positive Psychology and Buddhism}

\section{Enlightenment and Human Flourishing}

Although Buddhism and PP both aim at human flourishing, they have somewhat different conceptions of what flourishing entails. From its inception, PP has drawn on a conception of human flourishing that includes elements of subjective well-being, virtue, and meaning. Peterson and Seligman (2004) developed a neo-Aristotelian classification of character strengths and virtues they imagined might lie at the heart of human flourishing. Seligman's (2011) later PERMA model proposed a list of factors purported as the core constituent elements of human

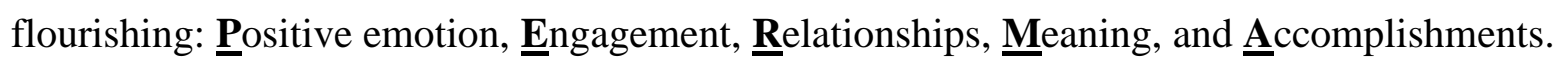

The Buddhist ideal of enlightenment - a state of subjective well-being marked by inner peace, nonhatred, nongreed, and the virtues of generosity, equanimity, and compassion — has elements in common with PP's conception of flourishing. Yet Buddhist enlightenment also includes metaphysical, transcendent, and supernatural connotations beyond what an empirical, naturalistic psychology can embrace (see Nelson \& Canty, Chapter 2, this volume). Contemporary authors (Batchelor, 1997; Flanagan, 2011; Segall, 2020) have proposed naturalized models of enlightenment that bring it more in line with PP. They view enlightenment as a set of positive mental states and virtues that jointly constitute flourishing. Even though this conception of enlightenment is compatible with PP, it probably is not the view of most Buddhists today, and critics worry naturalizing enlightenment diminishes it as an ideal. 


\section{Buddhist Influences on Positive Psychology}

Buddhist ideas have had a major impact on PP, especially the ideas that: (a) mindfulness, lovingkindness, and compassion are cultivatable qualities that enhance well-being; (b) attachment and aversion are causes of surplus suffering that can be remediated through attitudes of acceptance; (c) notions of a single "true self" can benefit from increased fluidity and relationality; and (d) self-transcendent spiritual experiences can enhance meaning and growth.

Mindfulness. Mindfulness found its way into contemporary Western psychology through the work of psychologists and scholars who pursued Buddhist practices and sought to share their application through accessible writings and clinical interventions (Helderman, 2019). One of the most influential has been Jon Kabat-Zinn (1982, 1990, 2005), who defined mindfulness as "paying attention in a particular way: on purpose, in the present moment, and non-judgmentally" (2005, p. 4), a definition that combines metacognitive awareness with an attitude of nonjudgmental acceptance. Even though this definition differs from the classical Theravada formulation (Bodhi, 2011; Gethin, 2011), it adheres closely to the Zen understanding.

Kabat-Zinn's Mindfulness-Based Stress Reduction (MBSR) offered mindfulness meditation as a vehicle for health and wellness. His 8-week MBSR format became the prototype for Mindfulness-Based Cognitive Therapy for recurrent depression (Segal, et al. 2013), Mindfulness-Based Eating Awareness Training for eating issues (Kristeller, 2019), and many other mindfulness-based interventions designed to address a large variety of mental disorders and medical conditions. Other interventions with mindfulness components include Marsha Linehan's (1993) Dialectal Behavioral Therapy for borderline personality disorder (which grew out of her experiences with Zen Buddhism) and Steven Hayes's Acceptance and Commitment Therapy (which had an independent origin; Hayes et al., 2003). 
Mindfulness has had a profound influence on clinical and neuropsychological research. The American Mindfulness Research Association's database has tracked over 7,000 articles on mindfulness in scientific publications through February 2020 (AMRA Library, 2021). This field has grown so large that Goldberg et al. (2021) recently reviewed results from 44 meta-analyses, representing 336 randomized controlled trials of mindfulness-based interventions $(N=30,483)$.

The integration of mindfulness with cognitive-behavioral therapy led to the emergence of the so-called "third-wave" of cognitive-behavioral therapies. However, as Ivtzan (2016) has pointed out, most mindfulness-based intervention studies measure outcomes in terms of symptoms and problems (not growth, flourishing, and well-being). Although mindfulness studies relevant to PP (rather than clinical psychology) are exceptions to the rule, there are now several promising mindfulness-based PP interventions designed to promote well-being in nonclinical populations, including Mindfulness-Based Strengths Practice (Niemiec \& Lissing, 2016) and the Mindfulness-Based Flourishing Program (Ivtzan et al., 2016b).

Mindfulness-Based Strengths Practice is a manualized 8-week program that integrates Peterson and Seligman's (2004) character strengths approach with Kabat-Zinn's MBSR. It encourages participants to reflect on their signature character strengths during formal meditation and throughout the day. Participants learn to appreciate and celebrate their character strengths; recognize when these strengths are operating, overused, or underused; and utilize their strengths to solve problems and navigate life. The program emphasizes developing authenticity and virtuous character. Niemiec and Lissing (2016) have reported promising results of three case studies and two nonrandomized pilot studies of its efficacy. Similarly, a pilot study of an online version of the intervention (Ivtzan et al., 2016a) found significant medium-to-large improvements in life satisfaction, flourishing, engagement, and signature strengths. In sum, 
Mindfulness-Based Strengths Practice is a promising mindfulness-based PP intervention that merits further testing with randomized assignment, active controls, and larger sample sizes.

The Mindfulness-Based Flourishing Program is an 8-week online program consisting of videotaped presentations and guided meditations. It combines mindfulness training with didactic content on self-awareness, positive emotions, self-compassion, self-efficacy, autonomy, relationships, and savoring. Research suggests the program significantly improves mindfulness, well-being, gratitude, compassion, self-efficacy, autonomy, meaning, and savoring compared to waitlist controls (Ivtzan et al., 2016b; Ivtzan et al., 2018), as well as work-based meaning, authenticity, flow, self-efficacy, and athletic motivation and training satisfaction (Young \& Ivtzan, 2019). The Mindfulness-Based Flourishing Program is a promising PP intervention that requires further validation in studies with active control comparators.

Several studies demonstrate the impact of more traditional mindfulness-based interventions on spiritual well-being. These studies find mindfulness practice can lead to meaningful increases in people's spiritual engagement, even when presented in a secular context, and this engagement plays a key role in improving its practitioners' well-being and selffunctioning. These studies find that mindfulness training increases love, spirituality, gratitude, and appreciation of beauty (Pang \& Ruch, 2019) and an increased spirituality that contributes to reduced distress and self-reported medical symptoms (Carmody et al., 2008). Greeson et al. (2011) found a significant proportion of improvement in post-MBSR depressive symptoms was uniquely explained by changes in spirituality and mindfulness. Geary and Rosenthal (2011) found that MBSR improved health care workers' spirituality and well-being, and Garland et al. (2007) found cancer patients who received MBSR improved more in spirituality than controls. A study on coping with early-stage cancer (Henderson et al., 2012) found MBSR superior to both a 
nutritional and usual-care control in terms of quality of life, spiritual well-being, and meaningfulness. Kristeller and Jordan (2018) found participation in a mindfulness-based eating intervention resulted in marked improvement in spiritual well-being that explained mindfulness's impact on eating regulation.

Self-Compassion. Kristin Neff (2003) introduced self-compassion to mainstream and PP. Neff's conception of self-compassion is deeply rooted in Buddhist psychology. Self-compassion has three facets, which Neff calls self-kindness, common humanity, and mindfulness. Selfkindness is the capacity to look on one's failings and suffering with compassion and understanding and without critical self-flagellation. Common humanity is the recognition that one's failings and sufferings are common to all humankind. Mindfulness is the ability to observe one's failings and sufferings intimately without suppression, minimization, or exaggeration.

Neff (2014) and Germer and Neff (2019) summarized a large body of research on selfcompassion. This research shows people higher in self-compassion are happier, more optimistic, and more satisfied with their lives and their relationships. They also are more emotionally connected, accepting, autonomy-supporting, willing to compromise, and concerned for others. A meta-analysis of 27 randomized controlled trials of self-compassion interventions found these interventions promote substantial increases in self-compassion and mindfulness and decreases in rumination, self-criticism, disordered eating, stress, depression, and anxiety (Ferrari et al., 2019). Importantly, Neff (2003) has differentiated self-compassion from self-esteem, the latter of which she views as being more closely linked with narcissism, inflated self-views, and the need to perceive oneself as superior to others. By comparison, levels of self-compassion are more closely associated with measures of happiness and optimism than levels of self-esteem are. 
Neff and Germer (2013) developed an 8-week Mindful Self-Compassion training program based on the MBSR structural model that includes mindfulness, lovingkindness, and compassion meditation practice and emphasizes compassionate listening, meeting difficult emotions, exploring challenging relationships, and embracing life with savoring, gratitude, and selfappreciation. Research shows Mindful Self-Compassion training can increase self-compassion, compassion for others, mindfulness, personal self-efficacy, and life satisfaction and can decrease stress, anxiety, depression, negative self-directed thinking, and diabetic distress (Bluth et al., 2016; Dundas, et al., 2017; Friis et al., 2016: Neff \& Germer, 2013). These studies, which are described more fully in Appendix 14.S1, suggest self-compassion is a teachable skill that can substantially enhance people's well-being.

Lovingkindness. The Theravada practice of lovingkindness (metta) meditation, introduced to the English-speaking West by Thich Nhat Hanh (2014) and Sharon Salzberg (1995), focuses on opening the heart to love and caring for others (Kristeller \& Johnson, 2005). It heightens the ability to experience empathy, benevolence, and compassion toward oneself and others, and is similar to compassion meditations in Tibetan Buddhism that have also received empirical attention (see Davidson \& Harrington, 2002; Goleman, 2003; Lutz et al., 2007, 2009).

The practice of lovingkindness meditation involves the silent self-recitation of the wish that sentient beings be happy, healthy, safe, and well. These wishes for well-being are first directed to oneself and then in turn to loved ones, neutral parties, people one finds difficult, and all beings. As one recites each wish, one mindfully observes the mind, heart, and body's reactions to each wish, before proceeding to the next meditative focus of lovingkindness. Buddhists consider lovingkindness to be one of the four Brahmaviharas (heavenly abodes) that help practitioners develop attitudes of benevolence and nonharming towards others. 
Lovingkindness meditations are often incorporated into mindfulness-based interventions of various kinds, including MBSR, Mindfulness-Based Cognitive Therapy, and Mindfulness SelfCompassion Training.

Several studies have examined the effects of lovingkindness meditation on well-being. In a seminal study, Fredrickson et al. (2008) found workplace lovingkindness meditation training led to significantly higher daily levels of amusement, awe, contentment, gratitude, hope, interest, joy, love, and pride relative to a no-treatment control. Increases in daily positive emotions led to increases in mindfulness, environmental mastery, self-acceptance, purpose in life, and positive relations with others, which in turn led to increases in life satisfaction. More broadly, Zeng's (2015) meta-analysis of 24 lovingkindness studies found medium-sized effects for increases in daily positive emotions. Studies have also found associations between lovingkindness practice and self-compassion, perceived social integration, decreased self-criticism, and altruistic behavior (Fredrickson et al. 2019; Leiberg et al. 2011; Shahar et al., 2015). Two studies suggest a possible link between practicing lovingkindness meditation and preserving telomere length (Hoge et al. 2013; Le Nguyen et al. 2019), suggesting it promotes people's physical health too.

Although the above studies support the benefits of lovingkindness meditation, only Le Nguyen et al. (2019) compared lovingkindness to an active control. Two studies suggest lovingkindness-based improvements in well-being may not be superior to improvements in wellbeing resulting from physical exercise (Galante et al., 2016) or listening to classical music (Sorenson et al, 2019). Although lovingkindness and self-compassion interventions show the promise of testing Buddhist ideas and techniques with modern research methodology, both require future testing with active controls. All the studies in this section are described in greater depth in Appendix 14.S1. 
Self and Self-Transcendence. Many humanistic and psychodynamic approaches to meditation (Epstein, 2001; Fromm, 1994; Rubin, 1996) entail a shift in self-experiencing that reveals a more porous, ephemeral, and relational self that is in line with the Buddhist idea of nonself. This changed self-experiencing typically emerges with meditative practice over time. This perspective runs counter to Western beliefs in an unchanging "true self." Letting go of rigid, essentialist notions of a "true self" can free one from limiting self-narratives, opening one up to deepening connections with others and with Nature.

There are also rapid, dramatic shifts in self-perception that emerge from self-transcendent spiritual experiences (James, 1902/1936). As Stace (1961) states, an experience "of only a few moments' duration" can transform a life previously felt to be "meaningless and worthless" into one imbued with "meaning, value, and direction" (pp. 60-61). Mahayana Buddhism employs a variety of meditative techniques to help practitioners attain self-transcendent experiences that evoke a vital sense of connection to the world and act as renewed sources of meaning.

Because it is hard to induce self-transcendent states experimentally through meditationmeditation may pave the way for them but can never produce them on demand —experimental psychology has turned to psychedelic experiences as possible analogues to Buddhist meditative states of self-transcendence. This is not without controversy, as Buddhism eschews intoxicants, and some Buddhist teachers suggest these experiences are not good analogs to meditative selftranscendent states. Nevertheless, Buddhist ideas concerning emptiness and self-transcendence have had a major impact on how these experiences are often interpreted (Huxley, 1954; Leary et al., 1964; Watts, 1962).

It is beyond the scope of this chapter to review the vast literature on psychedelics and well-being. Although early studies (Grof, et al., 1973; Pahnke, 1963) demonstrated the value of 
psychedelic spiritual experiences for personal growth, changes in how the law classified psychedelics in the 1970s put a temporary halt to research. Studies by researchers at the Johns Hopkins University Center for Psychedelic and Consciousness Research and the New York University Langone Center have recently revived the field (Pollan, 2018).

Researchers at the Johns Hopkins's group (Griffiths et al., 2008, 2011, 2018; MacLean et al., 2011) have found psilocybin reliably produces self-transcendent, sacred, and ineffable experiences that participants rate as among most significant spiritual experiences of their lives. Participants report long-lasting improvements in mood, attitude, life satisfaction, altruism, interpersonal closeness, gratitude, forgiveness, meaning, purpose in life, optimism, death transcendence, sanctification of strivings, daily spiritual experiences, religious faith, and coping. These studies are described in greater detail in Appendix 14.S1.

\section{Conclusion}

The Buddhist tradition has had a strong influence on PP, especially with regard to mindfulness, self-compassion, lovingkindness, and self-transcendence. It has encouraged psychologists to think of mindfulness, altruism, compassion, and kindness as teachable skills that can foster personal and collective flourishing. Engaging in these skills often enhances spiritual well-being, even when spiritual well-being is not explicitly addressed. These ideas have been integrated into interventions that have demonstrated promising potential for helping people lead flourishing and fulfilling lives. 


\section{References}

American Mindfulness Research Association Library. (2021). https://goamra.org/Library.

Batchelor, S. (1997). Buddhism without beliefs: A contemporary guide to awakening. Riverhead.

Bluth, K., Gaylord, S., Campo, R., Mullarkey, M., \& Hobbs, L. (2016). Making friends with yourself: A mixed methods pilot study of a mindful self-compassion program for adolescents. Mindfulness, 7, 479-492. https://doi.org/10.1007/s12671-015-0476-6

Bodhi, B. (2011). What does mindfulness really mean? A canonical perspective. Contemporary Buddhism, 12(1), 19-39. https://doi.org/10.1080/14639947.2011.564813

Braun, E. (2013). The birth of insight: Meditation, Buddhist modernism, and the Burmese monk Ledi Sayadaw. University of Chicago Press.

Carmody, J., Reed, G., Kristeller, J., \& Merriam, P. (2008). Mindfulness, spirituality, and healthrelated symptoms. Journal of Psychosomatic Research, 64(4), 393-403. https://dois.org/10.1016/j.jpsychores.2007.06.015

Davidson, R. J., \& Harrington, A. (Eds.). (2002). Visions of compassion. Oxford.

Dundas, I., Binder, P. E., Hansen, T. G. B., \& Stige, S. H. (2017). Does a short self-compassion intervention for students increase healthy self-regulation? A randomized control trial. Scandinavian Journal of Psychology, 58(5), 443-450. https://doi.org/10.1111/sjop.12385

Epstein, M. (2001). Going on being: Buddhism and the way of change. Broadway Books.

Ferrari, M., Hunt, C., Harrysunker, A., Abbott, M. J., Beath, A. P., \& Einstein, D. A. (2019). Self-compassion interventions and psychosocial outcomes: A meta-analysis of RCTs. Mindfulness, 10, 1455-1473. https://doi.org/10.1007/s12671-019-01134-6

Fields, R. (1986). How the swans came to the lake. Shambhala.

Flanagan, O. (2011). The Bodhisattva's brain. MIT Press. 
Fredrickson, B. L., Arizmendi, C., Van Cappellen, P., Firestine, A. M., Brantley, M. M., Kim, S. L., Brantley, J., \& Salzberg, S. (2019). Do contemplative moments matter? Effects of informal meditation on emotions and perceived social integration. Mindfulness, 10(9), 1915-1925. https://doi.org/10.1007/s12671-019-01154-2

Fredrickson, B., Cohn, M., Coffey, K., Pek, J., \& Finkel, S. (2008). Open hearts build lives: Positive emotions, induced through loving-kindness meditation, build consequential personal resources. Journal of Personality and Social Psychology, 95(5), 1045-1062. https://doi.org/10.1037/a0013262

Friis, A. M., Johnson, M. H., Cutfield, R. G., \& Consedine, N. S. (2016). Kindness matters: A randomized controlled trial of a mindful self-compassion intervention improves depression, distress, and HbA1c among patients with diabetes. Diabetes Care, 39(11), 1963-1971. https://doi.org/10.2337/dc16-0416

Fromm, E. (1994). The art of being. Continuum International.

Galante, J., Bekkers, M.-J., Mitchell, C., \& Gallacher, J. (2016). Loving-kindness meditation effects on well-being and altruism: A mixed-methods online RCT. Applied Psychological Health and Well-Being, 8, 322-350. https://doi.org/10.1111/aphw.12074

Garland, S. N., Carlson, L. E., Cook, S., Lansdell, L., \& Speca, M. (2007). A non-randomized comparison of mindfulness-based stress reduction and healing arts programs for facilitating post-traumatic growth and spirituality in cancer outpatients. Supportive Care in Cancer, 15, 949-961. https://doi.org/10.1007/s00520-007-0280-5

Geary, C., \& Rosenthal, S. L. (2011). Sustained impact of MBSR on stress, well-being, and daily spiritual experiences for 1 year in academic health care employees. Journal of Alternative and Complementary Medicine, 17(10), 939-944. https://doi.org/10.1089/acm.2010.0335 
Germer, C., \& Neff, K. (2019). Mindful self-compassion (MSC). In I. Ivtzan (Ed.), The handbook of mindfulness-based programs (pp. 357-367). Routledge.

Gethin, R. (1998). The foundations of Buddhism. Oxford University Press.

Gethin, R. (2011). On some definitions of mindfulness. Contemporary Buddhism, 12, 263-279. https://doi.org/10.1080/14639947.2011.564843

Goldberg, S., Riordan, K, Sun, S., \& Davidson, R. (2021). The empirical status of mindfulnessbased interventions: A systematic review of 44 meta-analyses of randomized controlled trials. Perspectives on Psychological Science. Advance online publication: https://doi.org/10.1177/1745691620968771

Goleman, D. (2003). Destructive emotions: How can we overcome them? Bantam Books.

Greeson, J. M., Webber, D. M., Smoski, M. J., Brantley, J. G., Ekblad, A. G., Suarez, E. C., \& Wolever, R. Q. (2011). Changes in spirituality partly explain health-related quality of life outcomes after mindfulness-based stress reduction. Journal of Behavioral Medicine, 34(6), 508-518. https://doi.org/10.1007/s10865-011-9332-x

Griffiths, R., Johnson, M., Richards, W., McCann, U., \& Richards, B. (2008). Mystical-type experiences occasioned by psilocybin mediate the attribution of personal meaning and spiritual significance 14 months later. Journal of Psychopharmacology, 22(6), 621-632. https://doi.org/10.1177/0269881108094300

Griffiths, R., Johnson, M., Richards, W., Richards, B., McCann, U., \& Jess, R. (2011). Psilocybin occasioned mystical-type experiences: Immediate and persisting dose-related effects. Psychopharmacology, 218, 649-665. https://doi.org/10.1007/s00213-011-2358-5

Griffiths, R. Johnson, M. Richards, W., Richards, B., Jesse, R., ... Klinedinst, M. (2018). Psilocybin-occasioned mystical-type experience in combination with meditation and 
other spiritual practices produces enduring positive changes in psychological functioning and in trait measures of prosocial attitudes and behaviors. Journal of Psychopharmacology, 32(1), 49-69. https://doi.org/10.1177/0269881117731279

Grof, S., Goodman, L., Richards, W. \& Kurland, A. (1973). LSD-assisted psychotherapy in patients with terminal cancer. International Pharmacopsychiatry, 8, 129-144.

Hadot, P. (1995). Philosophy as a way of life (M. Chase, trans.). Blackwell.

Han, C. (2021). Be the refuge. North Atlantic Books.

Hahn, T. N. (2014). No mud, no lotus. Parallax Press.

Harvey, P. (1990). An introduction to Buddhism: Teachings, history, and practices. Munshiram Manoharlal Publishers.

Hayes, S., Strosahl, K., \& Wilson, K. (2003). Acceptance and commitment therapy. Guilford. Helderman, I. (2019). Prescribing the Dharma. University of North Carolina Press.

Henderson, V. P., Clemow, L., Massion, A. O., Hurley, T. G., Druker, S., \& Hébert, J. R. (2012). The effects of mindfulness-based stress reduction on psychosocial outcomes and quality of life in early-stage breast cancer patients: A randomized trial. Breast Cancer Research and Treatment, 131(1), 99-109. https://doi.org/10.1007/s10549-011-1738-1

Hoge, E., Chen, M., Orr, E., Metcalf, C., Fischer, L., Pollack, M., De Vivio, I., \& Simon, N. (2013). Loving-kindness meditation practice associated with longer telomeres in women. Brain, Behavior and Immunity, 32, 159-163. https://doi.org/10.1016/j.bbi.2013.04.005

Huxley, A. (1954). The doors of perception. Harper \& Row.

Ivtzan, I. (2016). Mindfulness in positive psychology: An introduction. In I. Ivtzan and T. Lomas (Eds.), Mindfulness in positive psychology (pp. 1-12). Routledge. 
Ivtzan, I., Niemiec, R., \& Briscoe, C. (2016a). A study on the effects of mindfulness-based strengths practice (MBSP) on wellbeing. International Journal of Wellbeing, 6(2), 1-13. https://doi.org/doi:10.5502/ijw.v6i2.1

Ivtzan, I., Young, T., Martman, J., Jeffrey, A., Lomas, T., Hart, R., et al. (2016b). Integrating mindfulness into positive psychology: A randomised controlled trial of an online positive mindfulness program. Mindfulness, 7(6), 1396-1407. https://doi.org/10.1007/s12671-

\section{$\underline{016-0581-1}$}

Ivtzan, I., Young. T., Lee, H., Lomas, T., Daukantaite, D., \& Kjell, O. (2018). Mindfulness based flourishing program: A cross-cultural study of Hong Kong Chinese and British participants. Journal of Happiness Studies, 9, 2205-2223. https://doi.org/10.1007/s10902-017-9919-1

James, W. (1902/1936). The varieties of religious experience. Modern Library.

Kabat-Zinn, J. (1982). An outpatient program in behavioral medicine for chronic pain patients based on the practice of mindfulness meditation. General Hospital Psychiatry, 4, 33-47.

Kabat-Zinn, J. (1990). Full catastrophe living. Delacorte Press.

Kabat-Zinn, J. (2005). Wherever you go, there you are. Hyperion.

Kristeller, J. (2019). Mindfulness-based eating training (MB-EAT). In I. Ivtzan (Ed.), The handbook of mindfulness-based programmes (pp. 191-203). Routledge.

Kristeller, J., \& Johnson, T. (2005). Cultivating loving kindness: A two-stage model of the effects of meditation on empathy, compassion, and altruism. Zygon, 40, 391-407. https://doi.org/10.1111/j.1467-9744.2005.00671.x

Kristeller, J. L., \& Jordan, K. (2018). Mindful eating: Connecting with the wise self, the spiritual self. Frontiers in Psychology, 9, Article 1271. https://doi.org/10.3389/fpsyg.2018.01271 
Kristeller, J. L., \& Rapgay, L. (2013). Buddhism: A blend of religion, spirituality, and psychology. In K. Pargament, J. Exline, \& J. Jones (Eds.), APA handbook of psychology, religion, and spirituality. (Vol. 1, pp. 635-652). American Psychological Association.

Le Nguyen, K. D., Lin, J., Algoe, S. B., Brantley, M. M., Kim, S. L., Brantley, J., Salzberg, S., \& Fredrickson, B. L. (2019). Loving-kindness meditation slows biological aging in novices: Evidence from a 12-week randomized controlled trial. Psychoneuroendicrinology, 108, 20-27. https://doi.org/10.1016/j.psyneuen.2019.05.020

Leary, T., Alpert, R., \& Metzner, R. (1964). The psychedelic experience. University Books.

Leiberg, S., Klimecki, O., \& Singer T. (2011). Short-term compassion training increases prosocial behavior in a newly developed prosocial game. PLoS One, 6(3), ei7798. https://doi.org/10.1371/journal.pone.0017798

Linehan, M. (1993). The cognitive-behavioral treatment of borderline personality disorder. Guilford Press.

Lutz, A., Dunne, J., \& Davidson, R. (2007). Meditation and the neuroscience of consciousness: An introduction. In P. D. Zelazo, M. Moscovitch, \& E. Thompson (Eds.), The Cambridge handbook of consciousness (pp. 499-551). Cambridge University Press.

Lutz, A., Greischar, L. L., Perlman, D. M., \& Davidson, R. J. (2009). BOLD signal in insula is differentially related to cardiac function during compassion meditation in experts vs. novices. Neuroimage, 47, 1038-1046. https://doi.org/10.1016/j.neuroimage.2009.04.081

MacLean, K. A., Johnson, M., \& Griffiths, R. (2011). Mystical experiences occasioned by the hallucinogen psilocybin lead to increases in the personality domain of openness. Journal of Psychopharmacology, 25, 1453-1461. https://doi.org/10.1177/0269881111420188

McMahan, D. L. (2008). The making of Buddhist modernism. Oxford University Press. 
Neff, K. (2003). Self-compassion: An alternative conceptualization of a healthy attitude toward oneself. Self and Identity, 2(2), 85-102. https://doi.org/10.1080/15298860309032

Neff, K. (2014). The science of self-compassion. In C. Germer \& R. Siegel (Eds.), Compassion and wisdom in psychotherapy (pp. 79-92). Guilford Press.

Neff, K., \& Germer, C. (2013). A pilot study and randomized controlled trial of the mindful selfcompassion program. Journal of Clinical Psychology, 69(1), 28-44.

Niemiec, R., \& Lissing, J. (2016). Mindfulness-based strengths practice (MBSP) for enhancing well-being, managing problems, and boosting positive relationships. In I. Ivtzan \& T. Lomas (Eds.), Mindfulness in positive psychology (pp. 15-26). Routledge.

Pahnke, W. (1963). An analysis of the relationship between psychedelic drugs and the mystical states of consciousness [Doctoral dissertation, Harvard University]. https://maps.org/images/pdf/books/pahnke/walter_pahnke_drugs_and_mysticism.pdf

Pang, D., \& Ruch, W. (2019). The mutual support model of mindfulness and character strengths. Mindfulness, 10(8), 1545-1559. https://doi.org/10.1007/s12671-019-01103-Z

Peterson, C., \& Seligman, M. (2004). Character strengths and virtues. Oxford University Press. Pollan, M. (2018). How to change your mind. Penguin.

Prothero, S. (1996). The White Buddhist: The Asian odyssey of Henry Steel Olcott. Indiana University Press.

Rahula, W. (1959). What the Buddha taught. Evergreen.

Rubin, J. B. (1996). Psychotherapy and Buddhism: Toward an integration. Plenum Press.

Rutschman-Byler, J. M. (2014). Soto Zen in Meiji Japan [Master's thesis]. University of California-Berkeley.

Salzberg, S. (1995). Loving-kindness. Shambala. 
Segal, Z., Williams, M., \& Teasdale, J. (2013). Mindfulness-based cognitive therapy for depression ( $2^{\text {nd }}$ ed.). Guilford Press.

Segall, S. (2020). Buddhism and human flourishing. Palgrave MacMillan.

Seligman, M. E. P. (2011). Flourish. Simon and Schuster.

Shahar, B., Szsepsernwol. O., Zilcha-Mano. S., Haim, N., Zamir, O., Levi-Yeshuvi, S., and Levit-Binnun, N. (2015). A wait-list randomized controlled trial of loving-kindness meditation programme for self-criticism. Clinical Psychology and Psychotherapy, 22(4), 246-256. https://doi.org/10.1002/cpp.1893

Skilton, A. (1994). A concise history of Buddhism. Windhorse Publications.

Sorenson, S., Steindl, S., Dingle, G., \& Garcia, A. (2019). Comparing the effects of lovingkindness meditation (LKM), music, and LKM plus music on psychological well-being. Journal of Psychology, 153, 267-287. https://doi.org/10.1080/00223980.2018.1516610

Stace, W. T. (1961). Mysticism and philosophy. MacMillan and Company.

Young, T., \& Ivtzan, I. (2019). The Flourishing Mindfulness Programme: Overview and result from six randomised control trials. In I. Ivtzan (Ed.), Handbook of mindfulness-based programmes (pp. 403-413). Routledge.

Watts, A. (1962). The joyous cosmology: Adventures in the chemistry of consciousness. Vintage.

Westerhoff, J. (2018). The golden age of Buddhist philosophy. Oxford University Press.

Williams, P. (2000). Buddhist thought. Routledge.

Zeng, X., Chiu, C., Wang, R., Oei, T., \& Leung, F. (2015). The effect of loving-kindness meditation on positive emotions: A meta-analytic review. Frontiers in Psychology, 6, Article 1693. https://doi.org/10.3389/fpsyg.2015.01693 


\title{
Chapter 15
}

Positive Psychology and Religion/Spirituality Across Cultures in Europe, Non-U.S. North America, and South America

\author{
Clàudia Rossy ${ }^{1}$ \\ María Gámiz ${ }^{1}$ \\ Silvia Recoder ${ }^{1}$ \\ Iris Crespo $^{1}$ \\ Maria Fernández-Capo ${ }^{1}$ \\ Edward B. Davis ${ }^{2}$ \\ Ethan K. Lacey ${ }^{2}$
}

${ }^{1}$ Department of Basic Sciences, Universitat Internacional de Catalunya (UIC), Barcelona, Spain

${ }^{2}$ School of Psychology, Counseling, and Family Therapy, Wheaton College, Wheaton, IL, USA

Correspondence concerning this chapter should be addressed to Clàudia Rossy. Email: crossy@uic.es 


\begin{abstract}
The purpose of this chapter is to synthesize and critique the research on positive psychology and psychology of religion/spirituality (R/S) that has been conducted in Europe, non-U.S. North America (Canada and Central America), and South America. In light of the importance of considering cultural differences and similarities across religions and continents, this chapter synthesizes how cultural factors may affect research and practice on positive psychology and the psychology of R/S in these regions of the world. We start with a brief overview of the sociocultural and religious backdrop of these regions. Then we turn to review the main findings in each area, specifying how and what topics have been emphasized in the research on positive psychology and R/S. Finally, we consider practical implications for research and clinical practice, as well as some gaps in the current literature and future directions for research.
\end{abstract}

Keywords: positive psychology, religion, spirituality, Europe, America 


\section{Chapter 15: Positive Psychology and Religion/Spirituality Across Cultures in Europe,}

\section{Non-U.S. North America, and South America}

In an interview with an atheist Italian intellectual and founder of the newspaper $L a$ Repubblica, Pope Francis (Vatican, 2013) addressed the spiritual dimension of human life:

Let me ask you a question: You, a secular nonbeliever in God, what do you believe in? You are a writer and a man of thought. You believe in something, you must have a dominant value. Don't answer me with words like honesty, seeking, the vision of the common good — all important principles and values — but that is not what I am asking. I am asking what you think is the essence of the world, indeed the universe. You must ask yourself, of course, like everyone else, who we are, where do we come from, where we are going. Even children ask themselves these questions. And you? This quote highlights that finding meaning in life, searching for purpose, and experiencing hope and transcendence reflect universal aspects of human existence. This fact is underscored by the presence of religion/spirituality $(\mathrm{R} / \mathrm{S})$ across a wide variety of cultural settings as an important part of many people's lives. The fields of positive psychology and psychology of R/S both emphasize clinical implications of existential and core issues for human beings, including virtues, relational experiences, and meaning making (Davis et al., Chapter 1, this volume). In addition, within positive psychology, R/S is one of the universal character strengths that promotes well-being and contributes to a life worth living (Peterson \& Seligman, 2004). Nevertheless, the contribution of R/S to well-being may vary across cultures. For example, cultural dimensions such as country, world region, economic level, race/ethnicity, and gender can influence the results of studies on R/S and well-being and the effectiveness of different psychotherapies focused on R/S and well-being (see Captari et al., Chapter 26, this volume). 
Culture is defined as a community of members who share beliefs, traditions, behaviors, values, habits, and institutions, despite a degree of individual variability and change over time (Jensen, 2021; see Mattis, Chapter 9, this volume). Jensen (2021) has proposed three main reasons to acknowledge culture in psychological research and clinical practice: (a) humans are a uniquely cultural species and the human brain is continually adapting to a cultural context, (b) the digital and globalizing world promotes that people have influences from different cultures, and (c) there is a need for cross-cultural studies to improve the ecological validity of psychology interventions.

However, scientific research at the intersection of positive psychology and R/S is not equally represented across different continents and cultures. Much of this research has been focused on participants living in the U.S. and Western Europe, and it has largely oversampled Christians. Yet increasing evidence indicates the relevance and often beneficial effects of R/S on well-being are not limited to Christians or the Western world but extend globally across a diverse range of religions and cultures (Tay \& Diener, 2011).

The purpose of this chapter is to examine research on positive psychology and R/S that has been conducted in Europe, non-U.S. North America (Canada and Central America), and South America. In light of the importance of considering cultural differences and similarities across religions and continents, this chapter synthesizes how cultural factors may affect research and practice on positive psychology and the psychology of R/S in these world regions. We start with a brief overview of the sociocultural and religious backdrop of these regions (see also Appendix 15.S1, Table 15.S1). Then we turn to reviewing the research in each region, such as what topics, populations, and contexts have been examined in that region's research on positive psychology and R/S. Finally, we consider implications for research and clinical practice, gaps in the current literature, and future directions for research. 


\section{Overview of the Cultural and Religious Background of the Reviewed Regions}

\section{Europe}

Europe is the second-smallest world continent, covering about $2 \%$ of the Earth's surface. The total population is almost 749 million ( $9.51 \%$ of the world's population), and it is the second-most densely populated continent (second to Asia). Politically, it is comprised of 48 sovereign countries, of which Russia is the largest (World Population Review, 2021).

Religion has profoundly influenced the history, societies, cultures, and laws of the European continent. Christianity has played a particularly predominant role (Davie, 2006), both for good (e.g., influencing art, literature, science, and philosophy) and ill (e.g., motivating wars, conquests, and oppression). Christianity still has the most adherents (74.5\%), with Catholics tending to cluster in southern Europe, Protestants in northern Europe, and Orthodox Christians in eastern Europe (Pew Research Center, 2017). Even so, notwithstanding the historical influence of religion, Europe has become a relatively secular continent, with an increasing number of irreligious, atheist, and agnostic people (who collectively comprise about $18.8 \%$ of Europe's population). Although the Asia-Pacific region has the largest share of the world's religiously unaffiliated population (75.9\%), Europe has the largest share in the Western world (12.4\%; Pew Research Center, 2015).

\section{Non-U.S. North America (Canada and Central America)}

Canada is situated above the northern part of the continental U.S. It covers 3.8 million square miles and is the second largest country in the world (behind Russia: $6.61 \mathrm{mi}^{2}{ }^{2}$; World Population Review, 2021). Canada was an indigenously inhabited territory until French and British colonization began in the $16^{\text {th }}$ and $17^{\text {th }}$ centuries. French and British influence on Canadian culture remains strong (e.g., Canada's two official languages are French and English). Canada has the tenth largest economy in the world, and its population is around $38,000,000$ ( $0.48 \%$ of the world's population; World Population Review, 2021). 
Historically, Christianity has been the predominant religion in Canada, but the number of Canadians who identify as Christian is declining. Presently, around 55\% of adults identify as Christian (including roughly 29\% Catholic and 18\% Protestant), with a high and growing percentage of Canadians identifying as religiously unaffiliated (29\%). Most Canadians (64\%) say religion's influence on Canada's public life is waning (Lipka, 2019).

Moving south, Central America is the region that includes the eight countries between the United States on the north and Colombia on the south. Its eight countries are Mexico, Belize, Costa Rica, El Salvador, Guatemala, Honduras, Nicaragua, and Panama. The combined population of Central America is 181.7 million, with Mexico and Guatemala having the largest populations in the region (128.9 million and 17.9 million, respectively; Worldometer, 2021). Its countries tend to be rather economically poor (Ventura, 2021).

Although Central America is technically part of North America, it has more in common culturally with South America, given shared ties to ancient cultures (e.g., the Aztec, Olmec, Mayans, and Incas) and European colonial rule (World Population Review, 2021). Hence, Central America often is considered a part of the broader region labeled Latin America-Carribbean, comprised of the Central American, South American, and Caribbean countries (Pew Research Center, 2014). In the $16^{\text {th }}$ century, the Central American region was subjected to Spanish colonial rule, and thus its countries are still largely influenced by Spanish culture (Woodward \& Bushnell, 2021).

Central American countries tend to be highly religious, and they are overwhelmingly comprised of Christian adherents (ranging from $86 \%$ to 91\%). Given the region's historical ties to Spanish culture, Central America countries have historically been comprised of predominantly Catholic Christian adherents. For example, in Mexico, currently $81 \%$ of adults identify as Catholic and 9\% as Protestant; this gap is evident in Panama (70\% vs. 19\%) and Costa Rica (62\% vs. $25 \%$ ) as well. In other Central American countries, the gap between 
Catholic and Protestant affiliates is decreasing, as more adults "switch" to a Protestant tradition. These countries include El Salvador (50\% Catholic, 36\% Protestant), Guatemala (50\%, 41\%), Honduras (46\%, 41\%), and Nicaragua (50\%, 40\%; Pew Research Center, 2014).

\section{South America}

South America is comprised of 14 countries: Argentina, Bolivia, Brazil, Chile, Colombia, Ecuador, Guyana, Paraguay, Peru, Suriname, Uruguay, Venezuela, French Guiana, and the Falkland Islands. It has a population of 434.3 million people. Culturally, it has similar historical origins as the Central American countries, with ties to thriving indigenous civilizations that were subjugated to European colonial rule (mostly by Spain and Portugal), starting in the $16^{\text {th }}$ century. Contemporary cultures in South America often reflect a fusion of indigenous and colonial influences (World Population Review, 2021).

Yet when it comes to religion, South American cultures heavily reflect European colonial influences. In most South American countries, the vast majority of adults are adherents of Christianity, mostly ranging from $81 \%$ in Chile to $96 \%$ in Paraguay. The only exception is Uruguay, which has a uniquely low proportion of Christian adherents (57\%) and an uniquely high proportion of religiously unaffiliated people. The overwhelming majority of Christians in South America are Catholic affiliated, but the number of adults "switching" to Protestant affiliation (mostly a Pentecostal tradition) is rising steadily, especially in Brazil (61\% Catholic, 26\% Protestant) and Chile (64\%, 17\%). As in Central America, most South American countries are highly religious (Pew Research Center, 2014, 2015), and many are rather economically poor (Ventura, 2021; World Population Review, 2021).

\section{Purpose of the Current Review}

The overview above serves not only to familiarize the reader with the cultural and religious characteristics of Europe, non-U.S North America, and South America. From the backdrop of these distinct and varied cultures, we sought to conduct a literature review that 
examined and synthesized the past three decades of these regions' empirical research on positive psychology and the psychology of R/S.

\section{Method of Review}

We conducted three sets of database searches (one for each region) in PsycINFO. Specifically, we sought to identify relevant peer-reviewed scholarly articles that were published from January 1, 1990 until January 1, 2021. We searched for records with titles or abstracts that contained keywords associated with R/S (i.e., relig*, spirit*, worship*, pray*, faith, sacred) and positive psychology (i.e., altruis*, compassion*, forgiv*, gratitude, hope, humility, patience), crossing these terms with the names of the specific countries in each region. Nonempirical articles, empirical articles that did not intersect positive psychology and the psychology of R/S, and topically relevant empirical articles that did not include at least one sample from a geographic region of interest were excluded from the review.

The database searches that were performed for each region yielded a combined total of 937 unique records (Europe: $k=580$; non-U.S. North America: $k=343$; South America: $k$ $=64)$. After examining the titles and abstracts of each record, 226 articles were retrieved for further screening. We then reviewed the full texts of those articles, which resulted in the exclusion of 116 articles that did not meet the inclusion criteria. Metadata (e.g., title, year of publication), key details about the research context (e.g., country/countries, study focus), methodology (e.g., research design, sampling approach), participants (e.g., sample type, size, and age), and main findings were extracted from each retained article (see Appendix 15.S2).

\section{Results of the Review}

Across the three regions, a total of 110 articles met inclusion criteria (see Appendix 15.S3 for citations). The largest proportion were from Europe $(k=50)$, followed by non-U.S. North America $(k=34)$, and South America $(k=12) ; 14$ studies were multiregional. 


\section{Publication Activity, Research Context, and Utilized Methodology}

In Europe, 50 articles met inclusion criteria; all but five have been published since 2010. Almost all the studies used quantitative methods (94.0\%); there were only 3 qualitative or mixed-methods studies (6.0\%). Altogether, there were 11 longitudinal studies $(22.0 \%)$ and four experiments $(8.0 \%)$. Germany and Poland were the two European countries that had the most publications on positive psychology and the psychology of R/S.

In non-U.S. North America, 34 articles met inclusion criteria; again, all but nine were published since 2010. Regarding methodology, 15 studies (44.1\%) used quantitative methods and 19 studies (55.9\%) used qualitative or mixed methods. There were three longitudinal studies $(8.8 \%)$ and two experiments (5.9\%). Canada was the country with the most research published on positive psychology and R/S, with $61.8 \%(k=21)$ of this region's articles.

In the region of South America, 12 articles met inclusion criteria, three published prior to 2011. Regarding methodology, 7 studies (58.3\%) used quantitative methods and 5 studies $(41.7 \%)$ used qualitative or mixed methods. There was one longitudinal study $(8.3 \%)$ but no experiments. Brazil was the country with the most studies in the region $(k=7,58.3 \%)$.

\section{Topics and Variables of Interest}

Identifying the topics and variables examined in each region's articles on positive psychology and psychology of R/S can offer clues about the role and influence of culture on complex entities such as R/S. Thus, we next endeavored to identify the topics of positive psychology and psychology of R/S that were studied in each article (see Appendix 15.S2).

\section{Positive Psychology}

Forgiveness. In European studies, the topic of forgiveness has often been examined ( $k$ $=16,32.0 \%)$. This topic has been studied in a variety of populations, such as cancer patients (van Laarhoven et al., 2012), soldiers (Büssing et al., 2018), married adults (Jose \& Alfons, 2007), and inpatients in alcohol abuse treatment (Braun et al., 2018). 
Forgiveness has also been studied in non-U.S. North America $(k=6,17.6 \%)$ and South America $(k=5,41.7 \%)$. For example, it has been examined in adult Canadian survivors of childhood sexual abuse (Gall, 2006), ethnic minority youth in Canada (Kubiliene et al., 2015), and two samples of disaster survivors in Colombia (Chen et al., 2021).

Hope. The topic of hope has been studied quite extensively in Europe $(k=15$, 30.5\%), non-U.S. North America $(k=10,29.4 \%)$, and South America $(k=4,33.3 \%)$. Across European studies, hope has been studied as a coping strategy (Wirth \& Büssing, 2016; Wnuk, 2015; Zarzycka et al., 2019) and linked to coping efficacy (Ferreira-Valente et al., 2020), perceived posttraumatic growth (Kroo \& Nagy, 2011), life satisfaction (Oliver et al., 2017), meaning in life (Wnuk \& Marcinkowski, 2014), lower anxiety (Zarzycka et al., 2019), and lower suicide risk (Stefa-Missagli et al., 2020). Similarly, in non-U.S. North America and in South America, hope has been studied as a coping and social behavior (Aquino \& Zago, 2007; Gall et al., 2009; Gall \& Bilodeau, 2017) and associated with positive youth development (e.g., confidence and connectedness; Tirrell et al., 2019), benevolent God representations (Gall et al., 2007), self-efficacy at work (Duggleby et al., 2009), forgiveness and well-being (Chen et al., 2021), and happiness and faith (Holt \& Reeves, 2001).

Other Character Strengths and Virtues. Across all three regions, many other character strengths have been examined as well, such as compassion $(k=15)$, altruism $(k=$ 17), and gratitude $(k=3)$. For example, compassion has been identified as a core motivation for family caregivers of cancer patients in Mexico (Juarez et al., 2014), for palliative care professionals in Germany and the UK (Wasner et al., 2005), and for charity donors in Portugal (de Abreu et al., 2015). Additionally, in a large multiregional study across 36 countries (including all three regions examined in this chapter), altruism has been robustly and cross-culturally associated with higher religiousness and more positive attitudes toward outgroups (Ashton \& Lee, 2019). Within this chapter's regions of interest, altruism has been 
linked to authenticity in two Canadian samples (Cornwell et al., 2017) and to religiousness in four samples of Belgian adults (Saroglou et al., 2005). Furthermore, gratitude has been associated with life satisfaction among Catholic pastoral workers in Germany (Büssing et al., 2017) and with well-being among adolescents in Canada (Bosacki et al., 2018). Moreover, increases in gratitude have been found among participants in alcohol treatment in Poland (Charzyńska, 2015) and among teletherapy participants in Romania (Tulbure et al., 2018).

Well-being and Other Positive Psychology Constructs. Of the 110 studies of positive psychology and R/S included in this review, the topic of well-being has been examined in 66 studies $(60.0 \%)$. Across cultures and samples, there is evidence that people's psychological well-being is related to their religious/spiritual well-being (e.g., higher perceived closeness with God and lower spiritual struggle or distress). Studies have found that people often utilize R/S to cope with a variety of stressors, including substance use disorders (Braun et al., 2018), cancer (Testoni et al., 2016), natural disasters (Chen et al., 2021), and economic crises (Hendricks et al., 2018). Longitudinal studies in these regions have found that people's utilization of R/S typically has positive effects on their well-being (e.g., Gall \& Bilodeau, 2017; Oliver et al., 2017; Opsahl et al., 2019), although this is not always the case (e.g., Braun et al., 2018; Gall et al., 2009). Longitudinal studies in these regions have also found evidence R/S may lead to increased life satisfaction (Marques et al., 2013), positive emotions (Inman, 2014), hope and meaning (Aquino \& Zago, 2007), and character strengths and virtues (e.g., forgiveness and gratitude; Charzyńska, 2015). Furthermore, experimental studies have found that R/S can cause increased prosociality (Purzycki et al., 2016) and moral behavior (Shariff \& Norenzayan, 2011) and can be incorporated effectively into psychotherapy (Tulbure et al., 2018; Wang et al., 2016).

Besides well-being, several other positive psychology constructs have been examined, including life satisfaction $(k=13)$, meaning/purpose in life $(k=12)$, social support (positive 
relationships; $k=9)$, happiness $(k=6)$, and positive emotions $(k=6)$. For example, a crosscultural comparison of 41 nations found evidence happiness is tied to the quality of people's social relations and to macrolevel social structures and institutions (Haller \& Hadler, 2006).

\section{Religion/Spirituality}

Regarding the psychology of R/S topics studied, some similarities and differences were evident in the three regions (see Appendix 15.S1, Table 15.S2). Across all three regions, religious/spiritual belief was studied most frequently, ranging from $41.7 \%$ of studies in South America to 50.0\% in non-U.S. North America. Religious/spiritual attendance (public religious/spiritual practices) were also examined with similar regularity (between $30.0 \%$ and $33.3 \%$ across regions). Private religious/spiritual practices (e.g., prayer) were studied slightly more often in Europe and South America than in non-U.S. North America (38.0\% and 41.7\% vs. $23.5 \%$, respectively); the same was true for religious/spiritual importance (28.0\% and $25.0 \%$ vs. $14.7 \%$ ). Religous/spiritual affiliation (identity) was studied much more often in South America than in Europe or non-U.S. North America (41.7\% vs. $14.0 \%$ and $17.6 \%)$.

\section{Discussion}

The results of this review show there is growing scholarly interest in positive psychology and the psychology of R/S in Europe, non-U.S. North America, and South America. The varied contexts, populations, and topics that were studied illustrate the breadth of how researchers from different cultures are integrating positive psychology and R/S.

Across the three reviewed regions, a wide range of settings, samples, and topics were examined. European studies tended to focus on health contexts and issues (e.g., healthcare professionals, nursing students, cancer, COVID-19, chronic pain) and mental health difficulties (e.g., mental disorders, substance use disorders, anxiety, depression, suicide). Similarly, studies in non-U.S. North American frequently examined R/S as a resource to cope with stressors (e.g., discrimination, physical health problems, trauma, or abuse) or treat 
mental health difficulties. Articles in Europe and non-U.S. North America often focused on studying a special populations, such as youth, older adults, soldiers, prisoners, racial/ethnic minorities, refugees, and patients with mental or medical difficulties. South American studies were also quite eclectic in the settings and samples they examined. For example, studies were conducted with students, rural community members, older adults, religious leaders, spiritist mediums, cancer patients, healthcare professionals, caregivers, and disaster survivors.

Regarding the psychology of R/S, consistent with the model proposed by Saroglou (2011) — in which the R/S is conceptualized as having four cross-culturally evident dimensions (believing, bonding, behaving, and belonging) — there were similar and different patterns of religious/spiritual salience that were evident in the three regions, based on how often particular religious/spiritual topics were studied. The cognitive dimension of religious believing seems most salient across all three regions, because religious/spiritual belief was consistently the most studied topic. The experiential dimension of religious bonding (e.g., private religious/spiritual practices) seems slightly more salient in Europe and South America than in non-U.S. North America. The social dimension (e.g., religious/spiritual affiliation and identity) of religious belonging seems more salient in South America than in Europe or nonU.S. North America. The behavioral dimension of religious behaving (e.g., religious service attendance) seems similarly salient across the three regions. As shown in Table 15.S3 of Appendix 15.S1, most of the European and Canadian studies tended to focus on individually oriented religious/spiritual phenomena (religious believing, bonding, and behaving). Central and South American studies were more apt to incorporate examination of socially oriented religious/spiritual phenomena (religious belonging) as well. This distinction fits with European and North American tendencies toward cognitivism, experientialism, and individualism, as compared to Central and South American tendencies toward collectivism and familialism. These possibilities offer clues about the role culture may have in the 
experience of R/S in these regions. R/S may be more socially incorporative in Central and South America, whereas in Europe and Canada, it might be more individually focused.

This possibility makes sense in light of the multiregional findings of Diener et al. (2011). In prosperous nations that have achieved high material and social well-being (like Canada and most European countries), R/S is less prevalent, perhaps because the nonreligious can more easily achieve life satisfaction, receive social support, and experience positive emotions and feelings of being respected. On the contrary, the pattern of R/S and well-being is largely reversed in difficult versus benign societies. The nonreligious in poor societies, like many countries in Central America and South America, are at the biggest disadvantage, with noticeably lower positive feelings and higher negative feelings, as well as substantial deficits in social support and feelings of respect. In Diener et al. (2011), R/S predicted social support, respect, and purpose/meaning in life, each of which in turn predicted subjective well-being. That is perhaps why religious people in poorer societies were more likely to prosper in terms of subjective well-being if they lived in religious rather than nonreligious societies (Diener et al., 2011). It may also help explain why people in Central and South America tend to be more committed to R/S than most people in Europe and Canada (Pew Research Center, 2018).

Notably, the research activity of psychology of R/S and positive psychology (and consequently, its applicability to clinical practice) may be driven largely by the economic and social resources available to conduct valuable and rigorous studies. Of the three regions we studied, Europe was the most scientifically advanced region in terms of publication activity on positive psychology and R/S. Within non-U.S North American publications, there is a disproportion between the research activity in Canada versus other countries. South America is the region with fewer articles published compared to the other two regions. Taking into account the economic situation of many Central and South American countries, which often tend to be poorer than Canada and European countries, it is reasonable that there may be less 
publications in these regions because they have less financial and human resources available for research. Yet compared to the decreasing rates of R/S in Canada and Europe, Central and South America have a much higher percentage of religiously affiliated and practicing people. This means that, in Central and South America, R/S is still quite present as a way of life and a cultural tradition, but it is not a common focus of scholarly study. Even so, research is needed to inform scientific understanding and applied practice in Central and South America (as well as in Canada and Europe), so how can this problem be addressed? The next sections share ideas toward this end and offer suggestions to guide clinicians and researchers more broadly.

\section{Practical Applications and Recommendations}

\section{Clinical Practice}

Awareness and understanding of the impact of cultural factors is one of the key factors clinicians need to incorporate in their work, especially when it comes to working with religious/spiritual clients and using religious/spiritually integrated interventions (Vieten \& Lukoff, 2021; see Captari et al., Chapter 26, this volume). For example, maybe psychological interventions that integrate R/S in Canada and Europe should be more oriented to individually focused concerns (e.g., to address spiritual struggle or dryness), whereas in Central and South America, interventions may need to focus more on helping people nurture their social connections (e.g., to their family and community). Given the cultural salience of $\mathrm{R} / \mathrm{S}$ in Central and South America, it may also be important and effective for clinicians to collaborate with religious leaders and communities (Wang et al., Chapter 29, this volume).

\section{Future Research}

Investigating cultural factors affecting the integration of positive psychology and the psychology of R/S is not easy (see Mattis, Chapter 9, this volume). For instance, it often is challenging to (a) differentiate what is R/S and what is culture, (b) isolate R/S from culture, and (c) determine the extent to which both dimensions are implicitly attached to specific 
values, virtues, and character strengths. However, studies that examine these and other challenging questions are needed to advance scientific understanding of R/S and promote personal and societal well-being. Toward this end, we offer a few recommendations.

Conduct Culture-Focused Studies. Among the studies in this review, the ones that specifically focused on how cultural factors may affect R/S and positive psychological phenomena were scarce. As Lomas (2015) has described, one plausible explanation of this is the frequent presumption of universality. Lomas defined the universalizing perspective as focused on commonalities of people across cultures. The alternative option is the relativizing perspective, which focuses on the differences between people in different cultures. Both perspectives have strengths and weaknesses. For instance, the relativistic perspective allows a detailed understanding of the impact of culture on well-being and R/S, but a universalistic perspective can contribute general knowledge about people's common humanity and allow identification of cross-cultural similarities. Both types of culture-focused studies are needed.

Consider Religion as Culture vs. Religion as an Element of Culture. As Saroglou and Cohen (2011) have explained, R/S may be considered part of a culture, may constitute culture, or may interact with culture in influencing cognitions, emotions, and actions. One important consideration when starting to investigate a particular culture is to establish the degree to which religion in that specific group is part of the culture or is just an element that could be present or not. For instance, in some countries there is only one religion and there is no option for the citizen to choose their religion (or even to choose not to be religious), whereas in other countries, it is easy to find great diversity of religions and beliefs. Before studying particular cultures, these relevant issues need to be explored and understood.

Develop and Culturally Validate Measures. In order to conduct strong research on culture, R/S, and positive psychology, there is a need for measures that are psychometrically sound and culturally valid. Extant research in these regions does not attend enough to issues 
of measurement, such as the need for cultural validation and establishing cross-cultural construct equivalence. Because the study of cultural factors in positive psychology and the psychology of R/S is somewhat new, researchers need (a) to develop and use the same nomenclature to facilitate communication and cross-study, cross-cultural comparisons and (b) to create psychometrically strong instruments that allow the valid measurement of cultural factors and the ability to compare construct-equivalent results in different cultures and countries (becuase of demonstrated measurement invariance across specific cultural groups).

Conduct Intracultural and Intercultural Studies. Cross-cultural studies may be a key method to contrast and define the role of culture in the relationship of R/S and positive psychology. In this chapter, a total of 14 cross-cultural, multiregional studies were identified. However, to find greater relevance of cultural factors between countries across the world, a first step may be to conduct cross-cultural studies between countries in the same continent or region (e.g., within North America, Central America, or South America) and then compare them with other regions of the world. Intracultural studies may help clarify cultural nuances and variations within each continent- - before conducting larger-scale, cross-cultural studies across multiple continents. In addition, this could not only promote research on these topics in all cultures, but it could also allow the poorest countries - often where R/S are saliently practiced - to raise awareness of the benefits of R/S for people in their cultural context.

\section{Conclusion}

Positive psychology and the psychology of R/S are clearly in a position of advancing science and practice at the intersections of culture, R/S, and well-being. Even so, in the culturally and religiously complex, diverse regions of Europe, non-U.S. North America, and South America, there is a lot of work to be done. We are eager to see how scholars and practitioners take up this exciting opportunity in the decades to come, as our world becomes more culturally complex and diverse yet more globally interconnected and interdependent. 


\section{References}

Aquino, V. V., \& Zago, M. M. F. (2007). The meaning of religious beliefs for a group of cancer patients during rehabilitation. Revista latino-americana de enfermagem, 15, 42-47. https://doi.org/10.1590/S0104-11692007000100007

Ashton, M. C., \& Lee, K. (2019). Religiousness and the HEXACO personality factors and facets in a large online sample. Journal of Personality, 87(6), 1103-1118. https://doi.org/10.1111/jopy.12459

Bosacki, S., Sitnik, V., Dutcher, K., \& Talwar, V. (2018). Gratitude, social cognition, and well-being in emerging adolescents. Journal of Genetic Psychology, 179(5), 256-269. https://doi.org/10.1080/00221325.2018.1499607

Braun, B., Weinland, C., Kornhuber, J., \& Lenz, B. (2018). Religiosity, guilt, altruism and forgiveness in alcohol dependence: Results of a cross-sectional and prospective cohort study. Alcohol and Alcoholism, 53(4), 426-434. https://doi.org/10.1093/alcalc/agy026

Büssing, A., Baiocco, F., \& Baumann, K. (2018). Spiritual dryness in Catholic laypersons working as volunteers is related to reduced life satisfaction rather than to indicators of spirituality. Pastoral Psychology, 67, 1-15. https://doi.org/10.1007/s11089-017-0798$\underline{5}$

Büssing, A., Frick, E., Jacobs, C., \& Baumann, K. (2017). Self-attributed importance of spiritual practices in Catholic pastoral workers and their association with life satisfaction. Pastoral Psychology, 66(3), 295-310. https://doi.org/10.1007/s11089$\underline{016-0746-9}$

Charzyńska, E. (2015). Sex differences in spiritual coping, forgiveness, and gratitude before and after a basic alcohol addiction treatment program. Journal of Religion and Health, 54(5), 1931-1949. https://doi.org/10.1007/s10943-015-0002-0 
Chen, Z. J., Bechara, A. O., Worthington Jr, E. L., Davis, E. B., \& Csikszentmihalyi, M. (2021). Trauma and well-being in Colombian disaster contexts: Effects of religious coping, forgivingness, and hope. The Journal of Positive Psychology, 16(1), 82-93. https://doi.org/10.1080/17439760.2019.1663254

Cornwell, J. F., Franks, B., \& Higgins, E. T. (2017). How the "truth" self relates to altruism: When your problem is mine. Social Cognition, 35(2), 204-226. https://doi.org/10.1521/soco.2017.35.2.204

Davie, G. (2006). Religion in Europe in the 21st century. European Journal of Sociology, 47(2), 271-296. https://doi.org/10.1017/S0003975606000099

de Abreu, M. E., Laureano, R. M., da Silva, R. V., \& Dionísio, P. (2015). Volunteerism, compassion and religiosity as drivers of donations practices. International Journal of Nonprofit and Voluntary Sector Marketing, 20(3), 256-276. https://doi.org/10.1002/nvsm.1526

Diener, E., Tay, L., \& Myers, D. G. (2011). The religion paradox: If religion makes people happy, why are so many dropping out? Journal of Personality and Social Psychology, 101(6), 1278-1290. https://doi.org/10.1037/a0024402

Duggleby, W., Cooper, D., \& Penz, K. (2009). Hope, self-efficacy, spiritual well-being and job satisfaction. Journal of Advanced Nursing, 65(11), 2376-2385. https://doi.org/10.1111/j.1365-2648.2009.05094.x

Ferreira-Valente, A., Damião, C., Pais-Ribeiro, J., \& Jensen, M. P. (2020). The role of spirituality in pain, function, and coping in individuals with chronic pain. Pain Medicine, 21(3), 448-457. https://doi.org/10.1093/pm/pnz092

Gall, T. L. (2006). Spirituality and coping with life stress among adult survivors of childhood sexual abuse. Child Abuse \& Neglect, 30(7), 829-844. https://doi.org/10.1016/j.chiabu.2006.01.003 
Gall, T., Basque, V., Damasceno-Scott, M., \& Vardy, G. (2007). Spirituality and the current adjustment of adult survivors of childhood sexual abuse. Journal for the Scientific Study of Religion, 46(1), 101-117. https://doi.org/10.1111/j.1468-5906.2007.00343.x

Gall, T. L., \& Bilodeau, C. (2017). “Why me?”: Women's use of spiritual causal attributions in making sense of breast cancer. Psychology \& Health, 32(6), 709-727. https://doi.org/10.1080/08870446.2017.1293270

Gall, T., Kristjansson, E., Charbonneau, C., \& Florack, P. (2009). A longitudinal study on the role of spirituality in response to the diagnosis and treatment of breast cancer. Journal of Behavioral Medicine, 32(2), 174-186. https://doi.org/10.1007/s10865-008-9182-3

Haller, M., \& Hadler, M. (2006). How social relations and structures can produce happiness and unhappiness: An international comparative analysis. Social Indicators Research, 75(2), 169-216. https://doi.org/10.1007/s11205-004-6297-y

Hendriks, T., Graafsma, T., Hassankhan, A., Bohlmeijer, E., \& de Jong, J. (2018). Strengths and virtues and the development of resilience: A qualitative study in Suriname during a time of economic crisis. International Journal of Social Psychiatry, 64(2), 180-188. https://doi.org/10.1177\%2F0020764017749624

Holt, J., \& Reeves, J. S. (2001). The meaning of hope and generic caring practices to nurture hope in a rural village in the Dominican Republic. Journal of Transcultural Nursing, 12(2), 123-131. https://doi.org/10.1177\%2F104365960101200206

Inman, M. L. (2014). The effects of religious-body affirmations and religious commitment on men's body esteem. Psychology of Religion and Spirituality, 6(4), 330-337. https://doi.org/10.1037/a0036795

Jensen, L. A. (2021). The cultural psychology of religiosity, spirituality, and secularism in adolescence. Adolescent Research Review, 6, 277-288. https://doi.org/10.1007/s40894-020-00143-0 
Jose, O. A., \& Alfons, V. (2007). Religiosity and forgiveness among first-married and remarried adults. Mental Health, Religion and Culture, 10(4), 379-394. https://doi.org/10.1080/13674670600785545

Juarez, G., Branin, J. J., \& Rosales, M. (2014). The cancer caregiving experience of caregivers of Mexican ancestry. Hispanic Health Care International, 12(3), 120-129. http://dx.doi.org/10.1891/1540-4153.12.3.120

Kroo, A., \& Nagy, H. (2011). Posttraumatic growth among traumatized Somali refugees in Hungary. Journal of Loss and Trauma, 16(5), 440-458. https://doi.org/10.1080/15325024.2011.575705

Kubiliene, N., Yan, M. C., Kumsa, M. K., \& Burman, K. (2015). The response of youth to racial discrimination: Implications for resilience theory. Journal of Youth Studies, 18(3), 338-356. https://doi.org/10.1080/13676261.2014.963535

Lipka, M. (2019). 5 facts about religion in Canada. https://www.pewresearch.org/facttank/2019/07/01/5-facts-about-religion-in-canada/

Lomas, T. (2015). Positive cross-cultural psychology: Exploring similarity and difference in constructions and experiences of wellbeing. International Journal of Wellbeing, 5(4), 60-77. https://doi.org/10.5502/ijw.v5i4.437

Marques, S. C., Lopez, S. J., \& Mitchell, J. (2013). The role of hope, spirituality and religious practice in adolescents' life satisfaction: Longitudinal findings. Journal of Happiness Studies, 14(1), 251-261. https://doi.org/10.1007/s10902-012-9329-3

Oliver, A., Tomás, J. M., \& Montoro-Rodriguez, J. (2017). Dispositional hope and life satisfaction among older adults attending lifelong learning programs. Archives of Gerontology and Geriatrics, 72, 80-85. https://doi.org/10.1016/j.archger.2017.05.008 
Opsahl, T., Ahrenfeldt, L. J., Möller, S., \& Hvidt, N. C. (2019). Religiousness and depressive symptoms in Europeans: Findings from the Survey of Health, Ageing, and Retirement in Europe. Public Health, 175, 111-119. https://doi.org/10.1016/j.puhe.2019.07.011

Peterson, C., \& Seligman, M. E. P. (2004). Character strengths and virtues: A handbook and classification. American Psychological Association and Oxford University Press.

Pew Research Center (2014). Religion in Latin America: Widespread change in a historically Catholic region. https://www.pewforum.org/2014/11/13/religion-in-latin-america/

Pew Research Center (2015). Future of world religions: Population growth projections, 2010-2050. https://www.pewforum.org/2015/04/02/religious-projections-2010-2050/

Pew Research Center (2017). Five centuries after reformation, Catholic-Protestant divide in Western Europe has faded. https://www.pewforum.org/2017/08/31/five-centuriesafter-reformation-catholic-protestant-divide-in-western-europe-has-faded/

Pew Research Center (2018). The age gap in religion around the world. Author. https://www.pewforum.org/2018/06/13/the-age-gap-in-religion-around-the-world/

Purzycki, B. G., Apicella, C., Atkinson, Q. D., Cohen, E., McNamara, R. A., Willard, A. K., ... \& Henrich, J. (2016). Moralistic gods, supernatural punishment and the expansion of human sociality. Nature, 530(7590), 327-330. https://doi.org/10.1038/nature16980

Saroglou, V. (2011). Believing, bonding, behaving, and belonging: The big four religious dimensions and cultural variation. Journal of Cross-Cultural Psychology, 42(8), 1320-1340. https://doi.org/10.1177/0022022111412267

Saroglou, V., \& Cohen, A. B. (2011). Psychology of culture and religion: Introduction to the JCCP special issue. Journal of Cross-Cultural Psychology, 42(8), 1309-1319. https://doi.org/10.1177/0022022111412254

Saroglou, V., Pichon, I., Trompette, L., Verschueren, M., \& Dernelle, R. (2005). Prosocial behavior and religion: New evidence based on projective measures and peer ratings. 
Journal for the Scientific Study of Religion, 44(3), 323-348.

https://doi.org/10.1111/j.1468-5906.2005.00289.x

Shariff, A. F., \& Norenzayan, A. (2011). Mean gods make good people: Different views of God predict cheating behavior. The International Journal for the Psychology of Religion, 21(2), 85-96. https://doi.org/10.1080/10508619.2011.556990

Stefa-Missagli, S., Unterrainer, H., Giupponi, G., Wallner-Liebmann, S., Kapfhammer, H., ... \& Pompili, M. (2020). Influence of spiritual dimensions on suicide risk. Archives of Suicide Research, 24(4), 534-553. https://doi.org/10.1080/13811118.2019.1639571

Tay, L., \& Diener, E. (2011). Needs and subjective well-being around the world. Journal of Personality and Social Psychology, 101, 354-365. https://doi.org/10.1037/a0023779

Testoni, I., Visintin, E. P., Capozza, D., Carlucci, M. C., \& Shams, M. (2016). The implicit image of God: God as reality and psychological well-being. Journal for the Scientific Study of Religion, 55(1), 174-184. https://doi.org/10.1111/jssr.12252

Tirrell, J. M., Geldhof, G. J., King, P. E., Dowling, E. M., Sim, A. T., Williams, K., ... \& Lerner, R. M. (2019). Measuring spirituality, hope, and thriving among Salvadoran youth: Initial findings from the Compassion International Study of Positive Youth Development. In Child \& Youth Care Forum (Vol. 48, No. 2, pp. 241-268). Springer. Tulbure, B., Andersson, G., Sălăgean, N., Pearce, M., \& Koenig, H. (2018). Religious versus conventional internet-based cognitive behavioral therapy for depression. Journal of Religion and Health, 57(5), 1634-1648. https://doi.org/10.1007/s10943-017-0503-0

Van Laarhoven, H. W., Schilderman, J., Verhagen, C. A., \& Prins, J. B. (2012). Comparison of attitudes of guilt and forgiveness in cancer patients without evidence of disease and advanced cancer patients in a palliative care setting. Cancer Nursing, 35(6), 483-492. https://doi.org/10.1097/NCC.0b013e318243fb30 
Vatican (October 1, 2013). Pope Francis talks spirituality in new interview https://world.time.com/2013/10/01/pope-francis-talks-spirituality-in-new-interview/

Ventura, L. (2021, May 21). Poorest countries in the world 2021. Global Finance. https://www.gfmag.com/global-data/economic-data/the-poorest-countries-in-theworld

Vieten, C., \& Lukoff, D. (2021). Spiritual and religious competencies in psychology. American Psychologist. Advance online publication. https://doi.org/10.1037/amp0000821

Wang, D. C., Aten, J. D., Boan, D., Jean-Charles, W., Griff, K., Valcin, V., ... \& Wang, A. (2016). Culturally adapted spiritually oriented trauma-focused cognitive-behavioral therapy for child survivors of restavek. Spirituality in Clinical Practice, 3(4), 224236. https://doi.org/10.1037/scp0000101

Wasner, M., Longaker, C., Fegg, M. J., \& Borasio, G. D. (2005). Effects of spiritual care training for palliative care professionals. Palliative medicine, 19(2), 99-104. https://doi.org/10.1191\%2F0269216305pm995oa

Wirth, A. G., \& Büssing, A. (2016). Utilized resources of hope, orientation, and inspiration in life of persons with multiple sclerosis and their association with life satisfaction, adaptive coping strategies, and spirituality. Journal of Religion and Health, 55(4), 1359-1380. https://doi.org/10.1007/s10943-015-0089-3

Wnuk, M. (2015). Determining the influence religious-spiritual values on levels of hope and the meaning of life in alcohol co-dependent subjects receiving support in self-help groups. Journal of Substance Use, 20(3), 194-199.

Wnuk, M., \& Marcinkowski, J. T. (2014). Do existential variables mediate between religiousspiritual facets of functionality and psychological wellbeing. Journal of Religion and Health, 53(1), 56-67. https://doi.org/10.1007/s10943-012-9597-6 
Woodward, R. L., \& Bushnell, D. (2021). Central America. Encyclopedia Britannica. https://www.britannica.com/place/Central-America

World Population Review (2021, November 19). World population review. https://worldpopulationreview.com/

Worldometer (2021, November 19). Central America population.

https://www.worldometers.info/world-population/central-america-population/

Zarzycka, B., Śliwak, J., Krok, D., \& Ciszek, P. (2019). Religious comfort and anxiety in women with cancer: The mediating role of hope and moderating role of religious struggle. Psycho-Oncology, 28(9), 1829-1835. https://doi.org/10.1002/pon.5155 
Chapter 16: Positive Psychology and Religion/Spirituality Across Cultures in Africa,

\title{
Asia, and Oceania
}

\author{
Richard G. Cowden \\ Human Flourishing Program, Harvard University \\ Victor Counted \\ School of Psychology, Western Sydney University \\ Man Yee Ho \\ Department of Social and Behavioural Sciences, City University of Hong Kong
}

Correspondence concerning this chapter should be addressed to Richard G. Cowden.

Email: rcowden@fas.harvard.edu 


\begin{abstract}
Culturally responsive cross-pollination of positive psychology (PP) and the psychology of religion/spirituality (PRS) has the potential to enrich both subfields and augment their impact. To strengthen the interaction of PP and the PRS beyond the traditional boundaries of the West, this chapter explores the current overlap between these two psychology subfields in Africa, Asia, and Oceania. After providing a brief overview of each region, we apply a systematic approach to identify and evaluate research that intersects PP and the PRS in each region. Our search revealed a total of 128 scholarly articles over the last three decades. Topical emphases were classified into three overarching themes: (a) well-being, (b) character strengths and virtues, and (c) positive adaptation. Although recent growth in publication outputs within each region is an encouraging sign for the next wave of research, the quality of the existing empirical evidence is limited by over-reliance on cross-sectional observational studies. Based on the findings, we highlight some of the ways that culturally responsive cross-pollination of PP and the PRS could benefit people living within Africa, Asia, and Oceania. We also offer suggestions for broadening the scope and enhancing research that intersects both subfields in these culturally and religiously diverse regions.
\end{abstract}

Keywords: Africa; Asia; Oceania; positive psychology; religion and spirituality 


\section{Chapter 16: Positive Psychology and Religion/Spirituality Across Cultures in Africa,}

\section{Asia, and Oceania}

Positive psychology (PP) developed into a mainstream psychology subfield about two decades ago. At that time, its emphasis on strengths, virtues, and well-being represented a fundamental shift in the way psychologists had traditionally approached science and practice (Downey \& Henderson, 2021). Beyond the rebalance that PP has brought to the field's historic emphasis on psychopathology, PP has served as a bridge that connects psychology to other disciplines, partly because many PP concepts have historical roots in philosophy and theology. For example, the four cardinal virtues — prudence, justice, fortitude, and temperance - have been integrated into the character and virtue domain in VanderWeele's (2017) framework of human flourishing (see Ratchford et al., Chapter 4, this volume).

PP has also had an important role in linking subfields within psychology, most notably through its ties with the psychology of religion/spirituality (PRS). A direct indication of this is provided in Peterson and Seligman's (2004) groundbreaking work on character strengths and virtues, in which they classified spirituality as one of 24 universal character strengths. Many other character strengths in their classification scheme (e.g., forgiveness, gratitude, humility) are emphasized by major religious/spiritual (R/S) traditions (e.g., Christianity, Islam) as desirable attributes that promote personal transformation and support the development of virtuous character (Cowden, Counted, et al., 2021).

Despite the apparent conceptual threads that connect PP with the PRS, crossfertilization between these subfields of psychology has been limited (Worthington et al., in press; see also Davis et al., Chapter 1, this volume). To illustrate, a broad set of searches in the PsycINFO database for scholarly articles with titles and abstracts containing key terms in the PRS (i.e., religion, spirituality, worship, pray, faith, sacred) and several prominent R/Srelevant character strengths in PP (i.e., altruism, compassion, forgiveness, gratitude, hope, 
humility, patience) published during the last 10 years revealed that fewer than $5 \%$ of records intersected both PP and the PRS. Hence, growth in each subfield seems more often to have transpired independently rather than through cross-pollination of both subfields (Worthington et al., in press). The current chapter examines the degree to which this pattern is consistent across different world regions, focusing specifically on Africa, Asia, and Oceania.

This chapter is guided by the premise that culturally responsive cross-pollination ${ }^{3}$ of PP and the PRS would enrich both subfields and contribute toward their greater scientific and societal impact. To stimulate and enhance culturally responsive cross-pollination of PP and the PRS outside the traditional boundaries of the West, the current chapter explores the extent of overlap between these two subfields in Africa, Asia, and Oceania. After briefly describing the historical background, demographic composition, and religious landscape of these three regions, we summarize the results of a systematic literature search of research that intersects PP and the PRS in each region. We discuss some of the ways culturally responsive crosspollination of PP and the PRS could enrich clinical practice in Africa, Asia, and Oceania. Lastly, we offer suggestions for expanding this overlap through culturally sensitive and methodologically rigorous research within these regions.

\section{Historical Background and Religious Landscape of Africa, Asia, and Oceania}

Africa, Asia, and Oceania share sociopolitical heritages marked by foreign invasion, colonization, and intergroup conflicts, which have shaped contemporary societies within each region. Although many countries in these three regions face a similar range of social, economic, and political issues that are tethered to culture and history, each region also possesses unique features that are important for contextualizing patterns of research and for identifying potential areas in which scholarship at intersections of PP and the PRS might

\footnotetext{
${ }^{3}$ For the purposes of this chapter, we define culturally responsive cross-pollination as the deliberate integration of differentiable scientific fields (e.g., PP and the PRS) in ways that are sensitive to the contextual particularities and needs of the cultures that those fields study or serve.
} 
contribute to addressing the needs of that region. Selected population demographics and the religious composition of Africa, Asia, and Oceania are presented in Appendix 16.S1 (see Table 16.S1 and Table 16.S2).

\section{Africa}

The African continent is home to the second largest population of people in the world -1.4 billion people (almost $20 \%$ of the global population of 7.9 billion; see Appendix 16.S1, Table 16.S1). The genetic, cultural, linguistic, and phenotypic diversity in Africa is unparalleled, with over 2,000 distinct ethnolinguistic groups of people represented across the continent's more than 50 countries (Reed \& Tishkoff, 2006). Although Africa was richly diverse before colonization by European nations, colonialism only added further complexity to human life in the region (Worthington \& Cowden, 2017).

Towards the latter part of the $19^{\text {th }}$ century CE, many European nations were competing with one another over the potential economic benefits of colonial expansion in Africa. By the end of the so-called "Scramble for Africa" in the early $20^{\text {th }}$ century CE, almost all of Africa was controlled by Western European empires that were dominant at the time (e.g., Britain, Belgium, and France). During that unprecedented period of expansive colonization, conflicts arose between indigenous groups and colonial powers, who often used military force to gain control of territories or overcome resistance to colonial rule (Pakenham, 1991). Throughout Africa, indigenous citizens were forced to live under a foreign rule of law that was repressive and authoritarian. Colonialists also imposed arbitrary territorial borders that partitioned ethnic groups and placed ethnically fragmented populations under a single system of governance, thereby disrupting traditional ways of living and leading to tensions between ethnically distinct indigenous groups (Michalopoulos \& Papaioannou, 2017). Even though colonial rule in Africa ended in the 1970s, the artifacts of nearly a century of largescale colonization have shaped many challenges (e.g., inequalities, fragile intergroup 
relations, and political instability) that continue to impact much of the African continent (Besley \& Reynal-Querol, 2014).

Colonialism also precipitated a marked shift in ethnocultural dynamics of Africa. The influx of Europeans, Indians, Chinese, and other non-native Africans altered the racial, cultural, and linguistic diversity of the region. Even when African countries acquired independence, many people who had come to Africa during colonization stayed, and their descendants remain a part of the demographic configuration of Africa. Western ideas, values, and belief systems that were introduced through foreign invasion permeated native societies and expanded the sociocultural fabric of Africa. For example, traditional African religions were dominant in the region before the colonization of Africa (Aderibigbe, 2015). However, during colonial expansion, many European empires (e.g., Britain) integrated the proselytism of Africans to Christianity as part of the vision they had for the region. Today, approximately $50 \%$ of Africans identify as adherents of the Christian faith (see Appendix 16.S1, Table 16.S2). About $40 \%$ of the African population affiliate religiously with Islam. Christianity is concentrated in sub-Saharan Africa, whereas Islam is most prevalent in Northern Africa. Although traditional African religions are in the minority on the continent, they continue to influence the religious life of many people in Africa. One way this occurs is through religious syncretism, which is perhaps most poignantly illustrated by the growth of independent Christian denominations that fuse indigenous R/S with Christian theology (Cowden, Laher, et al., in press).

\section{Asia}

Asia is the world's geographically largest and most populous continent, with approximately $60 \%$ (nearly 4.7 billion) of the global population living in the region (see Appendix 16.S1, Table 16.S1). Asia can be geographically divided into five subregions: East Asia (e.g., China, Japan), Central Asia (e.g., Kazakhstan, Uzbekistan), Western Asia (e.g., 
Israel, Turkey), South Asia (e.g., India, Sri Lanka), and Southeast Asia (e.g., Indonesia, Singapore).

The Asian region is a diverse cultural landscape that has been shaped by a complicated history of dynastic cycles, regional conquests, transcultural encounters, and periods of colonial rule (Murphey \& Stapleton, 2019). Of particular relevance to the contemporary status of societies within Asia is the so-called "Silk Road," a network of trade routes that emerged near the $2^{\text {nd }}$ century BCE and connected Asia from east to west (and even south into India). Commerce and migration through these pan-Asian routes influenced parochial cultures and exposed local people to alternative religions. For example, Buddhism emerged in Northeast India around the $6^{\text {th }}$ century BCE and later spread to Central and East Asia, in part through monks who began to travel along the trade routes (Foltz, 2010). Another key period in the history of Asian culture was European colonialism, which occurred between the $15^{\text {th }}$ and $20^{\text {th }}$ centuries CE and permanently embedded Western influences, ideas, and religious beliefs into the culture of many nations and territories in Asia (e.g., India, Philippines). Legacies of colonialism continue to have residual effects on the sociopolitical environments in historically colonized parts of Asia. A contemporary illustration of this phenomenon is the tension and conflict that recently transpired in the former British territory of Hong Kong (now part of China), over concerns that newly proposed legislature would undermine territorial independence from the Chinese government.

All five of the major world religions (i.e., Buddhism, Christianity, Hinduism, Islam, Judaism) originated from Asia. It is also the birthplace of numerous other religious traditions (e.g., Confucianism) that are localized to parts of Asia. Hinduism is the oldest and second largest mainstream religion in Asia (see Appendix 16.S1, Table 16.S2), although its footprint is limited to specific countries in South Asia (e.g., India, Nepal). Buddhism is especially prevalent in China, Japan, Sri Lanka, South Korea, and several Southeast Asian countries 
(e.g., Cambodia, Malaysia). Islam emerged in the $7^{\text {th }}$ century CE and became the primary religious tradition in the subregion of Western Asia, where most of the world's Islamic adherents live today. Large Muslim populations also exist in countries within Central, South, and Southeast Asia, owing to the eastward spread of Islam that began with early Islamic conquests in the $7^{\text {th }}$ century $\mathrm{CE}$ and has continued to the present (through commerce and migration). Even though Christianity arose in Western Asia and maintains a presence throughout Asia, today it is a minority religion within most of the Asian continent. More people in Asia are religiously unaffiliated than in any other region of the world, and a majority of these unaffiliated people live in China (see Appendix 16.S1, Table 16.S2).

\section{Oceania}

The Oceania region is comprised of fourteen independent countries that are divided into four main subregions: Australasia (i.e., Australia and New Zealand), Melanesia (e.g., Fiji, Papua New Guinea), Micronesia (e.g., Palau, Guam), and Polynesia (e.g., Tonga, Samoa). Oceania has the smallest population among the six inhabited continents, with fewer than 50 million people living in the region $(<1 \%$ of the global population; see Appendix 16.S1, Table 16.S1).

The first inhabitants in the region are said to have arrived by sea from different regions of Asia, bringing along their indigenous religious beliefs and practices that had sustained them for thousands of years (Swain \& Trompf, 1995). It was only around the $17^{\text {th }}$ century CE that Dutch and Spanish explorers arrived in the region. The establishment of colonies in Oceania led to more explorations by European settlers (who wanted to see this new stretch of lands). Some of the first settlers were British convicts who were transported from Britain and Ireland to the new colony of Australia, both as a punishment for their crimes and as a chance for them to build a new and more positive life. Shortly after European settlers 
made Oceania their home, the so-called "Australian Gold Rush" (which began around the middle of the $19^{\text {th }}$ century CE) opened these colonies up to the world (Monaghan, 1966).

Alongside these historical developments and migratory experiences are the continuities and changes that have characterized the R/S life of the region's indigenous people and new settlers. Before the arrival of immigrants from Asia and Europe, Pacific Islanders in the region practiced their own Polynesian religions. Small island nations in Polynesia, Melanesia, and Micronesia worshiped their family and village gods, but European settlers brought their Christian faith to the new colonies. After the Immigration Restriction Act of 1901 was abolished in 1966, trade with Asia opened doors for new settlers, many of whom practiced Eastern religions (e.g., Buddhism, Hinduism) or Islam. By that time, the influence of European settlers - many of whom came as Christian missionaries in the company of colonial powers - helped Christianity become the dominant religion in the region. Over the years, many indigenous groups have abandoned their traditional religions and belief systems to embrace Christianity (Ernst, 2012). Although Christianity remains the dominant faith tradition in Oceania (see Appendix 16.S1, Table 16.S2), the region has seen a recent decline in Christian adherents (Bouma \& Halafoff, 2017). The dynamics between colonialism, culture, and ongoing migratory processes have continued to shape the religious landscape of Oceania. This complexity makes a compelling case for studying the role of R/S in the region.

\section{Method of Review}

We performed a literature review to collate and descriptively map empirical research that has intersected PP with the PRS in the regions of Africa, Asia, and Oceania during the last three decades. Three sets of database searches (one for each region) were conducted in PsycINFO to identify relevant peer-reviewed scholarly articles that were published between January 1, 1990 and December 31, 2020. We searched for records with titles or abstracts that 
contained keywords associated with R/S (i.e., relig*, spirit*, worship*, pray*, faith, sacred) crossed with a combination of recognized regional/subregional terms (e.g., Africa, Northern Africa, sub-Saharan Africa) and the names of specific countries where there are researchintensive universities. The official name of a country was included in a regional search if it had at least one local university listed in the World University Rankings ${ }^{4}$. The number of country names that were included in each regional search was higher for Asia (32) compared to Africa (10) and Oceania (2). Nonempirical articles (e.g., reviews, conceptual or theoretical articles, perspectives, viewpoints, or commentaries), empirical articles that did not intersect PP and PRS, and topically relevant empirical articles that did not include at least one sample from a geographic region of interest were excluded from this review.

The database searches that were performed for each region yielded a combined total of 2,296 records (Africa: $k=377$; Asia: $k=1,732$; Oceania: $k=187$ ). After examining the titles and abstracts of the records within each region, 310 articles were retrieved for further screening. We then reviewed the full texts of those articles, which resulted in the exclusion of 181 articles that did not meet the inclusion criteria. Metadata (e.g., title, year of publication), key details about the research context (e.g., country of origin, purpose), methodology (e.g., research design, sampling approach), participants (e.g., sample size, age), and primary results were extracted from the articles that were retained.

\section{Results of the Review}

Across the three regions, a total of 128 unique articles met criteria for inclusion (one article met criteria for inclusion in both Africa and Asia). The largest proportion of these articles was from Asia $(k=64)$, followed by Africa $(k=44)$ and Oceania $(k=21)$. A complete reference list of articles included in this review can be found in Appendix 16.S1.

${ }^{4}$ The World University Rankings provide a comparative performance index of research-intensive universities located around the world. Rankings are based on five domains of performance, two of whichresearch and citations - each contribute $30 \%$ to a university's overall performance score (Times Higher Education, 2019). 


\section{Publication Activity}

Research intersecting PP and the PRS within Africa, Asia, and Oceania has grown exponentially over the last 30 years. More than $65 \%$ of the articles that met criteria for inclusion in each region were published in the last decade. Close to 5\% of scholarly articles were published in the 15 years between 1990 and 2004. Oceania had a higher percentage of publications written by scholars from a single country in its region (approximately $80 \%$ ) compared to Africa (around 30\%) or Asia (about 50\%). Less than 5\% of the publications in each region came from collaboration among researchers from multiple countries in the same region. Asia had the highest number of publications involving collaboration with nonregional scholars (roughly 20\%). More than 50\% of the publications in Africa were authored entirely by non-African scholars (primarily U.S. researchers). Authorship solely by nonregional scholars occurred less frequently in the other regions, particularly in Oceania where it was rare for publications to be authored exclusively by researchers outside that region.

Almost 50\% of the publications in the Africa region acknowledged a funding source that supported the research; roughly $60 \%$ of those sources were tied to authors affiliated with universities outside Africa. Less than $20 \%$ of studies in Asia and Oceania acknowledged a funding source. Funding sources in each region were diverse. For example, studies in Africa were supported by local universities, national and regional funders (e.g., South Africa's National Research Foundation, African Development Bank), and nonregional universities and foundations (e.g., Michigan State University, the John Templeton Foundation).

\section{Research Context and Methodologies}

For each region, over $75 \%$ of articles involved samples from a single country in that region. When multicountry samples were recruited, studies mostly consisted of samples from countries outside the region rather than within the region. The percentage of studies that targeted the general adult population was higher in Africa (about 35\%) compared to Asia and 
Oceania (almost 20\% each). Africa had the highest percentage of publications with adult clinical samples (approximately 25\%), where many studies were linked to critical public health issues in the region (e.g., HIV/AIDS). Asia was the region with the largest proportion of studies focused on university students (roughly 35\%). Oceania had the highest number of studies sampling religious adherents (nearly 30\%), whereas Africa had the lowest $(<10 \%)$.

More than $50 \%$ of the publications in each region used quantitative designs, with the highest number found in Asia (approximately 90\%), followed by Oceania (about 75\%). Asia had the largest quantity of cross-sectional studies (almost $85 \%$ ); by comparison, both in Africa and Oceania, roughly half the studies were cross-sectional. Fewer than $15 \%$ of studies in each region used longitudinal designs, most of which were in the Oceania region. Fewer than $10 \%$ of studies in each region used experimental designs (e.g., randomized controlled trials). Approximately $35 \%$ of studies within Africa employed qualitative approaches, which were used less frequently in Asia and Oceania. Fewer than $15 \%$ of scholarly articles in each region used mixed methods (see Tsang et al., Chapter 8 , this volume).

In each region, less than $30 \%$ of studies used probability techniques to sample participants. Across regions, more than $70 \%$ of quantitative studies used nonprobability sampling, and approximately $20 \%$ of cross-sectional studies used probability sampling. Although sample sizes varied considerably across studies in each region, the Africa region had the highest percentage of articles with sample sizes over 500 (about $45 \%$ of studies). In each region, at least $60 \%$ of studies with low sample sizes ( $\leq 50$ participants) used qualitative or mixed methods designs. Nearly all studies in Asia and Oceania included both male and female participants, whereas about $20 \%$ of studies in Africa had unigender samples. Over $75 \%$ of studies in each region sampled only adults. Young adults aged 18 to 35 years were sampled most frequently, whereas children below age 10 years were sampled most rarely. 


\section{Topical Emphases}

We synthesized information about the scope, purpose, and main findings of the included articles, to provide a topical overview of PP and PRS research in Africa, Asia, and Oceania. Due to regional distinctions in emphases, findings are presented by region.

\section{Africa}

Well-Being. In the Africa region, almost $60 \%$ of the reviewed studies focused on well-being. Slightly more of those studies addressed aspects of eudaimonic well-being (primarily meaning in life) compared to those that focused on hedonic indices of well-being (predominantly life satisfaction). Although research on well-being was common, less than $5 \%$ of studies applied a holistic approach to well-being by assessing functioning across several core domains of life. Almost $25 \%$ of studies on well-being also focused on other thematic areas of PP that emerged during our review (i.e., character strengths and virtues, positive adaptation). The most frequently studied populations were university students, adult samples with medical illnesses, and adherents of major religions (e.g., Christianity, Islam).

Approximately $30 \%$ of the African studies on well-being intersected with religiosity. Operationalizations of religiosity ranged from broad (e.g., multidimensional assessment capturing beliefs, practices, and commitment) to narrow (e.g., a single-item measure of religiousness). About $30 \%$ of studies focused on specific aspects of R/S life that routinely form part of conceptualizations of religiousness (e.g., religious importance, religious beliefs, religious practices), with slightly fewer addressing spirituality. Religious affiliation formed a central part of nearly $25 \%$ of the studies on well-being. A small number of studies examined religious service attendance, religious coping, or perceptions of religion or its teachings.

Character Strengths and Virtues. In the Africa region, nearly $30 \%$ of studies examined character strengths and virtues alongside PRS concepts. Half of those studies focused on hope, with the rest examining prosocial behavior, forgiveness, or love. Almost 
$70 \%$ of the studies on character strengths and virtues also addressed aspects of well-being (e.g., meaning or purpose in life) and positive adaptation (e.g., coping, resilience). Only one study investigated multiple character strengths or virtues.

Many studies on character strengths and virtues addressed specific aspects of R/S life. The most common was religious practices (roughly 40\%), whereas religious affiliation, belief in God or the divine, faith, perceptions of God or the divine, and religious beliefs appeared less frequently. Only one study focused broadly on religiousness, and three studies investigated spirituality. Three studies examined intersections of character strengths and virtues with religious coping. None of the studies reported on religious service attendance, but one study addressed the topic of religious community.

Positive Adaptation. About one-fifth of the region's articles studied the intersections of $\mathrm{R} / \mathrm{S}$ with processes and outcomes indicative of positive adaptation. Close to $75 \%$ of those studies examined intersections between R/S and coping; the remainder centered on resilience or personal growth. Half of the articles assessed overall R/S, although many also assessed specific aspects of R/S life (e.g., religious affiliation, R/S beliefs, religious practices, faith, perceptions of God or the divine). A few studies explored religious coping as part of the broader coping process.

\section{Asia}

Well-Being. In the Asia region, more than $70 \%$ of studies examined some form of well-being alongside R/S concepts. At least half those studies emphasized specific indices of hedonic well-being (e.g., happiness or satisfaction with life), and around one-third applied a broader conceptualization of subjective well-being (e.g., quality of life). Approximately 10\% of articles reported findings based on measures that broadly captured specific dimensions of well-being (e.g., psychological well-being). A similar proportion of studies addressed aspects of eudaimonic well-being (e.g., meaning or purpose in life). Close to $10 \%$ of studies 
investigated facets of well-being in conjunction with specific character strengths or virtues. Studies on well-being most often sampled students. However, some studies sampled older adults or adherents of major world religions (e.g., Islam, Judaism).

About $60 \%$ of studies on well-being intersected with the PRS via the superordinate construct of religiousness. Like Africa, operationalizations of religiousness varied from narrow (e.g., single-item index of self-perceived religiousness) to broad (e.g., multidimensional measure of intrinsic and extrinsic religious motivation). Nearly $10 \%$ of studies assessed spirituality. Roughly $20 \%$ of articles explored specific R/S dimensions, such as R/S beliefs, religious commitment, and prayer. Studies on perceptions of God or attitudes towards religion were uncommon. Approximately $10 \%$ of studies focused on religious affiliation. A smaller number of articles explored religious support. Associations between religious service attendance and well-being were rarely investigated. Other topics that were examined infrequently included mysticism, spiritual transcendence, and spiritual distress.

Character Strengths and Virtues. Less than one-fifth of publications in the Asia region focused on character strengths and virtues. Several character strengths or virtues were represented across those studies, including forgiveness, hope, gratitude, and altruism. Roughly $60 \%$ of the studies that examined the intersection of R/S with character strengths and virtues assessed overall R/S. The other studies assessed certain R/S dimensions (e.g., R/S beliefs, participation), usually centering on dimensions of religion rather than of spirituality.

Positive Adaptation. A small proportion of studies (around 10\%) in the Asia region addressed topics of positive adaptation. About half those studies emphasized R/S coping as part of navigating the impact of negative life events. The other studies explored the role of $\mathrm{R} / \mathrm{S}$ in fostering adaptive psychological functions or attributes (e.g., emotional regulation) that could support recovery from adversity or trauma. 


\section{Oceania}

Well-Being. In the Oceania region, close to $60 \%$ of studies on PRS and PP examined aspects of well-being. More than $70 \%$ of these articles focused on subjective well-being. The remainder assessed other indices of well-being, including life satisfaction, psychological well-being, or R/S well-being. Fewer than $20 \%$ of studies examined the role of overall R/S in promoting well-being, but many others (approximately 40\%) examined specific R/S dimensions (e.g., religious affiliation, religious identity). Almost $30 \%$ of the articles explored the salience of religious coping in enhancing or sustaining well-being. One study examined the link between spiritual and subjective well-being. All studies on well-being recruited participants living in either Australia or New Zealand.

Character Strengths and Virtues. About $25 \%$ of studies in the Oceania region examined the intersection of R/S with character strengths and virtues. Most of them (roughly $60 \%$ ) examined connections between prosociality (e.g., charity, altruism) and either R/S affiliation or R/S identity. These were also the most methodologically rigorous studies from the region. The remaining studies investigated compassion or forgiveness, usually in association with spirituality.

Positive Adaptation. Nearly 25\% of studies examined processes and outcomes of positive adaptation. Almost all centered on the role of R/S in coping with adversities (e.g., migration, health problems) and in building psychosocial resilience. One study specifically examined religious coping and its link with stress-related growth, whereas another explored the connection between R/S well-being and styles of psychological adjustment. Many of the studies focused on migrants and religious minorities (e.g., Muslims) at the margins of societies in that region, with some also emphasizing spiritual considerations in healthcare. 


\section{Discussion}

During the last decade, the regions of Africa, Asia, and Oceania have seen encouraging growth in research that cross-pollinates PP with the PRS. This trend suggests there is great potential for further development of research that intersects these two subfields. In the sections that follow, we synthesize and discuss the findings of our review. We also offer suggestions for further cross-pollinating PP and the PRS, hoping that doing so would lead toward each subfield's greater scientific and social impact in Africa, Asia, and Oceania.

\section{Reflections on the Findings}

In all three regions, research on $\mathrm{R} / \mathrm{S}$ and indices of well-being predominated topically. Across the reviewed studies, scholars used a variety of frameworks to operationalize wellbeing (e.g., eudaimonic, hedonic). Studies on well-being largely emphasized a single dimension of well-being (e.g., life satisfaction). Across regions, assessing well-being using a holistic, multidimensional approach would help advance understanding of the linkages between R/S and well-being. Similarly, it was rare for studies in any of the regions to examine aspects of R/S well-being alongside other dimensions of well-being. Many R/S traditions emphasize R/S well-being as an integral part of living a fulfilling life, and it is likely that even a comprehensive measure of well-being will be unable to provide a complete picture of well-being if the R/S dimension is not also assessed. Research that integrates R/S well-being and other dimensions of well-being would advance scientific understanding of the nature and determinants of well-being in Africa, Asia, and Oceania. Such integration could contribute to the development, refinement, and application of culturally responsive mental health treatment approaches that intersect PP and the PRS in these contexts. Research along these lines would do well to be grounded in integrative theories that can provide an overarching framework for bringing together PP and R/S (e.g., existential PP or PP from the viewpoint of a single R/S tradition; see also Davis et al., Chapter 18, this volume). 
A majority of the populations that reside in all three regions are adherents of a major world religion (e.g., Buddhism, Christianity, Islam, Hinduism), each of which values similar character strengths and virtues (Peterson \& Seligman, 2004). Hence, we were surprised that disproportionately few publications in each region intersected character strengths and virtues (e.g., altruism, forgiveness, gratitude, hope, love) with the PRS. Character strengths and virtues that have been examined extensively in the West (e.g., humility, wisdom) received little attention alongside R/S concepts in Africa, Asia, and Oceania. Character strengths and virtues are a bridge that connects R/S values and ideals with other areas of PP (e.g., wellbeing), which suggests remarkable expansion in both PP and the PRS could be achieved if research on character strengths and virtues increased in the future (see Ratchford et al., Chapter 4, this volume).

Limited expertise in the PRS within Africa, Asia, and Oceania may be influencing the apparent lack of research depth in these regions. This deficiency in depth can be characterized by a lack of comprehensiveness, complexity, and methodological rigor in research at the intersection of PP and the PRS in these regions. Of note, research in all three regions has tended to overlook the potential "dark side" of R/S by focusing almost entirely on positive aspects of R/S. Considerable research in the West has now explored the phenomenon of R/S struggles (i.e., tensions, strains, and conflicts about sacred matters), with some evidence suggesting that R/S struggles can lead to psychological and spiritual growth (e.g., Exline et al., 2017). If scholars in Africa, Asia, and Oceania were to examine the full spectrum of R/S experiences more deliberately, scientific understanding of linkages between R/S and many PP concepts (e.g., meaning in life) would be greatly enhanced.

Although the thematic area of positive adaptation is represented in research on PP and the PRS within Africa, Asia, and Oceania, the emphasis was largely on coping and resilience. Few studies went beyond the role of R/S in facing distress or remaining psychologically 
buoyant while navigating adversity. Further research is needed to understand how R/S might facilitate personal growth or otherwise enable people to thrive in response to negative life events, particularly studies focused on forms of distress (e.g., suffering) that many R/S traditions emphasize as having the capacity to bring about personal or R/S transformation (Cowden, Davis et al., 2021). For example, culturally responsive research that crosspollinates PP and the PRS in these three regions could expand the corpus of empirical literature on suffering by providing additional insight into how non-Western worldviews shape perspectives of suffering, responses to suffering, and adaptive processes that enable people to transcend suffering. More generally, research that draws on both subfields to develop an understanding of R/S growth following hardships, challenges, and adversity could provide a foundation for interventions, public health initiatives, and policies that address key social issues in each of the three regions (Long \& VanderWeele, Chapter 25, this volume).

We found that, in all three regions, research intersecting PP and the PRS emphasized or was conducted with samples from major world religions. In each region, traditional or indigenous religions that are in the minority (but have played an important role in shaping local culture and the R/S life of contemporary societies) were underrepresented, particularly in Africa and Oceania. Traditional or indigenous religions often espouse a worldview that is fundamentally different from that of major religious traditions. For example, harmonious interdependency between the living and the spirits of deceased ancestors is a central part of many traditional African religions. Among adherents of these religions, when mental and physical health difficulties occur, those difficulties are often interpreted as being rooted in disruption to that harmony (Cowden, Laher, et al., in press). There is much that the subfield of PP could gain by understanding aspects of the human experience that are prioritized by traditional or indigenous religious beliefs systems, many of which remain (in some form of another) part of the R/S life of people who live in the regions of Africa, Asia, and Oceania. 


\section{Considerations for Practitioners}

Increased cross-pollination of PP and the PRS could enhance the competence and effectiveness of mental health practitioners who draw on PP in their work. More than $80 \%$ of people in Africa, Asia, and Oceania are adherents of a R/S tradition (see Appendix 16.S1, Table 16.S2), which can have an important influence on how people view, interact with, and make sense of the world. For example, R/S can shape how clients understand well-being, prioritize certain aspects of well-being, and interpret and face potential threats to well-being (e.g., suffering). Practitioners who explore and attend to the culturally embedded R/S attitudes, beliefs, and experiences of clients are likely to acquire a more holistic understanding of the people they are serving. In the process, practitioners would be better equipped to implement personalized treatment approaches that are more culturally responsive and tailored to the needs of clients.

Practitioners in Africa, Asia, and Oceania might consider strengthening R/S competencies through resources that offer training and guidance about providing mental health care that is responsive to clients' R/S beliefs, practices, needs, and values. A growing number of training programs are available to build R/S competencies (e.g., Spiritual Competency Training in Mental Health program), but it will be important for practitioners to be prepared with R/S competencies that are relevant to the culture and context in which they provide services. Development of R/S competencies needs to be encouraged and supported by professional psychology bodies in countries within each of these geographic regions.

As PP practitioners in each region pursue an enriched clinical approach through crosspollination with the PRS, there may be value in harnessing spiritually integrative treatment approaches to facilitate therapeutic change. One promising model is integrative meaning therapy (Wong, 2010), a holistic approach that intersects PP, clinical psychology, and existentialism. A central tenet of integrative meaning therapy is that R/S is an essential part of 
being human, and $\mathrm{R} / \mathrm{S}$ is recognized as a powerful resource that clients can mobilize to transcend the challenges of life towards more complete flourishing. Integrative meaning therapy also has a multicultural orientation that makes it an appealing model for use in culturally and linguistically diverse contexts. Although integrative meaning therapy may not be a suitable treatment for all clients, it does highlight the potential for PP and R/S to be integrated coherently into practice, to support clients from different cultures and contexts.

\section{Suggestions for the Next Wave of Research}

If the next wave of research that intersects PP and the PRS in Africa, Asia, and Oceania is to have a substantive impact both locally and internationally, at least two important developments in research practice are needed. First, the rigor of quantitative research traversing these two subfields must be increased in each region. Most publications in each of the three regions reported findings from quantitative cross-sectional designs, a methodological approach that makes it difficult to establish the direction of cause and effect. Consistent with calls to strengthen the science of both PP and the PRS (see Gallagher \& Lopez, 2021; VanderWeele, 2021), future research that cross-pollinates these subfields would benefit from carefully designed longitudinal studies grounded in frameworks for causal inference. That could substantially improve the methodological rigor and causal conclusions that can be drawn from quantitative research intersecting the two subfields (see also Tsang et al., Chapter 8, this volume).

Second, well-designed research programs are needed to build a stream of culturally responsive empirical evidence that cross-pollinates PP and the PRS, as well as to facilitate theoretical advances that apply to the societies within each region. In Africa and Asia, where many publications were authored exclusively by scholars outside each region, local researchers who form collaborative partnerships with well-established international scholars could acquire expertise in designing and implementing systematic programs of research. 
Collaborations with nonregional scholars could also enrich the existing body of evidence germane to each region, particularly when researchers from outside the region have expertise in a particular phenomenon that is of shared interest. For example, several studies in the Oceania region focused on migrants from countries in Asia (mainly Western Asia) but were authored entirely by researchers residing in Oceania. International research collaborations that merge regional knowledge of culturally appropriate research methodologies with sophisticated quantitative approaches to conducting research are likely to provide a useful balance of mixed-methods evidence that is sensitive to the sociocultural dynamics of each region and meets international standards of research excellence.

\section{Conclusion}

To catalyze, expand, and strengthen culturally responsive cross-pollination of PP and the PRS in non-Western contexts, we evaluated the extent of existing overlap between these two subfields in Africa, Asia, and Oceania. During this process, we discovered that relatively little research exists at the intersection of PP and the PRS in each of these geographic regions. Even when relevant research has been conducted, the internal and external validity of the findings has been limited by weaknesses in the utilized methodologies. Although the current body of literature in each region might lack quality and depth, that means there are numerous opportunities to pursue high impact, culturally responsive research that cross-pollinates PP and the PRS. We encourage researchers and practitioners to take advantage of what the subfields of PP and the PRS have to offer one another and integrate them in ways that are sensitive to the cultural and contextual nuances of Africa, Asia, and Oceania. Greater crosspollination of PP and the PRS in each of these regions could have important implications for transforming the scientific and social impact of psychology, both locally and internationally. 


\section{References}

Aderibigbe, I. S. (2015). Religious traditions in Africa: An overview of origins, basic beliefs, and practices. In I. S. Aderibigbe \& C. M. J. Medine (Eds.), Contemporary perspectives on religions in Africa and the African diaspora (pp. 7-29). Palgrave Macmillan.

Besley, T., \& Reynal-Querol, M. (2014). The legacy of historical conflict: Evidence from Africa. American Political Science Review, 108(2), 319-336. https://doi.org/10.1017/S0003055414000161

Bouma, G. D., \& Halafoff, A. (2017). Australia's changing religious profile-Rising Nones and Pentecostals, declining British Protestants in superdiversity: Views from the 2016 census. Journal for the Academic Study of Religion, 30(2), 129-143.

\section{https://doi.org.10.1558/jasr.34826}

Cowden, R. G., Counted, V., \& Ramkissoon, H. (2021). Transcending place attachment disruption: Strengthening character during a pandemic. In V. Counted, R. G. Cowden, \& H. Ramkissoon, Place and post-pandemic flourishing: Disruption, adjustment, and healthy behaviors. Springer.

Cowden, R. G., Davis, E. B., Counted, V., Chen, Y., Rueger, S. Y., VanderWeele, T. J., Lemke, A. W., Glowiak, K. J., \& Worthington, E. L., Jr. (2021). Suffering, mental health, and psychological well-being during the COVID-19 pandemic: A longitudinal study of U.S. adults with chronic health conditions. Wellbeing, Space and Society, 2, 100048. https://doi.org/10.1016/j.wss.2021.100048

Cowden, R. G., Laher, S., \& Worthington, E. L., Jr. (in press). South Africa: The psychology of religion up to 2020. In K. L. Ladd, J. Basu, V. DeMarinis, Ü. Ok, \& W. Zangari (Eds.), Cambridge international handbook of the psychology of religion. Cambridge University Press. 
Downey, C. A., \& Henderson, R. E. (2021). Speculation, conceptualization, or evidence? A history of positive psychology. In C. R. Snyder, S. J. Lopez, L. M. Edwards, \& S. C. Marques (Eds.), The Oxford handbook of positive psychology (3rd ed., pp. 8-17). Oxford University Press.

Ernst, M. (2012). Changing Christianity in Oceania: A regional overview. Archives de Sciences Sociales Des Religions, 157(1), 29-45. https://doi.org/10.4000/assr.23613

Exline, J. J., Hall, T. W., Pargament, K. I., \& Harriott, V. A. (2017). Predictors of growth from spiritual struggle among Christian undergraduates: Religious coping and perceptions of helpful action by God are both important. The Journal of Positive Psychology, 12(5), 501-508. https://doi.org/10.1080/17439760.2016.1228007

Foltz, R. (2010). Buddhism and the Silk Road. In R. Foltz (Ed.), Religions of the Silk Road: Premodern patterns of globalization (pp. 37-58). Palgrave Macmillan.

Gallagher, M. W., \& Lopez, S. J. (2021). Strengthening positive psychology. In C. R. Snyder, S. J. Lopez, L. M. Edwards, \& S. C. Marques (Eds.). The Oxford handbook of positive psychology ( $3^{\text {rd }}$ ed., pp. 3-7). Oxford University Press.

Michalopoulos, S., \& Papaioannou, E. (2017). The Scramble for Africa and its legacy. In M. Vernengo, E. P. Caldentey, \& B. J. Rosser, Jr. (Eds.), The new Palgrave dictionary of economics (pp. 1-11). Palgrave Macmillan.

Monaghan, J. (1966). Australians and the Gold Rush. University of California Press.

Murphey, R., \& Stapleton, K. (2019). A history of Asia ( $8^{\text {th }}$ ed.). Routledge.

Pakenham, T. (1991). The Scramble for Africa, 1876-1912 (1 ${ }^{\text {st }}$ ed.). Random House.

Peterson, C., \& Seligman, M. E. P. (2004). Character strengths and virtues: A handbook and classification. American Psychological Association. 
Reed, F. A., \& Tishkoff, S. A. (2006). African human diversity, origins and migrations. Current Opinion in Genetics \& Development, 16(6), 597-605. https://doi.org/10.1016/j.gde.2006.10.008

Swain, T., \& Trompf, G. W. (1995). The religions of Oceania. Psychology Press.

Times Higher Education. (2019). Methodology for overall and subject rankings for the Times Higher Education World University Rankings 2020. Times Higher Education. https://www.timeshighereducation.com/sites/default/files/the_2020_world_university _rankings_methodology_pwc.pdf

VanderWeele, T. J. (2017). On the promotion of human flourishing. Proceedings of the National Academy of Sciences, 114(31), 8148. https://doi.org/10.1073/pnas.1702996114

VanderWeele, T. J. (2021). Effects of religious service attendance and religious importance on depression: Examining the meta-analytic evidence. The International Journal for the Psychology of Religion, 31(1), 21-26.

\section{https://doi.org/10.1080/10508619.2020.1748932}

Wong, P. T. P. (2010). Meaning therapy: An integrative and positive existential psychotherapy. Journal of Contemporary Psychotherapy, 40, 85-93. https://doi.org/10.1007/s10879-009-9132-6

Worthington, E. L., Jr., \& Cowden, R. G. (2017). The psychology of forgiveness and its importance in South Africa. South African Journal of Psychology, 47(3), 292-304. https://doi.org/10.1177/0081246316685074

Worthington, E. L., Jr., Cowden, R. G., Davis, E. B., \& Exline, J. J. (in press). Virtues in positive psychology and the psychology of religion and spirituality: Existing overlap and promising possibilities. In L. J. Miller (Ed.), The Oxford handbook of psychology and spirituality ( $2^{\text {nd }}$ ed.). Oxford University Press. 


\title{
Chapter 17
}

\section{Religion, Spirituality, and Youth Thriving:}

\section{Investigating the Roles of the Developing Mind and Meaning-Making}

\author{
Pamela Ebstyne King \\ Peter L. Benson Associate Professor of Applied Developmental Science \\ PhD Psychological Science Program Chair \\ Susan Mangan \\ Postdoctoral Research Associate \\ Thrive Center for Human Development \\ School of Psychology \& Marriage and Family Therapy \\ Rodrigo Riveros \\ Postdoctoral Research Associate \\ Thrive Center for Human Development \\ School of Psychology \& Marriage and Family Therapy \\ Fuller Theological Seminary
}

\section{Author Note}

Correspondence concerning this article should be addressed to Dr. Pamela Ebstyne King, Thrive Center for Human Development, School of Psychology \& Marriage and Family Therapy, Fuller Theological Seminary, 180 N. Oakland Ave., Pasadena, CA, 91101. Email:

pamking@fuller.edu.

Pamela Ebstyne King: https://orcid.org/0000-0002-2853-7657

The authors have no conflict of interest. 


\begin{abstract}
In this chapter, we draw on positive Developmental Psychology, Psychology of Religion and Spirituality, and Developmental Neuroscience to explore how youth religiousness and spirituality contribute to thriving through the process of meaning-making. Thriving involves the individual, relational, and aspirational development necessary to pursue life purpose that is meaningful to the self and one's surroundings. Meaning-making is the process of constructing and internalizing abstract beliefs (about oneself, the world, and one's priorities) into salient values that contribute to the moral development necessary to thrive. When youth consider abstract ideas in the context of their actions, transcendent emotions, and the broader world, then their meaning-making can result in values-based goals and behaviors. Adolescents are naturally motivated to explore identity-related issues of meaning, values, roles, and belonging. In particular, meaning-making occurs when youth are given the opportunity to reflect in enriching dialogue with caring adults. More than most youth contexts, religion and spirituality provide young people with opportunities to seek and form meaning by being prompted to process transcendent beliefs and emotions through the narratives, intergenerational relationships, and transcendent experiences that religion and spirituality often provide.
\end{abstract}

Keywords: meaning-making, spirituality, religion, adolescence, transcendent emotions 


\section{Religion, Spirituality, and Youth Thriving: \\ Investigating the Roles of the Developing Mind and Meaning-Making}

A burgeoning body of research reveals the many ways religion and spirituality (hereafter, R/S) contribute to the positive development of youth (Hardy et al., 2019; King \& Boyatzis, 2015; Schnitker et al., 2019). With increasing awareness of the importance of promoting trajectories of human thriving that contribute to flourishing societies, scholars are looking for approaches to thriving that include not only individual well-being and life satisfaction but also a beyond-theself orientation and actions that strengthen the surrounding systems. One such approach, the reciprocating self, orients human development towards a telos (i.e., ultimate goal) that furthers an ongoing, mutually beneficial fit between self and society and requires continual individual, relational, and aspirational development (King \& Mangan, in press). Although research affirms the importance of moral ideals and spiritual commitments in this process (King et al., 2020; Schnitker et al., 2019), little is understood about how youths' beliefs turn from ideas into lived action. Meaning-making may be one helpful explanation (Furrow et al., 2004; Immordino-Yang et al., 2019; see Park \& Van Tongeren, this volume). Synthesizing brain development research in this area, we explain how meaning-making is the process of constructing and internalizing salient beliefs into a youth's narrative identity and core values. In turn, these values guide prosocial behaviors that are a mechanism and marker of thriving.

It is already well-documented that a person's narrative identity helps incorporate one's beliefs and values into goals that motivate prosocial and purposeful behaviors indicative of thriving. Narrative identity is the evolving story that "people have in their minds about how they have come to be the particular people they are becoming" (McAdams, 2021, p. 122). One's narrative identity integrates a reconstruction of their past and imagined projection of their future 
into a single narrative arc, which provides a sense of unity, purpose, and temporal coherence. Building on the work of McAdams, Schnitker (Schnitker et al., 2019; Ratchford et al., Chapter 4, this volume) and King (King et al., 2020) have proposed the term transcendent narrative identity to designate narrative identities that offer a beyond-the-self context for someone's life, providing a unifying spiritual, religious, or moral purpose to their narrative identity. What is less welldocumented or explained is how transcendent beliefs are incorporated into one's narrative identity. Thus, the focus of this chapter is explaining how R/S facilitate the process of youth meaning-making through exposure to transcendent beliefs and emotions in the context of caring relationships. The convergence of ideological, social, and transcendent resources promotes thriving, as reciprocating selves develop and grow personally and relationally, including the moral and spiritual commitments required to contribute meaningfully beyond the self.

In this chapter, we draw on research from Positive Psychology, Positive Youth Development, Developmental Neuroscience, and the Psychology of R/S to describe how youth are poised for constructing meaning that leads to enduring, values-aligned goals. These goals motivate behaviors that enable youth to become fulfilled, contributing members of society. Our interdisciplinary approach highlights how biological influences (e.g., brain maturation) coalesce with religious and spiritual development to create meaning. Our overall goal is to explain why youth have a propensity to engage in meaning-making and how R/S have the potential to provide the coherent belief systems, caring relationships, and transcendent experiences that lead to transcendent narrative identity and emotions and thereby to purposeful, thriving adults.

\section{Background: Relational Developmental Systems Theory and Thriving}

To describe how religious and spiritual development co-act with child and adolescent brain development and promote thriving through meaning-making, we draw on Relational 
Developmental Systems (RDS) metatheory. RDS considers the breadth of human development, including religious and spiritual development, in the context of the diverse systems in which humans live (King et al., 2021; Lerner, 2018; Overton, 2015; Davis et al., Chapter 18, this volume). Human development occurs not only through the bidirectional interactions between an individual and the world around them. It also occurs at all levels of the developing system—from the cellular to the social to the perceived transcendent. For instance, human cognitive abilities (e.g., our imagination) and attachment patterns (shaped by our relationships with our parents) influences our religious/spiritual development, such as how we view and relate to the divine. RDS emphasizes the multifaceted nature of religious/spiritual development, including the roles of the neurological changes that emerge with expanding cognitive and social-emotional skills. For example, the ability to take another person's perspective (or imagine God's perspective) and feel empathy for another are reasoning and emotional processing capacities that enable young people to engage in more abstract ideas and feelings of transcendence. Additionally, capacities like these help youth become involved in diverse and deeper relationships, which in turn nurture young people's meaning-making processes and capacities.

RDS is predicated on the worldviews of relationalism and holism, which emphasize the interconnectivity of all things. For instance, the well-being of one people group has direct or indirect effects on other people groups. Consequently, thriving aims for the entire developmental system to flourish over time (King \& Mangan, in press; Overton, 2015). At a practical level, this suggests that youth thriving involves contributions beyond-the-self-whether to one's family, peer group, community, the environment, or the greater good (Lerner, 2018). Although many conceptualizations of flourishing, well-being, and happiness within Positive Psychology emphasize the individual, from an RDS perspective, thriving involves ongoing mutually 
beneficial relationships between individuals and their contexts (see Davis et al., Chapter 18, this volume). To emphasize this, we draw on the theoretical telos of a reciprocating self (Balswick et al., 2016; King \& Mangan, in press), which infers that the purpose of human development is to grow as differentiated authentic individuals who are in mutually beneficial interdependence with others. This process requires ongoing moral and spiritual development that sustains a good fit between individuals and their broader contexts (King \& Mangan, in press; Lerner et al., 2003; Schnitker et al., 2019). This highlights how a developing person increases their capacity to thrive through individual, relational, and aspirational growth over time (see Appendix 17.S1, Figure 17.S1). Aspirational refers to the evolving moral ideals and spiritual commitments that guide and motivate contributions to the greater good through changing circumstances and contexts. To summarize, to thrive is to develop and adapt in authentic, mutually beneficial relationships that nurture one's contextually adaptive values, purpose, and contributions. Given that purpose involves an enduring goal that is meaningful to the self and makes a valuable contribution to the greater good (Damon et al., 2003), continually clarifying what is meaningful and of value to oneself and one's surroundings is essential for identifying and pursuing purpose.

To illustrate this dynamic process of thriving through individual, relational, and aspirational development in the pursuit of an enduring life goal that is meaningful to self and society, consider a transgender youth raised in an Orthodox Jewish community. This young person may struggle to feel seen and loved for who they are (individual), may struggle to feel close and respected by their religious community (relational), and may experience limited opportunities to pursue their faith in their current context (aspirational). Given such a potential discord, this young person will need to sort through beliefs, their relationships, and their understanding of God in order to determine what is most important and of value, especially as 
they navigate issues of gender and religious identity. In short, and hopefully with the assistance of caring adults, they will sort through what is meaningful and their sources of meaning in order to navigate a good fit between who they are as a transgender person and as someone who is religiously committed to Judaism. This process may be lead them to a Reformed synagogue or other Jewish community that not only is more supportive of this transgender young person's uniqueness but also allow more opportunities for them to pursue their sense of life purpose and contribute meaningfully to their religious community. For this young person to thrive, their personal strengths and social world need to align to support a mutually beneficial relationship, where the young person can reciprocate - receive and give - among the people they live. R/S provide rich opportunities (and at times challenges as described above) for the meaning-making necessary for this young person to cultivate an ongoing good fit between themselves and the world in which they are embedded, as they develop and thrive more fully as reciprocating selves.

\section{Religious and Spiritual Development}

From an RDS perspective, religious and spiritual development are distinct but overlapping constructs. They each occur as an individual interacts with the micro- and macrosystems around them, including beliefs, relationships, and perceptions of transcendence. Religiousness involves engaging in the doctrines, community, and rituals of a religious tradition. Religious development, however, is specifically the changes in one's capacities to engage in the beliefs, relationships, and practices associated with religion (King \& Boyatzis, 2015).

Spirituality, on the other hand, refers to one's experience of and response to transcendence (King et al., 2020). Transcendence-whether experiencing God or another form of ultimate reality - involves our thoughts, emotions, and other senses. Transcendence requires individuals to connect their perceptions with broader macrosystem-level beliefs in a way that encourages 
complex social emotions such as admiration, compassion, and moral elevation. For instance, youth might experience transcendence when they feel a connection with others or God while singing in a worship service, when they are confronted by natural beauty, or when they are volunteering. Transcendent ideas and emotions can inspire devotion to beliefs, resulting in the integration of values and life goals with personal identity, resulting in fidelity, which in turn motivates values-aligned behaviors of contribution that are characteristic of thriving (Appendix 17.S1, Figure 17.S2; King et al., 2014; Lerner et al., 2003; Riveros \& Immordino-Yang, 2021). In contrast to many definitions of spirituality that emphasize an individual's search or experience of transcendence (which can sometimes result in overlooking changes in identity and behaviors), this approach is referred to as Reciprocating Spirituality. Reciprocating Spirituality emphasizes the bidirectional influence between the individual and transcendent, which results in structural changes in the individual and leads to transcendent, values-aligned identity and behavior. For example, ideas and feelings about transcendence may result in a transcendent narrative identity informed by being a part of a reality bigger than daily life, and it may inspire devotion to act accordingly. Thus, spiritual development refers to the changes in one's capacities to experience transcendence in a way that informs identity and worldview and motivates valuesaligned behavior (King et al., 2014; King et al., 2020).

From this perspective, both religious and spiritual development occur through the developing young person's interactions with the world (see Davis et al., Chapter 18, this volume). Religion provides a context ripe with a set of beliefs, practices, and community that usually promote transcendence and spiritual development. However, spiritual development may or may not occur within the context of religion. Because spiritual development requires transcendence, it involves the engagement of transcendent beliefs and emotions that are often 
shared in relationships and pursued through practices. Thus, religious and spiritual contexts each include ideological, social, and transcendent milieus that provide beliefs and narratives, transcendent emotions, and relationships that support meaning-making (King et al., 2020).

\section{Meaning-Making}

Meaning-making is central to spirituality. It represents the process of constructing and internalizing cognitive and affective experiences of transcendence as enduring beliefs, which are incorporated into one's narrative identity. For the purposes of this paper on youth R/S and thriving, meaning-making refers to the process of internalizing beliefs into one's transcendent narrative identity. These salient beliefs inform people's views of themselves in relationship to the world, forming values-aligned goals that guide their actions accordingly (Furrow et al., 2004). Park's (2010) Meaning Making Model addresses how people assimilate adversity into their meaning system or accommodate the meaning system to adversity (see Park \& Van Tongeren, Chapter 6, this volume). For example, this adversity-based assimilation and accommodation is usually referenced with regard to spiritual coping, wherein individuals draw on their religious and/or spiritual beliefs, communities, and practices as strategies for making meaning out of loss, death, and struggle. However, within this chapter, we address the relationship between meaning-making and spirituality beyond the context of coping in order to understand how meaning-making incorporates beliefs into narrative identity and results in values-based goals that are necessary for the purposeful pursuits that help youth thrive.

For youth, meaning-making does not occur exclusively in contexts of R/S, but it is more broadly recognized as the process of identifying beliefs that are of enduring salience to an individual. These ideals are then incorporated into one's narrative identity, serving to motivate and guide behavior accordingly. Such enduring meaningful thoughts and their accompanying 
moral emotions are ascribed motivational properties and serve as values (Merrill \& Fivush, 2016). When a belief is meaningful, the experience of salience generates fidelity to it and signifies its integration into one's narrative identity. This then motivates actions consistent with one's ideals (Erikson, 1968; Furrow et al., 2004; King et al., 2014). For instance, an experience of Allah through an answered prayer may generate further devotion to Allah and motivate commitment to living out one's Muslim beliefs. Fidelity to a belief system involves a commitment to act on those beliefs and has the propensity to develop values-aligned goals that underlie the pursuit of noble purposes. Consequently, spirituality is seminal to thriving because the cognitive and emotional stimulation of transcendence spurs on an other-oriented meaningmaking process that results in fidelity, informs a transcendent narrative identity, values-aligned goals, and purposeful behaviors (Erikson, 1968; Lerner et al., 2003; King et al., 2020). In Appendix 17.S1, Figures 17.S3 and 17.S4 illustrate the thriving process and portray the cycle of R/S contexts prompting meaning-making, which leads to values that are informed by one's transcendent narrative identity and to embodied feelings that guide and motivate pursuit of purpose. Purposeful activities then engage people deeper in the beliefs, relationships, and experiences of transcendence, which further supports the process of thriving. As such, meaningmaking is a critical task of spiritual and religious development in youth. Understanding the brain growth that takes place across later childhood and adolescence gives insight into the timeliness of seeking belonging, connection, and meaning, which all are central to spirituality and thriving.

\section{Brain Readiness: Minds and Meaning-Making}

Developmental neuroscience explains how the child and adolescent brain is equipped for and nurtured through the process of making-meaning that is necessary for thriving. The following section overviews the interplay between youths' brain development and social support 
in relation to meaning-making. Broadly speaking, although meaning-making can promote brain and psychosocial development, it also is shaped by brain development, as well as by opportunities for guided reflection in the context of caring and nurturing relationships.

To illustrate, imagine the socially distanced birthday of a 4-year-old girl. Family and friends have brought or mailed their gifts. Some relatives are on a video call as she opens gifts. She excitedly jumps with every toy she unwraps, politely thanking the giver. Next, she opens the box that her aunt sent. Knitted mittens. She is caught by surprise as she was expecting another toy. She sees her aunt's and parents' faces, and understands that a reaction is expected from her; however, she does not know how to respond. Her parents understand her reaction and say: "Oh, she wants you to keep your hands warm this winter, doesn't she?" "Yes, I guess so," she replies, still surprised. "It feels good to receive mittens then, right? It means your aunt cares for you very much," says mom. "Oh yeah, I think so," she says, smiling and a bit more convinced.

What these parents did was as mundane as powerful. Throughout this brief exchange, they helped their daughter build meaning by connecting an unexpected gift with its more abstract, underlying significance. The parents could have merely focused on her behavior ("say 'thanks' to your aunt"). Instead, they guided her in a pattern of thinking that promotes the experience of social emotions such as gratitude, as well as prosocial behaviors in response.

\section{Building Age-Appropriate Narratives}

In the process of meaning-making, children and adolescents build narratives — stories they tell themselves - that connect life events with their beliefs and values. A youth's meaningmaking is influenced by their developmental stage (Merrill \& Fivush, 2016), particularly by their abilities to engage in abstract thinking and experience social emotions. Adults can be supportive of children's and adolescents' abstract meaning-making by providing opportunities for reflection 
and guidance (Immordino-Yang et al., 2019). The newly developing abstract patterns of thinking and feeling may in turn promote neural and mental growth (Riveros \& Immordino-Yang, 2021).

Building abstract life narratives promotes healthy psychosocial development. Life narratives are thought to be organized into more encompassing master narratives about who we are and aspire to be (McAdams, 2021). Abstract narrative meaning-making therefore helps nurture values-oriented thinking and organize youths' transcendent narrative identity (Merrill \& Fivush, 2016). It motivates actions and purposes (McAdams, 2021), as future goals connect with personal values and ideally become intrinsically meaningful over time.

Among many ways in which life narratives and meaning-making are practiced, a particularly well-suited medium for meaning-making can be in warm, safe social relations that afford opportunities for practice (Merrill \& Fivush, 2016). When children and adolescents share narratives with close others, they can elaborate on experienced or imagined events, while the listener can help scaffold, edit, and inspire more abstract interpretation of those events (Palacios et al., 2015). As the capacities for abstract thinking are still maturing during childhood and adolescence, adults' guidance can be fundamental (Immordino-Yang et al., 2019). Conversations with valued and trusted adults can expose children and adolescents to a more values-based, wiser lens of interpreting life events (Grossmann et al., 2010) and can inspire abstract and contextually adaptive meaning-making that allows youth to navigate changing circumstances purposely.

\section{Child and Adolescent Brain Development}

From year 3 onwards, children's capacities for meaning-making start developing from a flexible yet concrete mindset, focused on what is physically observable (Gopnik et al., 2017). Progressively, children's increasing knowledge about others' emotions and psychological state enrich their interpretations (Nelson et al., 2013). Children's proclivities for meaning-making 
become more abstract and values-oriented as their cognitive and social-emotional skills increase (Immordino-Yang et al., 2019). Thus, the progressive advancement in children's meaningmaking is likely to be undergirded by their brain development (Krogsrud et al., 2016).

After the relatively upward trajectory of children's neural and psychological growth, adolescence becomes an inflection point. Initiated by hormonal changes in puberty, adolescents' brains start a "dual" trajectory of development (Suleiman \& Dahl, 2017). On the one hand, there is significant growth in brain areas located underneath the cortex, such as the basal ganglia, which are critically involved in basic motivations (Telzer et al., 2018). Research has found that an injury in the basal ganglia may leave a youth feeling purposeless (Riveros et al., 2019). The initial growth of the basal ganglia is paralleled by an abrupt onset of adolescents' susceptibility towards social and identity-relevant rewards (Suleiman \& Dahl, 2017). This provides adolescents motivation to explore new roles and environments while building values congruent with their identity (Fuligni, 2019). On the other hand, adolescents experience significant growth in multimodal cortical brain areas, and these areas integrate complex cognitive and emotional information, support abstract meaning-making, and facilitate the experience of complex social emotions such as compassion and admiration (Immordino-Yang et al., 2019).

In short, developmental proclivities to process happenings, motivations, and emotions in terms of their broader meaning enable children and adolescents to embed concrete, contextspecific perceptions and actions into abstract narratives that transcend the here-and-now. These proclivities change following region-specific and nonlinear brain developmental trajectories, even as they are also being influenced by social-relational opportunities for connecting reflections with beliefs and values about who adolescents are and aspire to be (Riveros \& Immordino-Yang, 2021). The adolescent's brain is biologically tuned to cognitive and social- 
emotional opportunities for meaning-making (Pfeifer \& Peake, 2012). Opportunities for abstract meaning-making, therefore, have an outsized effect on adolescents' neurobiological growth (Larsen \& Luna, 2018). Those opportunities, when properly guided by a trusted adult, can have a favorable impact on adolescents' development (Immordino-Yang et al., 2019). Attuned adults are well-positioned to scaffold youth in constructing values and emotion-rich representations of perceptions and actions, forming the stage upon which youth can meaningfully engage with R/S.

\section{Religion and Spirituality as Meaning-Making Milieu}

$\mathrm{R} / \mathrm{S}$ have the potential to maximize this critical period of brain development for thriving. As youth mature, they are increasingly biologically oriented to opportunities for building meaning and processing identity-related information. In the following section, we explain how the meaning-making necessary for thriving can occur through the abstract belief systems, transcendent emotions, and caring relationships available through R/S.

\section{Abstract Beliefs}

Religious and spiritual contexts spur the contemplation of abstract ideas, which encourage youth meaning-making. Specifically, beliefs that address issues of identity, belonging, and meaning are especially stimulating for maturing youth. As their cognitive capacities increase, they are naturally enticed to consider immaterial and supernatural notions of God or the divine, contemplate existential questions such as issues of life and death, and reflect on their life in the context of their broader community and world. The brain's development during adolescence helps youth experience abstract reflection as rewarding and stimulating, meeting a maturing young person's needs for clarifying identity and purpose (Pfeifer \& Peake, 2012).

Congregations and youth groups are fruitful environments for the discussion of abstract ideas, helping youth create meaning that contributes to their identity. Furthermore, religion offers 
a coherent ideological belief and value system, which can readily function as a master narrative through which young people can identify, organize, and internalize abstract beliefs (Syed et al., 2020). More often recognized as a resource for adolescent identity, narratives are also important for the construction of childhood self-concept (King \& Boyatzis, 2015; Morris et al., 2011; Syed et al., 2020). Master narratives facilitate internalization of beliefs in a coherent and effective manner, as opposed to a system of unrelated abstract beliefs. In turn, these master narratives lend significance to personal narratives. When youth locate their personal narrative identities within grander religious or spiritual narratives, their own personal stories are "sanctified" and take on deeper, deeper significance as transcendent narrative identities (King et al., 2020; Ratchford et al., Chapter 4, this volume; Schnitker et al., 2019; Syed et al., 2020).

In an increasingly globalized world, youth are frequently exposed to many belief systems, and the cacophony of values and expectations can be overwhelming. Although other contexts such as families, schools, and clubs have implicit values and beliefs, settings that intentionally articulate and promote moral beliefs through active engagement and reflection are rare (for exceptions, see Larson et al., 2020). However, when youth are given the opportunity to engage with these types of abstract ideas and values, thriving may occur. Because thriving involves attitudes and actions that benefit others, the moral beliefs and caring relationships that are usually characteristic of R/S offer opportunities for constructing meaning that can inform youths' values-aligned goals that motivate prosocial behaviors. For example, Catholic students who participated in community service within their Jesuit school and process their service in the context of the life and actions of Jesus Christ may adopt a religious rationale and motivation for their actions. Religious beliefs that address issues of justice, the environment, and human dignity and diversity are particularly salient to adolescents, enabling them to make sense of the world 
and their place in it. Thriving occurs when moral beliefs and emotions are incorporated into the young person's narrative identity and motivate values-aligned behaviors.

\section{Self-Transcendent Emotions}

One way to enhance transcendent beliefs, and therefore increase meaning-making, may be through feeling more self-transcendent emotions. Youth who experience self-transcendent positive emotions are more likely to behave prosocially, a key characteristic of thriving (Yaden et al., 2017). Broaden-and-build theory indicates that positive emotions open our minds, making us more receptive to new ideas and better at solving issues creatively. This helps us build new resources and skills that give us opportunities to experience even more positive emotions, build even more skills, and so on (Fredrickson, 2001). One category of positive emotions, known as self-transcendent positive emotions, also include an awareness and feeling of oneness with other people; this includes emotions such as awe, compassion, love, joy, elevation, and gratitude (Yaden et al., 2017). Feeling self-transcendent emotions decreases self-focus, increases connectedness to others, and it fosters altruistic thinking and prosocial behavior (Yaden et al., 2017). These emotions are even associated with a decrease in materialism and envy, as well as lower rates of depression and higher academic achievement (Froh et al., 2011).

Religious and spiritual contexts encourage self-transcendent emotions (see Van Cappellen et al., Chapter 20, this volume). Many programs, organizations, and institutions (e.g., political parties, scouting) offer ideology and rich social environments, but few promote experiences of transcendence where people perceive and experience a reality beyond themselves (King et al., 2014; Yaden et al., 2021). Religious and spiritual contexts often do this naturally. For instance, people who pray more feel more gratitude (Lambert et al., 2009) and awe, which increase religious and spiritual feelings and behaviors (van Cappellen \& Saroglou, 2012). 
In addition, religious and spiritual practices offer effective means of experiencing selftranscendent emotions. Engaging in practices such as prayer or meditation provides youth an opportunity to experience spiritual, self-transcendent connection with nature and with divine beings or entities (King et al., 2014; Yaden et al., 2021). For example, Eastern traditions often emphasize the growing awareness of an essential unity of all beings and the universe. Many expressions of R/S provide conduits for self-transcendent emotions that can help internalize abstract beliefs into salient commitments that motivate values-based goals conducive to thriving.

\section{Caring Relationships}

Furthermore, R/S provide relationships that support meaning-making through dialogue, modeling, and opportunities for young people to be a part of something beyond themselves. R/S are particularly effective in generating social capital and offering spaces for explicit conversations that support youths' reflection and integration of beliefs and values (Fasoli, 2020; Hardy et al., 2019; King \& Boyatzis, 2015; Larson et al., 2020). Generally, religious traditions have specific processes for mentoring their young, usually involving various forms of teaching, socialization, and connection. For example, within the Hindu religion, gurus are considered to be self-realized masters and often viewed as embodiments of the divine. In some forms of Judaism, sages are important role models of right living and wisdom. In the Christian tradition, youth are often discipled by youth pastors or adult church volunteers.

Intentional mentoring can provide specific teaching but also caring relationships that scaffold the process of meaning-making through dialogue (Fasoli, 2020; Merrill \& Fivush, 2016). Within caring relationships, adults can actively reflect and discuss moral ideals, the practicalities of living them out, and their implications for the broader world. For instance, in the example above with the 4-year-old girl receiving birthday gifts, the adult explicitly connected the 
positive feelings of receiving a gift and the behavior of saying thank you with the belief that thanking someone is a good thing to do because relationships are valued. The parent scaffolded the meaning-making process by connecting the child's emotions and behaviors with a belief that has the potential to be internalized as a value for the child. Young people often learn beliefs and internalize values when others help them make connections between their current behaviors and prospective beliefs. Religious communities can also offer youth opportunities to lead and serve. Dialogue in which adults openly connect the youth's behaviors with moral and societal implications can model how abstract beliefs can be lived out for the benefit of others.

In addition, religious communities provide living and historic examples of how abstract beliefs can be enacted (Erikson, 1968; King \& Boyatzis, 2015; King et al., 2020). Adults can be formative sources of inspiration by being explicit about how their beliefs motivate their actions. For example, adults can explain how their own values helped them overcome challenges and can discuss both the positive and negative emotions and experiences that accompany living out one's convictions. As such, religious communities can provide an important social context with caring adults, where moral ideals can be explored, exemplified, and then embodied by youth.

Unique to $\mathrm{R} / \mathrm{S}$ is the possibility of experiencing a caring perceived relationship with divine beings or entities. Youths' perceived relationship with divine beings can be an important relational developmental system that impacts their meaning-making and sense of identity, while also motivating values-aligned behaviors conducive to thriving. Even adolescents who have had poor attachment relationships with their caregivers will oftentimes develop a security-enhancing perceived attachment relationship with God during adolescence. That surrogate attachment relationship can help them with moral meaning-making and guide and motivate thriving. 


\section{Implications for Clinical and Religious Practitioners}

The research synthesized here indicates multiple application ideas for clinical and religious practitioners. Specifically, practitioners can provide opportunities for youth to talk about abstract ideas, such as hosting discussion groups or book clubs. Additionally, practitioners can provide opportunities for youth to experience transcendent emotions such as awe, gratitude, and elevation. For instance, awe can be induced by having youth spend time in nature, interact with big ideas (e.g., God), or just look at pictures of inspiring scenery and discuss their beauty (Passmore \& Holder, 2016). ${ }^{5}$ Adults can enhance this experience by helping youth connect their thoughts and emotions to their growing ideas of their own identity, life goals, and place in their broader world. For example, many youths aspire to be vegetarian. From the approach put forth in this chapter, dietary commitments tend to be less enduring unless they become internalized by reflecting on how an individual's behavior may impact the world around them. When beliefs and behaviors become an integrated part of a larger master narrative, they take on more significance (Syed et al., 2020). In addition, when abstract ideals, such as "it's bad to eat meat," are connected to actual behaviors (e.g., not eating meat) that have implications for the broader world, youth experience rewarding emotions and thus are more apt to internalize such beliefs as values.

Practitioners can also connect youth to spiritual guides. Spiritual mentoring is an example of a caring relationship in which youth learn about transcendent ideas, practice abstract meaningmaking, experience social emotions, and witness inspiring role models. For instance, religious and spiritual settings provide many resources that offer the opportunity for youth to reflect on transcendent ideas. These ideas could emerge from sacred texts, spiritual writings, and individual

\footnotetext{
${ }^{5}$ For more ideas about activities youth can do to evoke self-transcendent emotions such as awe and gratitude, see Berkeley's Greater Good Science Center website: https://ggia.berkeley.edu/.
} 
and communal practices and then be explored in dialogue with a caring adult. In this way, spiritual mentoring can influence adolescents' thoughts, emotions, and general well-being. Adult relationships are key to supporting youth as they try to make sense of the ordinary and extraordinary in life. Mental health professionals, educators, and other practitioners can support youth development by directing them towards engaging in the religious/spiritual communities in their lives. Although congregations and religious youth groups are usual options, racial/ethnic communities and extended families should also be considered as resources for spiritual mentors, beliefs, and practices. For future directions in this area, see Table 17.S1 in Appendix 17.S1.

\section{Conclusion}

In this chapter, we aimed to explain the link between meaning-making, R/S, and thriving in youth development. Synthesizing existing research, we suggest that spirituality is seminal to thriving because the cognitive and emotional stimulation of transcendence spurs on otheroriented meaning-making that results in a transcendent narrative identity, values-aligned goals, and prosocial behaviors necessary for thriving. Whereas adolescents are increasingly motivated to engage with the kind of abstract ideas, transcendent emotions, and caring relationships that are suited for meaning-making, children also benefit from more relational scaffolding that can help them internalize values. R/S not only provide these socio-affectively rich opportunities to construct meaning and internalize values, but they generally also offer beyond-the-self beliefs and norms. The research reviewed in this chapter is intended to aid both researchers and practitioners in encouraging meaning-making as a way to promote positive spiritual and religious development and thriving. 


\section{References}

Balswick, J. O., King, P. E., \& Reimer, K. S. (2016). The reciprocating self: Human development in theological perspective. InterVarsity Press.

Damon, W., Menon, J., \& Bronk, K. C. (2003). The development of purpose during adolescence. Applied Developmental Science, 7(3), 119-128.

Erikson, E. H. (1968). Identity: Youth and crisis. W. W. Norton.

Fasoli, A. D. (2020). Conversations and moral development. L.A. Jensen (Ed.), Oxford handbook of moral development (pp. 482-500). Oxford University Press.

Fredrickson, B. L. (2001). The role of positive emotions in positive psychology: The broadenand-build theory of positive emotions. American Psychologist, 56(3), 218-226. http://dx.doi.org/10.1037/0003-066X.56.3.218

Froh, J. J., Emmons, R. A., Card, N. A., Bono, G., \& Wilson, J. A. (2011). Gratitude and the reduced costs of materialism in adolescents. Journal of Happiness Studies, 12(2), 289302. https://doi.org/10.1007/s10902-010-9195-9

Fuligni, A. J. (2019). The need to contribute during adolescence. Perspectives on Psychological Science, 14(3), 331-343. https://doi.org/10.1177/1745691618805437

Furrow, J. L., King, P. E., \& White, K. (2004). Religion and positive youth development: Identity, meaning, and prosocial concerns. Applied Developmental Science, 8(1), 17-26. https://doi.org/10.1207/S1532480XADS0801_3

Gopnik, A., O’Grady, S., Lucas, C., Griffiths, T., Wente, A., . . \& Dahl, R. (2017). Changes in cognitive flexibility and hypothesis search across human life history from childhood to adolescence to adulthood. Proceedings of the National Academy of Sciences, 114(30), 7892-7899. https://doi.org/10.1073/pnas.1700811114 
Grossmann, I., Na, J., Varnum, M. E. W., Park, D. C., Kitayama, S., \& Nisbett, R. E. (2010). Reasoning about social conflicts improves into old age. Proceedings of the National Academy of Sciences, 107(16), 7246-7250. https://doi.org/10.1073/pnas.1001715107

Hardy, S., Nelson, J., Moore, J. \& King, P. E. (2019). Processes of religious and spiritual influence in adolescence: A review of the literature, Journal of Research on Adolescence, 29(2), 254-275. https://doi.org/10.1111/jora.12486

Immordino-Yang, M., Darling-Hammond, L., \& Krone, C. (2019). Nurturing nature: How brain development is inherently social and emotional, and what this means for education. Educational Psychologist, 54, 185-204. https://doi.org/10.1080/00461520.2019.1633924

King, P. E., \& Boyatzis, C. J. (2015). Religious and spiritual development. In M. E. Lamb \& R. M. Lerner (Eds.), Handbook of child psychology and developmental science: Socioemotional processes (Vol. 3, pp. 1-48). Wiley.

King, P. E., Clardy, C. E., \& Ramos, J. S. (2014). Adolescent spiritual exemplars: Exploring spirituality in the lives of diverse youth. Journal of Adolescent Research, 29(2), 186-212. https://doi.org/10.1177/0743558413502534

King, P. E., Hardy, S., \& Noe, S. (2021). Developmental perspectives on adolescent religious and spiritual development. Adolescent Research Review. Advance online publication. https://doi.org/10.1007/s40894-021-00159-0

King, P. E., \& Mangan, S. (in press). Hindsight in 2020: Looking back and forward to PYD and thriving. APA handbook of adolescent and young adult development.

King, P. E., Schnitker, S. A., \& Houltberg, B. (2020). Religious groups and institutions as a context for moral development: Religion as fertile ground. In L. Jensen (Ed.), Handbook of moral development (pp. 592-612). Oxford University Press. 
Krogsrud, S. K., Fjell, A. M., Tamnes, C. K., Grydeland, H., Mork, L., ... \& Walhovd, K. B. (2016). Changes in white matter microstructure in the developing brain: A longitudinal diffusion tensor imaging study of children from 4 to 11 years of age. NeuroImage, 124, 473-486. https://doi.org/10.1016/j.neuroimage.2015.09.017

Lambert, N. M., Fincham, F. D., Braithwaite, S. R., Graham, S. M., \& Beach, S. R. (2009). Can prayer increase gratitude? Psychology of Religion and Spirituality, 1(3), 139-149. https://doi.org/10.1037/a0016731

Larsen, B., \& Luna, B. (2018). Adolescence as a neurobiological critical period for the development of higher-order cognition. Neuroscience \& Biobehavioral Reviews, 94, 179195. https://doi.org/10.1016/j.neubiorev.2018.09.005

Larson, R. W., Walker, K. C., \& McGovern, G. (2020). Youth programs as contexts for development of ethical judgment and action. L.A. Jensen (Ed.), Oxford handbook of moral development (pp. 552-569). Oxford University Press.

Lerner, R. M. (2018). Concepts and theories of human development $\left(4^{\text {th }}\right.$ ed.). Routledge.

Lerner, R. M., Dowling, E. M., \& Anderson, P. M. (2003). Positive youth development: Thriving as the basis of personhood and civil society. Applied Developmental Science, 7(3), 172180. https://doi.org/10.1207/S1532480XADS0703_8

McAdams, D. P. (2021). Narrative identity in the life story. In O. P. John \& R. W. Robins (Eds), Handbook of personality (4 ${ }^{\text {th }}$ ed. pp. 122-141). Guilford Press.

Merrill, N., \& Fivush, R. (2016). Intergenerational narratives and identity across development. Developmental Review, 40, 72-92. https://doi.org/10.1016/j.dr.2016.03.001

Morris, A. S., Eisenberg, N., \& Houltberg, B. J. (2011). Adolescent moral development. In B. Brown \& M. Prinstein (Eds.), Encyclopedia of adolescence (pp. 48-55). Academic Press. 
Nelson, J. A., de Lucca Freitas, L. B., O’Brien, M., Calkins, S. D., Leerkes, E. M., \& Marcovitch, S. (2013). Preschool-aged children's understanding of gratitude: Relations with emotion and mental state knowledge. British Journal of Developmental Psychology, 31(1), 42-56. https://doi.org/10.1111/j.2044-835X.2012.02077.x

Overton, W. F. (2015). Processes, relations, and relational-developmental-systems. In W. F. Overton, P. C. M. Molenaar, \& R. M. Lerner (Eds.), Handbook of child psychology and developmental science: Theory and method (Vol. 1, pp. 1-54). Wiley.

Palacios, J., Salem, B., Hodge, F., Albarrán, C., Anaebere, A., \& Hayes-Bautista, T. (2015). Storytelling: A qualitative tool to promote health among vulnerable populations. Journal of Transcultural Nursing, 26, 346-353. https://doiorg.ccl.idm.oclc.org/10.1177/1043659614524253

Park, C. L. (2010). Making sense of the meaning literature: An integrative review of meaningmaking and its effects on adjustment to stressful life events. Psychological Bulletin, 136(2), 257-301. https://doi.org/10.1037/a0018301

Passmore, H. A., \& Holder, M. D. (2016). Noticing nature: Individual and social benefits of a two-week intervention. The Journal of Positive Psychology, 12(6), 537-546. https://doi.org/10.1080/17439760.2016.1221126

Pfeifer, J. H., \& Peake, S. J. (2012). Self-development: Integrating cognitive, socioemotional, and neuroimaging perspectives. Developmental Cognitive Neuroscience, 2(1), 55-69. https://doi.org/10.1016/j.dcn.2011.07.012

Riveros, R., Bakchine, S., Pillon, B., Poupon, F., Miranda, M., \& Slachevsky, A. (2019). Frontosubcortical circuits for cognition and motivation. Frontiers in Psychology, 9, Article 2781. https://doi.org/10.3389/fpsyg.2018.02781 
Riveros, R., \& Immordino-Yang, M. H. (2021). Toward a neuropsychology of spiritual development in adolescence. Adolescent Research Review, 6, 323-333. https://doi.org/10.1007/s40894-021- 00158-1

Schnitker, S. A., King, P. E., \& Houltberg, B. (2019). Religion, spirituality, and thriving: Transcendent narrative, virtue, and telos. Journal of Research on Adolescence, 29(2), 276-290. https://doi.org/10.1111/jora.12443

Suleiman, A., \& Dahl, R. (2017). Leveraging neuroscience to inform adolescent health:. Journal of Adolescent Health, 60, 240-248. https://doi.org/10.1016/j.jadohealth.2016.12.010

Syed, M., Pasupathi, M., \& McLean, K. (2020). Master narratives, ethics, and morality. In L. Jensen (Ed.), Oxford handbook of moral development (pp. 500-515). OUP.

Telzer, E. H., van Hoorn, J., Rogers, C. R., \& Do, K. T. (2018). Social influence on positive youth development: A developmental neuroscience perspective. Advances in Child Development and Behavior, 54, 215-258. https://doi.org/10.1016/bs.acdb.2017.10.003

Van Cappellen, P., \& Saroglou, V. (2012). Awe activates religious and spiritual feelings and behavioral intentions. Psychology of Religion and Spirituality, 4(3), 223-236. https://doi.org/10.1037/a0025986

Yaden, D. B., Haidt, J., Hood Jr, R. W., Vago, D. R., \& Newberg, A. B. (2017). The varieties of self-transcendent experience. Review of General Psychology, 21(2), 143-160. http://dx.doi.org/10.1037/gpr0000102

Yaden, D. B., Giorgi, S., Kern, M. L., Adler, A., Ungar, L. H., Seligman, M. E., \& Eichstaedt, J. C. (2021). Beyond beliefs: Multidimensional aspects of religion and spirituality in language. Psychology of Religion and Spirituality. https://doi.org/10.1037/rel0000408 


\title{
Chapter 18
}

\section{Religious/Spiritual Development and Positive Psychology: Toward an Integrative Theory}

\author{
Edward B. Davis ${ }^{1}$, James M. Day ${ }^{2}$, Philip A. Lindia ${ }^{3}$, and Austin W. Lemke ${ }^{1}$ \\ ${ }^{1}$ School of Psychology, Counseling, and Family Therapy, Wheaton College \\ ${ }^{2}$ Psychological Sciences Research Institute, Universite Catholique de Louvain \\ ${ }^{3}$ School of Biblical \& Theological Studies, Wheaton College
}

\begin{abstract}
Author Note
We have no known conflicts of interest to disclose.

The authors wish to thank Sarah A. Schnitker, Everett L. Worthington, Jr., Pamela E.
\end{abstract}

King, Seyma N. Saritoprak, Kamlesh Singh, Mahima Raina, and Doug Oman for their very helpful feedback on this chapter. We are grateful for how you helped strengthen it.

This publication was made possible through the support of Grant 61865 from the John Templeton Foundation. The opinions expressed in this publication are those of the authors and do not necessarily reflect the views of the John Templeton Foundation.

Correspondence concerning this chapter should be addressed to Edward B. Davis, Wheaton College, 501 College Ave, Wheaton, IL 60187. Email: ward.davis@ wheaton.edu.

\section{Citation:}

Davis, E. B., Day, J. M., Lindia, P. A., \& Lemke, A. W. (in press). Religious/spiritual development and positive psychology: Toward an integrative theory. In E. B. Davis, E. L. Worthington, Jr., \& S. A. Schnitker (Eds.), Handbook of positive psychology, religion, and spirituality. Springer Nature. 


\begin{abstract}
Throughout the history of psychology, scholars and practitioners have sought to understand religious/spiritual (R/S) development and its intersections with well-being. Several models of R/S development have been proposed, but they have neither been well-integrated with each other nor studied and applied broadly in the field of positive psychology. This chapter's purpose is to draw on existing longitudinal research on $\mathrm{R} / \mathrm{S}$ development to propose an integrative theory for guiding developmental science and practice on religion, spirituality, and positive psychology. This Positive Religious and Spiritual Development (PRSD) theory posits that people's religiousness/spirituality (a) is motivated by goals designed to meet psychological needs (e.g., for acceptance, predictability, and competence); (b) consists of mental/neural representations (stored beliefs, emotions, action tendencies, and physiological responses) and R/S habits that develop and change through relational experiences at the micro-, meso-, and macrolevels; (c) is influenced by numerous contextual factors (e.g., age, sex/gender, culture, and faith tradition), including personal and sociocultural assets and liabilities (risk and resilience factors); and (d) interacts bidirectionally with people's holistic well-being via psychological, social, behavioral, and physical pathways. We offer illustrative examples of PRSD theory, highlight some of its caveats and limitations, and discuss applications for clinical practice and religious ministry. Keywords: religion, spirituality, development, positive psychology, theory
\end{abstract}




\section{Chapter 18}

\section{Religious/Spiritual Development and Positive Psychology: Toward an Integrative Theory}

It was a brisk California evening at Stanford University. With its world-renowned scientific reputation and close ties to neighboring Silicon Valley, this might be the last place you would expect students to have a deeply religious/spiritual (R/S) experience. But they did. They had come to hear the 2015 "Last Lecture on a Meaningful Life," delivered annually by a globally impactful luminary. This year's speaker was Oprah Winfrey, world-famous TV host, producer, actress, and philanthropist. After sharing about her own R/S journey, Winfrey (2015) exhorted:

Let every step you take move you in the direction of the one thing all religions can agree on-love... I'm not telling you what to believe, or who to believe, or what to call it, but there is no full life, no fulfilled, meaningful, sustainably joyful life without a connection to the spirit. I haven't seen it happen. And the way to sustainability is through practice. You must have a spiritual practice. What is yours? For some people it is going to church... [for others, it is] prayer, conscious kindness, empathy, consistent compassion, gratitude. All [are] spiritual practices [that help you in] becoming more of who you are.

\section{Chapter Purpose and Overview}

Winfrey’s advice echoes a refrain sung across cultures for millennia—religion/spirituality often plays a meaningful role in human development and flourishing (Day, 2010; VanderWeele, 2017). Within the field of psychology, scientists and practitioners have spent over a century exploring how people's religiousness/spirituality develops across their lifespan (Day, 2010) and interacts with their well-being (Koenig et al., 2012). Many theories of R/S development have been proposed (Friedman et al., 2012; King \& Boyatzis, 2015), but they have neither been wellintegrated with each other nor studied and applied broadly in the field of positive psychology. 
This chapter's purpose is to draw on existing longitudinal research on R/S development to propose an integrative theory for guiding developmental science and practice on religion, spirituality, and positive psychology. We begin by reviewing and proposing key definitions. Second, we summarize limitations of existing R/S development theories. Next, we conduct a scoping review of extant longitudinal studies of R/S development, which reveals reasons why an integrative theory is needed. Then we introduce our Positive Religious and Spiritual Development (PRSD) theory, including illustrative examples and discussion of its caveats and limitations. Finally, we describe a few applications for clinical practice and religious ministry.

\section{Conceptual Definitions of Key Terms}

Drawing on the work of Pargament et al. (2013), Harris et al. (2018), Mahoney et al. (2021), and George and Park (2016), we propose the following two integrative definitions of spirituality and religiousness. Spirituality refers to people's search for meaning and connection with whatever they perceive as sacred, typically including supernatural entities (e.g., deity/deities, saints, ancestors, karma, or fate/destiny) or aspects of life viewed as a manifestation of the divine (e.g., close human relationships) or as having transcendent or divine-like qualities (e.g., nature or the universe). Religiousness refers to people's search for sacred meaning (sense of transcendent significance, purpose, and coherence) and connection in the context of culturally sanctioned codifications (e.g., of beliefs, values, and morals), rituals (e.g., prayer, meditation, and collective worship), and institutions (e.g., families, faith communities, schools, and organizations). Because development is "the progressive series of changes in structure, function, and behavior patterns that occur over the lifespan of a human being or other organism" (VandenBos, 2015, p. 304), R/S development refers to progressive changes in the structure, function, and patterns that characterize people's search for sacred meaning and connection. 
Developmental theories often differentiate between a developmental factor (a variable or condition that influences development), developmental function (the role an entity, action, phase, or other phenomenon plays in development), and developmental sequence (order of changes in structure or function). Most contemporary developmental theories adopt a developmental systems framework (Overton, 2010), viewing development as "the result of bidirectional interaction between all levels of biological and experiential variables" (VandenBos, 2015, p. 305).

\section{Limitations of Existing R/S Development Theories}

Many R/S development theories already exist, and most fall into three categories: stagestructural (usually cognitively focused) theories, motivational theories, and relationally focused theories. Appendix 18.S1 contains summaries of some major theories from these categories.

Naturally, each existing category of R/S development theories has limitations. For example, stage-structural theories are often critiqued because they tend to (a) focus too narrowly on individuals and on cognitions (and not enough on motivations, emotions, relationships, and ecologies); (b) underplay contextual and cultural influences on R/S development; (c) assume people develop in a normative (universal), discontinuous (discretely stage-like), and linear (sequential) way; (d) undervalue the unique nuances and complexities embedded in people's R/S expressions; (e) understate the wide variation of R/S expressions at any one age; (f) imply children are limited to immature forms of religiousness/spirituality; and (g) use constructs that are difficult to operationalize concretely and apply cross-culturally (King \& Boyatzis, 2015).

The other categories of R/S development theories seek to address several of these limitations. For instance, motivational theories such as Pargament's (2013) theory of spirituality focus on explaining why people are R/S. Pargament (2013) argues people are R/S because they are motivated to discover, conserve, and occasionally transform their relationship with "the 
sacred" (p. 257), especially when coping with stress or loss. Pargament's (2013) theory of spirituality also helps explain how religiousness/spirituality interacts with people's well-being. He argues that integration (i.e., "the linkage of differentiated elements," Siegel, 2020, p. 461) is the marker and mechanism of healthy religiousness/spirituality. Specifically, Pargament (2013) posits that healthy religiousness/spirituality is marked and facilitated by R/S destinations (goals) and pathways (habitual ways of meeting goals) that "work together in synchrony [and are] marked by breadth and depth, responsivity to life situations, flexibility and continuity, and a concept of the sacred that is large enough to encompass the full range of human potential and luminous enough to provide the individual with a powerful guiding vision" (p. 267).

Even so, like the stage-structural theories, Pargament's (2013) theory of spirituality can be criticized for being overly individualistic, underemphasizing the roles of context and culture in R/S development, and failing to explain how a person's R/S goals and habits develop and change through multilevel relational experiences with their ecologies. Some relationally focused theories such as positive youth development theory (Benson et al., 2006; Lerner et al., 2015) and reciprocating spirituality theory (King \& Boyatzis, 2015; King et al., Chapter 17, this volume) have sought to address these limitations. Yet these relational developmental systems theories typically have focused on youth and in some cases only address R/S development peripherally.

\section{Drawing on Existing Longitudinal Research to Develop an Integrative Theory}

With all these limitations in mind, we wondered if there was a need for an overarching framework that could capitalize on the strengths of existing theories while simultaneously addressing their limitations. The extant empirical literature needed to guide this effort so that any theory we developed was both scientifically grounded and truly needed. We focused only on longitudinal studies (to see how R/S development occurs across time) and quantitative studies 
(due to their large-sampled findings). On May 28, 2021, we conducted a PsycINFO search using the terms "(religious development) OR (spiritual development) OR (faith development)" and the limiters peer-reviewed, empirical study, longitudinal study, and quantitative study. This strategy yielded 345 hits. We screened out around half these articles (170, 49.3\%) because they: (a) were a test-development or intervention study, (b) were misclassified as longitudinal, or (c) included religion/spirituality as only a very peripherally relevant variable. Ultimately, we retained 175 longitudinal studies, and those studies' details and findings are summarized in Appendix 18.S2.

Generally speaking, religiousness/spirituality and its dimensions (e.g., R/S attendance, importance, practices) influenced people's development positively — that is, R/S developmental factors usually led to better psychological, social, behavioral, and physical well-being. These effects were evident across cultures, contexts, and developmental periods. The positive developmental functions of religiousness/spirituality (and its dimensions) were compatible with Koenig et al.’s (2012) causal-pathways theory. That is, religiousness/spirituality positively affected well-being and development via four pathways: psychological (emotional, cognitive, and motivational) processes, social (relational) processes, behavioral processes, and physical (physiological) processes. Also, consistent with a developmental systems framework (King et al., 2021), there was bidirectional interaction across all systemic levels, including people's physical body (genetics and physiology), mental activity (thoughts, emotions, motivations, and identities), behaviors, relationships (with parents, peers, partners, and perceived deities), and sociocultural contexts (their school, community, faith tradition, and culture; Pargament et al., 2013).

To see whether an integrative R/S development theory was needed (and if so, what to include in it), we created Appendix 18.S3, which records (a) if each study assessed multiple R/S dimensions, (b) if contextual influences (moderators) of R/S development were identified, (c) if 
the interaction between religiousness/spirituality and well-being was studied, and (d) what theory or theories were explicitly or implicitly used to test hypotheses related to R/S development. As can be seen, 103 of the 175 studies (58.9\%) measured multiple R/S dimensions; the most often assessed were R/S attendance (public R/S practices; $k=102,58.3 \%$ ), R/S importance ( $k=49$, $28.0 \%$ ), R/S affiliation ( $k=35,20.0 \%$ ), and private $\mathrm{R} / \mathrm{S}$ practices (e.g., prayer frequency; $k=33$, 18.9\%). One-hundred twenty-five studies (71.4\%) identified contextual influences on R/S development. Over 90 moderators were identified; the most frequently identified were age $(k=$ 42), family contextual influences $(k=39)$, sex/gender $(k=20)$, faith tradition (e.g., R/S affiliation; $k=15)$, peer contextual influences $(k=12)$, and adverse life events $(k=12)$. Most studies ( $k=146,83.4 \%)$ examined the relationship between $\mathrm{R} / \mathrm{S}$ development and well-being.

The most pertinent finding of this review was that 112 distinct theories were used to guide the conceptual frameworks of the 175 studies. Nearly $80 \%$ of those theories were used to guide only one study of R/S development. Only five theories were used more than four times: parent religious socialization theory, Pargament's (2013) theory of spirituality, positive youth development theory (Benson et al., 2006), Erikson's (1963) psychosocial stage theory, and Big 5 personality theory. Roughly one-third of the reviewed studies $(k=58,33.1 \%)$ were atheoretical.

\section{Positive Religious and Spiritual Development (PRSD) Theory}

It was clear the existing psychological literature on $\mathrm{R} / \mathrm{S}$ development was characterized by a low degree of integration. The over 100 utilized theories were neither well-integrated with each other nor well-used in guiding developmental science on R/S development. Also, although the association between R/S development and well-being was examined in over $80 \%$ of the reviewed studies, none were published in a positive psychology journal (e.g., Journal of Positive Psychology or Journal of Happiness Studies), and very few utilized positive psychology theories 
(e.g., PERMA well-being theory; Seligman, 2011). In sum, existing theories and research on R/S development were not well-integrated, either internally or externally (with positive psychology).

Hence, we concluded an integrative theory of R/S development was needed. We sought to develop a theory that applied across the lifespan and across cultures, contexts, and forms of religiousness/spirituality. We wanted to build on existing theory and research as much as possible, so that the theory's conceptual and scientific foundations were robust. We wanted it to have practical relevance across disciplines and subdisciplines, especially mainstream psychology and positive psychology. Thus, we wanted the connections between people's R/S development and holistic well-being to be a prominent focus. However, like the theory of positive youth development (Benson et al., 2006; Lerner et al., 2015), we wanted this to be a strength-based theory, focusing on positive trajectories of R/S development. Ultimately, we chose to name the model Positive Religious and Spiritual Development (PRSD) theory (see Figure 18.1).

To reiterate, $R / S$ development refers to progressive changes in the structure, function, and patterns that characterize people's search for and response to sacred meaning and connection. As a lifespan developmental theory, PRSD theory focuses on "systematic intraindividual changesfrom [at or near birth] to the end of life_ of [a person's] behavior, and of the systems and processes underlying those changes and that behavior" (Overton, 2010, p. 4). It focuses especially on positive $R / S$ development, which is defined as R/S development that is contextually adaptive across time and situations, where contextual adaptiveness refers to "individual-context relations benefiting both the person and his or her ecology" (Lerner et al., 2015, p. 608).

\section{Mental/Neural Representations and R/S Habits: What Religiousness/Spirituality Is}

Consistent with the interdisciplinary metatheory of interpersonal neurobiology (Siegel, 2020), PRSD theory views all human experiences — including religiousness/spirituality — as 
emerging from interaction among the mind ("an embodied and relational process that regulates the flow of energy and information", Siegel, 2020, p. 5), embodied brain (entire distributed nervous system - the physical mechanism of energy and information flow), and relationships (how energy and information is shared between entities). In particular, PRSD theory is based on the recognition people's life experiences (a) lead their mind to develop mental representations (stored $\underline{\text { beliefs, }}$ emotions, and a action tendencies ["BEATs," Dweck, 2021, p. 90] that carry symbolic meaning psychologically) and (b) lead their embodied brain to develop neural representations (patterns of neural firing that carry symbolic meaning physically). These mental/neural representations guide all human experience and behavior (Siegel, 2020), forming the psychological and physical structures upon which one's religiousness/spirituality functions.

Because the mind is a self-organizing system (Overton, 2010; Siegel, 2020), people develop $R / S$ habits — patterns of thinking, feeling, relating, and behaving that characterize a person's search for sacred meaning and connection across time and situations. These R/S habits include behavioral R/S habits (e.g., private and public R/S practices, such as prayer and R/S attendance) and psychosocial R/S habits, which can be further categorized into cognitively focused R/S habits (e.g., R/S beliefs, values, orientations, and meaning) and socioemotionally focused R/S habits (e.g., R/S identity, affiliation, emotions, and relationships). This conceptualization of R/S habits into behavioral, cognitive, and socioemotional dimensions is compatible with Saroglou's (2011) Big Four theory, which identifies four basic dimensions of religiousness/spirituality: behaving, believing, belonging, and bonding. Notably, large-scale cross-cultural research has found evidence these R/S dimensions are universally present and functionally equivalent across cultures and faith traditions, although they vary in interrelatedness, relative salience, and modes of expression (Saroglou et al., 2020). 
Taken together, mental/representations and R/S habits form the structure of a person's religiousness/spirituality. Next, we explore how these mental/representations and R/S habits develop, function, and change, based on people's needs, goals, and relational experiences.

\section{Needs, Goals, and Relationships: The Why and How of Religiousness/Spirituality}

Consistent with Dweck's (2021) motivational personality theory, PRSD theory assumes religiousness/spirituality is universally motivated by goals designed to meet psychological needs. Motivations are the psychological (mental) and physiological (neural) processes that energize and direct behavior (Reeve, 2017). Needs are what play the energizing (driving) function of religiousness/spirituality, need-related goals are what play the directive function, mental/neural representations (BEATs) are the latent structures comprising people's religiousness/spirituality, and need-driven R/S habits are its more manifest structural elements (Figure 18.1; Dweck, 2021).

Although people certainly are motivated by physiological needs (e.g., thirst, hunger), because PRSD theory is a psychological theory, it centers on psychological needs that motivate people's search for sacred meaning and connection. Drawing on Dweck's (2021) motivational personality theory, PRSD theory assumes there are three basic needs that are present at or near birth (acceptance, predictability, and competence) and four later-emerging compound needs that are formed from combinations of the basic needs - trust (acceptance and predictability), control (predictability and competence), self-esteem/status (acceptance and competence), and self-

coherence (acceptance, predictability, and competence). A need is a mental or physical condition that is universally valued and essential for well-being, and a basic need must additionally be irreducible to other needs and apparent at or near birth (Dweck, 2021). In PRSD theory, needs for acceptance, trust, and self-esteem/status tend to drive socioemotional R/S habits; needs for predictability, trust, and control tend to drive cognitively focused R/S habits; and needs for 
competence, control, and self-esteem/status tend to drive behavioral R/S habits (Dweck, 2021;

Saroglou, 2011). The need for self-coherence (the nexus of all the needs) drives all three types of R/S habits, as people's minds strive toward optimal complexity, homeostatic equilibrium (balance), and maximal integration (linking of differentiated parts; Overton, 2010; Siegel, 2020).

But how do people's R/S habits develop and change across the lifespan? Consistent with relational developmental systems metatheory (King et al., 2021; Overton, 2010), PRSD posits that the structural building blocks of people's religiousness/spirituality (mental/neural representations and R/S habits) develop and change through bidirectional interaction between a person and multiple levels of their dynamic social-cultural contexts. Representations and habits are specifically formed and transformed through goal-pursuing relational experiences between people and their surrounding social ecologies. This includes experiences at the microlevel (with individual entities, such as family members, friends, romantic partners, and perceived supernatural entities), mesolevel (with groups and institutions, such as a peer group, school, neighborhood, and faith community), and macrolevel (with large-scale systems, such as culture, faith tradition, geographic region, and available media). A person's pursuit of need-fulfilling goals at various relational levels leads them to develop mental/neural representations of what has happened in prior goal-relevant pursuits. Those representations guide the selection and pursuit of future goals, and their recurrent use leads to the development of habits - characteristic patterns of responding cognitively, emotionally, relationally, behaviorally, and physiologically. These habits can be R/S or non-R/S (involved vs. uninvolved in searching for sacred meaning or connection). This dynamic is bidirectional in that people's habits guide their relational experiences, which in turn can lead to change in representations (Dweck, 2021; Siegel, 2020).

Personal and Sociocultural Factors (Moderators): Contextual Influences on R/S Development 
All developmental processes and outcomes-including developmental change itself-are influenced by a wide array of contextual developmental factors called moderators. Within PRSD theory, there are three main categories of these contextual moderators: (a) personal/internal assets and liabilities (physical and psychological resilience and risk factors, such as genetic and psychological strengths and vulnerabilities); (b) sociocultural/external assets and liabilities (social and cultural resilience and risk factors, such as interpersonal, community, and cultural strengths and vulnerabilities); and (c) other sociocultural factors, such as a person's age, sex, gender identity, sexual identity, race/ethnicity, socioeconomic status, disability status, adverse life experiences, generational cohort, and cultural context (local or national; Lerner et al., 2015). Causal Pathways and Integration: How Religiousness/Spirituality Interacts with Well-Being Consistent with causal-pathways theory (Koenig et al., 2012), PRSD theory assumes a person's religiousness/spirituality bidirectionally influences their well-being via psychological (cognitive, emotional, and motivational), social, behavioral, and physical (physiological) pathways. PRSD theory is also compatible with positive psychological theories of well-being. Resonant with VanderWeele's (2017) flourishing theory, it views people's holistic well-being (flourishing) as comprised of five interrelated facets: physical well-being (physiological flourishing), mental well-being (psychological flourishing), social well-being (relational flourishing), character well-being (virtuous flourishing), and R/S well-being (transcendental flourishing). The mental and social facets are compatible with Seligman's (2011) PERMA

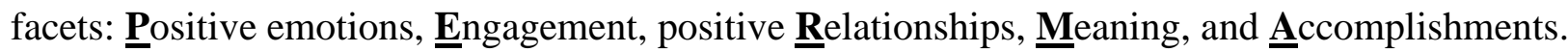
Finally, resonant with interpersonal neurobiology metatheory (Siegel, 2020), PRSD theory posits that contextually adaptive internal and external integration are both the structural markers and functional mechanisms of positive R/S development (Davis et al., 2021). To 
reiterate, contextual adaptiveness refers to person-context linkages that benefit the person and their surrounding multilevel context (Lerner et al., 2015). Internal integration refers to contextually adaptive linkage among a person's mental representations, neural representations, R/S habits, and non-R/S habits. External integration refers to contextually adaptive linkage between a person's religiousness/spirituality (representations and R/S habits) and context.

\section{Three Fictitious Examples Illustrating How PRSD Theory Works}

Childhood. Maria (a 9-year-old girl who lives in a favela in Rio de Janeiro, Brazil) was born in a highly R/S and Roman Catholic Christian family. Although socioeconomically poor (sociocultural risk factor), Maria's family is very close and happy (sociocultural resilience factor). Throughout her life, Maria has experienced consistently loving and supportive relationships with her parents, five siblings, extended family members, neighbors, and peers (relational experiences). As a result, she has developed positive mental representations (BEATs)—stored positive beliefs about herself, others, and the world ("I am loved, accepted, and competent; other people are good, responsive, and trustworthy; and the world around me is good, predictable, and full of opportunities"); stored representations of recurrently activated positive

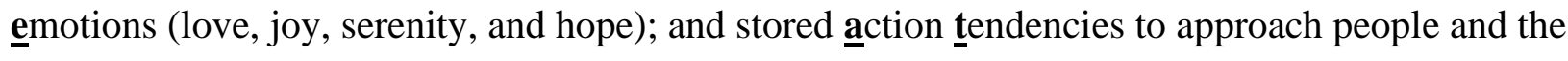
world with curiosity, confidence, and love (Siegel, 2020). These positive mental representations underlie her secure attachment style and high trait agreeableness and optimism (personal assets).

Maria's mental representations (and their associated neural representations) have also been shaped by religious socialization from Maria's parents, extended family members, local community, and Brazilian culture (which is very religious and predominantly Catholic; Pew Research Center, 2018). Through this socialization (multilevel relational experiences), Maria has developed mental representations that God and the saints are benevolent, powerful, and 
responsive and that others, the world, and herself are loved and cared for by God and the saints. She has developed a corresponding set of R/S beliefs (cognitively focused R/S habits) that help her make sense of her thoughts, emotions, and experiences (e.g., "God is powerful and cares about my family, community, and me"; "God always takes care of us"; and "God loves other people, so I should too"). Furthermore, Maria and her family attend weekly Sunday mass at their neighborhood's largest church, she participates in the church's children's group, and Maria prays whenever she feels stressed or uncertain (behavioral and socioemotionally focused R/S habits).

In sum, across time and situations, Maria's R/S habits meet her psychological needs and benefit both Maria and her relational context (e.g., family, church, and neighborhood; contextual adaptiveness). They contribute to Maria's holistic well-being (mental, physical, social, virtuous, and transcendental well-being) by providing a sense of meaning (coherence, purpose, and significance; psychological pathway), relational connection (with family, church members, neighbors, God, and the saints; social pathway), moral behavioral guidance (to love others; behavioral pathway), and physiological serenity (homeostatic equilibrium; physical pathway).

Adulthood. Mahmoud (a 45-year-old, Sunni Muslim man) lives in Cairo, Egypt with his wife and their five children. Growing up, Mahmoud had close and loving relationships with his parents and relatives (sociocultural resilience factor), which helped him develop positive mental representations (BEATs) of himself, others, the world, and Allah. From an early age, Mahmoud has had R/S beliefs (cognitively focused R/S habits) that help fulfill his needs for predictability, trust, and control (e.g., the shahadah - the belief "there is no god but Allah, and Muhammad is the messenger of Allah"). His public and private R/S practices (behavioral R/S habits; e.g., salah prayers five times a day, zakat yearly almsgiving, syiam fasting during Ramadan, and frequent mosque service attendance) have consistently helped fulfill his needs for competence, control, 
and self-esteem/status. Also, his strong R/S identity and positive R/S relationships with family members (wife, children, and relatives), mosque members, and Allah (socioemotionally focused $R / S$ habits) have helped fulfill his needs for acceptance, self-esteem/status, and self-coherence.

Since young adulthood, Mahmoud has owned a prosperous restaurant in a busy part of Cairo. His wife and children help him operate the restaurant. He and his family are known for their R/S devotion and their kindness, joy, and generosity. Two Muslim values guide their business decisions and daily life_-tawakkul (relying on and trusting in Allah) and shukr (gratitude). Those values lead his family to give generously to others in need (e.g., community and mosque members). Mahmoud's psychosocial and behavioral R/S habits have consistently promoted his holistic well-being via psychological pathways (meaning and purpose), behavioral pathways (moral and virtuous behaviors), and social pathways (identity and social support).

Older adulthood. Anika is a 65-year-old, married, Hindu woman who lives in rural India. She and her husband have six children, the last of whom just married. Throughout middle adulthood, Anika focused on fulfilling her duties and responsibilities to her extended family (e.g., her husband, children, parents [before their deaths], and eventually grandchildren). She pursued fulfilling these worldly duties in a wholehearted but dispassionate way, by cultivating habits of niṣkāma karma (i.e., dispassionate action; behavioral R/S habit) and anasakti (i.e., an attitude of nonattachment to events, experiences, and results; cognitively focused $R / S$ habit). She engaged in several other practices as well, including behavioral R/S habits of aștānga yoga (eight-step yoga), bhajans and kïrtans (communal religious chanting and singing), and mantra japa (mantra repetitions throughout the day). Through these and other practices, Anika gradually achieved greater levels of sat-chit-anada (state of bliss, truthful existence, and elevated consciousness) and holistic well-being (see Singh et al., Chapter 13, this volume). 
Now that all their children are married and their parents have passed away, Anika and her husband are looking forward to dedicating even more of their lives to meditation and svādhyāya (self-reflection and study of scriptures; behavioral and socioemotionally focused $R / S$ habits). To a greater degree than ever before, Anika will be able to unburden herself from concerns with matters of her extended family. She is trusting that the pious, compassionate (yet dispassionate and nonattached) frame of mind she seeks to cultivate (cognitively focused $R / S$ habit) will influence those in her community and extended family. Yet how this happens will not be her concern, as she seeks to trust Brahman (the Supreme Being) more and more, in small and large matters. She seeks to learn more about her atman (true self) via self-inquiry (socioemotionally focused R/S habit), leading to enhanced sat-chit-anada and holistic well-being, as her atman is revealed and achieves greater liberation and union with Brahman (Singh et al., this volume).

\section{Important Caveats and Limitations of PRSD Theory}

Before proceeding, some caveats and limitations to PRSD theory are worth noting. First, PRSD theory accounts well for the multilevel, multidimensional aspects of religion/spirituality but not as well for its multivalent nature (Pargament et al., 2013). Religion/spirituality is not beneficial for all people, at all times, and across all cultures and contexts. On the contrary, it can have harmful effects (e.g., R/S struggles can cause or exacerbate negative health outcomes) or expressions (R/S beliefs can fuel prejudice, discrimination, oppression, abuse, or violence; Vieten \& Lukoff, 2021), and those effects and expressions can vary across people, time, cultures, and contexts. Nevertheless, like the theory of positive youth development before it (Benson et al., 2006), PRSD theory is a strength-based approach, focusing on positive trajectories of R/S development. That is, it focuses on what can go right in R/S development, why it goes right, and how various developmental processes interact bidirectionally in promoting people's well-being. 
Second, although PRSD theory focuses on how and why religiousness/spirituality can enhance people's well-being, humans almost universally have life experiences that undermine their well-being. These experiences can happen within or outside R/S contexts, and they can happen to people who have or have not experienced previous periods of positive R/S development. For instance, adverse life experiences either within a R/S context (e.g., sexual abuse by a R/S leader) or outside one (e.g., sexual assault outside a R/S community) can lead to negative changes in people's mental/neural representations of themselves, others, and the world (Charuvastra \& Cloitre, 2008). Those changes can lead to shifts in people's R/S habits, which in turn can be contextually adaptive or maladaptive. Maladaptively, someone may experience R/S struggles that negatively affect their well-being. Conversely, if they experienced harm in a R/S context (e.g., a sexual minority coerced into a sexual orientation change effort), it may actually be adaptive to step away from religion/spirituality temporarily or for good.

Finally, people may have experienced religiousness/spirituality as need-fulfilling and health-enhancing for a period of their life, but over time it is not as need-fulfilling or healthenhancing. For example, their needs may become better fulfilled via other avenues (their family, friends, work, etc.), or their R/S habits may not be as beneficial to their well-being (e.g., they used to rely heavily on R/S strategies to cope with stress, but they come to rely more effectively on other coping strategies). Similarly, someone's religiousness/spirituality may have been contextually adaptive for a period of their life, but as they or their context changes, it is no longer adaptive. For instance, people may come to find the R/S communities and codifications that once supported their need-fulfillment and well-being now feel too constraining, simplistic, or vacuous. Therefore, in their quest for sacred meaning and connection, they may change their R/S habits, which might mean either deconverting or becoming $\mathrm{R} / \mathrm{S}$ in different ways (adopting a "spiritual 
but not religious" identity, shifting to a more progressive tradition/denomination, or becoming more private and personal in their R/S practices; Saroglou et al., 2020). In sum, when people's religiousness/spirituality is no longer as need-fulfilling, health-enhancing, or contextually adaptive, they may change their R/S habits as a result. These changes will not necessarily have negative effects on their well-being; they could instead have beneficial or benign effects.

\section{Practical Applications}

\section{Clinical Practice}

Help R/S clients draw on their religiousness/spirituality as a resilience resource. The vast majority of people in the world are R/S (Pew Research Center, 2018). By extension, most clients seen in clinical practice are R/S as well, even though psychologists often are not R/S themselves and report having little training in addressing clients' religiousness/spirituality (Vieten \& Lukoff, 2021). One application of PRSD theory is that it is clinically useful for mental health practitioners to develop R/S competencies, especially when it comes to helping R/S clients draw on their religiousness/spirituality to enhance their resilience and well-being.

\section{Assess how R/S clients' mental health problems are affecting their R/S development.}

A core process emphasized in PRSD theory is the bidirectional interaction of R/S development and well-being. This interaction has important clinical implications. For example, it highlights the fact that people's mental health problems can negatively affect their R/S functioning (Vieten \& Lukoff, 2021). Hathaway (2003) has referred to such effects as "clinically significant religious impairment" (p. 113; i.e., "a reduced ability to perform religious activities, achieve religious goals, or experience religious states, due to a psychological disorder," p. 113). One way this impairment can manifest is when mental health difficulties impair or impede someone's positive R/S development. Practitioners can assess for such a possibility and then intervene as needed. 
Promote R/S clients' positive R/S development. The most basic clinical application of PRSD theory is for mental health professionals to promote their R/S clients' positive R/S development. Clinicians can do so directly (e.g., by using spiritually integrated interventions as part of clinical treatment; Captari et al., this volume) or indirectly (e.g., by encouraging clients to nurture their positive R/S development both between sessions and following treatment).

Clients often complain their R/S beliefs, practices, commitments, and affiliations are ignored, belittled, or even pathologized by mental health practitioners (Vieten \& Lukoff, 2021). Likewise, clients often complain that R/S practitioners (e.g., pastoral counselors or R/S leaders) use one-size-fits-all approaches that impose rigid religious dogmas and ignore the person's unique contexts and personalized R/S goals, needs, and quests. PRSD theory emphasizes that one marker and mechanism of positive $\mathrm{R} / \mathrm{S}$ development is harmonious synchrony between individuals and the contexts in which they are embedded. Effective and culturally sensitive clinical practice needs to foster—not frustrate—-this vibrant synchrony (Pargament, 2013).

\section{Religious Ministry}

\section{Help faith community members understand how their R/S habits and well-being}

interact. Faith community members often do not have a clear understanding of why and how their R/S habits influence their well-being and vice versa. Therefore, effective R/S ministry can involve helping members understand these interconnections and make appropriate changes, such as changing their R/S habits to become more health-enhancing, well-integrated, and contextually adaptive. Similarly, by improving members' holistic well-being, effective R/S ministry may lead to adaptive changes in members' R/S habits (Davis et al., 2021; Wang et al., this volume).

Address impediments to members' positive R/S development. In addition, effective $\mathrm{R} / \mathrm{S}$ ministry may need to help members recognize and remove impediments to their positive R/S 
development. Such impediments could be internal (e.g., ways their R/S habits are not wellintegrated, health-enhancing, or contextually adaptive) or interpersonal (e.g., relationships or relational habits that are hindering their need-fulfilling, health-enhancing R/S development).

Nurture members' positive R/S development. Again, the most basic application of PRSD theory in R/S ministry is to nurture members' positive R/S development. This can occur through a variety of avenues, such as (a) strategic R/S interventions at the microlevel (R/S ministry to individuals, couples, or families) and macrolevel (R/S ministry to larger groups of members), (b) effective R/S ministry that enhances members' well-being and fulfills their basic and compound needs, and (c) caring R/S leaders who model well-integrated, health-enhancing R/S habits and help their community members cultivate such habits (Wang et al., this volume).

\section{Conclusions}

In this chapter, we have drawn on existing theory and research to present an integrative lifespan framework - positive R/S development (PRSD) theory — that can guide developmental science and practice on religion, spirituality, and positive psychology. PRSD theory is a lifespan theory, and it recognizes R/S development as both continuous (ongoing) and discontinuous (discrete) and as influenced by both nature and nurture (Overton, 2010). PRSD theory posits that positive R/S development involves the contextually influenced, bidirectional interaction among people's goal-relevant mental/neural representations, need-fulfilling relational experiences, wellintegrated and contextually adaptive R/S habits, and holistic well-being. Ultimately, we hope PRSD theory will be used to help individuals, families, communities, and societies flourish more fully, as we all seek to live the type of meaningful, fulfilling, and sustainably joyful life Oprah Winfrey (2015) and others inspire us to pursue. In so doing, may our search for sacred meaning and connection help us all increasingly in "becoming more of who [we] are" (Winfrey, 2015). 


\section{References}

Benson, P. L, Scales, P. C., Hamilton, S., \& Sesma, A., Jr. (2006). Positive youth development: Theory, research, and applications. In R. M. Lerner \& W. Damon (Eds.), Handbook of child psychology: Theoretical models of human development (pp. 894-941). Wiley.

Charuvastra, A., \& Cloitre, M. (2008). Social bonds and posttraumatic stress disorder. Annual Review of Psychology, 59, 301-328. https://doi.org/10.1146/annurev.psych.58.110405.085650

Davis, E. B., Granqvist, P., \& Sharp, C. (2021). Theistic relational spirituality: Development, dynamics, health, and transformation. Psychology of Religion and Spirituality, 13(4), 401-415. https://doi.org/10.1037/rel0000219

Day, J. M. (2010). Religion, spirituality, and positive psychology in adulthood. Journal of Adult Development, 17, 215-229. https://doi.org/10.1007/s10804-009-9086-7

Dweck, C. S. (2021). Toward an integrative theory on motivation, personality, and development. In O. John \& R. Robins (Eds.), Handbook of personality (4 ${ }^{\text {th }}$ ed., pp. 83-103). Guilford.

Erikson, E. H. (1963). Childhood and society (2 ${ }^{\text {nd }}$ ed.). Norton.

Friedman, H., Krippner, Riebel, L., \& Johnson, C. (2012). Models of spiritual development. In L. J. Miller (Ed.), The Oxford handbook of psychology and spirituality (pp. 207-220). Oxford University Press. https://doi.org/10.1093/oxfordhb/9780199729920.013.0014

George, L. S., \& Park, C. L. (2016). Meaning in life as comprehension, purpose, and mattering. Review of General Psychology, 20(3), 205-220. https://doi.org/10.1037/gpr0000077

Harris, K.A., Howell, D.S. \& Spurgeon, D.W. (2018). Faith concepts in psychology: Three 30year definitional content analyses. Psychology of Religion and Spirituality, 10(1), 1-29. http://dx.doi.org/10.1037/rel0000134 
Hathaway, W. L. (2003). Clinically significant religious impairment. Mental Health, Religion, \& Culture, 6, 113-129. https://doi.org/10.1080/1367467021000038174

King, P. E., \& Boyatzis, C. J. (2015). Religious and spiritual development. In M. E. Lamb \& R. M. Lerner (Eds.), Handbook of child psychology and developmental science (pp. 9751021). Wiley. https://doi.org/10.1002/9781118963418.childpsy323

King, P. E., Hardy, S., \& Noe, S. (2021). Developmental perspectives on adolescent religious and spiritual development. Adolescent Research Review. Advance online publication. https://doi.org/10.1007/s40894-021-00159-0

Koenig, H. G., King, D. E., \& Carson, V. B. (2012). Handbook of religion and health $\left(2^{\text {nd }}\right.$ ed.). Oxford University Press.

Lerner, R. M., Lerner, J. V., Bowers, E. P., \& Geldhof, G. J. (2015). Positive youth development and relational-developmental-systems. In R. M. Lerner (Ed.), Handbook of child psychology and developmental science ( $7^{\text {th }}$ ed.; Vol. 1, pp. 607-651).

Mahoney, A., Wong, S., Pomerleau, J. M., \& Pargament, K. I. (2021). Sanctification of diverse aspects of life and psychosocial functioning: A meta-analysis of studies from 1999 to 2019. Psychology of Religion and Spirituality. https://doi.org/10.1037/rel0000354

Overton, W. F. (2010). Life-span development: Concepts and issues. In R. M. Lerner (Ed.), Handbook of life-span development (Vol. 1, pp. 1-29). Wiley.

Pargament, K. I. (2013). Searching for the sacred: Toward a nonreductionistic theory of spirituality. In K. I. Pargament (Ed.), APA handbook of psychology, religion, and spirituality (Vol. 1, pp. 257-273). APA. https://10.1037/14045-014

Pargament, K. I., Mahoney, A., Exline, J. J., Jones, J. W. \& Shafranske, E. P. (2013). Envisioning an integrative paradigm for the psychology of religion and spirituality. In K. 
I. Pargament (Ed.), APA handbook of psychology, religion, and spirituality (Vol. 1, pp.

3-19). American Psychological Association. https://10.1037/14045-001

Pew Research Center (2018). The age gap in religion around the world. Author.

https://www.pewforum.org/2018/06/13/the-age-gap-in-religion-around-the-world/

Reeve, J. (2017). Understanding motivation and emotion ( $7^{\text {th }}$ ed.). Wiley.

Saroglou, V. (2011). Believing, bonding, behaving, and belonging: The Big Four religious

dimensions and cultural variation. Journal of Cross-Cultural Psychology, 42(8), 1320

1340. https://doi.org/10.1177/0022022111412267

Saroglou, V., Clobert, M., Cohen, A. B., Johnson, K. A., Ladd, K. L., . . \& \& Tapia Valladares, J. (2020). Believing, bonding, behaving, and belonging. Journal of Cross-Cultural Psychology, 51(7-8), 551-575. https://doi.org/10.1177/0022022120946488

Seligman, M. E. P. (2011). Flourish. Simon and Schuster.

Siegel, D. J. (2020). The developing mind ( $3^{\text {rd }}$ ed.). Guilford Press.

VandenBos, G. R. (Ed.). (2015). APA dictionary of psychology ( $2^{\text {nd }}$ ed.). American

Psychological Association. http://dx.doi.org/10.1037/14646-000

VanderWeele, T. (2017). On the promotion of human flourishing. Proceedings of the National Academy of Sciences, 114, 8148-8156. www.pnas.org/cgi/doi/10.1073/pnas.1702996114

Vieten, C., \& Lukoff, D. (2021). Spiritual and religious competencies in psychology. American Psychologist. Advance online publication. http://dx.doi.org/10.1037/amp0000821

Winfrey, O. (2015, April). 2015 Harry's last lecture on a meaningful life [Video]. Stanford University. https://news.stanford.edu/news/2015/april/oprah-rathbun-lecture-042315.html 
Figure 18.1: Positive Religious and Spiritual Development (PRSD) Theory

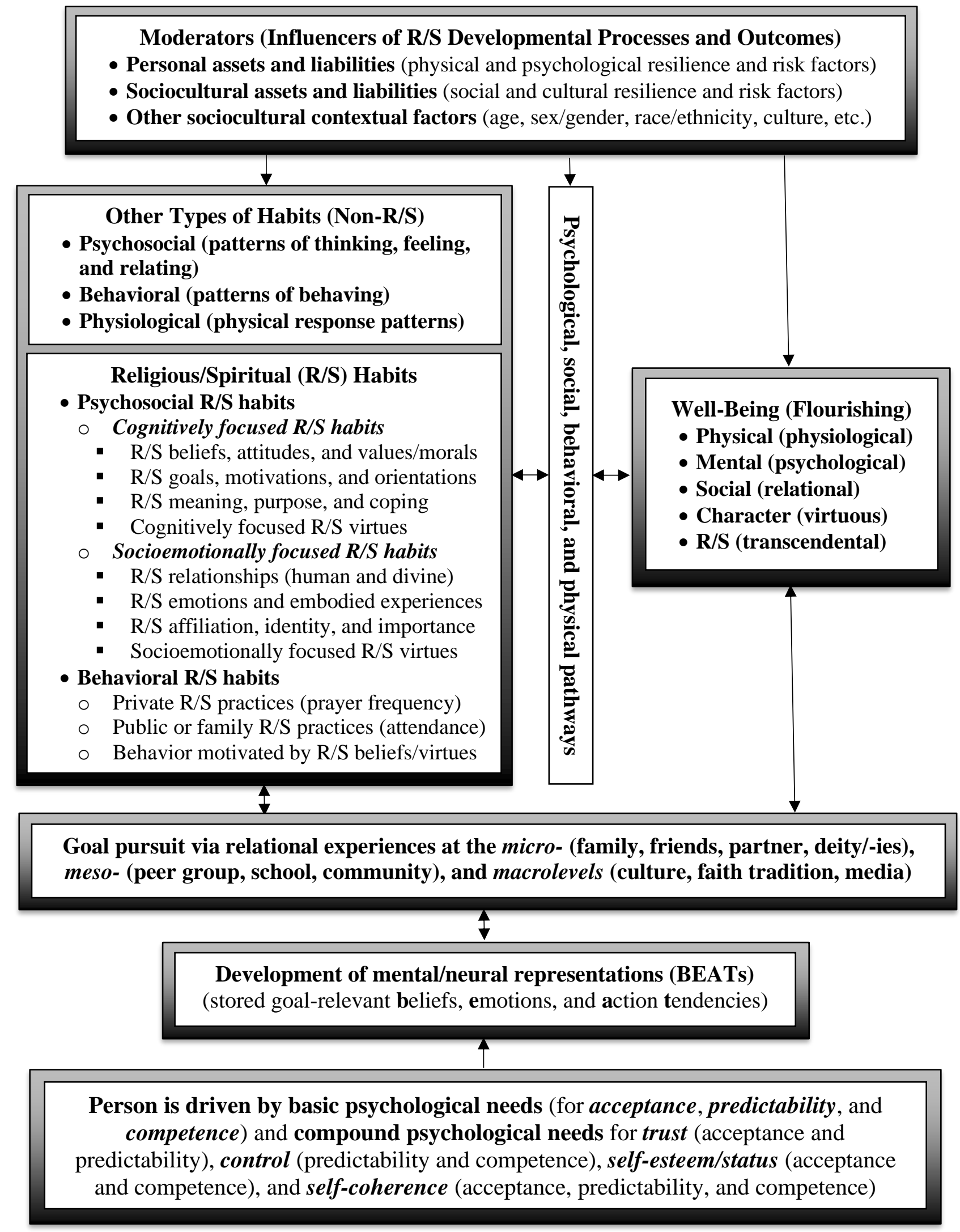




\title{
Chapter 19:
}

\section{The Scientific Study of Life Satisfaction and Religion/Spirituality}

\author{
Elizabeth Krumrei Mancuso \\ Pepperdine University \\ Rosemond Travis Lorona \\ Point Loma Nazarene University
}

Correspondence concerning this chapter should be addressed to Elizabeth Krumrei Mancuso. Email: elizabeth.mancuso@pepperdine.edu 


\begin{abstract}
One way in which religion and spirituality are relevant to the science and practice of positive psychology is through their links to life satisfaction. This chapter reviews scientific research on the relationship between religion/spirituality and life satisfaction. After providing conceptual definitions, we offer an overview of findings from systematic reviews, meta-analyses, and largescale studies, which collectively indicate there is a small, positive relationship between religion/spirituality and life satisfaction. We highlight longitudinal evidence indicating that religion/spirituality predict future life satisfaction, but we acknowledge that the size and nature of this relationship can differ between individuals, communities, and countries. We discuss these and other nuances. Next, we explore reasons for the links between religion/spirituality and life satisfaction, including the possibility that religion/spirituality can promote social connection, meaning, hope, coping, and physical health, all of which promote life satisfaction. At the same time, we take a nonreductionistic approach that acknowledges the unique contributions of religion/spirituality to life satisfaction—contributions that cannot fully be explained on the basis of indirect influences. We review the predominant limitations in this body of research and offer suggestions for advancing the study of religion/spirituality and life satisfaction. Finally, we conclude with applied implications for clinicians and religious/spiritual leaders.
\end{abstract}

Keywords: life satisfaction, religion, spirituality, positive psychology, well-being 


\section{Chapter 19: The Scientific Study of Life Satisfaction and Religion/Spirituality}

In his classic book Man's Search for Meaning, Viktor Frankl (1946/2006) asserted: "Happiness... cannot be pursued; it must ensue, and it only does so as the unintended side-effect of one's dedication to a cause greater than oneself or as the byproduct of one's surrender to a person other than oneself" (pp. xiv-xv). His seminal thoughts make clear that people cannot pursue happiness directly; instead, happiness results from people dedicating their lives to someone or something beyond themselves. Religion and spirituality $(\mathrm{R} / \mathrm{S})$ provide an avenue by which billions of people find something worth dedicating their lives to. In a 1990 television interview, Frankl described that religious individuals are not satisfied with just finding a meaningful task to complete; religious individuals go a step further by including an awareness of a Task Giver (i.e., Divinity). As such, R/S allows people to strive for what Frankl referred to as ultimate meaning. Positive psychology provides an excellent context for gaining and applying knowledge about how R/S might contribute in such ways to the good life and to individual and communal thriving (Miller-Perrin \& Krumrei-Mancuso, 2015). One aspect of this endeavor involves advancing an understanding of how and why R/S relate to life satisfaction (LS).

LS is a key topic of interest to positive psychology. Consistent with Frankl's thinking, many religions emphasize that when people surrender themselves to a self-transcendent purpose, they are able to experience meaningful living. For instance, Buddhists pursue Nirvana, the extinguishing of personal desire, which can end their suffering. Christianity emphasizes that whoever loses their life for the sake of Christ will find abundant life. These examples illustrate that people draw on their religious/spiritual frameworks to achieve rich and deep LS.

The scientific study of LS falls in the arena of subjective well-being, which is typically conceptualized as including affective and cognitive components (Diener et al., 2009; Lindert et 
al., 2015). The affective component involves the experience of pleasant/unpleasant emotions (see Van Cappellen et al., Chapter 20, this volume) and the cognitive component involves mental judgments of LS (this chapter). LS can be defined as a person's subjective perceptions of contentment, satisfaction, or happiness in life. LS can be assessed as a global synopsis of life or as an aggregate of satisfaction with major life domains (e.g., work, study, relationships, leisure, health). LS has been considered an important outcome in its own right and has been associated with a wide variety of positive social, physical, and mental health outcomes (Pavot \& Diener, 2008). Although the extent to which people experience pleasant and unpleasant emotions likely contributes to LS (Diener et al., 2006), this cognitive form of subjective well-being is distinct from affective well-being. For example, Lyubomirsky and Lepper (1999) noted that perceptions of whether someone has a happy life are powerfully driven by cultural expectations of what characterizes a happy life. As a result, whether people appraise themselves as being happy people is somewhat independent of the extent to which they view their lives are satisfactory. Furthermore, LS is empirically distinct from the experience of positive and negative emotions, in that these factors relate differently to age and other outcomes (see Diener et al., 2009 for review).

This chapter explores LS in relation to R/S. The literature includes fairly broad conceptualizations of spirituality, encompassing morality, well-being, meaning, or purpose, which all could overlap with LS. Therefore, we focused on research that tethers spirituality (and thereby religion) to what people hold sacred. This approach is consistent with the conceptualization offered by Davis et al. (Chapter 18, this volume). They define spirituality as people's search for meaning and connection within whatever they perceive as sacred and religiousness as spirituality that takes place within culturally sanctioned codifications. This chapter includes many forms of R/S, such as the extent to which people view R/S as important or 
engage in public/private religious/spiritual practices, religious/spiritual coping, and so forth. We acknowledge that distinct forms of R/S can relate differently to LS. Out of an extensive empirical literature examining links between R/S and LS, this chapter discusses highlights about how, why, and in what contexts R/S relate to LS. We will note how researchers can advance the science of R/S and LS and what some key implications are for clinicians and religious/spiritual leaders.

\section{Religion and Spirituality Relate to Life Satisfaction, but Context Matters}

Thorough systematic reviews have suggested R/S typically relate to higher LS (Koenig \& Larson, 2001; Koenig et al., 2012). The size of this relationship was quantified as small $(r=.12)$ by an early meta-analysis of 142 effect sizes from studies that used a broad definition of LS (Hackney \& Sanders, 2003). The aspect of R/S most strongly related to LS was personal devotion, followed by ideological religion, and then institutional religion. However, it is possible the nature and strength of this relationship can differ based on particular characteristics of individuals, communities, and countries.

On the level of the individual person, life circumstances and religiosity are important for understanding the relationship between R/S and LS. With representative global data from the Gallup World Poll (collected from 2005-2011), a comparison between religious and nonreligious individuals found that religious individuals were lower in LS than nonreligious individuals (Geerling \& Diener, 2020). However, it could be that people with more difficult living conditions tend to be more religious. When controlling the difficulty of individuals' life circumstances (e.g., lower income, less education, and worse health statuses), the same global data collected from 2005 to 2009 indicated religiosity was associated with slightly more LS (Diener et al., 2011). Additionally, as might be expected, longitudinal studies have found evidence that R/S predict increased LS to a greater extent for people who are stronger in their 
religious identities (Bradshaw \& Kent, 2018; Kent et al., 2018; Lim \& Putnam, 2010).

Beyond individual characteristics, community-level R/S can impact the relationship between R/S and LS for individuals and communities. R/S can be associated with LS in some regions of a country but not others, as has been observed in longitudinal research in Germany (Sinnewe et al., 2015). Furthermore, because standard of living factors can relate to levels of R/S in communities and geographic areas, these factors must be taken into account when examining links between R/S and LS on a community or similar large-scale level. In the U.S., the more religious a state is, the higher LS is in that state, when accounting for difficulty of societal circumstances (Diener et al., 2011). These findings, along with others that have examined the interplay of community and individual R/S in relation to individual well-being (e.g., Gebauer et al., 2017), encourage more research on how dynamics within families, neighborhoods, religious communities, cities, states, or regions affect the relationship between R/S and LS.

Similar themes can be observed on a global level. R/S are associated with LS in some countries but not others (Snoep, 2008), suggesting country-level characteristics impact the relationship between R/S and LS. When taking into account the difficulty of societal circumstances, world data from the Gallup Poll has indicated no overall relationship between country-level religiosity and country-level LS (Diener et al., 2011). However, cultural religiosity can affect individual people's experiences (see Gebauer \& Sedikides, 2021). The literature suggests that the positive relationship between personal R/S and LS is stronger in more religious countries, whereas personal R/S can be unrelated or even negatively related to LS among people living in less religious countries (Diener et al., 2011; Eichhorn, 2012; Lun \& Bond, 2013; Okulicz-Kozaryn, 2010; Stavrova et al., 2013). The social value hypothesis (Gebauer et al., 2012; Gebauer et al., 2017) could help explain some of these seemingly contradictory findings in 
multination comparisons. Religiosity may foster more well-being in cultures where religiosity is socially valued, but not in cultures where religiosity possesses little to no social value.

To summarize, on average, the existing evidence suggests there is a small, positive relationship between R/S and LS. However, this average is made up of individuals, communities, and countries among whom the strength of this relationship can vary greatly. Further, a mismatch between people's individual R/S and their community and cultural contexts can even result in negative links between R/S and LS. Thus, clinicians and religious leaders should be aware of the nuances of R/S within the communities they work, in order to have an accurate understanding of how R/S may relate to LS. In addition, they should attend to whether the social climate of R/S support or undermine the R/S of the individuals they work with, which could thereby affect LS.

Of note, the vast majority of research on this topic has examined individuals within a given country. More work is needed to examine links between R/S and LS on community and

global levels. As new methods emerge for using big data to examine links between economic and sociopolitical factors and subjective well-being (e.g., Diener \& Seligman, 2018), it would be valuable to include an examination of R/S in these endeavors.

\section{Are The Links Between Religion/Spirituality and Life Satisfaction Causal?}

Although it is difficult to say whether R/S and LS directly impact each other, longitudinal research shows whether changes in R/S are associated with subsequent changes in LS and vice versa. A challenge is that both R/S and LS tend to be fairly stable across time, which can make it difficult to observe their effects on each other. Despite this challenge, most longitudinal research suggests R/S are associated with subsequently higher levels of LS. A recent meta-analysis of nine independent samples observed a small, positive effect of R/S on LS $(r=.10)$. The metaanalysis only included studies examining whether positive aspects of R/S (e.g., importance of 
religion to the person, public and private religious participation, and positive religious coping) predicted future levels of LS while controlling preexisting levels of LS (Garssen et al., 2021). In essence, the existing research suggests R/S are associated with increased LS over time.

Furthermore, some research has examined how change in R/S relates to change in LS. A 16-year study of the general population in Germany indicated that people who became more involved in religious activities experienced long-term gains in LS, whereas those who became less religious experienced long-term losses in LS (Headey et al., 2010). Similarly, among samples of adolescents and adults in the U.S., religious service attendance (Chen \& VanderWeele, 2018; Crosby et al., 2020), prayer or meditation (Chen \& VanderWeele, 2018), and listening to religious music (Bradshaw et al., 2015) have been associated with higher subsequent LS. Among inter-city U.S. adults recovering from substance abuse, spirituality—but not religiosity—was predictive of higher subsequent LS (Laudet \& White, 2008).

Together, these studies suggest R/S may enhance people's LS. Additionally, long-term research from Germany has shown that R/S can buffer individuals from negative consequences of stressors. Attending religious services once a week mitigated the negative impact of becoming unemployed on LS (Lechner \& Leopold, 2015). Interestingly, joining a new religious movement (also referred to as a "cult") helped people who had previously experienced a dip in LS recover to a stable level of LS comparable to the general population (Namini \& Murken, 2009).

Research on developmental changes across the lifespan is also useful for considering the question of causality. On average, R/S decline during adolescence, but young people experience unique patterns of religious/spiritual change. Research from the U.S. and Middle East has found that youth who remain high in R/S over time tend to experience more LS than youth who either are low in R/S or decline in R/S over time (Kor et al., 2019; Wright et al., 2018). 
Highlighting the importance of cultural context, spirituality — but not religious service attendance — was associated with higher subsequent LS within a sample of adolescents in Portugal (Marques et al., 2013). In addition, research from Canada has shown that youth with higher levels of spirituality are more likely to experience trajectories of high and stable LS than trajectories of moderate or low LS (McDougall et al., 2016). Interestingly, research in Hong Kong has found that adolescents who are higher in spirituality tend to experience sharper declines in LS than less spiritual adolescents (Shek \& Liang, 2018). These researchers theorized that perhaps youth with higher spirituality have greater expectations about life, resulting in relatively stronger drops in LS. Yet even with these sharper declines in LS, more spiritual adolescents still exhibited higher LS than their less spiritual counterparts (Shek \& Liang, 2018).

Although the majority of developmental research on this topic has focused on adolescents, longitudinal studies have also examined older adults. The trends among older adults are similar to those found among adolescents in that various forms of R/S are associated with greater LS over time (e.g., Cowlishaw et al., 2013; Hu et al., 2018; Krause \& Hayward, 2013).

Taken together, research from numerous countries and developmental periods suggests that R/S benefit LS. R/S can also serve as protective factors when stressors might otherwise negatively impact LS. Large-scale, prospective studies provide strong support for the question of causality. Yet, in the absence of experimental research designs, it remains difficult to tease apart direct from indirect effects. The literature suggests increases in R/S and LS are associated with positive changes in other domains as well. For example, Cotton et al. (2006) observed that, among patients with HIV/AIDS, increases in LS were associated not only with increases in R/S but also with improvements in housing conditions, social support, self-esteem, and optimism.

Based on the longitudinal research conducted to date, religious leaders and clinicians 
might bolster people's LS by supporting them in their religious/spiritual pursuits. Interestingly, almost all extant research is framed in terms of the influence of R/S on LS. This is understandable, given that the field of positive psychology has historically focused on uncovering potential contributors to LS (Garssen et al., 2021). At the same time, there is evidence that increases in LS are associated with increases in R/S. For example, Cotton et al. (2006) observed among patients with HIV/AIDS that increases in LS over a 12- to 18-month period were associated with increases in spirituality during the same period. Consistent with a broaden-and-build theory of positive emotions (Fredrickson, 2001), it is plausible the relationship between R/S and LS is bidirectional. That is, the relationship between R/S and LS may involve a bidirectional causal pathway resulting in an upward spiral of positivity (see also Van Cappellen et al, Chapter 20, this volume; Davis et al., Chapter 18, this volume). More work is needed to address the relatively neglected side of this cycle involving how LS impacts religious/spiritual behaviors, experiences, and beliefs. It is possible that people who are more satisfied in life may be more likely to choose to become or stay religious (Lim \& Putnam, 2010). LS may also keep spirituality from becoming unfulfilling (Büssing et al., 2018). Future research can further elucidate if, when, and how changes in LS might result in changes in R/S. For example, increases in LS may result in people engaging more with their religious communities, deriving greater satisfaction from their belief systems, or being more resilient to spiritual struggles. Implications from such research would be particularly helpful to clinicians and religious leaders in their work with religious/spiritual individuals.

\section{How and Why Religion and Spirituality Predict Greater Life Satisfaction}

As reviewed, the most common finding in the literature is that $\mathrm{R} / \mathrm{S}$ are associated with higher LS. Here, we discuss theories about how R/S might contribute to LS. 


\section{Social Resources}

Researchers commonly offer social explanations for the link between R/S and LS, because religious/spiritual individuals tend to participate in religious communities that afford them social interaction and support. The most convincing support for this idea comes from national panel studies that follow representative samples of people over time and thereby can account for stable characteristics of the study participants (e.g., their personalities). Such research suggests a key factor in the link between religious service attendance and LS is the friendships that people form in their religious communities (Lim \& Putnam, 2010). At the same time, studies from numerous countries suggest a strong influence of religious service attendance on LS remains even after accounting for the importance of social resources (Kortt et al., 2015; Sinnewe et al., 2015). Moreover, dozens of cross-sectional studies have found that R/S are associated with more LS, even after accounting for the important influence of social factors. This research has been conducted predominantly among Christian samples from North America and Europe, but also among Jews from Israel (Lazar \& Bjorck, 2016), Muslims from Pakistan (Suhail \& Chaudhry, 2004), and religiously diverse people from Ghana (Addai et al., 2014).

Taken together, this literature suggests R/S afford people more opportunities for giving and receiving social interaction and support, which has positive implications for LS. However, the relationship between R/S and LS does not seem to be explained fully by social factors. Social relationships and interactions grounded in R/S impact LS uniquely from people's general degree of social activity and the size of their social networks. Religious/spiritual social networks may be particularly effective at providing a sense of identity and belonging because they draw on shared beliefs, core values, and perceived divine relationships. For example, praying to a higher power with others is more closely aligned with LS than spending social time with others or praying 
alone (Lim \& Putnam, 2010). R/S may also strengthen social bonds via theistic mediation, whereby individuals draw on a higher power as a neutral party to help resolve interpersonal conflict. Theistic mediation is particularly helpful when individuals believe the higher power wants what is best for everyone involved and is present to strengthen and help each person. Thus, clinicians and religious leaders might encourage interpersonal relationships in religious communities, communal religious rituals, and relational religious/spiritual activities to uniquely impact LS in favorable ways. Helping religious/spiritual individuals capitalize on relationship skills that draw on their religious/spiritual beliefs can also be a meaningful way to bolster LS.

\section{Thought Processes}

$\mathrm{R} / \mathrm{S}$ can offer ways of thinking about life and events that promote LS. R/S are unique in that they can go beyond naturalistic explanations in addressing life's big questions. When faced with existential mysteries, R/S can offer individuals a sense of a meaningful world and their purpose, identity, or worth in it (see Park \& Van Tongeren, Chapter 6, this volume).

Longitudinal research has indicated that R/S are associated with more LS precisely because they can offer a sense of meaningfulness (Cowlishaw et al., 2013). Furthermore, religious meaning may predict LS beyond a general sense that the world is meaningful, manageable, and orderly (Dezutter et al., 2010). Additionally, R/S can offer positive perceptions of one's self, selfefficacy, and future. Deriving a sense of control in life through collaboration with God is associated with higher LS (Krause, 2005). As such, R/S may promote LS by offering people a sense of security, hope, or terror management because they trust in God and/or an afterlife.

$\mathrm{R} / \mathrm{S}$ also offer distinctive resources for coping with distress and creating meaning out of adversity (Pargament, 1997). If individuals believe in the protection of a higher power or that their suffering is part of a larger spiritual plan, they may experience greater LS during hard 
times. Clinicians can draw on these beliefs therapeutically. Frankl (2006) illustrated this in his work with a Rabbi who had lost his wife and children at Auschwitz. Frankl suggested perhaps the Rabbi survived his children so he could be purified through his suffering to become worthy of joining his children in heaven. The Rabbi experienced great relief from this new perspective his suffering was not in vain but had deep religious purpose and offered hope for reuniting him with his children. Empirical literature supports that interpreting suffering through a religious lens results in greater LS. For instance, when older Blacks in the U.S. believe that religion sustains Black people in the face of racial injustice, they experience more LS (Krause, 2004). Therapeutic or religious community settings are ideal venues for helping individuals explore how their R/S contribute to - or perhaps undermine - their sense of meaning, purpose, identity, self-efficacy, and hope. Such discussions offer opportunities for exploring deep themes underlying LS.

\section{Physical Health}

$\mathrm{R} / \mathrm{S}$ can promote people's LS by impacting their physical health. Many religions encourage their adherents to treat their bodies as sacred, practice moderation in food and alcohol, and take periodic rest (e.g., Sabbath). Thus, R/S could promote LS through behavioral and lifestyle choices that positively impact physical health (see Masters et al., Chapter 21, this volume). Clinicians and religious leaders can encourage religious/spiritual individuals to practice such behaviors in authentic ways that promote spiritual growth, physical health, and LS.

Parallel to the themes discussed, longitudinal research has supported that R/S relate to LS even after accounting for the influence of physical health (Bradshaw et al., 2015; Koenig \& Vaillant, 2009). This theme is further supported by a substantial body of cross-sectional research indicating the same. Much of this research has been conducted in the U.S., although these findings have also been confirmed in other countries such as Israel (Lifshitz et al., 2019), Ghana 
(Addai et al., 2014), and Greece (Chliaoutakis et al., 2002). These findings suggest that, similar to social resources and cognitive appraisals, physical health is one of the avenues through which $\mathrm{R} / \mathrm{S}$ contribute to LS, but that it does not tell the full story.

\section{Analysis}

As reviewed, literature from various nations offers explanations for the positive link between R/S and LS based on social resources, thought processes, and physical health. Another theme is that R/S promote LS by offering opportunities for people to experience positive emotions (see Van Cappellen et al., Chapter 20, this volume). In addition, as posited in Davis et al.'s (Chapter 18, this volume) positive religious/spiritual development theory, people's psychological needs may drive their R/S and thereby their well-being. For instance, religious/spiritual social resources can fulfill psychological needs for acceptance, trust, and selfesteem/status and thereby account for increases in LS. Religious/spiritual thought processes can fulfill psychological needs for predictability and control, also accounting for increases in LS.

Of note is that some theories in the literature address $\mathrm{R} / \mathrm{S}$ in reductionistic ways, suggesting R/S merely represent more basic cognitive, social, physical, or emotional processes. Reductionism devalues R/S by shifting the emphasis to these other factors. If underlying psychological or physical processes are considered the crux of the matter, R/S are rendered irrelevant. However, the literature reviewed in this section suggests that in addition to working through non-R/S avenues, $\mathrm{R} / \mathrm{S}$ can promote $\mathrm{LS}$ in substantive ways that are unique and not redundant with the effects of non-R/S aspects of life. Such a nonreductionistic approach recognizes R/S as distinctive aspects of life that are motives in their own right. If people are considered spiritual beings, then R/S experiences contribute to LS by fulfilling basic needs and desires within individuals, not only by reflecting underlying non-R/S needs and desires. 
Clinicians and religious leaders may benefit from exploring their conceptualization of how R/S fit into the human experience. Some conceptualizations that give R/S a position of primacy alongside biopsychosocial experiences include Pargament's (2007) biopsychosociospiritual model and Piedmont's (1999) theory of spirituality as a dimension of personality.

\section{A Darker Side to Religion and Spirituality}

Although most research indicates positive links between R/S and LS, some forms of R/S involve struggle and strain. Individuals can experience the loss or violation of something they consider sacred, can feel abandoned by God, or can experience religious guilt and shame (Pargament, 1997). Surprisingly, there is not much research evidence to support that these strenuous forms of R/S are associated with lower LS. Although some studies have found spiritual struggles are associated with less LS cross-sectionally or at a later date (e.g., Hebert et al., 2009), this link does not seem to persist when taking into account people's preexisting levels of LS (Park et al., 2011; Wortmann et al., 2012). A recent meta-analysis of longitudinal research indicated higher levels of spiritual struggles are coupled with increases in negative psychological adjustment, but they are not associated with change in positive psychological adjustment, including LS (Bockrath et al., 2021). Further, cross-sectional research suggests LS is higher when spiritual struggles or doubts prompt spiritual growth or meaning-making (Zarzycka \& Zietek, 2019). Although more research is needed to confirm, it is possible LS may increase when spiritual struggles provide an opportunity for people to reexamine their faith or worldview in a constructive way.

\section{Future Research Directions}

Throughout this chapter, we have noted areas where more research would be helpful. Here we mention a few additional points. First, although in-depth measures of R/S and LS have 
been used, large scale studies - particularly multicountry, nationally representative, and panel studies - more commonly depend on few- or single-item measures. Because people may factor the domain of R/S into their global judgments of LS, the use of superficial assessments may lead to overestimation of the R/S-LS relationship. What complicates this issue further is that people differ cross-culturally in how they weigh the domain of R/S in their global judgments of LS (Theuns et al., 2012), which obfuscates an understanding of how the R/S-LS link differs between countries and cultures. Therefore, multicountry and longitudinal studies that use indepth and nuanced measures of R/S would be ideal.

Ongoing research can continue to parse out the aspects of R/S that are most influential to LS and how they relate to appropriate secular comparisons. Further, evaluating nonlinear relationships between R/S and LS could be enlightening, given there is some indication that religious individuals tend to be either very satisfied or dissatisfied with life (Okulicz-Kozaryn, 2010). Previous research has uncovered nonlinear links between R/S and mental health outcomes (e.g., nondepressed people with high or low church attendance were less likely to show depression at follow-up relative to moderate church attenders; Ronneberg et al., 2016). Similar work should be conducted in the examination of LS.

\section{Conclusions and Implications}

This chapter has offered highlights from the substantial literature base connecting R/S to LS. The findings underscore one way in which R/S contribute to human flourishing. Metaanalyses indicate the overall relationship between R/S and LS is small (Garssen et al., 2021; Hackney \& Sanders, 2003) but comparable to the size of the effect of other factors meaningfully related to LS, such as socioeconomic status, social support, and competence (Pinquart \& Sörensen, 2000). However, the nature and size of the relationship between R/S and LS can differ 
based on the unique characteristics of individuals, communities, regions, cultures, and countries.

This literature is relevant to clinicians who aspire to treat religious/spiritual clients as whole persons. With most of the research having been conducted in the general population, there is a need to assess clinical populations more closely. The positive impact of R/S on mental health may be even more robust among those facing stressful circumstances (Moreira-Almeida et al., 2006), and there is a strong base of clinical writings on the importance of R/S as sources of strength and support for psychotherapy clients (Pargament, 2007). At the same time, some groups, including sexual minorities, may see less strong or more nuanced relationships between R/S and health outcomes, due to stigma or minority stress (Lefevor et al., 2021). Although more research is needed to understand the links between R/S and LS among specialized populations, the current literature offers initial support for clinicians to affirm their clients' R/S as potential sources of LS. Importantly, clinicians can be aware of the potential individual differences and contextual factors highlighted earlier in this chapter, which may influence how R/S relate to LS.

Religious leaders may also benefit from this literature by encouraging religious beliefs, worldviews, theological interpretations, and practices that promote LS. Given that R/S also work through avenues such as relationships, hope, meaning, and health behaviors, religious leaders are encouraged to foster holistic approaches to R/S that nurture these LS-promoting aspects of life.

$\mathrm{R} / \mathrm{S}$ represent a critical aspect of many people's lives around the world. As subjective well-being (which encompasses LS) becomes an increasingly important measure of societal flourishing (Diener \& Seligman, 2018), positive psychologists can continue to examine the relationship between R/S and LS. This domain of study can promote insight that furthers the fundamentally intertwined goals of religious/spiritual leaders and clinicians who take holistic approaches to working with religious/spiritual individuals and communities. 


\section{References}

Addai, I., Opoku-Agyeman, C., \& Amanfu, S. (2014). Exploring predictors of subjective wellbeing in Ghana. Journal of Happiness Studies, 15(4), 869-890. https://doi.org/frb4

Bradshaw, M., Ellison, C. G., Fang, Q., \& Mueller, C. (2015). Listening to religious music and mental health in later life. The Gerontologist, 55(6), 961-971. https://doi.org/gg9mh7

Bradshaw, M., \& Kent, B. V. (2018). Prayer, attachment to God, and changes in psychological well-being in later life. Journal of Aging and Health, 30(5), 667-691. https://doi.org/frb5

Bockrath, M. F., Pargament, K. I., Wong, S., Harriott, V. A., Pomerleau, J. M., Homolka, S. J., Chaudhary, Z. B., \& Exline, J. J. (2021). Religious and spiritual struggles and their links to psychological adjustment: A meta-analysis of longitudinal studies. Psychology of Religion and Spirituality. https://doi.org/10/gk5z

Büssing, A., Baiocco, F., \& Baumann, K. (2018). Spiritual dryness in Catholic laypersons working as volunteers is related to reduced life satisfaction rather than to indicators of spirituality. Pastoral Psychology, 67(1), 1-15. https://doi.org/frb6

Chen, Y., \& VanderWeele, T. J. (2018). Associations of religious upbringing with subsequent health and well-being from adolescence to young adulthood. American Journal of Epidemiology, 187(11), 2355-2364. https://doi.org/10.1093/aje/kwy142

Chliaoutakis, J. E., Drakou, I., Gnardellis, C., Galariotou, S., Carra, H., \& Chliaoutaki, M. (2002). Greek Christian Orthodox ecclesiastical lifestyle: Could it become a pattern of health-related behavior? Preventive Medicine, 34(4), 428-435. https://doi.org/fhh9t3

Cotton, S., Puchalski, C. M., Sherman, S. N., Mrus, J. M., Peterman, A. H., Feinberg, J., Pargament, K. I., Justice, A. C., Leonard, A. C., \& Tsevat, J. (2006). Spirituality and religion in patients with HIV/AIDS. Journal of General Internal Medicine, 21(S5), S5- 


\section{S13. https://doi.org/bkcrfx}

Cowlishaw, S., Niele, S., Teshuva, K., Browning, C., \& Kendig, H. (2013). Older adults’ spirituality and life satisfaction: A longitudinal test of social support and sense of coherence as mediating mechanisms. Ageing and Society, 33(7), 1243-1262.

Crosby, R. G., III, Ritt, B., \& Slunaker, J. (2020). Motives for religious sacrifice: Classification, measurement, and longitudinal association with psychospiritual well-being. Psychology of Religion and Spirituality, 12(1), 1-12. https://doi.org/gk52

Dezutter, J., Robertson, L. A., Luyckx, K., \& Hutsebaut, D. (2010). Life satisfaction in chronic pain patients: The stress-buffering role of the centrality of religion. Journal for the Scientific Study of Religion, 49(3), 507-516. https://doi.org/dhhr79

Diener, E., Oishi, S., \& Lucas, R. E. (2009). Subjective well-being: The science of happiness and life satisfaction. In S. J. Lopez \& C. R. Snyder (Eds.), Oxford handbook of positive psychology (2 ${ }^{\text {nd }}$ ed., pp. 187-194). Oxford University Press.

Diener, E., \& Seligman, M. E. (2018). Beyond money: Progress on an economy of wellbeing. Perspectives on Psychological Science, 13(2), 171-175. https://doi.org/gdd3gn

Diener, E., Tamir, M., \& Scollon, C. N. (2006). Happiness, life satisfaction, and fulfillment: The social psychology of subjective well-being. In P. A. M. Van Lange (Ed.), Bridging social psychology: Benefits of transdisciplinary approaches. (pp. 319-324). Lawrence Erlbaum.

Diener, E., Tay, L., \& Myers, D. (2011). The religion paradox: If religion makes people happy, why are so many dropping out? Journal of Personality and Social Psychology, 101(6), 1278-1290. https://doi.org/b7h9zr

Eichhorn, J. (2012). Happiness for believers? Contextualizing the effects of religiosity on lifesatisfaction. European Sociological Review, 28(5), 583-593. https://doi.org/bzpvpd 
Frankl, V. E. (1946/2006). Man's search for meaning. Beacon Press.

Fredrickson, B. L. (2001). The role of positive emotions in positive psychology: The broadenand-build theory of positive emotions. American Psychologist, 56, 218-226. http://dx.doi.org/10.1037/0003-066X.56.3.218

Garssen, B., Visser, A., \& Pool, G. (2021). Does spirituality or religion positively affect mental health? Meta-analysis of longitudinal studies. International Journal for the Psychology of Religion, 31(1), 4-20. https://doi.org/ghgd2s

Gebauer, J. E., \& Sedikides, C. (2021). Cultural religiosity: A neglected but powerful dimension of culture. Current Opinion in Psychology, 40, 73-78. https://doi.org/gjmf

Gebauer, J. E., Sedikides, C., \& Neberich, W. (2012). Religiosity, social self-esteem, and psychological adjustment: On the cross-cultural specificity of the psychological benefits of religiosity. Psychological Science, 23, 158-160. https://doi.org/fznb7m

Gebauer, J. E., Sedikides, C., Schönbrodt, F. D., Bleidorn, W., Rentfrow, P. J., Potter, J., \& Gosling, S. (2017). The religiosity as social value hypothesis: A multi-method replication and extension across 65 countries and three levels of spatial aggregation. Journal of Personality and Social Psychology, 113(3), e18-e39. https://doi.org/gbvcpb

Geerling, D. M., \& Diener, E. (2020). Effect size strengths in subjective well-being research. Applied Research in Quality of Life, 15(1), 167-185. https://doi.org/dgxf

Hackney, C. H., \& Sanders, G. (2003). Religiosity and mental health: A meta-analysis of recent studies. Journal for the Scientific Study of Religion, 42(1), 43-55. https://doi.org/cchmch

Headey, B., Schupp, J., Tucci, I., \& Wagner, G. G. (2010). Authentic happiness theory supported by impact of religion on life satisfaction: A longitudinal analysis with data for Germany. The Journal of Positive Psychology, 5(1), 73-82. https://doi.org/dpbv6r 
Hebert, R., Zdaniuk, B., Schulz, R., \& Scheier, M. (2009). Positive and negative religious coping and well-being in women with breast cancer. Journal of Palliative Medicine, 12(6), 537545. https://doi.org/bq64gk

Hu, Y.-H., Chiu, C.-J., Wong, J. D., Lin, D.-C., \& Wray, L. A. (2018). The role of leisure activities in the relationship between marital transition in later midlife and psychological well-being trajectories. The International Journal of Aging \& Human Development, 86(4), 327-346. https://doi.org/g4df

Kent, B. V., Bradshaw, M., \& Uecker, J. E. (2018). Forgiveness, attachment to God, and mental health outcomes in older US adults: A longitudinal study. Research on Aging, 40(5), 456-479. https://doi.org/ggq3f6

Koenig, H., King, D., \& Carson, V. (2012). Handbook of religion and health (2 ${ }^{\text {nd }}$ ed.). Oxford.

Koenig, H. G., \& Larson, D. B. (2001). Religion and mental health: Evidence for an association. International Review of Psychiatry, 13(2), 67-78. https://doi.org/fnd6nn

Koenig, L. B., \& Vaillant, G. E. (2009). A prospective study of church attendance and health over the lifespan. Health Psychology, 28(1), 117-124. https://doi.org/d5858r

Kor, A., Pirutinsky, S., Mikulincer, M., Shoshani, A., \& Miller, L. (2019). A longitudinal study of spirituality, character strengths, subjective well-being, and prosociality in middle school adolescents. Frontiers in Psychology, 10, 377. https://doi.org/gg5fcf

Kortt, M. A., Dollery, B., \& Grant, B. (2015). Religion and life satisfaction down under. Journal of Happiness Studies, 16(2), 277-293. https://doi.org/frch

Krause, N. (2004). Common facets of religion, unique facets of religion, and life satisfaction among older African Americans. The Journals of Gerontology: Series B: Psychological Sciences and Social Sciences, 59(2), S109-S117. https://doi.org/dcwc7k 
Krause, N. (2005). God-mediated control and psychological well-being in late life. Research on Aging, 27(2), 136-164. https://doi.org/c5b7kg

Krause, N., \& Hayward, R. D. (2013). Prayer beliefs and change in life satisfaction over time. Journal of Religion and Health, 52(2), 674-694. https://doi.org/f4xb2p

Laudet, A. B., \& White, W. L. (2008). Recovery capital as prospective predictor of sustained recovery, life satisfaction, and stress among former poly-substance users. Substance Use \& Misuse, 43(1), 27-54. https://doi.org/cgntx5

Lazar, A., \& Bjorck, J. P. (2016). Religious support and psychological well-being: Gender differences among religious Jewish Israelis. Mental Health, Religion \& Culture, 19(4), 393-407. https://doi.org/frem

Lechner, C. M., \& Leopold, T. (2015). Religious attendance buffers the impact of unemployment on life satisfaction: Longitudinal evidence from Germany. Journal for the Scientific Study of Religion, 54(1), 166-174. https://doi.org/gdxtvq

Lefevor, G. T., Davis, E. B., Paiz, J. Y., \& Smack, A. C. P. (2021). The relationship between religiousness and health among sexual minorities: A meta-analysis. Psychological Bulletin. Advance online publication. https://doi.org/gk53

Lifshitz, R., Nimrod, G., \& Bachner, Y. G. (2019). Spirituality and wellbeing in later life: A multidimensional approach. Aging \& Mental Health, 23(8), 984-991. https://doi.org/gf36mj

Lim, C., \& Putnam, R. D. (2010). Religion, social networks, and life satisfaction. American Sociological Review, 75(6), 914-933. https://doi.org/cdpkq2

Lindert, J., Bain, P. A., Kubzansky, L. D., \& Stein, C. (2015). Well-being measurement and the WHO health policy Health 2010: Systematic review of measurement scales. European 
Journal of Public Health, 25(4), 731-740. https://doi.org/f7m2rj

Lun, V. M.-C., \& Bond, M. H. (2013). Examining the relation of religion and spirituality to subjective well-being across national cultures. Psychology of Religion and Spirituality, 5(4), 304-315. https://doi.org/gg9d2p

Lyubomirsky, S., \& Lepper, H. S. (1999). A measure of subjective happiness: Preliminary reliability and construct validation. Social Indicators Research, 46(2), 137-155. https://doi.org/bfpfhv

Marques, S. C., Lopez, S. J., \& Mitchell, J. (2013). The role of hope, spirituality, and religious practice in adolescents' life satisfaction: Longitudinal findings. Journal of Happiness Studies, 14(1), 251-261. https://doi.org/gg47gh

McDougall, J., DeWit, D. J., Nichols, M., Miller, L., \& Wright, F. V. (2016). Three-year trajectories of global perceived quality of life for youth with chronic health conditions. Quality of Life Research, 25(12), 3157-3171. https://doi.org/f93h3v

Miller-Perrin, C., \& Krumrei-Mancuso, E. (2015). Faith from a positive psychology perspective. Springer.

Moreira-Almeida, A., Neto, F. L., \& Koenig, H. G. (2006). Religiousness and mental health: A review. Revista Brasileira de Psiquiatria, 28(3), 242-250. https://doi.org/brnctc

Namini, S., \& Murken, S. (2009). Self-chosen involvement in new religious movements (NRMs): Well-being and mental health from a longitudinal perspective. Mental Health, Religion \& Culture, 12(6), 561-585. https://doi.org/cs9qsd

Okulicz-Kozaryn A. (2010). Religiosity and life satisfaction across nations. Mental Health, Religion \& Culture, 13(2), 155-169. https://doi.org/bbnbdk

Pargament, K. I. (1997). The psychology of religion and coping. Guilford Press. 
Pargament, K. I. (2007). Spiritually integrated psychotherapy. Guilford Press.

Park, C. L., Wortmann, J. H., \& Edmondson, D. (2011). Religious struggle as a predictor of subsequent mental and physical well-being in advanced heart failure patients. Journal of Behavioral Medicine, 34(6), 426-436. https://doi.org/dzg4w7

Pavot W. \& Diener E. (2008) The Satisfaction With Life Scale and the emerging construct of life satisfaction. The Journal of Positive Psychology, 3(2), 137-152. https://doi.org/dgn5p5

Piedmont, R. L. (1999). Does spirituality represent the sixth factor of personality? Spiritual transcendence and the Five-Factor Model. Journal of Personality, 67(6), 985-1013. https://doi.org/df88hs

Pinquart, M., \& Sörensen, S. (2000). Influences of socioeconomic status, social network, and competence on subjective well-being in later life: A meta-analysis. Psychology and Aging, 15(2), 187-224. https://doi.org/dbfzhx

Ronneberg, C. R., Miller, E. A., Dugan, E., \& Porell, F. (2016). The protective effects of religiosity on depression: A 2-year prospective study. The Gerontologist, 56(3), 421-431. https://doi.org/f8rj3m

Shek, D. T. L., \& Liang, L.-Y. (2018). Psychosocial factors influencing individual well-being in Chinese adolescents in Hong Kong: A six-year longitudinal study. Applied Research in Quality of Life, 13(3), 561-584. https://doi.org/gd67x6

Sinnewe, E., Kortt, M. A., \& Dollery, B. (2015). Religion and life satisfaction: Evidence from Germany. Social Indicators Research, 123(3), 837-855. https://doi.org/f7ppvg

Snoep, L. (2008). Religiousness and happiness in three nations: A research note. Journal of Happiness Studies, 9(2), 207-211. https://doi.org/dbzr65

Stavrova, O., Fetchenhauer, D., \& Schlösser, T. (2013). Why are religious people happy? The 
effect of the social norm of religiosity across countries. Social Science Research, 42(1), 90-105. https://doi.org/gdj4wf

Suhail, K., \& Chaudhry, H. R. (2004). Predictors of subjective well-being in an Eastern Muslim culture. Journal of Social and Clinical Psychology, 23(3), 359-376.

Theuns, P., Baran, B., Vaerenbergh, R. V., \& Tiliouine, H. (2012). A cross-cultural experimental approach to the contribution of health, religion and personal relations to subjective satisfaction with life as a whole. Psicologica, 33(3), 591-608.

Wortmann, J. H., Park, C. L., \& Edmondson, D. (2012). Spiritual struggle and adjustment to loss in college students: Moderation by denomination. International Journal for the Psychology of Religion, 22(4), 303-320. https://doi.org/gk56

Wright, A. W., Yendork, J. S., \& Kliewer, W. (2018). Patterns of spiritual connectedness during adolescence: Links to coping and adjustment in low-income urban youth. Journal of Youth and Adolescence, 47(12), 2608-2624. https://doi.org/gfkfvc

Zarzycka, B., \& Zietek, P. (2019). Spiritual growth or decline and meaning-making as mediators of anxiety and satisfaction with life during religious struggle. Journal of Religion and Health, 58(4), 1072-1086. https://doi.org/frcs 


\title{
Chapter 20: The Scientific Study of Positive Emotions and Religion/Spirituality
}

\author{
Patty Van Cappellen ${ }^{1}$, Ruixi Zhang ${ }^{1}$, and Barbara L. Fredrickson ${ }^{2}$ \\ ${ }^{1}$ Social Science Research Institute, Duke University \\ ${ }^{2}$ Department of Psychology and Neuroscience, University of North Carolina at Chapel Hill
}

\begin{abstract}
Author's Note:
Correspondence concerning this chapter should be addresses to Dr. Patty Van Cappellen, Duke University, Social Science Research Institute, Box 90420, Durham, NC, 27707-0420. Email: patty.vancappellen@duke.edu
\end{abstract}




\begin{abstract}
This chapter considers the role of positive emotions in religion/spirituality. We begin by reviewing key conceptual frameworks of positive emotions (e.g., Broaden-and-Build Theory of Positive Emotions) while focusing on self-transcendent positive emotions such as awe, gratitude, love, and compassion. We then review scientific research on the bidirectional relationship between religion/spirituality and positive emotions. First, we examine various pathways through which religion/spirituality promote the experience of positive emotions. For example, research has shown that religion/spirituality is related to specific valued emotions and particular emotionregulation strategies. In addition, religious/spiritual (R/S) practices provide the opportunity to experience positive emotions, partly through emotional embodiment. Second, we propose four effects of positive emotions related to religion/spirituality. Positive emotions support R/S beliefs, and when they are felt during R/S practices, they function as promoters of well-being, prosocial intentions and behaviors, and continued R/S practices (the Upward Spiral Theory of Sustained Religious Practice). We close by offering some applications of these findings for mental health practitioners, religious leaders, and religiously/spiritually oriented people.
\end{abstract}

Keywords: positive emotions, religion, spirituality, positive psychology, broaden and build 


\section{Chapter 20: The Scientific Study of Positive Emotions and Religion/Spirituality}

In The Brothers Karamazov, Fyodor Dostoyevsky alludes to a kind of spiritual ecstasy achieved through religious devotion: "My friends, pray to God for gladness. Be glad as children, as the birds of heaven" (Dostoyevsky et al., 1933, p. 335). Indeed, in literature, philosophy, as well as everyday life, religion/spirituality is closely associated with positive emotions.

However, despite the important role positive affect plays in religion/spirituality, the relationship between positive emotions and religion/spirituality has received little attention from empirical researchers. In this chapter, we first conduct a systematic review of existing research on the bidirectional relationship between positive emotion and religion/spirituality. We show that religious/spiritual (R/S) practices generate the experience of positive emotions via multiple means and reflect the expression of positive emotional experiences. We also discuss the practical implications of this relationship, focusing on four major effects of positive emotions in religion/spirituality: (a) supporting beliefs, (b) improving well-being, (c) promoting prosocial behaviors, and (d) maintaining R/S practices (the Upward Spiral Theory of Sustained Religious Practice, Van Cappellen et al., 2021).

\section{Positive Emotions}

Positive emotions are brief mental, physiological, and behavioral responses to changes in how someone interprets (appraises) their current circumstances (Fredrickson, 2013). Positive emotions tend to share three distinguishing characteristics: (a) they serve an adaptive (healthpromoting) function, (b) they engage an approach (appetitive) motivation, and (c) they involve pleasant feelings (Smith et al., 2014). There are a wide variety of positive emotions, including joy, gratitude, serenity (contentment), hope, interest, pride, amusement, inspiration, awe, admiration, or love (Fredrickson, 2013). 
According to the Broaden and Build Theory of Positive Emotions (Fredrickson, 2013), feeling momentary positive emotions broadens one's mindset (momentary repertoire of thoughts and actions) and enables one to think and act more creatively. As these brief moments of positive emotions accumulate and compound over time, positive emotions build enduring psychological (e.g., resilience, purpose in life), biological (e.g., reduced illness symptoms, cardiac vagal tone), and social (e.g., good quality relationships, received social support) resources for survival. In addition, positive emotions may have an "undoing effect" (Fredrickson et al., 2000, p. 237) on negative emotions. The effects of negative emotions often linger after the negative circumstances that caused the negative emotions are no longer present, but the experience of positive emotions speeds up recovery from these lingering effects (Fredrickson et al., 2000).

Yet positive emotions do not all have the same qualities and effects (Fredrickson, 2013; Sauter, 2010). One subset of positive emotions is called self-transcendent positive emotions, because they have a self-transcendent quality to them. These emotions include awe, gratitude, love, compassion, and admiration. Self-transcendent emotions are a subset of self-transcendent experiences, and as such, they are "marked by decreased self-salience and increased feelings of connectedness" (Yaden et al., 2017, p. 143). Indeed, Haidt (2003) has described them as emotions that rise above self-interest and often are associated with the welfare of others. As reviewed below, even more so than other positive emotions like pride or amusement, selftranscendent positive emotions seem universally central to R/S experience.

\section{Religion/Spirituality and the Promotion of Positive Emotions}

Although for many people it may not come as a surprise that religion/spirituality can generate the experience of positive emotions, empirical research on the topic is fairly nascent. Overall, extant studies suggest that R/S beliefs and practices are related to positive emotions 
(Myers, 2018), especially to self-transcendent emotions such as gratitude (Emmons \& Kneetzel, 2005; Kim-Prieto \& Diener, 2009). Daily experiences of spirituality are positively related to happiness (Ellison \& Fan, 2008), and deep religious and mystical experiences are often accompanied by intense positive emotions such as joy, peace, and reverence (Yaden et al., 2017). For example, in a cross-cultural study among Black, White, Latinx, and Korean members of Pentecostal and Presbyterian churches, linguistic analyses showed that narrative accounts of closeness to God and spiritual transformations were filled with references to positive emotions, especially gratitude (Abernethy et al., 2016).

How exactly does religion/spirituality promote positive emotions, especially selftranscendent ones? Below we emphasize two paths. One path targets R/S beliefs as a source of valuing self-transcendent emotion and of guiding adaptive emotion regulation. Another path targets R/S practices that provide opportunities to experience positive emotions; here we present new work from our team on the role of embodiment in religious practice.

\section{Emotion Regulation and Values}

$\mathrm{R} / \mathrm{S}$ beliefs shape the kind of emotions that the people who hold those beliefs value. In general, R/S people value positive emotions more than those who are less religious do (Vishkin et al., 2019). Specifically, Christians value high-arousal positive affect, whereas Buddhists value low-arousal positive affect (Tsai et al., 2007). Members of most major religious groups

(Christians, Muslims, Buddhists, and Hindus — but not Jews) report valuing the positive emotions of love, gratitude, and happiness to a greater extent than the positive emotion of pride (KimPrieto \& Diener, 2009). In addition, according to Belief Maintenance theory (Vishkin et al., 2020), people who embrace R/S beliefs tend to seek out certain affective experiences that are congruent with their beliefs. Therefore, they are particularly motivated to pursue self- 
transcendent positive emotions that strengthen their beliefs. For instance, in two large-scale, cross-cultural studies, Catholics, Jews, and Muslims rated how often they wanted to experience various emotions (e.g., awe, gratitude, and pride) and how often they usually experienced these emotions. Results showed that regardless of religious affiliations, religious people were more likely to value self-transcendent emotions (e.g., awe) than self-conscious emotions (e.g., pride; Vishkin et al., 2019).

In addition, religion/spirituality affects emotion-regulation strategies. Emotion regulation refers to the process in which people monitor and manage what emotions they have, when they have those emotions, and how they interpret, experience, and express them (Gross \& Thompson, 2007). Research in a sample of American Catholics, Israeli Jews, and Muslim Turks has found evidence of relationships between religion/spirituality and two adaptive emotion-regulation strategies: situational acceptance and cognitive reappraisal (Vishkin et al., 2019). First, people who are R/S tend to think of themselves as existing within a larger system that is organized by a higher order. They believe that instead of fighting against this order, they should learn to live harmoniously with it. Hence, people with higher (vs. lower) religion/spirituality are apt to use situational acceptance as an emotion regulation strategy, allowing them to come to terms with a given situation and gain secondary control by adjusting their mindsets. Second, people who are $\mathrm{R} / \mathrm{S}$ also tend to perceive their experiences (both personally significant events and seemingly mundane daily occurrences) as part of a larger meaning system. This tendency to reinterpret emotional events coincides with a specific emotion regulation strategy called cognitive reappraisal, which involves reframing an emotional event by changing its perceived meaning (Gross \& John, 2003). Again, people with higher (vs. lower) R/S were apt to engage more frequently in cognitive reappraisal (Vishkin et al., 2019). This adaptive strategy allows R/S 
people to think of their negative experiences differently and to look actively for the silver lining around the cloud, which often leads to positive emotional outcomes (Gross \& John, 2003).

Given that most of the work referenced in this section is recent, there is ample room for future research. In particular, researchers can explore the extent to which specific R/S practices represent practical exercises for certain emotion-regulation skills. For example, collective rituals can offer opportunities for large fluctuations in emotions (ranging from negative to positive and low arousal to high arousal) and for habitual patterns of meaning making out of emotional events. Religion/spirituality may also impact other "moments" in the emotion regulation process (Gross \& John, 2003), such as selecting different emotional situations or regulating the expression of an emotion. Commendably, considerable research on this topic has been conducted with people from different religious traditions and different countries. Previous research revealed that although the examined associations did not vary widely between religious traditions, they

did show some variations depending on the cultural/national context (e.g., U.S. Hindus compared to Indian Hindus), and these variations of course should be studied further.

\section{Embodied Religious/Spiritual Practices}

$\mathrm{R} / \mathrm{S}$ practices such as meditation, prayer, and collective worship provide opportunities to experience positive emotions. Many studies, including longitudinal studies of novice meditation practitioners, indicate that meditation increases positive emotions in the moment and in daily life (Fredrickson et al., 2017; Fredrickson et al., 2008). Similarly, the practices of prayer (Lambert et al., 2009) and church attendance (Krause, 2009) increase feelings of gratitude over time (see Long \& VanderWeele, this volume). Moreover, the physical space in which these R/S practices take place also influences emotional experiences during them. Naturally, many places of worship are designed to elicit awe through their monumental architectures (Joye \& Verpooten, 2013). 
Although there are many ways through which R/S practices can affect people's emotions, Van Cappellen's Belief, Affect, and Behavior Lab at Duke University has recently explored the role of an understudied aspect of religion/spirituality practices - the fact these practices involve embodied (physical) motions, gestures, and postures. Indeed, the body plays a central role in prayer, collective worship, meditation, and yoga. Postures both express and construct people's emotional experiences. This reciprocal relationship between body and affect is supported by decades of research in affective science (Barrett \& Lindquist, 2008) and grounded cognition (Winkielman et al., 2015). For example, expansive and upright postures are associated with positive feelings, whereas constrictive and slumped postures are related to negative emotions (LaFrance \& Mayo, 1978).

Van Cappellen and Edwards (2021a) have reviewed such literature and discussed the implications of prayer and worship postures (which are ubiquitous in R/S practice) for people's $\mathrm{R} / \mathrm{S}$ experiences. On the one hand, these postures visibly express emotions. On the other hand, adopting certain prayer and worship postures may support the experience of particular emotions. Van Cappellen and Edwards's (2021a) theoretical review supported both ideas. They examined two common types of postures that varied along two postural dimensions: postural orientation (upward vs. downward) and use of space (expansive vs. constrictive). Upward and expansive poses involve looking up and raising hands, whereas downward and constrictive poses involve looking down, kneeling down, clasping hands, or bowing one's head or bowing from the waist.

In one study, U.S. Christian community participants used a small mannequin to show how they would use their full body to express various prayer orientations (prayer, worship, praise, thanksgiving, repentance, confession, and anger toward God) and emotions that varied on the affective dimensions of valence and dominance. Postures were analyzed to derive 
measurements of the body's vertical, horizontal, and total space, and a coding system was developed to code for components of the body, such as head and arm positions. Results showed that postures representing prayer were systematically oriented more downwardly and constrictively than postures representing worship. Furthermore, postures representing praise and thanksgiving were more expansive and oriented upward, similar to postures representing positive emotions and dominance. By comparison, postures representing confession and repentance were more constrictive and oriented downward, similar to postures representing negative emotions and submission (Van Cappellen \& Edwards, 2021b).

In another study, U.S. Christian community participants reported about the kind of body postures they adopted while attending a Sunday worship service, as well as their overall affective experiences during that same service. Participants who adopted more frequent upward and expansive postures during the church service also reported feeling more positive and higharousal affect during the same service (more data were collected beyond emotions and can be found in Van Cappellen et al., in press). Moreover, other studies have found that directly manipulating physical postures modifies affective experiences. Across two experiments, adults who listened to a piece of emotionally ambiguous music while raising their hands and looking upward (upward and expansive posture) experienced more positive emotions than those who listened to the same or similar piece of music while clasping their hands in prayer and looking downward (downward and constrictive posture; Van Cappellen, Ladd, et al., 2020).

In sum, although each of these summarized effects was small, these studies suggest the embodied nature of R/S practices provides another pathway by which religion/spirituality connects with positive emotions_-by expressing and generating positive emotions. In addition, because postures are visible displays of people's emotions, they create opportunities to recognize 
the emotions others are feeling and then potentially join in, thereby spreading and amplifying the experience. Further research on R/S practices, including their embodiment and how they influence emotion intensity, frequency, fluctuation, or contagion is needed.

\section{Positive Emotions and the Promotion of Religion/Spirituality}

As reviewed above, positive emotions (experienced in any context) have the capacity to broaden the scope of attention, build enduring personal resources, and undo the effects of negative emotions (Fredrickson, 2013). In this section, we summarize research on the effects of positive emotions in relation to R/S and on the effects of positive emotions that are felt in the context of R/S practices. The first effect we describe is how positive emotions support people's $\mathrm{R} / \mathrm{S}$ beliefs. The other three effects we describe are the benefits that positive emotions experienced during R/S practices can have on health and well-being, prosocial intentions and behaviors, and continued engagement in these practices.

\section{Positive Emotions Support R/S Beliefs}

First, research shows that self-transcendent positive emotions promote R/S beliefs (e.g., "There is a higher plane of consciousness or spirituality that binds all people"). In a series of experiments, participants randomly assigned to experience awe (a self-transcendent emotion) reported greater spirituality than participants assigned to experience either amusement (a nonself-transcendent positive emotion) or no particular emotion (control condition; Saroglou et al., 2008). In two other experiments, inducing self-transcendent positive emotions of elevation (elicited by witnessing other people exemplifying moral beauty) and admiration (elicited by witnessing others' extraordinary talents and skills) led to increased spirituality, relative to amusement and control conditions. In addition, perceived meaning in life and positive worldviews (e.g., a basic belief in the benevolence of others) explained (mediated) the 
relationship between self-transcendent positive emotions and spirituality. This relationship was moderated by preexisting levels of religion/spirituality. Somewhat counterintuitively, elevation (as opposed to amusement) led participants who were initially less R/S to show a higher increase in spirituality than participants who were initially more religious (Van Cappellen et al., 2013). In sum, these studies suggest that self-transcendent positive emotions such as awe, elevation, and admiration may make people more open to spiritual and transcendental experiences.

\section{Positive Emotions Felt During R/S Practices Enhance Health/Well-Being}

A second effect of positive emotions is related to its impact on health and well-being, broadly defined. The observed relationship between religion/spirituality and subjective wellbeing (Koenig et al., 2012) has led psychologists to investigate specifically how religion/spirituality may affect well-being. Positive emotions, which have been shown to support well-being (Fredrickson, 2013), offer one possible pathway. Correlational studies have found that dispositional gratitude uniquely predicted subjective and psychological well-being amongst Iranian Muslims and Polish Christians (Aghababaei \& Tabik, 2012). Similar patterns were found in a series of studies conducted in Thailand; engaging in Buddhist practices and adhering to Buddhist values were related to higher happiness and lower negative affect, which predicted better health outcomes (Winzer \& Gray, 2019). Three other studies found that religion/spirituality promoted teleological explanations of an event, which in turn increased positive emotions and thereby enhanced well-being (Ramsay et al., 2019). Finally, in another large study, Catholic participants completed questionnaires after attending their Sunday service, assessing their church experiences and subjective well-being. Results showed that religion/spirituality was related to greater well-being because of increased positive emotions felt during church, especially self-transcendent emotions. The effects of positive emotions emerged 
above and beyond the measured social and cognitive benefits of attending church (Van Cappellen et al., 2014). Taken together, these results lend support to the theory that positive emotions are at least partly responsible for the relationship between religion/spirituality and health/well-being. However, additional evidence from longitudinal and/or experimental studies is needed to provide more convincing support for this theory (see Chapter 8, this volume).

\section{Positive Emotions Felt During R/S Practices Enhance Prosociality}

A third effect of positive emotions - particularly self-transcendent emotions-felt in the context of R/S practices may be to promote prosociality. Because self-transcendent positive emotions promote prosocial attitudes and behaviors (Stellar et al., 2017), one study tested whether self-transcendent positive emotions felt while engaging in R/S practices might be at least partly responsible for the well-established association between religion/spirituality and prosocial behaviors. In the aforementioned sample of Catholic participants who completed questionnaires after attending their Sunday church service, the relationship between their experience while at church and their prosocial behavior intentions was assessed. Participants were asked to imagine they had just won $€ 100,000$ (around $\$ 117,000$ US) at the lottery; then they were asked to describe what they would do with the money. Coders categorized these expenses as directed toward oneself or toward others (family, friends, or charities). Results indicated that higher levels of religion/spirituality were associated with greater reported willingness to share the money spontaneously with others. The self-transcendent emotion of love partially explained (mediated) the positive relationship between religion/spirituality and prosociality (sharing), whereas pride (a self-conscious emotion) was inversely associated both with religion/spirituality and with sharing (Van Cappellen, Saroglou, et al., 2016). 
In sum, preliminary evidence suggests that self-transcendent positive emotions felt while engaging in R/S practices may enhance people's prosocial attitudes, intentions, and behaviors. However, the correlational nature of this single study in a Catholic sample limits its generalizability. Future research should test this possibility in diverse samples and directly manipulate positive emotions felt during worship, in order to explore its impact on prosociality.

\section{Maintaining Practice: The Upward Spiral Theory of Sustained R/S Practice}

The final effect of positive emotions that we will highlight stems from their role in motivating behavior maintenance (Fredrickson, 2013). Specifically, positive emotions that are experienced during R/S practices may motivate, energize, and further sustain these practices over time. R/S practices (such as worshipping, praying, or meditating) are costly in time, energy, and resources. Although it is notoriously hard to maintain costly behaviors (e.g., exercising), R/S practices surprisingly are highly prevalent and often maintained over time, despite people's changing circumstances (e.g., moving, good or bad life events). One useful theory for explaining the social motivation for participating in collective R/S practices is Costly Signaling Theory (Atran \& Henrich, 2010), which posits that continued engagement in costly R/S practices signals to others that one is truthfully committed to the groups' beliefs and values. However, this hypothesis does not adequately explain the maintenance of private R/S practices (e.g., prayer), for which social signaling does not apply. We focus on the affective motivation to participate in private and collective R/S practices and suggest that when positive emotions are experienced while worshipping, praying, or meditating, they act as a motivational factor for fueling the continuation and likely recurrence of that practice. 


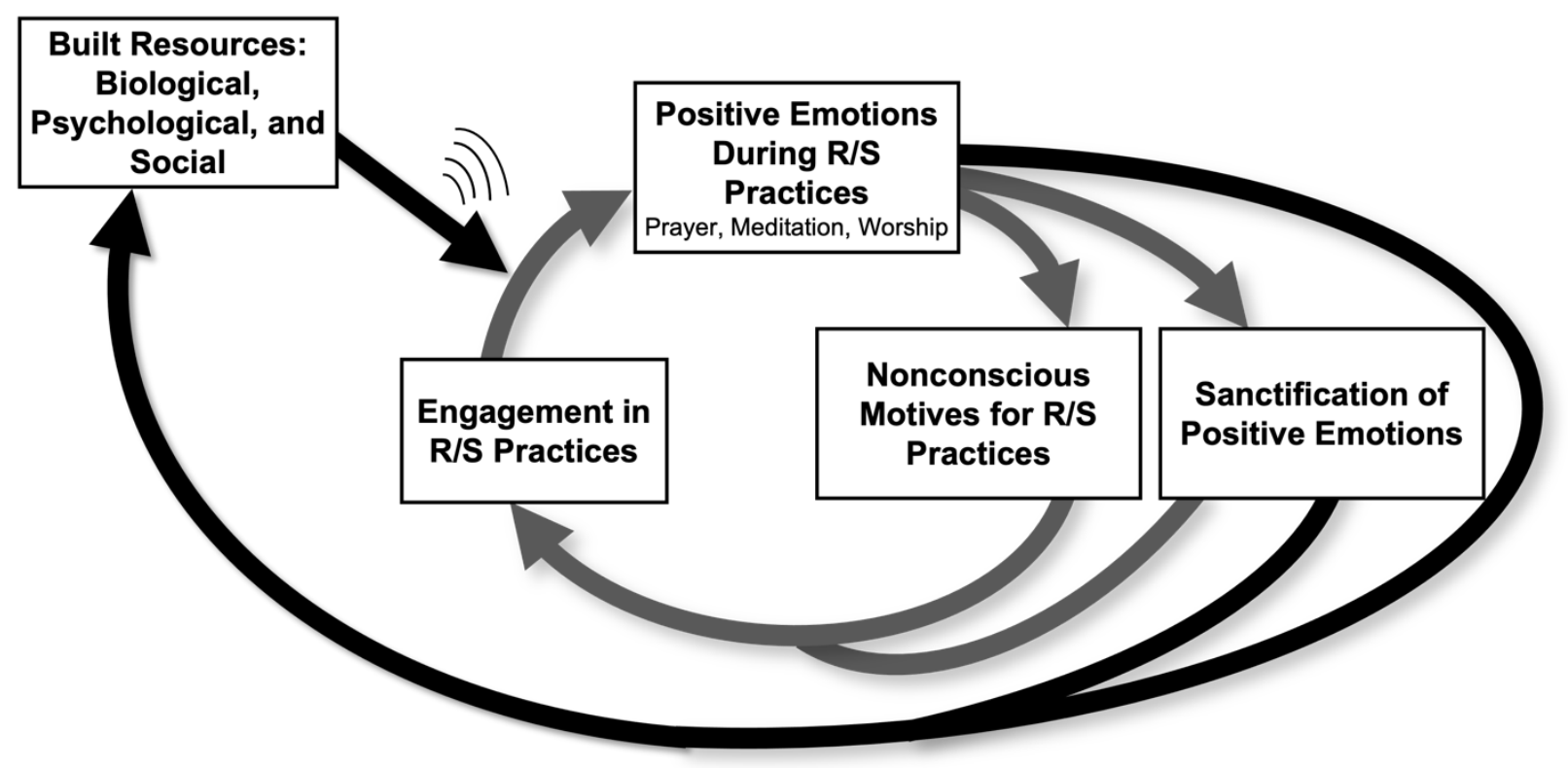

Figure 20.1. Upward Spiral Theory of Sustained R/S Practice.

The Upward Spiral Theory of Sustained R/S Practice (Van Cappellen et al., 2021) is an empirically based explanation of the mechanisms through which positive emotions that are experienced during R/S practices build long-term maintenance of those practices. Of course, pleasant activities are repeated simply because they are pleasant. However, other mechanisms explain the relationship between feeling positive emotions during an activity and repeating that activity, as represented in the light-grey inner loops of the Figure. First, positive emotions create nonconscious motives for engaging in these practices: cues related to the practice start looming larger in the environment and spontaneously pop into attention, increasing their salience in everyday life. Furthermore, positive emotions elicited by R/S practices are imbued with sacredness and connected to a broader (religious) meaning system. These sacred emotions might be more potent in driving engagement (relative to their "secular" versions) because what is considered sacred becomes salient, more powerful, and significant in people's lives, and it further drives their engagement in activities thought to promote this sacredness (Espinola \& 
Badrinarayanan, 2010). Over time, positive emotions felt in everyday life can also be connected back to religion/spirituality in a process of sanctification, acquiring transcendent quality and significance (Pargament \& Mahoney, 2005). Together, these processes energize people's motives for and engagement in R/S practices, providing repeated opportunities for concurrent positive emotional experiences.

Moreover, as represented in the black outer loops in the Figure, positive emotions that are experienced during R/S practices and accumulated over time will gradually build enduring psychological, biological, and social resources (cf. the aforementioned Broaden and Build Theory of Positive Emotions, Fredrickson, 2013), which will further enable the experience of positive emotions in the future. For example, sacred positive emotions experienced during collective worship will over time build social resources such as feelings of belonging within the group, which in turn will facilitate even greater enjoyment while worshipping with this group in the future. Other built resources include savoring (the ability to attend to, appreciate, and enhance positive emotions; Bryant \& Veroff, 2007) or psychological well-being. In a R/S context, further spiritual resources, such as developing positive attitudes toward God (e.g., trust in God, love of God, gratitude toward God), finding spiritual strength (Van Tongeren et al., 2019), meaning (Park et al., 2017), and support (Krause, 2016), may also be gained. This process of building resources that amplify the positive emotional response to repeated practices is responsible for the namesake of the theory, and it creates upward spirals between positive emotions and R/S practices.

In Van Cappellen et al. (2021), we review the available empirical evidence supporting several paths from the model represented in Figure 20.1. Here we only highlight areas that need further empirical studies. In particular, the behavior of meditation has received more empirical 
scrutiny in the context of this theory than the practices of individual and collective worship and prayer. For example, only one correlational study has demonstrated that more frequent expressions of praise and gratitude to God are associated with more frequent worship participation (Schneller \& Swenson, 2013). Additional correlational and experimental studies are needed to test the hypotheses that variations in positive emotions experienced during individual and collective R/S practices (a) create nonconscious motives (e.g., positive spontaneous thoughts, salience) and (b) predict long-term maintenance of the practice. In addition, the specific flavors of positive emotions and their types of sanctification warrant further research.

\section{Conclusion: Practical Implications and Applications}

Positive emotions_-especially self-transcendent positive emotions such as awe, gratitude, love - are valued and experienced frequently by people who identify as R/S and practice religion/spirituality. As reviewed above, these positive emotions are consequential. They not only feel good, but they also have individual and collective benefits. Each effect described above provides another compelling reason for cultivating positive emotions within and outside R/S practices. We describe implications and applications of these findings for three groups of people.

Although not all mental health practitioners may feel comfortable engaging with their client's R/S beliefs, they may still want to consider engaging with their clients' R/S practices. These practices, especially if they are habitual, may provide vehicles for the experience of positive emotions that can, over time, enhance clients' mental health and resilience. In addition, mental health practitioners may want to help clients identify other activities that for them can generate experiences of self-transcendent positive emotions (e.g., nature walks, counting blessings), which in turn may function to build clients' meaning and religion/spirituality. Finally, mental health practitioners can teach their clients useful skills for evoking, maintaining, or 
increasing positive emotions. We suggest that beyond helping clients build skills for coping with negative emotions, practitioners should spend time to helping clients choose activities (and appraise such activities) in ways that support the experience of meaningful positive emotions.

Religious leaders are well-positioned to evoke positive emotions during R/S activities. For example, they can carefully consider the content of the sermon or the prayers they lead, in order to promote the experience of self-transcendent positive emotions (e.g., thanksgiving and love). Music and the physical space in which R/S practices take place are also elements that can be conducive to positive emotions. In addition, the religious leader's own emotions can influence the mood of the entire group (Sy et al., 2005), providing another way to elicit positive emotions. Moreover, other aspects of R/S practices can be considered in order to amplify positive emotions. Collective practices allow for the experience of one person's positive emotions to spread to others (Neumann \& Strack, 2000) and get amplified by the group (cf. collective effervescence, Durkheim, 1912; Páez et al., 2015). We note that this process is facilitated by inperson collective activities because it allows for mimicry and biobehavioral synchrony. We also re-emphasize the role that embodied processes play in the visible expression and communication of positive emotions, as well as in the generation of positive emotions. In sum, we suggest that religious leaders consider strategically infusing positive emotions into their collective and embodied R/S practices, especially in contexts in which emotions can be shared and amplified.

Finally, religiously/spiritually oriented people can make efforts to seek out, notice, and savor the experiences of positive emotions that arise from their R/S practices. According to the research reviewed above, we already know that R/S people highly value the experience of selftranscendent positive emotions. Drawing on this value, R/S people can develop habits of engaging in R/S practices (and other activities) that promote the experience of positive emotions. 
Emotion regulatory skills can facilitate this process, such as demonstrating flexibility in choosing the right activity (e.g., leaving a group that becomes toxic) and developing the ability to attend to, appreciate, and enhance one's own habitual positive activities, as well as the positive experiences in one's life (cf. definition of savoring; Bryant \& Veroff, 2007).

In closing, we highlight the need for more research on the bidirectional relationship between religion/spirituality and positive emotions. On the one hand, future research can focus on expanding our understanding of the ways through which religion/spirituality, and in particular $\mathrm{R} / \mathrm{S}$ practices, foster the experience of positive emotions as well as the implications of experiencing such emotions specifically during religious/spiritual practices. On the other hand, we need much more research on how positive emotions are expressed in and further support R/S beliefs and practices. Overall, while the affective correlates of meditation and collective religious practice have received some attention, we do not yet know as much about the practice of prayer. Finally, these research efforts should continue the recent trend of recruiting culturally and religiously diverse samples of participants and moving beyond convenience samples of mostly Western Christians. We argue that such research is important because it may shed light on processes that ultimately benefit individual and collective good. 


\section{References}

Abernethy, A. D., Kurian, K. R., Uh, M., Rice, B., Rold, L., vanOyen-Witvliet, C., \& Brown, S. (2016). Varieties of spiritual experience: A study of closeness to God, struggle, transformation, and confession-forgiveness in communal worship. Journal of Psychology \& Christianity, 35(1), 9-21.

Aghababaei, N., \& Tabik, M. T. (2012). Gratitude and mental health: Differences between religious and general gratitude in a Muslim context. Mental Health, Religion \& Culture, 16(8), 761-766. https://doi.org/10.1080/13674676.2012.718754

Atran, S., \& Henrich, J. (2010). The evolution of religion: How cognitive by-products, adaptive learning heuristics, ritual displays, and group competition generate deep commitments to prosocial religions. Biological Theory, 5(1), 18-30. https://doi.org/10.1162/BIOT_a_00018

Barrett, L. F., \& Lindquist, K. (2008). The embodiment of emotion. In G. Semin \& E. Smith (Eds.), Embodied grounding: Social, cognitive, affective, and neuroscience approaches (pp. 237-262). Cambridge University Press.

Bryant, F. B., \& Veroff, J. (2007). Savoring: A new model of positive experience. Mahwah, NJ: Lawrence Erlbaum Associates.

Dostoyevsky, F., Robinson, B., \& Garnett, C. (1933). The brothers Karamazov. New York: Halcyon house.

Durkheim, E. (1912). Les formes élémentaires de la vie religieuse [The elementary forms of religious life]. Alcan. 
Ellison, C. G., \& Fan, D. (2008). Daily spiritual experiences and psychological well-being among US adults. Social Indicators Research, 88, 247-271. https://doi.org/10.1007/s11205-007-9187-2

Emmons, R. A., \& Kneetzel, T. T. (2005). Giving thanks: Spiritual and religious correlates of gratitude. Journal of Psychology and Christianity, 24, 140-148.

Espinola, A., \& Badrinarayanan, V. (2010). Consumer expertise, sacralization, and event attendance: A conceptual framework. Marketing Management Journal, 20(1), 145-164.

Fredrickson, B. L. (2013). Positive emotions broaden and build. Advances in Experimental Social Psychology, 47, 1-53. https://doi.org/10.1016/B978-0-12-407236-7.00001-2

Fredrickson, B. L., Boulton, A. J., Firestine, A. M., Van Cappellen, P., Algoe, S. B., Brantley, M. M., Kim, S. L., Brantley, J., \& Salzberg, S. (2017). Positive emotion correlates of meditation practice: A comparison of mindfulness meditation and loving-kindness meditation. Mindfulness, 8(6), 1623-1633. https://doi.org/10.1007/s12671-017-0735-9

Fredrickson, B. L., Cohn, M. A., Coffey, K. A., Pek, J., \& Finkel, S. M. (2008). Open hearts build lives: Positive emotions, induced through loving-kindness meditation, build consequential personal resources. Journal of Personality and Social Psychology, 95(5), 1045-1062. https://doi.org/10.1037/a0013262

Fredrickson, B. L., Mancuso, R. A., Branigan, C., \& Tugade, M. M. (2000). The undoing effect of positive emotions. Motivation and Emotion, 24(4), 237-258. https://doi.org/10.1023/A:1010796329158

Gross, J. J., \& John, O. P. (2003). Individual differences in two emotion regulation processes: Implications for affect, relationships, and well-being. Journal of Personality and Social Psychology, 85(2), 348-362. https://doi.org/10.1037/0022-3514.85.2.348 
Gross, J. J., \& Thompson, R. A. (2007). Emotion regulation: Conceptual foundations. In J. J. Gross (Ed.), Handbook of emotion regulation (pp. 3-24). Guilford Press.

Haidt, J. (2003). The moral emotions. In R. J. Davidson, K. R. Scherer, \& H. H. Goldsmith (Eds.), Handbook of affective sciences (pp. 852-870). Oxford University Press.

Joye, Y., \& Verpooten, J. (2013). An exploration of the functions of religious monumental architecture from a Darwinian perspective. Review of General Psychology, 17(1), 53-68. https://doi.org/10.1037/a0029920

Kim-Prieto, C., \& Diener, E. (2009). Religion as a source of variation in the experience of positive and negative emotions. The Journal of Positive Psychology, 4, 447-460. https://doi.org/10.1080/17439760903271025

Koenig, H., King, D., \& Carson, V. B. (2012). Handbook of religion and health (2 ${ }^{\text {nd }}$ ed.). Oxford University Press.

Krause, N. (2009). Religious involvement, gratitude, and change in depressive symptoms over time. International Journal for the Psychology of Religion, 19(3), 155-172. https://doi.org/10.1080/10508610902880204

Krause, N. (2016). Assessing supportive social exchanges inside and outside religious institutions: exploring variations among Whites, Hispanics, and Blacks. Social Indicators Research, 128(1), 131-146. https://doi.org/10.1007/s11205-015-1022-6

LaFrance, M., \& Mayo, C. (1978). Cultural aspects of nonverbal communication. International Journal of Intercultural Relations, 2(1), 71-89. https://doi.org/10.1016/0147$\underline{1767(78) 90029-9}$ 
Lambert, N. M., Fincham, F. D., Braithwaite, S. R., Graham, S. M., \& Beach, S. R. (2009). Can prayer increase gratitude? Psychology of Religion and Spirituality, 1(3), 139-149. https://doi.org/10.1037/a0016731

Myers, D. G. (2018). Religious engagement and living well. In J. P. Forgas \& R. F. Baumeister (Eds.), The social psychology of living well (pp. 137-160). Routledge.

Neumann, R., \& Strack, F. (2000). "Mood contagion": The automatic transfer of mood between persons. Journal of Personality and Social Psychology, 79(2), 211-223. https://doi.org/10.1037/0022-3514.79.2.211

Páez, D., Rimé, B., Basabe, N., Wlodarczyk, A., \& Zumeta, L. (2015). Psychosocial effects of perceived emotional synchrony in collective gatherings. Journal of Personality and Social Psychology, 108(5), 711-729. https://doi.org/10.1037/pspi0000014

Pargament, K. I., \& Mahoney, A. (2005). Sacred matters: Sanctification as a vital topic for the psychology of religion. The International Journal for the Psychology of Religion, 15(3), 179-198. https://doi.org/10.1207/s15327582ijpr1503_1

Park, C. L., Currier, J. M., Harris, J. I., \& Slattery, J. M. (2017). Trauma, meaning, and spirituality: Translating research into clinical practice. American Psychological Association. https://doi.org/10.1037/15961-000

Ramsay, J. E., Tong, E. M. W., Chowdhury, A., \& Ho, M. R. (2019). Teleological explanation and positive emotion serially mediate the effect of religion on well-being. Journal of Personality, 87(3), 676-689. https://doi.org/10.1111/jopy.12425

Saroglou, V., Buxant, C., \& Tilquin, J. (2008). Positive emotions as leading to religion and spirituality. The Journal of Positive Psychology, 3, 165-173. https://doi.org/10.1080/17439760801998737 
Sauter, D. (2010). More than happy: The need for disentangling positive emotions. Current Directions in Psychological Science, 19(1), 36-40. https://doi.org/10.1177/0963721409359290

Schneller, G. R., \& Swenson III, J. E. (2013). Talking to God: Psychological correlates of prayers of praise and gratitude. Christian Psychology, 39-50.

Smith, C. A., Tong, E. M. W., Ellsworth, P. C. (2014). The differentiation of positive emotional experience as viewed through the lens of appraisal theory. In M. M. Tugade, M. N. Shiota, \& L. D. Kirby (Eds.), Handbook of positive emotions (pp. 11-27). Guilford Press.

Stellar, J. E., Gordon, A. M., Piff, P. K., Cordaro, D., Anderson, C. L., Bai, Y., Maruskin, L. A., \& Keltner, D. (2017). Self-transcendent emotions and their social functions: Compassion, gratitude, and awe bind us to others through prosociality. Emotion Review, 9(3), 200207. https://doi.org/10.1177/1754073916684557

Sy, T., Côté, S., \& Saavedra, R. (2005). The contagious leader: Impact of the leader's mood on the mood of group members, group affective tone, and group processes. Journal of Applied Psychology, 90(2), 295-305. https://doi.org/10.1037/0021-9010.90.2.295

Tsai, J. L., Miao, F. F., \& Seppala, E. (2007). Good feelings in Christianity and Buddhism: Religious differences in ideal affect. Personality and Social Psychology Bulletin, 33, 409-421. https://doi.org/10.1177/0146167206296107

Van Cappellen, P., Saroglou, V., Iweins, C., Piovesana, M., \& Fredrickson, B. L. (2013). Selftranscendent positive emotions increase spirituality through basic world assumptions. Cognition \& Emotion, 27(8), 1378-1394. https://doi.org/10.1080/02699931.2013.787395 
Van Cappellen, P., Toth-Gauthier, M., Saroglou, V., \& Fredrickson, B. (2014). Religion and well-being: The mediating role of positive emotions. Journal of Happiness Studies, 1-21. https://doi.org/10.1007/s10902-014-9605-5

Van Cappellen, P., Saroglou, V., \& Toth-Gauthier, M. (2016). Religiosity and prosocial behavior among churchgoers: Exploring underlying mechanisms. The International Journal for the Psychology of Religion, 26(1), 19-30. https://doi.org/10.1080/10508619.2014.958004

Van Cappellen, P., Ladd, K. L., Cassidy, S., Edwards, M., \& Fredrickson, B. L. (2020). Documenting and measuring the effects of full-body postures associated with positive and negative affect. Manuscript under review.

Van Cappellen, P., Edwards, M. E., \& Fredrickson, B. L. (2021). Upward spirals of positive emotions and religious behaviors. Current Opinion in Psychological Science, 40, 92-98. https://doi.org/10.1016/j.copsyc.2020.09.004

Van Cappellen, P., \& Edwards, M. E. (2021a). The embodiment of worship: Relations among postural, psychological, and physiological aspects of religious practice. Journal for the Cognitive Science of Religion, 6(1-2), 56-79. https://doi.org/10.1558/jcsr.38683

Van Cappellen, P., \& Edwards, M. (2021b). Emotion expression in context: Full body postures of Christian prayer orientations compared to secular emotions. Journal of Nonverbal Behavior. https://doi.org/https://doi.org/10.1007/s10919-021-00370-6

Van Cappellen, P., Cassidy, S., \& Zhang, R. (in press). Religion as an embodied practice: Organizing the various forms and documenting the meanings of Christian prayer postures. Psychology of Religion and Spirituality.

Van Tongeren, D. R., Aten, J. D., McElroy, S., Davis, D. E., Shannonhouse, L., Davis, E. B., \& Hook, J. N. (2019). Development and validation of a measure of spiritual 
fortitude. Psychological Trauma: Theory, Research, Practice, and Policy, 11(6), 588596. https://doi.org/10.1037/tra0000449

Vishkin, A., Ben-Nun Bloom, P., Schwartz, S. H., Solak, N., \& Tamir, M. (2019). Religiosity and emotion regulation. Journal of Cross-Cultural Psychology, 50(9), 1050-1074. https://doi.org/10.1177/0022022119880341

Vishkin, A., Schwartz, S. H., Ben-Nun Bloom, P., Solak, N., \& Tamir, M. (2020). Religiosity and desired emotions: Belief maintenance or prosocial facilitation? Personality and Social Psychology Bulletin, 46(7), 1090-1106.

https://doi.org/10.1177/0146167219895140

Winkielman, P., Niedenthal, P., Wielgosz, J., Eelen, J., \& Kavanagh, L. C. (2015). Embodiment of cognition and emotion. In M. Mikulincer, P. R. Shaver, E. Borgida, \& J. A. Bargh (Eds.), APA handbook of personality and social psychology, Vol. 1. Attitudes and social cognition (pp. 151-175). American Psychological Association.

Winzer, L., \& Gray, R. S. (2019). The role of Buddhist practices in happiness and health in Thailand: A structural equation model. Journal of Happiness Studies, 20, 411-425. https://doi.org/10.1007/s10902-017-9953-z

Yaden, D. B., Haidt, J., Hood Jr, R. W., Vago, D. R., \& Newberg, A. B. (2017). The varieties of self-transcendent experience. Review of General Psychology, 21(2), 143-160. https://doi.org/10.1037/gpr0000102 
Chapter 21: The Scientific Study of Positive Psychology, Religion/Spirituality, and Physical Health

\author{
Kevin S. Masters ${ }^{1,2}$, Julia K. Boehm³ , Jennifer M. Boylan ${ }^{4}$, \\ Kaitlyn M. Vagnini ${ }^{1}$, and Christina L. Rush ${ }^{1}$ \\ ${ }^{1}$ Department of Psychology, University of Colorado Denver \\ ${ }^{2}$ Anschutz Health and Wellness Center, Anschutz Medical Campus, \\ University of Colorado \\ ${ }^{3}$ Department of Psychology, Chapman University \\ ${ }^{4}$ Department of Health and Behavioral Sciences, University of Colorado Denver
}

\begin{abstract}
Author Note
Kevin S. Masters https://orcid.org/0000-0001-6473-7018

Julia K. Boehm https://orcid.org/0000-0001-8360-9935

Jennifer Morozink Boylan https://orcid.org/0000-0003-2597-1367

Kaitlyn M. Vagnini https://orcid.org/0000-0001-6626-4592

Christina L. Rush https://orcid.org/0000-0003-2542-5366

The authors have no known conflicts of interest to disclose.

Correspondence concerning this chapter should be addressed to Kevin S. Masters, Department of Psychology, University of Colorado Denver, Denver, CO 80217-3364, United States. Email: kevin.masters@ucdenver.edu
\end{abstract}




\begin{abstract}
Possible relations between religion, spirituality, positive psychology, and physical health have interested humans throughout history. Only recently have these relations become the object of scientific study. In this chapter, we conducted a nonsystematic, narrative review of the modest but growing empirical literature, which suggests that positive psychological constructs such as life satisfaction, positive affect, purpose/meaning, and optimism are generally predictive of better physical health and functioning. Similarly, religion and spirituality (R/S) variables including religious service attendance, religious/spiritual coping, religious orientation, and prayer have demonstrated relations with better health outcomes. These relations are sometimes complex and possibly influenced by methodological considerations. Several possible pathways to account for these relationships have been proposed, including behavioral processes, social support, and direct physiological pathways. Whether these relations are causal remains a perplexing question to resolve, due to methodological challenges inherent in the nature of the variables themselves and to the practical difficulties of examining these variables via experimental investigation and longitudinal analysis. The possibility that positive psychology constructs may account for associations between R/S and health deserves further exploration, ideally using experimental and prospective longitudinal methods.
\end{abstract}

Keywords: religion, spirituality, positive psychology, health 


\section{Chapter 21:}

\section{The Scientific Study of Positive Psychology, Religion/Spirituality, and Physical Health}

Humans have long been interested in relations among religion/spirituality $(\mathrm{R} / \mathrm{S})$, positive psychological constructs, and physical health. Furthermore, many religions attempt to influence behavior through health-related prescriptions about food choices, sexual activity, substance use, and resting. Similarly, positive psychological constructs have been discussed in light of their presumed benefits on both mental and physical health (Ryff \& Singer, 1998). However, R/S and positive psychological constructs have only recently become objects of scientific investigation of their associations with physical health.

\section{Positive Psychology and Physical Health}

Broadly speaking, positive psychological constructs refer to the thoughts, feelings, behaviors, and characteristics that enhance well-being across time, situations, and cultures (Boehm \& Kubzansky, 2012). They encompass a variety of states and traits, including happiness, purpose in life, and optimism. In the current chapter, we highlight four positive psychological constructs whose relationships with physical health and mortality have been investigated most frequently, as evidenced by systematic reviews and meta-analyses: life satisfaction (evaluating one's life favorably; Pavot \& Diener, 2008), trait positive affect (experiencing frequent positive emotions; Pressman et al., 2019), purpose in life (having valued goals and activities in one's life; McKnight \& Kashdan, 2009), and trait optimism (expecting favorable outcomes in the future; Carver et al., 2010). Although the associations between these constructs and physical health has usually been examined independently, these four constructs tend to be moderately related (Kashdan et al., 2008). Moreover, there is considerable debate about how to assess these and related constructs in health-related studies (Ryff et al., 2020; VanderWeele et al., 2020). This 
nonsystematic, narrative review introduces readers to the most studied positive psychological constructs in relation to physical health.

In this chapter, we review how each of these four constructs is associated with objective health outcomes like morbidity (which refers to all physical symptoms, diseases, and medical conditions) and mortality. Given that how hard it is to conduct experimental investigations of large cohorts across long periods, we will focus on evidence from prospective longitudinal studies with initially healthy cohorts. These studies provide evidence suggesting whether positive psychological constructs are causally related to physical health. Similar studies have been conducted among people with chronic disease at baseline, to see whether positive psychological constructs are associated with reduced risk of secondary disease (e.g., Boehm \& Kubzansky, 2012). However, once disease processes are underway, the impact of positive psychological constructs on physical health may be attenuated (Boehm \& Kubzansky, 2012).

\section{Life Satisfaction}

Life satisfaction is associated with healthier and longer lives. Most reviews include life satisfaction in combination with positive affect and purpose in life (e.g., Steptoe, 2019), which makes it difficult to identify the unique health effects of life satisfaction. However, prospective longitudinal studies of initially healthy cohorts have found that higher levels of life satisfaction are associated with reduced risk of coronary heart disease (Boehm et al., 2011), other chronic diseases, and mortality (Feller et al., 2013; Rosella et al., 2019). For example, in a populationbased study of over 70,000 Canadians, individuals who were very dissatisfied with their lives

had the highest risk of chronic conditions and mortality across approximately 5 years, compared to those who were satisfied (Rosella et al., 2019). This finding is consistent with meta-analytic evidence that greater life satisfaction is associated with $12 \%$ reduced risk of mortality in initially 
healthy individuals (hazard ratio = .88; Martín-María et al., 2017).

\section{Positive Affect}

Several systematic reviews and meta-analyses demonstrate that higher levels of trait positive affect are associated with better health outcomes (e.g., Diener \& Chan, 2011; Pressman et al., 2019). The available evidence indicates positive affect not only has main effect associations with both morbidity and mortality, but it also buffers the association between stress and poor health. The existing evidence also suggests positive affect is associated with reduced risk of mortality, especially in older adults; however, this finding may be due to lower event rates in younger adults (Steptoe, 2019). Yet whether positive affect is associated with mortality independent of subjective health is unclear (Barger et al., 2020; Liu et al., 2016), given that some adjectives used to assess positive affect (e.g., energetic; vigorous) may themselves be indicators of health status. Diener and Chan (2011) report that effect sizes are small to moderate in size $(0.1$ to 0.2 standard deviation differences both in morbidity and mortality outcomes, when comparing low vs. high positive affect). Going forward, the scientific study of positive affect and physical health will be advanced by increased scholarly attention to: (a) utilizing high-quality measures, including measuring both low- (e.g., calm) and high-arousal (e.g., alert) positive affective states and using non-self-report measures (e.g., quantifying emotional-language use), (b) differentiating the temporal focus of assessment (e.g., state vs. trait affect), and (c) exploring and accounting for cultural differences in the value and utility of positive affect (Yoo \& Miyamoto, 2018).

\section{Purpose in Life}

Several prospective studies indicate that higher purpose in life is associated with lower mortality risk (Alimujiang et al., 2019), fewer cardiovascular events, less physician-reported chronic disease (Steptoe \& Fancourt, 2019), less Alzheimer's disease and mild cognitive 
impairment (Boyle et al., 2012), and lower risk of metabolic syndrome (Boylan \& Ryff, 2015). Effect sizes are typically small (e.g., hazard ratio for mortality $=2.43$ for lowest vs. highest purpose in life; Alimujiang et al., 2019) but comparable to traditional biomedical risk factors, such as body mass index (Boylan et al., 2017). Cohen and colleagues (2016) reviewed 10 prospective studies (combined $N>135,000$ ) and found that measures of purpose, meaning, life engagement, and ikigai (i.e., having a reason for being) were associated with a $17 \%$ reduced risk for all-cause mortality and cardiovascular events. Kim and colleagues (2019) updated Cohen's review, replicating the results and noting important future research directions: (a) addressing methodological limitations related to reverse causality (i.e., does being in good health cause higher purpose in life) and appropriate control of confounders, (b) empirically testing behavioral and biological mechanisms underlying the relationship, and (c) testing whether associations between purpose in life and health are consistent across sociodemographic groups.

\section{Optimism}

Optimism is commonly assessed with single items or the multi-item Life Orientation Test (LOT; Scheier et al., 1994). A recent meta-analysis of more than 60 studies examined whether optimism, assessed by the LOT, was associated with physical health outcomes, including biomarkers, disease, hospitalizations, and mortality (Scheier et al., 2021). They found that optimism is modestly associated with better physical health $(r=.03)$ when aggregated across outcomes and accounting for a range of covariates (e.g., sociodemographics, psychosocial confounders). However, some argue the LOT assesses two separate factors- the absence of pessimism and the presence of optimism. Both the absence of pessimism and presence of optimism showed small associations with physical health $(r \mathrm{~s}=.03$ and .01 , respectively; Scheier et al., 2021). Although effects appear small, they reflect associations adjusted for confounding 
factors, including the shared variance between optimism and pessimism and related psychosocial variables. Furthermore, small effect sizes are common when investigating associations between psychosocial factors and physical health. Moreover, small effects at the individual level have outsized impacts at the population level. Scheier et al. (2021) have aptly noted (p. 543): "A onepoint change in the pessimism direction of the pessimism subscale corresponds to an increase of 97,014 deaths from all causes (95\% CI [32,540, 162,641])" (p. 543).

When optimism is assessed with measures other than the LOT, associations with health and longevity remain. A meta-analysis of 15 studies demonstrated that optimism was associated with reduced risk of first-time cardiovascular events and all-cause mortality (Rozanski et al., 2019). Results were similar for men and women, as well as when adjusted for depression, socioeconomic status, and physical activity. Thus, optimism appears to be related to better health, but it is unclear if the active mechanism is optimism, the absence of pessimism, or both.

\section{Religion/Spirituality and Health}

Religion and spirituality (R/S) are considered overlapping constructs with distinct qualities. According to Yeary and colleagues (2020), "spirituality will be defined as one's experiences with the sacred, whereas religion refers to one's involvement in an organized system of beliefs and behaviors related to one's experience with the sacred" (p. 196). R/S constructs are multifaceted and encompass a variety of markers, such as religious service attendance, prayer, and religious/spiritual coping. For example, different types of religious/spiritual coping predict different health outcomes (Ai et al., 2007). To date, self-report measures are widely used when studying R/S and health, and much of the research focuses on religious service attendance. In this section, we consider four R/S indicators whose relation to physical health has been examined frequently: religious service attendance, religious/spiritual coping, religious orientation, and 
prayer. (See Table 18.S2 and Table 25.S2 in this volume for a summary of longitudinal studies of R/S and various mental and physical health outcomes.) As before, this section consists of a nonsystematic, narrative review of important R/S constructs.

\section{Religious Service Attendance}

Consistent evidence suggests that frequency of religious service attendance is associated with longevity and all-cause mortality (VanderWeele, Balboni, \& Koh, 2017). In a review of longitudinal studies of this association, Powell et al. (2003) found the strength of the relationship represented a $30 \%$ reduction in mortality, on average, after adjustment for demographic, socioeconomic, and health-related confounds. More recent high-quality, longitudinal prospective studies (e.g., Idler et al., 2017; Li et al., 2016; Wen et al., 2019) also support the association between service attendance and all-cause mortality. Comparing several meta-analytic studies, Lucchetti et al. (2011) found that effect sizes of attendance with all-cause mortality were similar in magnitude to those for recognized health behaviors such as mammography screening and consumption of fruits and vegetables (i.e., $25 \%$ reduction in mortality for service attendance vs. $26 \%$ reduction in mortality for mammography screening and fruit/vegetable consumption).

Service attendance may also predict cause-specific mortality, such as mortality due to cardiovascular disease (Chida et al., 2009; Shattuck \& Muehlenbein, 2020) or cancer (Li et al., 2016; Wen et al., 2019). However, after adjusting for health risk and demographic variables, not all high-quality studies find an association between attendance and cause-specific mortality (Hummer et al., 1999). Overall, there is strong evidence that attendance is associated with lower all-cause mortality and some evidence that it is also associated with cause-specific mortality. 


\section{Religious/Spiritual Coping}

Religious/spiritual coping assesses how individuals use R/S to cope with distressing life events or stressors. The most widely used measures of religious/spiritual coping are the RCOPE (Pargament et al., 2000) and Brief RCOPE (Pargament et al., 2011). Both versions operationalize religious/spiritual coping into two types. Pargament et al. (2011) explain: "positive religious coping reflects a secure relationship with a transcendent force, a spiritual connectedness with others, and a benevolent world view [whereas] negative religious coping reflects underlying spiritual tensions and struggles within oneself, with others, and with the divine" (p. 51).

There are limited data on religious/spiritual coping and mortality. Using data from the Black Women's Health Study, Vander Weele, Yu, et al. (2017) found that positive religious/spiritual coping led to reduced mortality, but effects were lessened after accounting for other religious/spiritual factors (religious service attendance, prayer, religious/spiritual orientation). There are also limited data on the association between religious/spiritual coping and health, although some studies point to a relationship. For example, positive religious/spiritual coping predicted beneficial outcomes among those with cardiovascular disease (Ai et al., 2007) and was associated with reduced incident hypertension in a large prospective study (Cozier et al., 2018). Negative religious/spiritual coping often predicts adverse outcomes in people diagnosed with cardiovascular conditions (e.g., Ai et al., 2007).

\section{Religious Orientation}

Religious orientation (Allport \& Ross, 1967; Gorsuch, 1994) may have associations with health as well. Extrinsic orientation (i.e., an orientation to religious engagement for social, psychological, material, and physical benefits) is associated with higher levels of depressive symptoms (Smith et al., 2003), and depression is in turn linked to worse physical health 
outcomes. Extrinsic orientation has also been found to be associated with exaggerated blood pressure reactivity to psychological stressors (Masters et al., 2004). In a majority Christian sample, proreligious orientation (i.e., motivation to engage in religion both for its own sake and for its social, psychological, material, and physical benefits) was associated with worse physical functioning, role limitations, and fatigue, compared to intrinsic, extrinsic, and nonreligious orientations (Hunter \& Merrill, 2013). However, in this same study, religious orientation and self-rated health were unassociated.

In other research, intrinsically religious adults (i.e., those motivated to engage in religion for its own sake) had healthier physical responses to stressors (Masters et al., 2004). In another adult sample, intrinsic religious beliefs were also associated with fewer medical complications and shorter hospital stays (Contrada et al., 2004). In a community-dwelling sample, intrinsically oriented and nonreligious people reported the best perceived health and lowest body mass (Masters \& Knestel, 2011). People with intrinsic and proreligious orientations were least likely to smoke tobacco or drink alcohol. In a study of cancer patients, there was a positive association between intrinsic religiousness and physical, functional, and social well-being (Pérez \& Rex Smith, 2015). In sum, intrinsic religious orientation is consistently associated with better health.

\section{Prayer}

Prayer frequency is associated positively with pain and illness coping, but its relations with markers of disease are somewhat dependent on several research design considerations (e.g., the population studied and methodology used). In a review, Moreira-Almeida and Koenig (2008) found evidence for cross-sectional associations between petitionary prayer (i.e., making a request of God) and higher physical pain, which the authors suggest is because people use prayer to ask for help when pain increases. Although prayer has been found to be associated with a higher 
likelihood of hypertension among adults in Chicago (Buck et al., 2009), prayer has also been found to be associated with better long-term post-operative adjustment in patients undergoing open heart surgery (Ai et al., 2010). Prayer was also associated with reduced risk of cognitive decline among midlife Arabic women (Inzelberg et al., 2013). Finally, prayer was associated with better prognosis among patients with advanced cancer (Paiva et al., 2014).

Using experimental designs, prayer has been associated with reduced pain and healthier cardiovascular responses to stress. For instance, in a randomized clinical trial of the effects of prayer on migraine headaches among Muslim patients, pain was significantly lower in the group who received pharmacological treatment plus prayer for 2 months than the group who received only pharmacological treatment for 2 months (Tajadini et al., 2017). Similarly, in a meditation study of migraine sufferers, compared to three other groups (internally focused secular meditation, externally focused secular meditation, progressive muscle relaxation), those who practiced spiritual meditation had greater decreases in the frequency of migraine headaches, anxiety, and negative affect, as well as greater increases in pain tolerance, headache-related selfefficacy, daily spiritual experiences, and existential well-being (Wachholtz \& Pargament, 2008). In a lab study of African American women recalling an incident of racism, prayer was associated with lower stress and diastolic blood pressure (Cooper et al., 2014). Likewise, in a randomized trial of Christians, devotional prayer led to lower blood pressure reactivity to an interpersonal challenge, relative to people in the secular meditation or control group (Masters et al., 2020). In sum, prayer appears to be associated with reduced stress, lower cardiovascular reactivity to stress, and better management of pain. Prayer is thought to encourage proximityseeking to perceived sacred beings that can provide comfort in times of distress (Granqvist, 2020). But relationships between prayer and health are complex because individuals are more 
likely to pray when their health is in decline. The literature would benefit from additional studies with experimental designs, as well prospective studies analyzing the function of prayer for health prior to and after disease onset. Analyzing the circumstances in which people pray, the meaning of prayer for them, study methodology (e.g., cross-sectional vs. longitudinal) are important.

\section{Possible Mechanisms and Pathways}

Given that R/S and positive psychological constructs have relationships with mortality and morbidity, what accounts for these relationships? What are plausible pathways that link R/S and positive psychological constructs with health outcomes, and might these relationships be causal? (See Chapter 18, this volume, for a theory explaining the links between R/S and health.)

There are at least three plausible and empirically supported pathways linking R/S or positive psychological constructs with health. The most well-established is the behavioral pathway. To the extent R/S and positive psychological constructs influence (non)engagement in behaviors that have probabilistic relationships with health outcomes, these constructs may be situated along a behavioral pathway affecting health. In the R/S and health literature, there are several studies demonstrating relationships of R/S with beneficial healthy behaviors (e.g., flu vaccination, cholesterol screening, breast self-exams) and lower likelihood of unhealthy behaviors (e.g., cigarette smoking, excessive alcohol use; Shattuck \& Muehlenbein, 2020; Yeary et al. 2020). Similarly, salubrious associations between positive psychological constructs and health behaviors have been found, including lower smoking rates, greater use of preventative measures (e.g., cancer screenings), better sleep quality, healthier diet, and higher physical activity (Boehm \& Kubzansky, 2012; Boehm et al., 2018; Kim et al., 2014; Steptoe, 2019).

A second pathway routes through social support. There is a well-established relationship between social support and health outcomes (Holt-Lunstad \& Uchino, 2015; Uchino et al., 
2018). If R/S and positive psychological constructs lead to broader and higher-quality social support, they would plausibly influence health via this pathway as well. For instance, one way religious attendance may influence health is through social support. In fact, studies that include social support as a predictor find it explains significant variance in the relationship between service attendance and health, although attendance remains a significant predictor (George et al., 2002; Kim \& VanderWeele, 2019). These studies suggest service attendance may enhance physical health partly by enhancing social support. Moreover, positive psychological constructs are closely linked with social integration and with having relationships with close others who provide support during challenging times (Diener et al., 2018). These social connections may provide support for engaging in preventive behaviors, help buffer the physiological consequences of stress, and protect physical health. Other evidence suggests positive emotions may be associated with healthier outcomes because happier people perceive more favorable social connections, which fosters an upward spiral that contributes to better health (Ramsey \& Gentzler, 2015; see Van Cappellen et al., Chapter 20, this volume).

The third pathway examines influences on physiology via psychological processes that are independent of social support. For example, in their meta-analysis, Shattuck and Muehlenbein (2020) found that prayer and meditation demonstrated several relationships with immune function parameters. Similarly, in a randomized experimental design, Masters and colleagues (2020) found dampened cardiovascular stress reactivity among participants who engaged in Christian devotional prayer, compared to people in the secular meditation and control groups. Previously, Masters et al. (2004) found that intrinsic religious orientation predicted dampened blood pressure reactivity among older adults. Various positive psychological constructs are also directly associated with cardiovascular, metabolic, and immune functioning 
(Boehm \& Kubzansky, 2012; Pressman et al., 2019; Steptoe, 2019). For example, one review found that positive affect was associated with indices of healthy immune functioning, including lower chronic inflammation and more robust vaccination responses (Marsland et al., 2007).

To what extent might these mechanisms and pathways be causal? Causality, in the domain of these constructs, is a complex and problematic topic. First, experimental research, which provides the strongest causal evidence, is difficult to conduct. Although some aspects of these topics (e.g., religious behaviors and practices; state affect) can be studied experimentally, others cannot (e.g., religious beliefs; personal dispositions). Health is also difficult to study in experimental designs, both for practical and ethical reasons. Often, health-related outcomes in experiments are measures of processes that have a relationship with health variables (e.g., cardiovascular reactivity to stress), but they are not themselves indicators of health per se. Prospective longitudinal studies are the next-best methodological option for investigating causality. Yet there are many challenges in conducting longitudinal studies of health, including recruitment, retention, need for repeated measurement, length of time, measurement of potential confounds, and measurement of health variables. Even with exquisitely constructed prospective longitudinal designs, this method of research remains observational and thorny to disentangle from a causal perspective. For example, it is difficult to determine whether life satisfaction predicts better health outcomes or whether better health outcomes predict life satisfaction. Temporal analysis helps but does not eliminate the problem. Epidemiologists encounter these issues and apply the Bradford Hill criteria (1965) when making judgments regarding cause and effect. Those criteria are useful for investigators conducting prospective longitudinal studies of R/S, positive psychological constructs, and health. For further recommendations, see VanderWeele et al. (2016), VanderWeele (2017), and Tsang et al. (Chapter 8, this volume). 


\section{Conclusions and Future Research Directions}

Several conclusions emerge regarding the evidence linking positive psychological constructs to health. First, life satisfaction, positive affect, purpose/meaning in life, and optimism are each associated with better physical health. Although these associations may appear relatively modest, they are comparable to that of other psychological characteristics (Friedman \& Booth-Kewley, 1987). Moreover, physical health outcomes are typically determined by multiple interacting factors (e.g., genetics, environment, behaviors), and statistical adjustments for these many factors can make the effect of any one factor appear small. However, at the population level, small individual effects can have a meaningful impact. In addition, the effect of positive psychological constructs may accumulate over the lifespan (Friedman \& Booth-Kewley, 1987; Kim et al., 2017). That said, theoretically informed research that integrates multiple positive psychological constructs is necessary to discern which constructs are most strongly related to physical health and in which contexts. Some posit that constructs most closely tied to regulatory processes, such as optimism and purpose in life, may be more relevant for health outcomes (Boehm \& Kubzansky, 2012). But a meta-analysis found that effect sizes of links with mortality were comparable across several positive psychological constructs (Martín-María et al., 2017).

Similarly, when considering the relationship between R/S and health, several key points emerged. The most robustly studied aspect of R/S in relation to mortality is religious service attendance, which is robustly positively related to mortality (see Chapter 25 , this volume). The existing studies of religious/spiritual coping and mortality suggest that positive religious/spiritual coping methods are associated with better physical health, whereas negative religious/spiritual coping methods are associated with worse adjustment and physical health. Intrinsic religious orientation is consistently associated with indices of better health. Finally, prayer is associated 
with benefits for coping with pain, as well as with reduced stress and cardiovascular reactivity; however, individuals may be more likely to pray when they are in poor health.

An interesting question regarding health outcomes and physiological processes is whether the effects of R/S on physiological processes can be accounted for by their relationships with positive psychological constructs. For example, religious/spiritual belief is often viewed as a source of meaning in life, and meaning or purpose in life are predictive of decreased mortality and certain favorable biomarkers for health processes (see Park \& Van Tongeren, Chapter 6, this volume). Does meaning or purpose that is based on religious/spiritual perspectives differ in its relationships with health variables? To what extent does R/S account for meaning's documented associations? These and many other important questions await empirical investigation.

Research on physical health in relation to positive psychological constructs and R/S has increased greatly in recent decades. Nevertheless, expanding this empirical investigation into worldwide populations and greater inclusion of different religious/spiritual perspectives or faiths will add significantly to both the depth and breadth of these areas of study. Whether the findings reported in this chapter will be found in future studies of more diverse populations remains to be seen, but future research will add significant nuance and complexity to these expanding fields. 


\section{References*}

Ai, A. L., Ladd, K. L., Peterson, C., Cook, C. A., Shearer, M., \& Koenig, H. G. (2010). Longterm adjustment after surviving open heart surgery: The effect of using prayer for coping replicated in a prospective design. The Gerontologist, 50(6), 798-809.

Ai, A. L., Park, C. L., Huang, B., Rodgers, W., \& Tice, T. N. (2007). Psychosocial mediation of religious coping styles: A study of short-term psychological distress following cardiac surgery. Personality and Social Psychology Bulletin, 33(6), 867-882.

Alimujiang, A., Wiensch, A., Boss, J., Fleischer, N. L., Mondul, A. M., McLean, K., Mukherjee, B., \& Pearce, C. L. (2019). Association between life purpose and mortality among US adults older than 50 years. JAMA Network Open, 2(5), e194270-e194270.

Allport, G. W., \& Ross, J. M. (1967). Personal religious orientation and prejudice. Journal of Personality and Social Psychology, 5(4), 432-443.

Barger, S. D., Broom, T. W., Esposito, M. V, \& Lane, T. S. (2020). Is subjective well-being independently associated with mortality? A 14-year prospective cohort study in a representative sample of 25139 US men and women. BMJ Open, 10(1), e031776e031776.

Boehm, J. K., Chen, Y., Koga, H., Mathur, M. B., Vie, L. L., \& Kubzansky, L. D. (2018). Is optimism associated with healthier cardiovascular-related behavior? Meta-analyses of 3 health behaviors. Circulation Research, 122(8), 1119-1134.

Boehm, J. K., \& Kubzansky, L. D. (2012). The heart's content: The association between positive psychological well-being and cardiovascular health. Psychological Bulletin, 138(4), 655691.

Boehm, J. K., Peterson, C., Kivimaki, M., \& Kubzansky, L. D. (2011). Heart health when life is 
satisfying: Evidence from the Whitehall II cohort study. European Heart Journal, 32(21), $2672-2677$.

Boylan, J. M., \& Ryff, C. D. (2015). Psychological well-being and metabolic syndrome: Findings from the Midlife in the United States national sample. Psychosomatic Medicine, 77(5), 548-558.

Boylan, J. M., Tsenkova, V. K., Miyamoto, Y., \& Ryff, C. D. (2017). Psychological resources and glucoregulation in Japanese adults: Findings from MIDJA. Health Psychology, 36(5), $449-457$.

Boyle, P. A., Buchman, A. S., Wilson, R. S., Yu, L., Schneider, J. A., \& Bennett, D. A. (2012). Effect of purpose in life on the relation between Alzheimer disease pathologic changes on cognitive function in advanced age. Archives of General Psychiatry, 69(5), 499-505.

Buck, A. C., Williams, D. R., Musick, M. A., \& Sternthal, M. J. (2009). An examination of the relationship between multiple dimensions of religiosity, blood pressure, and hypertension. Social Science and Medicine, 68(2), 314-322.

Carver, C. S., Scheier, M. F., \& Segerstrom, S. C. (2010). Optimism. Clinical Psychology Review, 30(7), 879-889.

Chida, Y., Steptoe, A., \& Powell, L. H. (2009). Religiosity/spirituality and mortality: A systematic quantitative review. Psychotherapy and Psychosomatics, 78(2), 81-90.

Cohen, R., Bavishi, C., \& Rozanski, A. (2016). Purpose in life and its relationship to all-cause mortality and cardiovascular events: A meta-analysis. Psychosomatic Medicine, 78(2), $122-133$.

Contrada, R. J., Goyal, T. M., Cather, C., Rafalson, L., Idler, E. L., \& Krause, T. J. (2004). Psychosocial factors in outcomes of heart surgery: The impact of religious involvement and 
depressive symptoms. Health Psychology, 23(3), 227-238.

Cooper, D. C., Thayer, J. F., \& Waldstein, S. R. (2014). Coping with racism: The impact of prayer on cardiovascular reactivity and post-stress recovery in African American women. Annals of Behavioral Medicine, 47(2), 218-230.

Cozier, Y.C., Yu, J., Wise, L.A., VanderWeele, T J., Balboni, T.A., Argentieri, M.A., Rosenberg, L., Palmer, J.R., \& Shields, A.E. (2018). Religious and spiritual coping and risk of incident hypertension in the black women's health study. Annals of Behavioral Medicine, 52(12), 989-998.

Diener, E. \& Chan, M.Y. (2011). Happy people live longer: Subjective well-being contributes to health and longevity. Applied Psychology: Health and Well-Being, 3(1), 1-43.

Diener, E., Seligman, M.E.P., Choi, H., \& Oishi, S. (2018). Happiest people revisited. Perspectives on Psychological Science, 13(2), 176-184.

Feller, S., Teucher, B., Kaaks, R., Boeing, H., \& Vigl, M. (2013). Life satisfaction and risk of chronic diseases in the European Prospective Investigation into Cancer and Nutrition (EPIC)-Germany Study. PLoS ONE, 8(8), e73462.

Friedman, H. S., \& Booth-Kewley, S. (1987). The 'disease-prone personality': A meta-analytic view of the construct. American Psychologist, 42(6), 539-555.

George, L.K., Ellison, C.G., \& Larson, D.B. (2002). Explaining the relationships between religious involvement and health. Psychological inquiry, 13(3), 190-200.

Gorsuch, R. L. (1994). Toward motivational theories of intrinsic religious commitment. Journal for the Scientific Study of Religion, 33(4), 315-325.

Granqvist, P. (2020). Attachment in religion and spirituality: A wider view. Guilford Press.

Hill, A. B. (1965). The environment and disease: Association or causation? Proceedings of the 
Royal Society of Medicine, 58, 295-300.

Holt-Lunstad, J., \& Uchino, B. N. (2015). Social support and health. In K. Glanz, B. Rimer, \& K. Viswanath (Eds). Health behavior: Theory, research and practice ( $5^{\text {th }}$ ed., pp. 183-204). Jossey-Bass/Wiley.

Hummer, R. A., Rogers, R. G., Nam, C. B., \& Ellison, C. G. (1999). Religious involvement and U.S. adult mortality. Demography, 36(2), 273-285.

Hunter, B. D., \& Merrill, R. M. (2013). Religious orientation and health among active older adults in the United States. Journal of Religion and Health, 52(3), 851-863.

Idler, E., Blevins, J., Kiser, M., \& Hogue, C. (2017). Religion, a social determinant of mortality? A 10-year follow-up of the Health and Retirement Study. PLoS ONE, 12(12), e0189134.

Inzelberg, R., Afgin, A. E., Massarwa, M., Schechtman, E., Israeli-Korn, S. D., Strugatsky, R., Abuful, A., Kravitz, E., Farrer, L.A., \& Friedland, R.P. (2013). Prayer at midlife is associated with reduced risk of cognitive decline in Arabic women. Current Alzheimer Research, 10(3), 340-346.

Kashdan, T. B., Biswas-Diener, R., \& King, L. A. (2008). Reconsidering happiness: The costs of distinguishing between hedonics and eudaimonia. The Journal of Positive Psychology, 3(4), 219-233.

Kim, E. S., Delaney, S. W., \& Kubzansky, L. D. (2019). Sense of purpose in life and cardiovascular disease: Underlying mechanisms and future directions. Current Cardiology, 21(11), 1-11.

Kim, E. S., Kubzansky, L. D., Soo, J., \& Boehm, J. K. (2017). Maintaining healthy behavior: A prospective study of psychological well-being and physical activity. Annals of Behavioral Medicine, 51(3), 337-347. 
Kim, E. S., Park, N., Sun, J. K., Smith, J., \& Peterson, C. (2014). Life satisfaction and frequency of doctor visits. Psychosomatic Medicine, 76(1), 86-93.

Kim, E. S., \& VanderWeele, T. J. (2019). Mediators of the association between religious service attendance and mortality. American Journal of Epidemiology, 188(1), 96-101.

Li, S., Stampfer, M. J., Williams, D. R., \& Vanderweele, T. J. (2016). Association of religious service attendance with mortality among women. JAMA Internal Medicine, 176(6), 777785.

Liu, B., Floud, S., Pirie, K., Green, J., Peto, R., \& Beral, V. (2016). Does happiness itself directly affect mortality? The prospective UK Million Women Study. The Lancet, 387(10021), 874-881.

Lucchetti, G., Lucchetti, A. L. G., \& Koenig, H. G. (2011). Impact of spirituality/religiosity on mortality: Comparison with other health interventions. Explore, 7(4), 234-238.

Marsland, A. L., Pressman, S. D., \& Cohen, S. (2007). Positive affect and immune function. Psychoneuroimmunology, 2, 761-779.

Martín-María, N., Miret, M., Caballero, F. F., Rico-Uribe, L. A., Steptoe, A., Chatterji, S., \& Ayuso-Mateos, J. L. (2017). The impact of subjective well-being on mortality: A metaanalysis of longitudinal studies in the general population. Psychosomatic Medicine, 79(5), $565-575$.

Masters, K. S., Emerson IV, R. W., \& Hooker, S. A. (2020). Effects of devotional prayer and secular meditation on cardiovascular response to a faith challenge among Christians. Psychology of Religion and Spirituality, Advance online publication.

Masters, K. S., Hill, R. D., Kircher, J. C., Lensegrav Benson, T. L., \& Fallon, J. A. (2004). Religious orientation, aging, and blood pressure reactivity to interpersonal and cognitive 
stressors. Annals of Behavioral Medicine, 28(3), 171-178.

Masters, K. S., \& Knestel, A. (2011). Religious orientation among a random sample of community-dwelling adults: Relations with health status and health-relevant behaviors. International Journal for the Psychology of Religion, 21(1), 63-76.

McKnight, P. E., \& Kashdan, T. B. (2009). Purpose in life as a system that creates and sustains health and well-being: An integrative, testable theory. Review of General Psychology, $13(3), 242-251$.

Moreira-Almeida, A., \& Koenig, H. G. (2008). Religiousness and spirituality in fibromyalgia and chronic pain patients. Current Pain and Headache Reports, 12(5), 327-332.

Paiva, C. E., Paiva, B. S. R., Yennurajalingam, S., \& Hui, D. (2014). The impact of religiosity and individual prayer activities on advanced cancer patients' health: Is there any difference in function of whether or not receiving palliative anti-neoplastic therapy? Journal of Religion and Health, 53(6), 1717-1727.

Pargament, K. I., Feuille, M., \& Burdzy, D. (2011). The Brief RCOPE: Current psychometric status of a short measure of religious coping. Religions, 2(1), 51-76.

Pargament, K. I., Koenig, H. G., \& Perez, L. M. (2000). The many methods of religious coping: Development and initial validation of the RCOPE. Journal of Clinical Psychology, 56(4), $519-543$.

Pavot, W., \& Diener, E. (2008). The Satisfaction With Life Scale and the emerging construct of life satisfaction. Journal of Positive Psychology, 3(2), 137-152.

Pérez, J. E., \& Rex Smith, A. (2015). Intrinsic religiousness and well-being among cancer patients: the mediating role of control-related religious coping and self-efficacy for coping with cancer. Journal of Behavioral Medicine, 38(2), 183-193. 
Powell, L. H., Shahabi, L., \& Thoresen, C. E. (2003). Religion and spirituality: Linkages to physical health. American Psychologist, 58(1), 36-52.

Pressman, S. D., Jenkins, B. N., \& Moskowitz, J. T. (2019). Positive affect and health: What do we know and where next should we go? Annual Review of Psychology, 70(1), 627-650.

Ramsey, M. A., \& Gentzler, A. L. (2015). An upward spiral: Bidirectional associations between positive affect and positive aspects of close relationships across the life span. Developmental Review, 36, 58-104.

Rosella, L. C., Fu, L., Buajitti, E., \& Goel, V. (2019). Death and chronic disease risk associated with poor life satisfaction: A population-based cohort study. American Journal of Epidemiology, 188(2), 323-331.

Rozanski, A., Bavishi, C., Kubzansky, L. D., \& Cohen, R. (2019). Association of optimism with cardiovascular events and all-cause mortality. JAMA Network Open, 2(9), e1912200.

Ryff, C. D., Boylan, J. M., \& Kirsch, J. A. (2020). Disagreement about recommendations for measurement of well-being. Preventive Medicine, 139, 106049.

Ryff, C. D., \& Singer, B. (1998). The contours of positive human health. Psychological Inquiry, 9(1), 1-28.

Scheier, M. F., Carver, C. S., \& Bridges, M. W. (1994). Distinguishing optimism from neuroticism (and trait anxiety, self-mastery, and self-esteem): A reevaluation of the Life Orientation Test. Journal of Personality and Social Psychology, 67(6), 1063-1078.

Scheier, M. F., Swanson, J. D., Barlow, M. A., Greenhouse, J. B., Wrosch, C., \& Tindle, H. A. (2021). Optimism versus pessimism as predictors of physical health: A comprehensive reanalysis of dispositional optimism research. American Psychologist, 76(3), 529-548.

Shattuck, E. C., \& Muehlenbein, M. P. (2020). Religiosity/spirituality and physiological markers 
of health. Journal of Religion and Health, 59(2),1035-1054.

Smith, T. B., McCullough, M. E., \& Poll, J. (2003). Religiousness and depression: Evidence for a main effect and the moderating influence of stressful life events. Psychological Bulletin, 129(4), 614-636.

Steptoe, A. (2019). Happiness and health. Annual Review of Public Health, 40(1), 339-359.

Steptoe, A., \& Fancourt, D. (2019). Leading a meaningful life at older ages and its relationship with social engagement, prosperity, health, biology, and time use. Proceedings of the National Academy of Sciences of the United States of America, 116(4), 1207-1212.

Tajadini, H., Zangiabadi, N., Divsalar, K., Safizadeh, H., Esmaili, Z., \& Rafiei, H. (2017). Effect of prayer on intensity of migraine headache: A randomized clinical trial. Journal of Evidence-Based Complementary and Alternative Medicine, 22(1), 37-40.

Uchino, B.N., Trettevik, R., Kent de Grey, R.G., Cronan, S., Hogan, J., \& Baucom, B.R.W. (2018). Social support, social integration, and inflammatory cytokines: A meta-analysis. Health Psychology, 37(5), 462-471.

VanderWeele, T. J. (2017). Outcome-wide epidemiology. Epidemiology, 28(3), 399-402.

VanderWeele, T.J., Jackson, J.W., \& Li, S. (2016). Causal inference and longitudinal data: A case study of religion and mental health. Social Psychiatry and Psychiatric Epidemiology, 51(11), 1457-1466.

VanderWeele, T. J., Balboni, T. A., \& Koh, H. K. (2017). Health and spirituality. Journal of the American Medical Association, 318(6), 519-520.

VanderWeele, T. J., Trudel-Fitzgerald, C., Allin, P., Farrelly, C., Fletcher, G., Frederick, D. E., Hall, J., Helliwell, J. F., Kim, E. S., Lauinger, W. A., Lee, M. T., Lyubomirsky, S., Margolis, S., McNeely, E., Messer, N., Tay, L., Viswanath, V., Węziak-Białowolska, D., \& 
Kubzansky, L. D. (2020). Current recommendations on the selection of measures for wellbeing. Preventive Medicine, 133, 106004.

VanderWeele, T. J., Yu, J., Cozier, Y. C., Wise, L., Argentieri, M. A., Rosenberg, L., Palmer, J. R., \& Shields, A. E. (2017). Attendance at religious services, prayer, religious coping, and religious / spiritual identity as predictors of all-cause mortality in the Black Women's Health Study. American Journal of Epidemiology, 185(7), 515-522.

Wachholtz, A. B., \& Pargament, K. I. (2008). Migraines and meditation: Does spirituality matter? Journal of Behavioral Medicine, 31(4), 351-366.

Wen, W., Schlundt, D., Andersen, S. W., Blot, W. J., \& Zheng, W. (2019). Does religious involvement affect mortality in low-income Americans? A prospective cohort study. $B M J$ Open, 9(7), e028200.

Yeary, K. H. K., Alcaraz, K. I., Ashing, K. T., Chiu, C., Christy, S. M., Felsted, K. F., Lu, Q., Lumpkins, C. Y., Masters, K. S., Newton, R. L., Park, C. L., Shen, M. J., Silfee, V. J., Yanez, B., \& Yi, J. (2020). Considering religion and spirituality in precision medicine. Translational Behavioral Medicine, 10(1), 195-203.

Yoo, J., \& Miyamoto, Y. (2018). Cultural fit of emotions and health implications: A psychosocial resources model. Social and Personality Psychology Compass, 12(2), e12372. *See the supplemental material (Appendix 21.S1) for a complete reference list that includes dois. 


\title{
Chapter 22
}

\section{The Scientific Study of Positive Psychology, Religion/Spirituality, and Mental Health}

\author{
Edward P. Shafranske \\ Graduate School of Education and Psychology, Pepperdine University
}

\begin{abstract}
Author Note
Edward P. Shafranske https://orcid.org/0000-0002-3354-1642

The author has no known conflicts of interest to disclose.

The author wishes to thank Edward (Ward) B. Davis for his editorial support and
\end{abstract} contributions to fine-tuning this chapter.

Correspondence concerning this chapter should be addressed to Edward P. Shafranske, Graduate School of Education and Psychology, Pepperdine University, 18111 Von Karman Avenue, Room 209, Irvine, CA, United States. Email: eshafran@pepperdine.edu

Citation:

Shafranske, E. P. (in press). The scientific study of positive psychology, religion/spirituality, and mental health. In E. B. Davis, E. L. Worthington, Jr., \& S. A. Schnitker (Eds.), Handbook of positive psychology, religion, and spirituality. Springer Nature. 


\begin{abstract}
In the past 20 years, over 11,000 published articles have examined the relationship between religion and spirituality $(\mathrm{R} / \mathrm{S})$ and mental health. This chapter summarizes that literature, drawing on recent meta-analyses and narrative reviews while focusing on mood, anxiety, and trauma-related disorders. Complex associations exist between R/S and mental health, with the preponderance of the research suggesting generally modest, positive effects. The impacts of religious/spiritual involvement, meaning making, religious coping, and spiritual struggles are considered. Following this review, the psychological processes in R/S are examined from the perspective of positive psychology. The interrelated contributions of positive emotion, virtues, and meaning making are identified as essential aspects of $\mathrm{R} / \mathrm{S}$ that promote well-being and are associated with mental health. Commonalities between religion and spirituality are found in their capacities to evoke positive emotions, support meaning making, and foster the cultivation of virtues. The intersections between secular (positive psychology) and sacred (religious/spiritual) pathways to mental health are highlighted, an integrative conceptual model is proposed, and recommendations for future research are offered.
\end{abstract}

Keywords: religion, spirituality, positive psychology, mental health 


\section{Chapter 22}

\section{The Scientific Study of Positive Psychology, Religion/Spirituality, and Mental Health}

In the past 20 years, over 11,000 journal articles have been published on the relationship between religion and spirituality (R/S) and mental health. This chapter presents a summary of this literature and discusses the health contributions of R/S via the lens of positive psychology.

Religion and spirituality are complex, interrelated phenomena. Historically, within the field of the psychology of R/S, religion referred to an individual's feelings, acts, and experiences concerning the divine (James, 1902/1982); however, the term later became associated with organized traditions of beliefs and practices. Religion served as an overarching construct involving individual and collective forms of religiousness. Today, both in the public's view and in academic study, religion denotes religious/spiritual involvement in institutional contexts, and spirituality refers to individualized, noninstitutional, or nontraditional beliefs and practices (Pargament et al., 2013; see Appendix 22.S1, for more discussion of these conceptual concerns).

\section{An Overview of the Empirical Research}

Several meta-analyses and narrative reviews on R/S and mental health have been conducted over the past 20 years (see Appendix 22.S2 for summaries), and those form the basis of this chapter's discussion. For instance, an exhaustive examination of over 3,000 quantitative studies found R/S generally was positively associated with better mental health and adaptation (Koenig et al., 2012). A recent review concluded R/S is strongly tied (in both positive and negative ways) to depression, suicidality, anxiety, psychosis, obsessions/compulsions, addictions, and other areas of mental health (Rosmarin \& Koenig, 2020, p. xvii; see also Koenig, 2018 and Park \& Slattery, 2013). Consistent with previous findings, meta-analyses of 48 longitudinal studies (Garssen et al., 2021, p. 13) and 67 studies from the German-speaking area 
(Hodapp \& Zwingmann, 2019, p. 1970) showed modest but significant associations between R/S and better mental health.

\section{A Closer Look at Mood, Anxiety, and Trauma-Related Disorders}

Due to space constraints, this chapter's review was narrowed to mood, anxiety, and trauma-related disorders. This narrowed focus took into consideration the mental disorders with the highest global prevalence rates (Dattani et al., 2021), the clinical features about which substantive research on R/S has been conducted, and the conditions with a strong theoretical underpinning for the relevance of $\mathrm{R} / \mathrm{S}$.

\section{Mood Disorders}

Most empirical studies have found positive effects of R/S on mood disorders (Mosqueiro et al., 2021), including lower depression and faster remission among more religious individuals (Bonelli et al., 2012; Koenig et al., 2012). However, these associations are modest, and R/S does not universally predict salutary outcomes. About half the reviewed prospective studies found a positive association between R/S and a better course of depression; however, $41 \%$ demonstrated no significant association, and $10 \%$ indicated more depression or mixed results (Braam \& Koenig, 2019). Braam and Koenig (2019) found that, although most patients who used religious coping reported positive outcomes (Ano \& Vasconcelles, 2005), religious coping was inversely correlated with depression in more than one-third of the studies. Spiritual struggles, doubts, and lack of meaning exacerbated depression and negatively impact coping (Rosmarin et al., 2014; Exline et al., 2011; Pargament et al., 2005), and the use of negative religious coping was almost universally associated with greater depression (Bockrath et al., 2021; Mosqueiro et al., 2020).

\section{Anxiety Disorders}


Associations between anxiety and R/S are complex, and findings are mixed. Engagement in religious/spiritual behaviors are poor predictors of anxiety, but religious/spiritual beliefs significantly influence mental health. Anxiety is inversely related to belief in a benevolent, loving God and to a secure perceived relationship with God (Rosmarin \& Leidl, 2020; Cherniak et al., 2021). Conversely, mistrust in God strongly predicts stress and anxiety (Rosmarin et al., 2009), a finding replicated in a clinical study in which reduction of mistrust in God over a 2week period was associated with decreased stress and worry (Rosmarin et al., 2010).

\section{Trauma-Related Disorders}

The consequences of trauma exposure are multifaceted and unique to each person, and trauma may indeed result in the development of one or more mental disorder. Trauma can shatter fundamental beliefs (Janoff-Bulman, 1992), evoke spiritual struggles (Pomerleau et al., 2020), and thereby lead to distress (Edmondson et al., 2011). Potentially traumatic experiences often test a person's R/S; they can strengthen or weaken someone's religious/spiritual beliefs, trust in God, or connections within a faith community (Pargament, 2007; Park et al., 2017).

Recovery from trauma exposure is complex and may involve the survivor using religious coping methods to find meaning, gain mastery and control, feel comfort from God, nurture intimacy with God and others, and experience perceived life transformation (Pargament et al., 2013). Religious/spiritual involvement has generally been found to be associated with better mental health outcomes following trauma and adversity (Chen \& Koenig, 2006; Kucharska, 2020; Shaw et al., 2005), and religious meaning making is associated with postdisaster resilience (Park, 2016). A meta-analysis of studies involving the U.S. military found that R/S was associated with lower PTSD symptoms (Rinehart, 2015). The use of prayer can offer a form of trauma disclosure that is linked to lower PTSD and depression (Tait et al., 2016), and collective 
religious rituals provide social cohesion in moments of elevated threat and, for some individuals, can lead to enhanced well-being (Ladd \& Spilka, 2013).

Although R/S is often a dimension of positive posttraumatic adaptation or even perceived posttraumatic growth (Ai et al., 2013; Tedeschi \& Moore, 2021), this is not universally the case. Religious distress mediates (casually explains) PTSD symptoms over time (Harris et al., 2012), and negative religious coping and spiritual struggles (e.g., turmoil with God; Currier et al., 2014, 2015,2019 ) are linked to negative aftereffects such as depression and anxiety (Ano \& Vasconcelles, 2005; Bockrath et al., 2021; Exline \& Rose, 2013). Trauma exposure can negatively impact R/S and weaken the potentially positive contributions of religious/spiritual beliefs, support, coping, and resources (Park et al., 2017). Most people do not change their R/S beliefs after trauma exposure, but significant changes can occur, such as survivors either increasing or decreasing their religious beliefs or practices (Falsetti et al., 2003; Leo et al., 2021).

\section{Positive Psychology, Religion, and Spirituality}

Positive psychology reorients psychological research and practice from psychopathology to well-being (Seligman, 2019) — from "What is wrong?" to "What works, what is right, and what is improving?” (Sheldon \& King, 2001, p. 216). It offers a place in psychology for building positive qualities and human flourishing (Seligman \& Csikszentmihalyi, 2000). Simply put, "positive psychology is the scientific study of optimal human functioning" (Linley et al., 2006, p.

8). It provides a lens to understand the processes and mechanisms that are embedded in R/S and contribute to mental health outcomes (see also MacDonald, Chapter 5, this volume).

\section{Positive Emotions}

Positive emotions signal well-being and set the stage for optimal functioning by broadening a person's momentary thought-action repertoires and building enduring personal 
resources (Fredrickson, 2001, 2012). These personal resources that are accrued across moments of positivity are durable and impact future behavior (Fredrickson \& Losada, 2005, p. 2). Experiences of positive affect undo lingering negative emotions, fuel resiliency, and build personal resources that contribute to resilience and aid in coping (Fredrickson, 2012), thereby contributing to better mental health (see Van Cappellen et al., Chapter 20, this volume).

R/S provides experiences that elicit positive (or negative) emotion. Geertz (1973) observed religion to be "a system of symbols which acts to establish powerful, persuasive, and long-lasting moods and motivations" (p. 70, emphasis added). Religious/spiritual experiences, whether in communal gatherings of worship, private moments of prayer or reflection, or personal or interpersonal experiences of self-transcendent emotions (e.g., awe and gratitude), often evoke powerful moods and positive emotions (Van Cappellen et al., 2021). The broaden-and-build theory provides a causal model of the R/S processes and mechanisms that contribute to mental health (Fredrickson, 2002, 2016; see Van Cappellen et al., Chapter 20, this volume).

Repeated experiences of positive emotion within religious/spiritual practices are selfreinforcing and contribute to "an upward spiral in which positive emotions and the broadened thinking they engender also influence one another reciprocally, leading to appreciable increases in emotional well-being over time” (Fredrickson, 2001, p. 223). Van Cappellen et al.'s (2021) Upward Spiral Theory of Sustained Religious Practice builds on this model (Van Cappellen et al., Chapter 20, Figure 20.1, this volume). Long-term adherence to religious/spiritual behaviors is also reinforced by regular experiences of optimism and hope (Ciarrocchi et al., 2008) and selftranscendent positive emotions like awe, gratitude, love, and peace (Van Cappellen et al., 2016).

Human flourishing has been found to be associated with participation in religious communities, through shared values and practices, which are reinforced by social ties and norms 
(Van Cappellen et al., 2017; VanderWeele, 2017). Collective religious/spiritual practices and sacred rituals may evoke feelings of unity and shared identity, including positivity resonance (Fredrickson, 2016), which can contribute to individual well-being and foster commitments to others. The experience of awe in a religious group setting has been found to spark a willingness to self-sacrifice (Naclerio \& Van Cappellen, 2021), which may build an enduring virtue over time. Position emotion evoked by religious/spiritual involvement contributes to well-being and sets in motion an upward spiral of experiences that can nurture mental health and flourishing.

\section{Virtues}

Virtues, together with strengths, are considered the essential "psychological ingredients... that lead to a good life" (Linley et al., 2006, p. 7). The consideration of virtue brings positive psychology into correspondence (or at least in the neighborhood) with religion and philosophy, because each weighs in on the question, "What is good for human beings as the particular creatures that we are"? (Fowers, 2005, p. 5; see also Ratchford et al., Chapter 4, this volume). The answer to this question transverses positive psychology's considerations of optimal functioning, religion's conceptions of meaning and purpose, and philosophy's grappling with the intrinsic worth in human life. Positive psychology cannot in itself answer this existential question; however, it can closely study human experiences and their impacts on subjective and objective experiences of well-being. Well-being, in the framework of positive psychology, goes beyond the absence of psychological symptoms or human languishing and sets its sights on flourishing. To flourish means not only to feel good but to do good, excel in daily life, and contribute to the world (Fredrickson \& Losada, 2005; Keyes, 2002; see MacDonald, Chapter 5, this volume). Flourishing nurtures both individual and civic virtues, and it moves individuals toward better citizenship (Seligman \& Csikszentmihalyi, 2000). Although coming from different 
paradigms, positive psychology and religion conjoin in asserting that a virtuous life promotes flourishing and leads to fullness of human potential. Virtue ethics provides practical wisdomthe ability to make wise decisions in light of the situation and what specific action is good for the person (Ciarrocchi, 2012; Fowers, 2005).

Psychological studies (see Washington-Nortey et al., Chapter 23, this volume; Cauble et al., Chapter 24, this volume; Long \& VanderWeele, Chapter 25, this volume) provide scienceinformed knowledge of virtues that are aligned with the teachings and practices of the major religions (Walsh, 1999) and represent a rapprochement with classical sources of human strength (McCullough \& Snyder, 2000). These studies illuminate the processes and mechanisms that contribute to mental health, and they offer practical guidance to address psychological problems in clinical practice (Jankowski et al., 2020; see also Captari et al., Chapter 26, this volume).

The development of virtues does not occur in a vacuum but rather develops within a relational context. "One's character is co-constructed from the very beginning” (Reilly \& Narvaez, 2018, p. 54) and typically relies upon observing virtuous members of the community, who serve directly or indirectly as models or mentors. R/S communities, given their shared beliefs, moral commitments, and private and collective practices, may serve as a primary mechanism to nurture a virtuous life (see Wang et al., Chapter 29, this volume). Self-regulation plays an integral role in cultivating virtues, achieving purposeful goals, and fostering well-being (Carver \& Scheier, 1998, 2017). R/S generally supports such self-control, and some forms of religious involvement promote self-regulation (McCullough \& Willoughby, 2009). The results of longitudinal and experimental research have found that "[religious] rituals (most notably, prayer), along with exposure to religious environments and institutions in the real world (e.g., 
religious schooling) influence self-control on the scale of weeks, months, and years" (Marcus \& McCullough, 2021, p. 167).

Religious/spiritual involvement supports the development of a virtuous life by instilling values, establishing meaningful goals, and encouraging optimism through positive emotions, private and communal practices, and community support through shared beliefs, values, and

collective rituals. Although not without challenges, the quest for a virtuous and flourishing life is often aligned with processes that foster and nurture well-being.

\section{Meaning}

Finding out what really matters in life (meaning making) and holding onto it in the face of adversity (meaning maintenance) contributes to well-being and mental health. Meaning in life is defined as "the extent to which one's life is experienced as making sense, as being directed and motivated by valued goals, and as mattering in the world" (George \& Park, 2016, p. 206; Park, 2013; Park \& Van Tongeren, Chapter 6, this volume). It offers purpose, significance, and coherence (Martela \& Steger, 2016; Heintzelman \& King, 2014) and anchors processes essential to a good life (Linley et al., 2006). Eudaimonia incorporates meaningful goal-directedness and complements hedonia as essential to self-fulfillment and flourishing (Disabato et al., 2016). Spiritual strivings, which involve the search for the sacred, orient meaning making to ultimate concerns, and as Emmons (1999) observed, "When it comes to psychological wellbeing, what people are striving for — the content of their aims and ambitions—does matter" (p. 49). Religious/spiritual belief systems provide global meaning (Park, 2013), infusing an ultimate (and sanctified) vision of what people should be striving for (Pargament \& Park, 1995). They offer a "sacred canopy" (Berger, 1967) and pathway to a good life. Religion supplies the grand metanarratives linking the believer to a universe and God(s) that transcend the individual. Global 
meaning influences the lived experiences of daily life in which ordinary encounters and stressful events evoke feelings and are interpreted. Involvement in R/S cultivates positive emotions and virtues through shared beliefs, commitments, and private and communal practices, thereby promoting well-being and flourishing. When an individual is flourishing-living consistent with their global meaning and experiencing positive emotions and well-being - the risk of anxiety, depression, and other mental health difficulties are reduced (Schotanus-Dijkstra et al., 2017).

Life is rarely a straight-line, upward journey of flourishing, however. Some people experience unstable meaning — the unbearable lightness of meaning-leading to threats to wellbeing, which are associated with higher levels of negative affect, including depression and anxiety (Steger \& Kashdan, 2013). If spiritual struggles (Exline \& Rose, 2013) and roadblocks to meaning are encountered, negative religious coping may result in negative psychological adjustment (Ano \& Vasconcelles, 2005; Bockrath et al., 2021; Smith et al., 2003).

Adversity and, at times trauma (which is all too common ${ }^{6}$ ), may also challenge global meaning and negatively impact well-being. Meaning making plays an important role when living with severe, persistent mental illness, such as schizophrenia or bipolar illness (De Fazio et al., 2015; Huguelet, 2017). Reconstituting global meaning can counter the negative psychological impact of adversity, because "meaning is a tool for adaptation, for controlling the world, for selfregulation, and for belongingness," and "indeed it is the best all-purpose tool on the planet" (Baumeister, 1991, pp. 357-358). Reengagement in global meaning is often bolstered by holding firm to religious/spiritual beliefs and practices, with the encouragement of a faith community.

\footnotetext{
${ }^{6}$ World Health Organization surveys in 24 countries found that over 70 percent of respondents had experienced at least one lifetime traumatic event, with an average exposure of 3.2 traumas per capita (Kessler et al., 2017).
} 


\section{The Intersections of Secular and Sacred Pathways and Mental Health}

$\mathrm{R} / \mathrm{S}$ seen through the lens of positive psychology share in its principles and jointly influence subjective well-being and mental health. Positive psychology reflects a secular approach in which positive emotions, fueled by hedonia and eudaimonia, and the cultivation of virtues, promote well-being to achieve optimal human functioning. R/S incorporate many of the processes and mechanisms identified by positive psychology, notably positive emotion, virtues, and meaning making, but they anchor well-being and flourishing in the search for the sacred.

Involvement in religion draws upon shared beliefs, sacred texts, and traditions, as well as established individual and collective practices and rituals. It also often provides engagement with a faith community of like-minded believers (see Wang et al., Chapter 29, this volume). Meaning making is sustained by shared worldviews, which offer beliefs about human suffering and provide a foundation for living a good and virtuous life. The synergy of shared beliefs, practices, and interpersonal and collective experiences potentially spark positive emotion and influence establishment and attainment of meaningful goals and positive mental health. Well-integrated religious faith provides coping resources to lessen anxiety and depression and to mitigate the potential impacts of trauma. However, religious doubt, negative religious coping, and spiritual struggles can exacerbate anxiety, depression, and ennui, negatively impacting mental health.

Spirituality, like religion, involves meaning making and includes spiritual beliefs, practices, and virtues that provide an orientation to well-being and to the sacred (however conceived). Although personal spirituality is not necessarily wedded to a religious institution, a person may draw upon religious influences within their broader culture or community. As with religious involvement, well-integrated spirituality provides resources to address the challenges faced in life, which impact well-being and mental health (MacDonald, Chapter 5, this volume). 
The pathways of positive psychology, religion, and spirituality intersect in their shared ambition to assist the individual in living a good life (see Appendix 22.S3 for some case illustrations). Positive emotions, virtues, and meaning making are often integral to optimal functioning, and as such, they routinely affect well-being and mental health. See Figure 22.1 for a proposed integrative conceptual model of religious/spiritual pathways to mental health.

Future studies on R/S and mental health will be advanced particularly by the use of: (a) longitudinal and experimental designs to allow for the investigation of causal associations between R/S and mental health; (b) designs that capture the dynamic nature of R/S (e.g., repeated measures over a period of time); (c) measures that assess the dimensions of R/S rather than reliance on single-item global measures; and (d) cultural and intersectional approaches (see Tsang et al., Chapter 8, this volume; Mattis, Chapter 9, this volume).

\section{Concluding Remarks}

"There are only two days of the year that nothing can be done. One is called yesterday and the other is called tomorrow, so today is the right day to love, believe, do, and mostly live." - The Dalai Lama

Religion and spirituality offer pathways to living a good life, flourishing, well-being, and mental health. We have seen that the associations between R/S and mental health are complex, and, for some, R/S is associated with heightened anxiety, depression, and struggle. Positive psychology furnished not only its own approach to optimal functioning but also illuminated many of the psychological processes that are embedded in R/S and contribute to their vibrancy. Religion, spirituality, and positive psychology point to "the farther reaches of human nature" (Maslow, 1971) and to the possibility of living a meaningful and virtuous life and with each day inspired "to love, believe, do, and mostly live." 


\section{References}

Ai, A. L., Richardson, R., Plummer, C., Ellison, C. G., Lemieux, C., Tice, T. N., \& Huang, B. (2013). Character strengths and deep connections following Hurricanes Katrina and Rita: Spiritual and secular pathways to resistance among volunteers. Journal for the Scientific Study of Religion, 52(3), 537-556. https://doi.org/10.1111/jssr.12043

Ano, G. G., \& Vasconcelles, E. B. (2005). Religious coping and psychological adjustment to stress: A meta-analysis. Journal of Clinical Psychology, 61(4), 461-480. https://doi.org/10.1002/jclp.20049

Baumeister, R. F. (1991). Meanings of life. Guilford Press.

Berger, P. L. (1967). The sacred canopy: Elements of a sociological theory of religion. Anchor Books.

Bockrath, M. F., Pargament, K. I., Wong, S., Harriott, V. A., Pomerleau, J. M., Homolka, S. J., Chaudhary, Z. B., \& Exline, J. J. (2021). Religious and spiritual struggles and their links to psychological adjustment: A meta-analysis of longitudinal studies. Psychology of Religion and Spirituality. Advance online publication. https://doi.org/10.1037/rel0000400

Bonelli, R., Dew, R. E., Koenig, H. G., Rosmarin, D. H., \& Vasegh, S. (2012). Religious and spiritual factors in depression: Review and integration of the research. Depression Research and Treatment. Article ID 962860. https://doi.org/10.1155/2012/962860

Bonelli, R. M., \& Koenig, H. G. (2013). Mental disorders, religion and spirituality 1990 to 2010: A systematic evidence-based review. Journal of Religion and Health, 52(2), 657-673. https://doi.org/10.1007/s10943-013-9691-4. 
Braam, A. W., \& Koenig, H. G. (2019). Religion, spirituality and depression in prospective studies: A systematic review. Journal of Affective Disorders, 257, 428-438. https://doi.org/10.1016/j.jad.2019.06.063

Carver, C. S., \& Scheier, M. F. (1998). On the self-regulation of behavior. Cambridge University Press. https://doi.org/10.1017/CBO9781139174794

Carver, C. S., \& Scheier, M. F. (2017). Optimism, coping, and well-being. In C. L. Cooper \& J. C. Quick (Eds.), The handbook of stress and health: A guide to research and practice. (pp. 400-414). Wiley Blackwell. https://doi.org/10.1002/9781118993811.ch24

Chen, Y., \& Koenig, H. (2006). Traumatic stress and religion: Is there a relationship? A review of empirical findings. Journal of Religion and Health, 45(3), 371-381. https://doi.org/10.1007/s10943-006-9040-y

Cherniak, A. D., Mikulincer, M., Shaver, P. R., \& Granqvist, P. (2021). Attachment theory and religion. Current Opinion in Psychology, 40(1), 126-130. https://doi.org/10.1016/j.copsyc.2020.08.020

Ciarrocchi, J. (2012). Positive psychology and spirituality: A virtue-informed approach to wellbeing. In L. Miller (Ed.), Oxford handbook of psychology and spirituality. (pp. 425-436). Oxford University Press. https://doi.org/10.1093/oxfordhb/9780199729920.013.0027

Ciarrocchi, J. W., Dy-Liacco, G. S., \& Deneke, E. (2008). Gods or rituals? Relational faith, spiritual discontent, and religious practices as predictors of hope and optimism. Journal of Positive Psychology, 3(2), 120-136. https://doi.org/10.1080/17439760701760666

Currier, J. M., Drescher, K. D., \& Harris, J. I. (2014). Spiritual functioning among veterans seeking residential treatment for PTSD: A matched control group study. Spirituality in Clinical Practice, 1(1), 3-15. https://doi.org/10.1037/scp0000004 
Currier, J. M., Foster, J. D., \& Isaak, S. L. (2019). Moral injury and spiritual struggles in military veterans: A latent profile analysis. Journal of Traumatic Stress, 32(3), 393-404. https://doi.org/10.1002/jts.22378

Currier, J. M., Holland, J. M., \& Drescher, K. D. (2015). Spirituality factors in the prediction of outcomes of PTSD treatment for U.S. military veterans. Journal of Traumatic Stress, 28, 57-64. https://doi.org/10.1002/jts

Dattani, S., Ritchi, H., \& Roser, M. (2021). Mental health. Our World in Data. https://ourworldindata.org/mental-health

De Fazio, P., Gaetano, R., Caroleo, M., Cerminara, G., Giannini, F., Jaén Moreno, M. J., ... Segura-García, C. (2015). Religiousness and spirituality in patients with bipolar disorder. International Journal of Psychiatry in Clinical Practice, 19(4), 234-238. https://doi.org/10.3109/13651501.2014.1000929

Disabato, D. J., Goodman, F. R., Kashdan, T. D., Short, J. L., \& Jarden, A, D. (2016). Different types of well-being? A cross-cultural examination of hedonic and eudaimonic well-being. Psychological Assessment, 27(5), 1-12. http://dx.doi.org/10.1037/pas0000209

Edmondson, D., Chaudoir, S. R., Mills, M. A., Park, C. L., Holub, J., \& Bartkowiak, J. M. (2011). From shattered assumptions to weakened worldviews: Trauma symptoms signal anxiety buffer disruption. Journal of Loss and Trauma, 16, 358-385. http://dx.doi.org/10.1080/15325024 .2011.572030

Emmons, R. A. (1999). The psychology of ultimate concerns: Motivation and spirituality in personality. Guilford Press.

Exline, J. J., Park, C. L., Smyth, J. M., \& Carey, M. P. (2011). Anger toward God: Socialcognitive predictors, prevalence, and links with adjustment to bereavement and cancer. 
Journal of Personality and Social Psychology, 100(1), 129-148.

https://doi.org/10.1037/a0021716

Exline, J. J., \& Rose, E. D. (2013). Religious and spiritual struggles. In R. F. Paloutzian \& C. L. Park (Eds.), Handbook of the psychology of religion and spirituality ( $2^{\text {nd }}$ ed., pp. 380398). Guilford Press.

Falsetti, S. A., Resick, P. A., \& Davis, J. L. (2003). Changes in religious beliefs following trauma. Journal of Traumatic Stress, 16(4), 391-398. https://doi.org/10.1023/A:1024422220163

Fowers, B. J. (2005). Virtue and psychology. American Psychological Association.

Fredrickson, B. L. (2001). The role of positive emotions in positive psychology. American Psychologist, 56(3), 218-226. https://doi.org/10.1037/0003-066x.56.3.218

Fredrickson, B. L. (2002). How does religion benefit health and well-being? Are positive emotions active ingredients? Psychological Inquiry, 13(3), 209-213.

Fredrickson, B. L. (2012). The broaden-and-build theory of positive emotions. The Science of Well-Being, 359(1449), 1367-1377. https://doi.org/10.1093/acprof:oso/9780198567523.003.0008

Fredrickson, B. L. (2016). The eudaimonics of positive emotions. In J. Vitters $\varnothing$ (Ed.), Handbook of eudaimonic well-being. (pp. 183-190). Springer International Publishing. https://doi.org/10.1007/978-3-319-42445-3_12

Fredrickson, B. L., \& Losada, F., M. (2005). Positive affect and the complex dynamics of human flourishing. American Psychologist, 60(7), 678-686. https://doi.org/10.1037/hea0001026 
Garssen, B., Visser, A., \& Pool, G. (2021). Does spirituality or religion positively affect mental health? Meta-analysis of longitudinal studies. International Journal for the Psychology of Religion, 31(1), 4-20. https://doi.org/10.1080/10508619.2020.1729570

Geertz, C. (1973). Religion as a cultural system. In C. Geertz (Ed.), The interpretation of cultures (pp. 87-125). Basic Books.

George, L. S., \& Park, C. L. (2016). Meaning in life as comprehension, purpose, and mattering: Toward integration and new research questions. Review of General Psychology, 20(3), 205-220. https://doi.org/10.1037/gpr0000077

Harris, J. I., Erbes, C. R., Engdahl, B. E., Ogden, H., Olson, R. H. A., Winskowski, A. M. M., ... Mataas, S. (2012). Religious distress and coping with stressful life events: A longitudinal study. Journal of Clinical Psychology, 68(12), 1276-1286. https://doi.org/10.1002/jclp.21900

Heintzelman, S. J., \& King, L. A. (2014). Life is pretty meaningful. American Psychologist, 69(6), 561-574. https://doi.org/10.1037/a0035049

Hodapp, B., \& Zwingmann, C. (2019). Religiosity/spirituality and mental health: A metaanalysis of studies from the German-speaking area. Journal of Religion and Health, 58(6), 1970-1998. https://doi.org/10.1007/s10943-019-00759-0

Huguelet, P. (2017). Psychiatry and religion: A perspective on meaning. Mental Health, Religion and Culture, 20(6), 567-572. https://doi.org/10.1080/13674676.2017.1377956

James, W. (1902/1982). The varieties of religious experience. Penguin Books.

Jankowski, P. J., Sandage, S. J., Bell, C. A., Davis, D. E., Porter, E., Jessen, M., ... Owen, J. (2020). Virtue, flourishing, and positive psychology in psychotherapy: An overview and research prospectus. Psychotherapy, 57(3), 291-309. https://doi.org/10.1037/pst0000285 
Janoff-Bulman, R. (1992). Shattered assumptions. Free Press.

Kessler, R. C., Aguilar-Gaxiola, S., Alonso, J., Benjet, C., Bromet, E. J., Cardoso, G., ...

Koenen, K. C. (2017). Trauma and PTSD in the WHO World Mental Health Surveys.

European Journal of Psychotraumatology, 8, 1-32.

https://doi.org/10.1080/20008198.2017.1353383

Keyes, C. L. M. (2002). The mental health continuum: From languishing to flourishing in life. Journal of Health and Social Behavior, 43(2), 207-222. https://doi.org/10.2307/3090197

Koenig, H. G. (2018). Religion and mental health: Research and clinical applications. Academic Press.

Koenig, H. G., King, D. E., \& Carson, V. B. (2012). Handbook of religion and health (2 $2^{\text {nd }}$ ed.). Oxford University Press.

Kucharska, J. (2020). Religiosity and the psychological outcomes of trauma: A systematic review of quantitative studies. Journal of Clinical Psychology, 76(1), 40-58. https://doi.org/10.1002/jclp.22867

Ladd, K. L., \& Spilka, B. (2013). Prayer: A review of the empirical literature. In K. I. Pargament, J. J. Exline, \& J. W. Jones (Eds.), APA handbook of psychology, religion, and spirituality (Vol 1): Context, theory, and research. (pp. 293-310). American Psychological Association. https://doi.org/10.1037/14045-016

Leo, D., Izadikhah, Z., Fein, E. C., \& Forooshani, S. A. (2021). The effect of trauma on religious beliefs: A structured literature review and meta-analysis. Trauma, Violence, and Abuse, 22(1), 161-175. https://doi.org/10.1177/1524838019834076 
Linley, A. P., Joseph, S., Harrington, S., \& Wood, A. M. (2006). Positive psychology: Past, present, and (possible) future. Journal of Positive Psychology, 1(1), 3-16. https://doi.org/10.1080/17439760500372796

Marcus, Z. J., \& McCullough, M. E. (2021). Does religion make people more self-controlled? A review of research from the lab and life. Current Opinion in Psychology, 40, 167-170. https://doi.org/10.1016/J.COPSYC.2020.12.001

Martela, F., \& Steger, M. F. (2016). The three meanings of meaning in life: Distinguishing coherence, purpose, and significance. Journal of Positive Psychology, 11(5), 531-545. https://doi.org/10.1080/17439760.2015.1137623

Maslow, A. H. (1971). The farther reaches of human nature. Penguin Books.

McCullough, M. E., \& Snyder, C. R. (2000). Classical sources of human strength: Revisiting an old home and building a new one. Journal of Social and Clinical Psychology, 19(1), 110. https://doi.org/10.1521/jscp.2000.19.1.1

McCullough, M. E., \& Willoughby, B. L. B. (2009). Religion, self-regulation, and self-control: Associations, explanations, and implications. Psychological Bulletin, 135(1), 69-93. https://doi.org/10.1037/a0014213

Mosqueiro, B. P., Caldieraro, M. A., Messinger, M., da Costa, F. B. P., Peteet, J. R., \& Fleck, P. M. (2021). Religiosity, spirituality, suicide risk and remission of depressive symptoms: a 6-month prospective study of tertiary care Brazilian patients. Journal of Affective Disorders, 279, 434-442. https://doi.org/10.1016/j.jad.2020.10.028

Naclerio, M., \& Van Cappellen, P. (2021). Awe, group cohesion, and religious self-sacrifice. International Journal for the Psychology of Religion. Advance online publication. https://doi.org/10.1080/10508619.2021.1975423 
Pargament, K. I. (2007). Spiritually integrated psychotherapy. Guilford Press.

Pargament, K. I., \& Park, C. L. (1995). Merely a defense? The variety of religious means and ends. Journal of Social Issues, 51(2), 13-32. https://doi.org/10.1111/j.15404560.1995.tb01321.x

Pargament, K. I., Falb, M. D., Ano, G. G., \& Wachholtz, A. B. (2013). The religious dimension of coping: Advances in theory, research, and practice. In R. F. Paloutzian \& C. L. Park (Eds.), Handbook of the psychology of religion and spirituality ( $2^{\text {nd }}$ ed., pp. 560-579). Guilford Press.

Pargament, K. I., Murray-Swank, N. A., Magyar, G. M., \& Ano, G. G. (2005). Spiritual struggle: A phenomenon of interest to psychology and religion. In W. R. Miller \& H. D. Delaney (Eds.), Judeo-Christian perspectives on psychology (pp. 245-268). American Psychological Association. https://doi.org/10.1037/10859-013

Park, C. L. (2013). Religion and meaning. In R. F. Paloutzian \& C. L. Park (Eds.), Handbook of the psychology of religion and spirituality ( $2^{\text {nd }}$ ed., pp. 357-379). Guilford Press.

Park, C. L. (2016). Meaning making in the context of disasters. Journal of Clinical Psychology, 72(12), 1234-1246. https://doi.org/10.1002/jclp.22270

Park, C. L., Currier, J. M., Harris, J. I., \& Slattery, J. M. (2017). Trauma, meaning, and spirituality. American Psychological Association. https://doi.org/10.1037/15961-000

Park, C. L., \& Slattery, J. M. (2013). Religion, spirituality, and mental health. In R. F. Paloutzian \& C. L. Park (Eds.), Handbook of the psychology of religion and spirituality ( $2^{\text {nd }}$ ed., pp. 540-559). Guilford Press.

Pomerleau, J. M., Pargament, K. I., Krause, N., Ironson, G., \& Hill, P. (2020). Religious and spiritual struggles as a mediator of the link between stressful life events and 
psychological adjustment in a nationwide sample. Psychology of Religion and Spirituality, 12(4), 451-459. https://doi.org/10.1037/rel0000268

Reilly, T. S., \& Narvaez, D. (2018). Character, virtue, and science. Philosophy, Theology and the Sciences, 5(1), 51-79. https://doi.org/10.1628/ptsc-2018-0005

Rinehart, K. A. (2015). Spirituality as a moderator of posttraumatic stress disorder symptoms in military personnel: A meta-analysis [ProQuest Information \& Learning]. In Dissertation Abstracts International: Section B: The Sciences and Engineering (Vol. 75, Issue 10$\mathrm{B}(\mathrm{E}))$.

Rosmarin, D. H., \& Koenig, H. G. (2020). Handbook of spirituality, religion, and mental health ( $2^{\text {nd }}$ ed. $)$. Elsevier Academic Press.

Rosmarin, D. H., \& Leidl, B. (2020). Spirituality, religion, and anxiety disorders. In D. H. Rosmarin \& H. G. Koenig (Eds.), Handbook of spirituality, religion, and mental health., ( $2^{\text {nd }}$ ed., pp. 41-60). Elsevier Academic Press. https://doi.org/10.1016/B978-0-12$\underline{816766-3.00003-3}$

Rosmarin D. H., Malloy, M. C., \& Forester, B. P. (2014). Spiritual struggle and affective symptoms among geriatric mood disordered patients. International Journal of Geriatric Psychiatry, 29, 653-660. https://doi.org/10.1002/gps.4052

Rosmarin, D. H., Pargament, K. I., \& Mahoney, A. (2009). The role of religiousness in anxiety, depression, and happiness in a Jewish community sample: A preliminary investigation. Mental Health, Religion and Culture, 12(2), 97-113. https://doi.org/10.1080/13674670802321933

Rosmarin, D. H., Pargament, K. I., Pirutinsky, S., \& Mahoney, A. (2010). A randomized controlled evaluation of a spiritually integrated treatment for subclinical anxiety in the 
Jewish community, delivered via the Internet. Journal of Anxiety Disorders, 24(7), 799808. https://doi.org/10.1016/j.janxdis.2010.05.014

Schotanus-Dijkstra, M., Ten Have, M., Lamers, S. M. A., De Graaf, R., \& Bohlmeijer, E. T. (2017). The longitudinal relationship between flourishing mental health and incident mood, anxiety and substance use disorders. European Journal of Public Health, 27(3), 563-568. https://doi.org/10.1093/eurpub/ckw202

Seligman, M. E. P. (2019). Positive psychology: A personal history. Annual Review of Clinical Psychology, 15, 1-23. https://doi.org/10.1146/annurev-clinpsy-050718-095653

Seligman, M. E., \& Csikszentmihalyi, M. (2000). Positive psychology. An introduction. The American Psychologist, 55(1), 5-14. https://doi.org/10.1037/0003-066X.55.1.5

Shaw, A., Joseph, S., \& Linley, P. A. (2005). Religion, spirituality, and posttraumatic growth: A systematic review. Mental Health, Religion and Culture, 8(1), 1-11. https://doi.org/10.1080/1367467032000157981

Sheldon, K. M., \& King, L. (2001). Why positive psychology is necessary. American Psychologist, 56(3), 216-217. https://doi.org/10.1037/0003-066X.56.3.216

Smith, T. B., McCullough, M. E., \& Poll, J. (2003). Religiousness and depression: Evidence for a main effect and the moderating influence of stressful life events. Psychological Bulletin, 129(4), 614-636. https://doi.org/10.1037/0033-2909.129.4.614

Spero, M. H. (1992). Religious objects as psychological structures. University of Chicago Press.

Steger, M. F., \& Kashdan, T. B. (2013). The unbearable lightness of meaning: Well-being and unstable meaning in life. Journal of Positive Psychology, 8(2), 103-115. https://doi.org/10.1080/17439760.2013.771208 
Tait, R., Currier, J. M., \& Harris, J. I. (2016). Prayer coping, disclosure of trauma, and mental health symptoms among recently deployed United States veterans of the Iraq and Afghanistan conflicts. International Journal for the Psychology of Religion, 26(1), 3145. https://doi.org/10.1080/10508619.2014.953896

Tedeschi, R. G., \& Moore, B. A. (2021). Posttraumatic growth as an integrative therapeutic philosophy. Journal of Psychotherapy Integration, 31(2), 180-194. https://doi.org/10.1037/int0000250

Van Cappellen, P., Edwards, M. E., \& Fredrickson, B. L. (2021). Upward spirals of positive emotions and religious behaviors. Current Opinion in Psychology, 40, 92-98. https://doi.org/10.1016/j.copsyc.2020.09.004

Van Cappellen, P., Fredrickson, B. L., Saroglou, V., \& Corneille, O. (2017). Religiosity and the motivation for social affiliation. Personality and Individual Differences, 113, 24-31. https://doi.org/10.1016/j.paid.2017.02.065

Van Cappellen, P., Toth-Gauthier, M., Saroglou, V., \& Fredrickson, B. L. (2016). Religion and well-being: The mediating role of positive emotions. Journal of Happiness Studies, 17(2), 485-505. https://doi.org/10.1007/s10902-014-9605-5

VanderWeele, T. J. (2017). Religious communities and human flourishing. Current Directions in Psychological Science, 26(5), 476-481. https://doi.org/10.1177\%2F0963721417721526

Walsh, R. (1999). Essential spirituality. John Wiley \& Sons. 


\section{Figure 22.1}

A Conceptual Model of Religious/Spiritual Pathways to Well-Being and Mental Health

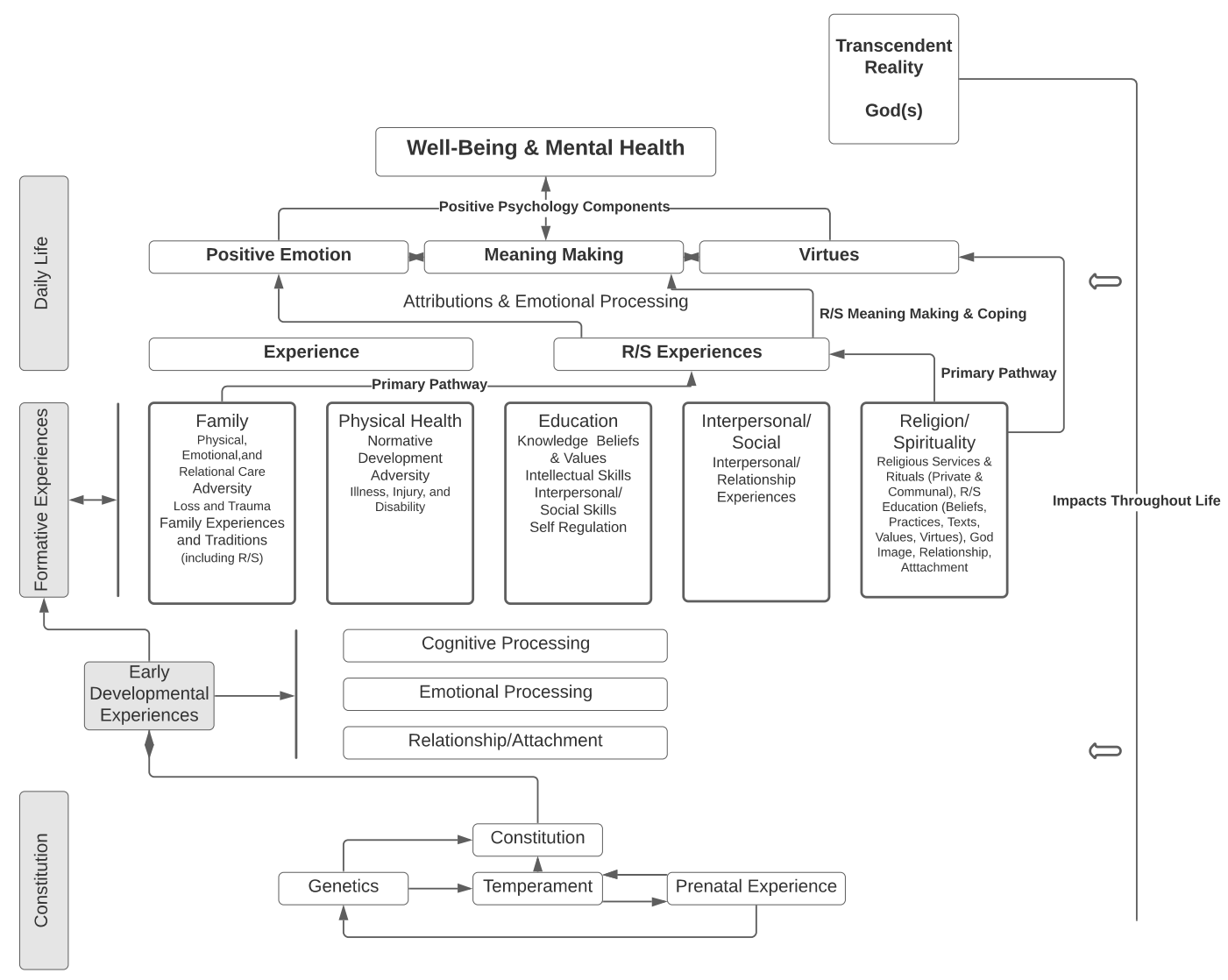

Note. This figure depicts a conceptual model of the developmental factors influencing individual religiousness and spirituality and, in turn, the religious/spiritual (R/S) pathways that contribute to well-being and mental health. A person's religiousness/spirituality is dynamic. It emerges from a host of constitutional, developmental, individual, social, and cultural factors, and it is affected by the person's present life circumstances. Both religious pathways and spiritual pathways involve positive emotions, support meaning making, and nurture virtues. However, this model allows considering the distinct impacts of religiousness (the religious pathway) and spirituality (the spiritual pathway) on well-being and mental health. Of note, this model includes the possibility of transcendent reality/God(s) as a force in human experience (Spero, 1992), even though this component of the model is of course not empirically verifiable from a positivistic perspective. 


\title{
Chapter 23
}

\section{The Scientific Study of Religion/Spirituality, Forgiveness, and Hope}

\author{
Melissa Washington-Nortey, Everett L. Worthington, Jr., and Rihana Ahmed \\ Virginia Commonwealth University
}

Correspondence concerning this chapter should be addressed to Melissa WashingtonNortey. Email: melissa.washington-nortey@kcl.ac.uk.

\footnotetext{
Washington-Nortey, M., Worthington, E. L., Jr., \& Ahmed, R. (2021). The scientific study of religion/spirituality, forgiveness, and hope. In Edward B. Davis, Everett L. Worthington, Jr., \& Sarah A. Schnitker (Eds.), Handbook of positive psychology, religion, and spirituality. Springer Nature.
} 


\begin{abstract}
Within both positive psychology and the psychology of religion and spirituality (R/S), forgiveness and hope are considered key virtues or character strengths. When relationships are beset by transgressions, engaging in forgiveness is related to hope that the relationship is repairable. When people participate in forgiveness interventions, they increase their hope. There are religious and spiritual, empirical, and theoretical reasons why forgiveness and hope are interrelated. We explore these reasons using an evidence-based theoretical model of forgiveness and relational spirituality, which is at the intersection of positive psychology and the psychology of $\mathrm{R} / \mathrm{S}$. The forgiveness and relational spirituality model combines basic and intervention research to conceptualize how R/S is related to forgiving. The model posits a sacred dimension to the inter-relationships among the victim, offender, and transgression, which are embedded in a particular forgiveness-relevant event. We incorporate hope into this theoretical model and review recent scientific research (2012 to 2020) on hope, forgiveness, and R/S conjointly $(k=27)$ and on forgiveness and R/S specifically $(k=72)$. Almost all this research has been cross-sectional and correlational. We hypothesize causal connections to guide future research and propose ways to guide practitioners as they help people navigate relational forgiveness with more hope.
\end{abstract}

Keywords: forgiveness, hope, relational spirituality, religion, theory 


\section{The Scientific Study of Forgiveness, Hope, and Religion/Spirituality}

Theologian Lewis B. Smedes eloquently argued, "Sometimes hate only nibbles at the edges of the heart; it does not always burn out the lining of the heart.... But whether your hate is a carcinoma growing hell-bent for death inside your soul, or only a pesky heartburn, it will hurt you if you do not use the right remedy... But eventually, unchecked hate will do you in. Such hate can be healed" (Smedes, 1984, pp. 27-28). Smedes (1996) advocated forgiveness as the healing potion acting between humans' power to imagine the future and their weakness at controlling it. "The answer to the problem of imagining a future we cannot control is hope. And the way to hope for a better future after a bad past is the way of forgiving" (Smedes, p. 171).

In short, forgiveness and hope are intertwined. Forgiveness is often motivated by the hope that relational problems will be healed through willpower that finds a different way through problems than conflict. When forgiveness occurs and is accepted by the one offended, it often motivates hope that the relationship, having survived a crisis, will now persevere.

People who seek counseling for relationship problems have often repeatedly transgressed against each other. Many relationships end because people cannot forgive their partner. Partners

lose hope. Willpower erodes. Ways have dead-ended. Perseverance finally evaporates. However, if people begin to forgive, hope can be rekindled, and other aspects of their relationship can flourish. In fact, a meta-analysis of randomized controlled trials using forgiveness interventions found that when people forgive, even though the interventions did not even mention hope, their level of hope increased at least as much as did their forgiveness (Wade et al., 2014).

Hope and forgiveness seem bound together. The five major religions value both (Rye et al., 2000), and empirical evidence on religion/spirituality (R/S) suggest a potential synergy. Yet few psychological theories have tied them together (cf. Ripley \& Worthington, 2014). 
In positive psychology, forgiveness and hope are usually studied as character strengths or virtues. Each is intertwined in various religions and in broader concepts of spirituality, making them popular foci in the psychology of R/S as well. With R/S frameworks, theorizing about how forgiveness and hope are connected is sparse, suggesting a need for theoretical attention. In this chapter, we build on an established theoretical model of forgiveness and relational spirituality, supported by basic and applied research (for a review, see Worthington \& Sandage, 2016). We make theoretical suggestions about how hope can be incorporated into this model, and we review recent research that studies hope. We seek to create a basic theory that relates forgiveness, hope, and $\mathrm{R} / \mathrm{S}$ and then to parlay that broadened theory into suggestions about interventions.

\section{Definitions}

Davis et al. (2015) have considered definitions of terms related to R/S. Spirituality is defined as a sense of closeness or connection with the Sacred. The Sacred is whatever a person believes is set apart as deserving veneration, such as God, the divine, ultimate reality, humanity, environment, the transcendent, or temporal objects like marriage or a holy book. Relational spirituality recognizes that such closeness or connection depends on relationships, so it must be contextualized within whatever people perceive to be sacred. Religion is defined as the system of shared basic beliefs about ultimate concerns, repetitive practices, and individual religious behaviors engaged in by a community of similarly minded (although not identically minded) individuals. Religiousness is one's search for and experience of whatever one perceives to be sacred, within the context of a religious tradition (Hill et al., 2000). Closeness or connection with something considered religiously sacred is called religious spirituality. It is differentiated from other types of spirituality that involve closeness or connection with other sacred objects like nature, humans, or something transcending the mundane. 
Worthington (2020b) recently evaluated key conceptual definitions and theoretical frameworks of forgiveness. Although forgiveness broadly conceived can include divine forgiveness (by a deity), self-forgiveness, and societal forgiveness, in the present chapter, we focus mostly on person-to-person forgiveness. Not all researchers agree on definitions or theories of forgiveness, but most agree that forgiveness involves internal prosocial changes toward a perceived transgressor. The internal experience of forgiveness includes reduced negative (and in some cases increased positive) emotions, motivations, behavioral intentions, and cognition towards the offender. It might eventuate in changed behaviors (Fehr et al., 2010), which are not, strictly speaking, part of forgiveness. The most frequently studied theory of forgiveness is stressand-coping theory (Worthington, 2006). At least two types of person-to-person forgiveness are identified (Worthington, 2020a). Decisional forgiveness is a behavioral intention statement that foregoes vengeance or getting even and intends to treat the offender as a valued and valuable person. Emotional forgiveness is the intentional replacement of negative unforgiving emotions with positive other-oriented emotions like empathy, sympathy, compassion, and love.

In this chapter, we use the forgiveness and relational spirituality model (see Worthington \& Sandage, 2016), which incorporates stress-and-coping theory within it. That model is directly applicable to the intersection of R/S, forgiveness, and hope. Hope is most often described using Snyder's et al.'s (1991) cognitive model of hope, recently supplemented with Rueger et al.'s (in press) model of persevering hope. In this chapter, we incorporate both conceptualizations. Snyder's model describes hope as the agency to bring about changes and the awareness of pathways to achieve those changes. Those types of hope are (theoretically) most important to the initiation of forgiveness. Rueger et al.'s (in press) persevering hope is both a motivation to persevere and an outcome, once forgiveness has occurred and positively affected the 
relationship. Hope can be based in sacred or human relationships. Interventions aimed at helping people recover hope and thereby motivate change in their psychological, physical, relational, or spiritual state have been found effective (for a meta-analysis, see Weis \& Speridakos, 2011).

In this chapter, we describe the forgiveness and relational spirituality model, hypothesize ways that hope might be incorporated in the model, and summarize recent research on the model and hope. With limitations of our research in mind, we draw implications and recommendations for basic and applied research at the intersection of forgiveness, hope, and R/S.

\section{Forgiveness and Relational Spirituality Model}

\section{Victims, Offenders, and Transgressions and Their Inter-Relationships}

The qualities of victim, offender, and transgressions are important in whether a victim forgives. So are the inter-relationships among these three members of the model.

Victims have personality, contextual, and other personal qualities that either promote or discourage them from forgiving. For example, personality traits like agreeableness and empathy are related to forgiving, but traits like neuroticism and vengefulness mitigate against forgiving. People forgive more easily in well-functioning relationships but struggle to forgive in conflictual relationships. Likewise, offenders contribute their own personal and contextual considerations into whether victims can forgive. For example, victims struggle to forgive offenders who are narcissistic, psychopathic, or Machiavellian (i.e., the dark triad); who cannot empathize with those whom they have hurt; or who are in a derogated outgroup. Victims might struggle due to the victim's perception of the offender or because offenders do not engage in the relationshipvaluing acts (e.g., taking responsibility for wrongdoing, apologizing, and offering to make amends). When offenders are in contexts that do not value forgiveness (e.g., in active warfare, status-hungry gangs, or a network of vengeful friends), forgiveness is rarely an option. 
Transgressions similarly affect whether victims forgive. Offenses that are severe, have longlasting effects (e.g., offenses causing spinal cord or traumatic brain injuries), and are committed by trusted others are particularly hard to forgive (for reviews, see Worthington \& Wade, 2020).

\section{The Forgiveness and Relational Spirituality Model Adds a Sacred Dimension}

The forgiveness and relational spirituality model (Davis et al., 2008; Worthington \& Sandage, 2016) adds a sacred dimension to how people handle transgressions. Our chapter is more concerned with the inter-relationships among this model's elements (victim, offender, and transgression), because these elements relate to what the victim perceives as sacred. An initial review of this research (through 2014) was summarized in Worthington and Sandage (2016).

A brief explanation of each inter-relationship. When it comes to navigating transgressions, the Sacred-victim relationship can exhibit many characteristics. For example, it can be characterized by spiritual devotion or struggle (Pargament \& Exline, 2021), religious attachment security or insecurity (Granqvist, 2020), or positive or negative religious coping (Pargament, 2007). The Sacred-offender relationship is seen from the victim's perspective. Victims are more likely to forgive offenders who are religiously/spiritually similar to themselves (for a review, see Worthington \& Sandage, 2016). The Sacred-transgression relationship relates to whether a victim perceives (appraises) the transgression to have religious or spiritual meaning. A divorce or murder of a loved one might be perceived as a loss of something sacred, and if so, it might be hard to forgive. A perceived desecration of something held sacred is often even more difficult to forgive. Yet if forgiveness is sanctified or imbued with spiritual significance (by the victim or by the victim's religious community), this sanctification can make forgiveness easier.

Theoretically hypothesized roles of temporal hope within the model. We might divide hope into two types. Transcendent hope is trust in a transcendent being or force someone 
believes is likely to lead ultimately to good outcomes. (Albeit, "good" is perceived from the vantage-point of the transcendent being.) Temporal hope (i.e., hope regarding earthly relationships) describes anticipated, valued outcomes in a relationship with another human being, either now or in the future. Both transcendent and temporal hope are pertinent to a Sacred-victim relationship. If a person trusts the Sacred (i.e., transcendent hope), then that trust can empower hope. Based on this transcendent hope, both Snyder's cognitively oriented agency and pathways to change (Snyder et al., 1991) might be activated, and temporal hope could empower forgiveness of a transgressor. Similarly, persevering hope (Rueger et al., in press)—hope when goals do not seem reachable - might be maintained even in the face of a sacred loss (e.g., a murdered loved one). Transcendent hope that is rooted in ultimate good might motivate forgiveness of a murderer. Thus, aspects of R/S might produce hope and motivate action. Both transcendent and temporal hope might be at both endpoints of that causal chain-activating forgiveness and resulting from forgiveness. That hope can lead to better relational, mental, and physical health. Hence, hope can act as a causal path between R/S and temporal outcomes. But Sacred-victim relationships characterized by hope that a sacred being might intervene in relationship differences might build stronger R/S. Therefore, hope can be either a causal agent or product of R/S, or it can be a mediator (i.e., causal path) between R/S and temporal outcomes.

A Sacred-offender relationship is a victim's perception of the degree of similarity the offender's religious/spiritual life has to their own. A perception characterized by hostile religious differences—-such as antagonists in a war over religious differences-may make victim-offender forgiveness virtually impossible. When people of similar religious beliefs, values, and practices harm each other, such as when a church splits, research has shown that the victims are more willing to forgive (Greer, Worthington, Van Tongeren et al., 2014). Thus, seeing the spiritual 
similarity of victim and offender can promote hope for forgiveness and reconciliation. Even if reconciliation is not seen as possible, persevering hope can remain, often motivated by R/S.

The Sacred-transgression relationship also might be either infused with hope or bereft of it. If a religious community interprets an event that occurs within it, such as the death of a beloved community member, as God calling the person to a just reward, then hope can flourish in that community. But if a sacred loss occurs, such as a religious person feeling that divorce irreparably damaged his or her relationship with the Sacred, then hopelessness can reign.

\section{Empirical Foundation for Our Reviews of Research}

We began with Davis et al.'s (2013) meta-analyses of R/S and forgiveness research through 2011. Forgivingness (or trait forgiveness) is a disposition to forgive across time and situations. State forgiveness is a one-off forgiving response to an unjust act. Davis et al. analyzed 64 samples reporting effect sizes of the relationship between R/S and forgivingness $(n=99,177)$ and 50 samples relating $\mathrm{R} / \mathrm{S}$ and state forgiveness $(n=8,932)$. $\mathrm{R} / \mathrm{S}$ was related to forgivingness $(r=.29)$ and state forgiveness $(r=.15)$. To avoid duplicating Davis et al.'s (2013) meta-analysis, we reviewed studies from 2012 to date that included hope, forgiveness, and R/S. We also reviewed studies since then that dealt with forgiveness and R/S (but not hope). The latter was not our focus but informed the model. Finally, to inform implications for interventions, we reviewed select interventions that assessed forgiveness and/or hope as outcomes.

\section{Method of the Review}

We searched PsycINFO, PubMed, Academic Search Complete, and ERIC for relevant articles using this string of keywords: "hope" AND "forgiv*" AND ["religi* OR "spirituality" OR "R/S" OR "Coping"]. We included articles if they (a) examined R/S, forgiveness, and hope; (b) were published in English between 2012 and 2020 (inclusive); and (c) were empirical 
(quantitative or qualitative). We excluded nonempirical documents (e.g., newsletters and editorials), non-English publications, and reviews. We found 38 studies published between 2012 and 2020 (inclusive), of which 27 met inclusion criteria. Given the broad nature of R/S, we included studies that examined a wide range of $\mathrm{R} / \mathrm{S}$ dimensions, such as religious service attendance, commitment, activity, involvement, interventions, centrality, coping, and denomination. We also included spiritual transcendence, spiritual appraisals, relational spirituality. For each study, we coded information related to the specific forgiveness and/or hope construct(s) examined, other constructs examined, guiding theory, main study aim, method and study design (qualitative, quantitative, cross-sectional, longitudinal, etc.), sample characteristics, measures/instruments used, and main findings (see Appendix 23.S1, Table 23.S1).

Second, we searched "forgiv*" AND the religious terms identified above. Our initial search produced 2,299 articles from 2012 and 2020 - 1,946 after duplicates were removed. Two independent reviewers screened them using inclusion-exclusion criteria indicated in Appendix 23.S1. "Fit" by both reviewers resulted in 72 studies (see Appendix 23.S1, Table 23.S2).

Third, we sought to provide some guidance for practitioners (e.g., religious counselors, clergy, pastoral counselors, and lay leaders). Thus, we also nonsystematically reviewed 15 intervention studies that had assessed hope and/or forgiveness as outcomes (see Table 23.S3).

\section{Results of the Reviews}

\section{Review of Hope in Relation to the Forgiveness and Relational Spirituality Model}

Worthington and Sandage's (2016) book, Forgiveness and Spirituality in Psychotherapy, summarized 25 studies that directly evaluated aspects of the model. Of those, six studies assessed hope. We extended the model using findings from our and Worthington and Sandage's (2016) review. Where possible, we illustrate our points with a recent study rather than an older study. 


\section{Hope and the Victim-Sacred Relationship}

A victim's relationship with the Sacred depends on the victim's religious beliefs, values, and behaviors and R/S-related virtues, which in turn are associated with increased forgiveness, better relationships, and improved mental and physical health. We hypothesize that hope is causally involved in those connections. In the research reviewed in Table 23.S1, few studies used experimental or longitudinal designs. Thus, it is hard to determine if hope produces forgiveness and other outcomes, forgiveness produces hope and other outcomes, or a more complex causal chain exists. Even so, the victim-Sacred relationship was related to hope in four areas.

Times of intense suffering. During intense suffering, well-being and life satisfaction might depend on disengaging from the pain and anguish of suffering, employing positive religious coping, and finding cause for hope - either in restored relationships or in one's relationship with the Sacred. For example, Chen et al. (2021) studied 272 survivors in civil war conflict (Study 1) and 1,651 people (Study 2) who were displaced to an area in which a devastating landslide killed and injured many—piling on three potentially traumatizing factors. In Study 1, survivors' trauma was associated with greater negative religious coping and lower hope, forgivingness, and well-being. When people suffer, the world looks bleaker, and hope and forgiveness erode. In Study 2, findings were replicated but positive coping was related to higher well-being, suggesting that the way people cope may affect hope and psychological well-being.

Ways people treat others. Sutton et al. (2014) studied Pentecostal and charismatic Christians. Love of God predicted neighborly love. Hope was treated as a predictor, not an outcome. Attachment to God and religious spirituality were related to hope and forgiveness.

Engaging a complex of virtues. Bushlack and Bock (2018) found evidence that hope and forgiveness are linked to wisdom and mindfulness, which are related to fewer psychological 
symptoms and higher well-being. Practical wisdom was related to higher mindfulness, hope, and forgiveness, which in turn were related to lower stress, anxiety, and depression. Forgiveness and hope and were not merely motivators of functional living; they were related to better health.

Personal spirituality's benefits. Berthold and Ruch (2014) assessed 20,538 people categorized as (a) nonreligious people, (b) religiously affiliated people who do not practice their religion, and (c) religious people who actively practice religion. Those who practiced their religion - presumably those with a closer relationship to the Sacred — were happier, felt their life had more meaning, and had higher levels of hope, forgiveness, gratitude, and kindness.

Summary. A close victim-Sacred relationship seems to mirror a good human-human relationship (e.g., a strong marriage or good friendship). When hurts or disappointments are perceived, a close victim-Sacred relationship is associated with higher hope and forgiveness.

\section{Hope and the Offender-Sacred Relationship}

Yet it is not merely the victim's relationship with the Sacred that is connected to forgiveness and hope. Victims judge whether offenders are ingroup or outgroup members. Identity similarity to the victim may often foster willingness to forgive. Worthington and Sandage (2016) reviewed six studies that reported positive correlations between forgiveness and spiritual similarity of the offender to the victim. Hope was also correlated with differentiation of self, commitment to social justice, positive religious coping, and recent spiritual transition.

\section{Hope and the Transgression-Sacred Relationship}

Our review of studies examining a spiritual desecration and forgiveness revealed no studies that included hope. Worthington and Sandage (2016) found seven studies showing that transgressions perceived as spiritual losses or desecrations were difficult to forgive. However, none of those studies assessed correlates with hope. 


\section{Recent Research on the Forgiveness and Relational Spirituality Model}

In the research on forgiveness and R/S since Davis et al.'s (2013) meta-analysis, all aspects of the forgiveness and relational spirituality model have been studied. Because our review is focused on the potential expansion of the model by including hope, we will not review each aspect of the model in the studies that did not include hope. Rather, we refer readers to Table 23.S2 in Appendix 23.S1. We make three summary comments based on that research.

First, most existing research has studied the Sacred-victim relationship; less scholarly attention has been dedicated to the Sacred-offender or Sacred-transgression relationship. Greer, Worthington, Van Tongeren et al. (2014) found that people experienced more hurt when offenders were ingroup than outgroup members, but people forgave ingroup members more readily. McElroy et al. (2014) found that desecration predicted intergroup forgiveness beyond the effects of religious commitment, personality characteristics, hurtfulness, and closeness.

Second, as of yet, there is limited cross-cultural examination, application and generalizability of this model. Cultural understandings of forgiveness and its meanings differ, as do concentrations of religions. Better understanding of culture is vital. Some research has been conducted in Iran (Ghorbani et al., 2017) and Hong Kong (Ho et al., 2017). More is needed.

Third, relatively few longitudinal studies have been done. Little is known about how forgiveness changes over time and how changes in R/S and forgiveness affect one another.

\section{Interventions to Promote Forgiveness}

Context. New studies must be seen in context of evidence on forgiveness interventions. In a meta-analysis, Wade et al. (2014) found that (a) REACH Forgiveness (Worthington, 2020a) and the Process Model (Freedman \& Enright, 2020) have been the most used, investigated, and 
efficacious models; (b) time spent trying to forgive predicts total forgiveness; and (c) the longer people try to forgive, the more hope and the less depression and anxiety they experience.

Intervention studies between 2012 and 2020 aimed directly at forgiveness. Most often, the REACH Forgiveness model has been either accommodated to Christian settings (e.g., Greer, Worthington, Lin et al., 2014) or used in secular form within explicitly Christian settings (for a review, see Worthington, 2020a). In religious settings, secular interventions work similarly to religiously accommodated versions, probably because highly religious people draw on their own R/S even when participating in secular interventions (Rye \& Pargament, 2002). Greer, Worthington, Lin et al. (2014) used a Christian-accommodated REACH Forgiveness intervention and found that completing a 6-hour do-it-yourself workbook increased forgiveness in Christians who had been hurt by other Christians. Hernandez et al. (2012) found that after a religiously integrated, forgiveness seminar, participants were more likely to forgive offenders.

\section{Interventions Aimed at Spiritual Practices Hypothesized to Increase Forgiveness}

Some interventions have aimed to increase people's R/S practices in order to promote forgiveness. Vasiliauskas and McMinn (2013) conducted a randomized controlled trial with Christians (two experimental conditions: prayer or religious devotion). Participants who prayed for their offender increased empathy, religious commitment, and forgiveness the most (see also Toussaint et al., 2016). Those in the religious-devotion intervention reported less increased forgiveness than those in the prayer condition but more than those in the control condition.

\section{Forgiveness Interventions Assessing Hope}

To provide an applied perspective regarding forgiveness, hope, and R/S, we nonsystematically reviewed 15 studies (see Table 23.S3). One salient finding of this review was that changes in hope as an outcome did not parallel changes in forgiveness. For example, 
Freedman and Enright (1996) treated people trying to forgive incest. Treatment lasted a mean of 60 hours. By treatment's end, people were more forgiving and hopeful. By follow-up, the enormous improvements in forgiveness had been maintained, but changes in hope had eroded to pretreatment levels. This finding indicated that (a) hope and forgiveness were distinct constructs and that (b) hope (such as Snyder's agency and pathways hope) might motivate change, but (c) hope as an outcome that reflects expectations about the relationship's future seems dependent on more complex life circumstances. Forgiveness was specific to an event (i.e., the incest). Once that incident was forgiven, participants considered it dealt with and seemed to move forward.

\section{Discussion}

\section{What We Know-Theoretical Integration of R/S, Forgiveness, and Hope}

We have sought to show that hope might be included in the forgiveness and relational spirituality model. We theorized about how such inclusion might work. We reviewed research on hope in each sacred inter-relationship. Overall, virtually none of the existing research would lead a basic researcher in positive psychology or the psychology of R/S to doubt the model itself, but almost all the research has been cross-sectional and done within Western countries. Therefore, the extant research has not strongly supported the model either. Virtually no research, on its own merits, has opened a new vista for basic researchers. We found correlational support for hope's involvement in the model but not support for how hope might be related to forgiveness within the sacred relationships. No studies tested mechanisms (e.g., by hope affecting stress or coping, making the Sacred-victim relationship stronger, or operating via another mechanism). For the model to continue to be heuristic, researchers need to conduct new, hope-relevant investigations.

Sacred-victim relationships are related to transcendent and temporal hope, as the Sacred superintends the temporal relationship. Sacred-offender relationships that indicate spiritual 
similarity of the offender to the victim can also be important. When the victim deems the offender is spiritually dissimilar, transgressions are harder to forgive (Greer, Worthington, Lin et al., 2014). But when transgressions are inflicted by a trusted ingroup member-such as sexual abuse by a clergy member - they can be devastating, because transgressions damage trust.

Our review of research on hope, forgiveness, and R/S, which reveals robust correlations among these variables, supports the hypothesis that forgiveness is bidirectionally related to hope within the context of R/S. That is, hope helps people forgive, forgiveness helps them maintain or regain a sense of hope, and R/S helps support both forgiveness and hope. Transgressions damage trust in relationships, especially when they are serious, hurtful, repeated, and piled onto other transgressions (Worthington \& Sandage, 2016). Damage to trust is magnified even more when a transgression is appraised as a desecration of something sacred (Pargament et al., 2005).

\section{What Theory Suggests We Would Like to Know}

$\mathrm{R} / \mathrm{S}$ is theorized to be involved with both forgiveness and hope, because people develop a relationship with whatever they hold to be sacred. Relationships are the basis for hope- -hope to pursue meaningful goals and to persevere with fortitude when goals seem unattainable. Thus, relationships with the Sacred are a ground for hope. Also, relationships among people can be damaged by transgressions. When repair of relationships seems impossible — especially when people appeal to the Sacred for repair-the relationship with the Sacred (as well as between the people involved) can be damaged, because the victim might think sacred trust was violated. Damaged relationships that seem either to degenerate further or to stagnate can disappoint people; they may come to believe that whatever they hold to be sacred is not powerful or loving enough to redeem the relationship, which then could lead to religious/spiritual struggles 
(Pargament \& Exline, 2021). Temporal relationships can be the ground for gaining or losing trust in the Sacred, which can affect hope that is perceived to emanate from divine sources.

Theory suggests hope can affect people's sense of agency or open new pathways toward reconciliation (Snyder et al., 1991). Forgiveness also can help people become more willing to persevere when they might not see a way forward (Rueger et al., in press). Agency, pathways, and persevering hope can motivate forgiveness. Theory also suggests forgiveness can lead to a resurgence of hope, which may in turn lead to a desire to reconcile. Yet even if reconciliation may look promising in the aftermath of forgiveness, persevering hope that envisions a positive future for the relationship likely depends on other contextual variables. Recall the Freedman and Enright (1996) findings in which forgiveness was maintained but hope was not. Our review's findings are consistent with causal reasoning in which hope can affect forgiveness and forgiveness can affect hope. But to date, these causal pathways have not yet been tested in longitudinal or experimental studies. We suggest three hypotheses to guide such research.

\section{R/S Can Produce Hope, Which Produces More Forgiving}

$\mathrm{R} / \mathrm{S}$ can produce hope when the R/S is characterized by trust, dedication, and other measures of the Sacred-victim relationship. That in turn can empower people through a sense of agency, through knowledge of pathways, and through a motivation to persevere when one does not see desired goals as reachable. In this relational context, people can therefore risk forgiving.

\section{R/S Can Promote Forgiveness and Hope, which Produce Better Mental Health}

Both forgiveness and hope can be religiously inspired - not only by a positive Sacredvictim relationship but also by Sacred-offender relationships in which offenders are perceived to have similar sacred relationships as the victim has. Hope can decrease depression because depression is characterized by hopelessness. Hope can also provide confidence in oneself or in a 
sacred agent, which thus can mitigate anxiety. Spirituality_one's private experience of closeness or connection with the Sacred - can motivate people to let religion affect them. Both hope and forgiveness produce better relationships between ingroups and outgroups. Those improved relationships can in turn reduce stress (for reviews, see Worthington, 2006) and thereby lead to better mental and physical health (for reviews, see Toussaint et al., 2015).

\section{Forgiveness Can Lead to Hope, Which Can Lead to Closer Relationships with the Sacred}

Emotions and motivations change through forgiveness, especially when forgiving is religiously motivated. These changes in emotions and motivations build hope. Hope feeds into a spiritual closeness to God (i.e., a more trusting Sacred-victim relationship), which promotes further willingness to forgive, trust in God, and pursue a better future. However, a large caveat is that the Sacred-victim relationship depends on much more than forgiveness (for reviews, see Worthington \& Wade, 2020). If some other relational variables poison the relationship, forgiveness alone will not keep it positive, and if the relationship fails, it will likely cause religious/spiritual struggle rather than a closer relationship to the Sacred.

\section{Causal Pathways Need to Be Mapped through Longitudinal Research}

Overall, the theoretical connections among R/S (closeness with the Sacred), forgiveness, and hope seem to act along multiple causal and correlative paths, as our three hypotheses posit. Generative theory is needed to identify the conditions under which each causal or correlational path is active. Longitudinal and experimental research is needed to map the causal sequences. Only with additional theory and research will other generative hypotheses be possible.

\section{Applications of the Hope-Inclusive Relational Spirituality Model of Forgiveness}

Psychotherapy. Worthington and Sandage (2016) applied the forgiveness and relational spirituality model in brief and longer-term psychotherapy, couple and family therapy, and group 
psychotherapy. Most of that application was with secular clients, which included religious, spiritual-but-not religious, and nonspiritual/nonreligious clients. The model did not specifically consider religious or spiritual populations, such as people from different religions or different denominations within a religion. Theology was not included. The model can be profitably expanded to religious and spiritual populations by considering and articulating more fine-grained descriptions of people's religious and spiritual beliefs, values, practices, and behaviors.

Clinical practice with religious and spiritual clients (and to inform treatment when clients do and do not want their religious/spiritual beliefs, values, and practices explicitly included in their treatment) could be improved if hope were considered more explicitly. On one hand, many patients say that their religious or spiritual beliefs, values, and practices are not treated with respect by mental health providers or that the use of "secular" evidence-based practices feels like clinicians are using a one-size-fits-all psychological approach instead of a patient-sensitive approach (Vieten \& Lukoff, 2021). On the other hand, some people who receive psychological treatment from R/S-identified professionals complain that sometimes such clinicians spiritualize psychotherapy and treat helping as a one-size-fits-all spiritual problem that has a prescribed spiritual cure (e.g., prayer for healing or divine comfort). Relational spirituality seeks to discern the person's relationship with the Sacred (and the relationship between the offender and the Sacred and the transgression and the Sacred) to design a holistic patient-responsive treatment.

Clergy counseling. Counseling by clergy must be contextualized within broader interventions in the religious or spiritual community. Those interventions include religious/spiritual education about forgiveness and hope (to congregants across the lifespan), ministry by lay helpers, provision of material and social resources to congregants, and leadership from clergy. The hope-inclusive forgiveness and relational spirituality model can inform 
preparation of clergy's public expositions of sacred texts, which then can influence the congregation through psychoeducation. In addition, a relational theology can help form the theology that is used to inform ministry and spiritual practice throughout the congregation.

\section{Recommendations for Research}

Limitations to our reviews of research should be seen in light of Davis et al.'s (2013) meta-analysis. They noted that we need more theory-driven research, culturally diverse samples, longitudinal designs, and intervention studies. Those limitations still hold in 2021, and we again

offer those as goals for the next decade. We emphasized the need for more theory and research of the hope-inclusive forgiveness and relational spirituality model. Forgiveness and hope are not clinical variables whose deficit yields diagnoses. However, they deserve their place in studies of the mechanisms involved in interventions. Both basic and applied research are sorely needed.

\section{Conclusions}

In the present chapter, we have outlined a hope-inclusive forgiveness and relational spirituality model. Over the last decade, hope has been correlationally related to important aspects of the evidence-based relational spirituality model of forgiveness. In this chapter, we have theorized that hope might also be related via Sacred-victim, Sacred-offender, and Sacredtransgression inter-relationships with forgiveness. Future research must investigate the hopeinclusive forgiveness and relational spirituality model, using causal-testing research designs.

We began with Smedes's (1996) observation that people can imagine the future but not control it. Smedes (1996) suggested that hope was the answer to that dilemma. We have suggested that interventionists might help religious and spiritual people to experience more positive outcomes from their wrestling over whether to forgive if they consider the motivating role of hope and the effects forgiving can have on long-term hope. 


\section{References}

Berthold, A., \& Ruch, W. (2014). Satisfaction with life and character strengths of non-religious and religious people: It's practicing one's religion that makes the difference. Frontiers in Psychology, 5, Article 876. https://doi.org/10.3389/fpsyg.2014.00876

Bushlack, T. J., \& Bock, T. (2018). Validating the "Centering for Wisdom Assessment”: Assessing the role of contemplative practices in the cultivation of practical wisdom. Journal of Psychology and Theology, 46(3), 143-167.

Chen, Z. J., Bechara, A. O., Worthington Jr, E. L., Davis, E. B., \& Csikszentmihalyi, M. (2021). Trauma and well-being in Colombian disaster contexts: Effects of religious coping, forgivingness, and hope. The Journal of Positive Psychology, 16(1), 82-93. https://doi.org/10.1080/17439760.2019.1663254

Davis, D. E., Hook, J. N., \& Worthington, E. L., Jr. (2008). Relational spirituality and forgiveness. Journal of Psychology and Christianity, 27, 293-301. https://doi.org/10.1037/a0033638

Davis, D. E., Rice, K. G., Hook, J. N., Van Tongeren, DeBlaere, C. Choe, E., \& Worthington, E. L., Jr. (2015). Development of the Sources of Spirituality Scale. Journal of Counseling Psychology, 62(3), 503-513. https://doi.org/10.1037/cou0000082

Davis, D. E., Worthington, E. L., Jr., Hook, J. N., \& Hill, P. C. (2013). Research on forgiveness and religion/spirituality: A meta-analytic review. Psychology of Religion and Spirituality, 5(4), 233-241. https://doi.org/10.1037/a0033637

Fehr, R., Gelfand, M. J., \& Nag, M. (2010). The road to forgiveness: A meta-analytic synthesis of its situational and dispositional correlates. Psychological Bulletin, 136, 894-914. https://doi.org/10.1037/a0019993 
Freedman, S. R., \& Enright, R. D. (1996). Forgiveness as an intervention with incest survivors. Journal of Consulting and Clinical Psychology, 64, 983-992. https://doi.org/10.1037/0022-006x.64.5.983

Freedman, S. R., \& Enright, R. D. (2020). A review of the empirical research using Enright's process model of interpersonal forgiveness. In E. L. Worthington, Jr., and N. G. Wade (Eds.), Handbook of forgiveness ( $2^{\text {nd }}$ ed., pp. 266-276). Routledge.

Ghorbani, N., Watson, P. J., Kashanaki, H., \& Chen, Z. J. (2017). Diversity and complexity of religion and spirituality in Iran: Relationships with self-compassion and self-forgiveness. The International Journal for the Psychology of Religion, 27(4), 157-171. https://doi.org/10.1080/10508619.2017.1340100

Granqvist, P. (2020). Attachment in religion and spirituality: A wider view. Guilford Press.

Greer, C. L., Worthington, E. L., Jr., Lin, Y., Lavelock, C. R., \& Griffin, B. J. (2014). Efficacy of a self-directed forgiveness workbook for Christian victims of within-congregation offenders. Spirituality in Clinical Practice, 1(3), 218-230. https://doi.org/10.1037/scp0000012

Greer, C. L., Worthington, E. L., Jr., Van Tongeren, D. R., Gartner, A. L., Jennings, D. J. II, Lin, Y., Lavelock, C. R., Greer, T. W., \& Ho, M. Y. (2014). Forgiveness of in-group offenders in Christian congregations. Psychology of Religion and Spirituality, 6(2), 150161. https://doi.org/10.1037/a0035186

Hernandez, B. C., Vonderfecht, H., Smith, S. B., Cress, P. K., Davis, R., \& Bigger, D. (2012). Development and evaluation of a faith-based psychoeducational approach to forgiveness for Christians. Journal of Religion \& Spirituality in Social Work: Social Thought, 31(3), 263-284. https://doi.org/10.1080/15426432.2012.679842 
Hill, P. C., Pargament, K. I., Hood, R. W., Jr., McCullough, M. E., Swyers, J. P., Larson, D. B., et al. (2000). Conceptualizing religion and spirituality: Points of commonality, points of departure. Journal for the Theory of Social Behavior, 30, 51-77. https://doi.org/10.1111/1468-5914.00119

Ho, M. Y., Worthington, E. L., Jr., \& Davis, D. E. (2017). Be a peace maker: Examining the relationship between religiousness and intergroup forgiveness. Peace and Conflict: Journal of Peace Psychology, 23(4), 427-431. https://doi.org/10.1037/pac0000266

McElroy, S. E., Rice, K. G., Davis, D. E., Hook, J. N., Hill, P. C., Worthington, E. L., Jr., \& Van Tongeren, D. R. (2014). Intellectual humility: Scale development and theoretical elaborations in the context of religious leadership. Journal of Psychology and Theology, 42(1), 19-30. https://doi.org/10.1177/009164711404200103

Pargament, K. I. (2007). Spiritually integrated psychotherapy. Guilford Press.

Pargament, K. I., \& Exline, J. J. (2021). Working with spiritual struggles in psychotherapy. Guilford Press.

Pargament, K. I., Magyar, G. M., Benore, E., \& Mahoney, A. (2005). Sacrilege: A study of sacred loss and desecration and their implications for health and well-being in a community sample. Journal for the Scientific Study of Religion, 44, 59-78. https://doi.org/10.1111/j.1468-5906.2005.00265.x

Ripley, J. S. \& Worthington, E. L., Jr. (2014). Couple therapy: A new hope-focused approach. InterVarsity Press.

Rueger, S. Y., Worthington, E. L., Jr., Davis, E. B., Chen, Z. J., Cowden, R. G., Moloney, J. M., Eveleigh, E., Stone, L. B., Glowiak, K. J., \& Lemke, A. W. (in press). Development and initial validation of the Persevering Hope Scale: Measuring wait-power in four 
independent samples. Journal of Personality Assessment.

Rye, M. S., \& Pargament, K. I. (2002). Forgiveness and Romantic Relationship in College: Can It Heal the Wounded Heart? Journal of Clinical Psychology, 58(4), 419. https://doi.org/10.1002/jclp.1153

Rye, M. S., Pargament, K. I., Ali, M. A., Beck, G. L., Dorff, E. N., Hallisey, C., Narayanan, V., \& Williams, J. G. (2000). Religious perspectives on forgiveness. In M. E. McCullough, K. I. Pargament, \& C. E. Thoresen (Eds.), Forgiveness: Theory, research, and practice (pp. 17-40). Guilford Press.

Smedes, L. B. (1984). Forgive and forget: Healing the hearts we don't deserve. Harper \& Row.

Smedes, L. B. (1996). The art of forgiving. Moorings.

Snyder, C. R., Harris, C., Anderson, J., Holleran, S., Irving, L. M., Sigmon, S. T., \& Harney, P. (1991). The will and the ways: Development and validation of an individual-differences measure of hope. Journal of Personality and Social Psychology, 60(4), 570-585. https://doi.org/10.1037/0022-3514.60.4.570

Sutton, G. W., Jordan, K., \& Worthington, E. L., Jr. (2014). Spirituality, hope, compassion, and forgiveness: Contributions of Pentecostal spirituality to godly love. Journal of Psychology and Christianity, 33(3), 212-226.

Toussaint, L., Kamble, S., Marschall, J. C., \& Duggi, D. B. (2016). The effects of brief prayer on the experience of forgiveness: An American and Indian comparison. International Journal of Psychology, 51(4), 288-295. https://doi.org/10.1002/ijop.12139

Toussaint, L. L., Worthington, E. L., Jr., \& Williams, D. R. (Eds.). (2015). Forgiveness and health: Scientific evidence and theories relating forgiveness to better health. Springer Science + Business. https://doi.org/10.1007/978-94-017-9993-5

Vasiliauskas, S. L., \& McMinn, M. R. (2013). The effects of a prayer intervention on the process 
of forgiveness. Psychology of Religion and Spirituality, 5(1), 23-31. https://doi.org/10.1037/a0029324

Vieten, C., \& Lukoff, D. (2021). Spiritual and religious competencies in psychology. American Psychologist. Advance online publication. https://doi.org/10.1037/amp0000821

Wade, N. G., Hoyt, W. T., Kidwell, J. E. M., \& Worthington, E. L., Jr. (2014). Meta-analysis of psychotherapeutic interventions to promote forgiveness. Journal of Consulting and Clinical Psychology, 82(1), 154-170. https://doi.org/10.1037/a0035268

Weis, R., \& Speridakos, E. C. (2011). A meta-analysis of hope enhancement strategies in clinical and community settings. Psychology of Well-Being: Theory, Research and Practice, 1(5), 1-16. http://www.psywb.com/content/1/1/5

Worthington, E. L., Jr. (2006). Forgiveness and reconciliation. Brunner-Routledge.

Worthington, E. L., Jr. (2020a). An update of the REACH Forgiveness model to promote forgiveness. In Everett L. Worthington, Jr. \& Nathaniel G. Wade (Eds.), Handbook of forgiveness ( $2^{\text {nd }}$ ed., pp. 277-287). Routledge. https://doi.org/10.4324/9781351123341-26

Worthington, E. L., Jr. (2020b). Understanding forgiveness of other people: Definitions, theories, and processes. In Everett L. Worthington, Jr. \& Nathaniel G. Wade (Eds.), Handbook of forgiveness ( ${ }^{\text {nd }}$ ed., pp. 11-21). Routledge. https://doi.org/10.4324/9781351123341-2

Worthington, E. L., Jr., \& Sandage, S. J. (2016). Forgiveness and spirituality in psychotherapy: A relational approach. American Psychological Association.

Worthington, E. L., Jr. \& Wade, N. (Eds.). (2020). Handbook of forgiveness ( $2^{\text {nd }}$ ed.). Routledge. 


\title{
Chapter 24:
}

\section{Religion/Spirituality and the Twin Virtues of Humility and Gratitude}

\author{
Madalyn R. Cauble ${ }^{1}$, Iman Abdulkadir Said ${ }^{1}$, Aaron T. McLaughlin ${ }^{1}$, Sarah Gazaway ${ }^{1}$, \\ Daryl R. Van Tongeren ${ }^{2}$, Joshua N. Hook ${ }^{3}$, Ethan K. Lacey ${ }^{4}$, Edward B. Davis ${ }^{4}$, and Don E. \\ $\operatorname{Davis}^{1}$ \\ ${ }^{1}$ College of Education and Human Development, Georgia State University \\ ${ }^{2}$ Psychology Department, Hope College \\ ${ }^{3}$ Psychology Department, University of North Texas \\ ${ }^{4}$ School of Psychology, Counseling, and Family Therapy, Wheaton College \\ Correspondence concerning this chapter should be addressed to Madalyn Cauble. Email: \\ madalyncauble@gmail.com
}




\begin{abstract}
In this chapter, we review the literature on religion/spirituality $(\mathrm{R} / \mathrm{S})$ and the twin virtues of humility and gratitude. We focused on articles that were published in peer-reviewed journals and included both a measure of religion/spirituality and humility or gratitude. We focus our review by exploring two questions: (1) how is R/S associated with humility and gratitude, and (2) how might humility and gratitude work in tandem (consistent with the social oil hypothesis of humility)? We found cross-sectional evidence linking R/S and humility and gratitude. Additional work is needed to test recent theory on how humility and gratitude may work together to promote and protect strong relationships, especially at the collective level. We suggest ways to catalyze research in this area, including the potential for future work aligning humility and gratitude. We also identify implications for practice both in clinical settings and religious communities. Keywords: religion, spirituality, gratitude, humility, virtue
\end{abstract}




\section{Chapter 24: Religion/Spirituality and the Twin Virtues of Humility and Gratitude}

The study of virtue is central to positive psychology, and it has also received substantial scholarly attention in the psychology of religion/spirituality (R/S). To gain a richer understanding of virtues, any theoretical approach must account for religious/spiritual contexts (Snow, 2019). Virtues involve acting in aspirational ways, often in the face of contextual pressures that make doing so difficult, and religious traditions can influence how people understand and practice virtues in their relationships, families, schools, workplaces, and communities (VanderWeele, 2017; see Ratchford et al., Chapter 4, this volume). For example, Saroglou (2011) contends that one of the central features of religion is to shape moral behaviors by prescribing virtuous actions and proscribing immoral ones. For example, religious communities may endorse virtues with social norms and a meaning system that encourages people to live virtuously, while also providing them social support to persist in acting virtuously even when it is difficult.

A limitation of prior work in positive psychology is that scholars often focus on one virtue at a time without considering how virtues may compliment or interact with each other in the context of relationships and group interactions (Davis, 2019). Some scholars (e.g., Emmons, 2016; Lavelock et al., 2017) consider humility and gratitude to be interrelated, superordinate relational virtues. As such, these twin virtues provide the cognitive and motivational structure to support a variety of other virtuous behaviors. Therefore, the purpose of this chapter is to provide a focused review of research on R/S and the virtues of humility and gratitude. First, we define terms and review our key research questions. Second, we describe the method of our search and review evidence pertaining to our primary research questions. Finally, we discuss limitations, directions for future research, and implications for clinical practice and religious communities. 


\section{Definitions}

Religion and spirituality (R/S) have been defined various ways. Spirituality has been defined as a "search for or relationship with the sacred" (Harris et al., 2018, p. 1). People may practice spirituality within or outside the context of a formal religious community, and they may seek greater spiritual connection with various sources of sacredness, including God, nature, humanity, or the transcendent (Davis et al., 2015). Religiousness has been defined as "ritual, institutional, or codified spirituality, which is culturally sanctioned" (Harris et al., 2018, p. 1).

Both spirituality and religion can be understood as relational and cultural constructs. Accordingly, people's relationships and cultural background influence how they understand and seek connection with whatever they perceive as sacred. According to Saroglou (2011), R/S has four main functions: believing, bonding, behaving, and belonging. An important aspect of how individuals relate to whatever they perceive as sacred is how people understand what kind of life is worth pursuing (i.e., beliefs), and these belief systems often include notions of virtue (i.e., culturally esteemed and prescribed ways of thinking, feeling, and behaving). Relational virtues, such as gratitude and humility, involve people's way of understanding how they ought to form, maintain, and repair relationships with other people and with perceived sacred entities (Sandage et al., 2020).

Humility is a multidimensional construct that (a) intrapersonally involves having an accurate view of one's strengths and limitations and (b) interpersonally involves being otheroriented rather than self-focused (McElroy-Heltzel et al., 2019). In addition to regulating negative interpersonal behaviors in alignment with modesty norms, some define humility as involving an orientation toward the betterment of relationships (e.g., Hook et al., 2013).

Behaviors that indicate humility may vary by context (Davis et al., 2016). For example, in a 
qualitative study of leaders, Owens and Hekman (2012) found evidence that humility is behaviorally expressed in three main ways: (a) acknowledging mistakes and limitations, (b) exhibiting teachability, and (c) appreciating the contributions of others.

The latter behavior points to an overlap between humility and gratitude. Gratitude is a positive emotion that occurs when people recognize that another being (a human, animal, or divine being) has intentionally done something to benefit them. Gratitude has been studied as a state, mood, or disposition (Ma et al., 2017), but to date, most studies have conceptualized and assessed it as a disposition - the tendency to feel gratitude across a range of situations, contexts, and relationships. The more people perceive a gift as good, unobligated, and costly, the more gratitude they feel (McCullough et al., 2001). In contrast, people high in entitlement may tend to overlook the contributions of others or view positive acts as obligations rather than gifts (Navarro \& Tudge, 2020). Thus, low humility often may lead to ingratitude, which then undermines trust and cooperation (Emmons, 2016). In contrast, a stance of viewing all things in life as spiritual gifts that find their ultimate source in sacred entities (e.g., God or nature) may provide religious/spiritual individuals with a persistent sense of gratitude that curbs entitlement.

\section{Key Research Questions for Review}

We had two key research questions that organized this chapter's review. The first research question was whether and how R/S are related to the virtues of humility and gratitude? This question is important, given debates whether R/S causes greater prosociality (Galen, 2012). Religious communities define and seek to promote virtuous behavior (Saroglou, 2011), but do they succeed? There is meta-analytic evidence that religiousness is weakly correlated with selfenhancement and social desirability bias (Sedikides \& Gebauer, 2010). People high in religiousness may think of themselves as virtuous, but they may not actually be much more 
virtuous than people who are lower in religiousness (Davis et al., 2013). Therefore, an initial place to start is to compare correlations of R/S with humility and gratitude, followed by looking for any evidence regarding causal direction or important moderators.

The second research question was based on the social oil hypothesis of humility. Prior theory on humility suggests that humility is especially important in contexts that involve a potential for conflict, such as power struggles, intellectual disagreements, or cultural differences (Van Tongeren et al., 2019). According to the social oil hypothesis, humility "reduces relational wear and tear in situations in which conflict is highly likely or there is a substantial power differential between partners. Namely, [this hypothesis] predicts that consistently expressing humble behaviors will buffer a relationship from deterioration in relationship quality that often accompanies competitive traits or conflict" (Van Tongeren et al. 2019, p. 464). Furthermore, even when offenses occur, humility may also promote relational-repair behaviors.

For several reasons, religious communities and contexts are important places to study the social oil hypothesis. First, they often involve some degree of tradition or hierarchy (Worthington, 1988). Second, they not only seek to promote commitment to ideological beliefs, but these beliefs are often sanctified, which has the potential to intensify group dynamics (Graham \& Haidt, 2010). Third, given that religious groups have strong traditions that promote conformity and cohesion, belonging to a religious group may create a potential for conflict, both within the community and with outsiders. Sanctification of religious ideas may result in both benefits (e.g., cohesion, belonging, and meaning) and costs (e.g., outgroup derogation, prejudice, and interpersonal conflict; for a review, see Hall et al., 2010). To the degree that religious groups promote humility (and perhaps gratitude) as a virtue for guiding divine and human relationships, it may help them optimize the benefits and costs of ideological commitment. 
Although this second question was primarily focused on R/S and humility, we scoured the literature on R/S and gratitude for clues about how gratitude and humility might work together to help people navigate relationships and manage conflict. If gratitude and humility are conceptually interrelated, then practicing gratitude might be an important way for people to express humility in their relationships. The find-remind-bind theory of gratitude (Algoe, 2012) overlaps with theorizing on the role of humility in strengthening and repairing social bonds (Davis et al., 2013). In particular, relational offenses may undermine trust and deter cooperation, but when offended people turn their mind towards the benefits they receive in relationship with their perceived offender, this altered focus might provide motivation to continue investing in the relationship. In research on couples, gratitude can help protect marriages from deterioration due to conflict (Fincham \& Beach, 2010). Likewise, in some forgiveness interventions, participants learn to engage gratitude as an other-oriented emotion that helps increase emotional forgiveness (Wade et al., 2014). On the other hand, some evidence suggests the benefits of gratitude may require mutual investment and can have drawbacks when only one partner practices gratitude with a partner who is low in gratitude (McNulty \& Dugas, 2019). Thus, we looked for potential inroads for studying gratitude as a hidden factor in the social oil hypothesis of humility. Because this area of the literature is early in its development and therefore somewhat sparse, we ultimately will explore future directions that may help illuminate this association further.

\section{Method of the Search}

On December 4, 2020, we conducted searches of PsycINFO that included the following search terms: relig* AND gratitude; God AND gratitude; gratitude to God; relig* AND humility. This search resulted in 367 abstracts for the search on humility and 427 for the search on gratitude. We included articles that were published in a peer-reviewed journal and included at 
least one measure of R/S and one measure of humility or gratitude. We identified 62 studies that focused on humility and R/S and 32 studies that focused on gratitude and R/S.

\section{Results}

\section{Overview of Samples and Research Designs}

A summary of the results is presented in Appendix 24.S1 (see Tables 24.S1 through 24.S4). For studies on humility, the vast majority $(k=47)$ involved convenience samples of undergraduate/graduate students or MTurk/Qualtrics-Panels workers. The remaining studies $(k=$ 15) recruited other types of samples, including general community samples or specificpopulation samples (e.g., religious leaders, mental health practitioners, psychotherapy patients, married couples, organizational employees). Most studies recruited U.S. samples $(k=48)$, but 14 studies that recruited from samples in other countries (e.g., Iran, Pakistan, Malaysia, Poland, Romania, Canada, and New Zealand). Most studies $(k=53)$ used a cross-sectional/correlational design; however, 9 used an experimental design, longitudinal design, or both $(k \mathrm{~s}=4,4$, and 1$)$.

For the gratitude studies, nearly half the studies recruited convenience samples of undergraduate or graduate student participants $(k=14)$, and the remaining studies recruited general community samples $(k=12$; e.g., of adults, older adults, or youth) or special-population community samples $(k=6$; e.g., married couples, adults with recurrent depression, or adults with heart failure). Most studies involved U.S. samples $(k=21)$, but 11 studies recruited samples in

other countries (e.g., Iran, Taiwan, China, India, Vietnam, Poland, Australia, and the U.K.). Most studies used a cross-sectional/correlational design $(k=28)$, but three studies used a longitudinal design, and one additional study used an experimental and longitudinal design.

\section{Overview of Measures}

The vast majority of studies in the current review relied on self-report measures of trait 
(i.e., dispositional) humility or trait gratitude. Several studies also measured gratitude to God, and a few of these studies (e.g., Lin, 2014, 2017) conceptualized dispositional gratitude as a higher-order construct that included gratitude to God.

\section{R/S and its Association with Humility and Gratitude}

Supplemental Tables 24.S1 and 24.S2 provide an overview of studies and correlations between $\mathrm{R} / \mathrm{S}$ and humility. Correlations between $\mathrm{R} / \mathrm{S}$ and humility ranged from strongly negative to strongly positive (the mean correlation was .18 and median was .21). Measurement moderators are often helpful for making sense of such disparate findings. For example, when correlations were negative, R/S was often assessed with a measure of religious orientation or of negatively valenced religiousness/spirituality (e.g., religious/spiritual struggles, religious ethnocentricism, or anger toward God). In addition, it is important to consider the conceptual difference between self-reports of humility (which tend to be relatively stable over time) and other-reports of humility within a specific relationship (which may change quite a bit after major events such as betrayals or after noticeably self-sacrificial behaviors; Davis et al., 2016).

Supplemental Tables 24.S3 and 24.S4 provide an overview of studies and correlations between R/S and gratitude. Again, correlations ranged from weakly negative to strongly positive, with an average correlation that was weakly to moderately positive (the mean correlation was .27 and median was .25). As before, measurement moderators will likely help understand this wide variability in correlations. For example, because gratitude is an emotional construct, it likely is related quite strongly to emotional and relational facets of R/S (e.g., spiritual transcendence), which is what Table $24 . \mathrm{S} 4$ suggests. Table 24.S4 also suggests that if R/S was assessed as a measure of religious/spiritual well-being (which some have critiqued as conflated with psychological well-being; Finke \& Bader, 2017), the R/S-gratitude link tended to be stronger. 
Across both literatures, an important question involves the need to explore the causal direction of the association between R/S and virtue. Several research groups have proposed models that situate R/S and virtue in a causal chain, and most situate R/S engagement in a faith community as leading to greater virtue (e.g., Krause et al., 2014). These studies have yet to use designs that would support an inference of mediation — and recently, scientists have become increasingly critical of correlational, cross-sectional tests of mediation (Maxwell \& Cole, 2007). Thus, at this point, although various theories exist for the causal association between R/S and humility — and some studies have taken strides in a promising direction (e.g., using an experience sampling method; Olson et al., 2018) — these ideas have yet to be put to an adequate test.

In sum, we see evidence that humility and gratitude tend to correlate with measures of trait-based R/S, but correlations vary widely when $\mathrm{R} / \mathrm{S}$ is assessed as part of a coping process. We have very limited data to test causal theories about how R/S relates to these twin virtues of humility and gratitude. Most studies involved monomethod bias, which does little to allay critiques that R/S correlates with virtue due to social desirability bias (de Vries et al., 2014).

\section{The Social Oil Hypothesis}

Our second research question involved exploring evidence for the social oil hypothesis of humility. In particular, we hoped to explore potential inroads for examining how humility and gratitude may work in tandem to protect relationships from potential conflict. First, to the degree that humility functions to reduce the potential for religious conflict, we might expect it to correlate with other measures of religious tolerance or openness; indeed, that is what an inspection of Table 24.S2 generally reveals. For example, Davis et al. (2016) found that general humility and intellectual humility were negatively related to religious ethnocentrism $(r=-.28$ and -39 , respectively). Similarly, Krumrei-Mancuso and Newman (2020) found evidence that 
intellectual humility buffers the association between political orientation and religious polarization in a sample of MTurk workers $(N=587)$, but only among political conservatives.

Despite the potential benefits of humility for managing potential conflict, Zhang et al. (2018) tested a theory that humility in the religious domain may involve an existential tradeoff. Across two studies, they found that people assigned to imagine themselves in an ideologically dissimilar small group tended to feel reduced social belonging and meaning in life, but people who were higher in intellectual humility experienced this effect to a lesser extent.

Only one study has tested the social oil hypothesis through inducing humility after an invivo offense. In Van Tongeren, Stafford, et al. (2016), Christian participants shared their views on a contentious topic with a religious outgroup member. After purportedly receiving negative feedback from the outgroup member, participants completed a word-sorting task that was used to prime humility. Unknown to participants, words (humility or non-humility) flashed on the screen too quickly to be processed consciously. Finally, participants had a chance to retaliate against the outgroup member. They prepared a snack for the critic, and the dependent variable was the amount of hot sauce used in preparing that snack. As expected, people in the humility condition doled out less sauce (i.e., acted less aggressively) than people in the control condition, despite having received equivalent explicit ratings of negative feedback from the outgroup member.

We hoped to find studies exploring how gratitude and humility might work together to protect relationships from potential conflict, given prior theory linking the two virtues. One study of couples examined relationship satisfaction and gratitude to God for one's partner, but that study did not assess humility (Fincham \& May, 2021). Additional work is needed to test how humility and gratitude may play complimentary roles in protecting relationships from conflict. For example, in prior work by Fincham's lab, couples were trained to pray for the benefit of their 
partner (Fincham \& Beach, 2010). A next step might be to teach couples to pray for increased gratitude for their relationship. Sanctification of gratitude through prayer might amplify the power of typical gratitude interventions (Schnitker \& Richardson, 2019). Furthermore, based on our theorizing, partners who know their partner is regularly thanking God for their relationship ought to see each other as humbler, which ought to lead to positive relationship outcomes (e.g., higher relationship commitment, trust, and satisfaction).

Taken together, we found additional programmatic work on the social oil hypothesis of humility. Although humility may reduce the potential for conflict, it could also reduce the potential for a sense of solidarity and shared sense of meaning in life. Based on prior theory, we suspect that gratitude plays an important role in helping people practice and express humility in the context of conflict, but these ideas need rigorous empirical testing.

\section{Discussion}

In the current chapter, we framed humility and gratitude as twin relational virtues. The purpose of our review was to examine two questions about R/S and virtues while examining findings from both literatures - the R/S-humility literature and the R/S-gratitude literature. First, to what degree are religious/spiritual constructs associated with each virtue? Second, to what degree do we see evidence that these virtues may function as a social oil within the context of relationships, especially given that commitment to R/S can sometimes amplify conflict?

Our review yielded ample evidence of association between R/S and the two relational virtues. At this point, most research is based on cross-sectional, correlational designs, despite provocative theories that suggest engaging in religious communities may increase gratitude and humility, which in turn can promote better relationships and positive outcomes. These ideas certainly fit into larger theories attempting to explain the ways that religious involvement may 
promote better health and mental health (VanderWeele, 2017; see also Wang et al., Chapter 29, this volume). The field seems ripe for rigorously testing theories about how R/S may influence changes in gratitude and humility, as well as other outcome variables of interest.

Regarding the social oil hypothesis, some recent work on humility supports prior theorizing. Prior research shows that R/S can sometimes increase conflict, especially when people imbue their point of view with sacred meaning. Although some initial evidence suggests that humility is sometimes associated with religious openness or tolerance, our review found evidence that humility may involve a tradeoff between existential security (which gives greater meaning and belonging) and ideological span (which may reduce conflict). Prior work on quest religious orientation has found a similar pattern in which greater ideological openness and willingness to explore existential ideas may come at a cost to psychological well-being (Van Tongeren, Davis, et al., 2016).

Although we found additional support for the social oil hypothesis, some recent findings raised new questions. For example, humility may work somewhat differently in political conservatives than it does in political liberals. As eager as we may be to explore this unresolved issue, it may require careful and programmatic work. For example, moral values may affect how people understand humility—conservatives may emphasize respect for authority and tradition, whereas liberals may emphasize egalitarian structures (Davis \& Hook, 2019). Also, for some highly religious individuals, humility may involve a dependence on God, which within some traditions may be understood as viewing beliefs through the lens of scripture (Hill et al., 2018).

A glaring gap in these two fields is the need for more studies that test causal theories of how R/S may promote greater virtue. Given overlap in theory on humility and gratitude, we hoped to find more research on the social oil hypothesis at the intersection of R/S, humility, and 
gratitude. At this point, we found very little, but it was easy to see promising next steps. For example, although there is limited work manipulating humility experimentally, many strategies exist for helping people to practice gratitude.

\section{Limitations and Future Research}

The current review revealed several limitations in the existing literature on $\mathrm{R} / \mathrm{S}$, humility, and gratitude. Samples included mostly undergraduates or people from crowdsourced platforms, and most measures were self-reports, raising concerns about monomethod bias and social desirability. More studies are needed that recruit samples from diverse faith traditions and ideally from a variety of nations, to avoid conflating nation and religion (Vishkin et al., 2020).

Although some studies proposed conceptual models relating R/S with humility or gratitude, the field is currently plagued by problems associated with cross-sectional data. The field needs more programmatic work focused on debates at the intersection of positive psychology and the psychology of R/S. When and how does R/S promote prosociality or flourishing? To answer such questions, future work could draw on current theories of how flourishing occurs across the lifespan (Seligman, 2018) and draws support from key systems, including family, work, educational, and religious communities (VanderWeele, 2017). Under ideal circumstances, how do parents instill relational virtues through aligning these four systems to promote virtuous habits (Obeldobel \& Kerns, 2021)? Similar ideas are explored in further detail in Chapter 17 (King et al., this volume). These authors discuss how selftranscendent emotions (including gratitude), which are encouraged and engaged in religious/spiritual contexts, may increase meaning-making and thriving in youth and adolescents. The Positive Religious and Spiritual Development theory introduced in Chapter 18 (Davis et al., this volume) serves as an integrative theory that-through its applications across life stages, contexts, and cultures-may be useful for addressing such questions by attending to both $\mathrm{R} / \mathrm{S}$ and positive psychology. 
To answer such questions, scholars can continue to build on theory about how humility and gratitude function within interpersonal relationships, such as work on how observing others express gratitude can strengthen groups (Algoe et al., 2020) or how gratitude can protect relationships from envy, cynicism, or other relational threats (Solom et al., 2017). Given that gratitude is such a powerful motivator of prosocial behavior (Ma et al., 2017), what virtues set the stage for gratitude? Furthermore, although most studies in our review focused on individuals, an important next step is to test theories about gratitude within groups. Recent work suggests that gratitude may cause greater conformity to social norms (Ng et al., 2017) and may alert people to the value of relationships (Williams \& Bartlett, 2015). Based on recent theory on transcendent emotions (Stellar et al., 2017), it may be important to explore how religious communities use collective experiences of awe, which may help groups bond, promote humility, and set the stage for coordination of other prosocial acts. This shift to studying gratitude in groups also has the potential to address cultural critiques of positive psychology (Wong, 2019).

Given the need to test causal theories, we suggest more studies use intervention or intervention-like designs to test process models of how humility and gratitude may work in tandem. Perhaps studying how various faith traditions practice gratitude may reveal new ideas on how to amplify the currently small effects of gratitude interventions (Cregg \& Cheavens, 2021). Given the minimal intervention work on humility, it may be helpful to develop a theoretical model that situates humility as a higher-order virtue that involves the practice of other virtues in key situations, such as generosity in the face of scarcity, forgiveness after offenses, and gratitude to counter the tendency for negative thoughts to narrow one's attentional focus.

\section{Practical Implications}

What are this chapter's implications for practitioners? Table 24.S5 (in Appendix 24.S1) presents humility $(k=2)$ and gratitude $(k=2)$ and intervention studies that can be used by 
practitioners or religious leaders within their target populations. The table explores how each virtue is engaged, the intervention that was used, and the empirical findings.

In studies of clinicians, humility has been linked with social justice commitment, intercultural competence, and faith maturity (Bell et al., 2017). In clinical consultation groups focused on discussion of R/S issues, humility has been correlated with self-efficacy, emotional maturity, and positive attitudes towards addressing R/S in psychotherapy (Crabtree et al., 2020). Humility has also been linked with positive qualities in clergy. For example, in a study of pastors, exposure to religious diversity correlated to religious tolerance, and this association was stronger in those higher in intellectual humility (Hook et al., 2017). Similarly, in a study of Christian leaders, intellectual humility was related to a variety of indicators of willingness to collaborate with mental health professionals, as well as with emotional intelligence (Hodge et al., 2020). Humility interventions—-such as workbooks and workshops—-have shown further promise within populations of religious leaders, with evidence that they may improve awareness of one's own level of humility (Cuthbert et al., 2018) and might lead to changes in one's humility, especially for people with a secure attachment relationship with God (Jankowski et al., 2021).

Theory and research on gratitude suggests that attending to gifts and expressing gratitude are powerful ways to avoid the potential for negative rumination to cause a downward cycle. Based on Brad Owen's work on leaders (Owens \& Hekman, 2012), noticing and appreciating the contributions of others in a group or community is one of the tell-tale signs of humility in leaders. We also know from research on couples that negativity can cause negative cycles of thoughts, emotions, and behaviors, whereas gratitude and its expression help keep relationships healthy (Fincham \& May, 2021). Even when people lack motivation to act virtuously, practicing gratitude is a good step, because it enhances motivation to act prosocially. Although gratitude 
plays this role in all relationships, people in religious communities may have unique resources to amplify gratitude (Krause \& Ellison, 2009). Additionally, individuals within religious communities can draw on religious/spiritual teachings and traditions to reinforce and sanctify the importance of gratitude and can interlace rituals with experiences of gratitude (Schnitker \& Richardson, 2019). When people enjoy the benefits of a strong community, gratitude has the potential to build on itself via cycles of reciprocity.

\section{Conclusion}

Although many studies on R/S and humility and gratitude have been conducted, from the present review, we see clearly that there is a need for programmatic work testing theory on how humility and gratitude complement each other within religious/spiritual individuals and communities. We called for more work that explicitly asks people to practice humility or gratitude and explores ways of engaging religious/spiritual beliefs or practices to amplify virtuous behaviors. This work has the potential to improve our understanding how of R/S affects physical and mental health through strengthening relationships in families and communities. It also has the potential to help scientists and practitioners understand how relational virtues allow $\mathrm{R} / \mathrm{S}$ communities to balance tradeoffs associated ideological commitment and belonging. Going forward, there is a need for both stronger theories and stronger methodologies that test the causal implications of said theories, as well as for work that explores humility and gratitude practices within a chain that causally links religious/spiritual constructs with positive outcomes. 


\section{References}

Algoe, S. B. (2012). Find, remind, and bind: The functions of gratitude in everyday relationships: Gratitude in relationships. Social and Personality Psychology Compass, 6(6), 455-469. https://doi.org/10.1111/j.1751-9004.2012.00439.x

Algoe, S. B., Dwyer, P. C., Younge, A., \& Oveis, C. (2020). A new perspective on the social functions of emotions: Gratitude and the witnessing effect. Journal of Personality and Social Psychology, 119(1), 40-74. https://doi.org/10.1037/pspi0000202

Bell, C. A., Sandage, S. J., Morgan, T. D., \& Hauge, D. J. (2017). Relational spirituality, humility, and commitments to social justice and intercultural competence. Journal of Psychology \& Christianity, 36(3), 210-221.

Crabtree, S. A., Bell, C. A., Rupert, D. A., Sandage, S. J., Devor, N. G., \& Stavros, G. (2020). Humility, differentiation of self, and clinical training in spiritual and religious competence. Journal of Spirituality in Mental Health. Advance online publication. https://doi.org/10.1080/19349637.2020.1737627

Cregg, D. R., \& Cheavens, J. S. (2021). Gratitude interventions: effective self-help? A meta-analysis of the impact on symptoms of depression and anxiety. Journal of Happiness Studies, 22(1), 413-445. https://doi.org/10.1007/s10902-020-00236-6

Cuthbert, A. D., Davis, E. B., Aten, J. D., Short, A., Yarborough, C. A., Lavelock, C. R., ... \& Van Tongeren, D. R. (2018). Cultivating humility in religious leaders: The effectiveness of a spiritually integrated positive psychology intervention. Spirituality in Clinical Practice, 5(4), 227-239. https://doi.org/10.1037/scp0000185

Davis, D. (2019). Envisioning more virtuous virtues. Journal of Positive Psychology, 14 (1), 72 76. https://doi.org/10.1080/17439760.2018.1528383

Davis, D. E., \& Hook, J. N. (2019). Cultural humility: Conclusion to the special issue. Journal of 
Psychology and Theology, 47(3), 230-239. https://doi.org/10.1177/0091647119856463

Davis, D. E., Rice, K., Hook, J. N., Van Tongeren, D. R., DeBlaere, C., Choe, E., \& Worthington, E. L., Jr. (2015). Development of the Sources of Spirituality Scale. Journal of Counseling Psychology, 62(3), 503-513. https://doi.org/10.1037/cou0000082

Davis, D. E., Rice, K., McElroy, S., DeBlaere, C., Choe, E., Van Tongeren, D. R., \& Hook, J. N. (2016). Distinguishing intellectual humility and general humility. The Journal of Positive Psychology, 11(3), 215-224. https://doi.org/10.1080/17439760.2015.1048818

Davis, D. E., Worthington Jr, E. L., Hook, J. N., \& Hill, P. C. (2013). Research on religion/spirituality and forgiveness: A meta-analytic review. Psychology of Religion and Spirituality, 5(4), 233-241. https://doi.org/10.1037/a0033637

de Vries, R. E., Zettler, I., \& Hilbig, B. E. (2014). Rethinking trait conceptions of social desirability scales: Impression management as an expression of honestyhumility. Assessment, 21(3), 286-299. https://doi.org/10.1177/1073191113504619

Emmons, R. A. (2016). Is gratitude queen of the virtues and ingratitude king of the vices? In D. Carr (Ed.), Perspectives on gratitude: An interdisciplinary approach (pp. 141-153). Routledge.

Fincham, F. D., \& Beach, S. R. (2010). Of memes and marriage: Toward a positive relationship science. Journal of Family Theory \& Review, 2(1), 4-24. https://doi.org/10.1111/j.17562589.2010.00033.x

Fincham, F. D., \& May, R. W. (2021). Generalized gratitude and prayers of gratitude in marriage. The Journal of Positive Psychology, 16(2), 282-287. https://doi.org/10.1080/17439760.2020.1716053

Finke, R., \& Bader, C. D. (Eds.). (2017). Faithful measures: New methods in the measurement of 
religion. New York University Press.

https://doi.org/10.18574/nyu/9781479875214.001.0001

Galen, L. W. (2012). Does religious belief promote prosociality? A critical examination. Psychological Bulletin, 138(5), 876-906. https://doi.org/10.1037/a0028251

Graham, J., \& Haidt, J. (2010). Beyond beliefs: Religions bind individuals into moral communities. Personality and social psychology review, 14(1), 140-150. https://doi.org/10.1177/1088868309353415

Hall, M. E. L., Christerson, B., \& Cunningham, S. (2010). Sanctified sexism: Religious beliefs and the gender harassment of academic women. Psychology of Women Quarterly, 34, $181-185$.

Harris, K. A., Howell, D. S., \& Spurgeon, D. W. (2018). Faith concepts in psychology: Three 30year definitional content analyses. Psychology of Religion and Spirituality, 10(1), 1-29. https://doi.org/10.1037/rel0000134

Hill, P. C., Dunnington, K., \& Hall, M. E. L. (2018). Glad intellectual dependence on God: A theistic account of intellectual humility. Journal of Psychology and Christianity, 37(3), $195-204$.

Hill, P. C., Pargament, K. I., Hood, R. W., McCullough, J. M. E., Swyers, J. P., Larson, D. B., \& Zinnbauer, B. J. (2000). Conceptualizing religion and spirituality: Points of commonality, points of departure. Journal for the Theory of Social Behaviour, 30(1), 51-77. https://doi.org/10.1111/1468-5914.00119

Hodge, A. S., Hook, J. N., Davis, D. E., \& McMinn, M. R. (2020). Attitudes of religious leaders toward integrating psychology and church ministry. Spirituality in Clinical Practice, 7(1), 18-33. https://doi.org/10.1037/scp0000200 
Hook, J. N., Davis, D. E., Owen, J., Worthington Jr, E. L., \& Utsey, S. O. (2013). Cultural humility: Measuring openness to culturally diverse clients. Journal of Counseling Psychology, 60(3), 353-366. https://doi.org/10.1037/a0032595

Hook, J. N., Farrell, J. E., Johnson, K. A., Van Tongeren, D. R., Davis, D. E., \& Aten, J. D. (2017). Intellectual humility and religious tolerance. The Journal of Positive Psychology, 12(1), 29-35. https://doi.org/10.1080/17439760.2016.1167937

Jankowski, P. J., Sandage, S. J., Ruffing, E. G., Crabtree, S. A., Bell, C. A., \& Park, S. H. (2021). A mixed-method intervention study on relational spirituality and humility among religious leaders. Spirituality in Clinical Practice. Advance online publication. https://doi.org/10.1037/scp0000248

Krause, N., \& Ellison, C. G. (2009). Social environment of the church and feelings of gratitude toward god. Psychology of Religion and Spirituality, 1(3), 191-205. https://doi.org/10.1037/a0016729

Krause, N., Bruce, D., Hayward, R. D., \& Woolever, C. (2014). Gratitude to God, self-rated health, and depressive symptoms. Journal for the Scientific Study of Religion, 53(2), 341355. https://doi.org/10.1111/jssr.12110

Krumrei-Mancuso, E. J., \& Newman, B. (2020). Intellectual humility in the sociopolitical domain. Self and Identity, 19(8), 989-1016. https://doi.org/10.1080/15298868.2020.1714711

Lavelock, C. R., Worthington, E. L., Griffin, B. J., Garthe, R. C., Elnasseh, A., Davis, D. E., \& Hook, J. N. (2017). Still waters run deep: Humility as a master virtue. Journal of Psychology and Theology, 45(4), 286-303. https://doi.org/10.1177/009164711704500404 Lin, C.-C. (2014). A higher-order gratitude uniquely predicts subjective well-being: Incremental 
validity above the personality and a single gratitude. Social Indicators Research, 119(2), 909-924. https://doi.org/10.1007/s11205-013-0518-1

Lin, C.-C. (2017). The effect of higher-order gratitude on mental well-being: beyond personality and unifactoral gratitude. Current Psychology, 36(1), 127-135. https://doi.org/10.1007/s12144-015-9392-0

Ma, L. K., Tunney, R. J., \& Ferguson, E. (2017). Does gratitude enhance prosociality?: A metaanalytic review. Psychological Bulletin, 143(6), 601-635. https://doi.org/10.1037/bul0000103

Maxwell, S. E., \& Cole, D. A. (2007). Bias in cross-sectional analyses of longitudinal mediation. Psychological Methods, 12(1), 23-44. https://doi.org/10.1037/1082-989X.12.1.23

McCullough, M. E., Kilpatrick, S. D., Emmons, R. A., \& Larson, D. B. (2001). Is gratitude a moral affect? Psychological Bulletin, 127(2), 249-266. https://doi.org/10.1037/0033$\underline{2909.127 .2 .249}$

McElroy-Heltzel, S. E., Davis, D. E., DeBlaere, C., Worthington, E. L., \& Hook, J. N. (2019). Embarrassment of riches in the measurement of humility: A critical review of 22 measures. The Journal of Positive Psychology, 14(3), 393-404. https://doi.org/10.1080/17439760.2018.1460686

McNulty, J. K., \& Dugas, A. (2019). A dyadic perspective on gratitude sheds light on both its benefits and its costs: Evidence that low gratitude acts as a "weak link". Journal of Family Psychology, 33(7), 876-881. https://doi.org/10.1037/fam0000533

Navarro, J. L., \& Tudge, J. R. H. (2020). What is gratitude? Ingratitude provides the answer. Human Development, 64(2), 83-96. https://doi.org/10.1159/000511185

Ng, J. W., Tong, E. M., Sim, D. L., Teo, S. W., Loy, X., \& Giesbrecht, T. (2017). Gratitude 
facilitates private conformity: A test of the social alignment hypothesis. Emotion, 17(2), 379-387. https://doi.org/10.1037/emo0000249

Obeldobel, C. A., \& Kerns, K. A. (2021). A literature review of gratitude, parent - child relationships, and well-being in children. Developmental Review, 61, Article 100948. https://doi.org/10.1016/j.dr.2021.100948

Olson, R., Knepple Carney, A., \& Hicks Patrick, J. (2018). Associations between gratitude and spirituality: An experience sampling approach. Psychology of Religion and Spirituality, 11(4), 449-452. https://doi.org/10.1037/rel0000164

Owens, B. P., \& Hekman, D. R. (2012). Modeling how to grow: An inductive examination of humble leader behaviors, contingencies, and outcomes. Academy of Management Journal, 55(4), 787-818. https://doi.org/10.5465/amj.2010.0441

Saroglou, V. (2011). Believing, bonding, behaving, and belonging: The big four religious dimensions and cultural variation. Journal of Cross-Cultural Psychology, 42(8), 13201340. https://doi.org/10.1177/0022022111412267

Sandage, S. J., Rupert, D., Stavros, G., \& Devor, N. G. (2020). Relational spirituality in psychotherapy: Healing suffering and promoting growth. American Psychological Association. https://doi.org/10.1037/0000174-000

Schnitker, S. A., \& Richardson, K. L. (2019). Framing gratitude journaling as prayer amplifies its hedonic and eudaimonic well-being, but not health, benefits. The Journal of Positive Psychology, 14(4), 427-439. https://doi.org/10.1080/17439760.2018.1460690

Sedikides, C., \& Gebauer, J. E. (2010). Religiosity as self-enhancement: A meta-analysis of the relation between socially desirable responding and religiosity. Personality and Social Psychology Review, 14(1), 17-36. https://doi.org/10.1177/1088868309351002 
Seligman, M. (2018). PERMA and the building blocks of well-being. The Journal of Positive Psychology, 13(4), 333-335. https://doi.org/10.1080/17439760.2018.1437466

Snow, N. (2019). Positive psychology, the classification of character strengths and virtues, and issues of measurement. The Journal of Positive Psychology, 14 (1), 20-31. https://doi.org/10.1080/17439760.2018.1528376

Solom, R., Watkins, P. C., McCurrach, D., \& Scheibe, D. (2017). Thieves of thankfulness: Traits that inhibit gratitude. The Journal of Positive Psychology, 12(2), 120-129. https://doi.org/10.1080/17439760.2016.1163408

Stellar, J. E., Gordon, A. M., Piff, P. K., Cordaro, D., Anderson, C. L., Bai, Y., Maruskin, L. A., \& Keltner, D. (2017). Self-transcendent emotions and their social functions: Compassion, gratitude, and awe bind us to others through prosociality. Emotion Review, 9(3), 200207. https://doi.org/10.1177/1754073916684557

VanderWeele, T. J. (2017). On the promotion of human flourishing. Proceedings of the National Academy of Science, 114(31), 8148-8156. https://doi.org/10.1073/pnas.1702996114

Van Tongeren, D. R., Davis, D. E., Hook, J. N., \& Johnson, K. A. (2016). Security versus growth: existential tradeoffs of various religious perspectives. Psychology of Religion and Spirituality, 8(1), 77-88. https://doi.org/10.1037/rel0000050

Van Tongeren, D. R., Davis, D. E., Hook, J. N., \& Witvliet, C. V. O. (2019). Humility. Current Directions in Psychological Science, 28(5), 463-468. https://doi.org/10.1177/0963721419850153

Van Tongeren, D. R., Stafford, J., Hook, J. N., Green, J. D., Davis, D. E., \& Johnson, K. A. (2016). Humility attenuates negative attitudes and behaviors toward religious out-group members. The Journal of Positive Psychology, 11(2), 199-208. 
https://doi.org/10.1080/17439760.2015.1037861

Vishkin, A., Schwartz, S. H., Ben-Nun Bloom, P., Solak, N., \& Tamir, M. (2020). Religiosity and desired emotions: Belief maintenance or prosocial facilitation? Personality and Social Psychology Bulletin, 46(7), 1090-1106. https://doi.org/10.1177/0146167219895140

Wade, N. G., Hoyt, W. T., Kidwell, J. E., \& Worthington Jr, E. L. (2014). Efficacy of psychotherapeutic interventions to promote forgiveness: A meta-analysis. Journal of Consulting and Clinical Psychology, 82(1), 154-170. https://doi.org/10.1037/a0035268

Williams, L. A., \& Bartlett, M. Y. (2015). Warm thanks: Gratitude expression facilitates social affiliation in new relationships via perceived warmth. Emotion, 15(1), 1-5. https://doi.org/10.1037/emo0000017

Wong, P. T. (2019). Second wave positive psychology’s (PP 2.0) contribution to counselling psychology. Counselling Psychology Quarterly, 32(3-4), 275-284. https://doi.org/10.1080/09515070.2019.1671320

Worthington, E. L. (1988). Understanding the values of religious clients: A model and its application to counseling. Journal of Counseling Psychology, 35(2), 166-174. https://doi.org/10.1037/0022-0167.35.2.166

Zhang, H., Hook, J. N., Farrell, J. E., Mosher, D. K., Van Tongeren, D. R., \& Davis, D. E. (2018). The effect of religious diversity on religious belonging and meaning: The role of intellectual humility. Psychology of Religion and Spirituality, 10(1), 72-78. https://doi.org/10.1037/rel0000108 


\title{
Chapter 25:
}

\section{Theological Virtues, Health, and Well-Being: Theory, Research, and Public Health}

\author{
Katelyn N. G. Long ${ }^{1,2}$ and Tyler J. VanderWeele ${ }^{1,2,3}$ \\ ${ }^{1}$ Human Flourishing Program, Harvard Institute for Quantitative Social Science \\ ${ }^{2}$ Department of Epidemiology, Harvard T.H. Chan School of Public Health \\ ${ }^{3}$ Department of Biostatistics, Harvard T.H. Chan School of Public Health
}

\begin{abstract}
Author's Note:
Correspondence concerning this chapter should be addresses to Dr. Katelyn Long, Human Flourishing Program, Harvard University, 12 Arrow Street, Suite 102, Cambridge, MA 02138. E-mail: knlong@fas.harvard.edu. We thank Jessi Stegall, our colleagues at the Human Flourishing Program, T.H. Chan School of Public Health, and others we collaborate with on projects pertaining to love, hope, and optimism, and Brendan Case, Edward Brooks, and Ryan Gregg for their close review of the manuscript and providing insightful philosophical and theological reflections.
\end{abstract}




\begin{abstract}
This chapter uses a population health perspective to examine the role of the theological virtues of faith, hope, and love in promoting human health and well-being. We begin with an overview of public health's traditional focus on health-related exposures and outcomes and then summarize growing evidence that religion and spirituality play a vital role in health and well-being. Next, we review empirical evidence suggesting associations between faith, hope, and love and subsequent health and well-being, focusing on findings from the public health literature. We reflect on what aspects of these virtues are and are not captured by current measures, as well as what might be needed to improve measurement going forward. We describe the role of religious communities in fostering these virtues in the modern context - faith in an era of an increasing sense of meaninglessness, hope in an era of increasing despair and deaths of despair, and love in an era of increasing division. We close by discussing potential implications for public health and human flourishing.
\end{abstract}

Keywords: faith, hope, love, human flourishing, public health 


\section{Chapter 25: Theological Virtues, Health, and Well-Being: Theory, Research, and Public Health}

The field of public health is often described as the science and art of preventing disease, prolonging life, and promoting health (Winslow, 1920). To achieve these ends, public health research is often focused on the relationships between exposures and health outcomes, and in turn, this research informs public health interventions and policy. Important examples include the relationships between smoking and lung cancer, poor nutrition and stunted childhood growth, and sugary beverages and diabetes. Historically, exposures of most interest to public health researchers are those that might be classified as environmental (e.g., housing, water, sanitation) or biological (e.g., specific pathogens, nutrients, or contaminants). Likewise, key outcomes of interest have generally been those related to physical health, morbidity, and mortality.

Throughout the $20^{\text {th }}$ century, the focus on environmental and biologic exposures has allowed public health scientists, researchers, and policymakers to make tremendous gains in human survival. Polio immunization, small pox eradication, clean drinking water, improved sanitation, and safer food production are just a few of many landmark achievements. These laudable gains in public health gave way to new empirical projects that explored features of a person's life beyond the environmental and biologic exposures that impact health and wellbeing. These features, often called the social determinants of health, include aspects of life such as socioeconomic status, housing, education, and healthcare access. Similarly, outcomes of interest have extended beyond physical health to incorporate mental health and social well-being. This expanded consideration of exposures and outcomes offers promising vistas of inquiry for public health research, fostering curiosity about other features of the human experience that might profoundly shape human health and well-being. 
Building on these themes, a growing line of inquiry examines the role religion/spirituality (R/S) plays in population health (Idler, 2014; Oman, 2018). Early empirical efforts to advance understanding of R/S and health were somewhat limited by the use of cross-sectional data or small sample sizes. However, in recent years, empirical exploration has grown far more rigorous in its use of longitudinal data, larger sample sizes, and increasingly sophisticated analytical techniques. The resulting body of evidence, highlighted in this chapter, suggests that R/S factors play significant and complex roles in human health and well-being (Oman, 2018).

Of course, most people do not engage in religious community and spiritual practices chiefly to improve their physical or mental health. For most, the driving motivations are connection to God or the transcendent and orienting one's life around a sacred reality (see Davis et al., Chapter 18, this volume). Against this backdrop, a reasonable question might be: If the chief ends of religious communities/persons are in God, mature spiritual states of being, or eternal flourishing, why then do we observe R/S associations with so many outcomes pertaining to forms of flourishing in the here and now? What is it about R/S factors that drive these gains in health and well-being? In addition to mechanisms such as community support and shared practices, could it also be that religious virtues, although ultimately pointed towards spiritual and eternal ends, simultaneously cultivate improved corporeal health and well-being? Or, put another way, what role might religious or theological virtues play in promoting human flourishing?

We attempt an answer to these questions with an inquiry focused on the Christian theological virtues of faith, hope, and love. We recognize that these virtues are shared by a number of religions and cultures and that different religious traditions embrace a variety of other religious virtues. Yet out of respect for the complex and distinct ways that various faith traditions approach these concepts, we have limited ourselves to one faith tradition (that itself is highly 
diverse; see Hodge et al., Chapter 10, this volume). We begin by defining the theological virtues from a historical Christian perspective and by summarizing empirical evidence of associations between faith, hope, and love and subsequent health and well-being. We reflect on aspects of these virtues that are (and are not) captured by current measures and on what might be needed to improve measurement going forward. We describe the role of religious communities in fostering these virtues in the modern context — faith in an era of an increasing sense of meaninglessness, hope in an era of increasing despair and deaths of despair, and love in an era of increasing division. Last, we discuss potential implications for public health and human flourishing.

\section{What Are the Theological Virtues?}

A virtue might be understood as a habit in accord with reason to attain the good, and for the theological virtues, the object of that good is God (Aquinas, 1948). In the Christian tradition, the theological virtues of faith, hope, and love are extoled throughout the Old and New Testaments, and across history and cultures, theologians have identified them as the seminal virtues (among many) in the life of Christian. The notion of virtues predated Christianity. The ancient Greeks had a long-running discourse on virtues, most notably the Four Cardinal virtues (of wisdom, justice, fortitude, and temperance) described in Plato's Republic (Plato \& Lane, 2007). From the Greeks (Socrates, Plato, and Aristotle), to the Romans (Cicero and Seneca), to philosophers and theologians from the Jewish (Philo) and Christian traditions (St. Augustine and St. Thomas Aquinas), the idea of virtue, defined broadly as a habit of human rightness (Pieper, 1966), became a basic component of consciousness that seeped its way deep into the soil of Western thought (Pieper, 1966, 1997). Hence, in approaching the concepts of faith, hope, and love, Christian theologians committed to divine revelation have grappled with ways these concepts mapped onto then-existing notions of virtue - and the ways in which they differed. For 
example, $13^{\text {th }}$ century philosopher and theologian St. Thomas Aquinas concentrated much of his intellectual work on the integration of classic Greek philosophy (particularly that of Aristotle) with Christian theology. In his massive theological work, the Summa Theologiae, Aquinas outlined why faith, hope, and love, were understood as essentially "theological" in nature:

Such like principles [faith, hope, and love] are called "theological virtues": first, because their object is God, inasmuch as they direct us aright to God: secondly, because they are infused in us by God alone: thirdly, because these virtues are not made known to us, save by Divine revelation, contained in Holy Writ. (Aquinas, 1948, IaIIae Q. 62 a.1)

Building on the work of St. Thomas Aquinas, $20^{\text {th }}$ century philosopher Josef Pieper has described theological virtues as 'the enobling of man's nature that entirely surpasses what he 'can be' of himself' (Pieper, 1997, p. 99). Pieper (1997) goes on to argue that theological virtues have a supernatural dimension, because they are grounded in and realized only in participation with the Divine. In other words, a theological virtue has its source and fulfillment in God (Pieper, 1997, p. 100).

Throughout Christian history, the nature and expression of the theological virtues has been an ongoing intellectual and spiritual project, including discussion about the proper ordering of the virtues in relation to one another (Summa Theologiae, IaIIae Q. 62 a. 4). The theological virtues are also deeply valued in other religious traditions, including ways that both align with and diverge from Christian interpretations (for an expanded discussion on virtue, see Ratchford et al., Chapter 4, this volume). Although exploring such streams of discourse goes beyond the aims and scope of the present chapter, it remains the case that most Christian traditions across history have extoled faith, hope, and love as three virtues that are worthy of their "theological" distinction and are essential markers and mechanisms of spiritual growth in the Christian life. 


\section{Faith}

In the New Testament book of Hebrews, the author writes, "Now faith is the assurance of things hoped for, the conviction of things not seen" (English Standard Version Bible, 2001, Hebrews 11:1). Building on this passage, St. Thomas Aquinas added: "Faith is a habit of mind, whereby eternal life is begun in us, making the intellect assent to what is not apparent" (Summa Theologiae, IaIIae Q. 4 a.1). He also described how faith perfects the intellect (the power in us that is concerned with truth) by elevating and perfecting human reason, so that human persons can understand their faith and judge it to be true (Summa Theologiae IIaIIae QQ 1-16). Faith might thus be understood as the habit of mind to believe in God and all that God has revealed.

\section{Measurement}

A number of existing measures attempt to assess faith or belief explicitly (Hill \& Hood, 1999), some of which use a tradition-specific approach to assess specific elements of faith (e.g., Fullerton \& Hunsberger, 1982; VanderWeele, Long, et al., 2021). Other faith-related items are included in scales that aim to capture elements of spiritual well-being or belief across a range of traditions (for example, the Spiritual Well-Being Scale [Paloutzian \& Ellison, 1982] and Brief Multidimensional Measure of Religiousness and Spirituality [Idler et al., 2003]). (Also, see an extended list of measures in Appendix 25.S1, Appendix 7.S1, and Appendix 7.S2). Although many of these scales are intermittently employed (in full or in part) in medical and public health research, the vast majority of studies using these items are cross-sectional in nature (Koenig, 2012), making it difficult to infer causality, due to the analysis of data from a single time point.

Given these considerations and the limitations of the available data and research, perhaps the most substantive and well-researched proxy of faith is religious service attendance. Indeed, for many people, it is their faith (or the search for faith) that draws them into participation with 
religious community (Bonhoeffer, 2009). Religious service attendance is admittedly a very crude proxy for faith; however, many large-scale studies often include a question about the frequency of a person's religious service attendance, which allows for a robust window into the relationship between faith and health. Of course, people may attend services for social reasons, rather than because of faith, and faith may be present to some degree in those not attending (e.g., those who suffer from an illness or injury that keeps them from religiou service participation). But for many, it is their faith that brings them back to their religious community week after week, and it is also often the religious community that helps uphold, support, and strengthen their faith.

\section{Empirical Evidence}

As noted earlier in the chapter, religious community participation is associated with a number of key health and well-being indicators. For example, longitudinal studies with control for baseline confounders and outcomes have produced strong evidence for the effects of religious service attendance on better outcomes for suicide, smoking, substance use disorders, cancer and cardiovascular disease survival, divorce, social support, meaning and purpose, life satisfaction, charitable giving, volunteering, and civic engagement (for example, see Aksoy et al., 2021; Chen \& VanderWeele, 2018; Li et al., 2018; Lim \& Putnam, 2010; Strawbridge et al., 1997; VanderWeele et al., 2016; see Appendix 25.S2, for summaries of all the longitudinal studies reviewed in this chapter; also, see Appendix 18.S2 for summaries of many other longitudinal studies of religiousness/spirituality). Below we examine a few of the studies that shed light on the relationship between religious community participation and health.

In their study, Li et al. (2016) used longitudinal data from the first wave of the Nurses' Health Study to examine the relationship between attendance at religious services and subsequent mortality among a sample of over 74,000 women between 1992 and 2012. After 
adjusting for a wide range of demographic covariates, lifestyle factors, and medical history, religious service attendance was associated with 33\% reduction in all-cause mortality, as well as significant reductions in cardiovascular and cancer mortality. Li and colleagues (2016) found that social support, optimism, depressive symptoms, and smoking each explained some of the effect. Another study using the same dataset found that attending religious services once a week or more was associated with a five-fold lower rate of suicide, relative to those who never attended religious services (hazard ratio $=0.16$; 95\% CI: 0.06-0.46; VanderWeele et al., 2016).

Whereas the Nurses' Health Study is comprised of predominantly White participants, a study conducted with data from the Black Women's Health Study similarly found that attending religious services several times a week was associated with substantially lower rate of death (mortality rate ratio $=0.64,95 \% \mathrm{CI}: 0.51-0.80$ ), compared to those who never attended services (VanderWeele et al., 2017). Even more recent studies have also employed sensitivity analyses for unmeasured confounding, and these analyses indicated it was unlikely the results were due entirely to unmeasured factors (VanderWeele, 2021a; VanderWeele \& Ding, 2017). Furthermore, meta-analyses of longitudinal studies examining the effect of religious service participation have found a $27 \%$ (95\% CI: 16\%-37\%) reduction in all-cause mortality risk (Chida et al., 2009) and a significant improvement in mental health (Garssen et al., 2021) for people who attended services at least weekly, compared to those who did not attend at all.

\section{Section Summary}

The cumulative evidence between religious community participation and subsequent health and well-being has lead some to ask whether the medical and public health communities might consider encouraging participation in religious community for those who already positively self-identify with a religious or spiritual tradition (VanderWeele, Balboni, et al., 
2021). Whereas the evidence for this dimension of faith might be increasingly clear, there remains far more that is not well-understood about the nature of faith and how it operates in a person's life over time. The literature on longitudinal studies examining the effects on health of specific religious beliefs (not to mention of faith itself, theologically understood) is essentially nonexistent. In some ways it is remarkable that an indicator as crude as frequency of religious service attendance is so predictive of numerous health and well-being outcomes. As new measures of religious faith perhaps emerge in the years ahead, it will be important to remain attentive to the ways each religious tradition approaches the topic of faith—what faith entails and the kinds of thought and action it motivates — such that participants "hear" their own traditions echoed in the wording and emphasis of survey questions (see Hill et al., Chapter 7, this volume).

\section{Hope}

The theological virtue of hope orients life to one's ultimate end in God, and it enables one to lean on God's help in the midst of human inability and insufficiency (Pieper, 1997). Speaking of this reality in more poetic terms, the Hebrew prophet Isaiah writes: "But those who hope in the Lord will renew their strength. They will soar on wings like eagles; they will run and not grow weary, they will walk and not be faint" (New International Version Bible, 2015; Isaiah 40:31). This dialectic between strength and weakness is characterized well in St. Thomas Aquinas's writing about hope as a desire for some good that arises out of the perception this future good is difficult but not impossible to attain (Summa Theologiae IIaIIae Q. 17). Speaking to the inherent tensions imbedded within theological hope, Pieper wrote: "hope presupposes not only magnanimity but also humility" (Pieper, 1997, p. 127). Theological hope might thus be understood as the habit of fixing one's attention on one's future happiness in God and on God as providing the means to attain that end, even amidst difficulties. Whereas these themes are deeply 
embedded in the Christian tradition of theological hope, hope is often characterized quite differently in its common usage and in much of the empirical literature to date.

\section{Measurement}

The dominant measure of hope used in psychological research is Charles Snyder's Adult Hope Scale (Snyder et al., 1991), which is based on Snyder's cognitive theory of hope and thus views hope as arising from two interrelated cognitive dimensions: agency and pathways (Snyder, 1994; Snyder et al., 1991). In his conception, agency refers to determination and commitment to help a person move towards their goals, and pathways refer to a person's perceived ability to reach those goals and formulate new plans when obstacles arise. Some have critiqued Snyder's emphasis on agency by pointing out the way that hope implies confrontation with the limits of one's agency (Miceli \& Castelfranchi, 2010); however, his measure of hope has been widely used to evaluate associations with psychosocial, physical, and R/S well-being (Snyder, 2000).

\section{Empirical Evidence}

The current body of research around hope has found strong associations with a range of positive outcomes, including emotional adjustment, life satisfaction, positive affect, social support, sense of purpose, and quality of life (Snyder, 2000). Conversely, hopelessness has been associated with increased risk of poor mental health outcomes, including anxiety, depressive, and post-traumatic stress disorders (Gerard \& Booth, 2015; Weinberg et al., 2016).

The topic of hope is not new to those in the field of positive psychology, but in the field of public health, explorations of hope are much less common. A recent exception is Long, Kim et al.'s (2020) longitudinal study of nearly 13,000 older U.S. adults. This study explored the relationship between levels of hope and a wide range of subsequent physical health and wellbeing outcomes (Long, Kim, et al., 2020). The analysis controlled for baseline levels of hope, 
baseline levels of the outcomes, and a rich set of demographic, physical, and psychological factors. Long and colleagues found that participants with a higher sense of hope exhibited a range of better physical health outcomes over time, including $16 \%$ lower risk of all-cause mortality, fewer subsequent chronic conditions, and reduced likelihood of cancer and chronic pain. For health behaviors, those with higher hope had an increased likelihood of engaging in subsequently more physical activity and reduced probability of experiencing subsequent sleep problems. In line with existing psychological research on hope, the study found strong positive associations with all indicators of subsequent psychological and social well-being, including higher life satisfaction, sense of purpose, and social integration, as well as lower depression and loneliness. The study also explored what gives rise to hope, finding several predictors that included physical health, health behaviors (e.g., physical activity), social factors (e.g., frequency of contact with friends, volunteering), and all dimensions of psychological well-being and distress. Because many predictors of hope were modifiable, Long, Kim, and colleagues (2020) suggested that public health programs consider the promotion of hope-enhancing features of people's lives, which in turn could improve long-term population health and well-being.

\section{Section Summary}

The measure of hope used in the study described above was comprised of four statements about hopelessness, meaning that the study assessed participants' hopefulness by the extent to which they rejected these statements. Although Long, Kim, et al. (2020) found meaningful results, there remains a clear need to press further into what is meant by hope in a philosophical and theological sense, as well as to explore how hope differs from optimism - a term used liberally as a synonym of hope but which is arguably distinct (Bury et al., 2016). There is arguably no measure yet available for the assessment of the theological virtue of hope. For 
people from the Christian tradition, theological hope points towards a more final and powerful fulfillment of the good, and this hope is itself mysteriously grounded in the birth, life, death, and resurrection of Jesus Christ. Such ideas are distinctive to the Christian faith and not yet reflected in current measures of hope.

\section{Love}

Love is considered the highest theological virtue in the Christian tradition. St. Thomas Aquinas noted that whereas faith gives way to vision and hope gives way to possession, love endures - and continues in perfect form when a person is fully united with God (Summa Theologiae IIaIIae Q. 23). But what is love? How might it be defined? Many theologians broadly conceive of love as a desire for and/or commitment to the good of others, yet it remains an understandably challenging task to deliminate a word used to describe everything from one's enduring affection for a hamburger to one's devotion to a spouse or God. In light of this challenge, theologian Nicholas Thomas Wright has quipped: "The English word 'love' is trying to do so many different jobs at the same time that someone really ought to sit down with it and teach it how to delegate" (Wright, 2012, p. 183).

Although the definition of the word "love" is unquestionably varied across disciplines (e.g., Fredrickson, 2014), philosophers and theologians have suggested it may have a common core - namely, that we use "love" when one or both of two desires are consistently present: a desire for good for the beloved and/or the desire for some form of union with the beloved (Stump, 2006; Summa Theologiae I.II Q. 26.4). In fact, a case can be made that all ordinarylanguage uses of "love" denote a disposition either (i) towards desiring a perceived good or towards desiring union with it (either as an end itself or with it being a source of delight in itself) or (ii) towards desiring good for a particular object for its own sake (VanderWeele, 2021b). The 
theological virtue of love involves both these dispositions, with God as its object. Hence, under this construal, hope would thus be rendered: the habit of desiring union with God as its own end and of desiring good for God's sake. Under a Christian understanding, this would also, by implication, entail love for one's neighbor as part of God's will (Summa Theologiae II.II Q. 25), and thus it would entail the habit of desiring good for one's neighbor for their own sake and final union with one's neighbor in God as an end in itself.

\section{Measurement}

Despite repeated calls for public health to undertake an epidemiology of love (Levin, 2000, 2007), such a line of research has yet to coalesce, due in part to the wide range of scales used to measure love and those scales' disparate definitions, philosophical assumptions, and emphases. Among the hundreds (perhaps thousands) of love-related items that are sprinkled across surveys employed in social, psychological, and health science, some of the more commonly used measures include the Measurement of Romantic Love Scale (Rubin, 1970), Love Attitude Scale (Hendrick \& Hendrick, 1986), and Passionate Love Scale (Hatfield \& Sprecher, 1986). (See Appendix 25.S1 for an extended list.) However, the of lack of standardized measures and validated definitions of love, as well as the diversity of views about love across disciplines, continue to make comparision or shared understanding difficult to achieve.

\section{Empirical Evidence}

Using available measures, many studies have found significant relationships between forms of love and health/well-being (Graham, 2011; Levin, 2001). The vast majority of studies to date have been cross-sectional in nature, meaning that in addition to the challenges of measurement, there is also the potential for reverse causality. To that end, we focus our discussion on a selection of rigorous longitudinal studies that have examined two forms of love 
(forgiveness and parental love) and their effect on subsequent health and well-being (see an extended discussion on social integration and marriage in Appendix 25.S1).

\section{Forgiveness}

Forgiveness, which often involves replacing ill-will towards a perceived offender with goodwill towards that offender (Worthington, 2020), does not mean that someone either forgoes the pursuit of a just outcome or excuses wrongdoing. However, it does release the injured party from harboring the pain of resentment, suppression, and anger. To date, a large body of psychological studies have demonstrated positive outcomes associated with increased forgiveness (Worthington \& Wade, 2020), inspiring public health researchers to explore how this unique form of love might impact population health (Toussaint et al., 2015).

For example, a series of recent studies used cohort data from the Nurses' Health Study II (NHSII) to examine the role of forgiveness and subsequent health and well-being, controlling for a large range of covariates and for prior values of outcome variables. One study found that people who forgave others more frequently had better outcomes in a number of areas, including higher levels of positive affect $(\beta=0.18 ; 95 \%$ CI: $0.15-0.21)$ and more social integration $(\beta=$ 0.15; 95\% CI: 0.13-0.17). Higher forgiveness also led to lower levels of psychological distress, such as lower levels of depression, anxiety, hopelessness, and loneliness (Long, Worthington, et al., 2020). Two other studies — one incorporating data from the nurses' children and another examining the role of self-forgiveness and divine forgiveness on health and well-beingsimilarly found that higher forgiveness was associated with greater psychosocial well-being and less psychological distress (Chen et al., 2018; Long, Chen, et al., 2020). 


\section{Parental Love}

The love beween parents and children is one of the more universal forms of love. In a recent study using longitudinal data from the Midlife in the United States study, 3,929 participants were followed across a 10-year period (Chen, Kubzansky, et al., 2019). This study found that parental warmth during childhood (a proxy for love) was positively associated with increased flourishing $(\beta=0.21 ; 95 \% \mathrm{CI}=0.18-0.25)$ and inversely related to several negative health behavior outcomes (such as smoking and drug use) during adulthood. Another robust longitudinal study that employed data from the Growing Up Today study examined various aspects of parenting and outcomes for children (Chen, Haines, et al., 2019). This study found that, among young adults, greater parental authoritiveness (high levels of both warmth and discipline), led to greater emotional well-being, fewer depressive symptoms, and lower risk of overeating and risky behaviors. Importantly, when parental warmth was considered independently, it was found to be the most important aspect of parenting and subsequent child well-being. These studies suggest that the childhood experience of loving warmth between parent and child is a pivotal factor in a child's future flourishing.

\section{Section Summary}

Whereas there is indeed evidence that love impacts health in generally positive ways, a great deal remains unknown, due to the limits of cohesive definitions and measures of love, as well as to the relatively few robust longitudinal explorations of love and its long-term effects on health and well-being. More attention is also needed concerning nonromantic forms of love that might apply across different relationships, such as love of neighbor, love of God, love of friends, and even love of enemies, because these types of dyads contain varying forms of appropritate union and contribution towards the good of the other (Stump, 2006; VanderWeele, 2021b). 
Further work might also consider the proper ordering of loves, for example that love of neighbor flows from one's love for God (Aquinas, 1948).

\section{Prospects and Limitations}

As noted above, the measures used in existing empirical research related to faith, hope, and love are essentially only capturing specific facets of — or very crude proxies for — the corresponding theological virtues. To the best of our knowledge, there has been no longitudinal research employing multi-item measures that are intended to assess the theological virtues of faith, hope, and love. The existing longitudinal studies might then be seen as studies of the effects of faith, of hope, and of love as theological virtues, rather than as studies of these virtues themselves. More work on measurement development and refinement remains to be done, and to that end we have tried to provide above some tentative construct definitions of each of the theological virtues (because proposed construct definitions are too often lacking and arguably are a critical part of sound measure development). The theological virtues are, of course, oriented principally towards God, which of course, affect a host of other things in a person's life as well, and it is these effects that the existing empirical research helps uncover.

\section{The Role of Religious Communities in Fostering the Theological Virtues}

This chapter was written in the midst of the COVID-19 pandemic where the sense of societal suffering was palpable and painful. Prior to the pandemic, rates of meaningless (Stein et al., 2017), deaths of despair (Case \& Deaton, 2020), and deepening divisions along polticial, racial, and ideological lines (Brooks, 2019) were all intensifying - trends that were only exacerbated during the pandemic and its attendant challenges. Although it is true that religious communities can themselves be the source of great pain and suffering, the evidence we present in this chapter suggests that religious communities might also be one of the most important 
resources in our time. They can play a vital role in helping heal some of our collective pain, suffering, and division (see Wang et al., Chapter 29, this volume). It is in these sacred spaces that faith is nurtured - faith that can instill a deeper sense of meaning and purpose to help ground the meaninglessness that overcomes so many people. It is in religious community that the message of hope — deep hope, improbable hope, resounding hope—can push against the despair that drives so much suffering and premature death. And it is in religious community that the love of God and love of neighbor can take root, offering a radical alternative to the divisions that threaten to shred our homes, our communities, our nation, and our world.

\section{Implications for Public Health Research and Practice—and Human Flourishing}

We close with three recommendations for improved public health research and practice. The first need is for better measurement of faith, hope, and love, informed by philosophical and theological discourse and in conversation with other social science research. The second need is for empirical research that employs rigorous methodology, including the use of longitudinal data, sufficient covariate control, and control for baseline outcomes where possible. Not only should future empirical work consider the long-term impacts of faith, hope, and love on human health and well-being, but it should also explore the factors give rise to these virtues, which represent principal ends of many religious communities. Finally, there is a need to promote the development and refinement of ideas that lead to human flourishing. If faith, hope, and love are demonstrably linked to human health and well-being, then public health, government, and faith community leaders can play a role in promoting these goods from their various vantage points. This is not a governmental universal "prescription of religion" but rather an encouragement to take seriously empirical work that demonstrates avenues towards human flourishing and to support communities, programs, and policies that are already working towards these ends. 


\section{References}

Aksoy, O., Bann, D., Fluharty, M. E., \& Nandi, A. (2021). Religiosity and mental wellbeing among members of majority and minority religions: Findings from understanding society, The UK household longitudinal study. American Journal of Epidemiology. Advance online publication: $\underline{\text { https://doi.org/10.1093/aje/kwab133 }}$

Aquinas, T. (1948). Summa theologica. Complete English translation in five volumes. Ave Maria Press.

Bonhoeffer, D. (2009). Life together: The classic exploration of Christian in community. Harper San Francisco.

Brooks, A. C. (2019). Our culture of contempt: Op-Ed. The New York Times. https://www.nytimes.com/2019/03/02/opinion/sunday/political-polarization.html

Bury, S., Wenzel, M., \& Woodyatt, L. (2016). Giving hope a sporting chance: Hope as distinct from optimism when events are possible but not probable. Motivation and Emotion, 40(4), 588-601. https://doi.org/10.1007/s11031-016-9560-z

Case, A., \& Deaton, A. (2020). Deaths of despair and the future of capitalism. University Press.

Chen, Y., Haines, J., Charlton, B. M., \& VanderWeele, T. J. (2019). Positive parenting improves multiple aspects of health and well-being in young adulthood. Nature Human Behaviour, 3(7), 684-691. https://doi.org/10.1038/s41562-019-0602-X

Chen, Y., Harris, S. K., Worthington, E. L., \& VanderWeele, T. J. (2018). Religiously or spiritually-motivated forgiveness and subsequent health and well-being among young adults: An outcome-wide analysis. The Journal of Positive Psychology, 14(5), 649-658. https://doi.org/10.1080/17439760.2018.1519591 
Chen, Y., Kubzansky, L. D., \& VanderWeele, T. J. (2019). Parental warmth and flourishing in mid-life. Social Science \& Medicine (1982), 220, 65-72. https://doi.org/10.1016/j.socscimed.2018.10.026

Chen, Y., \& VanderWeele, T. J. (2018). Associations of religious upbringing with subsequent health and well-being From adolescence to young adulthood: An outcome-wide analysis. American Journal of Epidemiology, 187(11), 2355-2364. https://doi.org/10.1093/aje/kwy142

Chida, Y., Steptoe, A., \& Powell, L. H. (2009). Religiosity/spirituality and mortality: A systematic quantitative review. Psychotherapy and Psychosomatics, 78(2), 81-90. https://doi.org/10.1159/000190791

Fredrickson, B. (2014). Love 2.0: Creating happiness and health in moments of connection. Plume.

Fullerton, J. T., \& Hunsberger, B. (1982). A unidimensional measure of Christian orthodoxy. Journal for the Scientific Study of Religion, 21(4), 317-326. https://doi.org/10.2307/1385521

Garssen, B., Visser, A., \& Pool, G. (2021). Does spirituality or religion positively affect mental health? Meta-analysis of longitudinal studies. The International Journal for the Psychology of Religion, 31(1), 4-20. https://doi.org/10.1080/10508619.2020.1729570

Gerard, J. M., \& Booth, M. Z. (2015). Family and school influences on adolescents' adjustment: The moderating role of youth hopefulness and aspirations for the future. Journal of Adolescence, 44, 1-16. https://doi.org/10.1016/j.adolescence.2015.06.003 
Graham, J. M. (2011). Measuring love in romantic relationships: A meta-analysis. Journal of Social and Personal Relationships, 28(6), 748-771. https://doi.org/10.1177/0265407510389126

Hatfield, E., \& Sprecher, S. (1986). Measuring passionate love in intimate relationships. Journal of Adolescence, 9(4), 383-410. https://doi.org/10.1016/S0140-1971(86)80043-4

Hendrick, C., \& Hendrick, S. (1986). A theory and method of love. Journal of Personality and Social Psychology, 50(2), 392-402. https://doi.org/10.1037/0022-3514.50.2.392

Hill, P. C., \& Hood, R. W. (1999). Measures of religiosity. Religious Education Press.

Holy Bible: English Standard Version. (2001). Crossway Bibles.

Idler, E. L. (Ed.). (2014). Religion as a social determinant of public health. Oxford University Press. http://www.oxfordscholarship.com/view/10.1093/acprof:oso/9780199362202.001.0001/a cprof-9780199362202

Idler, E. L., Musick, M. A., Ellison, C. G., George, L. K., Krause, N., Ory, M. G., Pargament, K. I., Powell, L. H., Underwood, L. G., \& Williams, D. R. (2003). Measuring multiple dimensions of religion and spirituality for health research: Conceptual background and findings from the 1998 general social survey. Research on Aging, 25(4), 327-365. https://doi.org/10.1177/0164027503025004001

Koenig, H. G. (2012). Handbook of religion and health (2nd ed.). Oxford University Press. Levin, J. (2000). A prolegomenon to an epidemiology of love: Theory, measurement, and health outcomes. Journal of Social and Clinical Psychology; New York, 19(1), 117-136. https://doi.org/10.1521/jscp.2000.19.1.117 
Levin, J. (2001). God, love, and health: Findings from a clinical study. Review of Religious Research, 42(3), 277-293. https://doi.org/10.2307/3512570

Levin, J. (2007). Integrating positive psychology into epidemiologic theory: Reflections on love, salutogenesis, and determinants of population health. Oxford University Press.

Li, S., Kubzansky, L. D., \& VanderWeele, T. J. (2018). Religious service attendance, divorce, and remarriage among US nurses in mid and late life. PloS One, 13(12), e0207778e0207778. https://doi.org/10.1371/journal.pone.0207778

Li, S., Stampfer, M. J., Williams, D. R., \& VanderWeele, T. J. (2016). Association of religious service attendance with mortality among women. JAMA Internal Medicine, 176(6), 777785. https://doi.org/10.1001/jamainternmed.2016.1615

Lim, C., \& Putnam, R. D. (2010). Religion, social networks, and life satisfaction. American Sociological Review, 75(6), 914-933. https://doi.org/10.1177/0003122410386686

Long, K. N. G., Chen, Y., Potts, M., Hanson, J., \& VanderWeele, T. J. (2020). Spiritually motivated self-forgiveness and divine forgiveness, and subsequent health and well-being among middle-aged female nurses: An outcome-wide longitudinal approach. Frontiers in Psychology, 11. https://doi.org/10.3389/fpsyg.2020.01337

Long, K. N. G., Kim, E. S., Chen, Y., Wilson, M. F., Worthington Jr, E. L., \& VanderWeele, T. J. (2020). The role of hope in subsequent health and well-being for older adults: An outcome-wide longitudinal approach. Global Epidemiology, 2, 100018. https://doi.org/10.1016/j.gloepi.2020.100018

Long, K. N. G., Worthington, E. L., VanderWeele, T. J., \& Chen, Y. (2020). Forgiveness of others and subsequent health and well-being in mid-life: A longitudinal study on female nurses. BMC Psychology, 8(1), 104. https://doi.org/10.1186/s40359-020-00470-w 
Miceli, M., \& Castelfranchi, C. (2010). Hope: The power of wish and possibility. Theory \& Psychology, 20(2), 251-276. https://doi.org/10.1177/0959354309354393

Oman, D. (Ed.). (2018). Why religion and spirituality matter for public health: Evidence, implications, and resources. Springer.

Paloutzian, R. F., \& Ellison, C. W. (1982). Loneliness, spiritual well-being and the quality of life. In D. Perlman \& L. A. Peplau (Eds.), Loneliness: A sourcebook of current theory, research, and therapy (pp. 224-236). Wiley.

Pieper, J. (1966). The four cardinal virtues (R. Winston, C. Winston, L. E. Lynch, \& D. F. Coogan, Trans.; $1^{\text {st }}$ edition). University of Notre Dame Press.

Pieper, J. (1997). Faith, hope, love (Revised edition). Ignatius Press.

Plato, \& Lane, M. (2007). The Republic (D. Lee, Trans.; $2^{\text {nd }}$ ed.). Penguin Classics.

Rubin, Z. (1970). Measurement of romantic love. Journal of Personality and Social Psychology, 16(2), 265-273. https://doi.org/10.1037/h0029841

Snyder, C. R. (1994). Psychology of hope: You can get here from there. Free Press.

Snyder, C. R. (Ed.). (2000). Handbook of hope: Theory, measures \& applications. Academic Press.

Snyder, C. R., Harris, C., Anderson, J. R., Holleran, S. A., Irving, L. M., Sigmon, S. T., Yoshinobu, L., Gibb, J., Langelle, C., \& Harney, P. (1991). The will and the ways: Development and validation of an individual-differences measure of hope. Journal of Personality and Social Psychology, 60(4), 570-585. https://doi.org/10.1037/0022$\underline{3514.60 .4 .570}$

Stein, E. M., Gennuso, K. P., Ugboaja, D. C., \& Remington, P. L. (2017). The epidemic of despair among White americans: Trends in the leading causes of premature death, 1999- 
2015. American Journal of Public Health, 107(10), 1541-1547.

https://doi.org/10.2105/AJPH.2017.303941

Strawbridge, W. J., Cohen, R. D., Shema, S. J., \& Kaplan, G. A. (1997). Frequent attendance at religious services and mortality over 28 years. American Journal of Public Health (1971), 87(6), 957-961. https://doi.org/10.2105/AJPH.87.6.957

Stump, E. (2006). Love, by all accounts. Proceedings and Addresses of the American Philosophical Association, 80(2), 25-43.

Toussaint, L. L., Worthington, E. L., \& Williams, D. R. (Eds.). (2015). Forgiveness and health: scientific evidence and theories relating forgiveness to better health. Springer. https://doi.org/10.1007/978-94-017-9993-5

VanderWeele, T. J. (2021a). Effects of religious service attendance and religious importance on depression: examining the meta-analytic evidence. The International Journal for the Psychology of Religion, 31(1), 21-26. https://doi.org/10.1080/10508619.2020.1748932

VanderWeele, T. J. (2021b). On an analytic definition of love. Under Review.

VanderWeele, T. J., Balboni, T. A., \& Koh, H. K. (2021). Religious service attendance and implications for clinical care, community participation and public health. American Journal of Epidemiology. Advance online publication: https://doi.org/10.1093/aje/kwab134

VanderWeele, T. J., \& Ding, P. (2017). Sensitivity analysis in observational research: Introducing the e-value. Annals of Internal Medicine, 167(4), 268-274. https://doi.org/10.7326/M16-2607 
VanderWeele, T. J., Li, S., Tsai, A. C., \& Kawachi, I. (2016). Association between religious service attendance and lower suicide rates among US women. JAMA Psychiatry, 73(8), 845-851. https://doi.org/10.1001/jamapsychiatry.2016.1243

VanderWeele, T. J., Long, K. N. G., \& Balboni, M. J. (2021). Tradition-specific measures of spiritual well-being. In M. T. Lee, L. D. Kubzansky, \& T. J. VanderWeele (Eds.), Measuring well-being: Interdisciplinary perspectives from the social sciences and the humanities (pp. 482-498). Oxford University Press.

VanderWeele, T. J., Yu, J., Cozier, Y. C., Wise, L., Argentieri, M. A., Rosenberg, L., Palmer, J. R., \& Shields, A. E. (2017). Attendance at religious services, prayer, religious coping, and religious/spiritual identity as predictors of all-cause mortality in the black women's health study. American Journal of Epidemiology, 185(7), 515-522. https://doi.org/10.1093/aje/kww179

Weinberg, M., Besser, A., Zeigler-Hill, V., \& Neria, Y. (2016). Bidirectional associations between hope, optimism and social support, and trauma-related symptoms among survivors of terrorism and their spouses. Journal of Research in Personality, 62, 29-38. https://doi.org/10.1016/j.jrp.2016.03.002

Winslow, C.-E. A. (1920). The untilled fields of public health. Science, 51(1306), 23-33.

Worthington, E. L. (2020). Understanding forgiveness of other people: Definitions, theories, and processes. In E. L. Worthington, Jr., \& N. G. Wade (Eds.), Handbook offorgiveness $\left(2^{\text {nd }}\right.$ ed., pp. 11-21). Routledge.

Worthington, E. L., \& Wade, N. G. (Eds.). (2020). Handbook of forgiveness (2 ${ }^{\text {nd }}$ ed.). Routledge. Wright, N. T. (2012). After you believe: Why Christian character matters. HarperOne. 


\section{Chapter 26:}

Integrating Positive Psychology, Religion/Spirituality, and a Virtue Focus Within Culturally Responsive Mental Healthcare

Laura E. Captari ${ }^{1}$, Steven J. Sandage ${ }^{1,2}$, Richard A. Vandiver ${ }^{1}$, Peter J. Jankowski ${ }^{3}$, and Joshua N. Hook ${ }^{4}$

${ }^{1}$ The Albert and Jessie Danielsen Institute, Boston University, Boston, MA, United States

${ }^{2}$ MF Norwegian School of Theology, Religion, and Society, Oslo, Norway

${ }^{3}$ Counseling Program, Bethel University, Saint Paul, MN, United States

${ }^{4}$ University of North Texas, Denton, TX, United States

\section{Author Note}

This project was supported by a grant from John Templeton Foundation on "Mental Healthcare, Virtue, and Human Flourishing” (\#61603).

We have no conflicts of interest to disclose.

Correspondence concerning this chapter should be addressed to Laura Captari, The Albert and Jessie Danielsen Institute, 185 Bay State Rd., Boston, MA 02215. E-mail:

lcaptari@bu.edu 


\begin{abstract}
Although both positive psychology and religious/spiritual traditions share a common focus on human strengths and holistic development, in mental healthcare, there has been little systematic integration of these fields. In this chapter, we overview key terms and synthesize meta-analytic evidence for spiritually integrated interventions (SIIs), positive psychology interventions (PPIs), and virtue-based interventions (VBIs). Through the lens of virtue ethics, we propose that growth in virtuousness fosters flourishing, which consists of more than the absence of psychological distress and includes greater well-being, meaning in life, relational maturity, and community contribution. We detail a research prospectus guided by virtue ethics to support the development of an integrated line of applied clinical research. In particular, we call for practice-based studies that attend to diversity and equity considerations and address reductionistic misapplications of virtue (which we call virtue bypass). Finally, we discuss innovative clinical and community applications, including the utility of a dialectical and contextual perspective, the need to consider both individual and communal flourishing, and the potential for communities as intervention sites.
\end{abstract}

Keywords: positive psychology, spirituality, religion, virtue, clinical practice 


\section{Chapter 26: Integrating Positive Psychology, Religion/Spirituality, and a Virtue Focus Within Culturally Responsive Mental Healthcare}

In his groundbreaking book Authentic Happiness, Martin Seligman (2002) bemoaned: "Rely[ing] on shortcuts to happiness, joy, rapture, comfort, and ecstasy, rather than [achieving] these feelings by the exercise of personal strengths and virtues, leads to legions of people who in the middle of great wealth are starving spiritually" (p. 8). Recognizing this possibility, positive psychologists and psychologists of religion/spirituality have explored what constitutes and contributes to the good life-which often is referred to as human flourishing (VanderWeele et al., 2019). But what makes life good, and who decides? What if one person's pursuit of flourishing brings harm to another? And how do these concerns intersect with the realities of suffering and inequity? Such questions have important implications for mental healthcare in a diverse world (Sue et al., 2019). Drawing on systemic and intercultural sensibilities, this chapter explores some of the complexities and dilemmas inherent in integrating the contributions of positive psychology, religion/spirituality, and virtue. To begin, we give an overview of key definitions and orient readers to our dialectical, developmental framework. Next, we synthesize the evidence for psychological interventions that integrate these domains. Third, we offer recommendations for an integrated line of research and practice, emphasizing the need for emic approaches that promote culturally responsive care. Finally, we discuss innovative clinical and community applications, suggesting that helping people and systems grow in the virtues salient within their cultural and spiritual context can promote flourishing.

\section{Getting Oriented: Definitions and Conceptual Framework}

We understand mental health to exist on a continuum, ranging from illness (e.g., high symptoms, low well-being) to languishing (e.g., low symptoms, low well-being) to 
health/flourishing (e.g., low symptoms, high well-being; Keyes, 2002). Flourishing involves not only the amelioration of symptoms but also the presence of well-being - an umbrella term broadly including “all different forms of evaluating one's life or emotional experience, such as satisfaction, positive affect, and low negative affect" (Diener et al., 2017, p. 87). Although there are many subclassifications of well-being, here we highlight two broad categories: (a) hedonic well-being focuses on personal pleasure and enjoyment (e.g., feeling good), whereas (b) eudaimonic well-being more broadly emphasizes relational maturity, meaningful life purpose, and communal concerns (e.g., pursuing good). Taken together, holistic flourishing is a multidimensional, developmental process that integrates hedonic and eudaimonic well-being with personal beliefs, values, and cultural contexts (Jankowski et al., 2020; Lambert et al., 2015).

Shifting from a deficit-based, symptom-alleviating medical model to a more holistic, capacity-building framework respects the complexity of human experience and is resonant with core tenets of many religious/spiritual (R/S) traditions. We utilize a pluralistic definition of R/S that includes Spiritual, Existential, Religious, and Theological dimensions of experience (SERT; Sandage et al., 2020), partly in response to the growing number of people who identify as multireligious (i.e., interweaving aspects of multiple traditions), spiritual but not religious, or as neither spiritual nor religious. Even among this latter group, existential themes (e.g., death, loss, meaning) are often relevant. Thus, we consider whatever a person views as ultimately most important, whether that be a divine being and/or other spiritual entities, cherished principles and values, or other ultimate concerns. This framework opens broad conceptual space to consider both the salutary and harmful ways people relate to whatever they consider sacred or ultimate.

Our engagement with positive psychology draws significantly on critical and intercultural lenses, often termed positive psychology 2.0 (Chang et al., 2016; Wong, 2011). Positive 
psychology 2.0 calls for attention to eudaimonic well-being (e.g., cultivating virtue and meaning) as well as contextual (e.g., culture, religious) and systemic (e.g., oppression, empowerment) factors. Although positive psychology's historically etic approach has brought scientific rigor to researching human strengths, it is risky to decontextualize these constructs from sociocultural situatedness. The resulting expectation can be that positive psychology constructs are universally positive and promote well-being irrespective of race, ethnicity, gender, social location, and other factors. However, a one-size-fits-all approach can be problematic. For example, R/S people often understand strengths in light of their R/S worldview and utilize embedded R/S practices that are not always acculturated to psychological language. In addition, for people with less privilege, strengths often intersect with minority stress and structural oppression, such that there are some cases where a particular virtue could appear counter to flourishing (e.g., gratitude and humility may seem like colluding with oppression). Positive psychology 2.0 attends to these nuances.

Throughout this chapter, we integrate virtue ethics — a framework rooted in Aristotelian philosophy and expanded on by Confucius, Maimonides, Aquinas, Al-Ijī, and other diverse traditions (MacIntyre, 2007) — to contextualize positive psychology within each person's culture, worldview, and presenting concerns. A core premise of virtue ethics is that flourishing cannot be achieved solely through symptom reduction, but rather, is inextricably connected with virtue development. Virtues refer to "qualities of human character and excellence which enhance the capacity to live well" (Sandage \& Hill, 2001, p. 243); put differently, virtues are "morally based [thoughts, feelings, and] actions that enable an individual and his or her social world to thrive" (Lerner, 2019, p. 79). The developmental language of virtue orients people to reflect on who they are and are becoming in relation to others (McMinn et al., 2016). Virtues are often motivated by personal beliefs, values, and goals, so understanding a person's SERT context is vital. Virtue 
ethics prioritizes practical wisdom as a meta-virtue to navigate (a) particularities of when to draw on which virtue and (b) the complexity of considering multiple virtues in tandem. For example, navigating conflicts may require self-control, honesty, and forgiveness, and doing antiracist work often necessitates justice, courage, and creativity. Dose and behavioral manifestation of each virtue may vary based on identity characteristics, $\mathrm{R} / \mathrm{S}$ worldview, and the present moment.

\section{What We Know: Synthesizing the Empirical Evidence}

We searched seven major electronic databases to identify meta-analyses comparing (a) interventions integrating religion/spirituality, positive psychology, and/or virtues with (b) either an alternative active treatment condition or a no-treatment control. For the purposes of this review, intervention includes psychotherapy and other mental health supports (e.g., self-help programs). To maintain a reasonable scope, we did not include meta-analyses of spiritual direction or ministry, which tend to be more loosely defined. Our use of the term integrated specifies drawing substantively on the contributions of religion/spirituality, positive psychology, or virtue, which have been defined in detail above. Reviewing results available in English as of May 1, 2021, we identified 36 meta-analyses. Below we summarize our findings in three domains: (a) spiritually integrated interventions, (b) positive psychology interventions, and (c) virtue-based interventions. See Appendix 26.S1 (Tables 26.S1, 26.S2, and 26.S3) for more detail.

\section{Spiritually Integrated Interventions}

Integrating clients' spirituality is an important aspect of evidence-based practice, described as a three-legged stool synthesizing (a) research evidence with (b) clinical expertise and (c) clients' values, preferences, and contexts (Shafranske, 2013). Spiritually integrated interventions (SIIs) can be conducted in "virtually any psychotherapeutic traditionpsychodynamic, cognitive behavioral, family systems, humanistic, and existential" (Pargament et 
al., 2005, p. 161). Currently, the largest efficacy base is in cognitive behavioral approaches adapted by religion, including Christian, Muslim, Jewish, Hindu, and Buddhist contexts (Abu Raiya \& Pargament, 2010; Koenig et al., 2015; Milevsky \& Eisenberg, 2012); however, there also are more pluralistic approaches that incorporate whatever a person considers sacred (Koszycki et al., 2014; Rosmarin et al., 2019). Further, models have been developed to address comorbid distress, such as body-mind-spirit interventions for physical and mental illness (McGrady \& Moss, 2018) and meaning-centered therapy for end of life (Thomas et al., 2014). Our review identified seven meta-analyses examining the efficacy of SIIs (see Table 26.S1). Broadly speaking, the existing evidence suggests SIIs are effective in ameliorating mental health symptoms to a modest or moderate degree, especially when people "learn to apply their own religious/spiritual beliefs to their mental health" (Smith et al., 2007, p. 653). SIIs are particularly effective in improving R/S well-being, such as increasing R/S believers' sense of meaning and the quality of their relationship with whatever they view as sacred. However, much remains unclear about the conditions and mechanisms that make SIIs effective (or ineffective) in routine clinical practice. Although most research has focused on explicit integration, including helping clients draw on personally salient practices (e.g., prayer, meditation, sacred scriptures, time in nature), implicit integration may also occur, as a client internalizes their therapist's attuned, responsive presence, resulting in positive changes in the person's relational schemas of the divine (e.g., experiencing a higher power as present and responsive, rather than distant and harsh). Research is needed to explicate how clients' SERT concerns may intersect with other aspects of their identity and culture, as well as how SERT concerns may lead to vulnerabilities or harm when R/S beliefs or practices are used defensively. For instance, spiritual bypass (i.e., "the use of spiritual practices and beliefs as a way of avoiding dealing with unresolved psychological 
issues" (Picciotto \& Fox, 2018, p. 65), could be a barrier to virtue development. Thus, it is vital for clinicians to consider how religion/spirituality can both facilitate and hinder flourishing.

\section{Positive Psychology Interventions}

Positive psychology interventions (PPIs) have emerged in response to growing evidence that the medical model, which focuses on symptom reduction, often falls short of promoting flourishing. PPIs focus broadly on enhancing well-being and attend particularly to the experience of languishing ("emptiness and stagnation, constituting a life of quiet despair," Keyes, 2002, p. 210), through fostering positive states (e.g., emotions, cognitions, behaviors) using evidencebased pathways (e.g., savoring, meaning, strengths). PPIs can be used as stand-alone (e.g., selfhelp) interventions in nonclinical settings, as adjuncts to mental health treatment (e.g., assigned for use between sessions), or as components integrated in individual or group therapies. Distinct therapeutic approaches have been developed using a PPI lens, such as well-being (Fava et al., 2005) and positive (Seligman et al., 2006) psychotherapies. Contemporary non-PPI treatments have also begun to prioritize a well-being focus, including acceptance and commitment therapy (Trompetter et al., 2017) and mindfulness-based interventions (Weiss et al., 2016).

Our review identified 12 meta-analyses examining the efficacy of PPIs (see Table 26.S2). High-quality studies consistently support the use of PPIs to foster hedonic and eudaimonic wellbeing to a modest or moderate degree, but the evidence is less clear when it comes to reducing symptoms (e.g., anxiety, depression). Divergent findings between symptoms and well-being substantiate the need to conceptualize mental health as a continuum (i.e., not a category) that has related but distinct dimensions. Overall, PPIs appear more efficacious over longer periods of time (rather than as brief interventions) and in the context of psychotherapy (compared with selfhelp programs). PPIs may have differing relevance to people, based on clients' presenting 
concerns and level of distress. In mental healthcare, there is evidence for multiple change trajectories (Stulz \& Lutz, 2007). For some clients, attention to strengths early in treatment supports agency and instills hope. For other clients, especially when their distress is overwhelming, focusing on symptom management and reduction may be most helpful before trying to enhance well-being. Future research can help clarify which PPI ingredients carry the weight of change in symptoms and well-being, for which populations, at which points in time.

\section{Virtue-Based Interventions}

Although PPIs focus on increasing well-being, virtue-based interventions (VBIs) are guided by a specific developmental telos, which may or may not be explicitly framed using a SERT lens. VBIs explicitly target the development of virtues, based on emerging evidence that growth in virtues promotes positive mental health and flourishing. Virtue development is thought to interact with other mechanisms of change (e.g., positive affect, intrinsic motivation, prosocial behavior), fostering self-reinforcing upward spirals of engagement, agency, and meaning that catalyze well-being (Rusk et al., 2018). As Jankowski et al. (2020) have elaborated, "Virtues as change mechanisms consist of repeated acts of virtuousness and growing levels of dispositional virtuousness over time" (p. 296). This perspective resonates with the ancient wisdom of all five major R/S traditions, each of which emphasize personal growth and transformation as central to flourishing. To date, VBIs fostering forgiveness, gratitude, and self-compassion have been studied most frequently, with growing research on hope, kindness, and empathy. VBIs can be implemented as stand-alone (self-help) interventions in nonclinical settings, as a virtue focus integrated into psychotherapy, or as a virtue-specific therapeutic model. For example, lovingkindness meditation can be used as a personal practice or can be integrated in treatment (Galante et al., 2014), and the REACH Forgiveness model has similarly been utilized both for self-help 
and in therapy, with greater gains found when SERT context was integrated (Wade et al., 2014). Distinct virtue-based therapeutic approaches have also been developed, including compassionfocused psychotherapy (Gilbert, 2014) and hope-focused couples therapy (Worthington, 2013).

Our review identified 17 meta-analyses examining the efficacy of VBIs (see Table 26.S3). Despite emerging support both for increased well-being and for symptom reduction, the evidence is less well-established for VBIs than for SIIs or PPIs. VBIs have most often been (a) compared to a no-treatment controls, (b) tested in nonclinical (e.g., college student) samples, and (c) limited by short duration. However, the available evidence suggests that context and dosage are important. VBIs may be more readily integrated with students and community members (compared to psychiatric populations), and longer interventions appear to facilitate greater gains. One limitation to date has been interventional focus on a single virtue, when, in daily life, virtues likely interact with one other to promote well-being. For example, highly R/S people often embrace numerous interlocking virtues, so separating the effects of a single virtue is fraught. Exploration of underlying change mechanisms is needed in real-world clinical settings, where chronic distress is often intertwined with "significant struggles in... unforgiveness, hopelessness, envy, and/or self-criticalness" (Jankowski et al., 2020, p. 301). Ongoing empirical work can help explicate the nuances of how and in what ways a focus on virtue may promote flourishing.

\section{What's Next?: Advancing Integrative, Culturally Responsive Research}

Readers will notice some overlap in these three intervention areas. However, scant attention has been given to the development and implementation of positive psychology and virtue-based interventions that are tailored to and situated within specific SERT and cultural contexts. One exception is some Christian-focused interventions. To date, research has primarily (a) captured the experiences of majority populations who hold substantial societal privilege, 
relative to racial, ethnic, R/S, sexual, and gender minorities; (b) focused on individual wellbeing, with less attention to couple, family, and systemic functioning; and (c) examined each virtue in isolation, without accounting for potential synergistic effects of multiple virtues acting and developing concurrently. Next, we discuss priorities for elucidating the interplay of positive psychology, religion/spirituality, and virtue in daily life, particularly among nonmajority groups.

\section{Test Virtue-Flourishing Links, Considering Intervention Setting and Format}

To advance the framework of virtue ethics, intervention studies need to continue testing the hypothesis that virtue development promotes flourishing. We also know very little about potential mediating and moderating factors, even though scholars theorize that relational and emotion regulation capacities may play influential roles (Jankowski et al., 2020). To date, most interventions have been developed and tested as short-term, psychoeducational protocols with college students. Moving the science forward will require testing virtue-flourishing links in a variety of settings, including individual, couple and family, and group therapies (within mental health treatment), as well as in systems-wide interventions, such as within schools, workplaces, and R/S communities. If scientists really want to understand how to help people, systems, and communities flourish, they must investigate a number of areas: What interventions are most effective, relevant, and feasible for promoting virtue development? How does this vary based on personal, cultural, and contextual factors? Which people benefit from which interventions? Some individuals and groups may be more interested in a broad well-being-focused intervention rather than one aimed at virtue development, whereas people for whom R/S is very important may prefer approaches that translate psychological science into theologically relevant terms.

Furthermore, based on these differences, what delivery format(s) might work best? For example, in some more collectivistic contexts, family and group interventions may be more 
salient and effective. Technologically delivered interventions may be more engaging and effective among teens and young adults. In populations not acculturated to Western models of mental health, interventions delivered within a culturally trusted institution (e.g., an educational setting, faith community, or other network with a history of trust and reliability) may increase accessibility and effectiveness. Research is also needed to explore the impact of the person or people facilitating the interventions, whether that be a psychotherapist, R/S leader, teacher, or someone else in the community. There could be potential differences in effectiveness depending on the intervention's orientation toward collaboration and interaction (ranging from very structured/didactic to experiential/cocreated). Relatedly, it is likely that people who seek out an intervention may more invested — and thus experience greater gains in flourishing — compared to people for whom intervention participation is expected (e.g., to meet educational requirements).

\section{Attend to the Complexities of Real-World Clinical Practice}

One limitation of efficacy research is the gap between well-controlled trials and realworld practice, where (a) people often present with comorbid diagnoses (and hence are excluded from efficacy studies) and (b) therapists frequently integrate multiple treatment approaches and modalities to address clients' needs. Despite the evidence reviewed above, there has been a significant lag in dissemination to mainstream mental healthcare. This dearth is vital to address. Both etic and emic studies are needed to investigate generalizable patterns (and exceptions to those patterns) unique to particular persons and contexts. For example, although we know that attending to spirituality, well-being, and virtues in treatment is generally helpful, much remains unclear about the conditions and mechanisms of change that make these interventions effective (or ineffective) in day-to-day practice. What are the choice points between implicit and explicit integration of these domains in treatment? For which people? At what points in therapy? And 
what role might the therapist's embodiment of virtue play (i.e., are virtues taught or caught)?

Although few clients present to therapy with a primary goal of increasing virtue, many describe wanting to better manage emotions and navigate relationships, and evidence suggests that virtues such as humility, gratitude, and forgiveness are associated with affect regulation and secure attachment (Dwiwardani et al., 2014). Interweaving a virtue and well-being focuscontextualized to a client's SERT context—within clinical formulation and treatment has significant potential, but it is much more difficult to study and thus is less empirically developed. As one example, a recent practice-based study found that clients in psychodynamic therapy evidenced growth in humility, which predicted changes both in symptoms and well-being (Jankowski et al., 2021). For some clients, virtues may be a relevant explicit focus (e.g., integrating the REACH Forgiveness model into therapy); for others, virtues may emerge within the treatment process (e.g., the therapist embodies humility amidst an alliance rupture). In the former situation, cognitive and behavioral foci may facilitate virtue development, whereas in the latter, virtues may be internalized via emotional and relational processes of therapeutic action (Schore, 2014). In real-world practice, treatment responsiveness may matter more than fidelity to a particular intervention (Norcross \& Wampold, 2018). To the extent that is true, research is needed that (a) tracks facilitators and hindrances of dissemination and effectiveness, (b) incorporates longitudinal and mixed-method practice-based research designs, (c) uses personcentered analyses to identify subgroups that respond well (vs. poorly) to treatment, and (d) explicates conditions for and mechanisms of change in symptoms and well-being.

\section{Critically Consider Diversity, Equity, and Justice}

Expanding positive psychology beyond a Eurocentric perspective requires grappling with how virtue and well-being constructs intersect with structural inequality, minority stress, and 
intersectionality. Most existing models and self-report measures (e.g., of optimism, gratitude, hope) center the experiences of White individuals from a higher socioeconomic status and education level (Paquin et al., 2019). However, these constructs "are necessarily embedded in a cultural context" (Sandage et al., 2003, p. 571). The diverse ways that virtues are understood, valued, and embodied can vary significantly, often based on the intersections of a person's culture, R/S beliefs, and social location, to name just a few areas. Therefore, flourishing needs to be investigated with increased (a) attention to diverse cultural and SERT understandings and (b) consideration of systemic and sociocultural factors that impact disparities in flourishing. Holistic and communal forms of well-being more closely align with the cultural worldviews and values of many clients, rather than the medical model's focus on symptom alleviation (which dominates healthcare in Europe and the U.S.). Research is needed that (a) examines pathways to flourishing for all people, not just dominant group members, and (b) interrogates organizational dynamics that perpetuate prejudice and inequities in mental healthcare (Paquin et al., 2019).

The interplay between eudaimonic and hedonic well-being holds important implications in the face of injustice, as Seligman (2002) has noted: "People who are impoverished, depressed, or suicidal care about much more than just the relief of their suffering. These people caresometimes desperately—about virtue, about purpose, about integrity and about meaning” (p. xi). It is vital to explore how chronic oppression may tax people's resilience and compromise their expression of virtues. As one example, scholars have begun to explicate the concept of burdened virtues, capturing how inequitable societal conditions often necessitate oppressed groups developing "a set of virtues that carry a moral cost to those who practice them" (i.e., they support survival but not flourishing; Tessman, 2005, p. 1). We invite researchers to consider ways to (a) privilege the narratives of individuals who have been historically oppressed and systemically 
affected by poverty, racism, and sexism; (b) use community action research designs to engage these populations in every stage of scholarly work, from theory-building and measurement to intervention design and implementation; and (c) integrate clients' culturally embedded strengths and SERT perspectives about human suffering and well-being into psychotherapy approaches.

\section{Elucidate Problematic Applications Such as Virtue Bypass}

Positive psychology has historically focused on increasing positively valenced emotions, which can perpetuate the myth that enhancing positive aspects of one's life will resolve—or prevent-distress. This does not translate well into real-world clinical practice, where clients often present with long-standing suffering. Failing to acknowledge and process life's hardships can produce toxic positivity, "the excessive and ineffective overgeneralization of a happy, optimistic state across all situations" that "results in the denial, minimization, and invalidation of the authentic human emotional experience” (Quintero \& Long, 2019, para. 4). In contrast, attending to negatively valenced affect — what Lomas $(2018,2019)$ has called the virtues of anger and sadness - can enhance adaptation and well-being over time. Affective neuroscience research elucidates the adaptive evolutionary functions of rage, fear, and sadness (Panksepp \& Biven, 2012), a keen reminder that all emotions lend important insight into our desires and needs and thereby can help motivate action. Although the dichotomies of positive and negative are useful in research, a dialectical perspective is often more salient, valid, and useful in practice. Rather than viewing positive affect and virtue behaviors as universally beneficial, we need to explore the specific function in light of each person's intrapersonal dynamics and social location.

We propose the term virtue bypass to describe when virtue language or behaviors are used in ways counter to flourishing, such as to (a) oppress and subjugate others or (b) repress and deny one's own emotions and needs. Consider a sexual abuse survivor whose faith community 
urges them to forgive as an extension of divine grace. This person may rush to verbalize forgiveness as a trauma response that restores equilibrium, bypassing the virtue of justice and related emotions of rage and mourning. Evidence-based practice here must synthesize positive psychology contributions with evidence that "an optimistic bias can put victims in danger" and in some cases "forgiveness [can] increase likelihood of further transgressions" (Sinclair et al., 2020, p. 26). Or, consider a teen who courageously share pent-up hurt with their parents, only to have their parents snap back, "Stop being dramatic and show some gratitude for all we've done for you!" Here, emotional invalidation is being dressed up in the clothing of virtue, and attending to this parent-child relational dynamic is vital for systemic well-being. Helping the family stay with and process difficult emotions together may foster greater understanding, trust, and connectionfrom which gratitude could emerge bidirectionally. Taken together, a virtue ethics perspective orients us toward practical wisdom and contextual sensitivity, so we can discern whether virtue language is emerging out of authentic struggle with and acceptance of reality, or it is being used to avoid or contort reality. Intervention frameworks are needed that account for this complexity.

\section{In Real Life: Implementation in Clinical and Community Settings}

In mental healthcare systems, clinical decision-making is often guided by attention to disorders, dysfunction, and deficits, which can reduce people to their diagnosis. In one woman's words, "When all you ask about is my symptoms, it feels like nothing else about me is real!" Positive psychology offers critical contributions to a holistic view of flourishing by orienting clinicians toward each client's and family's strengths and adaptive capacities. Yet, integration in routine practice has been stymied by a primary focus in positive psychology on the individual, forgetting that "the conditions in the environments where people are born, live, learn, work, play, worship, and age affect a wide range of health, functioning, and quality-of-life outcomes and 
risks" (U.S. Department of Health \& Human Services, 2021, para. 1). Thus, we offer practical recommendations for psychotherapists, spiritual care providers, and others in helping roles.

First, maintain a dialectical, contextual perspective, recognizing that without cultural humility (Hook et al., 2017), we can miss important aspects of people's lived experience and can enact colonizing and oppressive dynamics. Determining which intervention setting and format is most appropriate for a particular person is an iterative, collaborative process. Be mindful of power dynamics and the ethical problems that are embedded in authoritatively communicatingeven as part of psychoeducation — that X, Y, and Z virtues will promote clients' well-being. A majority of positive psychology research has been conducted with college students, many of whom are White and hold social privilege; thus, findings cannot necessarily be generalized to nonmajority and clinical populations. We can reshift the center beyond Eurocentrism not only in the lab but also in real-world care settings, by using empirical literature as a jumping-off point to spark curiosity and joint exploration about embedded cultural strengths and SERT resources that can promote flourishing for the person in front of us. Norcross and Wampold (2018) have captured this complexity in describing the need to cocreate "a new therapy for each patient" ( $p$. 1889). This is different from assuming that virtue growth in a particular area should be the interventional focus. Thus, SIIs, PPIs, and VBIs are best understood as clinical resources to guide treatment, rather than scientifically proven parameters to be implemented unquestioningly.

Second, reflect on how your work fits into the continuum of catalyzing flourishing at both individual and communal levels. Virtues have intrapersonal and systemic impacts, and this influence flows both ways. Psychotherapy is only one of multiple potential interventional contexts, and people stand to benefit from a well-being focus within their workplaces, schools, and faith communities. One innovative way to disseminate psychological science is through 
adapting these interventions to faith and/or learning communities' needs (Wang et al., Chapter 29, this volume). Considering R/S communities as intervention sites, Bufford et al. (2018) collaborated with Christian church leaders to develop and test a grace-focused intervention (e.g., sermon series and small group program), and participants reported growth in grace and selfforgiveness. Targeting a broader systemic context, Griffin et al. (2019) developed a universitywide forgiveness initiative, including active (e.g., lectures, movie nights) and passive (e.g., social media) components, resulting in student-reported growth in forgiveness. Community-based interventions may have particular salience following mass traumas (e.g., natural disasters, school shootings) and in meaningfully addressing the impacts of intergenerational trauma. For example, African American communities are historically organized around the local church——"the oldest and most resilient social institution in Black America...[and] traditionally the only Blackcontrolled institution of a historically oppressed people" (Putnam, 2000, p. 68). Religious institutions and community leaders are often looked to for guidance amidst turmoil and uncertainty, so they are uniquely positioned to support positive adaptation (Captari et al., 2019).

Finally, consider pluralistic and intercultural applications relevant to our diverse, global society. People increasingly draw on ideas and practices from multiple traditions, as they differentiate and redefine a new spiritual path across their life (Ammerman, 2020). Hence, approaches adapted to specific religions are inadequate to meet many people's needs. At the same time, it is questionable "whether positive psychology interventions can ever be characterized as purely secular" (Rye et al., 2013, p. 503), as perspectives about a particular virtue are shaped by family and community SERT influences. Without assuming what's good for me is good for you, virtue ethics provides a shared language for engaging in meaningful dialogue about the relevance of positive psychology across cultural and worldview differences. For 
example, recognizing how the COVID-19 pandemic has disproportionately impacted racial and ethnic minorities, expressing anger and grief about the impacts of systemic racism could be more positive (e.g., creative, generative, and healing) than a focus on optimism or gratitude — and it is not up to us to dictate this. By letting clients lead us toward positive psychology resources embedded in their SERT framework, implementation science can advance at a grassroots level.

\section{Conclusions}

Positive psychology, religion/spirituality, and virtues each offer a unique lens that can help promote culturally responsive mental healthcare. In this chapter, we have applied virtue ethics to facilitate a rapprochement between these literatures, situating positive psychology within the broader landscape of each person's sociocultural and SERT context. We have synthesized the evidence base for spiritually integrated, positive psychological, and virtue-based interventions to guide readers in utilizing these approaches in their work. These interventions are applicable to clinical practice, $\mathrm{R} / \mathrm{S}$ and learning communities, and other applied settings (workplaces, humanitarian aid, etc.). Attending to an individual's struggles and strengths in light of (a) personal beliefs, values, and goals as well as (b) systemic and sociocultural processes helps us avoid the pitfalls of both the medical model and a one-size-fits-all approach. Whether you find yourself in a therapy room, classroom, religious community, or boardroom, consider creative ways to incorporate the strengths and resources of these domains to help people pursue the good life "through meeting suffering head on and transforming it into opportunities for meaning, wisdom, and growth" (Emmons, 2003, p. 156). Together, we can create more compassionate, just, and empowering communities that promote flourishing for all. 


\section{References}

Abu Raiya, H., \& Pargament, K. I. (2010). Religiously integrated psychotherapy with Muslim clients. Professional Psychology, 41(2), 181-188. https://doi.org/10.1037/a0017988

Ammerman, N. T. (2020). Rethinking religion: Toward a practice approach. American Journal of Sociology, 126(1), 6-51. https://doi.org/10.1086/709779

Bufford, R. K., McMinn, M. R., Moody, J. A., \& Geczy-Haskins, L. (2018). The effects of grace interventions in church communities. The Journal of Positive Psychology, 13, 512-521. https://doi.org/10.1080/17439760.2017.1350740

Captari, L. E., Hook, J. N., Aten, J. D., Davis, E. B., \& Tisdale, T. C. (2019). Embodied spirituality following disaster. In V. Counted \& F. Watts (Eds.), The psychology of religion and place (pp. 49-79). Palgrave Macmillan.

Chang, E. C., Downey, C. A., Hirsch, J. K., \& Lin, N. J. (2016). Positive psychology in racial and ethnic groups: Theory, research, and practice. American Psychological Association.

Diener, E., Heintzelman, S. J., Kushlev, K., Tay, L., Wirtz, D., Lutes, L. D., \& Oishi, S. (2017). Findings all psychologists should know from the new science on subjective well-being. Canadian Psychology, 58, 87-104. http://dx.doi.org/10.1037/cap0000063

Dwiwardani, C., Hill, P. C., Bollinger, R. A., Marks, L. E., Steele, J. R., Doolin, H. N., .. \& Davis, D. E. (2014). Virtues develop from a secure base. Journal of Psychology and Theology, 42(1), 83-90. https://doi.org/10.1177/009164711404200109

Emmons, R. A. (2003). Personal goals, life meaning, and virtue. In C. L. M. Keyes, \& J. Haidt (Eds.), Flourishing: Positive psychology and the life well-lived (pp. 105-128). APA.

Fava, G. A., Ruini, C., Rafanelli, C., Finos, L., Salmaso, L., Mangelli, L., \& Sirigatti, S. (2005). Well-being therapy of generalized anxiety disorder. Psychotherapy and Psychosomatics, 
74, 26-30. http://dx.doi.org/10.1159/000082023

Galante, J., Galante, I., Bekkers, M.-J., \& Gallacher, J. (2014). Effect of kindness-based meditation on health and well-being: A systematic review and meta-analysis. Journal of Consulting and Clinical Psychology, 82, 1101-1114. http://dx.doi.org/10.1037/a0037249

Gilbert, P. (2014). The origins and nature of compassion focused therapy. British Journal of Clinical Psychology, 53(1), 6-41. http://dx.doi.org/10.1111/bjc.12043

Griffin, B. J., Toussaint, L. L., Zoelzer, M., Worthington, E. L., Jr., Coleman, J., Lavelock, C. R., McElroy, A., Hook, J. N., Wade, N., Sandage, S., \& Rye, M. (2019). Evaluating the effectiveness of a community-based forgiveness campaign. The Journal of Positive Psychology, 14(3), 354-361. http://dx.doi.org/10.1080/17439760.2018.1437464

Hook, J. N., Davis, D., Owen, J., \& DeBlaere, C. (2017). Cultural humility. APA.

Jankowski, P. J., Captari, L. E., \& Sandage, S. J. (2021). Exploring virtue ethics in psychodynamic psychotherapy: Latent changes in humility, affect regulation, symptoms and well-being. Counselling and Psychotherapy Research. Advanced online publication. https://doi.org/10.1002/capr.12389

Jankowski, P. J., Sandage, S. J., Bell, C. A., Davis, D. E., Porter, E., Jessen, M., Motzny, C. L., Ross, K. V., \& Owen, J. (2020). Virtue, flourishing, and positive psychology in psychotherapy. Psychotherapy, 57(3), 291-309. https://doi.org/10.1037/pst0000285

Keyes, C. (2002). The mental health continuum: From languishing to flourishing in life. Journal of Health and Social Behavior, 43, 207-222. https://doi.org/10.2307/3090197

Koenig, H. G., Pearce, M. J., Nelson, B., Shaw, S. F., Robins, C. J., Daher, N. S., .. \& King, M. B. (2015). Religious vs. conventional cognitive behavioral therapy for major depression in persons with chronic medical illness: A pilot randomized trial. The Journal of Nervous 
and Mental Disease, 203(4), 243-251. https://doi.org/10.1097/NMD.0000000000000273

Koszycki, D., Bilodeau, C., Raab-Mayo, K., \& Bradwejn, J. (2014). A multifaith spiritually based intervention versus supportive therapy for generalized anxiety disorder. Journal of Clinical Psychology, 70(6), 489-509. https://doi.org/10.1002/jclp.22052

Lambert, L., Passmore, H.-A., \& Holder, M. D. (2015). Foundational frameworks of positive psychology: Mapping well-being orientations. Canadian Psychology, 56, 311-321. https://doi.org/10.1037/cap0000033

Lerner, R. M. (2019). Character development: Four facets of virtues. Child Development Perspectives, 13(2), 79-84. https://doi.org/10.1111/cdep.12315

Lomas, T. (2018). The quiet virtues of sadness. New Ideas in Psychology, 49, 18-26. https://doi.org/10.1016/j.newideapsych.2018.01.002

Lomas, T. (2019). Anger as a moral emotion. Counselling Psychology Quarterly, 32(3-4), 341395. https://doi.org/10.1080/09515070.2019.1589421

MacIntyre, A. (2007). After virtue: A study in moral theory. University of Notre Dame Press.

McGrady, A., \& Moss, D. (2018). Integrative pathways. Springer.

McMinn, M. R., McLaughlin, P. T., Johnson, B. C., \& Shoup, R. (2016). Psychotherapy and the theological virtues. Open Theology, 2, 424-435. https://doi.org/10.1515/opth-2016-0035

Milevsky, A., \& Eisenberg, M. (2012). Spiritually oriented treatment with Jewish clients. Professional Psychology, 43(4), 336-340. https://doi.org/10.1037/a0028035

Norcross, J. C., \& Wampold, B. E. (2018). A new therapy for each patient: Evidence-based relationships and responsiveness. Journal of Clinical Psychology, 74(11), 1889-1906. https://doi.org/10.1002/jclp.22678

Panksepp, J., \& Biven, L. (2012). The archaeology of mind. W. W. Norton. 
Paquin, J. D., Tao, K. W., \& Budge, S. L. (2019). Toward a psychotherapy science for all: Conducting ethical and socially just research. Psychotherapy, 56(4), 491502. https://doi.org/10.1037/pst0000271

Pargament, K. I., Murray-Swank, N. A., \& Tarakeshwar, N. (2005). An empirically-based rationale for a spiritually-integrated psychotherapy. Mental Health, Religion and Culture, 8, 155-165. https://doi.org/10.1080/13694670500138940

Picciotto, G., \& Fox, J. (2018). Exploring experts' perspectives on spiritual bypass: A conventional content analysis. Pastoral Psychology, 67(1), 65-84. https://doi.org/10.1007/s11089-017-0796-7

Putnam, R. D. (2000). Bowling alone. Simon and Schuster.

Quintero, S., \& Long, J. (2019). Toxic positivity: The dark side of positive vibes. The Psychology Group. https://thepsychologygroup.com/toxic-positivity/

Rosmarin, D. H., Salcone, S., Harper, D., \& Forester, B. P. (2019). Spiritual psychotherapy for inpatient, residential, and intensive treatment. American Journal of Psychotherapy, 72(3), 75-83. https://doi.org/10.1176/appi.psychotherapy.20180046

Rusk, R. D., Vella-Brodrick, D. A., \& Waters, L. (2018). A complex dynamic systems approach to lasting positive change: The synergistic change model. The Journal of Positive Psychology, 13, 406-418. http://dx.doi.org/10.1080/17439760.2017.1291853

Rye, M. S., Wade, N. G., Fleri, A. M., \& Kidwell, J. E. (2013). The role of religion and spirituality in positive psychology interventions. In K. I. Pargament (Ed.), APA Handbook of Psychology, Religion, and Spirituality: Vol. 2 (pp. 481-508). APA.

Sandage, S. J., \& Hill, P. C. (2001). The virtues of positive psychology: The rapprochement and challenges of an affirmative postmodern perspective. Journal for the Theory of Social 
Behaviour, 31, 241-260. http://dx.doi.org/10.1111/1468-5914.00157

Sandage, S. J., Hill, P. C., \& Vang, H. C. (2003). Toward a multicultural positive psychology: Indigenous forgiveness and Hmong culture. The Counseling Psychologist, 31, 564-592. https://doi.org/10.1177/0011000003256350

Sandage, S. J., Rupert, D., Stavros, G. S., \& Devor, N. G. (2020). Relational spirituality in psychotherapy: Healing suffering and promoting growth. APA.

Schore, A. N. (2014). The right brain is dominant in psychotherapy. Psychotherapy, 51, 388397. https://doi.org/10.1037/a0037083

Seligman, M. E. P. (2002). Authentic happiness. Free Press.

Seligman, M. E. P., Rashid, T., \& Parks, A. C. (2006). Positive psychotherapy. American Psychologist, 61, 774-788. http://dx.doi.org/10.1037/0003-066X.61.8.774

Shafranske, E. P. (2013). Addressing religiousness and spirituality in psychotherapy: Advancing evidence-based practice. In R. F. Paloutzian \& C. L. Park (Eds.), Handbook of the psychology of religion and spirituality (pp. 595-616). Guilford Press.

Sinclair, E., Hart, R. \& Lomas, T. (2020). Can positivity be counterproductive when suffering domestic abuse?: A narrative review. International Journal of Well-being, 10(1), 26-53. https://doi.org/10.5502/ijw.v10i1.754

Smith, T. B., Bartz, J., \& Richards, P. S. (2007). Outcomes of religious and spiritual adaptations to psychotherapy: A meta-analytic review. Psychotherapy Research, 17, 643-655. https://doi.org/10.1080/10503300701250347

Stulz, N., \& Lutz, W. (2007). Multidimensional patterns of change in outpatient psychotherapy. Journal of Clinical Psychology, 63, 817-833. https://doi.org/10.1002/jclp.20397

Sue, D. W., Sue, D., Neville, H. A., \& Smith, L. (2019). Counseling the culturally diverse: 
Theory and practice. Wiley.

Tessman, L. (2005). Burdened virtues: Virtue ethics for liberatory struggles. Oxford University Press.

Thomas, L. P. M., Meier, E. A., \& Irwin, S. A. (2014). Meaning-centered psychotherapy: A form of psychotherapy for patients with cancer. Current Psychiatry Reports, 16(10), 488-499.

Trompetter, H. R., Lamers, S. M. A., Westerhof, G. J., Fledderus, M., \& Bohlmeijer, E. T. (2017). Both positive mental health and psychopathology should be monitored in psychotherapy: Confirmation for the dual-factor model. Behaviour Research and Therapy, 91, 58-63. https://doi.org/10.1016/j.brat.2017.01.008

U.S. Department of Health \& Human Services. (2021). Social determinants of health. https://health.gov/healthypeople/objectives-and-data/social-determinants-health

VanderWeele, T. J., McNeely, E., \& Koh, H. K. (2019). Reimagining health—Flourishing. Journal of the American Medical Association, 321, 1667-1668. https://doi.org/10.1001/jama.2019.3035

Wade, N. G., Hoyt, W. T., Kidwell, J. E. M., \& Worthington, E. L., Jr. (2014). Efficacy of psychotherapeutic interventions to promote forgiveness: A meta-analysis. Journal of Consulting and Clinical Psychology, 82, 154-170. http://dx.doi.org/10.1037/a0035268

Weiss, L. A., Westerhof, G. J., \& Bohlmeijer, E. T. (2016). Can we increase psychological wellbeing? The effects of interventions on psychological well-being. PLOS ONE, 11, e0158092. https://doi.org/10.1371/journal.pone.0158092

Worthington Jr., E. L. (2013). Hope-focused marriage counseling. InterVarsity Press.

Wong, P. T. (2011). Positive psychology 2.0: Towards a balanced interactive model of the good life. Canadian Psychology, 52(2), 69-81. https://doi.org/10.1037/a0022511 


\title{
Chapter 27
}

\section{Meaningfulness and Religious/Spiritual Meaning Systems at Work:}

\section{A Multilevel Framework}

\author{
Bryan J. Dik and Alexandra J. Alayan \\ Colorado State University \\ Fort Collins, Colorado, USA
}

Correspondence concerning this article should be addressed to Bryan J. Dik:

bryan.dik@colostate.edu 


\begin{abstract}
In this chapter, we examine the intersections of positive psychology (in particular, meaningfulness), religious and spiritual meaning systems, and the human experience of working. Psychological research and application related to work has generally taken either an individual perspective (within vocational psychology and career development) or an organizational perspective (within industrial-organizational psychology, management, and organizational behavior), which usually is contextualized within broader cultural and economic frames. Accordingly, we used a multilevel integrative model to explore factors that influence how religious/spiritual meaning systems can contribute to the experience of meaningfulness at work. This approach accounts for the individual, job, organizational, and societal levels of experience. Researchers are invited to test segments of the model using appropriate statistical techniques such as multilevel modeling. Counselors, human resource professionals, and organizational leaders are encouraged to invite workers to draw from their religious/spiritual meaning systems to inform career decisions and influence organizational policies and goals.
\end{abstract}

Keywords: meaning, work, religion, spirituality, positive psychology 


\section{Chapter 27: Meaningfulness and Religious/Spiritual Meaning Systems at Work:}

\section{A Multilevel Framework}

For many working adults today, work represents the life domain with the clearest and most impactful integrative potential for positive psychology and the psychology of religion and spirituality (R/S). The positive psychology literature offers a rich bounty of research and applications to organizational and working life. These are summarized in comprehensive works like the Oxford Handbook of Positive Organizational Scholarship (Cameron \& Spreitzer, 2012) and the Oxford Handbook of Positive Psychology and Work (Linley et al., 2010) — not to mention myriad popular books, websites, and consulting offerings. The psychology of R/S has been slower to expand into the work domain. An edited volume exploring potential linkages (Hill \& Dik, 2012) proposed promising directions, but a decade later, these remain underexplored. This is the case despite a growing, global faith and work movement actively exploring the intersection of R/S and work (Dik, 2020). In response, and in part to invite renewed scholarly attention to this intersection, this chapter explores points of convergence between positive psychology and R/S at multiple levels (e.g., individual, job, organizational, societal) within the work domain.

To inform this exercise, we begin by outlining the territory. Positive psychology broadly refers to the science and practice of well-being and human flourishing, addressing topics such as strengths, virtues, talents, pleasure, and meaning. A research domain within organizational behavior and management has targeted "workplace spirituality," defined as "a framework of organizational values evidenced in the culture that promotes employees' experience of transcendence through the work process, facilitating their sense of being connected to others in a way that provides feelings of completeness and joy" (Giacalone \& Jurkiewicz, 2003, p. 13).

Naturally, workplace spirituality focuses on experiences that unfold at work, especially within an 
organizational context. Workplace spirituality scholars are particularly interested in eudaimonic (e.g., meaning and growth-centered) aspects of well-being. Our chapter draws on workplace spirituality research but more specifically targets how positive psychology intersects with R/S. Religion can be understood as "ritual, institutional, or codified spirituality, which is culturally sanctioned;" spirituality is "a search for or relationship with the sacred" (Harris et al., 2018, p. 14). These definitions make clear that $R / S$ both involve the sacred. The sacred connotes ideas of God, a higher power, transcendence, or other aspects of life considered sanctified, holy, or worthy of reverence. The sacred is arguably what makes $\mathrm{R} / \mathrm{S}$ unique, distinct from (and not reducible to) other phenomena. $\mathrm{R} / \mathrm{S}$ are multidimensional (e.g., substantive, functional) in nature and can be examined on multiple levels (e.g., individual, social, cultural; Harris et al., 2018).

A central integrative theme within the psychology of $R / S$ is meaning making, especially the notion of religious meaning systems (Park \& Van Tongeren, this volume). Meaning systems include beliefs, values, and goals — essentially a worldview — that function to make sense of diverse experiences and tie them to a sense of purpose. They are evolutionarily fundamental and they are complex, operating at both micro (e.g., sensory processes) and macro (e.g., construction of socially influenced cultural meanings) levels. Religious/spiritual meaning systems often frame daily experience in terms of a higher-order meaning that transcends the context of particular events. This can occur within any life domain, certainly including work (Park, 2012).

To facilitate our integrative summary, we adopt a meaning-systems approach. We will target the experience of meaningful work, defined as work that is perceived as personally significant and worthwhile (Lysova et al., 2019). This includes meaningfulness in work (which stems from what workers do within the work role) and meaningfulness at work (which is rooted in workers' sense of being part of something bigger than themselves; Pratt \& Ashforth, 2003). 
Research on meaningful work notes that meaning operates on multiple levels. These levels were recently summarized in a model proposed by Lysova et al. (2019), which examines meaningfulness as a function of individual-, job-, organizational-, and societal-level factors. These factors provide the structure we use to organize this chapter (see Figure 27.1).

\section{Individual-Level Factors}

Individual factors that may influence the intersection of positive psychology, R/S, and eudaimonic well-being at work include dispositional signatures, characteristic adaptations, and personal narratives (McAdams, 2015).

\section{Dispositional Signatures}

Dispositional signatures are stable characteristics and traits that reflect the uniqueness of an individual. Two key dispositional signatures are interests and personality. In vocational psychology, interests are described as what people habitually enjoy or the "motivations that determine life decisions" (Walsh, 1999, p. 373). Evidence suggests that interests are highly stable over time, more so even than personality traits (Low et al., 2005). Furthermore, when people enter environments congruent with their interests, they experience substantial job satisfaction and well-being (Dik \& Hansen, 2008). Research has examined the links between the Big Five personality traits and the experience of meaningfulness at work. This research reveals moderate positive associations for openness, conscientiousness, and extraversion, as well as a weak inverse association with neuroticism (Frieder et al., 2018; Woods \& Sofat, 2013). Diverse religious traditions describe individual differences in domains such as interests and personality as useful for identifying a person's specific calling in the world of work (Dik, Duffy, \& Tix, 2012).

\section{Characteristic Adaptations}


Characteristic adaptations include goals and strivings. They offer perhaps the most direct application of a meaning systems approach when considering how religious/spiritual worldviews can influence work pursuits and the experience of meaningfulness. Notably, Park's (2012) meaning-making model posits that people are motivated to align their global meaning framework (i.e., beliefs, goals, values, sense of purpose) with their daily experiences and expressions of meaning. The more aligned these global and daily meanings are, the better the well-being outcomes and sense of coherence. Park (2012) proposed that R/S can play a major role in how this meaning-making process intersects with career behavior via four pathways: career choice and development, on-the-job conduct, work-related stress and coping, and work-related wellbeing. As Park (2012) summarized, "because work plays such a central role in most human lives, it follows that living a work life consistent with core religious or spiritual beliefs and facilitating progress on ultimate goals leads to higher levels of well-being" (p. 35). Park's model has not been directly tested within the work domain. However, it postulates an important role that the coherence between people's experiences of work and their religious/spiritual meaning systems can play in their overall experience of meaningfulness and both general and spiritual well-being.

Specific positive psychology topics that apply to experiences of purpose and meaning in work include positive emotions, flow, job crafting, calling, strengths, and gratitude (see Dik et al., 2015, for a review). Each of these constructs can be framed as characteristic adaptations. Strengths are "positive traits or skills that promote optimal functioning" (Owens et al., 2018, p. 266), and they are often a focus in career counseling. Peterson and Seligman (2004) described character strengths as moral traits (e.g., optimism, perseverance, appreciation of beauty) derived from six broader virtues (e.g., courage, justice, wisdom) identified through their study of 
religious traditions. Research reveals that when harnessed at work, strengths predict greater wellbeing, meaning in life, and sense of calling (Harzer \& Ruch, 2012; Owens et al., 2018).

Calling may be especially relevant to the integration of R/S and work. In modern usage, calling is considered both a sacred and a secular concept (Steger et al., 2010), yet it has long been understood through a religious lens. Calling has been defined as involving three dimensions: (a) a transcendent summons toward (b) purposeful work that is (c) carried out for the common good or well-being of others (Dik \& Duffy, 2009). The transcendent summons dimension, a reference to the notion that a calling implies a "caller," is particularly relevant to individuals with religious/spiritual commitments. However, all three calling dimensions have been shown to have significant relationships with religiousness (Ponton et al., 2014). Both having and living a calling have consistently been found to predict positive well-being (e.g., life satisfaction) and career development (e.g., self-clarity, career decidedness, occupational selfefficacy; Duffy et al., 2016) outcomes. Religiously committed individuals who perceive a calling tend to invest greater time and energy into their work, experience emotions such as gratitude, and exhibit higher levels of work motivation (Bott et al., 2017). They may also engage in job crafting, a set of behaviors that involve modifying the tasks, relationships, and cognitive boundaries governing the work role. A goal of job crafting is to forge a closer connection between their work experience and their broader sense of purpose in life. Research has found that job crafting positively predicts job satisfaction and commitment (Leana et al., 2009). Overall, a calling can provide a strong connection between global meaning systems and meaning derived from work experiences, perhaps especially when it comes to religious/spiritual meaning systems.

\section{Personal Narratives}


Personal narratives are the stories people tell about their lives. Articulating a narrative provides the opportunity for people to construct meaning from their lives, including their working lives. Personal narratives are also useful for articulating unique career-related goals and purposes while concurrently identifying life patterns (Savickas, 1995). Narratives can help people explore their values and "make sense of their career and world" (Collin \& Young, 1992, p. 2). Park's meaning-making model can inform the process of exploring career themes within a personal narrative framework (Park, 2012). Specifically related to R/S, sanctification (i.e., viewing an aspect of life as possessing sacred significance) offers people an opportunity to view and construct their careers through a sacred lens (Hernandez \& Mahoney, 2012). Research reveals that sanctification of work predicts positive work-related and general well-being outcomes, including job satisfaction, organizational commitment, and positive affect (Carroll et al., 2014; Walker et al., 2008). Also, sanctification of work is associated with lower inter-role work-family conflict among working mothers (Hall et al., 2012) and decreased job burnout among nurses (Ada et al., in press). Several qualitative studies of work-related calling have revealed that discerning a calling is a complex process that ties deeply to people's sense of identity (Schabram \& Maitlis, 2017) and faith perspective (Hernandez \& Mahoney, 2012).

\section{Job-Level Factors}

Job-level factors refer to tasks, responsibilities, expectations, and benefits that characterize one's job. Those factors can enable and/or impede workers' ability to experience meaningfulness at work. 


\section{Type, Quality, and Amount of Work}

Characteristics and conditions of one's work experiences can influence well-being and meaningfulness at work. Duffy et al.'s (2016) Psychology of Working Theory posits that work can potentially fulfill three needs fundamental to the human experience: (a) the need for survival and power, (b) the need for social connection, and (c) the need for self-determination. The lynchpin of Psychology of Working Theory is the attainment of decent work, which consists of (a) a safe environment free of physical, mental, or emotional abuse; (b) work hours that allow for adequate rest; (c) alignment of organizational and personal values; (d) adequate compensation; and (e) access to adequate health care. Attaining decent work is not only important for meeting the most fundamental needs people have from work but also for meeting self-determination needs and for developing a strong sense of meaning through work (Duffy et al., 2016).

\section{Job Design}

The oft-cited Job Characteristics Model (Hackman \& Oldham, 1976) suggests that jobs offering autonomy, skill variety, task identity, and task significance are linked to the experience of work meaningfulness. Similarly, jobs that are structured in ways that promote a sense of purpose and are focused on having a positive impact on others are related to greater experiences of meaningful work (Grant, 2007). Many employees possess little personal control over the design of their job, and it is important to acknowledge the boundary conditions of these concepts. Yet when possible, successful job crafting (e.g., of the job's tasks, relationships, and social function) can often result in job modifications that foster meaningfulness (Berg et al., 2013). For example, a hospital custodian might craft the job into one that is defined less by its list of required tasks and more by its social interactions and contributions to the hospital's mission of providing high-quality healthcare (Wrzesniewski \& Dutton, 2001). The extent to which such 
changes are possible varies as a function of the job itself, depending on factors such as how close workers are to the people who benefit from their work - a key notion underlying the concept of a job’s “social fit” (Dik, Duffy, \& Steger, 2012).

\section{Organization-Level Factors}

Most working adults have careers that unfold within organizations. Organizations therefore represent a key environment where people can experience meaningfulness, including that which may come from support for expressing a religious/spiritual meaning system. Organizations are also highly complex. Here, we briefly examine four organization-level influences: leadership, organizational culture, policies and procedures, and social context.

\section{Leadership}

In theory, one of the primary ways a leader can help enhance workers' experiences of meaningfulness and well-being is by creating structures that facilitate stronger connections between employees' daily experience of their job and a broader sense of purpose. This sense of purpose may include their religious/spiritual commitments (Lysova et al., 2019). There are several pathways through which this may be achieved. One way is to reinforce recurrently the ultimate aspirations of the company and to invite employees to align that organizational mission with their own values while also articulating how their job duties support these broader purposes (Allan, 2017). Leaders who can articulate their own sense of meaningfulness and how it links to the organization's mission may be especially effective at inspiring employees to do the same.

Leadership styles such as transformational leadership (Wolumbwa et al., 2013) and empowering leadership (Lee et al., 2017) have demonstrated positive relationships with meaningful work and can serve to support positive integrative thinking among workers with religious/spiritual meaning systems. Meaning-sensitive leaders may also act as an architect of 
sorts, providing a blueprint that maps connections between employee tasks and the organization's ultimate aspirations (e.g., focusing on a single ultimate aspiration, shifting attention from an ultimate aspiration to a concrete purpose, setting milestones for achieving this purpose, and articulating the link between the ultimate aspiration and concrete purpose; Carton, 2017). Especially germane to the question of how leadership style intersects with R/S is spiritual leadership (Fry et al., 2017), a style in which a clear vision and the moral values of faith, hope, and love are instilled within the workplace by an authentic leader with a vibrant inner life or mindful practice. (These values were introduced as broadly relevant across diverse religious traditions, but clearly draw from the Christian New Testament [1 Corinthians 13:13]; see Long \& VanderWeele, this volume). The values of hope and faith catalyze leader and team effort toward organizational goals. Altruistic love fosters ethical and kind behavior among workers, as well as a sense of membership. A compelling vision supports a calling and meaningfulness with respect to the work. Finally, a calling and sense of membership together evoke organizational commitment, productivity, and life satisfaction. Research on the spiritual-leadership model remains nascent but is generally supportive of its propositions (Fry et al., 2017).

\section{Organizational Culture}

Organizational culture is linked to workers' experience of meaningfulness and refers to the shared values, meanings, and assumptions that govern everyday life within an organization (Schein, 2010). Evidence suggests that highly controlling hierarchical cultures decrease employees' sense of their work as meaningful over time (Lee et al., 2017). Also, perceptions that an organizational culture enables collective learning are positively associated with scores on a measure of workplace spirituality dimensions (inner life, meaningful work, sense of community; Sorakraikitikul \& Siengthai, 2014). Other emerging evidence suggests that the organizational 
culture traits of adaptability and mission are more closely associated with workplace spirituality dimensions than are other organizational traits (e.g., consistency/stability; Alas \& Mousa, 2016). Rich conceptual explorations of how organizational cultures intersect with R/S (Demerath et al., 1998) and meaningful work (Cardador \& Rupp, 2011) offer promising directions for extending its currently limited empirical base.

\section{Social Context at Work}

The type and quality of interactions with other people within an organization are critical to employees' experiences of work as meaningful. This is the case because of how workers rely on social cues and social support to inform their perceptions of meaningfulness (e.g., Colbert et al., 2016). (Social context overlaps with organizational culture, but the former refers specifically to the nature of interpersonal relationships at work. In contrast the latter refers to an organization's values and norms for how work is carried out.) Positive relationships elicit a sense of belonging and social identity (Rosso et al., 2010). They also foster a common purpose, which contributes to meaningful work (Lips-Wiersma \& Morris, 2009) and often is a fundamental aspect of religious/spiritual meaning systems. Positive social-moral climates can evoke these types of relationships and are characterized by an atmosphere of trust, respect, support, cooperation, and a self-transcendent orientation. All of these enable the experience of meaningfulness at work (Schnell et al., 2013).

\section{Organizational Policies and Procedures}

There are several ways an organization's policies and procedures can support workers' well-being, experience of meaningfulness, and integration of religious/spiritual meaning systems. For example, establishing structures that invite personal and professional development can stimulate personal role engagement and enhance one's experience of work as meaningful 
(Fletcher, 2016). Specifically pertaining to support for employees with religious/spiritual commitments, Bennett (2008) described four levels of organizational action. The first focuses simply on compliance with laws that protect employees' rights to express their religion. The second entails normalization strategies that intentionally promote a culture of tolerance for religious expression (e.g., support for employee-led Bible study or meditation groups).

"Utilization" describes the third level, in which employees are encouraged to draw from their religious/spiritual meaning systems to inform organizational procedures and goals. The fourth is maximization, which occurs when the organization draws from religious/spiritual meaning systems to inform the broader organizational mission and ultimate aspirations. Although not necessarily linked to religious/spiritual meaning systems, corporate social responsibility initiatives have been found to predict employees' experiences of meaningful work (Glavas \& Kelley, 2014). So have corporate volunteering programs (Rodell, 2013), although authenticity is required to achieve these effects (Bailey et al., 2017).

\section{Societal-Level Factors}

The job-level factor of decent work, described earlier, functions on a societal level as well, given the public policy and economic environments in which decent work is possible and expected. A large proportion of the global population, for example, live in developing countries where decent work is often difficult to obtain. The International Labor Organization (ILO, 2016) has proposed that decent work is a fundamental human right and advocates national policies to ensure the realization of that right. Workers with decent work have a way to meet their survival, social connection, and self-determination needs; this theoretically frees up their psychological resources to more fully leverage their global meaning systems in the work role. Of course, although decent work is a key precursor to meaningful work, it is possible to experience 
meaningfulness in its absence. Ashforth and Kreiner (1999) and others have demonstrated this in their research on so-called "dirty" work (i.e., stigmatized work that is widely viewed as unpleasant, distasteful, thankless, or morally dubious). Cultural values for meaningful work also differ across geographic region (e.g., Schwartz, 1999) and time. So does the salience of R/S and the norms for integrating religious/spiritual meaning systems with various life domains.

\section{Cross-Level Interactions}

In their multilevel, integrative model, Lysova et al. (2019) emphasized the complex interactions within and across levels in their framework that govern behavior as it unfolds. For example, individual-level factors interact with job-, organization-, and society-level factors such that meaningfulness is more likely to occur when an individual's motivations, values, and goals align with those of their environment at each level (job, organization, society). To illustrate, a computer programmer with a sense of calling to help people learn will likely experience meaningfulness when developing educational products in an organization with prosocial values and a strong learning culture in a region that prioritizes funding for education. This same programmer will probably experience less meaningfulness developing fast-food delivery software for a company that prioritizes efficiency in an economically struggling region. Similarly, organizational- and job-level factors interact, such as when leaders influence organizational cultures and policies in ways that shape job design within an organization. For example, a CEO for a company that touts a value for flexibility and work-life balance may instate a policy that permits employees to work remotely rather than endure long daily commutes. Societal-level factors such as economic growth rate can also influence all other levels, such as when an economic recession or global pandemic creates severe constraints that shift organizational cultures, job descriptions, and individual-level values simultaneously—but 
not always in the same direction. The number and varied types of factors reviewed in Lysova et al.'s (2019) model mean that such interactions are many and multifaceted. Thus, the model offers several key pathways for future research and theorizing to follow in pursuit of increased clarity. In practice, the point is that the factors do not operate in isolation; understanding individual attitudes and behavior regarding religious/spiritual meaning systems and meaningful work requires being mindful of this reality.

\section{Implications for Research and Practice}

In their landmark review of research on meaningful work, Rosso et al. (2010) suggested that "systematic examinations of the mechanisms through which spiritual life impacts the meaning of work" (p. 107) would represent an important contribution to the literature.

Unfortunately, that suggestion has gone relatively unheeded in the intervening years, but it still represents a fruitful avenue for research and theory that seeks to explore points at which positive psychology and R/S converge within the human experience of work. In this chapter, we used Lysova et al.'s (2019) multilevel framework to explore factors on the individual, job, organizational, and societal levels that can support meaningful work that may arise from integrating religious/spiritual meaning systems. We close by highlighting next steps for this area of research, and offer some applications for counselors, human resource professionals, and organizational leaders as well.

The multiple levels in the model raise issues that have been explored elsewhere (e.g., Hill et al., 2013), such as the concern about conceptual distinctions and measurement focus across different levels of analysis. Testing the full model presented here, given its complexity, would indeed be challenging. A good starting point, echoing recommendations by Lysova et al. (2019), is for researchers to test model segments that align closest with their interests using appropriate 
statistical strategies such as multilevel modeling. Multilevel modeling allows researchers to examine workers embedded within jobs, organizations, and/or regional contexts, isolating sources of variance predicting meaningful work on different levels while accounting for the other levels. This technique has infrequently been used in the meaningful work or psychology of R/S literatures, but it offers a relatively nuanced way to examine elements of the model, including its interactions. As this work proceeds, the model can be adjusted and potentially developed into a formal theory. Parallel to this type of quantitative work, narrative research approaches may also provide a useful window into how people connect their global meaning systems with their dayto-day experience at work, within the context of a particular job, organization, and culture.

As research continues and theoretical advances are made, applications to practice come into sharper focus. Career counselors can collaborate with clients to explore how their religious/spiritual frameworks can inform their career decision-making, with the goal of achieving alignment. Within organizations, human resource professionals and organizational leaders might collaborate to deploy Bennet's (2008) “utilization" strategy. Planning sessions in which employees deliberately appeal to their meaning systems (including religious/spiritual meaning systems) to shape organizational goals and objectives are an example of this. Corporate social responsibility initiatives offer a good starting point for encouraging employees' own personal meaning-making processes, guided by a clearly (and genuinely) articulated organizational mission or ultimate aspiration (Carton, 2017) to serve as a scaffold. For individuals who struggle with this process, a starting point may be reflecting on the direct or indirect social impact of their work (Dik et al., 2012). Ultimately, our sincere hope is that as research grows and theory improves, innovative applications will offer meaningful benefits to workers who contribute the collective acts of mutual service that define a healthy society. 


\section{References}

Ada, H. M., Dehom, S., D’Errico, E., Boyd, K., \& Taylor, E. J. (in press). Sanctification of work and hospital nurse employment outcomes: An observational study. Journal of Nursing Management. https://doi.org/10.1111/jonm.13162

Allan, B. A. (2017). Task significance and meaningful work: A longitudinal study. Journal of Vocational Behavior, 102, 174-182. https://doi.org/10.1016/j.jvb.2017.07.011

Alas, R., \& Mousa, M. (2016). Organizational culture and workplace spirituality. International Journal of Emerging Research in Management and Technology, 5, 285-314.

Ashforth, B. E., \& Kreiner, G. E. (1999). "How can you do it?": Dirty work and the challenge of constructing a positive identity. Academy of Management Review, 24, 413-434. https://doi.org/10.2307/259134

Bailey, C., Madden, A., Alfes, K., Shantz, A., \& Soane, E. (2017). The mismanaged soul: Existential labor and the erosion of meaningful work. Human Resource Management Review, 27, 416-430. https://doi.org/10.1016/j.hrmr.2016.11.001

Berg, J. M., Dutton, J. E., \& Wrzesniewski, A. (2013). Job crafting and meaningful work. In B. Dik, Z. Byrne, \& M. Steger (Eds.), Purpose and meaning in the workplace (pp. 81-104). American Psychological Association. https://doi.org/10.1037/14183-005

Bennett, M. F. (2008). Religious and spiritual diversity in the workplace. In M. Moodian (Ed.), Contemporary leadership and intercultural competence (pp. 45-59). Sage. https://doi.org/10.4135/9781452274942.n5

Bott, E. M., Duffy, R. D., Borges, N. J., Braun, T. L., Jordan, K. P., \& Marino, J. F. (2017). Called to medicine: Physicians' experiences of career calling. The Career Development Quarterly, 65, 113-130. https://doi.org/10.1002/cdq.12086 
Cameron, K., \& Spreitzer, G. M. (Eds.). (2012). The Oxford handbook of positive organizational scholarship. Oxford University Press.

Cardador, M. T., \& Rupp, D. E. (2011). Organizational culture, multiple needs, and the meaningfulness of work. In N. M. Ashkanasy, C. P. M. Wilderom, \& M. F. Peterson (Eds.), Handbook of organizational culture and climate ( $2^{\text {nd }}$ ed.; pp. 158-180). Sage.

Carroll, S. T., Stewart-Sicking, J. A., \& Thompson, B. (2014). Sanctification of work: Assessing the role of spirituality in employment attitudes. Mental Health, Religion \& Culture, 17, 545-556. https://doi.org/10.1080/13674676.2013.860519

Carton, A. M. (2017). “I'm not mopping the floors, I'm putting a man on the moon”: How NASA leaders enhanced the meaningfulness of work by changing the meaning of work. Administrative Science Quarterly, 63, 323-369. https://doi.org/10.1177/0001839217713748

Collin, A., \& Young, R. A. (1992). Constructing career through narrative and context: An interpretive perspective. In R. A. Young \& A. Collin (Eds.), Interpreting career: Hermeneutical studies of lives in context. (pp. 1-12). Praeger/Greenwood.

Colbert, A. E., Bono, J. E., \& Purvanova, R. K. (2016). Flourishing via workplace relationships: Moving beyond instrumental support. Academy of Management Journal, 59, 1199-1223. https://doi.org/10.5465/amj.2014.0506

Demerath, N. J., Hall, P. D., Williams, R. H., \& Schmitt, T. (Eds.). (1998). Sacred companies: Organizational aspects of religion and religious aspects of organizations. Oxford University Press.

Dik, B. J. (2020). Why Faith@ Work needs vocational psychology: Five key findings. Journal of Psychology and Christianity, 39, 141-149. 
Dik, B. J., \& Duffy, R. D. (2009). Calling and vocation at work: Definitions and prospects for research and practice. The Counseling Psychologist, 37, 424-450. https://doi.org/10.1177/0011000008316430

Dik, B. J., Duffy, R. D., Allan, B. A., O’Donnell, M. B., Shim, Y., \& Steger, M. F. (2015). Purpose and meaning in career development applications. The Counseling Psychologist, 43, 558-585. https://doi.org/10.1177/0011000014546872

Dik, B. J., Duffy, R. D., \& Steger, M. F. (2012). Enhancing social justice by promoting prosocial values in career development interventions. Counseling and Values, 57, 31-57. https://doi.org/10.1002/j.2161-007X.2012.00005.X

Dik, B. J., Duffy, R.D., \& Tix, A. P. (2012). Religion, spirituality, and a sense of calling in the workplace. In P. C. Hill \& B. J. Dik (Eds.), The psychology of religion and workplace spirituality (pp. 113-133). Information Age Publishing.

Dik, B. J., \& Hansen, J. C. (2008). Following passionate interests to wellbeing. Journal of Career Assessment, 16, 86-100. https://doi.org/10.1177\%2F1069072707305773

Duffy, R. D., Blustein, D. L., Diemer, M. A., \& Autin, K. L. (2016). The psychology of working theory. Journal of Counseling Psychology, 63, 127-148. https://doi.org/10.1037/cou0000140

Fletcher, L. (2016). How can personal development lead to increased engagement? The roles of meaningfulness and perceived line manager relations. The International Journal of Human Resource Management, 30, 1203-1226. https://doi.org/10.1080/09585192.2016.1184177

Frieder, R. E., Wang, G., \& Oh, I.-S. (2018). Linking job-relevant personality traits, transformational leadership, and job performance via perceived meaningfulness at work: 
A moderated mediation model. Journal of Applied Psychology, 103, 324-333. https://doi.org/10.1037/apl0000274

Fry, L. W., Latham, J. R., Clinebell, S. K., \& Krahnke, K. (2017). Spiritual leadership as a model for performance excellence: A study of Baldrige award recipients. Journal of Management, Spirituality \& Religion, 14, 22-47. https://doi.org/10.1080/14766086.2016.1202130

Giacalone, R. A., \& Jurkiewicz, C. L. (Eds.). (2003). Handbook of workplace spirituality and organizational performance. Sharpe. https://doi.org/10.4324/9781315703817

Glavas, A., \& Kelley, K. (2014). The effects of perceived corporate social responsibility on employees. Business Ethics Quarterly, 24, 165-202. https://doi.org/10.5840/beq20143206

Grant, A. M. (2007). Relational job design and the motivation to make a prosocial difference. Academy of Management Review, 32, 393-417. https://doi.org/10.5465/amr.2007.24351328

Hackman, J. R., \& Oldham, G. R. (1976). Motivation through the design of work: Test of a theory. Organizational Behavior and Human Performance, 16, 250-279. https://doi.org/10.1016/0030-5073(76)90016-7

Hall, M., Oates, K. L., Anderson, T. L., \& Willingham, M. M. (2012). Calling and conflict: The sanctification of work in working mothers. Psychology of Religion and Spirituality, 4, 71-83. https://doi.org/10.1037/a0023191

Harris, K. A., Howell, D. S., \& Spurgeon, D. W. (2018). Faith concepts in psychology: Three 30year definitional content analyses. Psychology of Religion and Spirituality, 10, 1-29. https://doi.org/10.1037/rel0000134 
Harzer, C., \& Ruch, W. (2012). When the job is a calling: The role of applying one's signature strengths at work. The Journal of Positive Psychology, 7, 362-371. https://doi.org/10.1080/17439760.2012.702784

Hernandez, K. M., \& Mahoney, A. (2012). Balancing sacred callings in career and family life. In P. C. Hill \& B. J. Dik (Eds.), Psychology of religion and workplace spirituality (pp. 135155). Information Age Publishing.

Hill, P. C., \& Dik, B. J. (Eds.). (2012). Psychology of religion and workplace spirituality. Information Age Publishing.

Hill, P. C., Jurkiewicz, C., Giacalone, R., \& Fry, L. (2013). From concept to science: Continuing steps in workplace spirituality research. In R. Paloutzian \& C. Park (Eds.), Handbook of the psychology of religion and spirituality (pp. 617-631). Guilford Press.

International Labor Organization (2016). Decent work agenda. Author.

Leana, C., Appelbaum, E., \& Shevchuk, I. (2009). Work process and quality of care in early childhood education: The role of job crafting. Academy of Management Journal, 52, 1169-1992. https://doi.org/10.5465/amj.2009.47084651

Lee, M. C. C., Idris, M. A., \& Delfabbro, P. H. (2017). The linkages between hierarchical culture and empowering leadership and their effects on employees' work engagement: Work meaningfulness as a mediator. International Journal of Stress Management, 24, 392-415. https://doi.org/10.1037/str0000043

Linley, P. A., Harrington, S., \& Garcea, N. (Eds.). (2010). Oxford handbook of positive psychology at work. Oxford University Press. 
Lips-Wiersma, M., \& Morris, L. (2009). Discriminating between 'meaningful work' and the 'management of meaning.' Journal of Business Ethics, 88, 491-511. https://doi.org/10.1007/s10551-009-0118-9

Low, K. S., Yoon, M., Roberts, B. W., \& Rounds, J. (2005). The stability of vocational interests from early adolescence to middle adulthood: A quantitative review of longitudinal studies. Psychological Bulletin, 131, 713-737. https://doi.org/10.1037/0033$\underline{2909.131 .5 .713}$

Lysova, E. I., Allan, B. A., Dik, B. J., Duffy, R. D., \& Steger, M. F. (2019). Fostering meaningful work in organizations: A multilevel review and integration. Journal of Vocational Behavior, 110, 374-389. https://doi.org/10.1016/j.jvb.2018.07.004

McAdams, D. P. (2015). The art and science of personality development. Guilford Press.

Owens, R. L., Baugh, L. M., Barrett-Wallis, R., Hui, N., \& McDaniel, M. M. (2018). Strengths across the lifespan: A qualitative analysis of developmental trajectories and influential factors. Translational Issues in Psychological Science, 4, 265-276. https://doi.org/10.1037/tps0000164

Park, C. L. (2012). Religious and spiritual aspects of meaning in the context of work life. In P. C. Hill \& B. J. Dik (Eds.), Psychology of religion and workplace spirituality (pp. 25-42). Information Age Publishing.

Peterson, C., \& Seligman, M. E. (2004). Character strengths and virtues: A handbook and classification. Oxford University Press.

Ponton, R., Brown, T., McDonnell, B., Clark, C., Pepe, J., \& Deykerhoff, M. (2014). Vocational perception: A mixed-method investigation of calling. The Psychologist-Manager Journal, 17, 182-204. https://doi.org/10.1037/mgr0000019 
Pratt, M. G., \& Ashforth, B. E. (2003). Fostering meaningfulness in working and at work. In K. Cameron, J. Dutton, \& R. Quinn (Eds.). Positive organizational scholarship (pp. 309327). Berrett-Koehler.

Rodell, J. B. (2013). Finding meaning through volunteering: Why do employees volunteer and what does it mean for their jobs? Academy of Management Journal, 56, 1274-1294. https://doi.org/10.5465/amj.2012.0611

Rosso, B. D., Dekas, K. H., \& Wrzesniewski, A. (2010). On the meaning of work: A theoretical integration and review. Research in Organizational Behavior, 30, 91-127. https://doi.org/10.1016/j.riob.2010.09.001

Savickas, M. L. (1995). Constructivist counseling for career indecision. Career Development Quarterly, 43, 363-373. https://doi.org/10.1002/j.2161-0045.1995.tb00441.x

Schabram, K., \& Maitlis, S. (2017). Negotiating the challenges of a calling: Emotion and enacted sensemaking in animal shelter work. Academy of Management Journal, 60, 584-609. https://doi.org/10.5465/amj.2013.0665

Schein, E. H. (2010). Organizational culture and leadership (4 ${ }^{\text {th }}$ ed.). Jossey-Bass.

Schnell, T., Höge, T., \& Pollet, E. (2013). Predicting meaning in work: Theory, data, implications. The Journal of Positive Psychology, 8, 543-554. https://doi.org/10.1080/17439760.2013.830763

Schwartz, S. H. (1999). A theory of cultural values and some implications for work. Applied Psychology, 48, 23-47. https://doi.org/10.1111/j.1464-0597.1999.tb00047.x

Sorakraikitikul, M., \& Siengthai, S. (2014). Organizational learning culture and workplace spirituality: Is knowledge-sharing behaviour a missing link? The Learning Organization, 21, 175-192. https://doi.org/10.1108/TLO-08-2011-0046 
Steger, M. F., Pickering, N. K., Shin, J., \& Dik, B. J. (2010). Calling in work: Secular or sacred? Journal of Career Assessment, 18, 82-96. https://doi.org/10.1177/1069072709350905

Walker, A. G., Jones, M. N., Wuensch, K. L., Aziz, S., \& Cope, J. G. (2008). Sanctifying work: Effects on satisfaction, commitment, and intent to leave. The International Journal for the Psychology of Religion, 18, 132-145. https://doi.org/10.1080/10508610701879480

Walsh, W. B. (1999). What we know and need to know: A few comments. In M. L. Savickas \& A. R. Spokane (Eds.), Vocational interests: Meaning, measurement and counselling use (pp. 371-382). Davies-Black Publishing. https://doi.org/10.1080/10508610701879480

Wolumbwa, F. O., Christensen, A. L., \& Muchiri, M. K. (2013). Transformational leadership and meaningful work. In B. Dik, Z. Byrne, \& M. Steger (Eds.), Purpose and meaning in the workplace (pp. 197-215). American Psychological Association.

Woods, S. A., \& Sofat, J. A. (2013). Personality and engagement at work: The mediating role of psychological meaningfulness. Journal of Applied Social Psychology, 43, 2203-2210. https://doi.org/10.1111/jasp.12171

Wrzesniewski, A., \& Dutton, J. E. (2001). Crafting a job: Revisioning employees as active crafters of their work. Academy of Management Review, 26, 179-201. https://doi.org/10.5465/amr.2001.4378011 


\section{Figure 27.1}

\section{A Multilevel Conceptual Model of Religiousness/Spirituality and Meaningful Work (Adapted}

From Lysova et al., 2019)

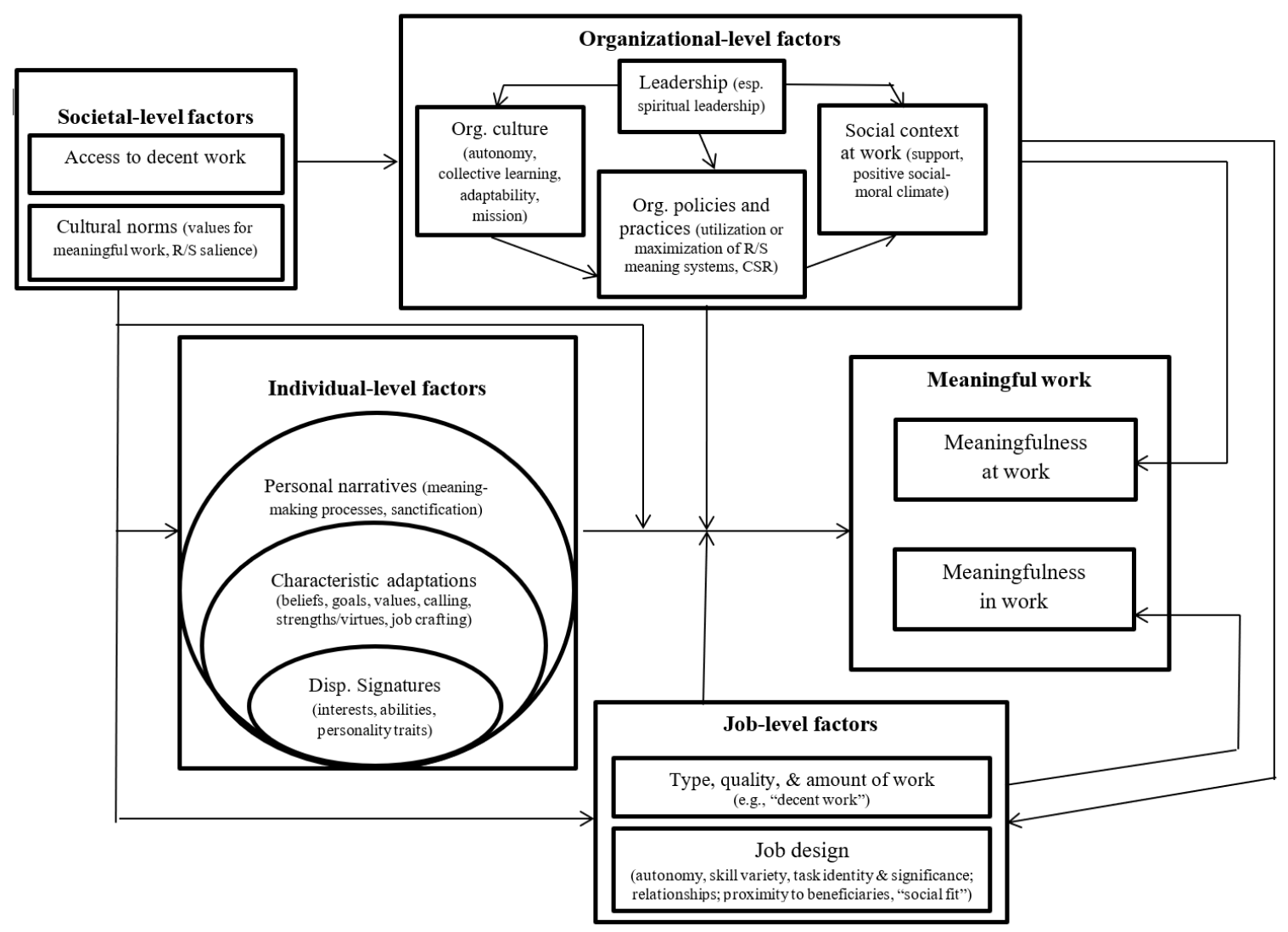

Note. This conceptual model is an integrative multilevel framework explaining factors that foster meaningful work and the integration of religious/spiritual meaning systems with work. It builds on the integrative model of meaningful work proposed by Lysova et al. (2019). 
Chapter 28: Positive Psychology and Religiousness/Spirituality in the Context of Couples and Families

Annette Mahoney, Jay R. Chinn, and James S. McGraw

Bowling Green State University

Correspondence concerning this chapter should be addressed to Annette Mahoney. Email:

amahone@bgsu.edu 


\begin{abstract}
In this chapter, we examine the intersections of positive psychology (PP) and religiousness/spirituality (R/S) in close relationships. Specifically, within Mahoney’s (2010) relational spirituality framework, we focus on the maintenance stage of healthy relationships and explore helpful roles that R/S can play for diverse types of couples and families. We briefly summarize extensive evidence that global markers of R/S (e.g., religious service attendance) are associated with relational well-being. We also highlight that global markers of R/S confound relatively commonplace positive religious/spiritual processes with less common but potentially toxic manifestations of R/S. We then delve into four specific religious/spiritual strengths that are empirically tied to better relational functioning: sanctification, spiritual intimacy, prayer for partner, and positive religious/spiritual coping. Next, we offer guidelines consistent with available scientific research that religious leaders, chaplains, couple and family educators, and psychotherapists might consider when working in community or clinical settings. Finally, we suggest ways to advance science and practice on the roles of R/S, for better and worse, within intimate and family relationships.
\end{abstract}

Keywords: couples, family, religion, spirituality, positive psychology 


\section{Chapter 28: Positive Psychology and Religiousness/Spirituality in the Context of Couples and Families}

The positive psychology (PP) movement offers uplifting insights on how social science can be leveraged to promote human well-being for individuals, couples, families, communities, and societies. As Chapter 4 of this handbook illustrates, many synergistic overlaps exist between religiousness/spirituality (R/S) and the virtues that PP has found to promote personal thriving. Similar intersections may exist between R/S and PP across diverse types of close relationships and families. In this chapter, we use Mahoney's (2010) relational spirituality framework and focus on the maintenance stage of healthy relationships, highlighting helpful roles that R/S can play for partners and parents. Specifically, we summarize extensive evidence linking global markers of higher R/S (especially higher religious service attendance) to relational well-being. Next, we delve into four specific religious/spiritual strengths associated with better relational well-being: sanctification, spiritual intimacy, prayer for partner, and positive religious/spiritual coping. We close by offering guidelines for helping professionals and recommendations for scientists to consider when they engage in efforts to enhance couple and family well-being.

\section{The Relational Spirituality Framework}

Mahoney's (2010) relational spirituality framework synthesizes empirical findings of global religious/spiritual markers and delineates specific religious/spiritual processes that can facilitate or undermine close relationship functioning. Analogous to Pargament and Mahoney's (2017) conception of spirituality as the discovery, conservation, and transformation of people perceive as sacred, Mahoney's (2010, 2013), relational spirituality framework heuristically sorted the R/S and couple/family research literature into three recursive, overlapping stages: (a) formation (i.e., creating and structuring a relationship), (b) maintenance (i.e., protecting an established relationship), and (c) transformation (i.e., reforming or exiting a distressed 
relationship). In addition, Mahoney differentiated specific religious/spiritual strengths or struggles that could emerge in individuals' relationships with (a) perceived supernatural figures (e.g., deity, immortal ancestor), (b) other individuals (e.g., romantic partner, spouse, child), and (c) the religious community (e.g., religious leaders and coreligionists). Each type of strength or struggle has the potential to shape close dyadic relationships (e.g., adult unions, parent-child relationships). Consistent with the emphasis of PP, this chapter elaborates the portion of the relational spirituality framework that addresses positive roles R/S can play in maintaining close relationships. Readers are referred elsewhere for scholarship on conflicts within and across families and religious communities (e.g., over the formation of adult unions and family units viewed as morally acceptable versus unacceptable; Mahoney \& Krumrei, in press) and for scholarship on ways R/S can exacerbate individual and family distress when people reform or exit distressed relationships (Ellison \& Xu, 2014; Mahoney, 2013; Mahoney \& Boyatzis, 2019).

Most scientific studies on faith and family life focus on families that adhere to a Western "traditional" family structure (i.e., married heterosexual couples with children), perhaps because such families tend to attend religious services more often than other types of family units. For example, according to 2011-2013 U.S. surveys, $49 \%$ of married mothers, $39 \%$ of single mothers, and $32 \%$ of cohabiting mothers attended religious services at least 2 to 3 times per month (Mahoney et al., 2015). Such group differences can be misinterpreted to mean that R/S is primarily or exclusively helpful to traditional families. However, in these same surveys, $79 \%$ of married mothers, $77 \%$ of single mothers, and $68 \%$ of cohabiting mothers reported R/S was somewhat or very important to their daily lives (Mahoney et al., 2015). More importantly, growing evidence indicates that higher R/S is tied to relational thriving for unmarried couples, same-sex couples, and families headed by single mothers (Mahoney \& Krumrei, in press). Thus, in this chapter, we highlight that greater R/S can be a valuable resource for many types of couple 
and family relationships, even though people in traditional family units may participate in organized religious groups more often than other people.

\section{Basic Research on Global Markers of Personal R/S and Relational Well-Being}

Since 1980, several hundred peer-reviewed studies have been published on associations between global markers of individuals' R/S (e.g., religious attendance or importance) and (a) forming and maintaining well-functioning adult unions, (b) becoming and being a better parent, and (c) sustaining close parent-adolescent relationships. Overall, this large body of work has found that higher involvement with organized religious groups is beneficial for couples and families. We refer readers to Ellison and Xu (2014), Mahoney (2010, 2013, 2021), Mahoney and Boyatzis (2019), Mahoney and Krumrei (in press), and Marks and Dollahite (2016) for extensive reviews of and specific citations from this rapidly expanding literature that we summarize below.

For couples, greater religious attendance and importance have been repeatedly correlated with less extra-relational infidelity, lower domestic violence, higher marital satisfaction, better relationship processes (e.g., relational commitment), and lower risk of divorce. For parenting, higher religious attendance and importance have been tied to less maternal substance use during pregnancy, higher parental satisfaction, and lower parental stress. For child-rearing attitudes and practices, higher religious attendance has been consistently linked to lower use of corporal punishment. Higher global R/S has also been tied to more positive childrearing methods, greater positive parental time spent with children, and lower risk of child physical abuse. Conservative Christian beliefs and affiliation have been tied to more frequent use of corporal punishment but not to higher rates of child physical abuse.

Taken together, higher R/S — especially religious attendance — has been consistently linked to desirable relational outcomes. Most of this research has been with married and unmarried opposite-sex couples or with single mothers. In addition, most findings are based on 
one or two global religious/spiritual items. Moreover, existing studies' typical cross-sectional methodology leaves open speculation about why and how greater involvement in religious groups is associated with better relational functioning. Perhaps greater involvement in a supportive religious community signals greater internalization of that religious community's valued family goals (e.g., getting and remaining married, having children) and virtues (e.g., altruism, generosity, commitment), which in turn protects and strengthens relational and family bonds. More longitudinal research is needed to explore these and other untested possibilities. Unfortunately, global religious/spiritual indices do not help identify what specific aspects of people's religious/spiritual thoughts, feelings, and relationships (with other people or deities) might be helpful versus harmful. Multi-item religious/spiritual measures that simply involve general questions about religious/spiritual activities (e.g., frequency of prayer or Scripture reading) also fail to disentangle adaptive versus maladaptive religious/spiritual processes. This confounding creates four major problems in understanding why faith matters for couples and families. First, skeptics can easily argue that any apparent association between virtues and higher global R/S is merely due to basic psychosocial strengths, such as prosocial morality, meaningmaking, or social support, each of which humans can develop and access within or outside of organized religious participation. From this conceptual vantage point, greater religious/spiritual engagement (such as attending religious services) is interchangeable with involvement in other cultural subgroups; it is not necessarily beneficial because of unique, substantive religious/spiritual beliefs, practices, or processes. Second, and conversely, critics can easily attribute associations between global religious/spiritual indices and relational vices to unique religious/spiritual beliefs that are taught by some religious groups, such the idea that scriptural passages or a deity condone spousal or child maltreatment. Third, global religious/spiritual indices allow scholars with an indiscriminately proreligious worldview to accentuate only the 
virtues tied to R/S (because global religious/spiritual indices can conceal rare but toxic forms of faith, especially in large national or community samples of mostly nondistressed individuals). Fourth, correlations between global indices of R/S and better relationship functioning are often small and/or insignificant, perhaps because the opposing effects of underlying positive and negative religious/spiritual processes often cancel each other out.

\section{Basic Research on Specific Relational Religious/Spiritual Strengths and PP Outcomes}

One solution to problems embedded within global religious/spiritual measures is for researchers to assess and disentangle religious/spiritual strengths from less-common religious/spiritual processes that are toxic. Illuminating both positive and negative religious/spiritual processes could also help integrate the aforementioned findings about the salutary links between R/S and PP, without losing sight of the potential dark sides of R/S.

We now highlight four specific relational religious/spiritual processes that mesh well with PP: sanctification, spiritual disclosure and intimacy, prayer for one's partner, and positive religious/spiritual coping. Unless otherwise noted, studies of these religious/spiritual strengths have thus far been conducted with U.S. samples of predominantly middle-class, White Christians. Such sampling is similar to overall U.S. demographics, but it obscures the roles these processes may (or not) play within various demographic subgroups or religious traditions.

\section{Sanctification of Couple and Family Relationships}

Sanctification is the degree to which a relationship is perceived (a) as a manifestation of God or Higher Powers (i.e., theistic sanctification) and/or (b) as imbued with sacred qualities (i.e., nontheistic sanctification). In a meta-analysis of correlational findings through mid-2019, Mahoney et al. (2021) found that greater sanctification of various types of close relationships is associated with more positive relational adjustment (i.e., average $r=.24, \mathrm{CI}=.20$ to .29 ) and lower rates of relational problems (average $r=-.12, \mathrm{CI}=-.06$ to -.18 ). Below we highlight a few 
findings from the around 55 qualitative and quantitative studies on sanctification and relational well-being in couples and family relationships that have been conducted as of mid-2021.

Married heterosexuals (Mahoney et al., 1999), same-sex couples, (Phillips et al., 2017), and dating and cohabiting couples (Henderson et al., 2018) often view their relationship as having sacred qualities and/or as being a manifestation of a deity's presence. For all three types of couples, greater perceived sanctification of the couple relationship has been tied to greater relationship satisfaction and commitment (Henderson et al., 2018; Phillips et al., 2017), even after controlling for positive relationship behaviors (e.g., forgiveness and sacrifice; Sabey et al., 2014) and stable traits of partners (Kusner et al., 2014). Greater sanctification has also been found to be linked to less partner-focused revenge (Davis et al., 2012), to buffer against the adverse impact of life stress on relationship quality (Ellison et al., 2011), and to predict more supportive partner behaviors and in turn greater relationship happiness (Rusu et al., 2015). Furthermore, cross-sectional and longitudinal evidence indicates that greater sanctification of marriage predicts better observed communication skills (by both spouses) and intimacy during conflictual marital interactions (Kusner et al., 2014; Rauer \& Volling, 2015) and emotionally vulnerable conversations (Padgett et al., 2019).

At least four studies have extended findings on sanctification of marriage beyond predominantly Christian U.S. couples. Consistent with findings on unmarried college students (Fincham et al., 2010), greater sanctification has been tied to lower infidelity thoughts and behaviors among married Iranians seeking counseling (Reich \& Kalantar, 2018). Also among married Iranians, greater sanctification of marriage uniquely predicted both greater marital satisfaction (after controlling for religious/spiritual coping; Fallahchai et al., 2021) and more frequent prayer for one's partner (Reich \& Kalantar, 2018). Furthermore, among Christian 
Orthodox couples from Romania, higher sanctification has been associated with better marital satisfaction and with more supportive marital interactions (Rusu et al., 2015).

The value of delving into specific religious/spiritual processes like sanctification is vividly illustrated by studies focused on sexuality within intimate unions. For decades, higher global R/S has been linked to greater sex guilt and more inhibition of sexual activity outside of marriage (Hernandez et al., 2013), implying that R/S mainly functions to suppress sexual wellbeing. However, greater sanctification of sexuality predicts greater sexual satisfaction crosssectionally among married and unmarried partners (Leonhardt et al., 2021) and longitudinally among newlyweds (Hernandez-Kane \& Mahoney, 2018). It also is tied to lower sex guilt among opposite-sex, same-sex, and cohabiting partners (Leonhardt et al., 2019; Phillips et al., 2017) and to lower odds of physical and emotional cheating, even after controlling for plausible alternate explanations (general R/S, problematic alcohol use, trait self-control; McAllister et al., 2020). Like with marriage, viewing parenting as sacred is commonplace, at least in the U.S. (Nelson \& Uecker, 2018). Moreover, both among married couples and single mothers, sanctification of parenting is tied to greater satisfaction with being a parent of school-aged children, even after controlling for global religious involvement and other demographics (Nelson \& Uecker, 2018). Among college students and their parents, sanctification has also been tied to more satisfaction within the parent-child relationship (Brelsford, 2013). Beyond satisfaction, viewing parenting as a sacred endeavor may intensify parental involvement and convictions about their preferred childrearing methods. Focusing on disciplinary situations, for example, sanctification by married parents has been linked to positive parenting techniques (e.g., to greater use of contingent praise and to teaching reparation) but not to punitive parenting techniques (e.g., to shaming or spanking; Volling et al., 2009). Furthermore, when a stronger belief in the sanctity of parenting is combined with greater use of nonpunitive strategies, evidence suggests it 
enhances children's conscience development (Volling et al., 2009). Focusing on fathers, viewing parenting as a sanctified role has been tied to fathers' greater involvement in their children's lives, even after accounting for personality and marital characteristics (yet children did not report feeling closer or more attached to their fathers; Lynn et al., 2016). More broadly, studies have found that greater sanctification of parenting also buffers parents against feeling stressed by their children's behavior problems, suggesting sanctification of parenting may help parents maintain confidence in the face of child noncompliance (Weyand et al., 2013).

\section{Spiritual Disclosure and Intimacy}

Whereas sanctification captures an individual's private view of a relationship, two or more people can engage in overt religious/spiritual behaviors that are tied to relational wellbeing as well, such as engaging in intimate dialogues about R/S. In an initial study on this topic, Brelsford and Mahoney (2008) assessed how much college students and parents openly shared their religious/spiritual views, resources, and struggles. Labeled spiritual disclosure, this process was associated with higher relationship satisfaction and lower verbal hostility, both in motherchild and father-child pairs. However, many people may avoid revealing information about their religious/spiritual thoughts or feelings to others, due to fear of being dismissed, ridiculed, or misunderstood (Brelsford \& Mahoney, 2008). Kusner et al. (2014) therefore created a measure to assess both dyadic spiritual disclosures and spiritual support (i.e., responding to a partner's spiritual disclosures in an empathic, nonjudgmental way), labeling this combined process spiritual intimacy. Greater spiritual intimacy predicted both partners displaying less negativity and more positivity during observations of couples discussing major conflicts, and associations persisted after accounting for couples' stable characteristics (e.g., education level, personality traits, and family backgrounds; Kusner et al. 2014). Moreover, in a longitudinal study, Padgett et 
al. (2019) found that spiritual intimacy predicted observations of new parents being more emotionally supportive of one another during emotionally vulnerable conversations.

\section{Prayer for Partner}

Individuals can privately turn to a perceived relationship with God to help them enact virtues that can help sustain their adult union (Fincham \& Beach, 2014). For example, several studies have found that, in generally well-functioning relationships, benevolent prayer for one's partner reliably facilitates that relationship's quality (Fincham et al., 2010; Fincham \& Beach, 2013, 2014). Indeed, in longitudinal studies of U.S. college students in a dating relationship, those who privately prayed for their romantic partner's well-being have reported increased relationship satisfaction and decreased risk of infidelity over time (for review, see Fincham \& Beach, 2013). Similarly, among Iranians seeking marital counseling, partner-focused prayer was tied to lower infidelity, even after controlling for sanctification (Reich \& Kalantar, 2018). Experimental studies have also found that praying for someone with whom one has a romantic or close relationship increases the praying person's levels of selfless concern, gratitude, and forgiveness of the person for whom they are praying (Fincham \& Beach, 2013). In addition, in a randomized experiment with a community sample of married African Americans, Beach et al. (2011) randomly assigned couples to one of three conditions: (a) an evidence-supported marital education program, (b) the same program supplemented with a module focused on partnerfocused prayer, and (c) self-help reading materials only. In the experimental condition, partnerfocused prayer enhanced marital outcomes for wives (but not husbands) over time, beyond the beneficial effects of the other two conditions. However, for both spouses, partner-focused prayer

predicted each partner's higher marital satisfaction, which also mediated (explained) the effect of partner-focused prayer on increased marital commitment (Fincham \& Beach, 2014). 


\section{Positive Religious/Spiritual Coping and Relationship Well-Being}

Rooted in Pargament's (1997) seminal book, extensive research exists on the role of positive religious/spiritual coping for individual well-being (Abu-Raiya \& Pargament, 2015). Measures of positive religious/spiritual coping largely assess how much people cope with stressful life events by drawing on a benevolent and secure relationship with God (divine coping) and on support from coreligionists (fellow religious believers). Such resources are often tied to better psychological adjustment, especially stress-related growth (Abu-Raiya \& Pargament, 2015; Pargament, 1997).

Likewise, positive religious/spiritual coping with personal and interpersonal stressors could potentially enhance relational well-being (Mahoney, 2010, 2013). Two studies of married couples offer preliminary support of this possibility. Specifically, for married Iranians, higher positive religious/spiritual coping predicted higher marital satisfaction (after controlling for prayer for partner; Reich \& Kalantar, 2018), sanctification of marriage, and global indices of R/S (frequency of prayer, religious pilgrimages, fasting, reciting the Quran; Fallahchai et al., 2021).

With regard to parenting, positive religious/spiritual coping has been related to parents' higher self-appraisals of parental competence, particularly among parents whose children have significant behavior problems (Weyand et al., 2013). However, such salutary links did not emerge for parents of at-risk preschoolers (Dumas \& Nissley-Tsiopinis, 2006) or children with autism (Tarakeshwar \& Pargament, 2001). Such null findings may reflect stress-mobilization coping processes where parents may call on God more often when they feel overwhelmed.

\section{Summary Observations}

To recap, greater sanctification of close relationships and benevolent prayer for a partner have been robustly tied to better marital, sexual, and parental satisfaction and commitment. These findings have emerged mostly in studies comprised of largely nondistressed couples and 
parents, as well as both inside and outside of marriage. Initial studies on spiritual disclosure, spiritual intimacy, and positive religious/spiritual coping suggest these specific religious/spiritual processes may also help enhance and sustain healthy relationships with loved ones. All these specific religious/spiritual processes are likely to be reciprocally linked to greater participation in a supportive religious community. Furthermore, being active in a religious group that affirms the type of family to which one belongs could be helpful in many ways, such as offering norms, role models, and classes that help build and reinforce positive couple, marital, and family dynamics.

\section{Applications of Relational R/S Strengths in Community and Clinical Contexts}

Next, we discuss possible applications of the research findings we have reviewed, particularly for religious leaders, chaplains, and couple and family educators or psychotherapists.

Awareness of religious/spiritual strengths. To date, nearly all studies on relational religious/spiritual strengths have involved national or community samples of generally wellfunctioning couples (opposite-sex or married-heterosexual) and single parents. One implication of this research is that helping professionals could consider integrating research findings about religious/spiritual relational strengths into psychoeducational prevention programs (e.g., couple or parenting enrichment programs) and facilitate participants' reflection and dialogue about the potential benefits of drawing on these religious/spiritual resources. Similarly, psychotherapists could help clients identify specific religious/spiritual beliefs about close relationships that could help motivate their use of interpersonal strategies for enhancing their relational and personal well-being. Next, we offer a few guidelines for such efforts, based on available research findings.

Avoid religious/spiritual stereotypes. One guideline is for practitioners and researchers to avoid stereotypes when anticipating who might benefit from relational religious/spiritual strengths. For instance, do not assume that religious/spiritual assets are only relevant to individuals embedded in "traditional" couples or families. Instead, when working across diverse 
types of couples and families, directly assess whether and how relational religious/spiritual strengths may facilitate personal and relational well-being. In addition, do not merely focus on simplistic religious/spiritual constructs such as religious affiliation or attendance. Moreover, assess constructs that are relevant for theists and nontheists alike, such as the nontheistic sanctification of couple and family relationships. Indeed, increasing numbers of people are involved in nontraditional intimate or family relationships. To illustrate, based on 2010 data, about $65 \%$ of U.S. women cohabited with a partner prior to their first marriage, about $40 \%$ of children were born to unmarried women, and $50 \%$ of children are expected to spend part of their childhood living with an unmarried couple (Mahoney \& Krumrei, in press).

Take religious/spiritual dialogue seriously. Another guideline is to take seriously the potential bonding power of two individuals vulnerably sharing their religious/spiritual thoughts, questions, or struggles with each other and then providing one another with empathic, nonjudgmental support about such disclosures. These spiritually intimate dialogues can lead to better relational satisfaction and emotion-focused communication skills. Leaders of relationship education programs can consider intentionally encouraging and facilitating spiritually disclosing, intimate dialogues between partners (and parents and children) about their respective religious/spiritual journeys and how these journeys inform their personal and relational aspirations. For example, individuals could be asked to share with loved ones whether, why, how, and to what degree they pray for each other and view their relationship as a sacred bond. Similarly, psychotherapists could consider exploring whether and with whom their clients experience spiritual intimacy, taking care to model genuine and nonjudgmental curiosity about the roles R/S plays in shaping their clients' desires and strategies to sustain healthy close relationships. The emergence of problematic conflicts during religious/spiritual dialogues could signal a need for relationship partners to approach—not avoid—unresolved tensions. 
Unpack religious/spiritual content. A third guideline is to unpack the content of individuals' religious/spiritual thoughts and ways of dialoguing with other people and with God/Higher power(s). Use religious language that matches the target audience (see Beach et al., 2008, for sample prayer script adapted for a particular religious tradition). As an exemplary illustration, Beach et al. (2011) examined the causal impact of adding partner-focused prayer to a well-established evidence-based marital education program (PREP). The researchers carefully tailored the language of sample partner-focused prayers to fit well with the worldview of their theologically moderate to conservative, African-American, Christian participants. Namely, the prayers emphasized God's love and were supplemented with agape-related biblical verses that resonated with the theme of selfless love. These partner-focused prayers improved relational outcomes, at least for wives (Beach et al., 2011). In contrast, Cobb and Sullivan (2015) found that over 2 years, wives who participated in uncontrolled premarital or relationship educational programs (71\% delivered in religious settings) showed declines in marital satisfaction that were 3.5 times steeper compared with wives who did not participate in any such programs, with no effects found for husbands. These results contrast Beach et al. (2011) and raise questions about how to address participants' religious/spiritual beliefs when disseminating relationship educational programs in religious settings. In psychotherapy, we suggest psychotherapists sensitively assess whether the content of clients' religious/spiritual beliefs or prayers aids or hinders partners' relational and personal well-being.

Possible clashes between R/S and PP. Although we highlight possible benefits of relational religious/spiritual resources, these processes can translate into relational processes that conflict with positive relationship dynamics that are often promoted by PP. For example, in a study of married heterosexual couples, greater perceived sanctity of the parent-infant bond led to decreased (not increased) egalitarianism in providing essential infant care (DeMaris et al., 2011). 
Also, in a small-scale study of mothers, greater sanctification of parenting was tied to more use of corporal punishment when mothers interpreted the Bible literally but less use of corporal punishment for mothers with more liberal biblical views (Murray-Swank et al., 2006). More studies are needed to clarify for whom and when specific religious/spiritual resources translate into virtuous attitudes and actions toward loved ones. In the meantime, couple and family educators and psychotherapists need to be prepared to address conflicts between their own and clients' views of the optimal means to build and maintain couple and family relationships.

Dark sides of R/S. As elaborated elsewhere (e.g., Johnson, 2015; Mahoney, 2013), dark sides of relational R/S exist that need to keep that in mind. To illustrate, we highlight four religious/spiritual risk factors that are counter examples to the four religious/spiritual strengths we emphasize in this chapter. First, individuals can perceive couple or family problems, such as a divorce or romantic break-up, as a desecration or sacred loss, which can intensify their subsequent personal and interpersonal distress. Second, in conflicts with loved ones, partners and parents can engage in religious/spiritual dialogues in which they "align" with divine beings or religious/spiritual teachings to justify a harmful position (i.e., spiritual one-upmanship), such as objecting to a sexual and/or gender minority family member (Etengoff \& Diaute, 2014). Third, instead of confronting problems productively, individuals can pray to God as an ineffectual means to tolerate or try to change another's dysfunctional behavior. Fourth, individuals can become embroiled in negative religious/spiritual coping, including distressing thoughts and feelings about supernatural figures (e.g., anger toward God, feeling punished by the devil), religious groups (e.g., conflicts with co-believers), or the self (e.g., feeling morally conflicted or confused about ultimate meaning). Studies suggest these four processes are relatively rare, but they each are tied to more relational and/or personal distress. Thus, although clinicians may be 
more likely to witness pathological manifestations of R/S than religious leaders in community settings, professionals working in either context need be willing to address the dark sides of R/S.

Collaborate with religious/spiritual groups. Although some people pursue their religious/spiritual journeys outside of a religious community, religious groups presumably represent salient social networks that can help individuals access religious/spiritual resources and work through religious/spiritual struggles. Mental health professionals and couple and family educators can collaborate with religious organizations to identify specific religious/spiritual factors that may enhance evidence-supported psychoeducational programs. Additionally, mental health professionals could offer religious groups insights from emerging research on personal and relational distress that some religious/spiritual teachings may create, and they can help religious organizations consider how to address such distress. Taken together, mutually beneficial collaborations could be built between practitioners and religious organizations, to help people cultivate healthy relationships (Mahoney et al., 2019).

\section{Recommendations for Science and Practice}

We close by articulating some key priorities for scientific research on R/S, close relationships, and PP. First, more research is needed that differentiates specific religious/spiritual factors from PP's virtues (e.g., forgiveness) that do not necessarily involve substantive religious/spiritual beliefs, practices, or elements. Second, studies need to disentangle specific religious/spiritual resource and risk factors that are confounded in global indicators of R/S. Third, diverse couples and families — beyond married heterosexuals with children — need to be included in studies of R/S and close relationships (e.g., couples without children, unmarried or repartnered/remarried parents, multigenerational families, same-sex couples); such efforts will broaden the sociocultural generalizability of scientific findings on how R/S can help relationships thrive. On a related note, kinship relationships across the lifespan need empirical 
attention (e.g., adult siblings, aunts/uncles, and friendships) to encompass the family networks of single adults without children. Fourth, researchers need to be clear that despite mean differences between subgroups (e.g., married vs. unmarried individuals), similar significant associations may exist between religious/spiritual strengths and relational well-being within subgroups. Fifth, research needs to establish pathways from relational religious/spiritual strengths to positive relational dynamics and then to individual and relational well-being. Taken together, progress on these recommendations could offer practitioners and policymakers deeper depth insights about why and how R/S matters for close relationships which could then be incorporated into evidencebased prevention and intervention programs for couples and families. 


\section{References}

Abu-Raiya, H., \& Pargament, K. I. (2015). Religious coping among diverse religions:

Commonalities and divergences. Psychology of Religion and Spirituality, 7(1), 24-33. https://doi.org/10.1037/a0037652

Beach, S. R. H., Fincham, F. D., Hurt, T. R., Mcnair, L. M., \& Stanley, S. (2008). Prayer and marital intervention: Toward an open, empirically-grounded dialogue. Journal of Social \& Clinical Psychology, 27(7), 693-710. https://doi.org/10.1521/jscp.2008.27.7.693

Beach, S. R. H., Hurt, T. R., Fincham, F. D., Franklin, K. J., McNair, L. M., \& Stanley, S. M. (2011). Enhancing marital enrichment through spirituality: Efficacy data for prayer focused relationship enhancement. Psychology of Religion and Spirituality, 3(3), 201216. http://dx.doi.org/10.1037/a0022207

Brelsford, G. M. (2013). Sanctification and spiritual disclosure in parent-child relationships: Implications for family relationship quality. Journal of Family Psychology, 27(4), 639649. http://dx.doi:10.1037/a0033424

Brelsford, G. M., \& Mahoney, A. (2008). Spiritual disclosure between older adolescents and their mothers. Journal of Family Psychology, 22(1), 62-70. https://doi.org/10.1037/0893$\underline{3200.22 .1 .62}$

Cobb, R. J. \& Sullivan, K. T. (2015). Relationship education and marital satisfaction in newlywed couples: A propensity score analysis. Journal of Family Psychology, 29(5), 667-678. http://dx.doi.org/10.1037/a0039580

Davis, D. E., Hook, J. N., Van Tongeren, D. R., \& Worthington, E. L. (2012). Sanctification of forgiveness. Psychology of Religion and Spirituality, 4(1), 31-39. https://doi.org/10.1037/a0025803

DeMaris, A., Mahoney, A., \& Pargament, K. I. (2011). Doing the scut work of childcare: Does 
religiousness encourage greater father involvement? Journal of Marriage and Family, 73(2), 354-368. http://doi:10.1111/j.1741-3737.2010.00811.x

Dumas, J. E., \& Nissley-Tsiopinis, J. (2006). Parental global religiousness, sanctification of parenting, and positive and negative religious coping as predictors of parental and child functioning. International Journal for the Psychology of Religion, 16(4), 289-310.

\section{http://doi:10.1207/s15327582ijpr1604_4}

Ellison, C. G., Henderson, A. K., Glenn, N. D., \& Harkrider, K. E. (2011). Sanctification, stress, and marital quality. Family Relations, 60(4), 404-420. http://doi.org/10.1111/j.1741$\underline{3729.2011 .00658 . x}$

Ellison, C. G., \& Xu, X. (2014). Religion and families. In J. Treas, J. Scott, \& M. Richards (Eds.), Wiley Blackwell companion to the sociology of families (pp. 277-299). Wiley.

Etengoff, C., \& Daiute, C. (2014). Family members' uses of religion in post-coming-out conflicts with their gay relative. Psychology of Religion and Spirituality, 6(1), 33-43. https://doi.org/10.1037/a0035198

Fallahchai, R., Fallahi, M., Moazenjami, A., \& Mahoney, A. (2021). Sanctification of marriage, religious coping, and dyadic adjustment in Iranian couples. Archive for the Psychology of Religion. Advance online publication. https://doi.org/10.1177/0084672421996826

Fincham, F. D., Lambert, N. M., \& Beach, S. R. H. (2010). Faith and unfaithfulness: Can praying for your partner reduce infidelity? Journal of Personality and Social Psychology, 99(4), 649-659. https://doi.org/10.1037/a0019628

Fincham, F. D., \& Beach, S. R. H. (2013). Can religion and spirituality enhance prevention programs for couples? In K. I. Pargament, A. Mahoney, \& E. P. Shafranske (Eds.), APA handbook of psychology, religion, and spirituality (Vol. 2): An applied psychology of 
religion and spirituality (pp. 461-479). American Psychological Association. https://doi.org/10.1037/14046-024

Fincham, F. D., \& Beach, S. R. H. (2014). I say a little prayer for you: Praying for partner increases commitment in romantic relationships. Journal of Family Psychology, 28(5), 587-593. http://doi.org/10.1037/a0034999

Henderson, A. K., Ellison, C. G., \& Glenn, N. D. (2018). Religion and relationship quality among cohabiting and dating couples. Journal of Family Issues, 39(7), 1904-1932. https://doi.org/10.1177/0192513X17728982

Hernandez, K. M., Mahoney, A., Pargament, K. I. (2013). The sacred dance between sexuality and religion: Scientific insights from the $21^{\text {st }}$ century. In D. L. Tolman \& L. Diamond (Eds.), APA handbook of sexuality and psychology (pp. 425-447). American Psychological Association. http://doi.org/10.1037/14194-013

Hernandez-Kane, K. M., \& Mahoney, A. (2018). Sex through a sacred lens: Longitudinal effects of sanctification of marital sexuality. Journal of Family Psychology, 32(4), 425-434. https://doi.org/10.1037/fam0000392

Johnson, J. A. (2015). Religion and men's violence against women. Springer.

Kusner, K. G., Mahoney, A., Pargament, K. I., \& DeMaris, A. (2014). Sanctification of marriage and spiritual intimacy predicting observed marital interactions across the transition to parenthood. Journal of Family Psychology, 28(5), 604-614. https://doi.org/10.1037/a0036989

Leonhardt, N. D., Busby, D. M., \& Willoughby, B. J. (2019). Sex guilt or sanctification? The indirect role of religiosity on sexual satisfaction. Psychology of Religion and Spirituality, 12(2), 213-222. https://doi.org/10.1037/rel0000245

Leonhardt, N. D., Busby, D. M., Hanna-Walker, V. R., \& Leavitt, C. E. (2021). 
Sanctification or inhibition? Religious dualities and sexual satisfaction. Journal of Family Psychology, 35(4), 433-444. http://dx.doi.org/10.1037/fam0000796

Lynn, M. G., Grych, J. H., \& Fosco, G. M. (2016). Influences on father involvement: Testing for unique contributions of religion. Journal of Child and Family Studies, 25(11), 32473259. https://doi.org/10.1007/s10826-016-0486-6

Mahoney, A. (2010). Religion in families 1999-2009: A relational spirituality framework. Journal of Marriage and Family, 72(4), 805-827. https://doi.org/10.1111/j.1741$\underline{3737.2010 .00732 . x}$

Mahoney, A. (2013). The spirituality of us: Relational spirituality in the context of family relationships. In K. I. Pargament, J. J. Exline, \& J. W. Jones (Eds.), APA handbook of psychology, religion, and spirituality (Vol. 1): Context, theory, and research (pp. 365389). American Psychological Association. https://doi.org/10.1037/14045-020

Mahoney, A. (2021). The science of children's religious and spiritual development. Cambridge University Press.

Mahoney, A., Pargament, K. I., Jewell, T., Swank, A. B., Scott, E., Emery, E., \& Rye, M. (1999). Marriage and the spiritual realm: The role of proximal and distal religious constructs in marital functioning. Journal of Family Psychology, 13(3), 321-338.

\section{https://doi.org/10.1037/0893-3200.13.3.321}

Mahoney, A., \& Krumrei, E. J. (in press). Questions left unaddressed by religious familism: Is spirituality relevant to non-traditional families? L. Miller (Ed.), The Oxford handbook of the psychology of spirituality ( $2^{\text {nd }}$ ed.). Oxford University Press.

Mahoney, A., \& Boyatzis, C. J. (2019). Parenting, religion, and spirituality. M. Bornstein (Ed.), Handbook of parenting ( $3^{\text {rd }}$ ed., pp. 516-552). Routledge.

Mahoney, A., Larrid, I. E., Payne, K. K., \& Manning, W. D. (2015). Religiosity and U.S. single, 
cohabiting and married mothers. (FP-15-06). National Center for Family \& Marriage Research. https://www.bgsu.edu/ncfmr/resources/data/family-profiles/mahoney-lamidipayne-religiosity-fp-15-06.html

Mahoney, A., Pomerleau, J., \& Riley, A. (2019). Transcending barriers to build bridges between family psychology and religious organizations. In B. H. Fiese, M. Celano, K. DeaterDeckard, E. N. Jouriles, \& M. A. Whisman (Eds.), APA handbook of contemporary family psychology: Applications and broad impact of family psychology (pp. 315-335). American Psychological Association. https://doi.org/10.1037/0000100-020

Mahoney, A., Wong, S., Pomerleau, J. M., \& Pargament, K. I. (2021). Sanctification of diverse aspects of life and psychosocial functioning: A meta-analysis of studies from 1999 to 2019. Psychology of Religion and Spirituality. Advance online publication. https://doi.org/10.1037/rel0000354

Marks, L. D., \& Dollahite, D. C. (2016). Religion and families: An introduction. Routledge. McAllister, P., Henderson, E., Maddock, M., Dowdle, K., Fincham, F. D., \& Braithwaite, S. R. (2020). Sanctification and cheating among emerging adults. Archive of Sexual Behavior, 49, 1177-1188. https://doi.org/10.1007/s10508-020-01657-3

Murray-Swank, N. A., Mahoney, A., \& Pargament, K. I. (2006). Sanctification of parenting: Links to corporal punishment and parental warmth among biblically conservative and liberal mothers. The International Journal for the Psychology of Religion, 16(4), 271287. https://doi.org/10.1207/s15327582ijpr1604_3

Nelson, J. J., \& Uecker, J. E. (2018). Are religious parents more satisfied parents? Individualand couple-level religious correlates of parenting satisfaction. Journal of Family Issues, 39(7), 1770-1796. https://doi.org/10.1177/0192513X17728983

Padgett, E., Mahoney, A., Pargament, K. I., \& DeMaris, A (2019). Marital sanctification 
and spiritual intimacy predicting married couples' observed intimacy skills across the transition to parenthood. Religions, 10, 177-193. https://doi.org/10.3390/rel10030177

Pargament, K. I. (1997). The psychology of religion and coping: Theory, research, practice. Guilford Press.

Pargament, K. I., \& Mahoney, A. (2017). Spirituality: The search for the sacred. In C. R. Snyder, S. J. Lopez, L. M. Edwards, \& S. C. Marques (Eds.), The Oxford handbook of positive psychology (3 $3^{\text {rd }}$ ed.). http://doi.org/10.1093/oxfordhb/9780199396511.013.51

Phillips, R. E., Avant, S., Kalp, D., Cenkner, D., Lucci, M., Herndon, R., \& Maccarelli, A. (2017). Initial validation of measures of sanctification in same-sex romantic relationships and sexual behavior. Journal for the Scientific Study of Religion, 56(4), 836-851. https://doi.org/10.1111/jssr.12488

Rauer, A., \& Volling, B. (2015). The role of relational spirituality in happily married couples' observed problem-solving. Psychology of Religion and Spirituality, 7(3), 239-249. https://doi.org/10.1037/rel0000022

Reich, N., \& Kalantar, S. M. (2018). The role of praying for the spouse and sanctification of marriage in reducing infidelity. Mental Health, Religion \& Culture, 21(1), 65-76. https://doi.org/10.1080/13674676.2018.1447555

Rusu, P. P., Hilpert, P., Beach, S. R. H., Turliuc, M. N., \& Bodenmann, G. (2015). Dyadic coping mediates the association of sanctification with marital satisfaction and well-being. Journal of Family Psychology, 29(6), 843-849. https://doi.org/10.1037/fam0000108

Sabey, A. K., Rauer, A. J., \& Jensen, J. F. (2014). Compassionate love as a mechanism linking sacred qualities of marriage to older couples' marital satisfaction. Journal of Family Psychology, 28(5), 594-603. https://doi.org/10.1037/a0036991

Tarakeshwar, N., \& Pargament, K. I. (2001). Religious coping in families of children with 
autism. Focus on Autism and Other Developmental Disabilities, 16(4), 247-260.

https://doi.org/10.1177/108835760101600408

Volling, B. L., Mahoney, A., \& Rauer, A. J. (2009). Sanctification of parenting, moral socialization, and young children's conscience development. Psychology of Religion and Spirituality, 1(1), 53-68. https://doi.org/10.1037/a0014958

Weyand, C., O’Laughlin, L., \& Bennett, P. (2013). Dimensions of religiousness that influence parenting. Psychology of Religion and Spirituality, 5(3), 182-191.

https://doi.org/10.1037/a0030627 


\title{
Chapter 29
}

\section{Positive Psychology in the Context of Religious Communities}

David C. Wang ${ }^{1}$, Mark R. McMinn², Zachary Wood ${ }^{1}$, and Collin Lee ${ }^{1}$

\author{
Biola University, Rosemead School of Psychology ${ }^{1}$ \\ George Fox University ${ }^{2}$
}

Correspondence concerning this chapter should be addressed to David C. Wang, Rosemead School of Psychology, Biola University, 13800 Biola Ave, La Mirada, CA 90639. Email: david.wang@biola.edu 


\begin{abstract}
This chapter explores the intersections of positive psychology, religion, and spirituality in the context of religious communities (e.g., churches, synagogues, mosques, and temples). We first argue that positive psychology provides an optimal vehicle for cultivating and demonstrating the mutual benefits of collaboration between psychology and religious communities by supporting adherents' religiousness/spirituality, virtue development, and holistic well-being within the context of their religious community. Second, we review empirical research on how positive psychology and religion/spirituality interact reciprocally in the context of religious communities. Finally, we discuss implications for ongoing research.

Keywords: positive psychology, religion, spirituality, religious communities, science and religion
\end{abstract}




\section{Chapter 29: Positive Psychology in the Context of Religious Communities}

It is surprising that psychology—-literally the "study of the soul" - does not seem to have a natural affinity for religious communities, where the life of the soul has been considered and nurtured for millennia. Instead, research suggests that psychologists are less likely to participate in a faith tradition than most of the general population (Shafranske \& Cummings, 2013; Vieten \& Lukoff, 2021), with some taking a confrontational approach to religious leaders, alleging religious leaders' perceived inadequacies in providing care for people with emotional needs (McMinn et al., 2005). Many explanations have been offered to help capture the nuances behind historic tensions between religion and psychology (or even between religion and science in general) - a topic that is beyond the scope of this chapter but is addressed elsewhere in this book (see Davis et al., Chapter 1, this volume; Nelson \& Canty, Chapter 2, this volume; Porter et al., Chapter 3, this volume). However, rather than focus on these areas of conflict, our premise is instead to suggest and expand on how these two communities (i.e., the psychological community and religious communities) would both benefit from greater collaboration with each other.

Before we enter into this discussion in earnest, we would like to first raise an important caveat - namely, that our use of the term religious communities may in fact hold different meaning or salience across different faith traditions. For example, within the Hindu tradition, Hinduism is understood to be more than a religion - it is a culture in and of itself, with practices and beliefs commonly integrated into all aspects of individual and community life (Hodge, 2004; see Singh et al., Chapter 13, this volume). Thus, the demarcation between those aspects of life that are understood to fall within the context of one's religious community and those that fall outside one's religious community may be much more fluid for some adherents, relative to others who are affiliated with faith traditions in which the categories of sacred and secular are 
modally less overlapping and/or are understood to be mutually exclusive. Nonetheless, with this important caveat in mind, we move forward with our case for positive psychology as a fertile context for greater collaboration between scientific and religious communities.

\section{Collaborative Opportunities with Positive Psychology}

Recent Gallup poll findings suggest that-for the first time since the polling began in the 1930s_-less than half of Americans hold membership in a religious community (Jones, 2021). Throughout the $20^{\text {th }}$ century, the rate of membership in a church, synagogue, mosque, or temple hovered around $70 \%$. However, this figure has dropped steadily during the $21^{\text {st }}$ century to the current rate of $47 \%$. Although this trend has garnered attention about the decline of religion in America, it is also noteworthy that nearly half of Americans still belong to religious communities. This reality arguably makes collaboration with religious communities one of the most optimal vehicles for promoting people's mental health and for disseminating psychological science into American society. At 47\%, membership in religious communities is approximately twice the rate of Americans who attend school (Schmidt, 2018). Apart from American political parties, one would be hard-pressed to find any U.S. social institution that involves such a high percentage of the population. Given how high religious importance and service attendance are in many other regions of the world (especially countries in Africa, South America, and South Asia; Pew Research Center, 2018), this potentiality is likely reflected in numerous cultures worldwide.

We offer several reflections on ways that positive psychology may be helpful in promoting collaboration between psychological and religious communities. These include caring for the emotionally wounded, cultivating virtue and purpose, and promoting human flourishing. 


\section{Caring for the Emotionally Wounded}

To begin, consider how society cares for those who are marginalized and wounded. In times of distress and loss, a vast number of individuals request help from clergy, often instead of seeking treatment from mental health professionals (Stanley et al., 2001). For example, Ellison et al. (2006) reported that over half $(56.7 \%)$ of those who frequently attend religious services view religious leaders as their first or second option when facing a mental health problem. One option in response to this tendency — an option unfortunately chosen with surprising frequency — is for psychologists to aver the inadequacies of clergy in providing care for people with emotional needs (McMinn et al., 2005). Yet this condescending reaction to clergy providing human services minimizes the long historical tradition of pastoral care, the often spiritual nature of recovery and growth, and the various obstacles people experience in seeking care from licensed professionals (religious/spiritual considerations notwithstanding; Worthington \& Scott, 1983).

Moreover, engaging in a turf battle over who best provides mental healthcare in times of need seems to minimize the importance of preventive community care that may naturally occur in the context of religious communities. Positive psychology is particularly pertinent in this regard. Edwards et al. (1999) detail several ways that psychologists and religious leaders can collaborate, such as workshops to promote specific and general well-being, congregational assessment, offering care to high-risk populations, and public education efforts. More detailed explorations of systematic collaborations, such as those by Benes et al. (2000) and Milstein et al. (2010), demonstrate how psychologists can cooperate with and support the work of clergy to enhance the access and quality of human services (rather than competing or quibbling about who should be providing which services in the first place). But beyond the issue of human services, a collaborative understanding of psychology and religion-especially one that is cultivated 
through collaboration between psychological and religious communities - opens the possibilities of authentic, constructive dialogue and genuine, mutual respect in our shared pursuit of big questions concerning matters of human existence. These include the meanings and mechanisms of flourishing, purpose, character/virtue, and lives well-lived-matters that are both promoted in positive psychology interventions and grounded deeply in religious/spiritual teachings and traditions (Rye et al., 2013). Indeed, such big questions transcend the scope and expertise of any single field or community; the very nature of the task calls for (and even requires) interdisciplinary, intercommunity collaboration (Milstein et al., 2010, 2017).

\section{Cultivating Virtue and Purpose}

Seligman's (2011) PERMA model of mental health alludes to a meaning-making process that is central to most religious and spiritual ways of understanding self, others, and the world (Slattery \& Park, 2011; see Park \& Van Tongeren, Chapter 6, this volume). Over the two decades since the "birth" of positive psychology, hundreds of positive psychology articles and books have been published. Many (perhaps most) of them, focus on topics of interest to both psychological and religious communities-well-being (Seligman, 2011), spiritual experiences (Lambert et al., 2013), lovingkindness (Frederickson et al., 2008), wisdom (McLaughlin et al., 2017), and forgiveness (Griffin et al., 2019), just to name a few. Moreover, rigorous longitudinal empirical studies have tied religious service participation to numerous aspects of human flourishing, including mental and physical health, happiness and life satisfaction, character and virtue, and meaning and purpose (VanderWeele, 2017; see Long \& VanderWeele, Chapter 25, this volume).

Although these topics can provide fertile contexts for dialogue and mutual learning, perhaps the most compelling opportunity for collaboration that positive psychology offers is in the area of character and virtue (Ratchford et al., Chapter 4, this volume). Religious communities 
have been seeking to understand and cultivate virtues for millennia. Take wisdom, for example. All major world religions claim wisdom is an essential characteristic of a life well-lived, even though they might differ in the particularities of what wisdom entails (Curnow, 2010). In Judaism, Islam, and Christianity, wisdom is viewed as a gift from God and a disposition that is fostered through study and faithful living; however, in other religious traditions such as Hinduism and Buddhism, wisdom is tied to spiritual practices that cultivate detachment from the physical world and a self-awareness that leads to harmony with nature (McLaughlin et al., 2017).

Virtue incorporates a focus on not only what is (which traditionally is the orientation and focal point of science) but also on what should be and on who we are to become (McMinn, 2017). Accordingly, any rigorous study of virtue must grapple with matters of teleology — that is, of human fullness, purpose, and calling. To this end, positive psychology needs the help of religious communities. At the same time, religious communities (which represent a key context in which virtue has been cultivated for millennia) can also benefit from the insights and trends uncovered by science, because scientific knowledge can help concretize the tenets of religious thought and practice (McMinn, 2017). For it is one thing to know about a religious principle (e.g., forgiveness) or even that a certain religious principle (e.g., nonattachment, lovingkindness) should be applied to a particular situation. However, it is entirely another matter to show either how the application of this principle can be embodied in real life or what underlying mechanisms and corresponding practices might help cultivate such religious dispositions over time.

To illustrate, in a landmark study, Emmons and McCullough (2003) found evidence of an upward spiral whereby gratitude cultivates prosocial behavior and social connectedness, and in turn these social networks build a sense of love and belonging. This study also found evidence that gratitude promotes spiritual awareness, broadens emotional and cognitive flexibility, and 
helps individuals resist stress (see Van Cappellen et al., Chapter 20, this volume). This type of upward spiral is intriguing in part because it brings spiritual experience and psychological experience together. By doing so, the study of virtue transcends self-interest and immediate personal benefit, placing virtues in the context of something deeper and richer than what science might naturally consider on its own (McMinn, 2017). Rather than being competitors, religious and scientific communities can form partnerships that synergistically promote holistic personal well-being and social good. For example, Maton et al. (2013) have highlighted the influence and functions that religious communities play in broader society and made a case for greater collaboration, including leveraging religious gatherings as a potential site for interventions.

\section{Promoting Human Flourishing}

Most collaboration described in psychology journals involves intentional efforts to bring psychology into religious communities. Yet it is worth repeating that religious communities have promoted human flourishing on their own for millennia. In recognizing this fact, it may be useful for psychologists to stand back and reflect humbly on the breadth and depth of positive psychology that has been occurring implicitly in religious contexts long before the birth of mainstream and positive psychology. Indeed, effective collaboration sometimes involves humble appreciation between collaboration partners before moving into productive work together. We illustrate this by considering examples from Christian and Buddhist spirituality.

Speaking from an ecumenical Christian perspective (i.e., incorporating various Christian religious traditions such as Roman Catholic, Eastern Orthodox, mainline Protestant, and Evangelical), Porter et al. (2019) identified three dimensions of growth and development that are modally cultivated by Christian spirituality: spiritual formation (growth in an individual's relatedness with God or another sacred being), character formation (development of habituated, 
virtuous dispositions such as kindness, generosity, compassion, and love), and moral formation (outward behavioral manifestations of virtue; e.g., forgiveness, service, love of one's enemies, etc.). Each of these dimensions of formation is understood as an interrelated facet of a larger process that is referred to as Christian formation proper (Collicutt, 2014) - the process whereby Christian adherents are continually formed into the likeness of Jesus (in character, deeds, etc.). Christian formation, regardless of whether it involves religious leaders or congregants, is understood to take place within the context of community, because relationships are essential for spiritual maturity (Porter et al., 2019). This is true whether it involves relationships within congregations, parishes, seminary communities, or more informal networks of intimate friends. Speaking of the latter, a spiritual classic written in the $12^{\text {th }}$ century by English monk Aelred of Rievaulx presents a discussion on the topic of spiritual friendships (Aelred of Rievaulx, 2010). In more recent history, theologian Dietrich Bonhoeffer (1954) added nuance to this conversation on formation and community by highlighting the paradoxical nature of formation, considering how it requires the Christian to maintain a harmony between spirituality cultivated in community and spirituality cultivated in isolation. He explained: "One who wants fellowship without solitude plunges into the void of words and feelings, and the one who seeks solitude without fellowship perishes in the abyss of vanity, self-infatuation and despair" (Bonhoeffer, 1954, p. 78).

Buddhist spirituality, although also emphasizing teachings such as the impermanence of all conditions and the illusory nature of life and the senses, is nonetheless similarly oriented towards shaping and transforming how people feel and live in the world. The Five Precepts, for example, are somewhat analogous to the second half of the Ten Commandments in Judaism and Christianity, outlining behaviors to avoid such as killing, stealing, and lying; the principle of not killing (which is sometimes translated as "not harming") has informed historical and 
contemporary expressions of nonviolent protest (High Commissioner's Dialogue Distribution, 2012). Moreover, virtues and emotions such as gratitude, serene joy, and pious confidence are among many emphasized in sacred texts such as the Theravāda vamsas (Berkwitz, 2003). In fact, although mindfulness has become somewhat of a buzzword in Western mental health, its origins as a religious contemplative practice were closely tied to the cultivation of lucid awareness (of self, objects, and the Buddha himself; see Segall \& Kristeller, Chapter 14, this volume) and were understood as a means of enacting lovingkindness to all beings (Oman, 2015). The cultivation of lovingkindness, among other virtues, involved a relational process that incorporated fellow Buddhists - relying on others for help and observing others model the provision of lovingkindness (Phillips et al., 2012). The visitation of sacred Buddhist heritage sites, for example, represents a salient communal means by which positive outcomes are cultivated. One qualitative study reported how heritage site visitors experienced the emotions of happiness, eudaimonic well-being, and awe, in response to interaction with both the sacred natural and built environment, other Buddhist visitors, and the local religious staff (Huang et al., 2019).

\section{Empirical Research on Collaboration}

Although an exhaustive review of research on collaboration between psychological and religious communities is beyond the scope of this chapter, we highlight illustrative empirical research that has considered clergy as first responders, the positive impact of engagement in religious communities, and positive psychology interventions in religious communities.

\section{Clergy as First Responders}

Much of the early research on the intersection of psychological and religious communities focused on the role of clergy as first responders in mental health crisis situations, especially as it pertains to highly religious individuals who may be more comfortable working 
with members of their own faith tradition or may be concerned their faith will not be adequately integrated or respected in the process of traditional counseling (VanderWaal et al., 2012). In support of this work, Milstein et al. (2010) developed a prevention-based model of Clergy Outreach and Professional Engagement (COPE) to delineate boundaries between clinical care provided by mental health professionals and religious care provided by clergy and to describe pathways of collaboration across these boundaries. This model has demonstrated effectiveness over time and across religious traditions (Ali et al., 2005; Milstein et al., 2017). It offers a promising guide for collaboration between positive psychology and religious communities.

However, the varied and complex psychological needs of parishioners can present challenges to clergy members, who may be overburdened by other clerical responsibilities and who may also lack specialized training to work with the many mental health concerns of their congregants (Uhder et al., 2017). With respect to access and care, clergy occupy an important and unique position within their communities in that they are known and trusted, usually offer free services, and may frame problems in spiritual terms that are more congruent with their congregants' worldview (Bohnert et al., 2010). Nonetheless, there is evidence suggesting that ministers are in fact interested both in consulting with mental health professionals about issues of congregational care (Lish et al., 2003) and in referring community members to mental health professionals if a mental health or substance abuse disorder is present (VanderWaal et al., 2012). Indeed, collaboration between clergy and the mental health community can help unlock greater access to mental health resources, particularly in under-resourced areas of the world. For instance, in the poor yet highly religious country of Haiti, Wang et al. (2016) found that children treated by clergy who were trained in spiritually oriented trauma-focused cognitive behavioral therapy evidenced comparable outcomes to children treated by a professional counselor. 


\section{Positive Impact of Engagement in Religious Communities}

In addition to their clergy or other religious leaders, religious people also often turn to their broader religious communities for support and care. For example, Tan's (2016) work on lay counseling among Christians presents a comprehensive synthesis of the research and best practices in this area, and this resource can be helpful for both Christian clergy and clinicians alike (Wang, 2017). Religious communities are known for providing rich instrumental social support (whereby individuals obtain practical assistance dealing with problems they are facing) and emotion-focused social support (whereby relational needs for connection and intimacy are met; Aldwin, 2007). Although conventional forms of social support like these are typically available within religious communities, some studies suggest that a distinct dimension of spiritual support may be present as well. Even after controlling for conventional social support, God support, and spiritual leader support, one study reported that Jewish religious community support remained significantly related to greater satisfaction with life and perceived physical health (Lazar \& Bjorck, 2008). Similarly, among Christians in India, support received from fellow adherents was predictive of improved emotional functioning, even after controlling for general social support (Torrecillas et al., 2020).

The seeking and provision of support and care within religious communities is a phenomenon observed across all major religions, including Buddhism (Phillips et al., 2012), Christianity (Salloum \& Lewis, 2010), Hinduism (Kent et al., 2020), Islam (Torrecillas et al., 2020) and Judaism (Lazar \& Bjorck, 2008). Indeed, a growing empirical base highlights the positive impact such support can provide. One study, for example, found that among a sample of predominantly Hindu South Asians living in the United States, the giving and receiving of congregational emotional support was associated with improved health outcomes (Kent et al., 
2020). In another study of adolescent Tibetan Buddhists, social support mediated (explained) the relationship between religious experience and subjective well-being (Ju et al., 2018).

Empirical studies seem to suggest that it is religious service attendance-rather than more intrinsically oriented or privately practiced religiousness/spirituality (e.g., prayer frequency, spiritual or religious identity, or religious coping) — that is most strongly associated with health (VanderWeele et al., 2017; see Long \& VanderWeele, Chapter 25, this volume). In fact, some longitudinal evidence indicates that religious service attendance may be a stronger predictor of health and longevity than any other social support variables, including marital status, number of close relatives, number of close friends, and hours spent in social groups (Li et al., 2016). Similarly, both cross-sectional and longitudinal data have indicated associations between religious attendance and lower frequencies of hypertension, functional disability, and overall mortality (Koenig et al., 2001). VanderWeele (2017) outlines several potential mechanisms of change that underscore the communal element of religion as central to religion's positive effects on health and well-being - mechanisms that include meaning in life, optimism, and forgiveness.

Beyond indicators of physical health, engagement in religious communities has also been associated with a variety of positive outcomes that are relevant to psychological well-being. Aside from social support, researchers suggest that another key pathway to emotional health that is inherent in religious communities is those communities' enhancement of members' general emotional coping skills (Idler et al., 2003). Accordingly, scholars have sought to advance understanding and psychometrically validate measures of religious coping that are adapted and contextualized for specific religious traditions, including Buddhism (Phillips et al., 2012), Hinduism (Tarakeshwar et al., 2003), and Islam (Abu Raiya et al., 2011). In addition, empirical 
evidence suggests that the impact of this enhanced capacity to cope is observable across religious contexts (VanderWeele, 2017; VanderWeele et al., 2017).

\section{Positive Psychology Interventions in Religious Communities}

Early positive psychology research within the context of religious communities was largely restricted to cross-sectional, nonexperimental studies that examined broad trends between religious/spiritual constructs and various positive outcomes related to physical and mental wellbeing. Although these studies helped establish this line of research, over the past decade, new studies have emerged — often using quasi-experimental designs — that seek to evaluate the effectiveness of religiously integrated positive psychology interventions deployed within religious communities. Several of these studies are presented in this section, because they represent a welcome evolution by elucidating potential mechanisms of change inherent in religious communities. They also uncover potential means by which religious communities can more effectively cultivate positive outcomes within their members (McMinn, 2017).

Many of the positive psychology interventions evaluated have been designed to cultivate character and virtue dispositions among adherents of specific religious traditions. Thus far, the results have been promising. For example, McLaughlin et al. (2017) developed a wisdom intervention designed to enhance participants' abilities in cognitive, affective, and moral domains. This intervention drew widely from the Christian tradition and incorporated elements that were educational, experiential, an process-oriented, such that proper attention was paid to cultivate both "practical and numinous aspects of wisdom" (p. 4). Two groups of church leaders from Quaker congregations completed the intervention, and significant positive effects were observed. Quantitative analysis indicated significant increases in practical wisdom, postformal thinking (i.e., the ability to consider nuance and complexity), and subjective well-being. 
Qualitative analysis highlighted themes relating to the relational nature of learning wisdom and to a self-reflective pursuit of greater harmony between what one knows and how one lives (McLaughlin et al., 2017). Similarly, Bufford et al. (2018) reported findings from two grace intervention studies conducted in Christian congregations; they found significant increases in grace and trait self-forgiveness. Likewise, Griffin et al. (2019) conducted a 2-week, communitybased forgiveness intervention and observed significant increases in forgiveness over time.

Other positive psychology intervention studies conducted in religious communities have evidenced mixed results, such as observing change that was positive but was statistically indistinguishable from that evidenced by comparison groups. To illustrate, Al-Seheel and Noor (2016) deployed an Islamic-based gratitude intervention and evaluated its effectiveness in cultivating happiness among Muslim students. Drawing from Islamic teaching on gratitude, their gratitude intervention incorporated expressions of gratitude and counting one's blessings, linking such gratitude explicitly to the providence of Allah. Results indicated that participants who practiced this religiously integrated, Islamic, gratitude intervention indeed evidenced significant increases in happiness, but these increases were empirically indistinguishable from the increases evidenced by those who practiced a similar nonreligious gratitude intervention. Likewise, Uhder et al. (2017) found a significant positive effect (on psychological well-being, spiritual wellbeing, life satisfaction, and favorable views of psychology and of interdisciplinary collaboration) from their gratitude intervention in a Christian church; however, again, this positive effect was statistically indistinguishable from what the waitlist comparison group evidenced.

\section{Implications for Ongoing Research}

McMinn et al. (2003) have helpfully distinguished between Basic Collaboration (which is a minimal competence for all psychologists) and Advanced Collaboration, which incorporates 
the pursuit of common values and goals, mutual trust, complementary expertise, and awareness of spirituality. The aforementioned work developing and evaluating positive psychology interventions deployed in religious communities represents a significant step toward Advanced Collaboration. However, the majority of intervention research thus far has been conducted in the context of a single-site local congregation (i.e., a field trial). Thus, more sophisticated, multisite effectiveness research is needed in which congregations are assigned to conditions, such that contamination within a congregation and issues related to generalization can be assessed.

Nevertheless, preliminary results from existing work have been promising, and even the studies reporting mixed results (e.g., observing positive effects in intervention groups that are indistinguishable from effects in comparison groups) introduce important gaps in scientific knowledge that future researchers should investigate. For example, what are the underlying ingredients of change (that facilitate positive psychology outcomes) that might be shared and/or unique to religious communities, compared to other social networks? Relatedly, what is the unique or additive benefit of explicitly incorporating sacred elements into positive psychology interventions (e.g., a general gratitude intervention vs. a gratitude to God intervention)? Concerning the development of future positive psychology interventions, special attention should be placed on not only the design of the intervention but also on the process by which these interventions are developed in collaboration with religious communities. Once sufficient data is available in this regard, researchers can begin to synthesize this information and articulate best practices on how interested parties (e.g., those interested in working with institutions of religious education with youth or adults) can develop interventions or curricula of their own in a datainformed manner. Furthermore, some initial evidence suggests that outcomes related to spiritual development and/or character and virtue development may in fact take many years to cultivate, 
rendering observable change difficult for short-term interventions that have timepoints separated by 6 months or less (Hall et al., 2015). Future research on such interventions should therefore explore developmental changes across longer periods of time.

\section{Conclusion}

Positive psychology offers compelling opportunities to cultivate and demonstrate the benefits of greater collaboration between scientific and religious communities. In this chapter, we have highlighted shared values and work between these communities while surveying the promising conceptual and empirical literature that attest to the potential benefits this partnership might bear for communities that may not otherwise have access to such resources and support. Indeed, in the wake of unprecedented human suffering (natural disasters, global pandemics, economic injustice, social, political, and racial unrest, and so on) our global communities are in urgent need of renewal of all forms_-biological, social, political, moral, and spiritual (Wang, 2021). The sheer scale and scope of the challenges that lie before us far surpass the capacity of any single community or field of study. We cannot think of two better communities to come together to tackle these problems in collaboration with each other than positive psychology and religious communities. Doing so would bringing together the fruits of robust scientific methodology and research alongside centuries of tradition, insight, and embodied exemplars of what constitutes a life well-lived (McMinn, 2017; VanderWeele, 2017). 


\section{References}

Abu Raiya, H., Pargament, K.I., \& Mahoney, A. (2011). Examining coping methods with stressful interpersonal events experienced by Muslims living in the United States following the 9/11 attacks. Psychology of Religion and Spirituality, 3, 1-14. https://doi.org/10.1037/a0020034

Aelred of Rievaulx (2010). Spiritual friendship. Cistercian Publications.

Aldwin, C. M. (2007). Stress, coping, and development ( $2^{\text {nd }}$ ed.). Guilford Press.

Ali, O., Milstien, G., \& Marzuk, P. (2005). The Imam's role in meeting the counseling needs of Muslim communities in the United States. Psychiatric Services, 56, 202-205. https://doi.org/10.1176/appi.ps.56.2.202

Al-Seheel, A. Y., \& Noor, N. M. (2016). Effects of an Islamic-based gratitude strategy on Muslim students' level of happiness. Mental Health, Religion \& Culture, 19, 686-703. https://doi.org/10.1080/13674676.2016.1229287

Benes, K. M., Walsh, J. M., McMinn, M. R., Dominguez, A. W., \& Aikins, D. C. (2000). Psychology and the church: An exemplar of psychology-clergy collaboration. Professional Psychology: Research and Practice, 31, 515-520. https://doi.org/10.1037/0735-7028.31.5.515

Berkwitz, S. C. (2003). History and gratitude in Theravāda Buddhism. Journal of the American Academy of Religion, 71, 579-604. http://www.jstor.org/stable/1466494

Bohnert, A., Perron, B., Jarman, C., Vaughn, M., Chatters, L., Taylor, R. J. (2010). Use of clergy services among individuals seeking treatment for alcohol use problems. The American Journal on Addictions, 19, 345-351. https://doi.org/10.1111/j.1521-0391.2010.00050.x

Bonhoeffer, D. (1954). Life together. Harper \& Row. 
Bufford, R. K., McMinn, M. R., Moody, J. A., \& Geczy-Haskins, L. (2018). The effects of grace interventions in church communities. The Journal of Positive Psychology, 13, 512-521. https://doi.org/10.1080/17439760.2017.1350740

Collicutt, J. (2014). The psychology of Christian character formation. SCM Press.

Curnow, T. (2010). Wisdom in the ancient world. Duckworth.

Edwards, L. C., Lim, B. R. K. B., McMinn, M. R., \& Dominguez, A. W. (1999). Examples of collaboration between psychologists and clergy. Professional Psychology, Research and Practice, 30(6), 547-551. https://doi.org/10.1037/0735-7028.30.6.547

Ellison, C. G., Vaaler, M. L., Flannelly, K. J., \& Weaver, A. J. (2006). The clergy as a source of mental health assistance: What Americans believe. Review of Religious Research, 48(2), $190-211$.

Emmons, R. A., \& McCullough, M. E. (2003). Counting blessings versus burdens: Experimental studies of gratitude and subjective well-being. Journal of Personality and Social Psychology, 84, 377-389. https://doi.org/10.1037//0022-3514.84.2.377

Frederickson, B. L., Cohn, M. A., Coffey, K. A., Pek, J., \& Finkel, S. M. (2008). Open hearts build lives: Positive emotions, induced through loving-kindness meditation, build consequential personal resources. Journal of Personality and Social Psychology, 95, 1045-1062. https://doi.org/10.1037/a0013262

Griffin, B. J., Toussaint, L. L., Zoelzer, M., Worthington, E. L., Jr., Coleman, J., Lavelock, C. R., McElroy, A., Hook, J. N., Wade, N., Sandage, S., \& Rye, M. (2019). Evaluating the effectiveness of a community-based forgiveness campaign. The Journal of Positive Psychology, 14(3), 354-361. https://doi.org/10.1080/17439760.2018.1437464 
Hall, T. W., Edwards, E., \& Wang, D. C. (2015). The spiritual development of emerging adults over the college years: A four-year longitudinal investigation. Psychology of Religion and Spirituality, 7, 1-12. https://doi.org/10.1037/rel0000051

High Commissioner's Dialogue Distribution. (2012). The Buddhist core values and perspectives for protection challenges: Faith and protection. https://www.unhcr.org/50be10cb9.pdf.

Hodge, D. R. (2004). Working with Hindu clients in a spiritually sensitive manner. Social Work, 49(1), 27-38. https://doi.org/10.1093/sw/49.1.27

Huang, K., Pearce, P. L., Wu, M.-Y., \& Wang, X.-Z. (2019). Tourists and Buddhist heritage sites: An integrative analysis of visitors' experience and happiness through positive psychology constructs. Tourist Studies, 19, 549-568. https://doi.org/10.1177/1468797619850107

Idler, E. L., Musick, M. A., Ellison, C. G., George, L. K., Krause, N., Ory, M. G., Pargament, K. I., Powell, L. H., Understood, L. G., \& Williams, D. R. (2003). Measuring multiple dimensions of religion and spirituality for health research. Research on Aging, 25, 327365. https://doi.org/10.1177/0164027503025004001

Jones, J. M. (March 29, 2021). U.S. church membership falls below majority for first time. Gallup News. https://news.gallup.com/poll/341963/church-membership-falls-belowmajority-first-time.aspx

Ju, C., Zhang, B., You, X., Alterman, V., \& Li, Y. (2018). Religiousness, social support and subjective well-being: An exploratory study among adolescents in an Asian atheist country. International Journal of Psychology, 53, 97-106. https://doi.org/10.1002/ijop.12270 
Kent, B. V., Stroope, S., Kanaya, A. M., Zhang, Y., Kandula, N. R., \& Shields, A. E. (2020). Private religion/spirituality, self-rated health, and mental health among US South Asians. Quality of Life Research, 29, 495-504. https://doi.org/10.1080/13557858.2019.1661358

Koenig, H. G., McCullough, M., \& Larson, D. B. (2001). Religion and health: A century of research reviewed. Oxford University Press.

Lambert, N. M., Stillman, T., \& Fincham, F. (2013). Autobiographical narratives of spiritual experiences: Solitude, tragedy, and the absence of materialism. The Journal of Positive Psychology, 8, 273-279. http://doi.org/10.1080/17439760.2013.789119

Lazar, A., \& Bjorck, J. P. (2008). Religious support and psychosocial well-being among a religious Jewish population. Mental Health, Religion and Culture, 11, 403-421. https://doi.org/10.1080/13674670701486142

Li, S., Stamfer, M., Williams, D. R., \& VanderWeele, T. J. (2016). Association between religious attendance and mortality among women. JAMA Internal Medicine, 176, 777785. https://doi.org/10.1001/jamainternmed.2016.1615

Lish, R., McMinn, M. R., Fitzsimmons, C., \& Root, A. M. (2003). Clergy interest in innovative collaboration with psychologists. Journal of Psychology and Christianity, 22, 294-298.

Maton, K. I., Sto. Domingo, M. R., \& Westin, A. M. L. (2013). Addressing religion and psychology in communities: The congregation as intervention site, community resource, and community influence. In K. I. Pargament, A. Mahoney, \& E. P. Shafranske (Eds.), APA handbook of psychology, religion, and spirituality (Vol. 2): An applied psychology of religion and spirituality (pp. 613-632). American Psychological Association. https://doi.org/10.1037/14046-032 
McMinn, M. R. (2017). The science of virtue: Why positive psychology matters to the church. Brazos Press.

McMinn, M. R., Aikins, D. C., \& Lish, R. A. (2003). Basic and advanced competence in collaborating with clergy. Professional Psychology: Research and Practice, 34, 197-202. https://doi.org/10.1037/0735-7028.34.2.197

McMinn, M. R., Runner, S. J., Fairchild, J. A., Lefler, J., \& Suntay, R. (2005). Factors affecting clergy-psychologist referral patterns. Journal of Psychology and Theology, 33, 299-309. https://doi.org/10.1177/009164710503300406

McLaughlin, P. T., McMinn, M. R., Morse, M., Neff, M. A., Johnson, B., Summerer, D., \& Koskela, N. (2017). The effects of a wisdom intervention in a Christian congregation. The Journal of Positive Psychology, 13,502-511. https://doi.org/10.1080/17439760.2017.1350739

Milstein, G., Manierre, A., \& Yali, A. M. (2010). Psychological care for persons of diverse religions: A collaborative continuum. Professional Psychology: Research and Practice, 41, 371-381. https://doi.org/10.1037/a0021074

Milstein, G. Middel, D., \& Espinosa, A. (2017). Consumers, clergy, and clinicians in collaboration: Ongoing implementation and evaluation of a mental wellness program. American Journal of Psychiatric Rehabilitation, 20, 34-61. https://doi.org/10.1080/15487768.2016.1267052

Oman, D. (2015). Cultivating compassion through holistic mindfulness: Evidence for effective intervention. In T. Plante. (Ed.), Psychology of compassion and cruelty: Understanding the emotional, spiritual, and religious influences (pp. 35-58). ABC-CLIO. 
Pew Research Center (2018). The age gap in religion around the world. Author.

https://www.pewforum.org/2018/06/13/how-religious-commitment-varies-by-countryamong-people-of-all-ages/

Phillips, R. E., III, Cheng, C. M., Oemig, C., Hietbrink, L., \& Vonnegut, E. (2012). Validation of a Buddhist coping measure among non-Asian Buddhists in the United States. Journal for the Scientific Study of Religion, 51, 156-172. https://www.jstor.org/stable/41349934

Porter, S. L., Sandage, S. J., Wang, D. C., \& Hill, P. C. (2019). Measuring the spiritual, character, and moral formation of seminarians: In search of a meta-theory of spiritual change. Journal of Spiritual Formation and Soul Care, 12, 5-24. https://doi.org/10.1177/1939790918797481

Rye, M. S., Wade, N. G., Fleri, A. M., \& Kidwell, J. E. M. (2013). The role of religion and spirituality in positive psychology interventions. In K. I. Pargament, A. Mahoney, \& E. P. Shafranske (Eds.), APA handbook of psychology, religion, and spirituality (Vol. 2): An applied psychology of religion and spirituality (pp. 481-508). American Psychological Association. https://doi.org/10.1037/14046-025

Salloum, A., \& Lewis, M. L. (2010). An exploratory study of African American parent-child coping strategies post-Hurricane Katrina. Traumatology, 16, 31-41. https://doi.org/10.1177/1534765609348240

Schmidt, E. (2018). For the first time, 90 percent completed high school or more. U.S. Census Bureau. https://www.census.gov/library/stories/2018/07/educational-attainment.html

Seligman, M. E. P. (2011). Flourish. Random House.

Shafranske, E. P., \& Cummings, J. P. (2013). Religious and spiritual beliefs, affiliations, and practices of psychologists. In K. I. Pargament, A. Mahoney, \& E. P. Shafranske (Eds.), 
APA handbook of psychology, religion, and spirituality (Vol. 2, pp. 23-41). American Psychological Association. https://doi.org/10.1037/14046-002

Slattery, J. M., \& Park, C. L. (2011). Meaning making and spiritually oriented interventions. In Aten, J. D., McMinn, M. R., \& Worthington, E. L., Jr. (Eds.), Spiritually oriented interventions for counseling and psychotherapy. American Psychological Association. https://doi.org/10.1037/12313-001

Stanley, S. M., Markman, H. J., Prado, L. M., Olmos-Gallo, P. A., Tonelli, L., St. Peters, M., et al. (2001). Community-based premarital prevention: Clergy and lay leaders on the front lines. Family Relations, 50, 67-76. https://doi.org/10.1111/j.1741-3729.2001.00067.x

Tan, S-Y. (2016). Lay counseling: Equipping Christians for a helping ministry. Zondervan.

Tarakeshwar, N., Pargament, K. I., \& Mahoney, A. (2003). Initial development of a measure of religious coping among Hindus. Journal of Community Psychology, 31, 607-628. https://doi.org/10.1002/jcop.10071

Torrecillas, J., Bjorck, J. P., Kamble, S. V., \& Gorsuch, R. L. (2020). Religious support and emotional functioning in India across three major religions. International Journal for the Psychology of Religion, 30, 18-34. https://doi.org/10.1080/10508619.2019.1614868

Uhder, J., McMinn, M. R., Bufford, R. K., \& Gathercoal, K. (2017). A gratitude intervention in a Christian church community. Journal of Psychology and Theology, 45, 46-57. https://doi.org/10.1177/009164711704500104

VanderWaal, C. J., Hernandez, E. I., \& Sandman, A. R. (2012). The Gatekeepers: Clergy involvement in referrals and collaboration with mental health and substance abuse professionals. Journal of Social Work and Christianity, 39, 27-51. 
VanderWeele, T. J. (2017). Religious communities and human flourishing. Current Directions in Psychological Science, 26, 476-481. https://doi.org/10.1177/0963721417721526

VanderWeele, T. J., Yu, J., Cozier, Y. C., Wise, L., Rosenberg, L., Shields, A. E., \& Palmer, J. R. (2017). Religious service attendance, prayer, religious coping, and religious spiritual identity as predictors of all-cause mortality in the Black Women's Health Study. American Journal of Epidemiology, 185, 515-522. https://doi.org/10.1093/aje/kww179

Vieten, C., \& Lukoff, D. (2021). Spiritual and religious competencies in psychology. American Psychologist. Advance online publication. https://doi.org/10.1037/amp0000821

Wang, D. C. (2017). Mobilizing and equipping the Church for soul care and counseling: A classic resource updated and revised. Journal of Psychology and Theology, 45, 144-146. https://doi.org/10.1177/009164711704500206

Wang, D. C. (2021). Where do we go from here? A call to the Christian mental health community to leverage the resources of science and faith for social action and community renewal. Journal of Psychology and Christianity, 40, 78-83.

Wang, D. C., Aten, J. D., Boan, D., Jean-Charles, W., Griff, K. P., Valcin, V., Davis, E. B., Hook, J. N., Davis, D. E., Van Tongeren, D. R., Abouezzeddine, T., Sklar, Q., \& Wang, A. (2016). Culturally adapted spiritually oriented trauma focused cognitive behavioral therapy for child survivors of Restavek. Spirituality in Clinical Practice, 3, 224-236. https://doi.org/10.1037/scp0000101

Worthington, E. L., \& Scott, G. G. (1983). Goal selection for counseling with potentially religious clients by professional and student counselors in explicitly Christian or secular settings. Journal of Psychology and Theology, 11, 318-329. https://doi.org/10.1177/009164718301100405 


\title{
Chapter 30:
}

\section{Building Spiritual Fortitude and Resilience Following Disaster:}

\section{Synthesizing the Contributions of Positive Psychology and Religion/Spirituality}

\author{
Laura E. Captari ${ }^{1}$, Laura Shannonhouse ${ }^{2}$, Jamie D. Aten ${ }^{3}$, and Jordan D. Snyder ${ }^{4}$ \\ ${ }^{1}$ The Albert and Jessie Danielsen Institute, Boston University, Boston, MA \\ ${ }^{2}$ Department of Counseling and Psychological Services, Georgia State University, Atlanta, GA \\ ${ }^{3}$ Humanitarian Disaster Institute, Wheaton College, Wheaton, IL \\ ${ }^{4}$ Department of Psychology, University of Wisconsin-Parkside, Kenosha, WI
}

\section{Author Note}

This project was supported by a grant from the John Templeton Foundation on "Christian Meaning-Making, Suffering and the Flourishing Life" (\#61467).

We have no conflicts of interest to disclose.

Correspondence concerning this chapter should be addressed to Laura Captari, The Albert and Jessie Danielsen Institute, 185 Bay State Rd., Boston, MA 02215. E-mail: lcaptari@bu.edu 


\begin{abstract}
This chapter examines the intersections of positive psychology and religion/spirituality in the context of disasters. We review the salience of religious/spiritual $(\mathrm{R} / \mathrm{S})$ processes to coping with mass trauma and summarize several strength-based disaster recovery theories that contribute to a holistic understanding of survivors' and communities' adaptation processes. Building on this review, we propose and describe the systemic model of Disaster Spiritual Fortitude and Resilience (DSFR). This is the first spiritually integrated disaster recovery model that (a) captures both strengths and vulnerabilities across holistic domains of human experience while also (b) considering across-time interactional effects between the individual and their multilevel socioecological context (at interpersonal, community, and larger societal levels). Implications for practitioners and community leaders working in disaster and humanitarian contexts are provided, along with recommendations for guiding science, practice, and policymaking.
\end{abstract}

Keywords: disasters; religion; spirituality; resilience; positive psychology 


\section{Chapter 30: Building Spiritual Fortitude and Resilience Following Disaster:}

\section{Synthesizing the Contributions of Positive Psychology and Religion/Spirituality}

"There are no greater treasures than the highest human qualities such as compassion, courage

and hope. Not even tragic accident or disaster can destroy them." — Daisaku Ikeda

Both environmental (e.g., severe weather) and human-caused (e.g., mass violence) disasters often result in cascading devastation. Losses can include the death of loved ones, no longer feeling safe, displacement from home, compromised infrastructure (e.g., emergency and medical services), disrupted rhythms of life (e.g., work and school), or destruction of or lack of access to sacred spaces (e.g., places of worship, nature). For example, as of December 2021, the COVID-19 pandemic death toll has reached 5 million people globally (World Health Organization, 2021). Over the last 2 years, physical distancing necessary to protect public health has limited gatherings and fueled isolation (Liu et al., 2020), and cumulative stressors (e.g., economic instability, inequitable access to healthcare) have disproportionately affected racial/ethnic minorities (Tai et al., 2021). In some places, overlapping disasters (e.g., wildfires displacing people during the pandemic) have posed particularly complex challenges.

What relevance do positive psychology and religion/spirituality have amidst such situations? In this chapter, we (a) review the salience of religious/spiritual (R/S) processes to coping with mass trauma and (b) synthesize key strength-based theories of disaster recovery. We then introduce our systemic model of Disaster Spiritual Fortitude and Resilience (DSFR), which holistically addresses survivors' needs while attending to strengths and capacities.

\section{The Salience of R/S Resources and Contexts Amidst Disaster}

Although many disaster recovery models focus on risks and vulnerabilities, survivors and the communities they live in also possess capacities that can help sustain and strengthen them, 
thus promoting fortitude and resilience. The terms fortitude and resilience capture related yet unique aspects of human experience. Fortitude refers to the capacity to endure persistent suffering with courage. Resilience speaks to adaptation and recovery. Disasters challenge our beliefs about the world, and survivors who identify as R/S often turn to their faith to make sense of their disaster-related suffering (Davis et al., 2018; Park, 2016). Studies have shown that it is not how devout disaster survivors are that makes the difference in their postdisaster adaptation, but rather it is how they engage with their faith that counts (e.g., McElroy-Heltzel et al., 2018). From a relational perspective, R/S engagement can be understood dialectically, including movements toward spiritual dwelling or spiritual seeking (Sandage, Rupert et al., 2020). Spiritual dwelling includes practices that foster security, communal affiliation, affect regulation, and spiritual grounding. Spiritual seeking (often in tension with dwelling) involves grappling with uncertainties and showing a willingness to question and reshape personal views and an appreciation for paradox and complexity. Incorporating R/S concerns in psychotherapy and community intervention can improve survivor outcomes, decrease stigma, and improve accessibility to resources. It can also link survivors with valuable social capital and spiritual support networks (Aten et al., 2019; Davis et al., 2018, 2019a; Pargament \& Cummings, 2010). However, the relationship between religion/spirituality and postdisaster adaptation and growth is not just salubrious. Survivors may experience a variety of moral, existential, and spiritual struggles, particularly when there is a gap between previously held beliefs (e.g., God is loving and able to protect me) and personal disaster impacts (Aten et al., 2012, 2019). Although common, R/S struggles can lead to chronic distress and mental disorders, if unaddressed (Pargament \& Exline, 2021). Practice-based research has found R/S struggles are linked to spiritual seeking (Sandage, Jankowski et al., 2020), suggesting R/S struggles can activate a quest 
for transformative spiritual evolution if responded to with validation and support during survivors' spiritual reorganization. However, when struggles are viewed as sinful and R/S communities stigmatize members who are doubting, survivors may feel demoralized and stuck.

\section{Trajectories of R/S Coping Following Disaster}

The diverse ways survivors engage with and understand the sacred can affect fortitude and resilience processes (Captari et al., 2019; McElroy-Hetzel et al., 2018). Appraisals of threat (negatively valenced) and/or challenge (positively valenced) can be impacted by personal R/S dynamics, the cause and type of disaster, and the survivors' personally experienced losses. Positive R/S coping (e.g., seeking God's love or assistance) may involve nurturing a secure attachment with one or more sacred entity, whereas negative R/S coping (e.g., feeling abandoned by God) often stems from attachment anxiety and/or avoidance in relation to the sacred. Although negative R/S coping has been associated with psychological, physical, and spiritual distress, more positive R/S engagement (e.g., finding meaning and hope) can buffer the relationship between disaster-related resource loss and psychological distress. Put differently, R/S domains can be a source of both struggle and strength—often in tandem (Aten et al., 2019).

The literature suggests four main trajectories of R/S coping following disaster. R/S survivors may (a) draw closer to the sacred and a R/S community to cope (e.g., spiritual dwelling), (b) grapple with spiritual dilemmas that lead to spiritual evolution over time (e.g., spiritual seeking), or (c) disaffiliate with religion/spirituality because of unsolvable spiritual dilemmas, pursuing other groups and support networks that can contribute to meaning. Finally, (d) previously non-R/S survivors may reflect on ultimate concerns following disaster and turn toward faith as a result (Counted et al., 2020). Contextual factors can also affect the relationship between survivors' experience and outcomes. Individual differences (e.g., race, ethnicity, gender, 
age), prior trauma, R/S variables (e.g., R/S attributions, coping, and importance) have been found to influence (i.e., moderate) or explain (i.e., mediate) spiritual and psychological outcomes (Feder et al., 2013; Shannonhouse et. al., 2019). Macrolevel (e.g., R/S community) and disasterrelated variables (e.g., resource loss, prior crises) are also critical to consider (Cook et al., 2013).

\section{Holistic and Strength-Based Disaster Recovery Theories: A Brief Review}

Disasters can indeed catalyze a process of spiritual and psychological growth over time, wherein losses, R/S struggles, and related vulnerabilities can be meaningfully addressed. A growing literature is examining the relevance of positive psychology in catalyzing community recovery and development processes in the wake of disaster (Morgado, 2020; Pargament \& Cummings, 2010; Park, 2016; Southwick et al., 2016). Despite this, no model to date has synthesized the intrapersonal, interpersonal, community, and larger societal influences that may contribute to positive R/S coping and adaptation. Below, we review four key theories that serve as building blocks to such a holistic, strength-based understanding of postdisaster adaptation.

\section{Disaster Recovery as a Dynamic, Evolving Process}

According to the Phases of Disaster Model (Zunin \& Meyers, 2000), disaster situations evoke a normative trajectory of reactions across time. Although survivors' responses may vary significantly, this framework offers a helpful heuristic for tracking common responses. In the pre-disaster phase, fear and uncertainty abound, frequently alongside minimization or denial of threat. Even when there is a warning period (e.g., a weather forecast of hurricanes), some individuals will prepare more than others, which can lead to guilt and self-blame among survivors who end up sustaining damage. Sudden and unpredictable disasters (e.g., a mass shooting) are often more disruptive to sense of personal agency and control. During the disaster impact phase, people may feel a range of intense emotions (e.g., terror, rage, helplessness), 
tempered by survival instincts that promote swift action. In the heroic phase, adrenaline-induced rescue behavior (e.g., altruistic behavior) is prevalent. This behavior can lead to dramatic shifts in emotion, described as the honeymoon phase, during which survivors feel incredibly bonded and fear tends to be offset by optimism. However, as the effects of stress, exhaustion, and loss become more apparent in the disillusionment phase, survivors may experience increased psychological distress and become more vulnerable to engaging in maladaptive coping (e.g., substance abuse). Finally comes the reconstruction phase, which often takes years and involves re-engaging with daily life in a forever-altered world and working through grief as a community. In cases of a protracted threat such as the COVID-19 pandemic, communities have experienced multiple impact points (e.g., waves of infection and lockdown), leading to a less linear process involving a confusing vortex of heroism, honeymoon, and disillusionment.

Integrating risks and vulnerabilities alongside human strengths, the Phases of Disaster model attends to the ways in which a communal focus, altruism, and optimism can help promote adaptation. However, this does not preclude survivors from also needing to grapple with and process the realities of death, displacement, and more ambiguous losses (e.g., of daily routines, freedom, dreams, possessions). Furthermore, it is vital to distinguish truly positive psychology from emotional avoidance and minimization, which can masquerade as unrealistic optimism or hope and try to circumvent the slow, hard work of reconstruction. The spiritual bypass literature similarly tracks the human propensity to avoid or circumvent consideration of psychological and physical needs, which can serve as a barrier to help-seeking behaviors (Fox et al., 2020).

\section{Resource Loss and Access as Critical Junctures}

Conservation of Resources (COR) theory explains individual differences in postdisaster adaptation as stemming from resource loss and access (Hobfoll, 1989). Resources are defined 
broadly as including material objects (e.g., house, belongings), personal characteristics (e.g., optimism, humor), relational conditions (e.g., companionship, feeling valuable to others), and energies (e.g., money, sleep). Within COR theory, the individual, family, and the larger community are all viewed as having resources (Hobfoll et al., 1995). Although resources lost at an individual level (e.g., losing one's job during the pandemic) can affect a person and their household, resources lost at a community or larger level (e.g., inability to gather for celebrations, funerals, and religious rituals) may also result in less-successful coping at the individual level. Research has found resource loss to predict increased anxiety, depression, and post-traumatic stress (Aten et al., 2019; Davis et al., 2018). In contrast, resource access can promote resilience, as resources help mitigate stressors and restore a sense of personal agency (Hobfoll, 2011). There is some evidence $\mathrm{R} / \mathrm{S}$ coping might be involved in these associations, such that negative $\mathrm{R} / \mathrm{S}$ coping may explain how resource loss provokes psychological distress, and positive R/S coping may buffer adverse effects of resource loss (Cook et al., 2013; Shannonhouse et. al., 2019).

Disasters can trigger "loss spirals" (Hobfoll, 1989, p. 519) particularly for vulnerable populations (e.g., children, older adults, or minorities), who often have fewer resources and greater levels of prior trauma and therefore are often more affected psychologically by disasters. However, increased access to resources can catalyze "gain spirals" (Hobfoll, 2011, p. 133) that bolster survivors' sense of agency, adaptive capacities, and community connections. In a study of racially diverse older adults, the demographic group hit hardest by the COVID-19 pandemic, those who were already socially isolated, had a low income, and had chronic health conditionsi.e., low levels of crucial resources-were particularly susceptible to further resource loss (Shannonhouse et al., 2021). In particular, losses in relational (i.e., not feeling valuable to others) and personal (i.e., losing one's sense of optimism) domains were tied to increased suicidality. 
COR theory's holistic framing of resources as physical, psychological, and relational—and as situated in individual and systemic contexts — captures much of the complexity of human experience. Assessing survivors' areas of resource loss and access can help tailor interventions strategically to address specific areas of need, rather than taking a one-size-fits-all approach.

\section{The Human Drive to Find Meaning Amidst Tragedy}

Meaning making is a vital process survivors engage in to make sense of disaster (Park, 2016). Park’s (2010) Meaning Making Model describes survivors' global meaning, which consists of beliefs, goals, and subjective feelings developed across the lifespan (e.g., a sense of safety, controllability, and justice). Global meaning also includes motives, hopes, and purpose, which may stem from a R/S tradition, salient identities, cultural groups, or family heritage. According to this model, when a disaster occurs, it can be interpreted in relation to survivors' global meaning system. If a survivor's situational meaning (specific to the disaster experience) is discrepant from their global meaning, they may feel distressed and be motivated to resolve this cognitive dissonance. Yet if their appraisal of the disaster is congruent with their global meaning,

they will not experience distress, so further processing is unneeded. The greater the discrepancy between global and situational meanings, the greater the distress experienced (Park, 2010, 2016).

$\mathrm{R} / \mathrm{S}$ survivors often draw on their faith to reduce discrepancies between global and situational meanings and restore a global view of the world as meaningful, their losses as comprehensible, and life as worthwhile (Hall et al., 2018). Engaging faith as a meaning system can include drawing on R/S beliefs, practices, and virtues (e.g., hope, courage, gratitude). In this context, successful resolution may result not only in a reduction of the discrepancy between global and situational meanings but also in perceived psychological or spiritual growth (Cook et al., 2013; Davis et al., 2019a). To illustrate, in the context of the COVID-19 pandemic, for a 
person whose global meaning includes theodicies of divine protection from suffering, losing loved ones may evoke situational attributions of divine judgement or abandonment, precipitating a search for revised meaning. In contrast, a person whose global meaning includes viewing adversity as an inevitable human experience amidst which God is present may possess greater inherent capacities to metabolize suffering, and this is often bolstered by the reinforcement of a faith community. Although Park’s (2010) Meaning Making Model focuses mainly on cognitive processes (and less on other aspects of human experience), it highlights the human drive to find meaning and how R/S beliefs can serve as risk or protective factors in disaster recovery.

\section{Recovery Processes as Unfolding Within Relationships}

Disaster unfolds in a social context, and survivors' social relationships can significantly influence their postdisaster adjustment process. The Social Ecology of Post-traumatic Stress theory points out that human-caused disaster events produce greater risk for post-traumatic stress, potentially because survivors' relational schemas (e.g., are other people safe or a source of threat?) are more substantially affected (Charuvastra \& Cloitre, 2008). Relatedly, perceived social support before and after a potentially traumatic event uniquely predicts psychological functioning across time and can buffer against the development of post-traumatic stress. This speaks to the ways in which meaning-making and resilience processes unfold within close relationships and community connections, which can provide instrumental support (e.g., practical, tangible help) and emotional support (e.g., facilitating coregulation of trauma-related affect and restoration of a sense of safety). However, close relationships can be the context for either (a) empathic support and facilitative processing of disaster-related shame, guilt, and anger or (b) emotional invalidation, conflict, and disagreements that further exacerbate postdisaster psychological vulnerabilities (Maercker \& Horn, 2013). At a broader societal level, community 
and government psychological responses can similarly be the context for either (a) collective grieving and memorializing of the disaster's broad-based impact and/or (b) segregation and prejudice toward nonmajority populations (who are frequently scapegoated as causing the disaster; e.g., in the U.S., COVID-19 was caricatured as the "China virus," leading to increased hate crimes toward Asians and Asian Americans).

A focus on postdisaster social ecology interweaves positive psychology and R/S perspectives. For many survivors, their faith community is a primary context within which they feel a sense of belonging and companioning through suffering (see Wang et al., Chapter 29, this volume). Virtues such as hope, fortitude, and courage are not only intrapersonal strengths but also are capacities that are nurtured in an interpersonal matrix. For example, a parishioner overwhelmed with despair after the death of loved ones in a community tragedy may draw hope from the steady, responsive presence of their church, synagogue, or mosque. In sum, although the Social Ecology model only deals with the psychosocial aspects of disaster response, it offsets individualistic perspectives of disaster recovery by highlighting interactions between survivors' postdisaster adaptation and their relational, community, and societal milieu (ecological system).

\section{A Systemic Model of Disaster Spiritual Fortitude and Resilience (DSFR)}

Each of these leading disaster theories offers a unique vantage point, but moving disaster science forward necessitates the development of a model that overtly synthesizes contributions from religion/spirituality and positive psychology. We also find it important to capture how social location, intersectionality, and structural oppression - as well as empowerment, liberation, and capacity-building — are inextricably intertwined with postdisaster adaptation. In our systemic Disaster Spiritual Fortitude and Resilience (DSFR) model (see Figure 30.1), we frame disaster recovery as a complex, dynamic, and multidimensional process that (a) involves relational, 
emotional, cognitive, and behavioral domains and (b) unfolds across time in intrapersonal, interpersonal, community, and larger societal contexts. Next, we describe this model in detail.

\section{Domains of Human Experience}

The DSFR model views fortitude and resilience as emerging from adaptive responses in four domains of human experience: (a) relational (e.g., expecting the sacred and close others to be unreliable and punishing vs. responsive and supportive), (b) emotional (e.g., fear and despair vs. hope and optimism), (c) cognitive (e.g., appraisals of threat vs. opportunity), and (d) behavioral (e.g., erratic/maladaptive vs. strategic/health-enhancing actions). These domains are shaped and reinforced across the lifespan based on experience, and past traumatic events can lead to both survival-oriented biases (e.g., hypervigilance to threat) and distinct strengths (e.g., creativity, adaptability). Each domain can be the site of psychological and R/S vulnerabilities that increase risk for maladjustment, or psychological and R/S strengths that catalyze fortitude and resilience. Thus, postdisaster adaptation involves a complex set of relational, emotional, cognitive, and behavioral interactions, unfolding over time within multilevel social contexts.

Drawing on the attachment and social neuroscience literatures, the relational domain captures how survivors' relational-spirituality dynamics contribute substantially to postdisaster psychological and spiritual outcomes, shaping "the course, intensity, and way of grieving" and overall adaptation (Stroebe et al., 2005, p. 58). Believers who have a sense of spiritual dwelling carry the relational expectation of their Higher Power(s) and R/S community as loving, emotionally present, and responsive to their suffering. Amidst tragedy or disaster, although they may be angry or confused, R/S survivors are often motivated to seek experiential closeness to whatever they deem sacred, as a means of comfort and support (Davis et al., 2019a, 2019b). Indeed, Massengale et al. (2017) found that secure attachment to God following Hurricane 
Katrina weakened the link between loss of tangible resources and psychological distress. In a longitudinal study of flood survivors, Davis et al. (2019b) found that divine attachment security facilitated emotional processing and spiritual meaning-making, including appraisals of God's providence and benevolence. In contrast, individuals with attachment insecurity in relation to the sacred may be at greater risk of psychological distress following traumatic death of a loved one (Captari et al., 2020) and disaster-related losses (Massengale et al., 2017). Taken together, survivors' relational schemas can either buffer or exacerbate a disaster's psychological impacts.

Secondly, the emotional domain captures both the deleterious impacts of trauma-related affect (e.g., fear, anxiety, guilt) and the health-enhancing influences of positive emotions (e.g., curiosity, love, hope; see Van Cappellen et al., Chapter 20, this volume). Although emotion dysregulation is a common factor in psychopathology, the broaden-and-build theory of positive emotions offers an additional perspective. Affect regulation includes an individual's ability to modulate emotional states in order to respond adaptively to their environment. In the context of disaster, emotions such as fear and anxiety can serve important adaptive functions in promoting survival-oriented and risk-reduction behaviors (e.g., evacuating, taking shelter, following health precautions). However, if not well-regulated, these emotions can be paralyzing and counterproductive over time. Within the DSRF model, the emotional domain also involves the upregulation of positive emotions, which can promote an upward spiral of cultivating psychological, R/S, and social resources (Van Cappellen et al., Chapter 20, this volume). Regulation can occur individually (self-regulation) and relationally (coregulation). Adaptive regulation allows survivors to experience emotions more fully (rather than dissociating or avoiding) and use them productively (as cues about underlying needs, desires, and motivations). Synthesizing contributions from the R/S coping and meaning-making literatures, the 
cognitive domain captures (a) the ways in which disasters may challenge or shatter previous beliefs about oneself, others, the world, and the sacred as well as (b) the role that positive or redemptive meanings can play in promoting resilience and adaptation. Many R/S survivors draw on their faith as they attempt to reconcile global meanings with painful disaster effects, often reducing dissonance by altering or expanding theodicies of suffering. This can include distressperpetuating negative appraisals, such as blaming themselves or society at large (e.g., viewing the disaster as punishment for sin) or accusing God (e.g., viewing the divine as wrathful, cruel, or untrustworthy). Alternatively, believers can reaffirm a divine purpose and plan (e.g., seeing God as at work amidst the disaster) and make positive attributions about unity, sanctification, or potential R/S growth. For instance, in a longitudinal disaster study, Davis and colleagues (2019a, p. 6) found evidence survivors engaged in resilience-promoting R/S appraisals about cause ("God did not cause the disaster but did allow it to happen"), purpose ("God has used the disaster to accomplish higher benevolent purposes"), and presence ("God has been a source of love, comfort, strength, and hope for survivors"). Metabolizing exposure to suffering, chaos, and death may occur via R/S narratives, rituals, and symbols that facilitate redemptive meaning-making.

Finally, the behavioral domain captures how in the context of danger, our bodies are physiologically primed to act, even as disasters upend our normal routines and rhythms of life. Survivors may exhibit erratic, disorganized action or antisocial behaviors and utilize maladaptive behaviors to cope. Or, on the contrary, they might restore a sense of personal agency and realistic control through engaging in adaptive coping strategies, altruism, and prosocial behaviors that meaningfully address community needs. As Vollhardt (2009) has noted, "Suffering may actually enhance the motivation to help other disadvantaged members of society, including outgroups" (p. 53). As one example, the resurgence of racial protests in the U.S. during the pandemic can be 
understood through this lens, as people spoke up and demanded justice for the bodies and lives of the oppressed. Yet altruistic action is not without its liabilities, and it can at times lead to counterproductive efforts (e.g., Penta et al., 2020) and exacerbated traumatization. Considering cultural norms, Lee and Kim (2020) found collectivism to be associated with greater prosocial behavior through the mediator of increased psychological closeness. This effect was particularly prominent among survivors reporting greater mortality salience, suggesting that awareness of life's fragility can orient people toward collaborative action in disaster recovery.

\section{The Influence of Systemic Contexts}

Although many disaster recovery models focus on individual vulnerabilities, research has documented that the aforementioned domains of human experience are nested within community, religious, and governmental contexts. Thus, as Figure 30.1 depicts, human experience unfolds within — and is influenced by — multiple levels of social ecology (see also

Davis et al., Chapter 18, this volume). Critically, practitioners and community leaders can look at each of these levels as potential intervention points. First, in the intrapersonal realm, personal strengths (e.g., resourcefulness, problem-solving) can buffer the psychological impacts of disaster-related losses and disruptions by facilitating social support, emotion regulation, benevolent religious appraisals, and prosocial behaviors. Alternatively, survivors' stress-andcoping trajectory may be burdened by personal vulnerabilities (e.g., past trauma history, insecure attachment, mental or physical health problems), increasing risk for isolation, emotional dysregulation, malevolent religious appraisals, and maladaptive behaviors.

Second, with regard to interpersonal contexts, survivors' social relationships (e.g., social support from a partner, family members, and friends) can offer powerful sources of fortitude and resilience. Couple and family systems enable coregulation of difficult affect, restore a sense of 
safety, and co-create benevolent, generative, and hope-filled meanings. For instance, parents can help their children adjust by developing a shared family narrative about surviving the disaster, drawing on R/S teachings and theodicies to make redemptive meaning of suffering and take prosocial action to support others. Alternatively, interpersonal contexts may exacerbate disasterrelated loss and lead to maladaptive coping due to heightened conflict, intimate partner violence, or abuse/neglect. Relationships that are unpredictable and unsafe (rather than stable and comforting) can fuel trauma-related affect, appraisals of threat, and desperate behaviors.

Third, community contexts, such as one's neighborhood, workplace, school, and faith community play vital roles. These formal and informal social networks have been described as a lynchpin in facilitating disaster preparation, response, and recovery at grassroots levels (Andrew et al., 2020). Schools are a safety net for vulnerable students, providing daily structure, socioemotional support, and nutritious meals (Lai et al., 2019). Workplaces offer daily continuity and opportunities for peer support. Churches, synagogues, and mosques are uniquely poised to help mitigate postdisaster social isolation because they are embedded within impacted communities and thus can serve as "social bridges [which] may in turn engender other sources of cohesion, such as trust, and further establishment of support networks and norms" (Vinson, 2004, p. 33). Community leaders' responses can help validate, contain, and support survivors in metabolizing suffering through adaptive coping, thereby providing contexts that facilitate mourning and meaning-making. Or, faith communities, learning environments, and local organizations can exacerbate disaster effects when leaders are reactionary, offer aid inequitably, or promulgate narratives that elicit fear and mistrust (rather than unity and collaboration). This can pit community members against each other, isolate vulnerable groups, and fuel maladaptive coping. Furthermore, communities themselves may be reeling from the effects of disasters and 
thus may be less able than usual to provide adequate support to individuals, families, and groups.

Finally, the responses of governments and larger society also influence fortitude and resilience processes. Public health initiatives and media responses can buffer negative outcomes through addressing multiple domains of human experience. This may include cultivating a sense of community cohesiveness and solidarity, down-regulating fear and anxiety by providing clear and direct information, fostering appraisals of rising to the challenge rather than minimizing or catastrophizing threat, and facilitating collaborative action to address survivors' needs.

Alternatively, organizations and governments may compound disaster impacts when responses are uncoordinated, reactive, mismanaged, or inequitable. Such responses can precipitate feelings of invisibility (especially for nonmajority groups), fuel emotional reactivity and traumatic stress, promote distress-perpetuating meanings, and provoke dysregulated or erratic behavior.

\section{Community Implications and Future Research Directions}

This systemic model of DSFR integrates the contributions of R/S resources with positive psychology perspectives in ways that consider both catalysts and impediments to postadaptation across multiple contexts. We invite clinicians, R/S leaders, and community/government stakeholders to use this model as an orienting framework to understand and assess survivors' strengths and vulnerabilities holistically and within their socioecological situatedness. Helping survivors identify and draw on culturally embedded strengths, R/S practices, and community supports can bolster their sense of fortitude amidst chaos and collectively orient people toward resilience. Below, we offer several practical recommendations to guide such a disaster response.

\section{Be responsive to survivors' needs in the moment, recognizing changes across time.}

For those involved in disaster response and spiritual care, it is vital to utilize a trauma-responsive approach, especially with respect to normative stress reactions and survivors' changing needs. 
For example, in the immediate aftermath, interventions that connect survivors with resources and help restore a felt sense of safety are advisable; practitioners can focus on offering survivors comfort, validation, and support. Survivors' meaning-making processes often unfold once their relational and emotional domains of experience are attended to, so practitioners should avoid jumping to theological concepts (e.g., quoting Scripture), which might be experienced as insensitive or invalidating. In research, mediation and moderation analyses can explore individual (e.g., R/S coping), familial (e.g., disaster narratives), and community (e.g., social support) variables that explain or influence postdisaster adaptation. Social network analysis, longitudinal studies, and ecological momentary assessment can also help explore these nuances.

\section{Consider overlapping disaster impacts and each person's sociohistorical and}

religious context. Disasters do not happen in isolation. Communities that are already vulnerable (due to historical trauma, oppression, or marginalization) are often hardest hit when a disaster strikes. Ongoing research is needed to explicate how these factors may affect how survivors view and relate to the sacred, as well as to develop culturally adapted forms of disaster spiritual and emotional care. Understanding differential effects of particular types of R/S coping can assist practitioners and R/S communities in offering effective culturally and spiritually responsive care.

\section{Intervene across multiple domains and contexts to bolster survivors' strengths and}

resources. Clergy responses can help foster R/S coping and adaptive meaning-making within their spiritual communities in the aftermath of disaster. Community leaders can work to procure additional resources from larger government agencies that can help supplement and rebuild community resources, taking care to offer equitable supports and identify those most vulnerable. In addition to providing direct psychological care, mental health practitioners can partner with faith communities, schools, and other groups to create opportunities for memorializing and 
mourning, drawing on relevant R/S and cultural practices to foster hope, comfort, and support.

Collaborate with and contribute to faith communities. Ongoing community

partnerships between clergy, emergency/disaster response personnel, community leaders (e.g., school principal, mayor), and mental health practitioners is a key aspect of disaster preparedness and response. Clinicians should not deploy individually or attempt to intervene on their own, and R/S leaders should not work outside their competency by trying to provide psychological care. Public health efforts can also be much more effective when clergy are involved, given that many people trust and look to their spiritual leaders amidst crises. Research efforts should always be grounded in community-based participation and support. Wheaton College's Humanitarian Disaster Institute (https://www.wheaton.edu/academics/academic-centers/humanitarian-disaster$\underline{\text { institute/) }}$ offers a hub for developing and enriching these types of community partnerships.

\section{Conclusion}

This chapter has synthesized the contributions of leading disaster theories and proposed a systemic model integrating positive psychological and R/S perspectives. We invite researchers, clinicians, and community stakeholders to use this model to inform empirical investigations and community-based interventions. By considering the interplay of human strengths and capacities for positive adaptation alongside R/S processes of struggle and transformation, we all can support individuals, families, and communities in not just surviving disasters but transcending them through seasons of mourning and disorientation toward horizons of new meanings, deeper connections, and hopeful possibilities. 


\section{References}

Andrew, S. A., Bacot, H., \& Craw, M. (2020). A multiorganizational cooperation framework for neighborhood disaster resilience. State and Local Government Review, 52(1), 53-66. https://doi.org/10.1177/0160323X19898516

Aten, J., Bennett, P., Hill, P., Davis, D. \& Hook, J. (2012). Predictors of God concept and God control after Hurricane Katrina. Psychology of Religion and Spirituality, 4(3), 182-192. https://doi.org/10.1037/a0027541

Aten, J., Smith, W., Davis, E. B., Van Tongeren, D., Hook, J., Davis, D., ... \& Hill, P. (2019). The psychological study of religion and spirituality in a disaster context: A systematic review. Psychological Trauma, 11(6), 597-613. https://doi.org/10.1037/tra0000431

Captari, L. E., Hook, J. N., Aten, J. D., Davis, E. B., \& Tisdale, T. (2019). Embodied spirituality following disaster: Exploring intersections of religious and place attachment. In V. Counted \& F. Watts (Eds.), Religion and place (pp. 49-80). Palgrave.

Captari, L. E., Riggs, S. A., \& Stephen, K. (2020). Attachment processes following traumatic loss: A mediation model examining identity distress, shattered assumptions, prolonged grief, and posttraumatic growth. Psychological Trauma,13(1), 94-103. https://doi.org/10.1037/tra0000555

Charuvastra, A., \& Cloitre, M. (2008). Social bonds and posttraumatic stress disorder. Annual Review of Psychology, 59, 301-328. https://doi.org/10.1146/annurev.psych.58.110405.085650

Cook, S., Aten, J., Moore, M., Hook, J. N., \& Davis, D.E. (2013). Resource loss, religiousness, health, and posttraumatic growth following Hurricane Katrina. Mental Health, Religion \& Culture, 16(4), 352-366. https://doi.org/10.1080/13674676.2012.667395 
Counted, V., Neff, M. A., Captari, L. E., \& Cowden, R. G. (2020). Transcending place attachment disruptions during a public health crisis: Spiritual struggles, resilience, and transformation. Journal of Psychology and Christianity, 39(4), 276-287.

Davis, E. B., Aten, J. D., Van Tongeren, D. R., Hook, J. N., Davis, D. E., DeBlaere, C., \& Shannonhouse, L. R. (2018). Advancing scientific research on disasters, religion, and spirituality. Journal of Psychology \& Christianity, 37(1), 87-93.

Davis, E. B., Kimball, C. N., Aten, J. D., Andrews, B., Van Tongeren, D. R., Hook, J. N., Davis, D. E., Granqvist, P., \& Park, C. L. (2019a). Religious meaning making and attachment in a disaster context: A longitudinal qualitative study of flood survivors. Journal of Positive Psychology, 14(5), 659-671. https://doi.org/10.1080/17439760.2018.1519592

Davis, E. B., Kimball, C., Aten, J. D., Hamilton, C., Andrews, B., .. \& Chung, J. (2019b). Faith in the wake of disaster: A longitudinal qualitative study of religious attachment following a catastrophic flood. Psychological Trauma, 11(6), 578-587. https://doi.org/10.1037/tra0000425

Feder, A., Ahmad, S., Lee, E., Morgan, J., Singh, R., Smith, B., Southwick, S., \& Charney, D. (2013). Coping and PTSD symptoms in Pakistani earthquake survivors: Purpose in life, religious coping and social support. Journal of Affective Disorders, 147(1-3), 156-163. https://doi.org/10.1016/j.jad.2012.10.027

Fox, J., Picciotto, G., Cashwell, C., Worthington, E. L., Jr., Basso, M. J., ... \& Zeligman, M. (2020). Religious commitment, spirituality, and attitudes toward God as related to psychological and medical help-seeking: The role of spiritual bypass. Spirituality in Clinical Practice, 7(3), 178-196. https://doi.org/10.1037/scp0000216

Hall, E., Shannonhouse, L., Aten, J., McMartin, J., \& Silverman, E. (2018). Religion-specific 
resources for meaning-making from suffering: Defining the territory. Mental Health, Religion \& Culture, 21(1), 77-92. https://doi.org/10.1080/13674676.2018.1448770

Hobfoll, S. E. (1989). Conservation of resources: A new attempt at conceptualizing stress. American Psychologist, 44(3), 513-524. https://doi.org/10.1037/0003-066X.44.3.513

Hobfoll, S. E. (2011). Conservation of resources theory: Its implication for stress, health, and resilience. In S. Folkman (Ed.), The Oxford handbook of stress, health, and coping (pp. 127-147). Oxford University Press.

Hobfoll, S. E., Dunahoo, C. A., \& Monnier, J. (1995). Conservation of resources and traumatic stress. In J. R. Freedy \& S. E. Hobfoll (Eds.), Traumatic stress (pp. 29-47). Plenum.

Lai, B., Esnard, A., Savage, R., Wyczalkowski, C., \& Shah, H. (2019). Trajectories of school recovery after a natural disaster. Risk, Hazards \& Crisis, 10, 32-51.

Lee, J., \& Kim, Y. (2020). The effect of collectivism, psychological closeness, and mortality salience on prosocial behavioral intentions. Journal of Risk Research, 24(6), 756-770.

Liu, K., Chen, Y., Lin, R., \& Han, K. (2020). Clinical features of COVID-19 in elderly patients: A comparison with young and middle-aged patients. The Journal of Infection, 80, 14-18. https://doi.org/10.1016/j.jinf.2020.03.005

Maercker, A., \& Horn, A. (2013). A socio-interpersonal perspective on PTSD. Clinical Psychology \& Psychotherapy, 20, 465-481. https://doi.org/10.1002/cpp.1805

Massengale, M., Davis, D., DeBlaere, C., Zelaya, D., Shannonhouse, L., .. \& Hill, P. C. (2017). Attachment avoidance to God exacerbates the negative effect of tangible resource loss on psychological resource loss. Mental Health, Religion \& Culture, 20(5), 489-501. https://doi.org/10.1080/13674676.2017.1359242

McElroy-Heltzel, S. E., Van Tongeren, D. R., Gazaway, S., Ordaz, A., Davis, D. E., Hook, J. N., 
... \& Stargell, N. A. (2018). The role of spiritual fortitude and positive religious coping in meaning in life and spiritual well-being following Hurricane Matthew. Journal of Psychology and Christianity, 37(1), 17-27.

Morgado, A. M. (2020). Disasters, individuals, and communities: Can positive psychology contribute to community development after disaster? Community Development, 51, 3-16. https://doi.org/10.1080/15575330.2020.1714685

Pargament, K. I., \& Cummings, J. (2010). Anchored by faith. In J. W. Reich, A. J. Zautra, \& J. S. Hall (Eds.), Handbook of adult resilience (pp. 193-210). Guilford Press.

Pargament, K., \& Exline, J. (2021). Working with spiritual struggles in psychotherapy. Guilford. Park, C. (2010). Making sense of the meaning literature. Psychological Bulletin, 136(2), 257301. https://doi.org/10.1037/a0018301

Park, C. (2016). Meaning-making in the context of disasters. Journal of Clinical Psychology, 72, 1234-1246. https://doi.org/10.1002/jclp.22270

Penta, S., Wachtendorf, T., \& Nelan, M. M. (2020). Disaster relief as social action. Sociological Forum, 35(1), 145-166. https://doi.org/10.1111/socf.12571

Sandage, S. J., Jankowski, P. J., Paine, D. R., Exline, J. J., Ruffing, E. G., Rupert, D., Stavros, G. S., \& Bronstein, M. (2020). Testing a relational spirituality model of psychotherapy clients' preferences and functioning. Journal of Spirituality in Mental Health. Advance online publication. https://doi.org/10.1080/19349637.2020.1791781

Sandage, S. J., Rupert, D., Stavros, G. S., \& Devor, N. G. (2020). Relational spirituality in psychotherapy: Healing suffering and promoting growth. APA.

Shannonhouse, L., Bialo, J., Majuta, A., Zeligman, M., Davis, D., McElroy-Heltzel, S., Aten, J., Davis, E., Van Tongeren, D., \& Hook, J. (2019). Conserving resources during chronic 
disaster: Impacts of religious and meaning-focused coping on Botswana drought survivors. Psychological Trauma, 11(2), 137-146. https://doi.org/10.1037/tra0000420

Shannonhouse, L., Snyder, J., Fullen, M., Davis, E., McElroy-Heltzel, S., .. \& \& Van Tongeren, D. (2021). Resource loss, religious coping, and suicide predictors/outcomes during the COVID-19 pandemic. Journal of Psychology and Christianity, 39(4), 313-327.

Southwick, S. M., Satodiya, R., \& Pietrzak, R. H. (2016). Disaster mental health and positive psychology. Journal of Clinical Psychology, 72(12), 1364-1368. https://doi.org/10.1002/jclp.22418

Stroebe, M., Schut, H., \& Stroebe, W. (2005). Attachment in coping with bereavement: A theoretical integration. Review of General Psychology, 9(1), 48-66. https://doi.org/10.1037/1089-2680.9.1.48

Tai, D., Shah, A., Doubeni, C., Sia, I., \& Wieland, M. (2021). The disproportionate impact of COVID-19 on racial and ethnic minorities. Clinical Infectious Diseases, 72(4), 703-706. https://doi.org/10.1093/cid/ciaa815

Vinson, T. (2004). Community adversity and resilience. Report submitted to the Ignatius Centre, University of New South Wales. http://hdl.voced.edu.au/10707/110419

Vollhardt, J. R. (2009). Altruism born of suffering and prosocial behavior following adverse life events: A review and conceptualization. Social Justice Research, 22(1), 53-97. https://doi.org/10.1007/s11211-009-0088-1

World Health Organization. (2021). WHO Coronavirus (COVID-19) dashboard. https://covid19.who.int/

Zunin, L. \& Myers, D. (2000). Training manual for human service workers in major disasters. U.S. Department of Health and Human Services. https://www.hsdl.org/?view\&did=4017 


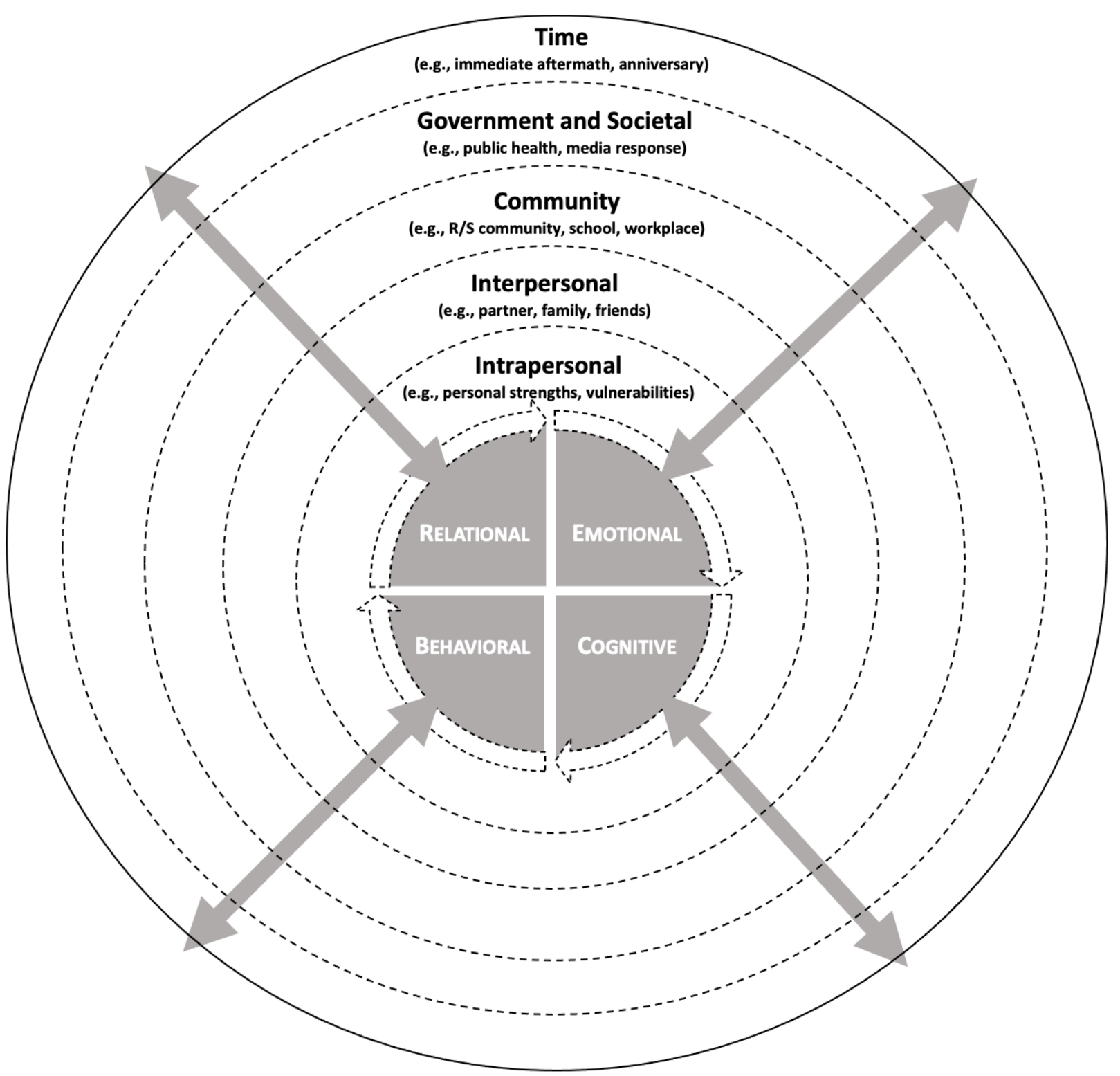

Figure 30.1. Systemic Model of Disaster Spiritual Fortitude and Resilience (DSFR).

Disaster adaptation and recovery involves relational, emotional, cognitive, and behavioral domains of experience unfolding across time and within multiple contexts: intrapersonal, interpersonal, community, and governmental/larger society. 


\title{
Chapter 31: Future Directions for the Positive Psychology of Religion and Spirituality
}

\author{
Edward B. Davis ${ }^{1}$, Sarah A. Schnitker ${ }^{3}$, Everett L. Worthington, Jr. ${ }^{2}$, and Ethan K. Lacey ${ }^{1}$ \\ ${ }^{1}$ School of Psychology, Counseling, and Family Therapy, Wheaton College \\ ${ }^{2}$ Department of Psychology and Neuroscience, Baylor University \\ ${ }^{3}$ Psychology Department, Virginia Commonwealth University
}

\begin{abstract}
Author Note
We have no known conflicts of interest to disclose.

This publication was made possible through the support of Grant 61865 from the John Templeton Foundation. The opinions expressed in this publication are those of the authors and do not necessarily reflect the views of the John Templeton Foundation.

Correspondence concerning this chapter should be addressed to Edward B. Davis, Wheaton College, 501 College Ave, Wheaton, IL 60187. Email: ward.davis@ wheaton.edu.
\end{abstract}




\begin{abstract}
In this chapter, we synthesize the content of this Handbook for Positive Psychology, Religion, and Spirituality and call for scientists and practitioners to devote focused attention to the intersections of positive psychology, religion, and spirituality. First, we summarize key themes from the Handbook's sections and chapters. We highlight deficiencies that are currently inhibiting progress at the intersections of positive psychology and the psychology of religion and spirituality. Finally, to address these deficiencies, we conclude the Handbook by proposing an integrated field — the positive psychology of religion and spirituality — and then offer a prospectus for guiding science and practice in this unified field.
\end{abstract}

Keywords: positive psychology, religion, spirituality, field development, future directions 


\section{Chapter 31: Future Directions for a Positive Psychology of Religion and Spirituality}

You have nearly reached the end of this Handbook of Positive Psychology, Religion, and Spirituality. In this final chapter, we highlight key themes that emerged across the Handbook and identify several deficiencies in theory and research uncovered along the way. We also propose the unification of positive psychology and the psychology of religion/spirituality $(\mathrm{R} / \mathrm{S})$ into an integrated field — the positive psychology of R/S—to address these deficiencies. Finally, we make recommendations about strategic priorities to guide science and practice in this field.

\section{Key Themes Across the Handbook}

\section{Positive Psychology and the Psychology of R/S are Not Yet Well-Integrated}

Despite their similar aims, foundations, and emphases, these two fields are not yet wellintegrated (Davis et al., Chapter 1). Handbook authors offered possible reasons for this gap.

Nelson and Canty (Chapter 2) critiqued the fields by averring that one historical and philosophical reason for this lack of integration is that both fields are hindered by a commitment to positivistic naturalism (i.e., the assumption that nothing exists beyond the natural world and all knowledge must be verified empirically by the scientific method). They, Porter et al. (Chapter 3) and Tsang et al. (Chapter 8) encouraged positive psychology and the psychology of R/S to transcend this commitment by adopting a more methodologically and epistemologically pluralistic approach that will welcome more diverse methods and sources of knowledge.

Davis et al. (Chapter 1) indicated there might be personal and professional reasons for this lack of integration as well. They noted that psychologists usually lack personal or professional familiarity with R/S (Vieten \& Lukoff, 2021) and often exhibit skepticism and potential bias against both R/S (Slife \& Reber, 2009) and positivity (Vaish et al., 2008). 
In addition, Davis et al. (Chapter 1) explained how these biases might influence the openness of editors and peer reviewers to publishing research on R/S in mainstream psychology journals, despite relatively more openness to publishing positive psychology research. Mattis (Chapter 9), Rossy et al. (Chapter 15), and Cowden et al. (Chapter 16) discussed other structural and logistical reasons for the lack of integration between the positive psychology and psychology of R/S. For instance, many countries and cultural groups lack the structural access, resources, and infrastructure to support such research. Moreover, the theories, measures, and applied tools in positive psychology and the psychology of R/S are overwhelmingly White-, Christian-, and Western-centric, as was mentioned in most chapters.

\section{There are Several Natural Bridges Between the Fields}

Despite these noteworthy barriers between the positive psychology and psychology of R/S fields, there are several natural bridges of intersection between them. Numerous authors highlighted the potential for virtues and character strengths to serve as a nexus for such integration (Ratchford et al., Chapter 4; Hill et al., Chapter 7; Shafranske, Chapter 22; Washington-Nortey et al., Chapter 23; Cauble et al., Chapter 24; Long \& VanderWeele, Chapter 25; Captari et al., Chapters 26 and 30; Wang et al., Chapter 29). As the least-developed area among the positive psychology search terms that were systematically reviewed in Chapter 1

(Davis et al.), there is indeed enormous room for growth in this domain. Several authors pointed to other prime candidates for bridging the fields, including meaning (Park \& Van Tongeren, Chapter 6; King et al., Chapter 17; Davis et al., Chapter 18; Shafranske, Chapter 22; Dik \& Alayan, Chapter 27), positive emotions (Van Cappellen et al., Chapter 20; Shafranske, Chapter 22; Captari et al., Chapter 30), human and perceived divine relationships (King et al., Chapter 17; Davis et al., Chapter 18; Mahoney et al., Chapter 28; Wang et al., Chapter 29), and health 
and well-being itself (Davis et al., Chapter 1; MacDonald, Chapter 5; Singh et al., Chapter 13; Mancuso \& Lorona, Chapter 19; Masters et al., Chapter 21; Shafranske, Chapter 22; Long \& VanderWeele, Chapter 25). These natural bridges between positive psychology and the psychology of R/S reflect three elements in Seligman's (2011) PERMA well-being framework: positive emotions, positive relationships, and meaning.

\section{R/S Are Robustly Linked to Positive Psychology Constructs and to Well-Being, but the Directionality, Mechanisms, and Boundary Conditions of These Links are Less Clear}

Another theme that echoed across the Handbook was that R/S are robustly linked to positive psychology constructs and to well-being. For instance, extant research indicates that R/S is consistently associated with each of the aforementioned natural bridges with positive psychology: (a) virtues and character strengths (e.g., forgiveness, hope, humility, gratitude, and love; Washington-Nortey et al., Chapter 23; Cauble et al., Chapter 24; Long \& VanderWeele, Chapter 25; Captari et al., Chapter 26), (b) meaning (Park \& Van Tongeren, Chapter 6; Dik \& Alayan, Chapter 27), (c) positive emotions (Van Cappellen et al., Chapter 20), and (d) positive relationships (King et al., Chapter 17; Davis et al., Chapter 18; Mahoney et al., Chapter 28). R/S is also robustly linked to overall health/well-being (MacDonald, Chapter 5; Davis et al., Chapter 18) and many of its facets, including life satisfaction (Mancuso \& Lorona, Chapter 19), physical health (Masters et al., Chapter 21), mental health (Shafranske, Chapter 22), relational health (Mahoney et al., Chapter 28), occupational health (Dik \& Alayan, Chapter 27), and community health (Long \& VanderWeele, Chapter 25; Captari et al., Chapters 26 and 30).

Notwithstanding, the directionality (causal direction of effects), causal mechanisms (mediators), and boundary conditions (moderators) of links between R/S and positive psychology constructs are less clear. One methodological refrain that emerged across chapters is that over 
$93 \%$ of the studies at the intersections of positive psychology and the psychology of R/S are cross-sectional/correlational (Tsang et al., Chapter 8), thereby precluding tests of causal effects (directionality; VanderWeele et al., 2020) or causal mechanisms (mediators; Maxwell \& Cole, 2007). Furthermore, boundary conditions for effects are largely unknown, especially when it comes to identifying cultural and contextual factors that may influence the strength or direction of the relationship between R/S and positive psychological traits or phenomena.

\section{Deficiencies Inhibiting the Integration of Positive Psychology, Religion, and Spirituality}

Chapter authors discussed deficiencies hindering the integration of positive psychology and the psychology of R/S. We highlight three major themes.

\section{Theoretical and Cultural Deficiencies}

First, there are several theoretical deficiencies uncovered in this Handbook. Although there are exceptions (e.g., Koenig et al., 2012; see MacDonald, Chapter 4, for a review), there is a relative dearth of theories that explain how, why, and when R/S and positive psychological traits and phenomena are linked. Several chapter authors addressed this need by proposing or elaborating on integrative theories, including (a) Mattis's (Chapter 9) Culture, Religiosity/Spirituality, and Positive Development theory; (b) Davis et al.’s (Chapter 18) Positive Religious and Spiritual Development theory; (c) Van Cappellen et al.'s (Chapter 20) Upward Spiral Theory of Sustained Religious/Spiritual Practice; (d) Shafranske's (Chapter 22) Religious/Spiritual Pathways to Mental Health model; (e) Dik and Alayan's (Chapter 27) R/S and Meaningful Work model; and (f) Captari et al.'s (Chapter 30) Systemic Model of Disaster Spiritual Fortitude and Resilience. Each of those theories also addressed another inadequacy in the extant literature on R/S and positive psychology—the lack of theories attending to multiple levels of analysis and the interactions among those levels (e.g., at the individual, relational, and 
macrosystemic levels). To date, most research at the intersections of R/S and positive psychology has been atheoretical (i.e., not guided by a particular theory; see Davis et al., Chapter 18) or has focused only on the individual level of analysis.

Relatedly, as Mattis (Chapter 9) underscored, most research at the intersections of R/S and positive psychology has been acultural (i.e., neither focusing on culture nor considering of culture meaningfully in its conceptual foundations, research questions, or data analyses and interpretation). In fact, positive psychology often seems to have culturally appropriated religious/spiritual beliefs, practices, and phenomena. For instance, apart from Peterson and Seligman (2004) acknowledging the religious origins of their taxonomy, positive psychology has largely ignored the religious moorings of virtues and virtue development (Nelson \& Canty, Chapter 2; Ratchford et al., Chapter 4). It also has largely disregarded the religious mechanisms that have helped people cultivate virtues and well-being for millennia - through historic religious practices, guided by historic religious texts, and nurtured in historic religious traditions and communities (Schiffman et al., Chapter 11; Singh et al., Chapter 13; Wang et al., Chapter 29). Positive psychology seems to have appropriated certain historically religious concepts (e.g., hope and forgiveness; Washington-Nortey et al., Chapter 23) and practices (e.g., mindfulness and meditation; Segall \& Kristeller, Chapter 14), secularized them, and demonstrated empirically that the secularized versions apply to and work effectively with nonreligious/nonspiritual people. ${ }^{7}$

Yet the psychology of R/S field is not faultless either. It has rarely attempted to establish the cross-cultural equivalence of its constructs and measures (Hill et al., Chapter 7), the crosscultural generalizability of its theories and findings (Rossy et al., Chapter 15; Cowden et al., Chapter 16), or the cross-cultural applicability of its theories and interventions (MacDonald,

\footnotetext{
${ }^{7}$ To be fair, religious/spiritual people and groups are often eager to appropriate secular, positive psychological research evidence, claiming it empirically validates their pre-existing religious/spiritual concepts and practices.
} 
Chapter 5; Mattis, Chapter 9; Captari et al., Chapter 26). Moreover, it frequently ignores the contributions of culture to R/S, fails to disentangle culture from R/S, and glosses over cultural variations of R/S (e.g., differences in how R/S is approached and practiced; Saroglou et al., 2020).

\section{Methodological and Analytic Deficiencies}

This Handbook also revealed methodological and analytic inadequacies at the intersections of positive psychology and the psychology of R/S. Many authors underscored this literature's over-reliance on cross-sectional studies, culturally homogenous (and small/underpowered) samples, and correlational analyses. Admittedly, this deficiency characterizes mainstream psychology and most (if not all) its subfields — not just positive psychology and the psychology of R/S (Tsang et al., Chapter 8). Nevertheless, there is a clear need for greater methodological and analytic sophistication. That of course includes a need for more longitudinal and experimental research, but it also includes a need for more studies that recruit large samples characterized by meaningful diversity within and across cultural groups (in terms of nationality, religious affiliation and importance, age, gender, sexual orientation, race/ethnicity, education level, socioeconomic status, marital status, and disability status). In addition, there is a need for more studies that use advanced statistical analyses (e.g., growth modeling [Grimm et al., 2017] or outcome-wide analyses [VanderWeele, 2017a; VanderWeele et al., 2020]), conduct sensitivity analyses (to test the robustness of effects to potential unmeasured confounding; VanderWeele \& Ding, 2017), and utilize open science practices (e.g., preregister study plans and hypotheses, share study data and materials, replicate study findings, and post manuscript preprints; https://www.cos.io/; Nosek et al., 2015; Tsang et al., Chapter 8). 


\section{Applied and Practical Deficiencies}

Finally, this Handbook illuminated the scarcity of practical applications and implications explored at the intersections of positive psychology and the psychology of R/S. Most of the literature at these intersections is basic science (i.e., "scientific research or theory that is concerned with knowledge of fundamental phenomena and the laws that govern them, regardless of the potential applications of such knowledge," VandenBos, 2015, p. 109), rather than applied science (i.e., "the use of scientific principles and theories to serve a practical human purpose rather than to extend knowledge for its own sake," VandenBos, 2015, p. 70). There is a great need to nurture and refine an applied positive psychology (Lomas et al., 2015) and an applied psychology of R/S (Pargament, 2013), but more broadly, there is an exigency to emphasize the applied and practical sides of integrating positive psychology and the psychology of R/S. Several chapters explored possible venues and vehicles for doing that, such as public health efforts (Long \& VanderWeele, Chapter 25), psychological and spiritual interventions (Captari et al., Chapter 26), work settings (Dik \& Alayan, Chapter 27), couples and families (Mahoney et al., Chapter 28), religious communities (Wang et al., Chapter 29), and humanitarian and disaster contexts (Captari et al., Chapter 30).

\section{Prospectus for a Positive Psychology of Religion and Spirituality (PPRS) \\ The Definition, Aims, and Scope of the PPRS Field}

To address these and other deficiencies at the intersections of positive psychology and the psychology of R/S, we propose formalizing a new, unified field - the positive psychology of R/S. Building on the work of Linley et al. (2006; who defined positive psychology as "the scientific study of optimal human functioning”, p. 8) and Davis et al. (Chapter 18, this volume; who defined R/S as "people's search for and response to sacred meaning and connection"), we 
define the positive psychology of $R / S$ (PPRS) as the field of psychological science and practice dedicated to understanding and promoting flourishing in and through people's R/S (search for and response to sacred meaning and connection; Davis et al., Chapter 18). In other words, the aims of the PPRS are to understand and promote flourishing in and through people's R/S.

When it comes to the topical scope of PPRS, we adapt the same four elements that Linley et al. (2006) identified as the pillars of positive psychology's scope: (a) wellsprings, (b) internal processes, (c) external processes, and (d) outcomes. We have slightly modified Linley et al.'s (2006) definitions and conceptualizations, in part to account for the multilevel nature of R/S.

(a) The wellsprings of interest to the PPRS are the distal precursors and contributors to the processes that facilitate or impede flourishing in and through people's R/S. These wellsprings might include individual level factors (e.g., a person's genetics and temperament), relational factors (e.g., family-of-origin experiences and other flourishingfacilitative versus flourishing-undermining experiences earlier in life), and macrolevel factors (e.g., a culture's history and customs). ${ }^{8}$

(b) The internal processes of interest to the PPRS are the proximal, internal ingredients that facilitate or impede flourishing in and through people's R/S, such as a person's physical and mental health, character strengths/virtues versus weaknesses/liabilities, and positive

\footnotetext{
${ }^{8}$ Due to space constraints, we have mainly provided examples at the individual level. However, as we show in some of the provided examples, wellsprings and processes can refer to multiple levels of analysis (e.g., dyads, groups, institutions, communities, cultures, and societies). For example, at the dyadic level of analysis (e.g., a parent-child dyad or a romantic couple), examples of wellsprings might include the individuals' respective genetics and temperament, their life experiences as individuals and as a dyad, and their respective families and cultures of origin. At the institutional level of analysis (e.g., a school, workplace, or religious community), examples of internal processes might include the institution's structure and dynamics, and its external processes would include the community and culture within which the institution is embedded. At the macrosystemic level of analysis (e.g., a community or culture), examples of wellsprings might include that community or culture's history, internal processes might include that community or culture's internal structure and dynamics, and external processes might include that community or culture's relations with other communities and cultures, as well as the broader societal context and historical period within which that community or culture is embedded.
} 
versus negative psychological traits. These internal processes might also be conceptualized and assessed at a relational, institutional, or cultural level, such as by examining the internal structure and dynamics of a family, institution, or culture.

(c) The external processes of interest to the PPRS are those proximal, external factors that facilitate or impede flourishing in and through people's R/S, such as flourishingfacilitative versus flourishing-undermining factors in the social and cultural ecology within which a person is embedded (in their current family, peer, romantic, and perceived divine relationships; school or workplace; community; social and cultural groups; and society). External processes can also be conceptualized and examined at an institutional level (e.g., church, mosque, temple, school, or workplace), such as by examining the broader community or culture within which the institution is embedded, as well as ways the broader community/culture engages with other communities and cultures.

(d) The outcomes of interest to the PPRS are those physical, psychological, social, religious/spiritual, institutional, cultural, and societal states that characterize flourishing.

\section{Strategic Priorities for Science in the PPRS Field}

Basic Science. Most fundamentally, we encourage scientists in the PPRS field to remedy the theoretical, cultural, methodological, analytic, applied, and practical deficiencies we already mentioned. Topically, there are three major strategic priorities to address in PPRS basic science.

Question 1: What is the nature and function of flourishing-facilitative R/S (and flourishing-undermining $\mathbf{R} / \mathbf{S}$ ) in various cultures and contexts? There likely are similarities and differences in what characterizes and facilitates (or impedes) flourishing across various cultures and contexts (VanderWeele, 2017b). By extension, there are presumably similarities and differences in the nature and function of flourishing-facilitative versus flourishing-undermining 
R/S. For instance, there may be some dimensions of R/S that only play a strong role in characterizing, facilitating, or impeding flourishing in certain cultures or contexts, whereas other dimensions play a strong role across all or most cultures and contexts. Large-scale, longitudinal research with geographically, culturally, and religiously diverse samples (e.g., the Global Flourishing Study of 240,000 people across 22 countries; Crabtree et al., 2021) and wellreplicated research across diverse contexts (e.g., the Religious Replication Project; PRSM Lab, n.d.) is greatly needed.

\section{Question 2: What are the determinants and consequences of flourishing-facilitative} $R / S$ (and flourishing-undermining $R / S$ ) in various cultures and contexts? In the same way, there probably are similarities and differences in what determines and results from flourishingfacilitative R/S and flourishing-undermining R/S (i.e., internal/external processes and outcomes). For example, there may be mediators (causal mechanisms), boundary conditions (moderators), and outcomes of flourishing-facilitative (or flourishing-undermining) R/S that only emerge in certain cultures and contexts, whereas others are evident across most cultures and contexts. To illustrate, even though meaning may be a causal mechanism of flourishing across most cultures and contexts (Park \& Van Tongeren, Chapter 6, this volume), cultures vary considerably in how often R/S is approached as a substantive source of meaning (e.g., $\mathrm{R} / \mathrm{S}$ is often mentioned as a source of meaning in the U.S. but rarely mentioned in most Asian or European countries; Pew Research Center, 2021). Hence, experimental and longitudinal PPRS research may find that R/S causally enhances meaning and flourishing in certain cultures but does not do so in others. To address such questions, team science approaches that coordinate the collection of data internationally at diverse sites should be adopted, as is being done through the Developing Belief Network's (n.d.) coordination across 30 international field sites with participants from multiple 
religious/spiritual traditions (see also Many Analysts of Religion Project, n.d.; Psychological

Science Accelerator, n.d.).

\section{Question 3: What are the internal and external processes that lead to (or impede)}

flourishing-facilitative $R / S$ at various levels of analysis? In addition, there is a need for PPRS

basic science research on the internal and external processes that cause (or undermine)

flourishing-facilitative R/S in individuals, dyads, families, groups, institutions, communities,

cultures, and societies. For instance, at the individual level, researchers could work to identify

the physiological (e.g., neurobiological) substrates of flourishing-facilitative and flourishing-

undermining R/S (Masters et al., Chapter 21, this volume) and examine how those substrates

interact bidirectionally with internal psychological processes (e.g., emotional, cognitive,

behavioral, and motivational factors) and external social and cultural processes (e.g., relational,

systemic, and societal factors). One research center that is pioneering this type of multilevel

research on R/S is the Institute for the Biocultural Study of Religion (https://www.ibcsr.org/).

Applied Science. Applied science in the PPRS field should of course address the aforementioned deficiencies. In addition, there are three major topical priorities to address.

Question 1: Why, when, and for whom are PPRS interventions effective? As Captari et al. (Chapter 26, this volume) have summarized, there is a growing evidence base supporting the effectiveness of spiritually integrated, positive psychological, and virtue-based interventions. However, there is a need for process research to identify the change mechanisms and contextual influencers of PPRS interventions' effectiveness (e.g., the Mental Healthcare, Virtue, and Human Flourishing Project; John Templeton Foundation, n.d.). There also is a need to identify the people for whom PPRS interventions are most (or least) effective, based on the recipient's characteristics, culture, and preferences, such as the recipient's presenting concerns (mental 
disorders; spiritual struggles) and religious/spiritual affiliation, salience, beliefs, and practices

(Shafranske, 2013; Captari et al., Chapter 26). Moreover, there is a need to ensure that all mental health practitioners develop clinical competencies in R/S (Vieten \& Lukoff, 2021; e.g., the

Religious and Spiritual Competencies in Mental Health Care Project; John Templeton Foundation, 2021b).

\section{Question 2: What are ways to promote flourishing-facilitative $R / S$ at multiple levels}

and in various cultures and contexts? Practically all PPRS interventions have been developed

(a) in North America, Europe, or Australia-Oceania; (b) from Western conceptual, religious, and valuative frameworks; (c) for WEIRD (Western, Educated, Industrialized, Rich, and

Democratic), White, and Christian people; and (d) to promote flourishing at the individual level. These deficiencies must be addressed in PPRS applied science. For example, there is a need for PPRS interventions that effectively promote the flourishing of couples (e.g., hope-focused couple therapy; Ripley \& Worthington, 2014), families (e.g., sanctification-focused family therapy; cf. Mahoney et al., Chapter 28, this volume), organizations (e.g., meaning-focused organizational interventions; cf. Dik \& Alayan, Chapter 27, this volume), religious communities (e.g., virtuefocused congregational interventions; McMinn, 2017; cf. Wang et al., Chapter 29, this volume), and societies (e.g., R/S-focused public health interventions; Long \& VanderWeele, Chapter 25, this volume). There also is a need for PPRS interventions that are found effective for utilizing in a diverse range of contexts and with geographically, religiously, and culturally diverse range of people (e.g., the Building More Forgiving Communities Around the Globe Project; Templeton World Charity Foundation, n.d.).

Question 3: How can the science-practice gap in PPRS be narrowed? One major problem in psychology and its subfields is the science-practice gap - the integration divide 
between the knowledge produced through scientific research and the knowledge consumed and used by practitioners and the public (Aguinis et al., 2020). Of note, although the science-practice gap usually refers only to the divide between science and clinical practice, we are using this phrase to refer to the divide between science and real-world practice in a much broader sense. Specifically, we use the phrase to refer to the divide between science and practice not only in clinical healthcare contexts but also in religious ministry, educational institutions, organizational contexts, public health, and other broadly defined areas of practice, including laypeople's everyday lives.

The science-practice gap is especially pronounced in the PPRS, partly due to the same barriers and deficiencies described above (Davis et al., Chapter 1; Nelson \& Canty, Chapter 2) and partly due to other factors. Some of these other factors include (a) the need for scientists to make knowledge more relevant, useful, and accessible; (b) the need for practitioners and the public to tell researchers what knowledge they want, need, and actually would use; and (c) the need for scientists and practitioners to welcome each other's valuable contributions more humbly and offer those contributions more proactively. To illustrate, (a) PPRS scientists can contribute scientific knowledge about R/S, virtues, and flourishing; (b) PPRS practitioners can contribute practical tips and tools for helping people cultivate their R/S, virtues, and flourishing; and (c) religious practitioners (e.g., religious leaders, institutions, and laypeople) can contribute theological and experiential knowledge about R/S, virtues, and flourishing and offer practical tools (e.g., religious texts and practices) that people across cultures have used for millennia to cultivate them. For lists of suggestions for science-practice integration that could easily be adapted for the PPRS, see Aguinis et al. (2020) and Geimer et al. (2020). 


\section{Strategic Priorities for Practice in the PPRS Field}

Lastly, we highlight six strategic priorities for practice in the PPRS field. These priorities are domains through which the PPRS might arguably be of greatest benefit to society and humanity.

Clinical Practice. The PPRS interventions mentioned above can be disseminated and used in a diverse array of clinical practice contexts, including healthcare settings (e.g., hospitals, outpatient clinics, inpatient programs, treatment centers, long-term care facilities), correctional facilities (e.g., prisons), and spiritual care contexts (e.g., spiritual direction centers, spiritual retreat centers, and spiritual study centers). Practitioners in all these settings can also cultivate their clinical competencies in positive psychology (Rashid \& Seligman, 2018) and R/S (Vieten \& Lukoff, 2021), including skills in how to help people build flourishing-facilitative R/S (Pargament, 2007) and reduce flourishing-undermining R/S (e.g., resolve religious/spiritual struggles; Pargament \& Exline, 2021).

Religious Ministry. PPRS interventions can be disseminated and used in religious ministry settings as well, but these interventions may often need to be culturally adapted to enhance their compatibility with religious groups' beliefs, values, practices, and worldviews (Soto et al., 2018). Additionally, religious leaders and laypeople can draw on the findings and tools of PPRS to supplement the benefits they glean from their pre-existing beliefs and practices. Furthermore, religious leaders and communities can use knowledge and tools from PPRS to enhance the effectiveness of their religious ministry efforts and programs (McMinn, 2017).

Character Education. Character refers to the totality of a person's morally relevant habits of thought, feeling, and behavior (Baehr, 2017), and virtues are habits of thought, feeling, or behavior that are consensually esteemed as morally good, contextually adaptive (beneficial to 
the person and their social context), and situationally coherent (prudent for specific times and places; Lerner, 2019). Character education (i.e., deliberate attempts to promote the development of virtues and good character) frequently happens in the context of schools, families, religious communities, colleges, and faith-based organizations. Character educators can incorporate the knowledge and tools of PPRS into their efforts and strategies (Berkowitz \& Hoppe, 2012).

Organizational Settings. People's work is one of the strongest contributors to their overall flourishing (VanderWeele, 2017b), and workers often draw on their R/S to enhance the meaningfulness of their work (Dik \& Alayan, Chapter 27, this volume). Organizations and their leaders can harness the findings of PPRS research (Hill \& Dik, 2012; Neal, 2013) to improve the flourishing, functioning, and performance of their employees, leaders, and overall organization.

Humanitarian and Disaster Contexts. The number of people affected by disasters and humanitarian crises has increased dramatically over the past 50 years. For example, since 1970 there have been over 22,000 natural and technological disasters (collectively causing 4.6 million deaths and nearly $\$ 5$ trillion in economic losses), and the number of natural disasters per decade has increased five-fold (World Meteorological Organization, 2021). Between 2000 and 2019 (i.e., the 20 years prior to the COVID-19 pandemic), over 4 billion people were affected by disasters (UNDRR, 2020), and during the COVID-19 pandemic, the number of people in need of humanitarian assistance and protection has increased exponentially (from 1 in 45 people globally [168 million] during 2019 to 1 in 33 people globally [235 million] during 2020; UNICEF, 2021).

There already is an extensive amount of PPRS research on humanitarian crises and disasters (Aten et al., 2019; Captari et al., Chapter 30), along with numerous practical tools and resources for use in humanitarian and disaster contexts. Many of these resources are available through Wheaton College's Humanitarian Disaster Institute (http://www.wheaton.edu/hdi). 
Policymakers, religious communities, and humanitarian aid and disaster organizations can draw on such studies and resources to help prepare for and respond to disaster-related and humanitarian needs.

Public Health. As Long and VanderWeele (Chapter 25) have discussed, public health is the scientific discipline focused on preventing disease, promoting flourishing, and prolonging life (Winslow, 1920). An enormous share of the global population (about 85\%) identifies as religious/spiritual (Pew Research Center, 2015), and for most people (around 68\%), R/S is an important part of their lives and identity (Diener et al., 2011). This is especially the case for people in Africa, South Asia, Latin America, and the Middle East (Pew Research Center, 2018). Global research suggests that people living in societies characterized by difficult life circumstances — including many countries in the same regions just mentioned - are particularly likely to self-identify as religious/spiritual and to experience higher flourishing to the degree they are religious/spiritual (Diener et al., 2011). In these and other societies worldwide, policymakers, government workers, and public health officials could draw on the findings of PPRS research (e.g., Chen \& VanderWeele, 2018; Long \& VanderWeele, Chapter 25, this volume) to inform their efforts in making policies and laws, designing public health initiatives and interventions, and engaging in public health prevention and management. Doing so could improve the lives, well-being, and longevity of literally billions of people around the globe (Idler, 2014).

\section{Conclusion}

"Take the first step in faith. You don't have to see the whole staircase, just take the first step." - Martin Luther King, Jr.

You have reached the end of the Handbook of Positive Psychology, Religion, and

Spirituality. Regardless of how religious or spiritual you are personally, we hope you now have a 
greater understanding and appreciation of how intimately intertwined R/S, positive psychology, and flourishing are. We hope you also are inspired to apply what you have learned and make your work, life, and the world a better place—-filled with more love, hope, and flourishing. Even if you don't know exactly how to do that, we encourage you to heed the visionary call of Dr. Martin Luther King, echoing through the halls of time and beckoning you just to take the first step in faith. 


\section{References}

Aguinis, H., Banks, G. C., Rogelberg, S. G., \& Cascio, W. (2020). Actionable recommendations for narrowing the science-practice gap in open science. Organizational Behavior and Human Decision Processes, 158, 27-35. https://doi.org/10.1016/j.obhdp.2020.02.007

Aten, J. D., Smith, W. R., Davis, E. B., Van Tongeren, D. R., Hook, J. N., Davis, D. E., ... \& Hill, P. C. (2019). The psychological study of religion and spirituality in a disaster context: A systematic review. Psychological Trauma: Theory, Research, Practice, and Policy, 11(6), 597-613. https://doi.org/10.1037/tra0000431

Baehr, J. (2017). The varieties of character and some implications for character education. Journal of Youth and Adolescence, 46, 1153-1161. https://doi.org/10.1007/s10964-017$\underline{0654-\mathrm{Z}}$

Berkowitz, M. W., \& Hoppe, M. A. (2009). Character education and gifted children. High Ability Studies, 20(2), 131-142. https://doi.org/10.1080/13598130903358493

Chen, Y., \& VanderWeele, T. J. (2018). Associations of religious upbringing with subsequent health and well-being from adolescence to young adulthood: An outcome-wide analysis. American Journal of Epidemiology, 187(11), 2355-2364. https://doi.org/10.1093/aje/kwy142

Crabtree, S., English, C., Johnson, B. R., Ritter, Z., \& VanderWeele, T.J. (2021). Global Flourishing Study: Questionnaire development report. Gallup. https://hfh.fas.harvard.edu/files/pik/files/globalflourishingstudy_report.pdf

Developing Belief Network. (n.d.). Research sites. https://www.developingbelief.com/research$\underline{\text { sites }}$ 
Diener, E., Tay, L., \& Myers, D. G. (2011). The religion paradox: If religion makes people happy, why are so many dropping out? Journal of Personality and Social Psychology, 101(6), 1278-1290. https://doi.org/10.1037/a0024402

Geimer, J. L., Landers, R. N., \& Solberg, E. G. (2020). Enabling practical research for the benefit of organizations and society. Industrial and Organizational Psychology, 13, 334338. https://doi.org/10.1017/iop.2020.55

Grimm, K. J., Ram, N., \& Estabrook, R. (2017). Growth modeling: Structural equation and multilevel modeling approaches. Guilford Press.

Hill, P. C., \& Dik, B. J. (2012). Psychology of religion and workplace spirituality. Information Age Publishing.

Idler, E. L. (Ed.). (2014). Religion as a social determinant of public health. Oxford University Press.

John Templeton Foundation. (2021a). Mental healthcare, virtue, and human flourishing. Author. https://www.templeton.org/grant/mental-healthcare-virtue-and-human-flourishing-2

John Templeton Foundation. (2021b). Catalyzing a cultural shift toward integrating religious and spiritual competencies in mental health care through training and systems-level change. Author. https://www.templeton.org/grant/catalyzing-a-cultural-shift-towardintegrating-religious-and-spiritual-competencies-in-mental-health-care-through-trainingand-systems-level-change

Koenig, H. G., King, D. E., \& Carson, V. B. (2012). Handbook of religion and health (2 $2^{\text {nd }}$ ed.). Oxford University Press.

Lerner, R. M. (2019). Character development: Four facets of virtues. Child Development Perspectives, 13(2), 79-84. https://doi.org/10.1111/cdep.12315 
Linley, A. P., Joseph, S., Harrington, S., \& Wood, A. M. (2006). Positive psychology: Past, present, and (possible) future. Journal of Positive Psychology, 1(1), 3-16. https://doi.org/10.1080/17439760500372796

Lomas, T., Hefferon, K., \& Ivtzan, I. (2014). Applied positive psychology. Sage.

Many Analysts of Religion Project (MARP) Team. (n.d.). A many analysts approach to the relation between religiosity and well-being. Author. https://osf.io/qbdce/

Maxwell, S. E., \& Cole, D. A. (2007). Bias in cross-sectional analyses of longitudinal mediation. Psychological Methods, 12(1), 23-44. https://doi.org/10.1037/1082-989X.12.1.23

McMinn, M. R. (2017). The science of virtue: Why positive psychology matters to the church. Brazos Press.

Neal, J. (Ed.). (2013). Handbook of faith and spirituality in the workplace. Springer.

Nosek, B. A., Alter, G., Banks, G. C., Borsboom, D., Bowman, S. D., ... Yarkoni, T. (2015). Promoting an open research culture: Author guidelines for journals could help to promote transparency, openness, and reproducibility. Science, 348, 1422-1425. https://doi.org/10.1126/science.aab2374

Pargament, K. I. (2007). Spiritually integrated psychotherapy. Guilford Press.

Pargament, K. I. (Ed.). (2013). APA handbook of psychology, religion, and spirituality (Vol. 2): An applied psychology of religion and spirituality. American Psychological Association. https://doi.org/10.1037/14046-000

Pargament, K. I., \& Exline, J. J. (2021). Working with spiritual struggles in psychotherapy: From research to practice. Guilford Press.

Peterson, C., \& Seligman, M. E. P. (2004). Character strengths and virtues: A handbook and classification. American Psychological Association. 
Pew Research Center. (2015). The future of world religions: Population growth projections, 2010-2050. https://www.pewforum.org/2015/04/02/religious-projections-2010-2050/

Pew Research Center. (2018). The age gap in religion around the world. Author. https://www.pewforum.org/2018/06/13/how-religious-commitment-varies-by-countryamong-people-of-all-ages/

Pew Research Center. (2021, November 18). What makes life meaningful? Views from 17 advanced economies. Author. https://www.pewresearch.org/global/2021/11/18/whatmakes-life-meaningful-views-from-17-advanced-economies/

PRSM Lab. (n.d.). Religious replication project. https://prsmlab.com/replication/

Psychological Science Accelerator. (n.d.). Psychological science accelerator: A distributed laboratory network. Author. https://psysciacc.org/

Rashid, T., \& Seligman, M. E. P. (2018). Positive psychotherapy: Clinician manual. Oxford University Press.

Ripley, J. S., \& Worthington, E. L., Jr. (2014). Couple therapy: A new hope-focused approach. InterVarsity Press.

Saroglou, V., Clobert, M., Cohen, A., Johnson, K., Ladd, K., .. \& V Valladares, J. T. (2020). Believing, bonding, behaving, and belonging: The cognitive, emotional, moral, and social dimensions of religiousness across cultures. Journal of Cross-Cultural Psychology, 51, 551-575. https://doi.org/10.1177/0022022120946488

Seligman, M. E. P. (2011). Flourish. Simon and Schuster.

Shafranske, E. P. (2013). Addressing religiousness and spirituality in psychotherapy: Advancing evidence-based practice. In R. F. Paloutzian \& C. L. Park (Eds.), Handbook of the psychology of religion and spirituality (pp. 595-616). Guilford Press. 
Slife, B. D., \& Reber, J. S. (2009). Is there a pervasive implicit bias against theism in psychology? Journal of Theoretical and Philosophical Psychology, 29(2), 63-79. https://doi.org/10.1037/a0016985

Soto, A., Smith, T. B., Griner, D., Rodriguez, M. D., \& Bernal, G. (2018). Cultural adaptations and therapist multicultural competence: Two meta-analytic reviews. Journal of Clinical Psychology, 74, 1907-1923. https://doi.org/10.1002/jclp.22679

Templeton World Charity Foundation. (n.d.). Building more forgiving communities around the globe through engagement to complete do-it-yourself REACH forgiveness workbooks. Author. https://www.templetonworldcharity.org/projects-database/building-moreforgiving-communities-around-globe-through-engagement-complete-do

UN Office for Disaster Risk Reduction (UNDRR). (2020). Human cost of disasters: An overview of the last 20 years (2000-2019). Author. https://www.undrr.org/publication/human-costdisasters-overview-last-20-years-2000-2019

UNICEF. (2021). Humanitarian action: Global annual results report 2020. Author. https://www.unicef.org/media/102401/file/Global-annual-results-report-2020humanitarian-action.pdf

Vaish, A., Grossmann, T., \& Woodward, A. (2008). Not all emotions are created equal: The negativity bias in social-emotional development. Psychological Bulletin, 134(3), 383403. https://doi.org/10.1037/0033-2909.134.3.383

VandenBos, G. R. (Ed.). (2015). APA dictionary of psychology (2 ${ }^{\text {nd }}$ ed.). American Psychological Association. http://dx.doi.org/10.1037/14646-000

VanderWeele, T. J. (2017a). Outcome-wide epidemiology. Epidemiology, 28(3), 399-402. https://doi.org/10.1097/EDE.0000000000000641 
VanderWeele, T. J. (2017b). On the promotion of human flourishing. PNAS, 114(31), 81488156. https://doi.org/10.1073/pnas.1702996114

VanderWeele, T. J., \& Ding, P. (2017). Sensitivity analysis in observational research:

Introducing the e-value. Annals of Internal Medicine, 167(4), 268-274. https://doi.org/10.7326/M16-2607

VanderWeele, T. J., Mathur, M. B., \& Chen, Y. (2020). Outcome-wide longitudinal designs for causal inference: A new template for empirical studies. Statistical Science, 35(3), 437466. https://doi.org/10.1214/19-STS728

Vieten, C., \& Lukoff, D. (2021). Spiritual and religious competencies in psychology. American Psychologist. Advance online publication. https://doi.org/10.1037/amp0000821

Winslow, C.-E. A. (1920). The untilled fields of public health. Science, 51(1306), 23-33.

World Meteorological Organization. (2021). WMO atlas of mortality and economic losses from weather, climate, and water extremes (1970-2019). Author.

https://library.wmo.int/doc_num.php?explnum_id=10902 